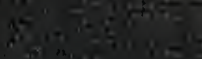




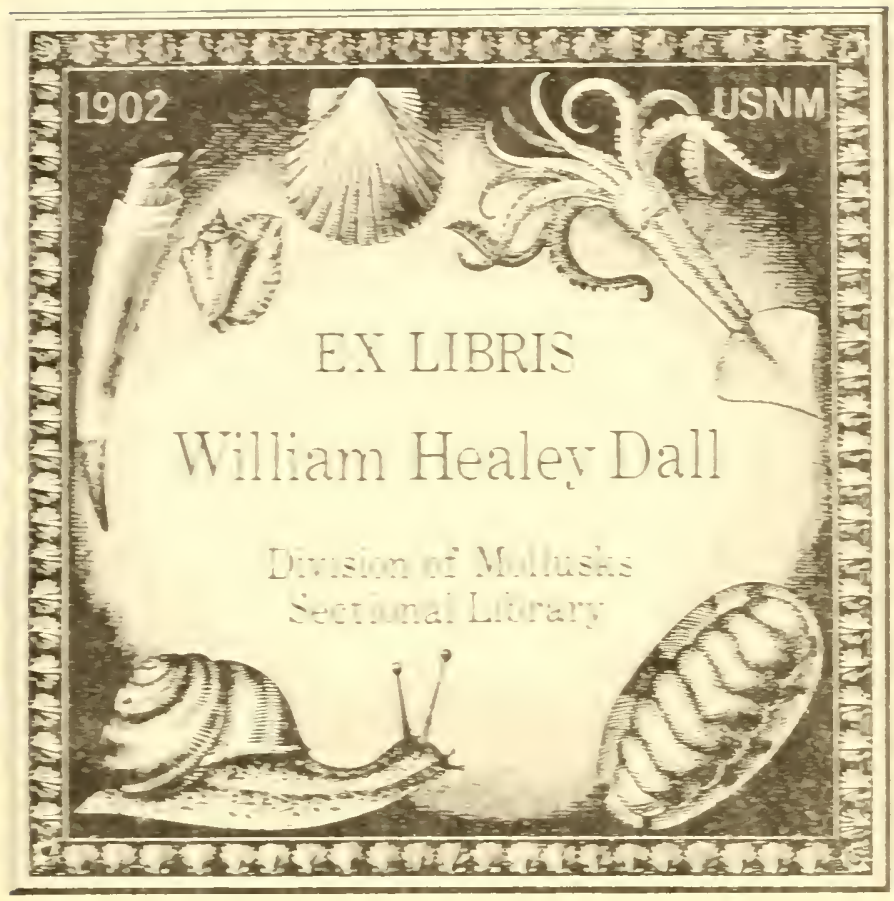








$$
\begin{aligned}
& 426 \\
& \text { I 48D38 Tautgences= ith } \\
& 1905 \\
& \text { Wallesks at } \\
& \text { MOLC } \\
& \text { Tonkin }- \\
& \text { Indo } \sin 2 \\
& 4 \text { antracts from. }
\end{aligned}
$$

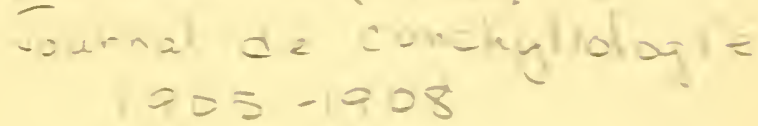





\section{Division of Molluevelta} Sectional Tibrary

\section{LISTE DES MOLLUSQUES}

récoltés par $M$. Le Capitaine de Frégate BLAISE

au ronkin

ET

DESGRIPTION D'ESPÈGES NOQUVELLES

PAR

Ph. DAUTZENBERG et H. FISGHER

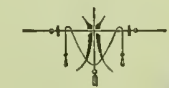

PAIIS

1903 
Extrait du $x^{\circ} 3$ du rol. Llll du domrual de Conchyliologie, II. Fiscukk, directeur, boulevard saint-.lliehel, 3̈l, Paris. 


\section{IISTE IOE- MOLLESQLE-}

récoltés par M. I0 Capitaine de Frógato Iblatso

:11 Tonkin,

et drecription despeces nouvelles.

P.AR

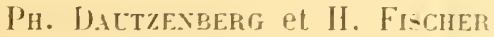

Les Mollusques dont nous allons nous occuper ont été recueillis par II. Liaise, capitaine de frécate. appelé par son service à visiter, de 19022 à 190't, différents points des còtes de la Baie dldons. les rivière qui -r déversent. plusieurs iles de l'Arhipel des Faî-T-i-Long. ainsi qu une grande partie de la rérgion qui sétend à l'Est du Fleuve kouge et de la Rivière Claire.

L'abondance des matériaux rapporté- par II. Hlaise nous a permis de constater que les noms des especes de cette région avaient été beaucoup trop nultipliés, certainauteurs ayant décrit comme espéces soit de -imple- rariétés, soit des formes indiriduelles, soit mème des exemplaires de différents àges d'une seule e-pèce : ces abus se sont surtout produit pour les Corbicules et les Naiades.

II. Blaise. en apportant à la préparation rles spécimen: recueillis un soin minutieux et en se donnant la peine de rapporter des séries nombreuses d'exemplaires de diffé rents àges. nous a permis d’acquérir une notion plu= exacte de l'étendue des rariations de chaque e-pèce : aus-i considérons-nous que se - récoltes ont fait faire à la con- 


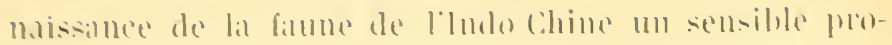
gris.

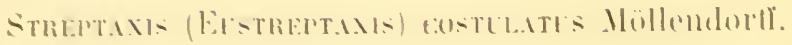

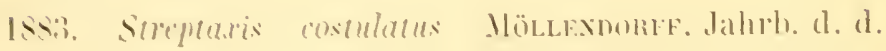
Malali. Cies. I. 1). 27ë. p)! 10. fig.s.

Ix:i. - Thiox. Man. ol Conch.. I.

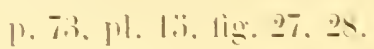

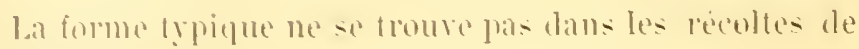
II. Iilai-e'.

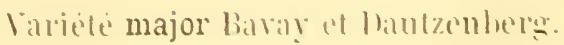

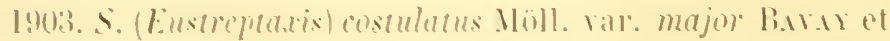

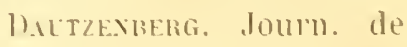

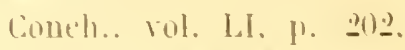

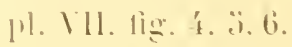

Tomkin. He kirien. Exemplaires morl- récolles dans les

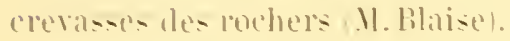

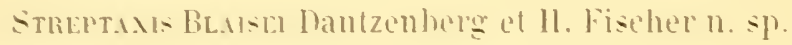

II. III, ligr. 1. 2, 3. 4.

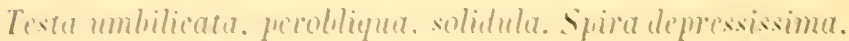

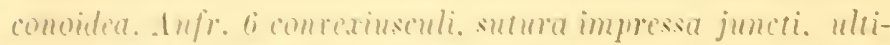

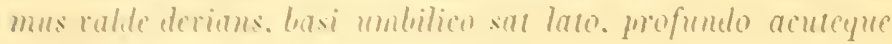
marginato municus. Inf. supurne confersim longiudinaliter

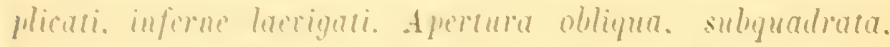

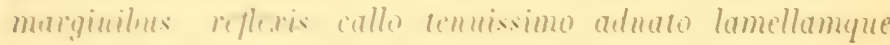
intrantem eminente junctis. Columelid obligua. Labrum

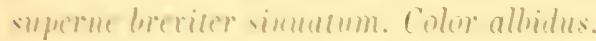

lomgit. i. dum. mujor 1: millim. : aprertur:l 6 mill. alta. cimill. lusis. 
Corquille ombiliquée, tres oblique, assez solide. Spire aplatie, légèrement ronoüde au sommet. Six tours un peu convexes, separes par une suture ascez profonde logerement camaliculée au dernier tour qui est fortement dévit. Base du dernier tour légèrement concave, pourve dun ombilic assez grand, frofond, ì jourtour subanguleux. Tours embryonnaires lisses; les suivants ornés de stries longitudinales nomlireuses, llexueuses, plus accusées rers la suture. Ces stries s'eflacent sur la partie inférieure de l'avant-dernier et du dernier tour. Ourerture subqua drangulaire. l'éristome assez épais, rélléclii. Bords reliés par une callosité mince, alpliquée, sur laruelle se développe un pli pénétrant. lamelleux et étroit. Columelle oblique. Bord columellaire rélléchi sur l'ombilic. Labre aminci et sinueux au sommet. Coloration blanchâtre uniforme.

Tonlin. Ile hrieu. In seul exemplaire (I. Blaise).

Kaliella Jocbixi Dautzenberg et H. Fischer n. sp.

(11. III, fig. 1:3, 14, 1\%, 16.)

Testa angustissime perforata, trochoidea, tenuissima ac snbpellucida. Anfr. of conrexiusculi, regulariter crescentes. oblique confertim striati. Lltimus haud descendens, ad peripheriam carinatus, basi conterus et ibi striis concentricis tenuissimis ornatus. A pertura subquadrata. Columella brecis, rectiuscula, peristoma acutum. Color undique fultus. Alı. כ, diam. maj. 6 ; apertura 2 mill. 1/2 alta ś mill. lata.

Coquille étroitement perforée, de forme trochoïde, trẻs mince et subpellucide. composée de 6 tours assez convexes croissant régulièrement et ornés destries d’accroissement obliques nombreuses. Dernier tour non descendant, caréné à la périphérie, à base convexe et ornée de stries concentriques fines, nombreuses et un peu ondulées. Ouverture subquadrangulaire. Columelle courte presque 
verticale. Bord basal et labre minces et tranchants. Colo ration fauve uniforme.

Tonkin. Baie d tiong: ile lirieu il. Blaiset.

Cette pretite e-pece. que nous dedion- à II. Joubin. profes-enr de Valacologie au Muséum d'histoire naturelle de Paris. eat remarquahle par la sculpture concentrique le la bave du dernier tour. tandis que tont le re-te de la roguille est orne de stries longitudinale.

Trochomorphi sapfot Heude.

PI. N, lig. 1, 2. 3.

Inti. H. (Trochomorpha) bicolor L. Mordet non r. Martens). Journ.Conch..vol.AXXIII. 1. 259.

InT. Trochomorpha bicolor MabiLle (non r. Martens). Bull. soe. Ilal. Fr. J. !s.

15yo. Helir saprea Hfore. Mém. Hist. Mat. Emp. Chinois p. I'́3, pl. х.11111. fig. 13. 13. 13.

14.91. Trothomorping bicolor P'. Fischer (non r. Martens. Catal. Indo-Chine in soc. Hist. Nat. Autun. l. lo (1) 2't du t. d̀ p).

1s91. H. illectotropisis sapeca H. P. Fischer, ibidem. p. 110 (p. 光) du t. à p.).

190'. Trochomorpha sapeca H. II. Fischer et Dattzesberg. Catal. Indo-Chine in Mission Parie. Etudesdiverses. III. p. 3̊x (p. ? clu t. à p.).

$190 \%$ '. II (momallei bautzenberg nos. H. Fischer el lotizevaerg ibid.

Tonkin. He hirieu I1. Blaise

Lasimilation de rette e-péce de l'Indo-Chine au $T r$. bicolor de sumatra. Borne. Jara. etc. propusée par le 
C. L. Morlet ne peut ètre maintenue : cest pourquoi nous arions rréé pour elle en 190't le nom Trochomorpha Hamonrillei bautzenbere mss. Celte forme indo-chinoise est plus grande, a lombilic plus ourert. les tours plus plans. et la carène du dernier tour plus aiğuë que le réritable $T$. bicolor: sa coloration est aussi fort différente. car tandis que chez le $T$. bicolor il existe au milieu des tours une bande lorune laissant voir de chaque còté un espace jaune clair. chez le $T$. Hamonrillei ciest le fond de la coquille qui est brun, arer une ligne blanche accompagnant la carène et une zone assez larg̣e bien définie. également blanche. régnant immédiatement au-dessous de la suture. On observe, de plus. chez le T. Hamonillei, sur lid partie brune du test. de nombreuses linéoles spirales blanches hydrophanes.

Mais nous estimons aujourd hui que le nom Trochomorpha Hamonillei ne peut ètre maintenu dans la nomenclature, car un examen très attentif des figures et de la description un peu sommaire de l'Helix sapeca, décrit par Heude, de Tay-Ninh (Cochinchine) nous donne à penser qu'il s'agit d'une seule et mème forme. a laquelle revient dès lors le nom plus ancien proposé par Heude.

Helix (Cumaexa) Gisbrielda Dautzenberg et d Hamonville.

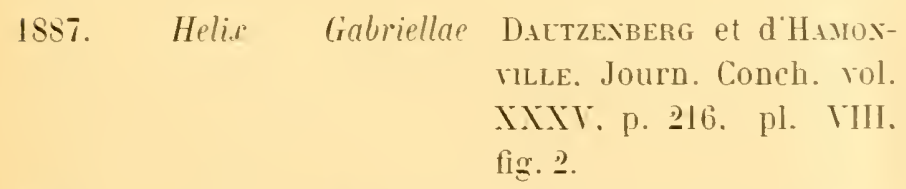

1857. - bathmophora Mabille. Surquelques.Moll. du Tonkin in Bull. Soc. Ilal. de Fr..p. 83. pl. II. tig. 6.7.

18ss. Hadra Gabriellae Axces, Le Naturaliste.p.ż. fig. 1 . 
1590. Helix Gabriellae PILsbry..Man. of Conch.Str. and Srst TI. p. 20\% pl. 't?. กig. 26. 27.

1s91. H. Hadra? - D. et d'H. P. Fischer. Catal. IndoChine. loc.cit.. p. 11 21 p. 24 du t. à $\mathrm{p}$.)

159'. Camaena - - Pilsbry. Man. of. Conch.. I. p. 10'́

I90'. - - - Fischer el DaltzeNberg. Catal. Indo-Chine in Mission Parie. loc. cit.. p. 399 (p. 10 du t.à p.).

1901. - bathmophora Iab. H. F1scher et DattzexBerfi. Catal. Indo-Chine in Yission Parie, loc. cit., p.399 (p. 10 du t. à p.).

La forme trpique ne figure pas dans les récoltes de II. Blaise.

Variété subhainanensis Pilsbry.

1890. Helir I Camaena rabriellae rar. subhainanensis PiL-BRY. Man, of. Conch. Helic. IV. p. 200., 11. 42. fig. 2s.

Tonkin. lle Kirieu (M. Blaise).

Helix (Chloritis) Balaxal L. Morlet.

var. cincta. U. et H. F. nom. nov.

PI JII. fig. ว. 6. $7,8,9$.

1suti. Helir Balansai L. Montet. Didzn. Moll. terr. et llur: du Tonkin p. 1 . 


\section{$-91-$}

1856. Helix Balansai L. Monlet. Coq. rec. au Tonkin par Jourdy in Journ. Conch.. p. 2.5s, 270. pl. XII. fig. $4.4^{\mathrm{a}} \cdot \mathrm{k}^{\mathrm{b}}$.

I887. - - I. M. Daltzenberg el d'HaMoNrille, Journ. de Conch., p. 2શะ.

1857. - - L. M. J. Mabille. Sur quelques Mollusques du Tonkin in Bull. Soc. Mal. Fr., p. s? (rar B.)

1S91. H. (Trachia) BalansaiL. I. P. Frscher. Catal. IndoChine, loc. cit..p. 110 (p. 26 du t. à p.)

190'. Chlorilis Balansai L. II. H. Fischer et Dattzenberg. Catal. Indo-Chine in Mission Parie. loc. cit., p. 400 (11 du t. à p.)

Tonkin. Ile lirieu. Archipel des Faï Tsi-Lonģ (M. Blaise) par temps pluvieux sur les arbustes poussant sur les rochers de l’ile. Nombreux exemplaires vivants nappartenant pas à la forme typique. mais à la rariété $̧$ s de L. Morlet (à test semi-transparent. hispide. bordé d'une zoue brune à la périphérie du dernier tour. tig. 4 $4^{\text {bl }}$ pour laquelle nous proposons le nom de rar. cincta. Yous représentons ici. (pl. III. fig. .’ à s/ un deces spécimens remarquablement frais arec une figure grossie montrant linsertion très régulière des poils (figg. 9).

Helix (Gayeselda?) Lami Dautzenberg et H. Fischer.

PI. III, fig. 10,11 , I2.)

Testa subtrochiformis, mediocriter sed perrie umbilicata. tenuissima, subpelluciela, nitidula. Spira conica, parum elata. 
Anfr. 4 1, 2 subplani, rapide crescentes, sntmra panlo impressa sejuneti, plicis inerementi obliquis irregularibusque ornati et minutissime corrugati. Infr. ultimus pone aperturam ri.r descendens, infra valde conrerus "l in peripheria acutissime carinatns. Apertura ampla, obligna, marginitms commicentibus. Peristoma expansum pauhlumeque reflexum. Columella leviter areuata, superne dilatata ibique nmbilicum ex parte obtegens. Labrum medio angulatum et infira angulum, arcuatum.

Color pallide fulrus, smperne lineis albis, opacis et infra cariuam zona fusca sat lata ormatus. Peristoma albescens.

Diam. maj. 17, min. 1', altit. 10 millim. (les traits qui accompragneut les ligures ont été, far erreur, tracés trop courts).

Coquille subtrochiforme, mérliocrement, mais profondémeut ombiliquée, très mince, subpellucide, assez luisante. Spire conique, peu élevée, composée de 4 1/2 tours presque plans, croissant rapidement el séparés par une suture bien marquée et étroitemenl marginée à partir de la moitió du dernier tour jusqu’a son extrémité. Surface présentant des plis d'accroissement obiquues très irréguliers el ornée d'une sculpture chagrince extrèmement fine. qui n’est visible quä l'aide d'mue forte loupe. Dernier tour ne descendant quà partir d'une fable distance de son extrémité, bien convexe au-dessous d'une rarène périphériale tres aiguë. Onrerture grande. oblique; bords un peu rapprochés mais non reliés par une pallosité. Péristome dilaté. un peu réfléchi aux bords. Columelle légèrement arpue élargie an sommel oi elle surplombe en partie l'ombilir. Labre anguleux, presque roslré à l'endroit où aboutit la ratrene, hien arefur an-descous de ce point.

Colobation d'un faure clair, orné de linéoles blamches, opapues: lume soulignant la suture, une antre regnant an milieu des tours, la troisiène areompagnant la carène. sous la louje, on apreroit encore d'antres linéoles blanches. 
mais très délicates. Enfin. une bande hrune, assez large et nettement limitée, est siluée immédiatement au-dessous de la carène. Péristome blanchàtre.

Tonkin. Ile Kíreu. In senl exemplaire (M. Blaise).

Nous prions notre collaborateur, M. Edouard Lamy, atlaché au Laboratoire de Malacologie du Muséum d'Histoire naturelle, d'accepter la dédirace de cette espèce qui se rapproche un peu des l'apmina par sa carène très aiguë qui a une tendance à rendre le labre rostré au milieu.

\section{Plectopylas libled.riy Ancey.}

1888. I'lectopylis Villedaryi Axcer, Le Naturaliste.p. \& (clu t. à p.), fig. 2.

1891. II. (I'lectopylis) - Anc. P'. Fiscuer, Catal. IndoCluine, loc. cit., p. 109 (2:; du t. ̀े p.).

1892. Plectopylis Villedaryi - Pissbrix, Man. of Conch., VIII, p. I:37, pl. 43, lig. 36i, $37,38,39$.

1897. - - - Gune, Science Gossip. IV, p. $1399, \mathrm{tig} .60^{\mathrm{a}}, 60^{\mathrm{b}}$.

1901. - - - Gicue, Journ. de Conch.. vol. XLIT, p. 2I?.

190'. - - - - II. Fischer et Dattzexbeng, Cat. Indo-Chine in Mission Pavie, loc.cit., p. 40? (p. 1:3 du t. aे p.).

Tonkin. Dong-Trieu, dans les racines des arloustes qui poussent sur des rochers semblables à ceux de la baie d'Along (M. Blaise).

He Kírieu. Exemplaires jeunes (II. Blaise).

V'après l'opinion de II. II. Pilshry, les Plectopylis Joxin Jabille et Villedaryi Ancey pourraient bien nètre que des variétés du $\mathrm{Pl}$. Schlumbergeri. 
Ch.lefotixis infantilis Gredler.

188\%. Vanina (?) infantilis Gredter,Jahrl.d.deutsch. Malak. Cies., p. I'is, pl. 3. fig. 2.

1886. Tanina infantilis (is. Tryox, Man. of. Conch., ll, P. 2016, p]. 6', fig. 18.

1887. - - Gir. .J. Mabille, Surqq.Moll.du Tonkin in Bull. Soc. Nlalac. Fr., p. 7\%.

1891. Sriophanta - Gir. P. Fischer, Catal. IndoChine, loc.cil.. p. 107 (p. 2.3 du t.à p.).

1893. Testa milmeata Dautzenberi, Moll, nouv. rec. au Tonkin par Mt. Dorr in Journ. Conch., rol. XXYIX.

189\%. Chalepotaxis infantilis Gr. ThYox. Man. of. Conch.. X1, p. $16 i \overline{7}$.

190't. - - Gir. H. Fischer el Daltzexberg, Catal. Indo Chine in Mission Pavie. loc. cit., 1). 10.2 (p. 13. du t. ̀े p.).

Tonkin. Ile Krieu, Baie d'Along (II. Blaise).

Gaxesela Vatheleti Bavay el Dautzenberg.

1899. Helix(Geotrochus) Tatheleti Bavay el Dattzexbeag. Journ. de Conch., rol. Xlıll, p. 36, fl. 1, fig. ?.

190'. Gamesella V'atheleti B. el I). H. Fischer et Dautzexberi, Catal. Indo-Chine in Mission Parie, loce cit., p. 103 (p. I't dul l. ì l.).

Tonkin. Ile kirieu, Baied'Ilong (II. Blaise). 
Helix (Eulota) sinilaris Férussac.

1822. Helix similaris Férussac, Tabl. syst.,p. 亿̇.

1831. - Woodiana Lea,Obs. G. Unio. I, p. 169, pl. XIX, fig. 69.

183:. - translucens Kuxo, Zool. Journ. $\mathrm{V}$, p. 339.

1820-1831. - similaris Férussacet Desha yes, 11 ist. nat. Nloll. I, p. 171, pl. 2:3b fig. 1 à $4 ;$ pl. 27 a fig. 1 à

183.i-184:3. Ilelix similaris Fér. D’Orbigny, Voy. Amér.mérid., p. 24.3.

1837. Bradybaena - Beck, Index p. 18.

\begin{tabular}{|c|c|c|c|}
\hline 1810. Helix & - & - & $\begin{array}{l}\text { Dufo, Ann. Sc. nat. } 2 \text { sér } \\
\text { XHV, p. } 198 .\end{array}$ \\
\hline 184:\%. - & - & 一 & $\begin{array}{l}\text { Catlow el Reeve, Conch } \\
\text { Nonencl., p. 13:;. }\end{array}$ \\
\hline 1846. & - & - & $\begin{array}{l}\text { Preiffer in Guemitz } \\
\text { Conch. Cal. Ze éd., p. 341 } \\
\text { pl. 60, fig. 13-16. }\end{array}$ \\
\hline 1818. & - & 一 & PFeIfFer, Mon.Hel.I,p.336 \\
\hline 19. & - & - & $\begin{array}{l}\text { Moussos, Java, p. 21.pl. II } \\
\text { fig. } 4, \ddot{3} \text {. }\end{array}$ \\
\hline
\end{tabular}

1830. II. (Eulota) similaris - Mörcu, Catal. Kierulf p. 2. 18:31. Helix:

- Reeve, Conch. Icon., pl. XXXIV, fig, 149a, et 149"

183̈1. - brardiana PFenfer, Proc. Zool.Soc. L. p. 203 (t. A. Morelet).

18:2. - similaris - ReEve, Conch. Icon., pl. CXXVII, fig. $767^{a}, 767^{\mathrm{b}}$.

1832. - Brardiana Pfr. Reere, Conch. Icon. pl. CVIII, fig. 60 '.

$18 \dddot{3}$

Premfare, Mon. Hel. III. p. 208. 
18.33. Helir Brardiana

\begin{tabular}{|c|c|c|c|c|}
\hline 8:ii). & - & \multicolumn{2}{|c|}{ similaris } & $\begin{array}{l}\text { Preiffeli, Mon. Hel. III, } \\
\text { p. 22.27. }\end{array}$ \\
\hline 汭. & ? Ilel & ir stimplson & & $\begin{array}{l}\text { PFemFer, Proc. Z. S. L. } \\
\text { p. 1 } 9 \text {. }\end{array}$ \\
\hline (î. & ? Ilel & - & & $\begin{array}{l}\text { REEVE, Conch. ICOH., pl. } \\
\text { CXCT, fig. } 1370 \text {. }\end{array}$ \\
\hline 8:i!). & ? Heli & - & & $\begin{array}{l}\text { Prenter, Mon. Hel. IV, } \\
\text { P. 28:1. }\end{array}$ \\
\hline 13ii?. & - & Brardic & ana & $\begin{array}{l}\text { PFenfarer, Mon. Hel. IV. } \\
\text { p. } 268 .\end{array}$ \\
\hline 8.i!. & - & simila & ris & $\begin{array}{l}\text { Preiffer, Mon. Hel. IN, } \\
\text { p. 2lit. }\end{array}$ \\
\hline s(it). & - & - & Fèr. & $\begin{array}{l}\text { A. Morelet Séries Collch. } \\
\text { I. p.:is. }\end{array}$ \\
\hline 1861 & Horcas. & - & - & $\begin{array}{l}\text { Y. M.datexs Nlbers Heli- } \\
\text { ceen. 2e éd. p. 107. } 108 \text {. }\end{array}$ \\
\hline 1861 & Melir & gemilabris & & $\begin{array}{l}\text { v. Martens, Mal. Bl. VIl, } \\
\text { p. } 33 \text { (teste ipso). }\end{array}$ \\
\hline $186 i 3$. & - & Areasiana & & $\begin{array}{l}\text { Crosst ef Debealx. Journ. } \\
\text { Conch., vol. XI, p. } 386 .\end{array}$ \\
\hline 1863. & - & Borbonica & & $\begin{array}{l}\text { Deshayes, Moll. Réunion } \\
\text { p. } 8 \%, \text { pl. 10, fig. 9-11. }\end{array}$ \\
\hline $186 \%$ & - & Arcasianc & & $\begin{array}{l}\text { Chosse el Debeatx, Journ. } \\
\text { Conch., vol. Xll, p. 3lti, } \\
\text { pl. I?. fig. h. (et var. solidula. } \\
\text { platior, teste v. Martens). }\end{array}$ \\
\hline $186 i \overline{.}$ & - & similaris & Fér. & $\begin{array}{l}\text { y. Martexs, Preuss. Exped. } \\
\text { Ostas., p. } 270,7,9 \text { (ral. } \\
\text { Stimpsoni), 43. 76. }\end{array}$ \\
\hline 865. & - & - & - & $\begin{array}{l}\text { Cox Mon. auslat. Land- } \\
\text { shells, p. } 38, \text { pl. 9, tig. l't. }\end{array}$ \\
\hline
\end{tabular}

Pfeiffer, Conch.Cah. De éd. llelix 2- rol. Sect. IX. p. 38:i, pl. 142. fig. 7, 8. peiffeli, Mon. Hel. III, ค. 227 . Pratrear, Mon. Hel. IV. 1. 28!! ค. 268 .

Perfater, Mon. Hel. IN, $-67$

p. :is.

$\checkmark$ Mantexs Mlbers lleliceen. 2e ed. p. 107. 108.

v. Mlartens, Mal. Bl. IIl.

CROSSE Et DEBEAT J.journ. Conch., vol. xI, p.380.

ion

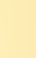


1868..?Helix Stimpsoni PfeIffer, Mon. IJel. Y, p. 378 .

18tis. Helix borbonica PFeIfFer. Mon. Hel. T, р. 004 .

1868. - Mrardiana PFemfer, Mon. Hel. V. р. .302.

1868. - similaris PFEIFFEn, ibidem, p. ̋̈02.

1868. II. (Dorcasia) similaris Fér. Nevul Proc. Z. S. L. p. 62. 1869. Helix similaris - Hodugo, Viaje al Pacifico, P. 20 .

1869. Galaxias - - Fracexfeld in Verh. k.k. Zool. Bot. Gies. Wien XIX, p. $87 i$.

1871. H.(Fruticicola) - _ StoLicsidJourn.Asiat.Soc. of Bengal, XLII, p. ㄲ6.

1871. Hygromia - - Pesse, Proc.Z.S.L.P. 474.

I874. ILeli. - - C Crosse, Journ. Conch, vol. ХХІІ, Р. 230.

187:. - - - \. Monelet, Séries Conch. IV, p.

1876. - - PFeiffer, Mon. Ilel. VII, p. 401.

1876. - Brardiana Pfenfer, Mon. Hel VII, 1. 402.

1876? - Stimpsoni PFeifrer, Mon. Ilel. VII, 1. 439 .

1876. - borbonica Desh. PFeiffer, Mon. Hel. VII, p. 439.

1891 II.(Fruticicola)similaris Fér. P. Fiscner, Catal. IndoChine, Ioc. cit., p. 111 (p. 27 du t. à p.).

1904. Eulota similaris Fér. I. Fischer et Dautzeneng, Catal. Indo-Chine in Mission Pavie, loc. cit., p. 403 (p. I't du t. à p.). 
Tonkin. Haïphong (.1. Blaise) dans les endroits humides: grandes herbes. vieux mus, vieilles barrières de bambous. cle. Par tempss sec, ces Hélices se ferment au moyen d'un épiphragme blane. Nous remaryuons dans ces récoltes une variété unicolore plus commune que la variété a vec bancle périphériale brune.

\section{Helix (Eılota) Jocrdil L. Morlet}

\begin{tabular}{|c|c|c|c|}
\hline 1880 i. & IIelix & Jomralyi & $\begin{array}{l}\text { L. Mon Let, Journ. Conch. } \\
\text { rol. XXXIT, p. } 7 \ddot{3} \text {. }\end{array}$ \\
\hline 18860. & - & - & 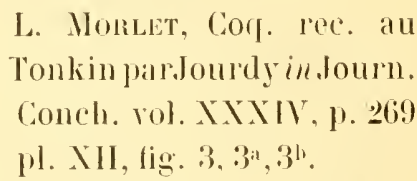 \\
\hline $188 \%$. & - & - & 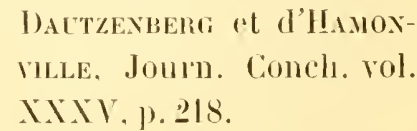 \\
\hline
\end{tabular}

188\%. - rorlicellina Hezde, Journ. Conch., vol. XXXVII, p. 22- (teste P. Fischer).

1891. II. (IIadra) Jomrdyi L. MorLet, Journ. Conch. rol. XXXIX, p. 2.2'牛.

\begin{tabular}{|c|c|c|c|}
\hline $1891 .-$ & - & L. II & $\begin{array}{l}\text { P. Fischer, Catal. Indo- } \\
\text { Chine,loc.cit., p. } 112 \text { (p. } 28 \\
\text { (lu t. d p.). }\end{array}$ \\
\hline 1892. II. (Eulota) & - & - & 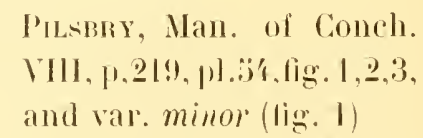 \\
\hline Is:'t. Eulota & - & - & P'LABRY ibid. IX p. Do'. \\
\hline 1901$)^{\prime}$ & -- & & 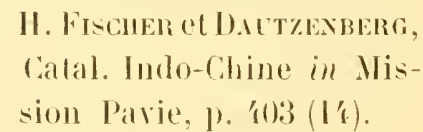 \\
\hline
\end{tabular}

Tonlin. Lam (11. Bhaise); Phu-lang-Thuong (.II. Blaise) sous des écorces, spécimens lypiques et un exemplaire 
arec une bande brune à la péripherie, pour lequel nous proposons le nom de var. zonata.

Möılexdorfra Buaısı Dautzenberg et H. Fischer n.sp. (PI. III, fig. 17, I8, 19.)

Testa parum solida, aisioidea, meliocriter sed percie umbilicata. Spira complanata. An/r 5 concexiusculi, sutura impressa juncti : primus levis, ceteri tuberculis piliferis, oblique seriatim ordinatis, exasperati. Tubercula in basi anfr. ultimi valde attenuata, mope umbilicum ommino exanescunt. Anfr. ultimus rotundatus, antice abrupte deflexus ac denique solutus, post labrum serobiculo profundo, elongato, superneque altero minore munitus. Serobicula insupra duo : alterum post marginem basalem, alterum post sinum marginis columellaris sita, a peristomate occultantur. A pertura fere horizontalis et subtriangularis. Peristoma continum et undique reflexum. IIargo columellaris superne emaryinatus medio dentem validum emittit. Labrum medio dentes duo : supero multo debiliore, infero ralidiore profundeque intrante, praebet. Margo basalis dente unico munitus.

Color undique pallide fulvus.

Diam. maj. 15, min. 12, altit. $61 / 2$ milim. Apertura 7 millim. alta, 8 millim. lata.

Coquille peu solide, discoïde, médiocrement et profondément oubiliquée. Spire plane, romposée de 3 tours un peu convexes, séparés par une suture bien marquée : le premier lisse, les autres finement chagrinés et parsemés de petits tubercules disposés en séries obliques et pourvus chacun d'un poil chitineux assez long. Les tubercules de la base du dernier tour s'afiablissent graduellement et disparaissent tout à fait à proximité de l'ombilic. Dernier tour à contour arrondi, descendant brusquement et détaché à l'extrémité. On observe au milieu, derrière le labre, une scrobiculation profonde, prolongée en arrière 
et une seconde scrobiculation beancoup plus faible située un peu au-dessus de la premiere. I'ne autre scrobiculation profonde existe derrie le sinus du labre el on en remarque une derniere sous le bord hasal. Ces deux dernières sont masquérs par le péristome qui est calleux, contimu el réfléchi el qui se développe un peu au-dessus de la cavité oublilicale. Bord columellaire échancré au sommet par un sinus arrondi et présentant au milieu un fort pli denti forme. Labre arondi. pourva an milien de denx plis dentiformes : le supérieur faible, l’inférieur fort el se prolongeant profondément daus louverture. Bord hasal pouru l'un pli dentiforme médiocre.

Coloralion d'un fauve clair uniforme; péristome el intéreur de louverture d'un faure carmócé.

Cetle espèce diflère dı Möl. lorotata Mabille par sa taille plus faible, son dernier tombien arombi, nettement caréné, jar une doublescrobiculation en arrière du labre (chez 10. 1\%. Iorotata il nen existe qu'une), par ses tuberules moins saillants, enfin par sa coloration plus claire. Elle dilline du ./. Mesaugeri bavay el Daulz. par sa spire aplatie nullement convexe, par la scrobiculalion double en arrière du lahre, far son ombilir moins recouvert par lexpansion du bord columellaire, fal ses tubercules plus nombreux. enfin far sa colololion plus raire.

Tonlin. lle lirieu (M. Blaise).

Noms arons sous les yeux des spécimens de la mème espeece, reneillis autrefois par l'abbe Vathelet dans la liaie ('Mlong (Collection Datutzenber's).

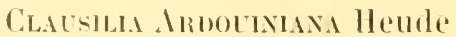

188.i. Clansilia Ardonimiana Hetre, Mém. conc. I'Hist. Nal. de l'Empire Chinois. Molluspues du theure Blen, 1. IIs, pl. ХXXı, fig. I. 
1887. Nenia horrida J. Mabille, Moll. Tonkin. diagn. p. 16.

1887. - - J. Mabilet, Sur qq. Moll. du Tonkin in Bull. Soc. Mal. Lir. p. 109, ,1. III, fig. 9, 10. 1891. - Ardonimiana P. Fiscuen, Catal. Indo-Chine, loc. cil., p. 118 (p. 3.̈ du 1. aे p.)

1891. Clausilia horrida Mab. P. Fischer, ibidem, P. I20 (p. 36 du t. à p.).

1899. C.(I'sendonenia) Ardoniniana Bavay el Dautzenberg, Journ. de Conch., vol. XLVII, p.30, pl.III fig. 3, 3:"

1902 Clausilia Ardoniniana II. SYkes, On Tonkinese Clausilia in. Proc. Mal. Soc., p. 189.

$190 \%$

II. H. Fischer et Dautzexberg, Catal. Indo-Chine in Mission Pavie, loc. cit., p. 408 (p. 19 du t. à p.).

190'. - horrida Mab. H. Fiscmer et Dautzenberg, ibrid. p. 1.10 (p. 19 du t. à p.).

Tonkin. Ile Lrieu (M.Blaise); Lede la Surprise (M.Blaise).

Var. minor Dautz et H. F. n. var.

(pl. IV, fig. 4,

Nous avons fait figurer pl. IV, fig. 4, : une curieuse variété minor de la mème provenance.

Charsha Barpanomes Möllendorfi. Subsp. microthyra Möll.

1901. Phaedusa (Oospira) rhopaloides subsp. microthyra. Möltexdonsf, Nachritsh. d. d. Malak. Ges., p. Illi. 
1902. Clausilia rhopalerides Möll. subsp. microthyra Möll. Srise, On Tonlinese Clan siliate in Proe. Mal. Soc. p. 1993, pl. III, lig. 7 .

190\% - - - subsp. microthyra Möll. H. Fiscier el D.LTtzexberi, Catal. Indo-Chine in Mis sion Pavie, lor. cil. p. 价 (f. . .l du l. à p.).

Tonkin. Ile lírieu (M. Blaise: exemplaires de petite laille: forme minor).

Baie d'Along, He de la Surprise (.I. Blaise). Baie de la Noix (M. Blaise) grande lorme dépassant mème la taille du tỵe de Möllendorli.

Clacsigla stenothira Möllendorft.

1901. Phadusa (Psendonenia) stemothyra MöLtexdorrs, Nachrichtsho. d. d. Malak. Ges. 1) 116.

1900. Clansilia stenothyra Möll. Srkis. On tonkinese Clausiliae in Proe. Mal. Soc. p. 193, pl. 11I, fig. 2.

1!0\%. Clansilia stenothyra Möl. H. Fiscmen el D.tetzexbeng, Catal. Indo-Chine in Mission Pavie, loce cit. p. 'slo (p. 21 d lu l. ì p.).

Tonkin. Baie d Jong : lle de la Surprise (M. Blaise).

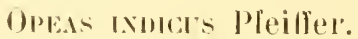

18:34. Bulimus............ Ilıtros, Jomen. Isiatic Soc. Bengal, 111, p. s'

1846. Bulimus indiens PrenFen, P. \%. S. L., P. 10 (= Achatima gracilis Bensoll mss. leste ipso 184s). 
18'6. Achatina gracilis

18ณ.'Bulimus

1849.

1849

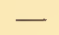

1849.

apex

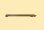

1819.: -

cerens

Bexsox mss. in Preiffer, P. Z. S. L., P. 40.

- Hult. PFeiffer, Mon. Helic. II, p. $1: 37$.

REEVE, Conch. Icon., pl. LXIX, fig. 4983 (figure médiocre).

Moussox, Moll. Java, p. 330, pl. II, tig. :3.

Moussox, Zeitschr. f. Malak., p. 180 (= gracilis, teste ipso).

Reeve, Conch. Icon. sp. :301 et Mon. Achatina, pl. XVII. lig. 81 .

18533. - gracilis Hutl. P'FenFER, Mlon. Hel. III. ए. 399.

18.99. - - - Premfer, Mon. Hel. IV, 1.

1861. Spriaxis - - Blaxford, Contr. 10 Ind. Malac. II, p. 16 (olss.).

1867. Stenogyra - - r. Martexs, Preuss. Exp. II, p. 83, 37̈; pl. 19, fig. :̈. pl. 22.2, fig. I3.

1868. Bulimus

- - Preiffer, Mon. Hel. Vi, p. 9 (i.

1870

Haxley el'Theobaln Conch. Indica II, p. 1?, pI. XXIII, fig.' (optima).

1871. Stenogyra - - Nevilt, Enum. Helic. et Pneum. in Ins. Ceylon detect., p. 3.

187'.

1874.? Subulina

Crosse, Journ. de Conch., vol. XXII, p. 2029.

JoKel, Afr. Moll. 1. 137. 
187't. Stenogyra gracilis Hutt. Lsen., Moll. Borneens.p.̈̈l.

\begin{tabular}{|c|c|c|c|}
\hline sit. S. Ople & - & $\longrightarrow$ & 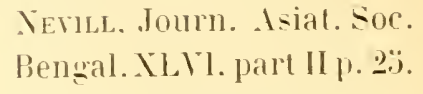 \\
\hline ï. Bulimus & - & - & $\begin{array}{l}\text { PFEIfFEr, Mon. Hel. VIII, } \\
\text { p. 13ï. }\end{array}$ \\
\hline Opeas & - & - & 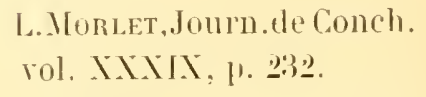 \\
\hline 91. & - & - & 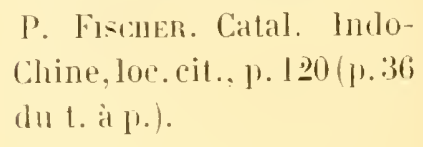 \\
\hline 9 '. - & gracile & - & $\begin{array}{l}\text { MöLLEXWORFF. P. Z. S. L., } \\
\text { l. Ï̈1. }\end{array}$ \\
\hline $00 .-$ & - & - & $\begin{array}{l}\text { Möllexdorff, Nachricht- } \\
\text {-H. d.d. Mal. (i.. p. 13' }\end{array}$ \\
\hline $10: 3$. & - & & $\begin{array}{l}\text { Iilatford, Land \& Freshw. } \\
\text { Moll. from siam in Proc. } \\
\text { Mal. Soc. L., p. 280. }\end{array}$ \\
\hline (1)' & gracilis & - & 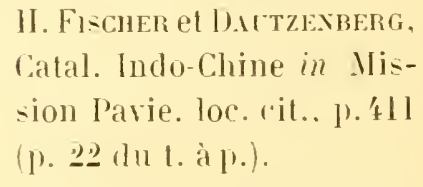 \\
\hline
\end{tabular}

Tonlin: Luc-Yam (.. B. Baise) I exemplaire itlentique à la figure de Hanley et Theobald et qui ressemble plus à la figure de Reeve représentant le s. subula quà celle du mème auteur représentant le gracilis; mais la figure qui peut ètre considérée comme typique pour lo. subula est Jutôl la figure d'O. octonoides de d’Orbigny.

Yous ne pourons éviter le reprendre pour cetterspecele nom imlions qui lui a été donné gar Pfeiffer en Iśli. bien que cet anteur ait connu alor's lexistence d'un Achatima gracilis Bemson mss.

Il est vrai yue Pfeiffer lui-mème a repris bientot après le nom d'espèce grarilis qüil altribur à Hutton fen réalité a dernier naturaliste a déerit romme Mnlimus..... sams 
nom spécifique, une espèce qui est bien la mème que l'Achatina gracilis Benson mss.); mais les noms manuscrits ne peurent d’après les règles de nomenclature aroir aucun droit a la priorité et c'est le nom publié le premier qui doit subsister, mème lorsque son auteur a modifié par la suite sa manière de roir.

\section{SpIraxis erecta Benson.}

1842. Achatina erecta Bexsox, Ann. \& Iag. X.H. W sel. IX. p. 4St.

184. - - Bens. Premfer, Mon. Hel. II. ए. $260 \%$.

IS'9. - - - ReEve. Conch. lcon.pl. l6. fig. 69.

18:30. - - - Pfenfer, Conch. Cab. 2 éd. p. 333, pl. 2.s, fig. 6. 7 .

18.i. Spiraxis $\quad-\quad-$ PfeIfFer. Proc. Z. S. L.p.? (obs.).

18:58. Subulina(Stenogyra) - Awss Genera of rec. Iloll. II, p. 111.

18:39. Spiraris erecta Bens. PfelfFer, Mon. Hel. IT, p. $\because 33$.

1878. S. (Euspiraris) - - Cussolx, Nomend. Ilel., 1. 3203.

1891. Spriraris (?) erectus - P. Fischer, Catal. IndoChine, loc. cit. p. l’2l (p. 3 i du t. à p.).

190'. Spiraxis

- - H. Fischer el Diltzenberg, Catal. Indo Chine in Mission Pavie. loc. cit., p. łll (p. 끌 du t. à p.).

Tonkin : Région du Haut Song Luc-Nam. Environs de Lam sous des pierres dans un bois des environs du poste de Lam (.II. Blaise). 
Arracla connexses Pfeifler.

183\%. Auricula Chinensis PFemfer, Proc. Zool. Soc., P. 1 i拄.

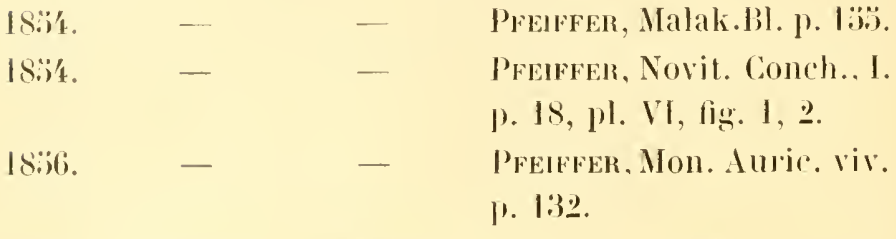

18.i8. Ellobium chinense 11. et A. Ansus, Cien. of rec. Noll. 11, 1. 237 .

1876. Amimala chinensis Premfen, Mon. Pneumonop. viv. 3 suppl., p. 33is.

187ti. - Reiniana Konest, Conch. Miscellen in .lahrl. d. d. Mal. Cies. III, 1. 1:31, pl. : , fig. 3, 6.

1878. - Chimensis Sowers in Reve, Conels. lcon. pl. II, lig. II.

1879. Auricula Reiniana Kobest, Fauna Japon. exlam. in AbhandI. der Senclenbers. Nit. Ges., XI, p. $35 \overline{\text { (p. }} 103$ du t. à p.).

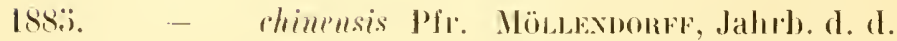
Mal. Ges., X11, p. 3i\%氵.

1891. - Chinensis P. Fiscanen, Ciatal. IndoChine, loc. cit., p. 123 (p. 39) du 1. à p.).

1898. - chinensis - liobelt, Mon. Conch. Call.

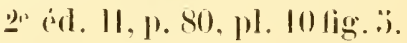
1898. - reiniana lionelt, Mon. Conch. (ah).

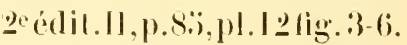

Igo'. - rhimensis Pfr. II. Fiscuen et Dactzexpeng, Catal. Indo Chine in Mission Pavie, loc. cil, p.'tl3 (J. 2) dut $\mathrm{t}$ ( ) p.). 
Tonkin. Cua-Cam, Haiphong. Eaux saumàtres, sur les bancs de rase qui bordent les rives du fleuve et qui déeourrent à marée basse. Elles s'enfoncent dans la vase mais doivent probablement à certaines heures de la journée monter sur les tiges des herbes qui poussent sur ces banes (M. Blaise).

Nos exemplaires correspondent mieux arec les figures de l'A. Reiniana yu'avec celles del'A. chinensis; mais nous avons reçu de 11 . Schmacker sous le $n o m$ de A. chinensis des exemplaires identiques aux nôtres, provenant de Macao. Nous croyons quil y a lieu de réunir ces deux espèces. Nos exemplaires d'A. Reimiana du Japon sont aussi identiques à ceux de l'Indo Chine.

L'A. chinensis diflère de l'A. anris Judae par sa taille plus faible, sa surface moins treillissée sur le dernier tour et son pli colmmellaire moins aseendant, phus transversal ef plus saillant.

\section{Lamodonta Bhonei Philippi.}

1846. Auricula Bromi Pmupr, Zeitschr. f. Mala koz., p. 98 .

18:2. - samdurichiensis Ernoux el Socleyet, Voy. Bonite., p. 3̈24, pl. 29, tig. 29!) : 32.

183̈6. Melampus Bronni PFentek, Mon. Auric, p. 4!).

1836. - Sandwichiensis Prenfer, Mon. Auric., p. :30.

18388. Ellobium Brounii 11. et A. ADaMs, Genera of rec. Noll. II, p. 237.

1871. Laemodona Bromi Ph. Martexs \& Laxgkavel, Donum Bismarckianum, 1. : $: 7$.

187'. - Bronni Ph. Jicieli, Afr. Moll., p. 178. 
1576. Melampms Bromi PfenfFer. Mon. Pneumon. 3e suppl., [1. 319. 42ł.

1s98. M. (Laimorlonta) brommii Ph. Kobert. Mon. Conch. Cab. 2e éd. H. 1\%. 200, 1). 23, fị. 1, 2.

La forme typique n'est pas représentée dans les récoltes de M. Blaise.

Var. producta Dautzenlerg et H. Fischer n. var.

P1. 11, tig. 20, 21.)

Tonlin. Anse du Cràne (11. Blaise.)

Nos exemplaires du Tonkin ont la taille du L. Bromi Philippi (qui n’a jamais été figuré) mais leur spire est jlus élevée. lls concordent exactement arec les figures de Jickeli (L. Bromi rar. comica Pease); mais il convient de remarquer que le véritable $L$. conica Pease est une espèce beaucoup plus petite et ne présentant quime hande blanche bien nette. Nous attribuons a la forme recueillie par M. Blaise le nom de L. Brommi rar. moulucta.

Le genre Laimolonta, caractérisé par 3 dents pariétales et une lame transversale et pénétrante à l’intérieur du labre, n’avait pas encore été cité en Indo-Chine.

\section{CAssmLla Atris-Felis Bruguière.}

1786. Toluta Coffea Limnaei Cummxitz (non Linné) Conch. Cab. IX, 2) p. 4̈̈ pl. 121 fig. 10 4:3-10\%'.

1789. Bulimus anris felis Brogrièz, Encrel. méth. I, p. 3я; (pl. 160, fig. ̈̈", $\ddot{\prime}^{\prime \prime}$.

1798. Ellobimm inflammamm Boltex, Mus. Boltenianum 1). 106 ; édit. altera, P. T́.

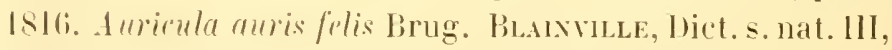
suppl. p. 1:3:. 
182?. Auricula felis

18으. - - -

182:; Voluta Colfeae (sic)

1830. Amicula felis Lk. Mexise, Synopsis, ed. alt, р. $3 \ddot{8}$.

1837. Cassirlulus

1837.

1838. Auricula felis

Férussac, Tabl. Syst, de la famille des Auricules, р. 109.

LAMARck, Anim.s. vert VI, 20 p. p. 138.

Wood (non Linné), Index test, p. 102, pl. 19, 1. 1\%.

Веск, Index, 1. 10:\%.

Beck, Index, p. 10:i.

Deshayes, in Lamalick, Anim. s. vert. 2éd. rill, p. 3206 .

1838. Auricula felis Lk Potizz el Murato, Gal. de Douai, 1, p. 201.

1839. A. (Cassidula) felis Axтох, Verzeichn. p. 18.

1840. Rhodostoma colfea Swansox, Treatise on Malac., p. 344.

1840. Sidula felis Catli Gray in Turtox Man. 2 èd., p. 21 .

1841. Auricula fusca Hombrox el Jacecixot, Voyage au Pöle Sud, pl. 9, fig. $7-9$; p. 3ł (texte publié par Rousseau en IS:3'ł).

1842. - telis Li Reeve, Conch. Syst. p. I06, pl. CLXXXYil, tig. 6 .

184'. - - Lk Küsten in Chenistz Coneh. Cab., 20éd. pl. 3, fig. 3.

'1847. Cassidula auris felis Br. Gray, Proc. Z. S. L. p. 179. 1830. Auricula felis Lk JaY, Catal, p. 206'.

1830. Cassidula auris felis Br. M. E. Gкат, Fig. Moll. Anim.,p. 17!, pl.30', fig.3, $3^{\mathrm{a}}$.

185̈2. - inflammata Bolt. MöreH, Catal Yoldi, p. 38. 
18.ip. Auricula felis Lk. Esdocx el Sorteret, Toy. Bonite, 1. 3̈t6, ,1. 29, tig. 21-23.

18.34. - fused Rocssent in Hombros et Jacolnotot, Voy. an Pòle Sud, p. 3\%.

1s:i6s. Cassidula anris felis Br. Prenfer, Mon. Aurirul. viv. p. 117.

18:i8. - coffea H. et A. Adass (non Lin.), P. Z. S. L..p. 31.

18.8. - auris felis Br. 11 . el A. Abans, Gen. of. rec. Moll., 11, p. 238, pl. 8:, fig. 2., 2.'.

1870. Anricula anris felis Br. Woomwan, Mannel de Mal. Iratl. fr., p. 31:i, fig. 1:32.

1874. Cassidula felis Lk. Isseu, Moll. Borneens.p.ö9. 187:. - auris felis Br. Monelet, Séries Conch. IV, 1. 272 .

1876. - roffea Ch. Tapparone Caneri (nou L.). Malac. Viagg. Magen1a, 1). 210.

1876. - auris felis Br. Prestren, Mon. Pneumonop. vis. suppl. 111, p. 33̈4. 1878. Arricula felis 1. Sowenв in ReEve, Conch. Icon., pl. IV, fig. 2:3(médio(cre).

1s!1. C'assidula amris folis Br. 1’. Fiscuer, Catal. IndoChine, loc. cit., 1. 12?3 (p. 39) du t. à p.).

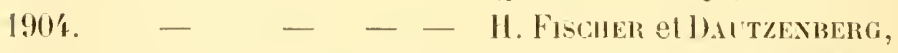
Catal. Indo-Chine in Mlission Pavie, loc. cil., p. 'tl:3 (1). ․ㅏ du t à p.).

Tonkin. He Krieu (II. Mlaise). 
.Cassidula plecotreatatones Möllendorfl.

188:i. Cassidula plecotrematoides Möllexnorff, Jahrb. d. d, mal. Ges., p. 33̈2, pl. 9, fig. C a, b, c.

1909. - - Möl. Il. Fischis et D.lutzenberg Catal. Indo-Chine in Mission Pavie, loc. cit., p. 亿13 (p. ㄴ. dlu 1. à p.).

Tonkin. Ile de la Cae-Ba, anse du Cràne, baie d'Along. Marée basse sous des cailloux reposant sur la vase (M. Blaise), exemplaires un peu plus petits que ceux de Hong Kong. - Archipel des Faï-Tsi-Long, ile aux Biches. (1I. Blaise), msime forme, coquilles recueillies au milieu de gros graviers à l'embouchure d'un petit ruisseau.

Plecothema proctigeiza II. el $\mathrm{A}$. Adams

183̈3. Plecotrema punctigera 11. et A. Aosus, Proc.Z. S. L., p 120.

18:̈6. - - Id. Prenter, Mon. Auric., 1). $10 \%$ el rar. $\beta$ punctis magis excacatis, long. 7 $1 / 2$, diant. :

186\%. - - C Crosse el Fischer, Faune inal. Cochinchine in Jourm. de Conch. vol. XII, p. 330.

187\%. - - - Issel, Moll. Borneens., p. 6 으. 187:. - - 1. Morelet, Séries Conch.

1876. - - - Pretrak, Mon. Pneumo nop. viv. 3e suppl. p. 34.7.

188\%. - $\quad$ - MöLlendorfF in Jahıb. d. d. Mal. Ges., XII, 1. 3.̈.1. 
|\$9।. Plecotrema punctigerum Ad. P. Fiscanes, Calal. IndoChine, loc. rit., p. I2' (p.40 du t. it p.).

1901.

- II. Fiscing el Diltzexberg Calal. Indo Chine in Mission Pavie, loc. rit. p. 1.3

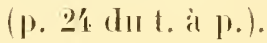

Tonkin. He aux Biches, He de la Surprise (M. Blaise). Anse du Cràne, Baie d’ Mlong, roquilles recueillies à marée basse sous des cailloux reposant sur la vase (II. Blaise). Tous possédonscetle espèce de Dong Trieu el de Wuang Yén (récoltes de $\mathrm{M}$. Dor' collection l)aulzenlerga). Il en existe au Muséum fi exemplaires collés sur mon carlon, recueil-
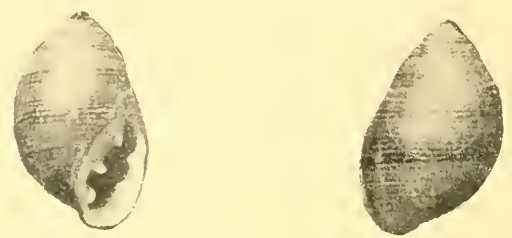

Plecotrema punctigernm II. et I. Ad. grossi 3 tois.

lis a Pexahuri, siam, bar M. Bocourt: leur taile (8 i $9 \mathrm{~mm})$ est plus forte que relle des exemplaires rapportés par II. Blatise.

Nous rroyons utile de représenter ici un des specimens provenant de l'ile anx Biches, car le l'lecotrema punctigerum lla jamais élé figuré a notre commaissance.

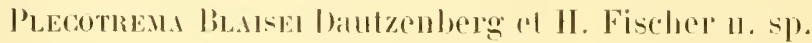
(PI. N1, lig. 1s, 19.)

Testa orato-conica, solida, hand nitens, spiraliter confertim sulcata ae striis incrementi tennissimis subdecussata. A pex 
acuminatus. Anfr. primi subplani, sutura pasne conspicua sejuncti. Ultimus antice $5 / 7$ allitudinis aequans, basi attematus et crista ralida, rimam umbilicalem circumalante, munitus. Aperma orato-oblonga $4 / 7$ altitudinis aequans, superne angulata, marginibus rallo arluato nitidoque junctis. Margo columellaris dentibus parietalibus 2 munitus : superus minor oblique descendens, inferus validior ac profunde intrans. Plica. columellaris transcersa sat ralida marginem antingit. Labrum inuss superne simuatum inferne rero inerassatum ar ibi bituberculatum. Color undique satnrate fuscus, peristoma pallidins.

Allitudo $7 \mathrm{~mm}$., lat. i mm., apertura $4 \mathrm{~mm}$. alta, $23 / 4 \mathrm{~mm}$. lata.

Coquille de forme ovale-conique, solide, mate, ornée de sillons décurrents nombreux el de stries daceroissement très fines qui produisenl de très hégères granulations. Sommet aigu. Tours supériéurs presque plans, séparés par une suture a peine visible. Dernier tour oroüde, occupant les $: 3 / 7$ de la hauteur de la coquille (lorsquion le regarde du côté antérieur) et présentant ì sa lose une crète saillante qui cutoure une légère fente ombiliale. ()urerture ovale allongée occujant les $\$ / 7$ de la hauleur. anguleuse dans le haut oì elle se termine par un petit canal très étroit. Bords reliés par une callosité luisante. appliquée. Bord columel laire frésentant 2 dents pariétales: la supérieure, petite courte et descendant oblinuement; l inférieure, plus forte, moins oblipue et se prolongeant profondément dans l’intérieur de lourerture. Pli columellaire transversal assez - fort et alleignant le bord du péristome. Labre présentant it l'intérieur et dans le haul un simus limité par une callosilé qui se prolonge vers le bas justu à sa jonction aver. lo hord columellaire. Cette callosité émet @ِ dentelons dont le supérieur est le plus fort.

Coloration d'un hrun foncé noirailre, miforme ou présentant parfois une zone subsulurale un peu plus claire. 
Péristome et dents de l'ouverture l'un ton gूris rarnéolé.

Tonlin. Ile krieu (M. Blaise). Baie d'Along, anse du Cràne (M. Blaise).

Cette espèce à une rertaine anatogie aver le Plecotrema siamensis 1. Morelet, dont nous figurons, à titre de comparaison, un sjécimen de la collection du Muséum de Paris provenant des récoltes de M. Bocourt à Pexaburi (Siam); mais le I\%. Blaisei en diflere par sa taille plus faible. ses sillons décurrents plus accusés, et surtout par la crète qui entoure la région ombilicale el dont il nexiste aucune trace 'hez le $1 \%$. siamensis.

Melanpls sincaporexsis Pfeiller.

18:3'. Melampus Sincaporensis Prenfer, Novitates Conch. I. 11. 4ti, pl. Xll, fig. 1: 16.

183:\% - - - Peerfen, Malak. B1., 1.8. 18.jit - sincuporensis PFenfer, Mon. Muric. 1. 任.

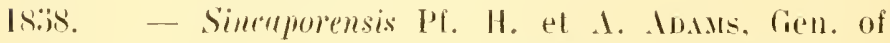
lee. Moll. 11. p. 216.3.

1876. - sincaporensis Prenfer, Mon. P'neumo nop. vir. 3e supp., p. 311.

1897. - Singaporensis V. Martexs, Moll. Weber,

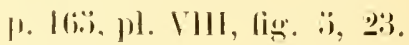
1895. - sincaporensis linbet, Mon. Conch. Cals. ‥ édit, II, p. I!)i, pl. 르, lig. I:i, lli.

Tonkin. Ile de la Surprise (M. Blaise).

\section{MelaMe's pelanelets Petit.}

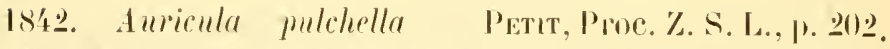

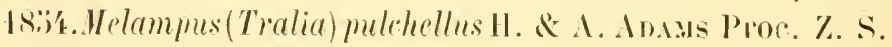

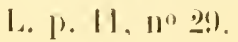


133̈t. Melampus pulchellus Petit P'Fenfere, Mon. Auric., p. 33\%.

18:38. Tralia (Tifata) pulchella H. \& A. An.sus, Gen. of rec. Moll., II, 1). 凅.

1876. Melampus pulchellus Petil PrenFer, Mon. Pneumon. 3: Suppl., 308.

1898.

- liobelt, Mon. in Conch. Cab. 20éd. II, p. 느1, pl. 206, lig. $8,9$.

Tonkin. Ile aux liches. Archipel des Faï-Tsi-Long (.M. Blaise); Yen-Lun, dans les gercures d'un vieil arbre échoué à la laisse de haute-mer (M. Blaise).

Les exemplaires que $\mathbf{M}$. Blaise a récoltés. sont identiques a dautres que nous arons reçus de M. Coneménos sous le nom de $M$. pulchellus Petit et provenant de Bom bay. Les flammules disparaissent avec l'àge et il ne reste que des bandes transversales daus les individus adultes.

\section{Melampes Adamsines pleiller.}

18:3'. Melampus Adamsianus Pretffer, Proe. Z. S. L., p. II.

18:3\%. - - Premper, Novit. Conch. 1, p. 18, pl. V, lim. 17-19.

IN:3i. - - P'TeIFFer, Mon, Auric., 1. 2 'י.

18:i8. Tralia (Pira) Adamsiana Pf. H. et A. Ands, Gen. of. lee. Moll. I1, 1). 2014.

186i3. Melampus Adamsianus Pf. Gissses, Faune nour. Cal. 1, 1. :̈7, ,1. VII, lig. 2.
1871.

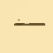
Pí. Gassies, faune nouv. Cial. II, p. IO:;.
1878

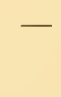
Pafenter, Mon. Pnenum.vis. 3" suppl., p. 30'́s.
$189 \%$.
- Pi. Crosse, Rev. N. Cal., p. 16il. 
1898. Melampus adamsiamus Pf. Kónest, Mon. Conch. Cab. 20 éd. II, p. I89, pl. 22, fig. 3.

Tonkin. Ilr aux liches, archipel des Faï-T'si-Long ( $\mathbf{l}$. Blaise).

Scalabes Cegalem Philippi.

1817. Scarabus Cecillii Pmulpr, Zeilschr. f. Malak., J. 12.2.

1830. Scarabus - A. Adams, Proc. Zool. Soc.. p. 149.

18.36. Pythia Cecillei Prenter. Mon. Auric. vis. р. 97.

18.:8. - - II. \& A. ADAms, Genera of rec. Moll., II, 1) 2 '10.

1860. Scarabus - Ph. Reeve, Conch. Icon., pl. III, fig. 20 .

1s66. Pythia - Ph. PFeiffen, Novit. Conch. III, ю. 3̈̈̈, pl. LXXXII, fig. 3, 4.

1876. - - Plifrer, Mon. Pneumonop. viv. 3 suppl., p. 342. 1898. - - Ph. Kiobelt, Mon. Conch. Cal. 2e éd. 11, p. 1'1, pl. 14, fig. :, 6 .

Tonkin. Song kinh Tay, Yen Jun, eau sammàtre sur les tiges des grandes herbes poussant sur les bancs de vase qui bordenl le lleuve (M. Blaise). lle de la Surprise (M. Blaise). lle aux Biches près d'un petil ruissean (M. Blaise).

Lamaka Buase Daulzenberg et II. Fischer n. sp.

$$
\text { (P. V, fig. 1, 2.) }
$$

Testa temuis, parra, orata, anyuste rimata, sat nitida, striis inerementiobsoletissimis tantum senlpta. Spira 1/.3 testae altitu- 
dinem subaeruans. Anfr. 4 convexi, gradati (superne paululum planulati) suturaque impressa juncti. A pertura otata, superne angulata, marginibus callo temi adnatoqne junctis; columella superne subtorta, parmm excatata. Labrum simplex, arcuatum. Color undique fulvus, cohmella albicante.

Altitul. \& millim; latit. 3 millim. A pertura $31 / 2 \mathrm{~mm}$. alta, 21/2 mm. lata.

Coquille mince, de petite taille, de forme ovale, pourvue d'une fente ombilieale étroite. Surface assez luisante, ornée seulement de stries d'accroissementà peine visibles. Spire oecupant á peu près le tiers de la hauteur totale de la coquille, composée de 4 tours convexes un peu élagés et séparés par une suture bien marquée. Ourerture orale, anguleuse au sommet, à bords réunis par une callosité mince et appliquée. Columelle peu excavée, un peu tordue dans le haut. Labre simple, arqué.

Coloration d'un fanve clair uniforme à l'exception de la région columellaire blanchàlre.

Tonkin. Régiondu bas Song Luc-Nam, environs de V'anlen. Coquilles recueillies en eau absolument douce dans les rizières des environs de Van-len. Elles grimpent aux tiges du riz et paraissent avoir les mèmes habitudes que tous les Mollusques des rizieres qui s'enfonçent dans la rase quand les rizieres sont i sec (M. Blaise).

Bien que de fort pelite taille, les exemplaires rapportés par M. Blaise paraissent bien adultes et ne nous semblent pouroir ètre rapprochés d'ancune des Limmées indo-ehinoises décrites jusqu à présent et qui sont toutes beaucoup plus grandes.

Planorbis saigonensis Crosse et Fischer.

1834. Planorbis compressus Huttox(non Michaud 1831) Journ. As. Soc. Beng. III 1. 91,93 . 
1863. Planorlis Saigonensis Crosse el Fiscuen, Journ. de Conch., vol XI, p. 362, pl. XIII, fig, 7 .

187.;. - compressus Hutt. A. Monelet (non Mich.), Séries Conch. IV, p. 2276.

1581. - confusus Rochebrice, BuH. Soc. Philom., ]. 32.

1886. - Saigmensis Cilessix, Conch. Cah. 2uéd. 1. 191, pl. 29, fig. 3.

1891. - Saigonensis P'. Frsculer, Catal. IndoChine, loc. cit., p. I6 (p. 42 du t. àp.).

1891. - confusus Roch. P. Fiscuer, ibid. p. I26 (19.2) 1904 - saigonensis H. Fischen el Daltzesibrg, Catal. Indo-Chine in Mission Pavie, loc. cit., p.任 (1). 2.") du l. à l.).

1904. Planorbis confusus H. Fischen el Dactzexber, ibidem, ]. 414(루).

Tonkin. Bas Luc-Nam, environs de Van-Ien, dans une rizière (M. Blaise).

Parmi les spécimens recueillis par M. Blaise, un ou deux ont une tendance à ètre carénés sur le dernier tour. ce qui les rapproche dı $\%$. confusus Rochebrune (compressus Hutlon 1834, non Michaud I831). L'avis de A. Morelet qui rattachait le $P$. saigonensis au $P$. compressus a titre de variété se trouve donc confirmé, mais il y a lieu de reprentre le nom de l'l.saigonensis pour désigner l'ensemble de ces deux formes.

P'LAxombis Dorrinste Wattebled.

1881. Dlanorbis Dorrianus Wattebled, J. de Conch. rol. XXXIl, p. 126, pl. I1. lig. … 
1891. Planorhis Dorrianus W. P'. Fiscuer, Catal. IndoChine, loc. cit., p. 126 (p. 侻 du t. à p.).

190 '.

- II. H. Fischer el Dautzexberg, Catal. Indo-Chine in Mission Pavie, loc. cit. p. 414 (p. 2 i du t. à p.).

Tonkin. Rizières de Luc-Nam (M. Blaise).

\section{Mrtra scutulata (Ch.) Gimelin.}

1788. Voluta scululata ele. Chemsitz, Conch. Cab. X. p.168,pl.1:31,fig. 1428, 1429.

1790. Jilra - Ch. Gmeds, Syst. Nat.éd. XIII. p.34:i2

$\begin{array}{llll}\text { 1811. } & - & - & \text { Lamarci, AnI du Mus. } \\ \text { XVII,p.212. } \\ \text { 182.2. }\end{array}$

182:;. Troluta scutulata L. Wood, Index testac., p.9s pl. 20, fig. 1202(mala).

1838. Mitra - Ll. Liener, lcon. Cof. viv. p. 6', pl. 17, fig. :37, 3̈7. 1841. - - Ch. Kïster, Mon. Conch. Cab. 20éd., p. 侻, pl.8, fig. I, 2. 184' - - ReEve, Conch.Icon, pl.XII, lig. 82.

1847. - - Lk. Sowersy, Thes. Conch.IT, p. 18, pl. 372, fig. 130, '131, 132.

1882. - - - Trion, Manl of Conch.IV, p. І:\%i, pl. 46, fig. 332-337.

Tonkiu, sans localité précise (I. Blaise) un exemplaire. 
Murex pinnates Wood.

1777. Purpura subalala, triquetra Manton, Conch. Cals. III, p. 318, pl. 111, fig. 1036, 1037?

1828. Murex pimatus Woon, Ind.testac. suppl..

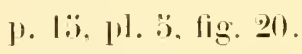

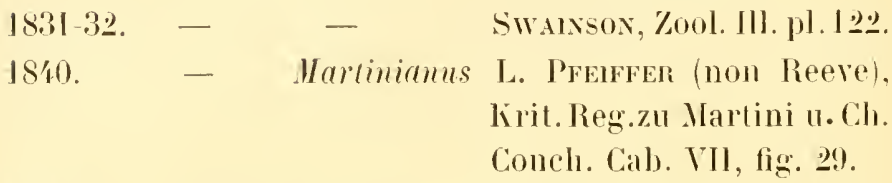

181:. - pimnatus Wood hiexen, Icon. Coq. vir.. p. 114, pl. : 3 , fig. 3,3 .

1813. - - Wood Desmayes in Lanarck,
Anim. s. Vert. 2e éd., IX,
p. 603. 1894. - - Sw. lï̈ster, Mon. Conch. Cab. 2. éd., p. 28, pl. 12, lig. 3,6 . 184\%. - - ReEve, Conch. Icon. pl. XII, fig. : 37.

1879. - - Sowerbr, Thes.Conch., IT, 1. 2-3, pl. 391, fig. 123.

1880. - - - Tryox, Man. of Conch., II, p. 87, jl. 41, fig. 320 (excl. fig. $330 \mathrm{H}$. pellucidus live). 1881. Typhis - - - de Rochebrexe, Suppl. aux documents sur la faune malac. de la Cochinchine et dı Cambodge, extr. Bull. Soc. Philomat. Paris, p. 19.

1891. Murer - $\quad$ P. Fischer, Calal. IndoChine, loc. cil.. p. 146 (62) (lu t. à p.).

Tonkin. Baic d'Mlong (M. Blaise). 
Plirura alveolata Reeve

1846. Purpura alceolata ReEv, Conch., Icon.pl. XI, fig. 60 .

1858. - - Rve Küster, Mon. Conch.Cab., 2.éd., p. 187 pl. $31^{\mathrm{a}}$, fig. ?.

1880. - hippocastanum var. alveolata live

Tryos, Man. of Conch., II,

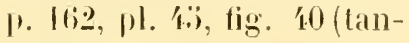
(110m).

Tonkin. Baie d'Along, I exemplaire (II. Blaise).

Cette espèce nous parait différer suffisamment du $I$. hippocastamum Lamarek pour ètre maintenue comme distincte. L'habitat (Panama n indiqué par Reeve après la description originale nous paraît fort douteux. M. Smith a d'ailleurs déjà signalé le l'alceolata de Formose, du Japon et de l'dustralie.

Clum gradata Jonas.

1816. Prurpma gradata Joxas Zeitschr.f.Malakoz. p. I'́.

18'6. - trigona ReEve, Conch. teon. pl. XI, fig. :33.

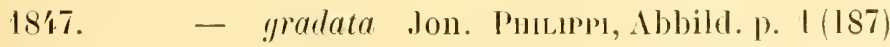
pl. 1, fig. I, 2.

18.00. - Grateloupiana Petut de la Saussaye,Journ. de Conch., I, ]. 402, pl. 13, fig. 1 (élabli sur un spécimen roulé).

18:8. - gradata Jon. Küster, Mon. in Conclı. Cals. 2 éd., p. 99, pl. 17. lig. 10, 11.

1880. Cuma - - Tryos, Man. of Conch., I1, p. 201, pl. 63, fig. 33ㄹ., 338 . 
1891. Purpura gradata don. P. Fischer, Calal. IndoChine, loc. cit., p. Ifs (lít du t. à p.).

Tonkin. Anse du Cirâne, Baie d’Along (II. Blaise).

\section{Coma manalca Philippi.}

1836. Purpure mulata var. Kémen(non Lk), Icon. Cor. viv. pl. 33;, lig. s', 8'.

1848. - jacanica P'HıLıp,Zeitschr.f.Malak, 1. 27 .

1838. - Phil. Kï̈ster, Mon. Conch. Cals., Q éd., p. 171, pl. 20, fig. 10, 11.

1880. - - - Trox, Man. of Conch., II, pl. 63, fig. 331.

Tonkin. Baie d Along. Anse du Cràne, nombreux exem. plaires recueillis à basse mer sur les roches qui bordent l'Anse (M. Blaise).

Sistrum rodtum Chemnilz.

179:3. Murex mulatus Chemstre, Conch. Cab. XI, р. І2я, ㄱ. 192, fig. 18:0)I8:i1.

189'. - Ch. Küster, Mon. in Conch. Cal, 2o éd., p. 121, pl. 36;. lig. 7,8 .

183:. Lurpura fiscella linser, (non Chemnit\%), loonogr. Cor. viv., p. 30, 1). 6, fig. I․ 12 (tantum).

1880. Sistrum undata Ch. Trrox (ex p.), Nan. of Concli. II, p. 189 (aucune figure satisfaisante). 
1891. Pẹtadactylus undatus Clı. P. Fischer, Catal. IndoChine, loc. cit., p. 1/49 (6ï du t. ì p.).

Tonkin. Baie d'Along, sur les rochers; Anse du Crâne (M. Blaise).

\section{Strombus Isabella Lamarck}

1822. Strombus Isabella Lamanci, Anim. s. Vert., VII, P. 207.

1832. - canarium L. var. a Deshaves, Eneycl. méth. III, p. 990 .

I82:\% - - - var. Diluwre, Deser. Catal., II, p. 671 .

1834. - tieniatus Quoy Qror et Gamard, Voy. Astrolabe, III, p. 7:8, pl.:̈l, fig. I'́, Г̈̈ (sub nom. Strombe rubané).

1843. - Isabella Lk. Deshares in Lamanck, Anim. s. Vert., 2e éd. KX, p. 700 .

\begin{tabular}{|c|c|c|c|c|}
\hline $184: 3$. & -- & - & & 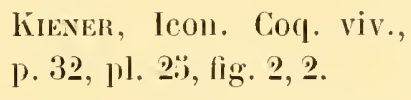 \\
\hline 184:. & - & - & Lk. & $\begin{array}{l}\text { Küster, Mon. in Conch. } \\
\text { Cab, 2e éd.1. } 41 \text {, pl. } 7 \text {, fig. } 3 .\end{array}$ \\
\hline .1847. & - & - & & $\begin{array}{l}\text { Sowerzв, Thes. Conch., I, } \\
\text { p. } 33 \text {, pl. VIIl, fig. } 68, \text { זl. }\end{array}$ \\
\hline 18301. & - & - & Lk. & $\begin{array}{l}\text { ReEve, Conch. Icon. pl. } \\
\text { XVlll, fig. } 31 .\end{array}$ \\
\hline
\end{tabular}

188.3. - canarium L. var. isabella Lk.

Tryon, Man. of Conch., VII, p. 110, pl. 2, fig. 20 (et pl. 3, fig. 2 I var taeniata) 
1889. Strombus Isabella Lli. L.MorLet, Journ.de Conch. XXXVI, p. 1位.

\begin{tabular}{|c|c|c|c|c|}
\hline 1890. & - & - & - & Crosse et Fisciner, Journ. \\
\hline 1891. & - & - & - & $\begin{array}{l}\text { P. Fischer, Catal. Indo- } \\
\text { Chine, loc. cil.. J. 20:8 ( } 7 \text { ' } \\
\text { du t. a b.). }\end{array}$ \\
\hline
\end{tabular}

Tonkin. Baie d'Along (M. Blaise).

Cenithum coxcisum Hombron et Jacquinot. (= morus Lamarek non Brug.).

182.2. Cerithum morus Lanarci (non Brug.), Anim. sans. Vert., I'Il, p. 73.

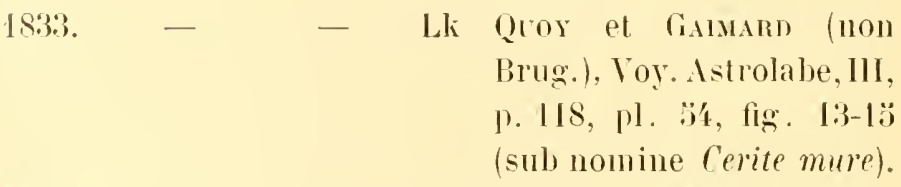

1838. - - Lk Potiez ET Miciaud (noll Brug.), Gal. de Douai, I. p. 336, pl. XXX1, fig. 2-7.26.

181. - roncisum Honbrox el Jacolixot, Voy. au Pôle Sul, pl. 2't, lig. I. 2; (p. 102, par Rousseau, 18:31).

1893. - morus Lk DeshaYes in Lamarck, Inim. s. Vert., Q2éd., IX, 1. 302 (non Brug.).

18:3\%. - obsrum Rousseat in Hombrox et Jacecinot Voy. alu Pòle Surl, p. 102.

18.\%. - morns di Sowerbi (non Brug.), Thes.Conch. II, p. 870 , pl. CLXXXIl, fig. 1:99, 160.161. 
186.). Cerithium morus Lk ReEve (non Brug.), Conch. Icon., pl. VII, fig. 位.

1887. - - - Triox (non Brug.) (ex parte), Man. of Conch., IX, p. 133, pl. 2't, fig. 32., 33 (tantum).

1891. - - - P. Fischer (non Brug., Cat. Indo-Chine, p.161(77). 1898. - - - Kobezt (non Brug.), Mon. in Conch. Cab. p. S0, pl. lï, fig. 6-7.

La forme typique ne se trouve pas dans les récoltes de M. Blaise.

\section{Var. nigrofasciata Sow.}

1863. Cerithium nigrofasciatum Sowerby mss. in Reeve, Conch. Icon., pl. IX, fig. ̈̈s.

Tonkin. Ile aux Biches, Archipel des Faï-Tsi-Long, spécimens de coloration normale et unicolores; Anse du Crâne, Baie d'Along, spécimens de petite taille (M. Blaise).

Nous expliquons plus loin, à propos du Potamides tulpr'culatus, que le nom de Cerithium morus a été attribué à cette espèce par Bruguiere et que Lamarck a employé à tort le mème nom morus pour l'espèce dont il est question ici. C'est pourquoi nous adoptons au lieu de l'. morus Lamarck le nom de C. concisnm Hombron et Jacquinot qui nous parait sans aueun doute s'appliquer ḋ la présente espèce.

Cenithum gemsulatci Hombron et Jacquinot.

18'1. Cerithium gemmulatum Hoмввох ет JасQекот, royage au Póle sud, pl. ㄴ't, fig. 3, ' $, 7,8,9,10,39$, 40, 41 . 
18\%彳. Cerithium concisum var. gemmulatum Rorssear in Ноnввом et Jiconinot, Voyage au l'òle Sud, p. 103 (olsservations).

Baie d'Along (M. Blaise).

II. Vignal dont la compétence pour le genre Cerithium est bien connue, considère cette forme comme intermédiaire entre les Cerithium concisum (= morus Lk non Brug.), C.moniliferum el C. rariegatum.

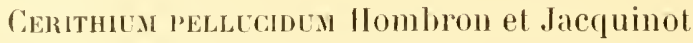
Var. depauperata Dautzenberg et H. Fischer.

181. Cerithium pellncirhm Honbroxetdacerixot, Voy. au Pòle Sud, pl. XXIII, fig. ำ, 2.j.

18:í. Rocsseat in Hombrox et Jacounot, Voy. au Póle sud, 1. 101.

183:\%. - patulum Sowenis, Thes. Conch. II, 1. $8 \overline{1}$, pl. CLXXIX, fig. 7'́.

18603. - Sow. Reeve, Conch., Icon., pli. I, $112.33^{34}, 33^{\mathrm{b}}$.

Isti7. giblerosum Fracexfeld, Moll. Novara, Il, palt. Ill, p. 7, pl. 1,fig. 7 I87I. - panulum Sow. v. Martens el LaNGKaYel, Donum Bismareli. p. 33.

1886. - - Sow. Y. Martexs, Moll. Mergui in Linn. Soc. Jourlı. XXI, p. 168 .

1587.

Tryox, Man of Conch., IX, p. 133, pl. 너, fig. 20-21, lig. 20 (pellucidum Homb). et Jac(puinot). 
1891. Cerithium patulum Sow. P. Fischer, Catal. IndoChine, loc. cit. P. Iti) (77 dı l. à p.).

1898. Cerithium (s.s.) patulum Sow. KoвeLt, Mon. in Conch. Call., 2o éd., p. 113, pl. 22.2. fig. 6,7 .

Tonkin. Baie d'Along (II. Blaise). Exemplaires diflérant du type par leur taille plus faible et leur sculpture moins nettenent tuberculeuse.

Lampina acstrabls Quoy el Gaimard.

1833. Cerithinm anstrale Quovel Cammard, Voy. Astrolalie, III, p. 131, pl. :3:;. fig. 7 (sul) nom. C. de Sydney).

184.1.

- Q. Lílexer, Icon. Coq. viy., 1. (6), pl. 8, lig. 2 .

181.3.

- Q. Deshayes in Lamarck, Anim. s. Vert., 2 éd. IX, 1. $32 \%$.

18:3:i.

- Q. Sowerry, Thes.Conch., ll, p. 884, jl. 18:i, fig. 266 .

1s66. Lampania australis REere (exparte), Conch. Icon., pl. I, fig. 价 lexcl. fig. 依).

186i7. - $\quad$ - AxGis, Australian Moll. in Proc. Z. S. L., p. 2018.

1886. C. (Lampania) australe Q. Watson, Challenger (ialstrop., p. $: 336$.

1887. Potamides (Lamparia) australis Trion(ex parle) Man. of. Conch., IX, p. If66, pl. 34. fig. 100, excl. fig. 99). 
1898. C. (Lampania) australe Q. Kiobert, Mon. in Conch. Cab., 2 éd., p. I3:, pl. 2:;, fig. 1 ì $\%$.

Tonkin. Ile aux Biches, archipel de Faï-Tsi-Long (M. Blaise).

Potanides (Terebrala) sulcatls Born. (non Murex sulcatus Cimelin = Tafon Adanson).

1778. Murex sulcatus Bons, Index rerum Mus. Caes. Vindob., p. 32't.

1779. Strombus Mangiom Scпï̈TE⿺, Flussconch., p. 383.

1780. Murex sulcatus Bons, Test. Mus. Caes. rindol)., 1. 30.

1790. - moluccamus (ivelix.Syst. Nat. Ed. XIII, 1. 33iti3.

1792. C'rithium sulcatum Brogutient, Encycl. méth., p. 186 .

1798. Strombus Mangos BoLtex, Mus. Boltenianum. p. 97.

1798. - Semistriatus Botrex, Mus. Boltenianum, 1. 97.

182.2. Cerithium sulcatum Brugr. Lasanick, Inim. s. Vert., III, p. 66.

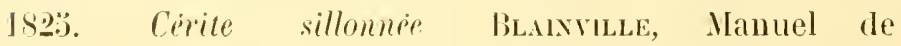
Mal., pl. 20. fig. :

1830. Cerithium sulcatum Sowerby, Genera of Sh. 1, lig. 3 .

1833. Cerite sillommie Qtoy et Gimuanw, Voy. Astrolabe, III. p. I2I, pl. $3^{\prime}$, fig. 2.2, 2.3.

1811. Cerithium sulcatum Br. Linxer, Icon. Coq. vir. p. 89, pl. 27, fig. I, I, 2, 2, 2 . 
1843. Cerithium sulcatum Br. Deshares in LAMARCK, Anim.s. Vert., és., IX, 1. $28 \%$.

18:2. - - Born. Möıcu, Catal. Yoldi, p.:̈7. 1852. - semistriatum Bolt. Mörch, Catal. Yoldi, p. :37. 183̈. - - semitrisulcatum Sow erву, Thes. Conch., II, 1. s84, pl. CLXXXY, lig. 206i3.

18\%3. - sulcatum Sowerby, Thes. Conch., II, p. 883, ן. CLXXXY, fig. 262 .

186:3. P'yrasus sulcatus ReEve, Conch. Icon., pl. I, fig. $\left.\right|^{a}, 1^{\mathrm{b}}, \mathrm{I}^{\mathrm{e}}$.

1863. - semisulcatus (Bolten) ReEve, Conch. Icon., pl. I, fig. 4. (var).

1887. Pot. (Terebralia) sulcatus Trion, Man. of Coneh., IX, p. 160, pl. 32, fig. $46,47$.

1857. - - semistrisulcatus Bolt. Tri Yon, Man. of Conch.,

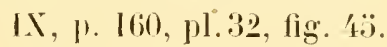

1891. - - sulcatus Born P. Fisenen, Catal. Indo Chine, loc.cit., 1).163(p. 79 du t. ì ए.).

I89S. C. (Pyr.) sulcatum Born Lionelt, Mon. in Conch. Cah., p'éd., p. 10, pl. 8, fig. 7,8 .

190' '. (Tereb.) sulcatus Born. II. Fischer et Dactzexberg, Catal. Indo-Chine in Mission Pavie, loc.cil., p. 低 (p. $2 \bar{\jmath}$ du t. à p.).

Tonkin. Ile aux Biches, Archipel des Faï-Tsi-Long (M.Blai se), nombreux exemplaires.

Baie d'Along, Anse du Crâne, sur les rochers à basse mer (M. Blaise).

Deshayes dans la 2o édition des Animaux sans vertèbres 
attribue la paternité du Cerithium sulcatum ì bluguière et dil que, d’après la loi de prioritéil faudrait reprendre lenom de molnrrams Gmelin qui est plus ancien. Cette erreur est d'autant moins explicable que Bruguière cite le I/urex suleatus Born farmi ses références.

Il y a lieu d'examiner si le Cerithium scmistriatum Bolteu (Stromlus) est une variété du P'otamides sulcatus ainsi Ifue Kiener l'a admis ou bien s'il doil constituer une espece distincte. Tryon, Reere, Sowerby maintiennent les deux espèces. T) après les spécimens que nous arons sous les yeux (collection bautzenberg), il nous semble qu il est impossible de fixer une ligne de démarcation entre les deux formes. Dans tous les cas, les noms semisulcatus et semilrisulratus sont à rejeter, et il faut adopter le nom de s'mistriatus pour la variété ou espèce lisse.

Potames timercelates (Limné) Burn.

(Cerithium borni sowerly emend.)

(Pl. I, fig. 16.)

1767. Strombus tuberculatus Lixé, Syst. nal. Edit. Xll. 1). 1213 .

ITAs. - - Limné Bors, Ind. Mus. Ciates. Vind., p. 오르.

І780. - - Bokx.Test.Mlus. Caes. Vindol., p. 노't. pl. 10, tig. 16, 17.

1790. - - Gimelix, Sỵst. nat. Eiit. XIII, 1. 33i2I.

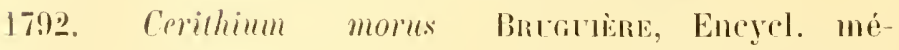
thoil. p. :80) (non Lamarek 18으르).

I82.2. - tuberculatum L. Lamarck, Inim. s. Tert. III, p. $7:$.

184. - carbonarium Phunere, \%eitsoler. I. Ma. lak.. p. 1'位. 
1S:32! Cerithium tomannense Exooux et Soluevet, Voy. Bonite, p. 60I, pl. 39, fig. 3. $4, \because)$.

18:33. Cerithium Bornii Sowerby, Thes. Conch., II, p. S69, pl. CLXXXII, lig. 17:\%.

186i. - Bornii Sow. ReEve, Conch.Icon., pl. II, lig. $2(i$.

1857. - carbonarium Hh. Thison, Man of Conch., 1X, 1. 133, ph. 21, fig. 2'tantum, excl. fig. 34.

$\mid 891$.

- P. Fischer, Catal. IndoChine, loc.cit., p. $161(77)$.

1898. - - - liobelt, Mon. in Conch. Cab. p. 276, pl. 17,fig. 3, 亿.

Tonkin. Ile Rousse, baie d'Along; ìle aux Biches, Archipel des Faï-Tsi-Long ( $\mathrm{M}$. Blaise).

Il résulte de l'examen des spécimens recueillis par M. Blaise que l'opercule de celle espèce : circulaire, multispiré à nucléus central, est relui d'un Potamides el non d'm Cerithium. Nous arons lait représenter pl. I, fig. Iti, un de ces spécimens, avec son opercule.

Le Cerithium tuberculatum Linné (Strombus), n'est acconpagné d’aucune référence.

Gimelin, p. 3:i21 a précisé l'espèce linnéenne en citant comme références: Seba, pl. :ö, fig. 21; Born, pl. 10, fig. 16. 17; Martini, pl. 1:37, fig. 1'90).

Lamarck a conservé le nom de $C$. tuberculatum pour l'espèce de Linné et de Gmelin en citant les mêmes références que celles ci-dessus mentionnées, plus celle de Lister, pl. 102', fig. 89 et le Cerithium morus Bruguière.

Ce Cerithium morus Bruguière, Encycl. méth., est accompagné des mèmes références : Lister, 1021, tig. 89; Seha, э.: fig. 201; Born, 10, lig. 16, 17; Martini, 1:37. fig. 1490. 
Il est done impossible d'attribuer le nom de C. Auberculatum à un autre espèce que celle figurée par Born, pl. 10, fig. 16. I 7 et à laquelle Sowerby a tommé en $18: 3 . ;$ le nom noweau de Cerithium Borni.

Lamarck a eu grand torl de donner le nom de morus a une espèce diflérente du C.morus de Bruguière dont il connaissait l'existence puisquil le cite comme synonyme du $C$. tuberculatum linné.

Le nom morus Lamarck ne peut done ètre maintenu pour aucun Cerithin puisquil existe un C.morus Bruguière plus ancien et il y aura lieu de sulstituer au nom morus Lamarck celui de Cerithium concisum llombron et Jacquinot, ainsi que nous l'arons fait en nous occupant plus baut de cette espece.

Trupaxotones axgchates fimelin.

(= fluriatilis Potiez et Mich. non Murex furiatilis Gmelin).

1780)! Turbo gramulatus minor ete. Martisi, Conch. Cab. IV. p. 3로. pl. CLV1l. fig. 1492.

1790! Wurer cingulatus Givels. Sirst. Nat. Ed.XIII, p. 3361 .

1798. Strombus Picta Boltex, Museum Boltenia1) 1 . р. 97.

I\$38. Cerithim fluriatile Potez el Muando, Galerie de Douai, I, p. 3(i:3. pl. XXXI, fig. 1!, 20.

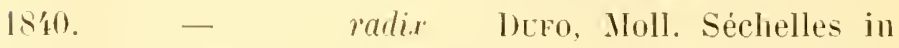
Ann. Sc. Nat., ?2 sér. XIV, p. 6i2.

184. - fluciatile P. el M. Liener, teon. Coq. vir.,

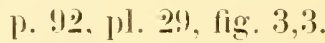

18'1： - - IlombroxetJacuchot, Voy. au Pòle Sud, pl. 23, fig. 10, II (variété se rapprochant 
de la var. microptera, mais avec le labre un peu moins détaché en arant. (Toir aussi Rousseau, 18\%'t.)

1841! Cerithium incisum Hoxibrox et Jacecixot, Voy. au Pòle sud. pl. 2?:3, fig. 8.9. (Voir aussi Roussean 18:3't.)

1843. - fluriatile P. et II. DeshaYes in LaMarcki. Anim. s. Vert., ㄹéd., IX. 1). 320 .

1849. - alatum Phicippr, Abbildungen, p. $\bar{\imath}$, pl. 1 , fig. 11 (c'est la variété eurypterus).

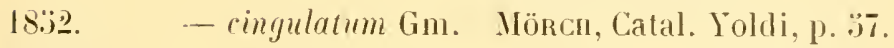
183̈́! - fluriatile Rorsseat in Howbrox et JAç̨inot, Voy. au Póle Sud, p. 98.

18\%'! - incisum Rousseal in Hombros et J.ç一ñot, Voy, au pòle Sud, p. 97.

18.3.\%. - fluciatile P. et II. Soweris. Thes. Conch., II, p. 891, pl. CLXXXVl, fig. 296 à 299 [la fig. 297 est la var. euryptera, et la fig. 299 la var. microptera].

1866. Tympanotonos fheriatilis P. et M. ReEre. Conch. Icon.. pl. Il, fig. $9^{d}, 9^{b}$.

1887. Pot. (Tympanotonos) flutiatilis Tr Yox, Man of Conch.IX, p. 139, pl. 31, fig. 38. 40 (pl. 39 var. microptera) pl. 3로, fig. 49. 3̈1, :32.

1891. Potamides fluciatilis P. et M. P. Fscher, Catal. IncloChine, loc. cit., 1). 16:3, (1. 79 du t. à p.). 


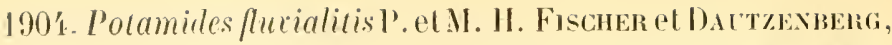
Calal. Indo-Chine in Mission Pavie, loc., cit. p. '́lti, (p. 27 (lı t. à p.) el var. enryptera.

Tonkin. Cua-Cam, Iläphong. Nombreux exemplaires en eau saumâtre sur les bancs de vase qui bordent le CuaCam et le Song Tam-Bac et qui découvrent à marée basse (II. Blaise).

Var. microptera liiener.

184. C'erithium microptera líreser, Icon. Coq. viv., p. 93, pl. 30, fig. $3,3$.

186ii. - fluciatile SowenBr, Thes. Conch., II, p. 891, pl. CLXXXY1, fig. 299 (var. microptera).

I866. Tympanotonos microptera Kien. Reeve, Conch. Icon., pl. Il, lig. $7^{\mathrm{a}}, 7^{\mathrm{b}}$.

1887. Pot. (Tympanotomos) fluciatilis Trios, Man. of Conch., Іх, p. 139, pl. 31, lig. 39 (var. microptera).

1898. C.(Tympanotonos) micropteraKobelt, Mon. in Conch., Cab. 2 éd., p. 7', pl. 14, fig. : :,6.

Tonkin. Anse du Cràne, baie d'Along (M. Blaise).

Mörch a eu raison de reprendre pour cette espèce le nom qui lui a été attribué dès 17!0 par Gmelin, lequel a indiqué comme référence la figure l19:2 de Martini qui est Irès probante.

Certthinea rhizoporaren A. Adams.

18:3'. Cerithidea Rhizoporarum A. Andxs, P. Z. S. L.p. Sï. 18:3. - rhizoporarum Ad. Sowerbr, Thes Conch., II. 1. 886, pl. CLXXXVI, fig. 273 
I866. Cerithidea rhizonorarum ReEve, Cunch. Icon., pl. II, fig. $133^{\mathrm{a}} \cdot 13^{\mathrm{b}}$.

1857. - - Mr. Trisos, Man. of Conch., IX, p. 162, pl.333,fig. $67,(i 2$.

1891. Pot. (Cerithidea) obtusus Lam. var. Rhizoporarum A.Ad.

P. Fiscuer, Catat. IndoChine, loc.cil., p. 16:3(p. 7?) du t. a p.).

1898. Cerilhium ('erithidea) rhi=oporarum A. Adams líbelt, Mon. in Conch.

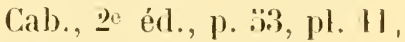
fig. 3, 3 .

1904. Cerithidea rhizoporarum. Id.H. Fischen el 1).дтtzexierg, Cilal. Indo-Chine in Mission Pavie, loc. cit., p. 416 ( $2 \bar{t}$ dlu t. à p.).

La forme Iypique n'existe pas dans les récoltes de II. Blaise.

\section{Var. Freitagi liobelı.}

18:18. Cerithium (C'erithidea) Freitagi linßelt, Mon. in Conch. Cab.. 2. éd., p. 1:32, pl. 22! lig. I, ‥

Tonkin. Ihe anx Biches, arrhipel des Faï-T'si-Long (II. Blaise).

Ceritunea Larghlaterti Philippi.

(= Formei .1. Molams).

18'S. Cerithium (lolamides) Largillierli PuLsur, Zeilschr. f. Malakoz,p. 이.

1899. Cerilhium Largillierli Pmopre, Mbloildungen p. 1:i, ,1. 1,fig. 7 .

18:3'. Cerithidea Fortmei 1. Amss, proe. \%. S. L., p. $8 . i$. 
183̈. Cerithidea (l'irenella) Largillierti Ph. A. Abass, Proc. Z. S. L., p. S's.

Is:i.;. Cerithim Montagnei Sowenвy (ex parte) (non dorb.) Thes. Conch. II, 1. 888 , p. CLXXXY1, lig. $286(\tan t u m)$.

Istit. Cerithidea Fortumei 1. Ad. Reeve, Conch.Icon.,pl.III. fign. $1: 3^{a}, 1: 3^{b}$.

1887. - - А. Id. Trios, Man.of Conch., К, p. 16, p1. 33, fig. 6i8, 66 (copie des ligures de Reere).

1898. Cerihlum (Cerilhidea) Largillierli P'h. Koвset, Mon. in Conch. Cab. 2 éd., 1. $(6.3$, pl. 13. fig. 2..3.

190'. Certhidea Fortunei A. Ad. II.Fischer el D.utzexiero, Catal. Indo-Chine in Mission Pavie, loc. vit., p. 4ll (27 du t. à p.).

Tonkin. Maïphong. Coquilles recueillies sur les banes de vase qui bordent les rives du Song Tam-Bac el du CuaCam et qui décourrent à marée basse. Eaux saumâtres, nombreux exemplaires(M. Blaise). Baied Along (M. Blaise).

Lassimilation de cette espece de Chine et d'Indo-Chine avec le C. Montagnei de Panama. indiquée par Sowerby et Tryon, nous paraît impossilıle ì admettre, dautant phus que le C. Largillierli est pourvu de varices el a le labre mon élalé, tandis que le C. Montagmei na pas de varices el a le labre largement étalé el se prolongeant a la base en avant du bord columellaire. Le C. Iargillierli nous paraìt par contre èlre identicue au C. Formuei.

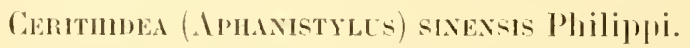

І899. Cerilhim sinense Phump, Mbbildungen, p. 18, 1) I, fig. I:3. 
1866.. Cerithidea sinensis Ph. ReEve, Conch. Ieon.,pl.11I, fig. 17 .

1887.

J. Mabille. Sur qฯ. Moll. du Tonkin in Bull. Soc. Mal. Fr., p. 1:38.

1857. C. (Aphanistylus) sinensis Tisox (ex parte), Man. of Conch., IX, p. 16', pl. :3', fig. 97 (excl. fig. 98).

1891. Pot. (1 phanistylus)Sinensis P. Frscuer, Catal. IndoChine, loc. cil., p. I6't (80). 1901.- - simensis H.Fischer el Dautzexbelig, Cat. Indo-Chine in Mission

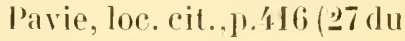
1. ¿̀ p.).

La forme typique n’est pas représentée dans les récoltes de M. Blaise.

Var. tonkiniana Nabille.

1887. Cerithidea tonkiniana J. Mabule, Sur qq. Moll. du Tonkin in Bull. Soc. Mal. Fr., p. 1:is.

Toukin. Région du delta Song'Tam-Bac, Hä̈phong. Coquilles recueillies en eau samatre sur les hancs de vase qui bordent les rives du Song Tam-Bac el du Cua-Cam el qui décourrent à basse mer; ces Mollusques montent sur les herbes qui poussent sur ces hancs (II. Blaise); Baie d'.llong (M. Blaise).

Les spécimens rapportés par M.Blaise concordent exactement aver ceux de la récolte de Balansa conservés an Muséum de Paris el sur lesquels J. Mabille à établi son C. tonkiniana; mais nous ne croyons pas qu'il y ait lieu de séflarer celle espèce du C. sinensis Phil. qui n’en diflère que par sa laille plus faible el sa forme un peu phus cylindrique: nous la considérons done comme une simple variélé : nous possédons en effet des spécimens de Shangaï 
(collection Dautzenberg) qui sont intermédiaires entre la figure de Philippi et nos spécimens du Tonkin. Le C. sinensis est d'ailleurs très variable sous le rapport de la seulpture : ses còtes sont plus ou moins rapprochées ou espacées sur les derniers tours et la base est plus ou moins aplatie ou convexe. Ces deux formes extrèmes sont d'ailleur's réunies sur le mème carton du Muséum.

Le Cerithidea (Aphanistylus) Moreleti décrit de l'Innami par Watleblet et dont il est question ci-apres est fort roisin du sinensis. auquel il a été réuni par Tryon à titre de variété. Le prentier se distingure en général du seeond par sa taille plus faible. ses còtes longitudinales un peu plus espacées et moins arquées, sa coloration plus uniforme. sa hase striée concentriquement (tandis que le $C$. simensis a la base lisse). Il est cependant malaicé de domner des earactères distinctifs absolus. car certains de nos spécimens de $C$ sinensis ne diffèrent guere du C. Horeleti que par leur spire à peine plus alténuée au sommel; par sa forme, le C. Moreleti se rapproche mème davantage du sinensis typique que de la variété tonkiniana.

Nous croyons toutefois plus purudent d'attendre la découverte de nouveaux matériaux avant de se prononcer -

Centhuea (Aphoxistyles) Moneleti Wattebled.

1ssti. Aphanistylus Moreleti Watterbed, Journ. de Conch., vol. XXXIT, p. .̈9. pl. II, fig. :

1857. Cerithidea (A phanistylus) sineusis Ph.var.Voreleti Watt. Trгox, Man. of Conch., IX. 1). 16i. pl. 31, fig. 98.

1891. Pot. (Aphanistyhes) Horeleti IV. P. Fisents, Calal. Indo-Chine, loc. cil. p. 16' (s0 (lu t. ì p.). 
1901. Pot.(Aphanistylus) Horeleti W. II. Fischer et DautzexBErg, Catal. Indo-Chine in Mission Pavie, loc. cil.

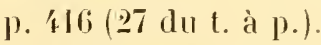

Tonkin. Région du Delta Song Tam-Bac (II. Blaise).

Tryon a réuni le C. Moreleti Wattebled comme variété au $C$. sinensis; nous maintenons provisoirement les deux espèces comme distinctes el renvoyons le lecteur à l'observation qui concerne le $C$. sinensis précédemment cité.

\section{Planaxis stlcates born.}

1780. Buccinum suleatum Bonx, Test. Mus. Caes. Vind.p. .:38, pl. X, fig. :3. (i.

1789. - - Born Bruguìke, Encycl. Méth., p. 2.\%.

1790. - pyramidale Gimelin, Syst. Nal. Ed. XIII, р. 3 4 88

1817. - sulcatum Brug. DiLlwry, Descr. Catal. II, p. 6il'

182.2. Planaxis suleata Lamarck, Anim. s. Vert. VII, p. औ̈l.

18묘. - umlulata Lamarci, Anim. s. Vert.

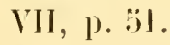

1823. - sulcata Lk. Buanvilue, Man.de Malac., 1. 40.;, pI. 16, fig. 4 .

1827. - - Crocin, Introd. to Conch. p. 33, pl. 16, fig. 18.

1830. - sulcatus Lk. Sowerbi, Gen. of Sh., p. 2, 1). 1, fig. 1 .

1832. - buccinoides Deshayes, Encycl. Méth., III, p. 776 .

1833. - sulcata Quor et Gumaro, Voy. Astrolabe, II, p. 186, pl.33, lig. 2:3-39. 


\begin{tabular}{|c|c|c|c|c|}
\hline 32 的. & Planari. & & us & $\begin{array}{l}\text { REETE, Conch. Syst., II, } \\
\text { p. } 238 \text {, pl. } 270 \text {. fig. } 1 .\end{array}$ \\
\hline 83. & - & & lcata & $\begin{array}{l}\text { Desnayes, in LAMARCK, } \\
\text { Anim. s. Vert. 2e Ed., 1X, } \\
\text { p. } 236 .\end{array}$ \\
\hline 13. & - & une & ulata & Deshayes, ibid.. 236. \\
\hline$\ddot{3} 2$. & - & & lcata & $\begin{array}{l}\text { Sowerbx, Conch. Manual } \\
\text { te Ed., p. 2’ö, fig. } 3603 .\end{array}$ \\
\hline$\ddot{99 .}$ & - & sulcat & $s$ Lk. & $\begin{array}{l}\text { Chexd, Man. de Conch, I, } \\
\text { p. } 303 \text {, fig. } 2142 .\end{array}$ \\
\hline 39. & - & undulat & is Lli. & Chexe, ibid., fig. 2140. \\
\hline$\pi$ & - & sulcatus & Born, & $\begin{array}{l}\text { Reeve, Conch. Icon., pl. I, } \\
\text { fig. } 4^{a} 4^{b} 4^{c} \text {. }\end{array}$ \\
\hline SI. & - & & Lk. & $\begin{array}{l}\text { De Rochebrixe, Docum. } \\
\text { sur la faune de la Cochin- } \\
\text { chine \& et du Cambodge. } \\
\text { Extr. Bull. Soc. Philomat., } \\
\text { Paris, p. 2l. }\end{array}$ \\
\hline 1887. & - & - & Born & $\begin{array}{l}\text { Tryox, (ex parte) Man. of } \\
\text { Conch. IX, p. } 276 \text {, pl. } 32 \text {, } \\
\text { fig. } 22,23 \text { (excl. var. plur. : } \\
\text { brericulus Desh., Sarigmi } \\
\text { Desh., obscums A. Ad., } \\
\text { breris Quoy). }\end{array}$ \\
\hline 1887. & - & - & Born & $\begin{array}{l}\text { Sowerby, Thes. Conch. } 1 \text {, } \\
\text { p. 171, pl. 483. fig. 10, } 11 \text {. }\end{array}$ \\
\hline $185 \%$ & - & - & Born & $\begin{array}{l}\text { L. Morlet, Journ. de } \\
\text { Conch. XXXYI, p. l'k't. }\end{array}$ \\
\hline 1891. & - & - & Born & $\begin{array}{l}\text { P. Fischer, Catal. Indo- } \\
\text { Chine, loc. cit., p. 16' } \\
\text { (80 du t. à p.). }\end{array}$ \\
\hline
\end{tabular}

Tonkin. Ile aux Biches, Archipel des Faï-T'si-Long, nombreux exemplaires (II. Blaise). 
Melayia Hamoxvllet Brot.

1857. Melania Hamonillei Brot, Journ. de Conch. XXXY, p. 32.
1887.

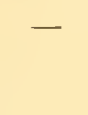
1891.

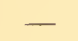
- Brot P. Fischer, Catal. Indo- Chine loc. cit. p. 167. (83 du t.à p.).
1893.
- Brot L. Morter, Journ. de Conch., rol. XLI, p. 13̈', pl. II, fig. 르, 으.
1904 .
- Brot h.Fischer el Dactzenberg. Catal. Indo-Chine in Mis sion Pavie. loc. cit. p. 417 (ㅇs dut. à p.).

Tonkin.Région du Song Lue-Nam. Haut Song-Bo(affluent du Song Luc-Nam), environs de Gang, ea ux douces légèrement ferrugineuses; sur des fonds de sable rasard; on les trouve parfois sur des troncs d'arlores ou des pierresimmergées dans le lit de la rivière(M. Blaise). Ruisseau sans nom, aflluent du Song Bo, eau douce. forèt de liha-Lé (décembre 1903) (1l. Blaise), exemplaires jeunes. Le nom local de cette espèce est Con òc vàn.

Cette espéce est fort variable sous le rapport de la sculpture : les cotes longitudinales des premiers tours sont souvent obsolètes ou absentes.

\section{Melaxia téberculata Müller.}

1773. Nerita tuberculata MüLler,Hist.Verm.,p. I91. 1779. Strombus costatus etc. Scnıöter, Fluss Conch. p. 373, pl. VIlI, fig. 14 . 
1786. Nerita mberculata ete. Canemsitz, Conch. Cab. IX, p. 183, pl. 134i, fig. I씨, 120.2 .

178!. Bulimus tuberculatus Bucgùìze, Encycl. Méth., p. 330 .

1801. Melanoides fasciolata Obivier, Voy. Emp. Othoman, atlas livr. P. VI, pl. 31 , fig. 7 .

Iร22. Melania - Oliv. Lamarck, Anim. s. Vert., IV, 2 p., p. 167 .

\begin{tabular}{|c|c|c|c|}
\hline 29. & - & truncatula & $\begin{array}{l}\text { LAMARck, Anim. s. Vert., } \\
\text { YI. } 2 \text { p.. p. I67. }\end{array}$ \\
\hline 893. & 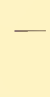 & fasciolata & 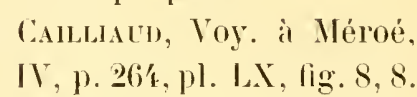 \\
\hline 30). & $\ldots$ & manriciae & $\begin{array}{l}\text { Jessox, Voy. Coquille, II, } \\
\text { p. 3i;'. }\end{array}$ \\
\hline
\end{tabular}

1834. - rirgulata Fér. Qcor et Gamman, Voy. Astrolabe,III, p.141,pl. :36, lig. I-1.

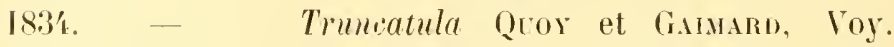
Astrolabe,III, p.It3, pl.̈̈li, fig. $\ddot{3}, 7$.
18:36. - pyramis Bessox. Journ. As. Soc. Bengal, $Y$, p. 780(non Reeve nec Hanley \& Theobald).
1838. - fasciolata Potiez et Michaub, Gal. de Douai, p. 260, pl. XXY'I, lig. $17,18$.
1838. - pmetata Potizz el Micnaud, (non Lk) Gal. de Douai,

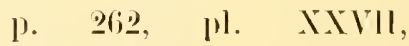 lig. I $13,16$.
18:38. - virgulala Fér. Deshayes in Lamarci, Anim. sans Vert., 2 Ed. VIII, p. $4: 37$.


18.28. Melania truncatula 1,k. Ротш: el Мichaud, Cial. de Douai I, p. 伍, pl. XXVll, fig. 니, 2․․‥

\begin{tabular}{|c|c|c|c|}
\hline 181. & - & & $\begin{array}{l}\text { DeLessert Rec.Coq.,pl.30, } \\
\text { fig. } 17^{\mathrm{a}}, 17^{\mathrm{a}} \text {. }\end{array}$ \\
\hline I81:. & - & tuberculata Nüll. & 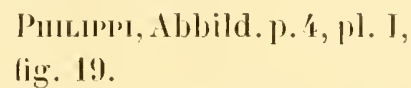 \\
\hline I84. & - & suturalis & 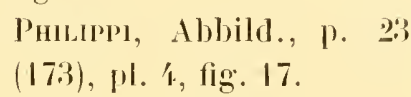 \\
\hline 1817. & - & rivularis & 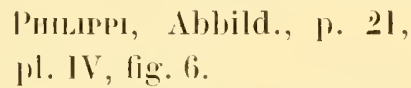 \\
\hline $189 \overline{0}$ & - & pyramis & 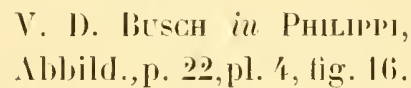 \\
\hline
\end{tabular}

1849. - tuberculata Müll. Morssox, Moll. de dava, p. 73, pl. XI, fig. 6, 7 .

18:38. Melanoides - 11. $\$$ A. MDavs, Gen. of rer. Moll. I, p. 297.

18:38. Melania Layardi Donis, Proc. 7. S.. p. 13:3. [8:39. - tuberculata Müll. Revve, Conch. Icon. pl. XIII, lig. 87, pl. XYl lig. 110.

18:i:. - virgulata Reeve, Conch. Icon., pl. XVI, fig. 109.

18:i\%. - punchlata Gratelortin Reeve, Conch. Icon., pl. XV., fig. 100).

18:9.9. - Layardi Dohrn ReEve, Conch. ICon., pl. $\mathrm{XV}$, fig. 10'.

18:39. gemmulata ReEve, Conch. ICon., pl. XIII, lig. 86.

1860. - beryllina Ввот, Rev. et Mag. de Zool. 1. 2(i2, pl. 17, fig. 18.

1860. - tuberculatavar. $\%$ Morelet, Séries Conch. II, p. 113.

1861. - Rothiana Morsson, Coq. rec. par Roth en Palestine, 1. 61. 
186я. Melania mberculata Müll. Bocrgulgxat, Malac. Mgé-

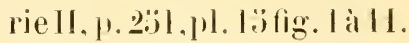

186.3. - rubropunctala Tristran, Proc. Zool. Soc., p. วัһl.

1870. - tigrina var. Hutton Haxley et Theobalo Conch. Indica, p. 屰, pl. I10. lig. I (? fig. 2).

1\$70. - Layardi Dohrn IIangeyel Tueobalo, Conch. Indica, p. $31, \mathrm{pl} .73$, lig. 8 et !)

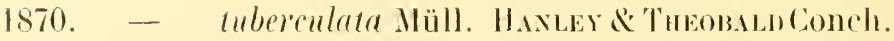
Indica, 1. 32, pl. LXXII, fig. 1 ì 4 .

1871, - - - Brot, Calal. Melanidae in Amer. Journ. of Conch. VI, p. 291 .

1871. - p!jramis Bens. Brot ibidem p. 2\$s!).

187?. - fasciolata Olivier Brot, Matér. III, p. 220, pl. I, fig. 7.

1872. - truncatula Lk. Ввот, Matér, III, P. 22, pl. I, fig. 13.

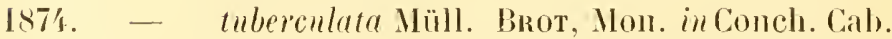

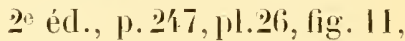
$11^{\mathrm{a}}$ aे $1 \mathrm{I}^{\mathrm{h}}$.

187\%. - mramis Bens. Bнот, Mon. in Conclı. Call.

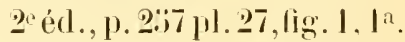

1874. - mberculata MüH. Jickesı, Fauma Moll. N. O. Ifr.p. 2.öl, pl. Ill fign. 7 ; pl. VII fig. 36 (coq. embryonnaire) (excl. syn. plur.)

187'. - rimlaris Ph. Isseu, Moll. borneens., p. 101 .

187i. - gemmulala Rye Morelet, Séries Coneh. IV. 1. 318. 
1891. Melania tuberculata Müll. L. MonLet, Journ. de Conch. Tol. XXXIX, p. 23't.

1891. - myramis Bens. J. Mortet, ibidem, 1. 23'.

1891. - tuberculata Müll. P. Fischer, Catal. IndoCline loc. cit., p. 169. (8;i du l. à p.).

1891. - virgulata Fér. var. P'. Fischer, ibidem, 1. I69) (8:3).

1891. - gemmulata Rve P. Fiscier, ibidem, p. I66; (82).

1897. - tuberculata Müll. Mıx Weber, Die Süss und Brackw. Moll. des Ind. Areh. p. : 66 .

1904. - - - - Il. Fischer el Daltzexberi, Catal. Indo-Chine in Mission Pavie, loc. cit. p. 418 (29) (tu t. aे p.).

190'. - myramis Bens. H. Fischer et Dautzenberg, ibidem p. 4.18 (29).

190'. - gemmulata Rve H. Fischer et Dautzenberg, ibidem 1). 417 (요).

190'. - virgulata Fér. var. H. Fischer et Dautzexbers, ibidem p. 418 (29).

Tonkin. Haut Song-Bo (M. Blaise). - Région du Song Luc-Nam, Luc-Nam, coquilles recueillies aux environs de Gang au pied du Nui-Gom, dans des mares destinées à recueillir les eaux de la montagne pour l'alimentation des rizières, eau absolument douce. peut èlre un peu ferrugineuse (M. Blaise). - Tam-Ra, environs de Luc-Nam, exemplaires jeunes (M. Blaise). - Rizières, Canal des Bambous (II. Blaise).

la plupart des auteurs qui se sont occupés de celte espèce ont considéré comme typique la forme du Nord de l'Afrique. Brot, dans sa monographie du Conch. Cab., indique le type du Nil, près d'Alexandrie. 
Or, Mïller dit expressément que son Nerita tuberculata provient de la cote du Coromandel et qu'il est alsondant en compagnie de Buccinum seabrum (qui est le Melania scabra).

Nous ne croyons pas qu’il soit possible de séparer du $\boldsymbol{M}$. tuberculata le $I$. pyramis Benson, qui est également de l'lnde et ne se distingue que par son test un peu plus solide et ses toursmoinsconvexes, et il y aurasansdoutelieu de réunir au $\boldsymbol{\|}$. uberculata, à litre de simples variétés les 1I. Grangeri Wattebled, M. l'ariei L. Morlet et peut-être même M. Lemyrei L. Morlet.

Littorina (Melaraphe) intermeda PhiJippi.

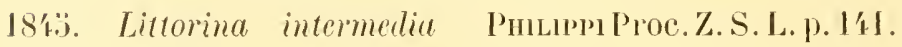

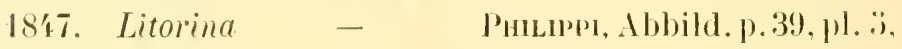
fig. 6-9.

18.i8. Littorina - Phil. ReEre, Conch. Icon., pl. XVIII, fig. I0I.

ISSI. Melaraphe - Phil. ve Rocuebroxe, Docum. sur la faune Malac. de la Cochinchine et du Cambodge, extr. Bull. Soc. Philomal. Paris, p. ำ.

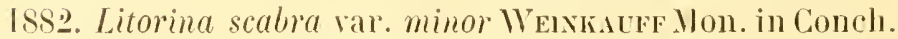
Cab., p. 37 (ex parte)pl.'́. fig. 16, 17, is (tantum).

1887. Littorina scabra vitr. intermedia Tnox, Man. of Conch.

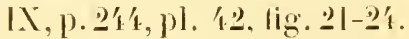

1851. Littorina intermedia

P. Fiscineli, Calal. IndoChine loc, eit. p. $170(86$ (lu t. à p.).

Tonkin. lle aux Biches, Baie d'Along (nombreux exemplaires); ile de la Surprise, baie de la Noix, Anse du Cuàne (.I. Blaise). - Région du has Delta : Cua-Cam. Maïphong, 
sur les enrochements qui bordent le Cua-Cam, eau saumitre (I. Blaise).

Cette forme diffère du L. scalra par sa petite taille et par sa sculpture consistant en stries très peu prolondes; elle nous paraît assez constante pour pouvoir ètre conservée comme espèce spéciale.

$$
\text { Littorina (Melahaphe) scabri Limué. }
$$

1738. Helix scabra Lowé, Syst. Nat., éd. X, 1. 770 .

\begin{tabular}{|c|c|c|c|}
\hline 1767. & - & - & $\begin{array}{l}\text { Lixié, Syst. Nat., éd. XIl, } \\
\text { p. lo't3. }\end{array}$ \\
\hline $1-00$ & - & & $\begin{array}{l}\text { Ginelin, Syst. Nat., éd. } \\
\text { XHI, р. } 3620 .\end{array}$ \\
\hline
\end{tabular}

179:. Helix scabra Linnaei Cumsit\%, Conch. Cab. X1, p. 283, pl. 2210, fig. 207', 2073 .

1829. Phasianella angulifera Lamarck, Anim. S. Yert. VII, p. :34.

\begin{tabular}{|c|c|c|c|c|}
\hline $18: 33$. & Littorina & - & & $\begin{array}{l}\text { Qcor el Gamind, Voy. } \\
\text { Astrol. 11, p. } 47 \text {, pl. } 33 \text {, } \\
\text { fig. 1-3. }\end{array}$ \\
\hline IS'3. & Phasianella & - & & 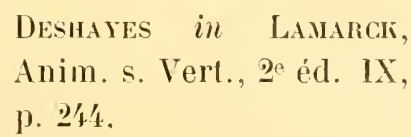 \\
\hline 181.7. & Litorina & - & & 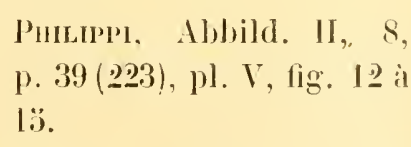 \\
\hline 1817. & - & scabra & $\mathrm{L}$. & $\begin{array}{l}\text { Pillippl, Abbild. II. 8, } \\
\text { p. } 37 \text { (2021), pl. V, fig. } 3-3, \text {, } \\
\text { 10, } 11 \text {, (tantum). }\end{array}$ \\
\hline $18 \%: 3$. & Helix & - & - & $\begin{array}{l}\text { HaNbeY, Ipsa Linn. Conch. } \\
\text { p. :36:\%. }\end{array}$ \\
\hline
\end{tabular}


1837. Litorina scabra L. Reeve, Conclı. Icon., 1.l. $\mathrm{V}, \mathrm{fig} .2 \mathrm{I}^{\mathrm{a}}, 2 \mathrm{I}^{\mathrm{b}}, 2 \mathrm{I}^{\mathrm{c}}$.

183ï. - ahenea ReEve, (ex p. non Lk). Conch. Icon., p]. 3, fig. I: $1: 3^{3}, 1 \%$.

18s1. Melaraphe scabra L. Rocuebrixe, Suppl. faune malac. Cochinchine et Cambodge, 1. 12.

1882. Litorina - - Weinkauff, Mon.in C.Cal)., 2. Ed., p. 37, pl. 4. fig. $7-10,12,16-18$.

1887. Littorina(Melaraphe)scabrat. Tryos, Man. of Conch. IX, P. 2 13, p]. 往, fig. $11-$ 1:3, $130-20$.

1889. Litlorina scabra? L. Monuet, I. de Conch., XXXYll, p. 1/4.

1891. - scabra P. Fiscmer, Catal. Indo Chine, loc. cit., p. $170(86$ (du t. à p.).

Tonkin. Ile de la Surprise; Baie de la Noix (M. Blaise). 11 est impossible a notre avis de distinguer le $L$. angulifera lk du L. scabra L. Liespèce de Lamarck est en effet basée sur des figures de Lister représentant une forme des Intilles qui possède une carène aussi accusée que les exemplaires typiques de l'Océan Indien. On rencontre d'autre part, dans locéan Indien, des individus à carène eflacée qui concordent parfaitement avec dautres spécimens des Indes Occidentales désignés ordinairement sous le nom d'ahemea Reeve.

LitTomiNa (Melabapie) Mel.aNosto.i. Garay.

183:. Littorina melanostoma 'Gras, Zool. Beechey Voy. p. 140. 


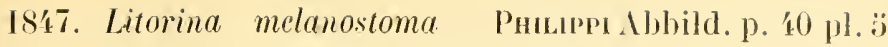
fig. 16.

1837. Littorina - Gray Revve, Conch. Icon., pl. IX, fig. $4 i^{a}$ el var. articulata Nevill, fig. 4:3

1881. Melaraphe melanostoma Gr. De Rochebrune, Docum. sur la faune malac. de la Cochinchine ef du Cambodge, extr. Bull. Soc. Philomat. Paris, p. 21.

1882. Litorina melanostoma Gr. Wesnkaufr, Mon. in Conch. Cab. 20 Ed.p. 41 pl.4 fig. 19. 1887. Littorina - - Tryon, Man. of Conch. IX p. 24: pl. 13, fig. 12 et 13 (var. articulata Nev.)

\begin{tabular}{|c|c|c|c|c|}
\hline 1889. & - & - & Gray & $\begin{array}{l}\text { L. Morlet, Journ. de } \\
\text { Conch. XXXVII, p. I } 7 .\end{array}$ \\
\hline s & - & - & $\mathrm{Gr}$. & $\begin{array}{l}\text { P. Fiscuer, Cat. Indo-Chine } \\
\text { loc. cit.p. } 17 \text { I } 87 \text { du t. ̀̀ p.) }\end{array}$ \\
\hline
\end{tabular}

Tonkin. IIaïphong, sur la vase (M. Blaise).

Tectarius nodulosus Gmelin.

1790. Trochus nodulosus Guects, Syst. Nat., Ed. N1I1 p. 338.

1817. Turbo trochiformis Diluwr, Descr. Cat. II., p. 8:6 (non Litorina trochiformis Philippi).

1833. Littorina pyramidalis Qror et Gamand, Voy. Astrolabe II, p. 482, pl. 33, fig. 12 à 15.

1843. - nodulosa Deshayes in Lamarck, 1 nim. s. Vert. 20 Ed. IX, ן. 20.: (non Pir. nec d'orb.) 
1846. Litorina myramidalis (1. et G. P'ntenpr, Abbild. p. 13, 1). II, fig. 10, 20.

18:37. Littorina

18.̈8. Tectarius
(1. el Ci. ReEve Conch. Icon, pl. II, fig. 14.

- Q. et G. H. el A Adums, Gen. of. rec. Moll. I, 1. 31:.

1882. Litorina (Tect.) nodulosus Gim. WenkactF, Mon. in Conch. Calb. p. 4i3. pl. ̈̈. lign. $\ddot{3}, \mathbf{6}$.

1887. Tectarius nodulosus Triox (ex-parte), Man. of, Conch. IX, p. 2:58, pl. 48, fig. 7 , $7:$, (tantum).

Tonkin. Ile aux Biches, Baie d’Along (M. Blaise).

Le Tectarius tubermlatus Gray, ćlabli sur la fig. 30 de la pl. (i de Wood (Index test.), est lit forme des Intilles, ì épines tris saillantes, que nous possédons de Cuba (Arango) el de la (iuadeloupe (Marie). Elle ne nous paraît ètre qu'une variété du $T$. tuberculatus d'Orbigny (= nodulosa d'Orb. non Desh. = dilatata d'Orl). $)$ quoique Philippi (Abbild.) considere les deux espèces comme distinctes : il ne sagit là que d'asperts différents produits par le déreloppement plus ou moins grand des tubercules.

La forme de l'océan Indien que nous possédons de Colombo (Cuillerel) el de Penang (Eudel) nous paraî ètre le T. malactanus Philippi ls't6 qui lui-mème se rattache intimement an $T$. vilis Menke, in Philippi 18łti, forme loutefois plus effilée el a lubercules plus gros sur le dernier tour. Cette dernière espece ne serait quime variété de la forme indo-chinoise rapportée par M. Blaise. Ces spécimens indo chinois nous semblent identiques an $T$. py/ramidalis Quoy et Ciamard, auquel on doit appliquer le nom plus ancien T. nodulosus fimelin. 
Tegtalus mlebgranes Philippi.

18's. Litorina millegrana Prntrpp, Abbild. HI, p. (ii; (:i!), pl. VII, fig. 1:i.

18\%.. Litlorina radiata Sollevet, Voy. Bonile, p.

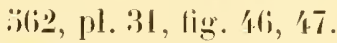

17:3. - granularis Reeve (non Giay), Conch. leon. pl. Xlll, fig. lisit, $68^{\mathrm{h}}$.

1837. - Feejeensis ReEve, Conch. Icon. pl. $\mathrm{XY}$, fig. $82, ;$, 82t.

18:8. - millegrana REETe, Conch. ICon, pl. XV1I, fig. 99.

18:39. Tectarius - Ph. H. el A. Andss, Cien. of. rec. Moll., I, p. 31:5.

18:9. Lilorina exigua DuNker, Malak. BI., 1. 220ti, pl. 2, fig. 3 .

1861. - - - IJnkel, Moll. Japon, P. 13, jl. II, fig. 3.

1871. - - Dkr Lischke, Jap. Mar. Moll. II, p. 70 .

187'. Liltorina (Melaraphe) viliensis Duxken, in Mus., Godelfroy, 1. 10:\%.

187\%. Litorina plena Calal. Mus. Goderfror, (hon Gould), V. p. 10:3.

1882. - millegrana Ph. Weiskatef, Mon. in Conch. Cab. Qe édition, p. 9s, pl. 1' ', lig. :i, 8.

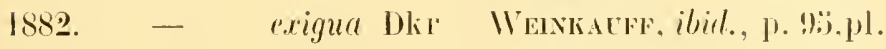
13, fig. 13, 14.

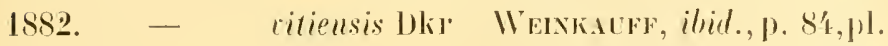
11, fig. 10, 11 .

1887. Tectarius granularis T'roon, (ex parte, non (iray), Man. of. Conch., IX, p. $2(60$, , 1. 48, fig. 8', 8.j. 
1891. Littorima radiata soul. P'. Fiscuen. Calal. Indo Chine, loc. cit., p. 171., (\$7 du t. iो p.).

Tonkin. Ile aux Biches, Bair d Ilong, (M. Blaise).

Bitulas Daltzenberga Waltebled (emend.).

18sti. Rithinia Dantzenbergiana W.tTteblew, Journ. de Conch., Vul, XXXIT, p. 66. 1). 1II, lig. 6.

1886. - subcarinala WatTEBLEd, ibid., p. 6i; pl. III, fig. 7 .

1891. - Dantenbergiana W. P. Fiscier, Catal. IndoChine, loc.-cit., 1\% 1720 (88 du t. ¿ il.).

1891. - subcarinata W. P. Fiscmen, ibid., p. 1720 (88 du t. à lr).

1900. Rythinia dantzonbergiana MöLtexdorre, Nachrichtsbl. d. d. Mal. Gies., p. I3!).

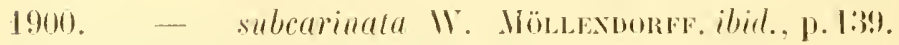
190'. Bithinia Dantzenbergiana W. H. Fischen el DActzexBlag Cialal. Indo-Chine, in Mlission l'avie, loc. cil.,

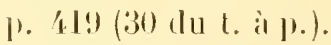

1901. - subearinala W. H. Fischen el Dattzexneri, ibiil., p. 1!! (30).

Tonkin. Région du Song Luc-Nam, Luc-Nan. Coquilles recueillies dans les rizières avoisinant Luc-Nam. Elles ont probablement les mêmes habitudes que les poissons de rizìres: elles doivent s'eufoncer dans la vase quand tes rizières commencent a se dessécher et ne reparaitre à la surface qu'à l'époque où l'eau y revient. Pendant la saison seche on ne froure an effel ancune lace de ces cofuilles dans les rizieres (11. Baise.) 
II nous semble que Möllendorff a en raison de penser que le $i$. subcarinata Wattebled n'est que le jeune B. Daut senbergiana.

Stexothyol moxillfera Beusom.

(PJ. Y fig. 13, 14, 1:3.)

18:36. Stenothyra monilifera Bexson, Inn. and Mag. N. 11. 2. Ser, XYII, p. 497.

1864. - - Bens. Chosse el Fischen, Journ. Conch. XII, p.33I.

1877. - - - Ilangey \& Theobild, Co. Ind. p. 17, pl. XXX'il fi心.

Coquille ovale, turbinée, assez solide, présentant une fente ombicale à peine indiquée. Spire tronquée au sommet. Trois tours subsistants convexes, séparés par une suture profonde et ormés de 17 à 18 sillons (sur le dernier tour) dans lesquels on distingue des séries d’impressions arrondies contiguës formant chaimette. Dernier tour légérement aplati en avant, descendant vers l'ouverture, oil il se contracte fortement. Péristome continu, très peu délaché. Ouverture petite, ohlique, subelliptique, anguleuse au sommet. Opercule assez épais, paucispiré.

Coloration d’un fauve verdàtre, péristome teinté de noir. Hauteur ' 1/2 largeur 3 mill.; ourerture : hateur 1 $1: 3 \mathrm{~mm}$., largeur I mm.

Tonkin. Région du llaut Luc-Nam, environs de Luc-Nam. Un seul exemplaire recueilli dans les rizières du poste de Luc-Nam. Des recherches faites dans les rizières avoisinantes n'ont pas permis d'en trouver d'autres spécimens (M. Blaisè).

Cette espèce est surtout remarquable par sa sculpture composée de sillons peu nombreux et articulés de petites cupules formant des chainettes analogues à celles qu'on 
observe chez certains bulliens appartenan aux gen res Sea phander, Philine, ainsi que chez les Actipon. Nous n'avons pas observédans le spécimen unique rapporté par $l l$. Blaise la bande claire signalée dans la diagnose originale; mais lous les autres caractères : taille, sculpture, etc., sont identiques.

Le lype de Bemson provenait de Mergui (Birmanie) et l'espèce a été signalée de la Corhinchine par MII. Crosse et Fischer, daprès desspécimens recueillis par M. Michau.

Palcuisa amplobromis Souleyel.

18.i․ Paludina ampulliformis Sovberet, Voy. Bonite, p. 3̈9. pl. 3I. fig. 220̈-27.

1863. - - Soul. Crosse et Fischer, Journ. de Conch., vol. Xl. p.373.

1870. - lecythis var. ampulliformis Souleyet.

Ilanley et Theobal, Conch. Indica, p. 32, pl. LXXYl, lig. 7.

187.;. - ampulliformis Soul. Morelet, sérjes Conch., p. 306 .

I8S6. - -.. - - $\quad$ L. Morlet, Col. du Tonkin (Jourdy) in Journ. de Couch., vol. Xxxil, p. 닐.

1891. - - P. Fiscalen, Catal, Indo chine, loc. rit., 1) 17!) (9:3 (la l. iो p.).

1898.

- r. MöllexwolsF, Die bin nen Moll. Annams, in. Nachriehtshl. d. 1. Mal. Ges., 1. 7!). 
190'Paludina ampulliformis Soul.II. Fischer et D.ATtzenbers, Catal. Indo Chine, in Mission Pavie, loc. cit., p. 4를 (33) clu t. à p.).

Tonkin. Rizières de Luc-Nam el rizières du Bas LucNam (nom vulgaire : con ôc búói) (1I. Blaise).

D’après A. Morelet, cette espèce ne différerait guère du I'. malleata Reeve (= abbreriata Reeve) du Japon.

Nous pensons que Paludina laosensis L. Norlet est très probablement synonyme de $I^{\prime}$. ampulliformis.

Paledina polizoxata frauenfeld.

1862. Tiripara polyzonata Fratenfelo, Verh. d.K.K. Zool., bot. Ges., p. $116 . ;$.

1863. Paludina

$186 \%$

$186: 3$.

1869.

1886.

1887. Vivipara
Fr. Reeve, Conch. Icon., ph. Vll, lig. $38^{z}, 38^{\mathrm{b}}, 38^{\mathrm{e}}$.

Fralenfeco, Verzeichniss d. Namen ron Paludinen in Yerh. d. li. K. Zool., bot., Ges., p. 6336 (76 du t. (i).).

Fr. v. Martens, Ueber Oslasiat. und neuholländische Paludinen, in Malak. Bl., p. 1 19.

Morelet, Lourn. de Conch., rol. XVII, p. 408.

L. Morlet, Coq. du Tonlin (Jourdy) in J. de Conch., XXXIY, p. 26?․ J. Mabille, Sur qq. Moll. du Tonkin, p. $19 ̋ 7$. 
1887. Pahulina polyzonata Dautzenberg el d'Ilamon vilte, Journ. de Conch., vol. XXXY, p. 219.

\begin{tabular}{|c|c|c|c|}
\hline I891. & - & - & $\begin{array}{l}\text { Fr. L. Morlet, Journ. de } \\
\text { Conch., vol. XXXIX, p. } \\
\text { 2́ï. }\end{array}$ \\
\hline 1891. & - & - & $\begin{array}{l}\text { - P. Fischen, Catal. Indo- } \\
\text { Chine, lot. cit., p. } 176(!) 2 \\
\text { du t. a p.). }\end{array}$ \\
\hline 1904. & - & - & 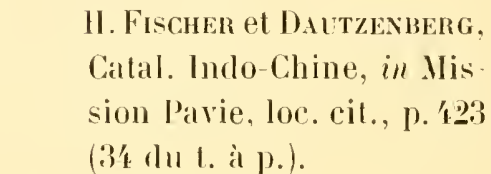 \\
\hline
\end{tabular}

Tonkin. Ruisseau sans nom, aflluent du Song Bo, forèt de Kha-Lé (décembre 1903) (II. Blaise), rizières, canal des Bambous (M. Blaise). Régions de Mai Xu, environs de Gang, Haut Song Bo (M. Blaise).

Var. Duchieri H. Fischer.

18!8. Paludina Duchieri II. Fischer, Notes sur la faune du Haut Tonkin, Moll. rec. par le $D^{\text {r }}$ Billet in Bull. scientifique de la France et de la Belgique, p. 2I, pl. XVIII, fig. 48, 49.

190'. - - II. F. H.Fisciler el Dautzexberi, Catal. Indo-Chine in Mission Pavie, loc. cit., p. 42.2 (33 du t. à p.).

Toukin. Rizières de Luc-Nam (M. Blaise); ruisseau sans nom, affluent du Song Bo, forèt de liha-Lé, décembre 190:3 (ll. Blaise). 
Les rẹcoltes de M. Blaise nous ont apporté la preuve qu'il existe tous les intermédiaires entre le $P$. polyzonata et le $I$. Duchieri: nous rattachons donc cette derniere forme à la première, en la considérant comme une simple variété, remarquable d'ailleurs par son épaisseur et son poids.

\section{Paludina quadiata Bensun.}

18\%2. Paludina quadrata Besson, Ann. \& Mag. N.II. fst Ser. IX, p. 487.

1816. ? -

- Bens. Pincmpre, Abbild. Il, p. 13\%,

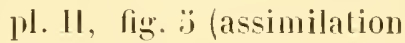
(douteuse).

18̈̈2. ? - - K Küster, Conch. Cab. 2 Ed., p. 22., pl. 4, fig. 1'1; pl. i. fig. 13, 1'

183\%. - - - Benson, Journ. Asiatic Soc. of beng. XXIV, P. 129 .

1863. ! - - Bens. Reeve, Conch. Icon.. pl. IV, fig. 17.

1863. - aeruginosa ReEve, Conch. Icon., pl.

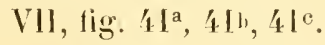

186.3. - quadrata Bens. v. Martexs, Ueber Ostasiatische und neuholländische Paludinen in Malak. B1., p. 1 \%:.

186:3. - aeruginosa Rve v. Martens, Ioc. cil., p. 146.

I860̈. - - - - Fracenfeld, Verz. Paludina in Verh. K. K. Zool., Bot. Ges. Wien, p. ว. 
1886. Paludina aeruginosa Rve L. Montet, Coq. du Tonkin (Jourdy) in Journ. de Conch., rol. XXXIV, p. 으.

185:\% - quadrata Bens. L. Mortet, Moll. Cambodge el Siam (Parie) in Journ. de Conch.. rol. XXXYH, P. 1:30,

1889. - aemiginosa Rve L. Monlet, ibid., p. I'9:.

I890. - qualrata Bens. Hevde, Mém. I1. N. Emp. Chinois. Moll. de la Vallée du fleuve Bleu. p. $176, \mathrm{pl}$. XL, fig. I0.

1890. - fantozatiana Hecoe, ibid., p $176, \mathrm{pl}$. XL, fig. 9.

18:00. - lapillorum IlEube, ibid., p. 177, pl. XL, fig. $11,11^{3}$.

1890. - aeruginosa Rve Ilevos, ibid. 1. 17:;, pl. XL, fig. $: \because,: \%$

1891. - quadrata Bens. P. Fiscier, Catal. IndoChine, loc. cit., p. 178 (9' du t. à p.).

189)1. - aeruginosa Rve P. Fischer, ibirl., p. ITs (94 du t. à p.).

I891. - - - L. Montet, Journ. de Conch., vol. XXXIX, p.24:\%.

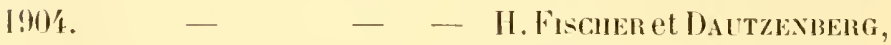
(ex parte), Catal. Indo-Chine il Mission Pavie, loc. cit. p. 42.2 (33) du t. à p.).

En l'absence de figuration originale et d'me description suffisante, il est fort difficile de se reudre compte de ce que ponvait être exactement le type lu Paludina quadrata Benson. Aussi les auteurs qui se sont occupés de cette 
espece ont-ils représenté sous le nom de $P$. quadrata des formes péu concordantes. Les plus anciennes figures, celles de Philippi, sont indiquées par eet auteur lui-mème comme d’une assimilation douteuse. Elles représentent une coquille courle, à tours assez convexes, ce qui ne concorde pas avee les mots anfractibus sex planulatis de la des cription originale de Benson. Reeve a représenté sous le nom de I'. quadrata une coquille presque lisse "orné parfois de 20113 cordoms plus ou moins obsolites m tandis que la descriplion de Benson porte liris subquimis. Enfin le R. P'. Ileude a représenté, toujours sous le nom de quadrata, unc coquille allongée à loul's subplans ef présentant environ:i earènes médiocrement saillantes. Nous possédons des spérimens d’une Paludine de Shanghä̈ fcollection Dautzenberg) qui concordent bien avec res dernières figures de lleude, mais qui démontrent qüil s'agit d’une espece fort variable sous le rupport llu nombre des carcines, puisfue nous en comptons : isurertains dentre eux, 3 ou mime seulement 2) sur les aulles.

11 nous semble que dans ces cilconstances it convient dadopter romme représentant le mieux le $P$. quadrata typique les figures pl. XL, fig. I0 et 10 de Heude. Mais autour de ce type viennent se grouper de nombreuses formes aflines et qui à notre avis ne peuvent ètre séparées comme especes distinctes. Celles qui se rapprochent le plus du type sont $P$. fantozatiana Heude et I'. lapillorum Heude, dont leur auteur lui-même dit, en parlant du l'. quadrata: "Les 2 espèces suivantes (fanto:atiana et lapillo rum) n’en sout (que des formes un peu dillérentes. ))

Par sa forme générale le $P$. quadrata se rapproche un peu du P. polyzonata; mais il en dilliere constamment par ses earènes bien moins accusées et ineolores, ainsi que par son bord columellaire plus arqué et dilaté de manière à recourril entiérement la fente ombilicale.

La forme typinfue de $P$ '. quadrara, définie par les réfé- 
rences de Benson 18't2 el de Ilende 1890 n'est pas représentée dans les récoltes de M. Baise.

Var. Reevei Dautzenbero et H. Fischer nom. nor.

Tonkin. Rizières de Luc- Nam (M. Blaise).

Le Paludina quadrata représenté par heeve est une variélé que nous désignerons sous le nom de var. Reerci, i carènes presque nulles el qui forme le passage entre le I'. quadrata typique et la var. aeruginosa.

\section{Viar. aeruginosa Reeve.}

Le $I$. aeruginosa décrit par Reeve en 186\%3, auquel nous rémnissons toutes les références postérieures de mème nom spécififue citées plus haut, à l'exception de celle du R. P. Heude (1890), ne dilfère dı l'. quadrata figuré par heeve en I863 et dont nous arons fait la var. Reexei, que par une diatation un peu phus grande du dernier tour et lia disparition romplète des carènes sauf celle de la périphérie. Reeve dit d'ailleurs de son l'. aeruginosa qu’il est très voisin du quadrata de la mème localité, d'une color"ation uniformément plus claire.

Il existe 'hez le l'. aeruginosa, sur les corlons, des cicatrices provenant de poils épidermiques. Nous n'en arons guère observé sur les quadrata typiques; mais ce caractère ne présente pas une importance ni une constance sulfisantes pour justifier une séparation spécifigue.

La var. aeruginosa ne se rencontre pas dans les récoltes de II. Blaise.

Var. bizonalis Möllendorff mss.

Tonkin. Riziores de Lue-Nam (Jl. Blaise).

MöIlendorff a distribué sous le non de l'aludina bizonalis une coquille qui ne diffère du I'. quadrata var. Reexei que par la présence de deux bandes marron régnant l'une immédiatement au-dessus, l'autre un peu au-dessous de 
de la suture. Sur la base du dernier tour, une 3 bande de mème couleur rìgne au-dessous de la périphérie.

Var. Heudei Dautzenberg et H. Fischer nom. nov.

Heude a figuré en 1890 sous le nom de $I^{\prime}$. aeruginosa Reeve une forme à tours convexes qui concorde bien yar sa forme générale arec l'aernginosa typique mais qui présente de nombreux cordons décurrents. Nous désignerons cette forme sous le nom de var. Meudei. Elle n'existe pas dans les récoltes de M. Blaise.

Ampularia gracilis hea.

18:36. Ampullaria gracilis Les, Proc. Ae. Sc., VIII, p. 110.

\begin{tabular}{|c|c|c|c|c|}
\hline $186^{\prime}$ '. & - & - & & $\begin{array}{l}\text { Les, Obs. G. Unio XI, } \\
\text { p. } 70 \text {, pl. } 22 \text {, fig. } 1\end{array}$ \\
\hline 1876. & - & - & Lea & $\begin{array}{l}\text { Crosie et Fischer, Joum. } \\
\text { de Conch., XXI, p. } 31: \text {. }\end{array}$ \\
\hline I89I. & - & - & & $\begin{array}{l}\text { P. Fischer, Calal. Indo } \\
\text { Chine, loc. cit., p. } 181 \text {, } \\
\text { (p. } 97 \text { du t. à p.). }\end{array}$ \\
\hline 903. & - & - & Lea & $\begin{array}{l}\text { BLanfolid, Land and } \\
\text { Freshw. Moll.from Siam in } \\
\text { Proc. Mal. Soc. L., p. } 281\end{array}$ \\
\hline 1904. & - & - & 一 & 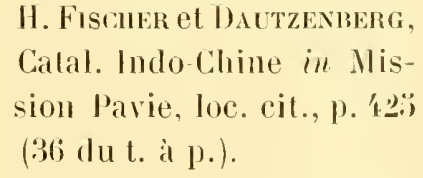 \\
\hline
\end{tabular}

Tonkin. Rizières du village de Van-len, Bas Luc-Nan (II. Blaise). - Rizières du village de Luc-Nanı (M. Blaise).

aMrellaria polita Deshayes.

1830. Ampullaria polita Desustes, Encycl.méth. Ir, p. 31 . 
1838. Ampullaria polita DeshaYes in landarCK, Anim. s. vert, 2: Ed. VIll. p. 淮。

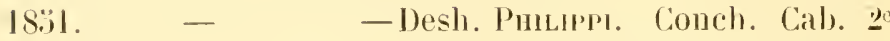
Ed., p. $\stackrel{2}{9}$, , pl. 8, fig. 1, I.

18... - - tirescens Deshave, Dict.class. Hist. Nat., pl. 87. lig. 2) (teste ipso et Morelet).

IS60. - polita Desh. v. Martexs, Proc. Z. S. L. p. I2.

186\%. - pagoda MoneLet. Journ. de Conch. vol. Xlll, p. 2297(teste ipso).

1S7\%. - polita Desh. A. Morelet, Séries Conch. IV, P. 291.

1887. - - - J. Mabilee, sur qq. Moll. du 'Tonkin, in Bull. Soc. Mal. Fr., l. 1.3.\%.

І88\%. - - - L. Morlet, Jourmal de Conch.,vol.XXX111,p.1:31.

I891. - - - P. Fiscuer, Calal. IndoChine, loc, cit.1). 181(97/1u t. à j.).

1892. - Brohardi Ginaxaer, Descr. d'ume nour. esp. d'Ampullaire, in Le Naturaliste. 1) 97 (monstruosité).

1901. - polita Desh., monstr. Brohardi (iangoer. Surti, Proc. Malac. Soc. London II p. 40, fign.

1903. - - Desh. Busxoms Land and Freshw. Moll. from Siam in Proc. Mal. Soc. I.. P. 291. 
190'. Ampullaria polita Desh. H. Fischen el Daltzexberg, Catal. Indo-Chine in Mlission Pavie, loc. cit. 1). 住: (37 du t. à p.)

Tonkin. Mare de la forêt de Tam-Ra, pr. Luc-Nam (Mi. Blaise). - Rizières du Tonkin (M. Blaise).

\section{Assminea BREVICLLA PfP}

$$
\text { (PI. V, tig. 9, 10, 11, 12.) }
$$

18:3. Hydrocena brecicula P'FenFer, Proc. Z. S. L., D. 306 .

\begin{tabular}{|c|c|c|c|}
\hline 18.38. & - & - & $\begin{array}{l}\text { II. et A. Mbdus, Gen. of } \\
\text { rec. Moll. II, p. } 299 .\end{array}$ \\
\hline $18: 38$. & - & - & $\begin{array}{l}\text { Prenfer, Mon. Pneumo- } \\
\text { nop. viv. Ier suppl., p. I:36. }\end{array}$ \\
\hline 186i:). & - & - & $\begin{array}{l}\text { Prenfrer, Mon. Pneumo- } \\
\text { nop. viv. 2e suppl., p. } 172 .\end{array}$ \\
\hline $186 \% 3$. & - & marginata & $\begin{array}{l}\text { A. Monelet, Journ. de } \\
\text { Conch., vol, Xlli. p. } 2266 \\
\text { (teste ipso). }\end{array}$ \\
\hline
\end{tabular}

1869. Ass. (Hydrocena) brevicula PEAse, Journ. de Conch, vol. XVll, p. I63.

187:3. Assiminea brevieula $\mathrm{P}[\mathrm{r}$ A. Monelet, Séries Conch. IV, p. 294 .

1876. Realia?

1891. Assiminea

1904.
- Premfer, Mon. Pneumonop. viv. 3 e suppl., p. 2910.

- Pfr P. Fisciner, Catal. Indo Chine, loc. eit., p. 183. (!)9 (lu t. à p.).

- Pfr H. Fischer el Dautzexberg, Catal. Indo-Chine, in Mis sion Parie, loc. cit., p. 1.26 (37 dut. à p.).

Tonkin. Song Kinh-Tay, Yen-Lun. Nombreux spécimens 
Peeneillis en eau saumatre sur les tiges des grandes herbes gui poussent sur les banes de vase bordant le fleuve el décourrant à basse mer.

Celle espèce nayant pas été figurée. nous en faisons représenter , l. I, fig. 9, 10, I1, deux exemplaires, l'un de coloration jame, l'autre, fig. I2, de coloration rouge. L'A. brevicula est caractérisé par sa taille relativenent grande, la solidité de son test, ses tours plans, son sillon subsutural bien accusé, sa columelle dilatée et rélléchie à la base, fortement déprimée au sommet ef formant un angle rentrant à sa jonction aver la callosité pariétale.

Assmined Fraxços Dautzenberg et II. Fischer n. sp. (l'l. V, tig. 3, 4, ï.)

Testa parta, orato-conoidea, solida, angustissime umbilicata, striis incrementi obsoletis ornata. Spira conoidea, apice obtusiusculo. Anfr. 5 comrexi, sutmra impressa discreti. A pertura oxata, obliqua, superne angulata, inferme subsinuata, marginibus callo crasso adnatoqne junctis. Columella arcuata umbilicum fere omnino obtegens. Labrum arcuatum, acutum.

Color pallide fulurs. Columella alba.

Longit 2, 7/10 $\mathrm{mm}$; latit $1,7 / 10 \mathrm{~mm}$. Apertura $1,1 / 2 \mathrm{~mm}$. alta, $11 / 10 \mathrm{~mm}$. Lata.

Coquille de petite taille, ovale, conoüde, trẻs étroitement ombiliquée, ornée senlement de stries d’accroissement obsolètes. Spire conoïde, un peu obtuse au sonmet, composée de "̈ tour's convexes, séparés par une suture bien accusée. Ouverture orale, oblique, un peu anguleuse au sommet et légèrement sinueuse à la base. Bords reliés par une callosilé appliquée épaisse. Colmmelle arquée, recourant presque enlièrement l’ombilic. Labre argué, tranchant.

Coloration d'un faure pàle. Columelle el callosité pariélale blanches. 
Tonkin. Song Luc-Nam, Ian-len. Cette espèce, que nous dédions à M. Francois, Chef des Travaux Pratiques à la Faculté des Sciences de l’aris, est représentíe par de nombreux spécimens recueillis sur les bancs de vase bordant les rives du lleuve. Ils se tiemment au pied des herbes à la laisse de mer haute et semblent virre sur des terrains humides mais non recourerts par les eaux. Dans cette partie du fleuve, bien que la marée se fasse sentir, les eaux sont absolument douces (M. Biaise).

Issminea interrepta Dautzenberg et II. Fischer n. sp. (Pl. V, lig. 6, 7, 8.)

Testa orato-conoidea, solida, imperforata, longitudinaliter tenuissime striata. Spira conica, apice acuminato, anfr. 7 snbplani, sutura impressa juncti ac paulo infia suturam carina transtersa filiformi ormati. A pertura otata, superne angulata, marginibus callo alnato junctis. Colmmella callosa, arcuata, superue appressa. Labrum aculum arenatum. Opereulum cornenm, tenue, paurispiratum.

Color fulrus, fascia subsuturali fusea et, in anfr. ultimo. fasciis insupra duabus intermplis et propis aperturam flammulis longitudinalibus pictus. A pex nigricans.

Lougit. Smm, latit. \& 1/2mm; apertura $31 / 2 \mathrm{~mm}$ alta. $3 \mathrm{~mm}$ lata.

Coquille ovale conoïde, solide, imperforée, ornée de stries d'accroissẹnent très fines. spire conique, acuminée au sommet, composée de $\bar{T}$ tours presque plans, séfrarés par une suture bien marquée et jrésentant sur les derniers tours, un peu an-dessous de la suture, une earène décurrente filiforme. Ouverture ovale, anguleuse au sommet. Bords réunis par ume callosité huisante, appliquée. Columelle arquée, déprimée au sommet. Lable arrué, tranchant. Opercule corné, mince, paucispiré.

Coloration faure, ornée d'une bande subsuturale brune, 
¿ laquelle viennent sadjoindre, sur le dernier tom: deux autres bandes interrompues de mème mance. A la fin de ce lour, les bandes somt remplacées par des flammules longitudinales étroites. Sommet noiràtre.

Tonkin. Song linh-Tay. Nombreux exomplaires recueillis en amont de Dong Trieu (M. Blaise).-Rizierestle Van len, I ex. (M. Blaise). Cetle espere est roisine par sal rolo ralion de l'A. rarinata Lea, mais elle ne présente pas la carène circumumbilicale terminée par me échancrurc du bord basal de louverture. Celle ci est entiere, of cest a peine si lou peut distingues chez quelques exemplares un léger cordon bordant de près la callosité columellaire.

Crabolnobes bobraxs J. Mabille.

1887. Cyclophorus dodrans .I. Mabmule, Moll. Tomk. diagn., J. I'́.

1887.

J. Maßille, Sur qा. Moll. du Tonlin in linll. Soc. Malac. de France, p. 19:i, pl. III, fig. 13.

1888. - -Mab. Axcer, Moll. du IlaulTonkin in Le Naturaliste, 1. S', fig. 3 (1. à p. p. 11).

1891. - songmaensis L. Morlet. Journ. de Couch., 1. $2 \overline{7}, 2.00 ;$ pl. I, lig. : :

1891. - - L.M.P. Fischer, Calal. IndoChine, loc. rit..p. Is\& (10) (lu t. aे l.).

IS91. - dorlrans Mab. P'. Fiscuen, ibidem.

ISIS. - - - II. Fiscuen, Moll, du IItTonlinin in linlt. Se. France et Bele... 1). 2.3. 
1904. Cly.cophorns dodrans Mab. H. Fischer el Dartzenberg, Catal. Indo-Chine in tlission Pavie, loc. cit., p. 4-28 (39) du l. à p.).

Tonkin. Mont Nui-tiom, près Luc-Nam (Il. Blaise).

\section{Crabornorits unices J. Mabille.}

(P. IV, lig. (i, 7, s.)

1887. Cyclophorus unicus J. Mabille, Moll. Tonk. diagn, p. 1'

1887.

- .. Mabille, Sur qq. Moll. du Tonkin, 1. 143.

1891. Cyclophorns unicus Mab. P. Frsener, Cant. Indo-Chine, loc. cil., p. Is8 (10' du 1. a p.).

19()$^{\prime}$.

11. Fischer et D.nutzendera, Cat. Indo-Chine in Mission Pavie, loc. cit., p. 4.?!) (40) (lı l. à p.).

Tonkin. He aux Biches, archipel des faï-Tsi-Long (M. Blaise).

Ce Cyclophorns, qui n’a pas été figuré par Mal)ille, mais dont nous avons examiné le tỵe conservé au Muséum de Paris, se distingue de ses congénères par sa forme solide, sa spire peu d́léée, ainsi que prar son périslome éprais, calleux, souvent dédoublé et étroitement rélléchi. II se mapproche un peu par sa forme générale du C.inooluhlus de Ceylan, tandis que sa coloration est analogue à celle du C. Jourdyi. ll se rapproche aussi par sa forme générale du C. Jerloni Benson des Nilgheries, mais a le péristome plus saillant el plus réfléchi et ne présente aucune trace de stries spirales sur la base. 
Crabophomes Jockmo L. Morlet.

I8s6. Cyclophorus Jomrdyi L. Mortet, Jour'l. de Conch., vol. XXXIV, p. 260. 2081, ,1. XIY, lig. I, $\mathrm{I}^{\mathrm{a}}, \mathrm{I}^{\mathrm{b}}, \mathrm{I}^{\mathrm{e}}$.

1887.

- L. M. Dautzenperg el D'HanoxVILLE, Journ. de Concli.. rol. XXXY, p. 르르.

1887. - $\quad-\quad$ MamLle. Sur qq. Moll. du Tonkin, p. I's.3.

1889. - - - - L.MlorLet Jomrn. de Conch. vol. XXXvll, p. 1:3'.

1891. - - - P. Fischer, Catal. IndoChine, loc. eil., p. $187(103$ du t. à p.).

$190^{\prime}$

H. Fiscier el Daltzexberg, Calal. Indo-Chine in Mission Pavie, loc. eit., p. 't28 (39 dul t. ì p.).

Tonkin. Ile Krien, archipel des Fä̈-Tsi-Long (M. Blaise).

Cette espece est fort roisine du C. cryptomphalus Benson, dont elle se rapproche par son bord columellaire sourent très dilaté sur l'ombilic; mais elle en dillère par sa forme moins transversale, son ombilic sensiblement plus étroit et par sa taille plus faible.

\section{P'Terociclos D.rieler L. Morlet.}

1886. P'terocychs Manieli l. Mortet, Journ. de Conch.,p. $2(i 1,283$, pl. XII, lig. $2,2,2 a, 2 b, 20$

1s87. Pterocyclos - L. M.J. MabiLle, sur qu. Moll. du Tonkin, I. I't3. 
1591. Pterocyclus Danieli L. M. P. Fischer, Catal. IndoChine, loc. cit., p. I89 (10.̈ du t. à p.).

$190 \prime$.

- - II. Fischen el Dautzexberi, Catal. Indo-Chine in Mlission Pavie, p. 130 (位 du 1. वे p.)

Tonkin. Lle Kirieu (I. Blaise); Dong-Trieu, région du Soug-Kinh-Thay. Les spécimens de cette seconde localité concordent avec ceux à ombilic plus ouvert que nous avons recus de II. Frühstorfer, sous le nom manuscrit de subsp. euryomphala Möllendorfi.

\section{Lagochilus scissimakgo Benson.}

(1'. I1, tig. 14, 13.)

I836. Cyclophorus? scissimargo Bexsox, Ann. Mag. N. II., $2{ }^{\mathrm{d}}$ Ser. XVll, p. 228.

1838. - ? - Bens. P'Feiffer, Mon. Pneum., fer Suppl., p. 61.

1860.

- - PFenter, Novit. Conch. I, p. I'1, pl. XXXVil. fig. 19-21.

1861. - - - Reeve, Conch. Icon., pl. XX. lig. 10\%.

186'. Lagocheilus

- - Blanfort, Ann. Mag. N. H., $3^{\mathrm{d}}$ Ser., XIII, p. 4.3 ?.

1863. Cyclophorus - - - Preifrer Mon. Pneumon., 2. Suppl., p. 76 .

1870. Lagocheilus scissimargo Bens. Hanlex el Theobald, Conch. Indica., p.3, pl. VI, lig. 7.

1876. Cycl. (Lagocheilus) scissimargo PFeiffer, Mon. Pneum., 3 Suppl., p. 122, 409. 
1889. Lagocheilus scissimargo Bens. L. MonLet, Journ. Conch. XXXYl, p. I:

1891. Lagochitus - - P. Fascuen, Catal. IndoChine, Joc cil., p. 190 (106 dul. a p p.).

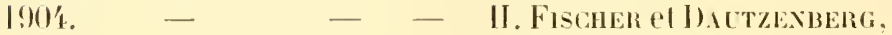
Caltal. Indo-Chine in Mission Pavie. loc. cil. p. 位 ('1으 du t. ì p.).

Nons figurons. pl. N, lig. 1', lï, un spécimen bien conforme an type de Benson, en provenance de That-kié (rolonel Messager). Celle forme typique n'est pas reprisentée dans les réeoltes de M. Blaise.

Il nous semble que les L. Mariei L. Norlet (.Iourn. de

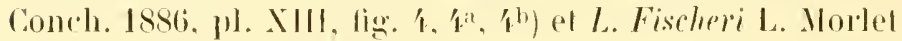

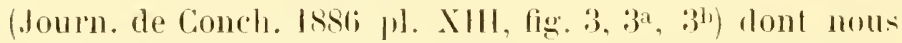
représentons ì noureat les types pl. IT, fig. !) It) et lli, I7 ne sont que des variélés aplaties du l. scissimargo benson, et que le L. Matgenmiilleri Nabille (Bull. Sor. Nal. Fr. IS87, pl. IV, fig. I 1) est strictrment symonyme du L. Mariei. Le L. altispirmm Möllendorfi mss. (in H. Fiscmen et DattzexBenc, Catal. Indo-Chine in Mission Parie, loce cit., p. 130, p. Al du l. à p.) me nous parat meme pas une variété du L. scissimargo.

Var. major bautzenberge et II. Fischer n. Var. (Pl. N, lig. 11, 1:2.)

Nous représentons ici, à litre de comparaison aree les spécimens réroltós par M. Blaise deux exemplaires qui uous ont été envoyés de That-Khé par M. Messager. Ils sont remarquables jar leur grande taille et nous proposons pour eux la variété nourelle major. 
Variélé carinata Dautzenberg el II. Fischer n. var.

(PI. II, Iig. 1:3.)

Cette nouvelle variété se caractérise par l'existence de plusieurs carènes bien arcusées; la coloration est d'un faure claire uniforme, dépourvu de taches.

Tonkin. Ile Krieu (.I. Blaive).

Pepxi Donn Dautzenberg.

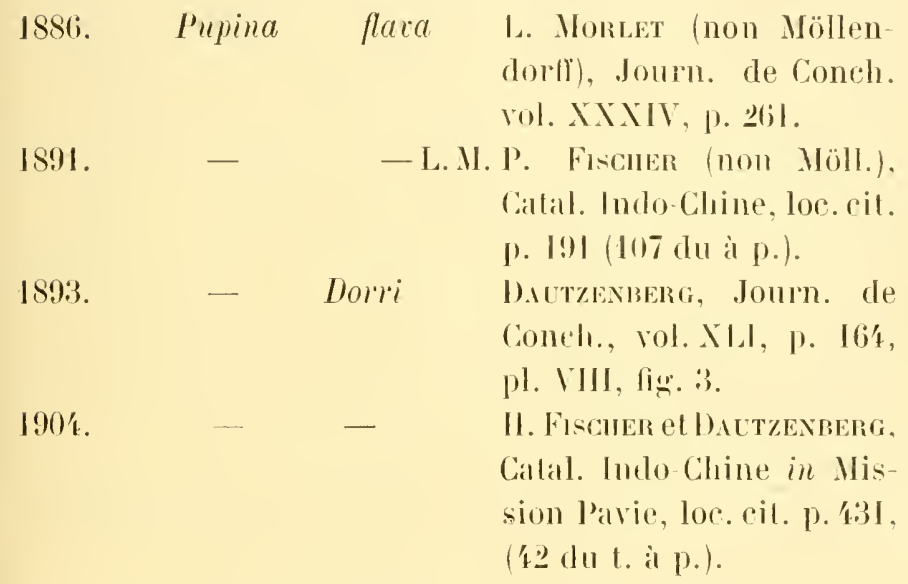

Tonkin. He Krieu. Baie d'Along (M. Blaise). DongTrieu, I ex. recueilli sur les ranes des arlousles qui poussent sur les rochers.

Hrgocrstis gravina Benson.

18:36. (mars) Megalomastoma gracidum Bexsox, Ann. Mag. N. II., 2. Ser. XVII, p. 229.

183̈6. (octobre) Cyclostoma pollex (iot tu, Proc. Boston Soc., p. 13.

183̈8. Megalomastoma gratidum Premres, Mon. Pneumon. viv. suppl., p. 79. 
18.i9. Hyboeystis gratila Bexsox, Inn. Mag. N. II. 3'ser. 11. 1. 91.

1860. - - Bens. Premfer, Novil. Conch. I, р. 123, 11. 3.і, lig. 1,2. (Sub. nom. Ilegalomastoma gratidum), el fig. 3,4 var. minor mocarnea.

1862. Cyclostoma pollex Goulu, Otia Conch., extr. P. Boston Soc. N. H., p. 2.21.

1S6:3. Hybocystis gratida Bens. Prenfer, Mon. P'nemm. vir., 2 suppl., p. $: 37$.

1876. - - - Premfer, 11011. Pneum. viv. 3 suppl., p. 391.

1887. Pollicaria - - Sowerbi, Thes. Conch. III. 1.l. $24 i 3$, fig. :i.

I887. Hybocystis C'rossei DAUTZEXBER el b'llayoxville, Journ. de Conch. 1. 르으, pl. I'lll, lig. 4 .

1887. - Rochebruni .I. Mabilee, Moll. Tonk. (liagil..p. I?.

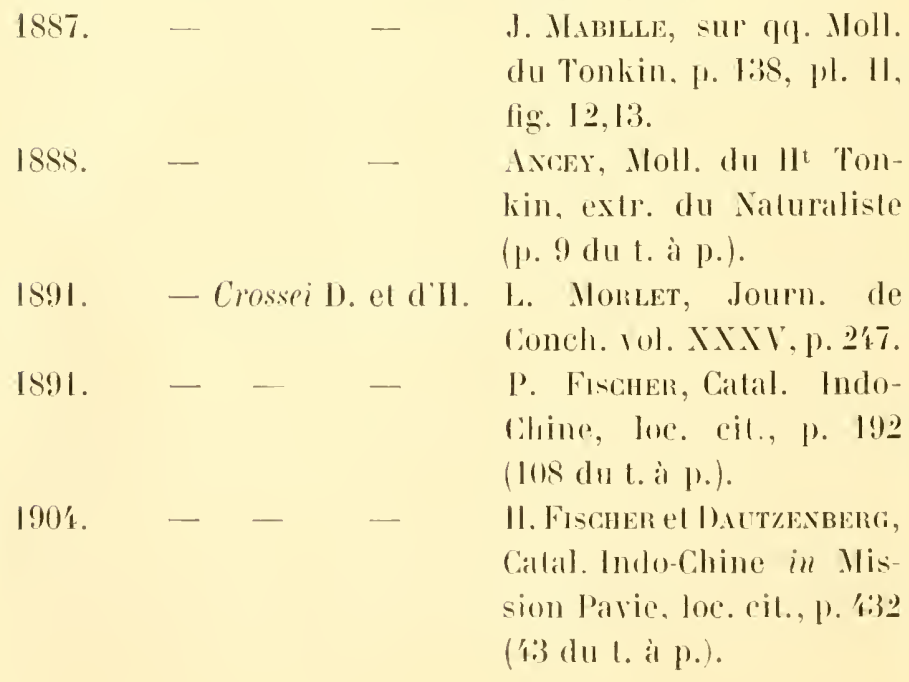


Tonkin. Mont Nui-Gom près Luc-Nam, 1 grand exemplaire (M. Blaise).

Il résulte de l'examen des nombreux matériaux rapportés du Tonkin par M. le Cl Messager qu’il est impossible de séparer l'Hybocystis Rochelrmei Mab. = Crossei D. et d'H. de I'I. gracida Benson, de la Birmanie méridionale. Les caractères différentiels indiqués par M. Ancey (dernier tour beaucoup plus haut et ouverture beaucoup plus grande) ne présentent en effet aucune constance, et nous avons entre les formes extrèmes toute une série de passages insensibles.

\section{Natica macelosi Lamarek.}

1781. Pellis

tigrina Cuessitz, Conch. Cab. I, p. 26ii, pl. GLXXXVII, fig. 189?, I893.

1790. Nerita canrena var. ๘. Giselix (non Linné) Syst. Nilt., éd. XllI, p. 3670.

1817

- - - L. Dllewry (non Limné), Deser. Catal. II, p. $9 \bar{\imath}$.

1822. Natica maculosa Laмarck, Anim.s.Vert. VI, 2 P., p. 202 .

1820. - javanica Lamarck, Anim.s. Iert. YI, 2 p., p. 204 .

1838. - maculosa DeshaYes, in LaMARck, Anim. s. Vert., 2e éd. VIll, p. 6 '1.

1838. - Javanica DeshaYes in Lamarck, ibid. p. 6 亿彳

181. - maculosa Lk. Delessert, Rec. de Coq. pl. 32, fig. 1' $\mathbf{t}^{\mathrm{a}}, 1 \mathbf{4}^{\mathrm{b}}$.

1841. - Jacanica - Debessert, Rec. de Coq., pI. 32, fig. $16^{\mathrm{a}}, 16^{\mathrm{b}}$. 
8.i2. Natica pellis tigrina Pmump, Mon. in Comels. Cab., 2e éd., p. 20, pl. 2, fig. 19. 20 et pl. I: lis. lig. I, I (rar. maculosa Lk), 2. 2 (var. jacanica Lk).

\begin{tabular}{|c|c|c|c|c|}
\hline ;ii. & - & maculosa & Lli & $\begin{array}{l}\text { ReEve, Conch. Icon., pl. } \\
\text { XlII, fig. :̈̈. }\end{array}$ \\
\hline 1860. & - & - & - & Ү. MARTEN, P.Z.S.L.J.IT. \\
\hline 1886. & - & - & - & $\begin{array}{l}\text { ThYox, llan. of Conch. } \\
\text { V111, p. 16, pl. } 3 \text {, fig. } 3 ; 3 .\end{array}$ \\
\hline $188 \pi$. & - & - & & $\begin{array}{l}\text { Soweris, Thes. Conch. } \mathrm{V} \text {, } \\
\text { p. St. pl. 4i.i., tig. } 13 .\end{array}$ \\
\hline 1889. & - & - & - & $\begin{array}{l}\text { L. MonLet, Journ. de } \\
\text { Coneh. vol.XXXII, p.li,i. }\end{array}$ \\
\hline Is89. & - & - & & $\begin{array}{l}\text { Crosse el Fiscmer, Jom'n. } \\
\text { de Conch. XXXYI, p. 28!). }\end{array}$ \\
\hline 91. & - & 一 & $\ldots$ & $\begin{array}{l}\text { P. Fiscuer, Catal. Indo- } \\
\text { Chine, loc. vit., p. l!) (110) }\end{array}$ \\
\hline
\end{tabular}

Tonlin. Mo-Son, entrée du Cua-Cam (1I. Blaise).

Le nom Pellis digriud nétant pas binominal dans Chemnilz et nayant pas été eonfirmé avant la date de 182.2, il nous semble que les règles de la monenclature exigent que le nom maculosa lamarek soit eonservé pour l'espere dont il est ruestion.

Le Tatica pellis tigrina de Reere et de Sowerby nest pas l'espèce figurér sous ce nom par Chemnitz, mais bien celle a laquelle Recluz a attribue le nom de $\mathrm{X}$. cariolaria et qui provient de l'Afrifue occidentale. Le 2 . javanira n'est quine variété de potite taille al allongée dı $N$. maculosa.

\section{P'seumothochatela Moghot l'feiffej}

lstie. Trochatella Mouhoti P'renfere, Proc. \%. S. L., pl. $2 \overline{7}$, p. XXXYl, lig. I'́ I86ii. - - Preiffer. Mon. Pnenm., 20 sแpl., p. ㄴ⒊ 
186iti. Trochatella Mouhoti Presfere, Novit. Conch. II,

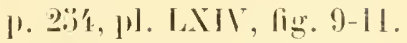

1867. - - Pfi v. M.natexs, Prellss. Exp. II, p. lis.

Isti. - - Plemer, Mon, Pneum. viv., 3e suppl., p. 2te?.

1s\$6. Melicina (Trochatella) Monhoti I. MonLet, Liste roq. ree. par II.Jourdy iniJourn. de Conch., rol. XXXIV, 1). 261 .

1887. Trochatella Mouhori P'fr. Mabiltes, sur qq. Moll. du Tonkin in Bull. Soc. Mal. Fi., p. 13\%.

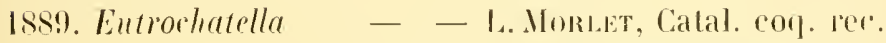
gar M. Pavie in Journ. de Comeh. vol. XXXYitp. 1:ī.

1891. H. (licotrochatella) - - I'. Fiscmer, Catal. IndoChine, lore rit. p. 1978 (11:3 dul to il p.).

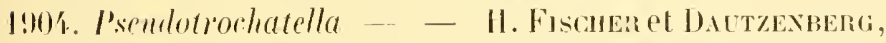
Catal. Indo-Chine in Mission lavie, loc. cit., p. 1333 ('1́. du l. à T.).

Tonkin. Ile Kirieu (Al. Blaise). Baie d'Along, ile de la Surprise (.I. Blaise).

Les spécimens rectieillis par M. Blaise ont le péristome plus épais que dans la forme typique.

Pshomotrochatela .Jotrovi (Crosse mss.) Daulzenberg.

1860. Helicina (Trochatella) Ciredleriana var. (non Möllendorli) L. Mor Let. Liste des coquilles rec. au Tonkin par M. Jourdy, in Journ. de Conch., vol. XXXIV, p.261. 
189:i. Geotrochatella Jourdyi Crosse mss. Dirtzexibrg, Journ. de Conch., rol. XLIII, p. 2:;, pl. V, fig. 1 .

1904. Psendotrochatella Jourdyi Crosse mss. 11. Fisciner et Dantzexberg, Catal. IndoChine in Mlission Pavie, loc. cil. p. 1333 俰 du t. à p.).

Tonkin. lle Kirieu (M. Baise).

Il est possible que la découverte de nombreux matériaux conduise plus tard à considérer celle espèce comme une simple variété à plis plus nombreux du P's. Monhoti.

Nebita inscilata Recluz.

\begin{tabular}{|c|c|c|c|}
\hline $107 \%$ & Nerita & insculpta & Recluz, Rev. Zool., p. 1̈̈2. \\
\hline 1811. & - & Listeri & $\begin{array}{l}\text { Reclez, Rev. Zool., p. } 177 \\
\text { (synon. de } N \text {. P'acifica teste } \\
\text { ipso). }\end{array}$ \\
\hline 1811. & - & georgina & RecLCz, Rev. Zool., p. 180. \\
\hline 11 & - & arcta & $\begin{array}{l}\text { Hombrox el Jacecinot, } \\
\text { Yoy. au Pòle sud, p. } 62 \\
\text { (18̈̈́), pl. 16, fig. 12, } 13 .\end{array}$ \\
\hline $18 \ddot{30 .}$ & - & insculpta & $\begin{array}{l}\text { Recuez, Journ. de Conch. } \\
\text { I, p. } 28 \text { ?. }\end{array}$ \\
\hline 850. & - & georgina & $\begin{array}{l}\text { Recluz, Journ. de Conch. } \\
\text { I, p. } 282, \text { pl. II, fig. } 10 .\end{array}$ \\
\hline 1830. & - & Pacifica & $\begin{array}{l}\text { Reculz, Journ. de Conch. } \\
\text { I, p. } 283 \text { (avec indication } \\
\text { erronée de pl. 11, fig. 10). }\end{array}$ \\
\hline 1852. & - & Georgina Recl. & $\begin{array}{l}\text { Socleset, Voy. Bonite, } \\
\text { Zool. II, p. ̈̈(66, pl. } 3 \text { ', fig. Ö } \\
\text { (sub nom. Nérite geor- } \\
\text { gienne). }\end{array}$ \\
\hline
\end{tabular}


18:31. Nerita arcta

Rocsseau in llombrox et IAconnot, Voy. al póle Sud, p. 62 (planches pa. rues en 1811).

1833. - Listeri Recl. ReEve, Conch. Iron., pl. VIII, fig, $36^{\mathrm{a}}, 36^{\mathrm{l}}$.

18:3. - Georgina - Reves, Conch. Icon., pl. XVII, fig. 7'缺,

1874. - Birmanica Ph. var. minor Dexker, Catal. Mus. GodelTroy, V. P. 14, il, 1). 90 .

187'. - pacifica Recl. Dexien, Catal. Mus. Godeliroy $V$, p. I i:i.

1874. - Hilleana Dkr Denker, Catal. Mus. Godeffroy $\mathrm{V}, \mathrm{p}$. 14\%.

1889. - insculpla v. Martens, Mon. in Conch. Cab., 2e éd., p. 88, pl. 11 , fig. 1-'

1891. -

- Recl. P. Fischer, Catal. IndoChine, loc. cit. P. 199 (11:) (lu t. à p.).

Tonkin. Région du Bas Delta, Haïphong, eau saumâtre, sur les pierres qui bordent le Cua-Cam (II. Blaise).

Le Nerita Georgina de Recluz est beaucoup plus finement sculpté que le $\mathrm{N}$. Georgina de Souleyet.

\section{Nerita chamaeleon Linné.}

1738. Nerita Chamaeleon Lixié, Syst. Nat.,éd. X, p. 779 .

1761.

1767.
LINNÉ, Mus. Lud. Ulr., p. 681.

Linvé, Sys. Nat., éd. XII, p. $12 \%$ 


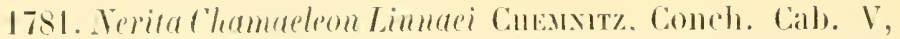
р. 310, p1. САххххи, lig. 1985-1991.

1790. - Thamaeleon

179:\%. - Stella

IsIT. - rlamacleom

1820.

IN:3. - -

18:38. - stolla Ch.

18'1. - strammlata

เง̂. - modesta

Isis. - scalirella

184: -

$18.0 \%$

18:\%' - -

15:ii. -

1s:ii. - sella Ch.
Givelix, syst. Nat., éd. XIII, p. 36s.

Chemser, Conch. Cah). $\mathrm{NI}$.

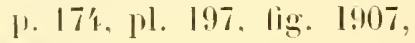
$190 \mathrm{~s}$.

D)Lifris, Deser. Catal. I, p. 100:3.

L.мынск, Inim. s. Vert. YI. Q․ 1. 1) 192.

Deshayes in Lamarci, Iniml. s. Velt., 2éd. VIII, 1. 606 .

1)eshises, ibid. P. 610.

Le Richlor, ReY. Zool.. P. :3'人。

Hombrox et Jacocisot, loy.aupolesud.pl. lti.fig. i. 6 (texte parm en 18:34).

Punlupr. Zeitsehr. f. Malakko\%. P. I'́.

P'mllippr. ibid.. P. I'.

RecL\%, Jom'n. de Conch., rol. 1, p. 20R(i.

- var. Rorssem in IJombrox et dacerixot, loy. all poile sud, p. lil (tes planches ont paru en (śl).

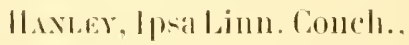

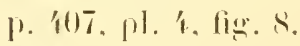

ReETe, Conch. I'oun., fl

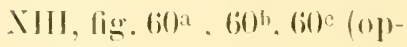
(ima). 
18.;\%). Terita squamulata Le G. Reeve, Conch. Icon., pl.

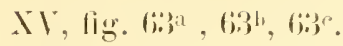

18:i:. - anmulata likeve, Comeh. Icon., [1]. Xlill, fig. $78^{a}, 7 s^{\prime \prime}$.

1878. I (Theliostyla) stella Ch. Trowatu, Jas Gebiss der schol. II, p. 19:i, pl. X'111 fig. - (radula).

1881. Pila stella (h. we Rochebroxe, Doeum. sur la famne malac. de lis Corhinchine et du Cambodge, extr. Bull. Soe. Philomat. Paris, p. 19.

1889. Nerila chamacleon Lin. L. Monuet, Journał de Conch., XXXYll, p. 1:̈.

1889. - - - v. Martexs, Mon. ill Conrlo. Calb. ㄹe éfl., p. I!), ए1. 2,

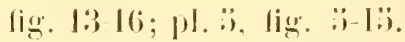

1891. - - L. P. Fiscaler, Catal. IndoChine, loc. cil., p. 198 (11' lu t. à p).

1891. - stella Ch. P. Fischere, ibirl. p. I9s, (II).

Tonkin. He aux Biches et anse dn Crune, Baie d'Along (II. Blaise).

Nerita Lixeata (Chemnitz) Gmelin.

1781. Teria lineata, ete. Chennitz, Conch. Cab. I. p. 297. 11. 191, fig. 19:8, 19:39.

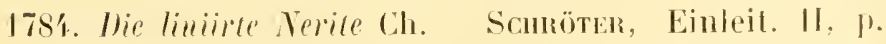
:33;i).

1790. Neritu lineala

Gimelix, Syst. Mat., élit.

NIII, p. 368'. 
1817. Nerita lineata Gils.

182. - - Gill

1838. - -

I81. - - Ch.

18:0. - -

I8:34. - - -

1878. N.(Pila)-
1880. Nerita -

186\%. - - -

I8:\%. - - -

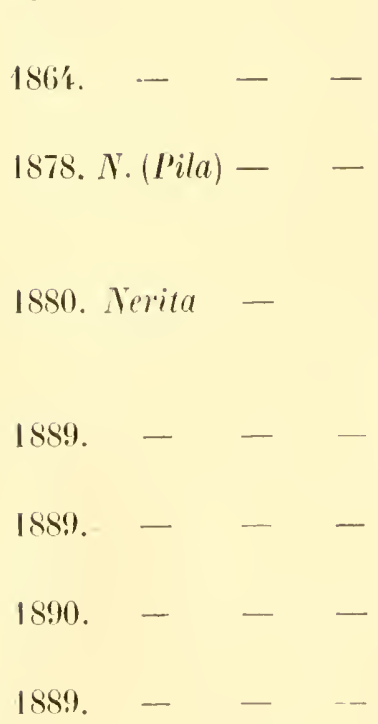

Dhlwy, Deser. Cat. II, p. 1001 .

Lamarck, Anim. s. Vert. หา, p., р. 19'.

DESHAYES iU LAMARCK, Anim. s. Vert., Edit. riil, p. 60s.

II OMBROX el JACQUXNT, Voy. au póle Sud, p. (in

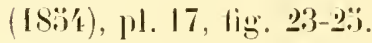

Reculz, Journ. de Conch.. I, p. 283.

Rousseat in Ilombrox \& Jacecinot, Voy. au Póle sud, p. (ii3.

Rieeve, Conch. Icon.. pl. III, fig. $133^{\mathrm{a}}, 13^{\mathrm{b}}$.

Crosse et Fischer. Journ. de Conch., XII. p. 330.

'Troscher, Das Gebiss der' schn. II. p. I90, pl. XVII, fig. .i. $\ddot{3}^{\mathrm{a}}$ (radula).

Texisox-Woons, Proc. Linu.. Soc. $\mathrm{N}$. S. W., I, 1. I20.

Crosse el Fischer, Journ. deConch., XXXYII, p. 290. L. Morlet, Journ. de Conch., XXXYll, p. 1:̈7. Crosse et Fischer, Journ. de Conch., XXXYlli, p. 17. ․ Mantexs, Mon, ill

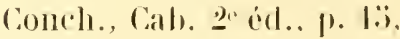
pl. I, fig. 3, 4; pl. 1. fig. $12-1 \%$. 
1891. Nerila lineata Ch.

P. Fiscuer, Calal. IndoChine, loc eit. p. 197 (11:3 (lu 1 . iे p.).

Tonkin. Anse du Cràne et ile des Biches, Baie d'Along (M. Blaise), Port Wallut (M. Baise).

Neritaxa Sowerbiaxa Recluz emend.

1842. Nerita Sorrerbiana Recuz, Proc. Z. S. L., p. 17\%.

1812. - pulchella Recucz, Proc. Z. S. 1.., p. $17: 3$.

18:33. Neritina mlehella Reeve, Conch. Icon., p]. XXI, fig. 91.

18\%氵. - Soterbiana Sowerвт, Thes. Conch. II, 1. : :

18:3̈. - mulchella Recl. Sowerbr, Thes. Conch.ll, 1.:330, pl.CXY, fig. $209-2011$.

18:3;. - Soxerbii Rec. ReEre, Conch.Icon.,p.XX, fig. 89 ! : $-899^{\mathrm{d}}$.

1879. - Soncerbiana Riecl. v. Mantexs, Mon. in Conch. Cab, 2ِérl., p. 171, pl. Is, fig. I-4 et 10 .

1881. - Soucrbii Recl. De Rochebnuxe, Bull. Soc. Philomal. Paris, p. 11.

1888. - Soucerbyana Rerl. Triox, Man. of Conch. X,

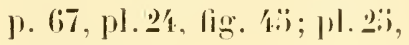
fig. 便-:il.

1891. Y. (Clithon) Sonerbyana Recl. P. Fiscmen, Calal. IntoChine, loc. cil., p. $200(116$ du 1. ̀े p.).

Tonkin. Baie d'Along, ean saumàtre à l'embonchure drs petits ruisseaux, espèce répandue parlout (M. Blaise). - Ile Rousse, archipel des Faï-Tsi-Long (II. Blaise). - lie 
aux Biches (M. Blaise). - Ile de la Cac-Ba, anse du Cranc, eall douce (M. BJaise).

Les nombreux spécimens récollés par M. liaise comprennent le grpe el les variétés maculofasciata, lactiflua intermittems, polysticta de v. Matrens, mulchella de Recluz, et hicn dautres auxquelles il ne nous semble pas ulile de dommer des noms.

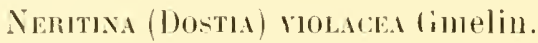

177!). Die lange schmale Nevite mit riolet blanen IIollen.

simröter, Flussoonth., j. :39:3.

1790. Terita riolacea Gimets.syst. Nal.éd Xlll, 1). 3(iski (basisur les lig. l:31. l:3' de lal pl. l:3 de Mallini).

18.20. Neritima crepulularia Laxanck, Inim. s. Viel. 11, 2. p., p. Is6i.

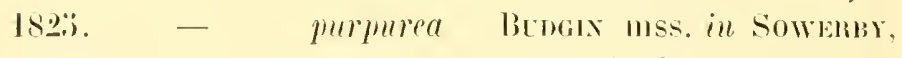
Cillal. 'Tamkerve, p. 10.j.

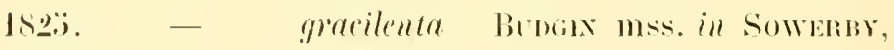
ibirl., p. 彷.

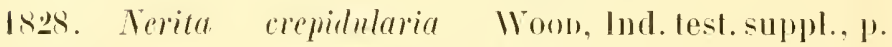
2.;, l1. א. lig. li.

18:30. Verilina phmata Mexke. Synopsis. p. 139.

15:30. - mitrula Mexis, symopsis, p. 倓.

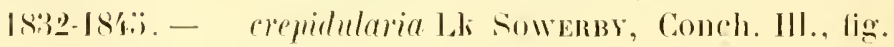
2.;. 2.i.

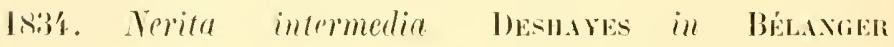
(moll Sowerly), loy. anx moles ()1., p. 㳕0, pl. I. lig. (i. 7 .

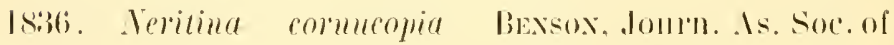
Jiengal リ. リ. T你. 


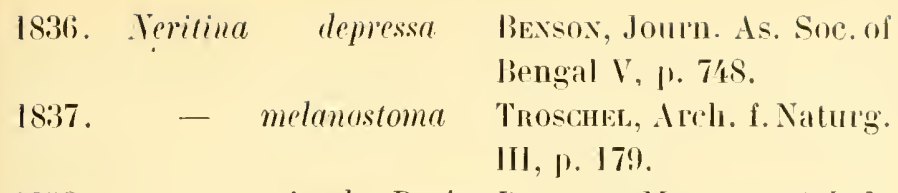

1838. - mitrula Beck. Potiez el Michalm, (ial. de Douai I, J. 303, ,1. XXIX, fig. $\ddot{B}, \mathbf{l}$.

1838. - crepulularia Deshayes in Lanabek, Anill.s. Vert., 2.'éd., VIII, p. $: 37$.

1838. - riolacea besmaYes in Limarck, ibiel, 1.. 880.

1892. Nerila Indica Sollevet, liev.\%ool.,p.269.

189.2. - Touranensis Soulevet, Rev.Zool.,p.269.

184:3. Veritina Siquijorensis Recuz, Pros. \%. S. L., 1. 198.

1843. - melanostoma Tr. Philirp, Mbbild. I, pl. I, lig. li:.

18\%:. Nerita riolacea (ill1. Recuz, Proc. \%. S. L., p. 119.

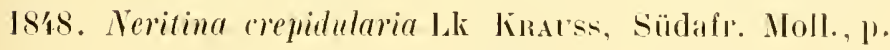
89.

1819. - Siquijorensis Recl. Sowrrm, Thes. Conch., II, p. :310, pl. 113, fig. I价, 1 1 1 i.

18:\%0. - crepidularia Lk Reccuz, Jounn. de Conch. vol. I, p. $60,69$.

18:30. - violacea Gill. Rectez, ibid., ]. 67.

18:0. - melanostoma Tr. Recizu, ibid., p. 70.

18:0. - touranensis Recurz, ibiel., [). 7I.

18:30. - exaltata Recucz, ilid., p. (ii), pl. III, fig. 3.

18:0. - pilooles: Reclez, ibirl., p. 666.

1831. - erepidularia Lk Metcalfe, Proc. \%. S. L., 1. 7 ?․ 
18:32. Nerita melanostma Tr. Eroocx el Sorteyet, Toy.

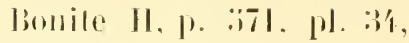
lig. : $: 32.3 \%$

18:\%. - Touramensis Sormerar, Voy. lionite II,

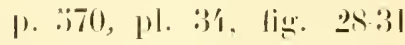
(sul). non!. Nérite in(lienne).

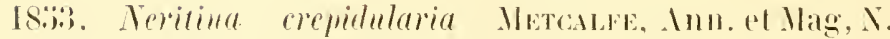
II. XI, p. (i!).

18:\%\%. - - Lk Sowersy, Thes. Conch. II,

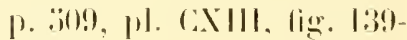
I'作.

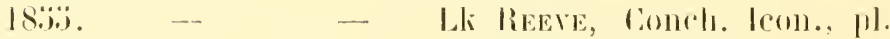
VIII, 1ig. 38;. $38^{\mathrm{h}}$.

18:i\%. - depressa Bens. Sowmeny, Thes. Eonch. II,

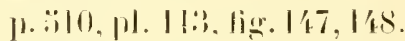

18:3i. - - liens. Reeve, Combh. Icon., ph. XVlll, fig. Sfia, sfir.

1856. Neritina Siquijoremsis Recl. Rews, Conch. lron.,

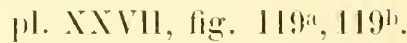

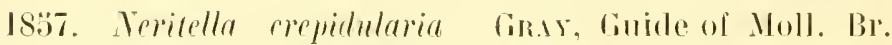
Mrs., p. 138, fig. Th (rarlula).

1860. Verilina - li ve Mantexs, Malali. BI. VII, リ. 份。

1stiti. N. (Mostia) depressa liens. Busward, dourn. As. Soe. Beng., p. 10, 11. Xll, lig. 17-19.

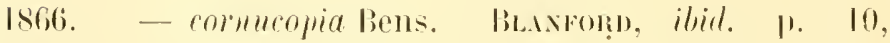
pl. XII, lig. 203-20:3.

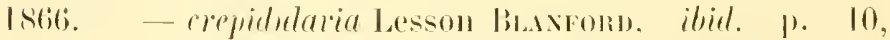

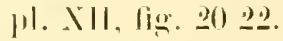

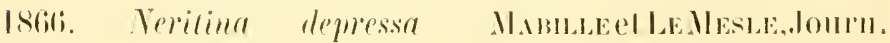

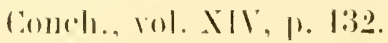


1872. Verilina cormeopia Monelet, Journ. de Conch. vol. XX, p. 1:37.

1872. - riolarea Gin. Moneme, ibid., p. 133, 137. 187'́ N. Mirrula) Schlïflii. Moussox,Jomrn.deConch., vol. XXII, p. 19.

187'. Terilina crepidularia v. Mnntess, Vorderasiat.

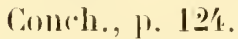

187'1. N. (Hostia) crepidularia Lk Isses, Moll. bornéens., p. 106 , 10s.

I87'. - - cormucopiae. Bens. Isses, ibid. p. IOS.

1878. Nerilina riolacea Gin. Mnaket, Sérieslonch. IV,

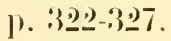

187i. - cormeopia Bens. Monelet, ibid., p. 32̈.

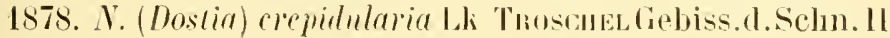
1. 182, pl. 16, fig. 23 (i"adula).

1878. - - exallata Recl. Trosines, ibid. p. 182.

1879. Nerilina crepidularia Lk r. Mantess, Mon. in Conch. Cials., ód., p. 37,

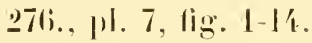

1889. - riolacea Gmel. I. Monset, Journ. Conch. vol. XXXY'll, p. I:37.

1889. - cornncopiae Bens. L. MonLet, Journ. Conch., rol. XXXVII, p. I:̈.

1891. M. (Mostia) violacea (im1. P'. Fisturn, Cat. IndoChine, loc. cil., P. 200 (lli du t. à j).).

1891. - - depressa Bens. P. Fiscims, ibid., J. 200 (I) 1 i).

1891. - - rormmequia Bens. P'. Fiscolen, ibid., p. 200 (I I i).

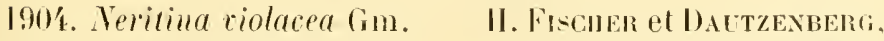
Calal. Into Chine in Mission Pavie, loc. cit., p. 134: (10̈ lu t. à p.). 


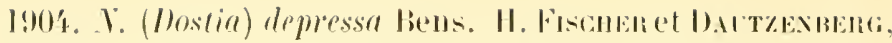
ibid., 1. 1334 ('i:i).

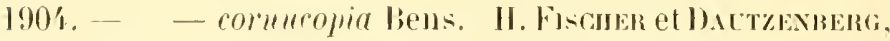

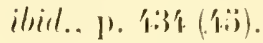

Tonkin. Région du bas Delta, Cua-Cam, Ilä̈phong, à marée basse en eall saumatre, sur les ball's de vase qui bordent le Cua-Cam et le song Tam-bac (M. Blaisc). Exemplaires le grande taille, i peristome rouge vif; exemplaires plus petils, de coloralion externe lome uniforme, à péristome oramgé sale. (Forme typhique el var. depressa liens.)

Région du somg limh-Thay, Dong-Trieu en eau plutot Houce que sammatre dans un petit ruissean roulant au pied les rochers de Dong-Trieu (M. Baise). Exemplaires de petile taille, a péristome orangé sale. Coloration externe fauve linement linéolée et ponctuée. D’antres exemplairts de taille plus forte, à péristome rouge vif (forme typinge et variété depressa bens.).

Région dh Bas Delta, lläphong, en ean saumàtre sur les bords du Song Tam-Bare ef du Cua-Cam (Al. Blaise). Exemplaires de petite laille, coloration fauve ponctuée. péris lome blanchàtre avec la callosilé columellaire orangé sale. (var. depressa benson).

Song limh-Tay, Yen-L,m. ell cau sammitre sum les tiges des grandes herbes qui poussent sur les banes de vase hordant le lleuve ef découranut à marée basse (.I. Baaise). Spécimens des deux formes ci dessus (forme lypique et var. depressa Bensoni).

Terbo conoxites dimelin.

Var. granulata (imelin.

17sl. Cochlea lumaris perforata gramulata, ele.

Cansunit\%, Conds. Cab. I.

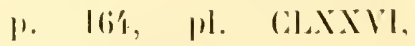

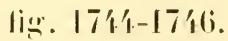




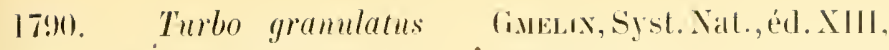
1., 3601 .

1829. Turbo coronatus var. S. Luмnck, Anim. s. Vert. 171. p. 16.

1893. - - - Deshipls in Lamaris, Inim.s. Vert., è éd. IX, 1). 198.

1846. - gramulatus Gm. Prntapr, Mon. it Conch.

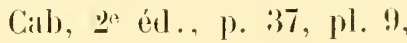
fig. $7,8,9$.

18:38. Lunclla gramulata Ch. II. et A. Amsus, Gen. of rec. Moll. I, р. 39)'.

1869. Turbo gramulatus Gm. Lischke. Japan. Meeres Conch. I, p. 87 .

1873

- P. Fischer in hieser, Mon. (i. 'Turbo, p. 78, pl. XXrlll, lig. 2; pl. Xl, lig. 3 (var. coreensis).

1888. - (Harmorostoma) corouatus val. gramulatus Gm. Turos, Man of Conch. X. 1. 217, pl. 14, fig. 18.

1890 - gramulatus Gim. Chosse et Fischer, Journ. deConch., XXXY111, p. 17. 1891. - - - P. Fiscuer, Cátal. IntoChine, loce cit., p. 202

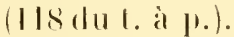

Tonkin. He aux Biches el anse du Crame, Baie d'Along, sur les rochers à marée basse (M. Blaise).

La variété granulata du Turlo coronalus dillère de la forme typique par son ombilic ouvert.

Mosodosta Labo limné.

1737. Turbo le Rétan doxssox, Voy. Sénégal, 1. I81, 11. 12, fig. 2, 2. 


\begin{tabular}{|c|c|c|}
\hline :38. & Trochus & $\begin{array}{l}\text { Lixé, syst. Nat, édit. X, } \\
\text { p. 7i:9. }\end{array}$ \\
\hline tifí. & - & $\begin{array}{l}\text { Lisé, Mus, Lud. Ulr., } \\
\text { p.6h!(explsyn.Cillaltieri). }\end{array}$ \\
\hline 767. & - & $\begin{array}{l}\text { Luxé, Syst. Nal. éd. Xll. } \\
\text { p. lezo. }\end{array}$ \\
\hline 80. & - & $\begin{array}{l}\text { Boms, Tes. Nus. Caes. } \\
\text { Vindob.,p. 33:3, pl. 12, fig.7. } \\
\text { s. }\end{array}$ \\
\hline 81. & Trochas Labeo et & 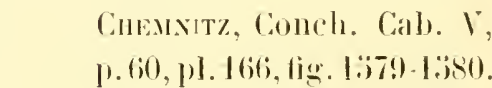 \\
\hline 90. & Labio & 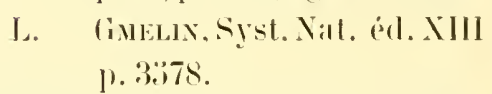 \\
\hline
\end{tabular}

181:3. - (Monodonta) Labio Binookss, Introd. lo Conch., i. 12:3, 11. 7, 1ig. 9\%.

1817. Troches labeo Ihlawix, Callal. II. p. 79?. 182.2. Monodonta labio Lamanck, Mnim. s. Vert.. 111., ]. 3'.

182:- Twro Bismortue, Mam, de Malac.,

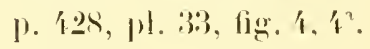

Is:i.). Trochus Lulico Woom, Index testac. p. 138, pl. 2!!, lig. 80.

1830. - Labeo Sowrms, (ien. of Shelis, fig. $\because 3$.

1832. - labio Desmaves, Encyel. Mélh. III, 1. 1080.

1813. Llonodonta labio L. Desmatres, in Lananck, Anim. s. Vert.. 2 éllit. IX, ). I76.

184ti. Trochus labio L. P'mupre, Mon. in Conch.

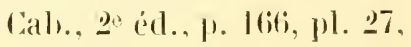

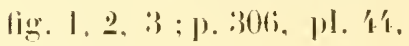
lig. S (var.).

1859. Monodomta labio Lk Cursu, Man. de Conch. I. 1. $338, \operatorname{lig} .263 \%$ 
1870. Monodonta labeo L. Woowwr, Manual, p. 277, pl. 10, fig. Il (sub noun. II. Labio).

1874. confusa Taparone Canerra, Zool. Viaggio della freg. llagenta p. I(i.i, pl. 1. tig. 8 (vils. = var. immanis Fischer).

1880. Trochus labio L. P. Fischen, foonogr. Cof. viv., p. 223, pI. 73, fig. I; pl. T', fig. 4, '.

1889. Monodonta labio Lin. Crusse et Fiscuen, Journ. de Conch. XXXYll, p. 290.

\begin{tabular}{|c|c|c|c|c|}
\hline 1889. & - & - & - & $\begin{array}{l}\text { Pissbry, Man. of Conch. } \\
\text { Xl, p. } 86, \text { pl. 19, fig. 9:3, } 96 \\
\text { et var. confusa pl. 22.2, } \\
\text { fig. } 37 .\end{array}$ \\
\hline 1889. & - & $\ldots$ & - & $\begin{array}{l}\text { L. MonLet, Journ. de } \\
\text { Conch. XXXvil, p. lis?. }\end{array}$ \\
\hline 1891. & - & - & & $\begin{array}{l}\text { P. Fischer, Catal. Indo- } \\
\text { Chine, loc. cil., P. 200'. } \\
\text { (120 du l. i l.). }\end{array}$ \\
\hline
\end{tabular}

Tonkin. He aux Biches, arrhipeldes Faï-T'si-Long, nombreux exemplaires (M. Blaise). - He de la Surprise, baie d'Along (M. Blaise). - Anse du Cràne, baie d'Alonğ (II. Blaise).

\section{Euchelus sp?}

Tonkin. Baie d Mlong, anse du Cràne.

II. Blaise a récolté à l’Anse du Cràne k spécimens jeunes qui ressemblent heaucoup à des exemplaires reçus de MIM. Sowerby et Fulton, sous le nom de Euchelus eden. tulus A. Ardams et provenant des ìles Andaman; mais nous ne pouvons préciser leur détermination, l'Euchelus edentulus n’ayam jamais élé figuré ni identilié d'une ma nière certaine. 


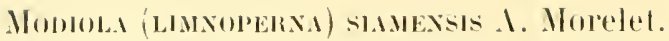

1866. Dreissena siamensis A. Monelet, Rev. Zool., ()). 17.

187.;. Modiola - M. Monelat, sériesconch., IV. 1. 336.i, pl. XYll, fig. $3,3,3$.

I8s!. Limnoperna - А. M. ne liodmbace, Suppl. and documentssur la faume malace de la Coehinchine et Ju Cambotge. extr. Bull. Soc. Philomat. Paris, 1. : i.

188!. Modiola - A. M. L. MonLet, Journ. de Contel, XXXYII, p. Itil.

1891. 12. (Limnopema) - I'. Fiscinen, Catal. IndoChine, loce eit. 11. 21:3

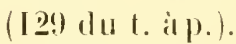

1898. Modiola - I. M. H. Fismen, Liste moll. reer. par le Dr Billet, extr. Bull. scient. France, p. 2oli.

Tonkin. Song Lue-Nan, Lann. Coquilles reeveillies dans des eatux absohmment douces sur des trones d'arbres ou des rochers décourrant a marée basse. Les coquilles de couleur claire sont mélangées aux roquilles de couleur sombre. Nombreux exemplaires (M. Barise). - Bas LacNam, village de Tan-len, tres nombreux exemplaires de cette espèce, connue des indigènes sous le nom de conmong-tay (M. Blaise).

La forme et la roloration de cetle espere sont assez variables.

MrTLES varuBHLS liauss.

1848. Mytilus variabilis linstess. Südaft. Moll. p.

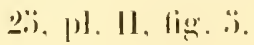


188!. Mytilus senegalensis Li Cubssix (ex. parte? non Krauss), Mon, in Concls. Cab., 201., 38, pl. 11 fig. : :, í.

Tonkin. Anse du Crâne, Baie d’Along, nombreux exemplaires sons les eailloux (.I. Haise).

Le Mytilus rariabilis est certainement fort voisin du $M$. senegaleusis; mais il nous parailrail cependant téméraire dans l'étal actuel de nos commaissances de les réunil comme Clessin a proposé de to faire.

Sertarer prooses (Reclaz) Clessin.

1889. Tichogonia (Seprifer)pilosus Rechuz mss. in Cisessix, Mon. in Conch.Cab., 20 Ed.,

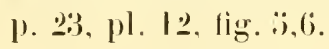

Tonkin. Anse du Grine, Baie d'Along (11. Baise).

Espèce caractérisée far sa forme étroite et allongée, son septum profondément entailté au milien en angle aign, ses còtes rayounantes bi- et trifurquées vers le bord.

Arca (Barbatia) necorvata Lischlie.

181'. Arca obliquata lieve (non Gray), Conch. Icon., pl. XIl, fig. 80 .

1844? - obtusa lieve,Conch.Icon.,pl.XII, lig. 77 .

1869. - elecurrata Lischise, Japan. Moll. I, ヤ. 148.

18!). A. (Barbatia) - Kiobert, Mon. ill Conch. Calb., 2. éd.. p. 120, pl. 31, lig. $: 3,6$.

Tonkin. Do-Son, S. O. de l'entrée du Cua-Cam. Sables et vase (Il. Baise). - Anse du Crinne, Baie d'Along (M. Blaise). 
arca (Barmata) Jlembanga Bruguiere.

1784. Area nicea maris rubri ete. Chemstz. Conch. Cab. VII, p. 191, pl. ."14, fig.

I7s'. - camdida Melblingii Chemstт\%, Conch. Cals. VII,

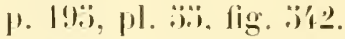

1789. - Helblingi Brogribse, Enerol. meth.. p. !!!).

17910. - orala linems, Syst. Nat., Edil. Xlll, p. 3307, (espece éta blie sur la figure de Chemnitz).

183:i. - otala Cimel. Deshares in Lamarck, Inim. s. Vert. ㄹód. Vt, p. 隹i.

183:i. - Melblingii Brug. Deshayes. Joc. cit., 1. 169. ls't. - nirea Ch. Reeve, Conch. Icon., pl. XIT, fig. 96.

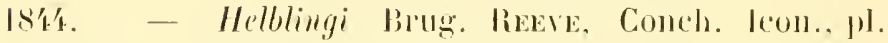
XIV, fig. 90.

1869. - mirea lih. Jsser, Malar. del Mar Rosso, p. !1.

1869. - Melblingi Brug. Issta ibid., p. 91.

1881. Barbatia Heblingii Brug. De Rocnebraxe, Docum. sur la faune malace de la Cochinchine el du Cambodme, extr. Bull. Soc. Pliilomal. Paris, p. ï.

1891. 4. (Barbatia) nirea Ch. lionest, Mon. in Conch.

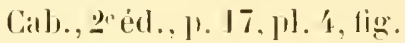
(i.

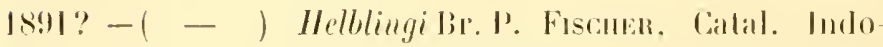
Chine, loce ril.. p. 221:; (p. 1:31 du t. à p.). 
Tonkin. Do-Son. Còté S. O. de l'entrée du Cua-Cam. Sables ef rase (II. Blaise).

Il est imposible, à notre avis, lorquion examine une série tant soit peu importante d'Arca du groupe de l'A. Helblingi, de maintenir l'A. orata (im. = nirea (Ch.) auct. comme une espirce distincte, car cette derniere n’en est quine forme plus trigone et un peu plus oblique: on rencontre tous les intermédiaires possibles entre ces deux formes extrèmes.

Aran (Barbata) vireschass Reeve.

18я. Area rirescens Rave.Conch.Icon.,pl.XY, (ig. 97 .

1891. - - Rue lionelt, Mon.in Conch.Cab.

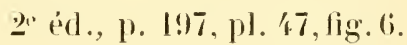

Tonkin. Mo-Son. Còté S. O. de l'entrée du Cua-Cam. Sables et vase (M. Blaise).

C'est avec quelque hésitation que nous rapportons à cette espèce l'exemplaire unique récolté far M. Blaise. 11 est plus quadrangulaire que le lype et présente sur la région postérieure des còtes rayonnantes subépineuses plus nombreuses.

Araca (Avomalogabou) gravosa Linné.

17:i8. Area granosa Linné, Syst. Nat.. éd. X. p. 69 \%.

1767. - - LiNxé, Syst. Nat, éd XIt, p. 11 1:2.

178'. - - Limaei Cinemstz, Conclı. Cab., III, pl. :̈li, fig. :3:37.

1790. Lrea granosa

L. Cinelis, Syst. Nat., ed. XIII, p. 3310.

1817. In,wr, Deser. Catal.. I, 1. . 2.3.3. 
1819. Ara granosa L. L.мmsis, Mnim. s. Vert. V.1. P. '仿.

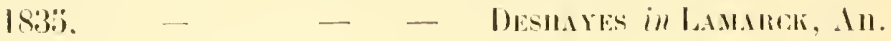

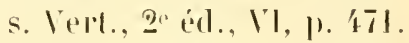

18я. - - - ReEve, conch. leon., 1). III, lig. I:i.

18.3i. Anomalocardia granosa L. Mïncr, Ciatal. Voldi.. II, 1. 行.

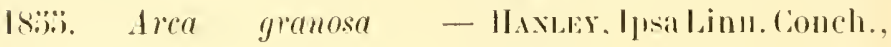
1). 9.i.

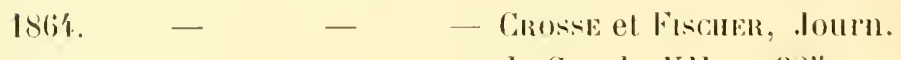
de Conch. XII, p. 32:":.

1869. - - L Lashise Japanische Meeres Iloll. І, 1. І 亿:.

188\%. Anomalocardia granosa - Doxken, Imr. Moll. Jajon. 1. 2.33.

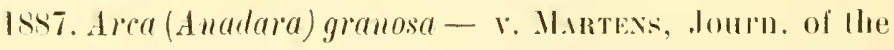
limn. Sore - Zoology XXI, 1). $217 \%$

Iss:. Anadara gramosa L. L. Monus, Jom'n. de Conch. XXXYII, p. li?.

І\$90. Lrea - - Crosse et Fischer, Jomm.

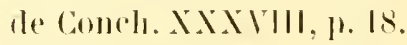

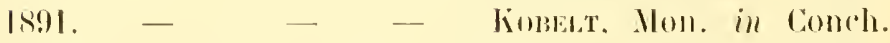
Calb., 2"éd., 1. 38. pl. 3. tig. 7 .

1891. A. (Aualara) granosa L. P. Fiscuen, Calal. IndoCline, loce cit., 1. 213

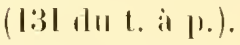

Tonkin. Ilon-(iay, Baie d'Mlome (II. Blaise).

Araca zabiensis Recre.

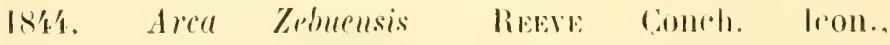
1). XVII. lig. I 20 . 
1891. Area (s. str.) Eebuensis liobelt, Monogr. in Conch. Cal)., 2"'éd., p. 86, pl. 23, fig. 7,8 .

Tonkin. Anse du Cràne. Baie d’Along à basse mer sous les cailloux (M. Blaise).

Cette espece ressemble beancoup au premier aspect à l'A. lactea des mers d'Europe, mais elle en dilfiere par son ligament très petit formant un losange sous les crochets.

\section{Inodonta Jotrovi L. Morlet.}
18s6. Anodonta Jourdyi
L. MORLET, Jour'H.
Concls., vol. XXXIV, p. 7ti

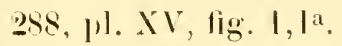

Tonkin. Bas Luc-Nam, villages de Luc-Nam et de Vanlen, exemplaires jemnes; entre Van-ten el l'embouchure du Song-Thuong (exemplaires énormes) (II. Blaise). Mares d'eau douce des environs d'Haïphong (M. Blaise). 
Les spécimens récoltés par M. Blaise entre Van Jen et lembouchure du song-Thuong atteignent une taille considérable : diam. umbono-rentral l'ä mm., diam. antéropostérieur $180 \mathrm{~mm}$. épaisseur $733 \mathrm{~mm}$.

\section{Axodonta lacida lleude.}

1877. Anodonta lucida Hecone, Mém. Emp. Chinois, pl. XX, fig. 4.;.

\begin{tabular}{|c|c|c|c|c|}
\hline 1889. & - & - & lende & $\begin{array}{l}\text { L. Morlet, Journ. Conch. } \\
\text { XXXYll, p. 167. }\end{array}$ \\
\hline 1890. & - & - & - & Paetel, Calal. III, p. I\$I. \\
\hline 1891. & - & - & - & $\begin{array}{l}\text { P. Fischer, Catal. Indo- } \\
\text { Chine, loc. cit.. p. 2ls (13,'t } \\
\text { du tà p.). }\end{array}$ \\
\hline 19000. & - & - & - & $\begin{array}{l}\text { Sumsox, Synopsis Naiades } \\
\text { p. (it). }\end{array}$ \\
\hline 1901. & - & - & - & 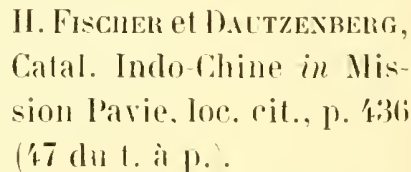 \\
\hline
\end{tabular}

Tomkin. Mares dean douce aux environs d'Haïphong (M. Blaise). Luc-Nam (M. Blaise). - Bas Luc-Nam, village de Van-len (M. Blaise).

II est probable que les citalions de l'I. Suinhoei Il. Adans dans l'tudo Chine doivent ètre rapportées à cette espèce.

Quant au veritable d. Suruhoei décrit de Formose par 11. Alams (Pror. Zool. Soc. Lond., 1866, p. 1:39), il se dislingue du lucida par sa forme plus ovale, moins rostrée du còté postérixus.

DIrSAS Phates Legeh.

I81:̈. Dipsas plicatns Lescu, Zoot. Miscellany I, 1. Iํ), 1)1. I.1II. 
1817. Cristaria tuberculata Schcmacher, Nour. Syst., 1. 107, pl. XX, fig. 2.

1823? Mytilus plicutus Solander in Grar, On the Structure of Pearls and on the Chinese mode of producing these in Ann. of Philosophy, new ser., rol. IX, p, 2-27-2?.

182:3. Barbala plicata Gisy, ibid. 1. 28.

182:;. Anodonta Dipsas Bunxwule, Man. de Malak. p. . 738 , pl. 66, fig. 2.

1830. Symplyynota Bi-alata Les, Tr. Am. Phil. Soc. new ser. III,p. '保, pl.XIV, fig. 2.1.

1831. - - I.ea. Obs. G. Unio I, p. jl. XIV, fig. 2't.

1833. Unio bi-alata DeshaYes, in LAMARCK, Anim. s. Vert., 2e éd. Vt, 1. :3:8.

1838. Margarita (Dipsas) plicatus Lea, Synopsis p. 20.

1839. Unio bialatus Deshares. Traité élém. de Conch., p. 19, pl. XXXl, fig. 3.

1839. Dipsas plicatus Sowerby, Conch. Manual, pl. IIII, fig. 14:?.

1843. Unio bialata Desh. Haxley, Biy. Sh. p. 214, pl. 2ำ, fig. '́.

184:i. Dipsas plicalus Cinese, Bibl. Conch. III (Leach), p. li, , l. 6. fig. I. 184:. Unio bialatus Desh. Cațlow el Reve, Conch. Nomencl., p. 86.

1817. Anodonta (Dipsas) herculea Mobendorrs, Bull. Phys. Math. Ac. Sc. Petersb. Yr, p. 3003 . 


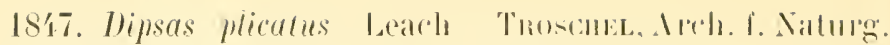
Nill, p. 207.

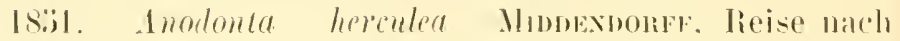

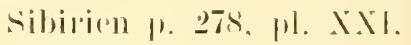
fig.:i. XXll, lig. 1, 2, XX17, fig. I. 2.

18.i2. Margarilana pliratus Les. Symopsis. p. Ati.

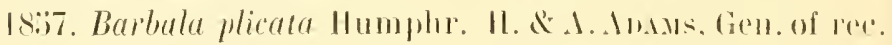

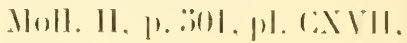
lim. 亿. '保.

18:39. - bialata Lea Cuexr, Mant. de Conch. II. 1. І 㣙, lig. 717 .

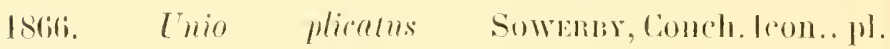
LI, lig.

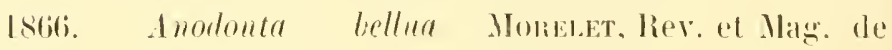
7001. Xl111, 1). 167\%.

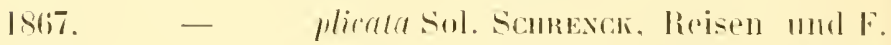
Ammi Lande II, p. 70', pl.

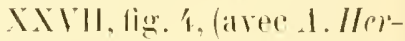
culea Midd. comme synonymor).

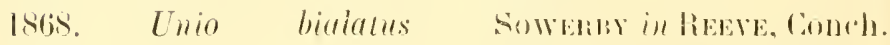

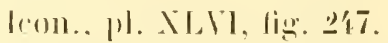

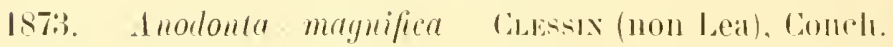

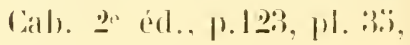
lig. 1,2.

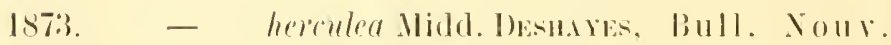
Areh. Mus.. p. I. pl. I. lim. I.

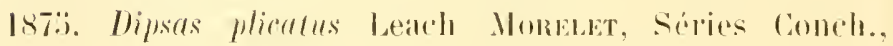
II, 1. 33:3.

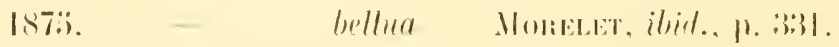

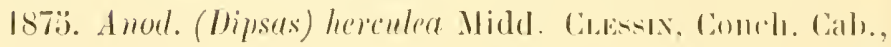

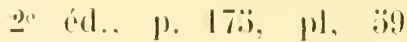
li $2,1,2$. 
1876. Anod. (Dipsas) plicata Sol. Cussin, ibid., p. 2010, 11. 21, fig. 1, 2.

1879. Llipsas plicala sol. Kobelt, Mh. Senck. Nat. (ies. XI, p. 429, pl. XV XY1, XYI, XVHI, fig. I.

1879. - - var. Clessini liobect (ex parte), ibirl. p. 往9, 尚. XY.

1881. - plicatus Leach Ileute, Conch. flur. Nanling., pl. XXXIl, lig. (iד, XXXI, fig. (ita, Ll, fig. 9', 9 '.

188\%. - occidentalis Herde, Coneh. fluv. Nank. IX, pl. LXT', fig. 12!).

1887. - bialata Lea Daltzexbers et d'HAnoxVILLE, Journ. Conch. XXXY, p. 2-29?.

1889. - plicatus Leach L. Monuet, Journ. Conch. XXXYI, 1). 168.

1890. Barbala occidentalis Ide PaEtel, Calal. III, P. I7i. 1890. - plicatula sol. PaEtel, ibil., P. $17 \%$. 1890. - bialata Desh. Paetel, ibid. p. 17:\%. 1890. Anolonta herenlea Midd. Westerisand, Fauna Pal. II, pl. YII, pl. 299.

1890. Barbala - - PAetel, Catal. 111, р. I7:. 1891. Dipsas plicatus Leach P. Fiscuen, Catal. Indo Chime, loc. cit., p. 22? ( I38 dul à p.).

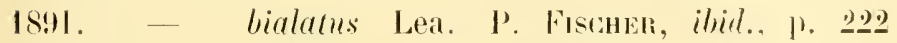
(13s).

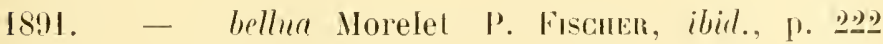
(1:38).

1893. Cristaria plirata v. Inensw, Mh. Senck. Nall lies. Xlill, p. 14.7.

1893. - herculea Midd. v. Інекахg, ibirl. p. I'́li. 


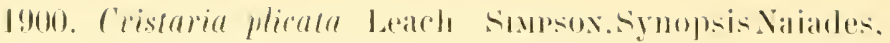
1). is:3.

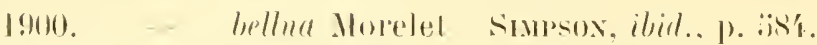

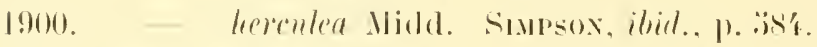

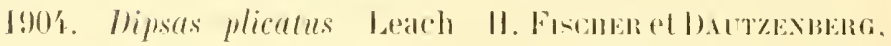
Calal. Indo Ciline in Mis sion Pavie, lox. eil.. p. 1:3 (4.8 du 1. a 1.).

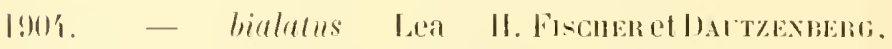
ibirl., 1. 偻 (18).

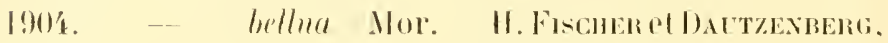

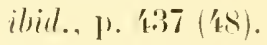

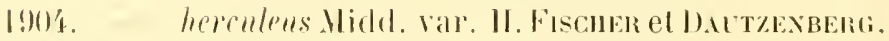
ibid., 1). 行 (你).

Tomkin. Las Lu Nam entre Van-len el lembonchune du Somge Thmong (M. Blaise).

La splendirle séric rapportée har M. Mlaise comprend des sperimens de diliérents diges, dont quelpues mons onl conserve leurs expansions alifomes. anssi bien en anal des roohets quien arriere. Les expansions sont parfois fordues el on peutse demander si les remous du courant ne seraient pas la cause des irregularités de croissance de

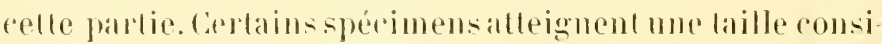

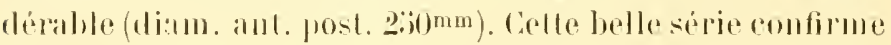
de lous poinls lopinion do Sehronk, suivant laguelle los U. plicalus, bialams, et herculens ne forment quime semle espèce. Nos spécimens me penvent d’aillems pas se sépaler

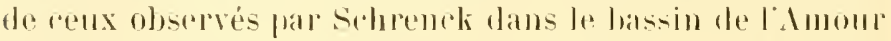

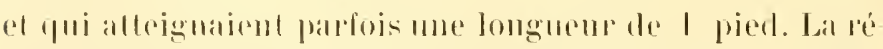

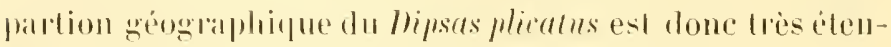

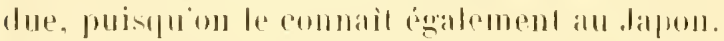

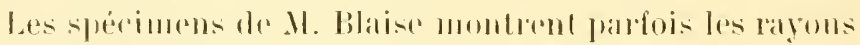

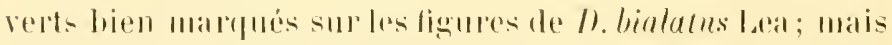

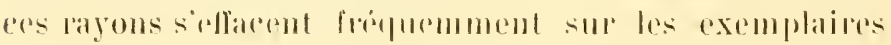


bien adultes; nous avons mème vu des jeunesspécimens qui hien montraient aucune trace.

Into (Hriopsis) Cumana Lea.

1832. Lnio Camingii Lea, Proc. Irad. X. Sc.. Philad. Y1, p. : i's.

18:2. Hargaron (Unio) Cumingi Les, Synopsis, p. 19.

18:37. U. (ILtaptera) Cumingii Lea HI. el 1. Mmms, Genera of ree. Moll. II, p. 198 .

1860.

Unio

LeA, Journ. Lead. N. Se, Phil. IV, p. 290, ph. XXXY.

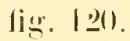
1860. - - Les, Obs. Ci. Inio VII,

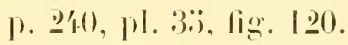

Is66. - Cumingi Lea Sowsers, Conch. Icon., 1) XIIX, fig. 므.

188. - Cumingii - Herbe, Conch. Huv. VIII. pl. IXIY.

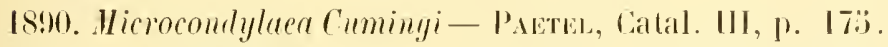
1900. IIyriopsis - - smusox, Syllopis Naiades, 1. :37!).

1901.

Unio

- Hevie, Obs. faune Imman, iil J. de Conch., vol. XLIX. p. 36 .

$190 \%$ 年. - - Paetel, Catal. III, p. 17:3. 1904. Hyriopsis Goliath Rolete, Nachrichtsbl. d. „l. Malak. Cies. p. 26, pl. I, 2.

Tonkin. Bas Luc-Nam entre Van-leu et l'embouchure du Song-Thuong (M. Blaise).

La magnilique série de spécimens de diflérents àges rapportée par M. Blaise proure que I'Hyriopsis Goliath Rolle de la Rivière Claire (Tonkin) n'estque l'état très adulle de l'U. Cumingi ayant perdu son expansion aliforme. Cette espèce vit en compagnie du Inip.sas plicatns Learh. 
l'épaissemr el la ridhe cololalion de sa nacre donnent à penser que cette aspece pourlat ète aratageusement utilisée dans ¡̈industrie nacriere.

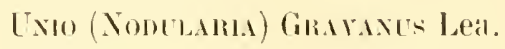

18:31. Inio

Grayamis:

Lat, THalls. Im. Phil. Soc. new ser., V. p. lik, pl. I. lig. 20 (i)

18:3'. Les, obs. li. lnio I, P. I Tis. pl. IX, lig. 2 (i).

1831. - lirayii (imatru, Cuvier, pl. XXI, lig. :3.

1838. Jlargarita (Unio) Grayanns Les. Synopsis, 1) I', 37. 18я. Inio firayams lea liseve, Comels. Syst. I. p. IIs, pl. LXXXYll, lig.

18\%. -

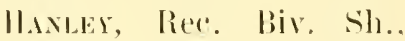
р. 17\%, sиppl. pl. XXIV. lig. :i.

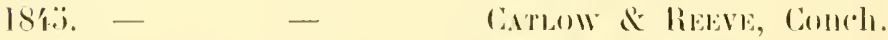
Xomenel. p. :3\%.

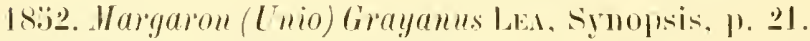

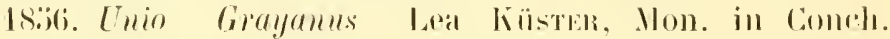
Cals.. p. 16ī, pl. 18, lim. : .

18:i7. L. (Iliplodon) Girayanus - II. et A. Ansus, Genel. of lee. Moll. II, p. 4! 97.

Istii. Unio Grayamus Lia sownss, Coneh. Ieon.. pl. $\mathrm{XXY}$, lig. $11 !$.

\begin{tabular}{|c|c|c|c|}
\hline ISTT. - & - & - & $\begin{array}{l}\text { ILETE, Comeh. Hus., HI. } 16 \text {, } \\
\text { fig. } 36 \text {. }\end{array}$ \\
\hline 1881\% - & - & - & 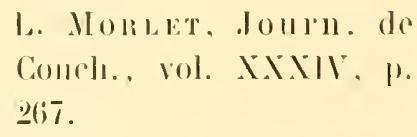 \\
\hline $185 \%$ & -- & & 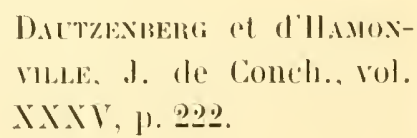 \\
\hline
\end{tabular}


1889. L.(Miplodon) Grayanus Lea Cunst, Man. de Conch. II, 1. 192 , fig. 700 .

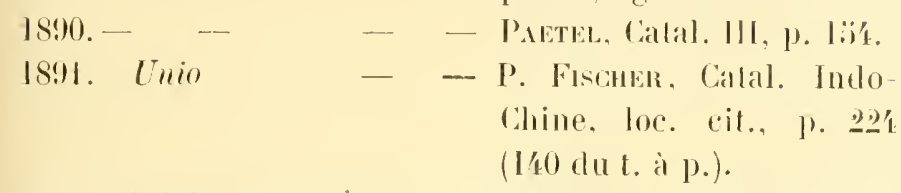

1900. Nodularia Grayana Lea Smmsox. Symopsis of the Naiades, p. 807 .

1901. Unio Grayams - Hecre, J. de Conch., rol. XIIX; p. $3 . \%$.

190\%. - - - H. Fischer el DAITZENberg, Calal. Indo-Chine in Mission Pavie, loc. cit., ]). '1.38 (19) du t. à p.).

190'. Unio(Nodularia)gracillimus Route, Nachrichtsbl. der deulshen Malak. dies. p. 26, pl. 4, lig. 6 .

Tonkin. Luc-Nam. Villages de Van-len et de Luc-Nam. Spécimens recueillis dans des eaux absolument douces sur les banes de rase qui se forment à l'rmbourhure des ruisseaux se jetant dans le Luc-Nam el qui décourrent à marée basse. Colte rspèce semble exister dans tout le Bas Luc-Nam; quelques spécimens ont élé recucillis à Vanlen, en aval du Luc-Nam (M. Blaise).

Les figurations de l'Unio Crayames fournies par Lea sont faiblement sculptées. La pluparl des exemplaires recueillis flar $\mathrm{M}$. Blaise concordent hien avec la forme typique lant comme taille que romme sculplure, mais nous en possédons d'autres de l'Mndo. Chine qui atteignent des dimensions de beaucoup supérieures et qui présentent des granulations plus fortes el répandues sur une bien plus grante portion de la surfare comme dans les ligures de Chenu ef de Heude. Il nous semble que l'U. (Nodularia) gracillimus Rolle s'éloigne trop pen du type pour pouvoir 
ètre considéré méme comme une variété de l' $L$. Grayams. Quant a l' $T$. Frühtorferi Dautzenberg, il se distingue fal sa forme raccourcie, son diamètre umbono-ventral sensiblement plus haut, ses plis très accurés, sa nacre saumonée, mais il est possible que malgré ces diflérences qui paraissent importantes à premiere rue, on soit anené à considérer plus tard cette forme comme une variété aberrante de l'U. Grayauns. Nous nous demandons de mème si de noureaux matériaux ne prouveront pas que I'l.glarliolus lleude n’est également qu une variété extrème de l'l. Grayanus.

Rappelons à ce propos que Heude a indiqué en 5901 (J. de Conch. p. 33) la présence de I $U$. gladiolus identique a celui de Chine dans les rivieres de Tourane, de Ilué et de Vinh.

Von Martens a décrit en 1902 dans le Vachrichtsblatt, mais sans les figurer, deux Lnio du groupe de l' $U$. Cirayams : $1^{\circ}$ Todularia (Lanceolaria) bilirata qui se distingue par deux carènes dorsales sur chaque valve el sa forme raccourcie et haute. 20 Nodularia (Lanceolaria) lieris fu il dit lui-même ne différer de l'Ĺ. Crayams que par l'absence de seulpture rherromuée. Nous arons sous les yeux des spécimens rapportés de Than-Mloï par M. Frühstorfer el qui nous paraissent correspondre aux descriptions de ces deux especes qui, à notre avis, seraient à rapprocher toutes deux de l'U. Frïhstorferi.

UNio Jocridi L. Morlet.

1886. Lnio Jomrdyi L. Mortet, Journ. de Conch., Vol. XXXN, p. 7 ;

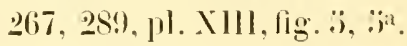

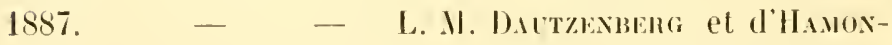
VILLE, J. de Conch., Tol. XXXY, p. 2.2.? 
1887. Unio Jourdy

$\begin{array}{lll}1890 . & - & -\end{array}$

1900. Nodularia

1904. Unio
L. M. J. Mabille, Sur qq. Moll. du Tonkin, in Bull. Soc. Mal. Fr., p. I63. Paetel, Catal. III, p. I:;6. P. Fischer, Catal. IndoChine, loc. cit., p. 206 (142 du t. aे p.).

- Simpsox, Symopsis of the Naiades, p. 816 .

H. Fischer et l)ALTZENBERG, Catal. Indo-Chine in Mission Pavie, loc. cit., p. 438 ('9) du t. à p.).

Tonkin. Bas Luc-Nam, villages de Luc-Nam et de Vanlen (M. Blaise) - eaux douces du Song Luc-Nam (M. Blaise). Cette espèce vit dans des eaux douces, sur les bancs de vase qui se forment àl'embouchuredes ruisseaux se jetant dans le Song Luc-Nam et qui découvrent à marée basse. Ces coquilles semblent exister dans tout le lbas Luc-Nam: quelques-unesont été recueillies à Van-Ien, en aval de LucNam,mais aucune n'a élé trouvée en amont decettelocalité, où il pourrait se faire qu’elles ne vivent pas àcause de la nature des fonds qui sont généralementformés de roches, de sable dur, de graviers et de cailloux, mais où la vase est très peu abondante. Lesindigènes appellent ces coquilles " con-vem».

Tonkin. - Mèmes localités (M. Blaise).

Var. corrugata Dautzenberg et H. Fischer, n. var.

Dans cette variété la sculpture tuberculeuse du sommet descend sur une partie de la surface.

L'Unio Jourdyi est voisin de l'U. Hendei Bazin mss. in Heude Journ. de Conch., 187ィ, p. 114 et représenté llém. Emp. Chinois, pl. I, fig.3 sous le nom de $U$. Cordierilleude; 
mais aubut que nous pouvons en juger en labsenore de spécimens de la localitín orginate frivieres de la province dre Nanking) l'L. Hendei serail plus rentlé en avand, phus allongé transversalement of plus acuminé a l'axtrumité postérieure.

L'L. Morri Wallebled est bien voisin de l'L Jourdyi el n'en est peut ìre qu’me variéti rostrée el à nacresumonée. šil étail démontré que les demx formes ne sonl que des varialions d'une méme espece, il y anrail lieu d'adop-

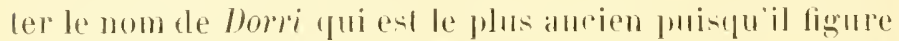

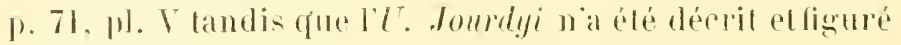
que 1. 7(i, pl. XIII.

Nous avous eru deroir abandonner le nom d'U thio gladiator Ancer (Le naturaliste ls8l). ne pourant noms rentre comple fante de liguration el d'une descriplion suflisante s'il s'agit de I'L. Jourdyi on de I'U. Micheloti L. Mos'let.

L'I'. Jourdyi est fort roisin jar sa formegénérale del't. fungio Benson de l'Inde el de Chine mais l'U pugjo est calatclérisé par sa seulputure délicale, son bord dorsal arqué el sa coloration rerte, landis fue les exemplaires seulplés d $L$. Jourdyi présentent des plis al des fuberules plus grosiers, ont le bord dorsal plus rectiligne el sont dime coluration brume.

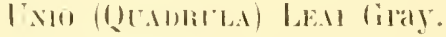

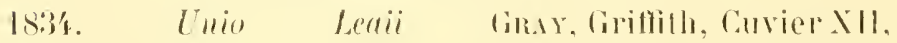

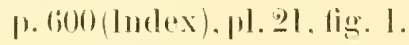

1836. Margaria (Lnio) Leaii Ls. symops. 1) IT.

\begin{tabular}{|c|c|c|c|}
\hline $18: 38$. & - & - & Gir. L\&, synops. p. 16, 37. \\
\hline I8仿. & Lliio & Leeai & 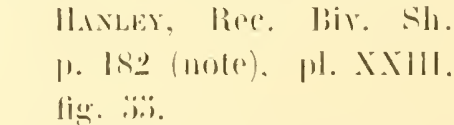 \\
\hline
\end{tabular}

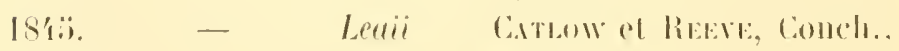
Nolli., I. 60. 


\begin{abstract}
18.;0. Lnio Leaii Jar. Calal., p. (il.
I8.;. Hargaron (Unio) Leaii (ir. Lea, Synops. p. 23.

18\%3. Cuio(Theliderma) Leaii Cir. Brssox, Journ. As. Soe.

Beng., XXIT, p. I36.

18:38. Unio (Iridea) Leaii Gray H. \& 1. ADaws, Genera of rec. Noll. II, ए. 496.

1861. - Leni Gray Kïster, Conch. Cab. Unio, p. 232, pl. 78, fig. 3 .
\end{abstract}

1864. - nodulosus var. Richtho/eni Sowwruy (non Wood), Conch. Icon., pl. IX, fig. 3르 (errata $=$ Leai (iray).

1875. - montames

1873. - Leaï (iray

187:\%. - Leleci

1873. - Richthoteni

187

1876

1881. - Leteci IIde
Heude (nom Ratinesque, nec Philippi), Conch. 1lur, pl. 11, lig. 11.

Heroe, Conch. Huv. I, pl. IV, fig. 10 (var. A), 1). II, liz. IB (var. B), lig. Ili (var. C). Harde, Conch. fluv, I, pl. IV, lig. I2, pl. V, lig. I't. v. Martens, S. B. Nal. Fr., p., 3.

v. Martexs, Mal. BI. XII, p. 187.

Preafere, Novit. Conch. I), р. 1:̈6. pl. CXXXYT, lig. 1, ... 3.

Cirenteh, Jahrb. d. d. Mal. (ies. YIII, 1). I22.

1881. - Leaivar cimnamomeus(ineder, Jahrb. d. d. Mal. lies. YIII, p. I르, pl. VI, fig. 16.

1882. - cormum lunae Heine, Conch. Hur., j)l. Lill, fig. $10 \%$. 
1852. Unio paschalis

1852. - rerruculosins

1882. - abortims (jur.) Hetwe, Conch, Hus. YIII, pl. Ixill, tig. 1ㄴ.

188\%. - monticole

1886. - Leai

1886. - - Giay

1890. - (Ividea) Leai Ciay Pater, Catal. Conch.

Samml. 111, 1. 1:37.

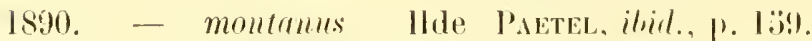

1890. - cormum lunar - PAETEL, ibid., P. 14:9.

1890. - paschalis - P.A.TE, ibid., p. li2.

1890. - vermenlosus - PAETE, ibril., 1. 171.

18:19. - (Iriden) Leai var. cinumomens Gredl.

PaEtla, ibiel., p. $1: 37$.

1890. - - Kichthofeni Pfr. PAETEL, ibid., 1. Ilii.

1890. - - Leai var. Leleci Paetel, ibid. p. 1:37.

1891. - Leni Gray L. Morlet, J. de Conch., vol. XXXIX, 1. 2.3s.

1891. - - - P. Fischer, Catal. IndoChine, loc. cit., p. 20?6 (1 192 du 1. à l).).

1900. Quadrula — - Simpsox, Synops. Naiarles. 1. 801 .

1900. - commum lumap llde Sisupsox, ibid., p. 802, el var. cinnumomens Gredl.

19900. - Leai var. Leleci - simpox, loce cil., P. sol.

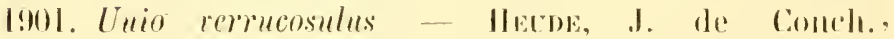
inl. NLIX, p. :3i;. 


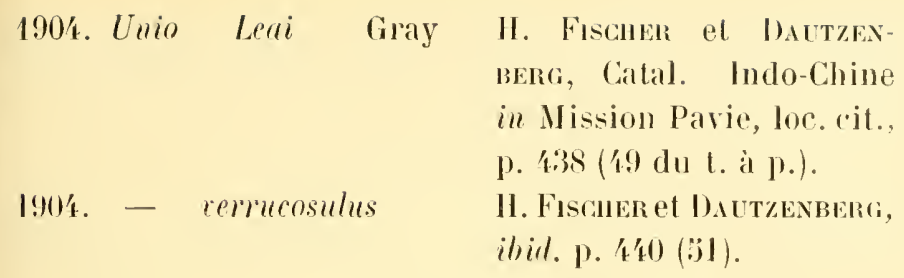

Tonkin. Bas Lue-Nam, village de Van-Ien (M. Blaise).

Nous partageons l'avis de M. Simpson qui pense qu'il y aura lien de réunir sous un mème nom plusieurs formes chinoise quit conserve tontefois comme espèces distinctes faute de matériaux suffisants. Les récoltes de M. Blaise nous amènent à réunir aujourdhui sous le nom d'U. Leai Gray les U. Leai Gray, Richthofeni v. Marts, Leleci Heude, montamus Heude (= monticola nom. subsı.), cornum lunae Ilde, paschalis IIde, rernculosus Hde et abortirus Hıle, ce dernier basé sur un exemplaire jeune. Parmi ces noms on peut retenir comme désignant des variétés:

$1^{\circ}$ Var. Richtofeni von Martens.

Bien représentée par Pfeilfer (Novit.) ctensuite par Reeve (cité pl. IX lig. 32. sous le nom de nodulosus, mais l'errata indique qu'il s'agil de l’ U. Leai Gray). C'est une forme ì hord dorsal très convexe el dilaté.

20 var. ponderosa Dautzenberg et II. Fischer n. var.

Forme lrès épaisse, très rentlée, très inéquilatèrale, à sonmets terminaux, pourvue sur chaque valve d'un carène bien accusee qui part du sommet et limite un corselet ormé de grros plis olhiques réguliẻrement espacés. Le bord ventral est arqué et lrès ascendant du còté postérieur ; le ligament parait ètre constamment plus court que chez l'U. Leai typique. L'U. Leleci Heude (Conch. Huv. jll IV lig. 12) pourrait constituer un passage entre le type et notre var. 
fonderosa, landis que la coquille représentée par Heude ll. I. fig. I's nous somble presque illentique à l' $L$. Leai typipue.

:3" var. paschalis Ileule.

Ne se distingue de l' I'. Leai typique que par sa srubl' ture moins grosierement verruqueuse ef phulot composte de plis disposés en cherrons.

LiL. modnlosus Wood appartient an même groupe gue 1\%. Leai : mais il est plus ovale. plus épais, plus lourd et son plateau cardinal est sensiblement plus large.

L' ${ }^{\prime}$. affmis Heude (nou tea nec sowerby), nous parait synonyme d' $L$. nodulosus. La substitution de nom $L$. simi laris proposée frar M. Simpson pour cause de double emploi devient donc inutile.

L'U. Leai et notamment la rar. paschalis possède parfois une nacre d’un joli ton carnéolé qui pourrait trouver son emploi dans l’industrie nacrière : les dents earlinales notamment seraient utilisables en hijouterie concurremment avec colles des Unio de l'Imérique du Nord.

Uxo (Qcadrula) Buasea Dautzenberge el H. Fischer n. sp. (P. I1, fig. 1,2.)

Testa elongala, orata, crassissima, antice abrupte truncata, concentrice imegulariter plicula, cersns mubones ralde tumida, bicarmata irregulariterque corrugata. postice decliris. compressa ae sublaerigata. Area postica oblique rude plicata. Hargo dorsalis umdulatus. Umbones terminales, erosi. Ligamentum ralidum, corneum. C'ardo crassissimus in ralra de tra dentem cardinalen fortem lacinialum dentempure lateralem dongatum ; in valra sinistra verodentes cardinales. quormm anticus debilis, postirns ralidusetlarinialus. dentestgue laterales 2 dengatos praebet. Impressio muscularis pastira rotmulata hand impressa, antira rero mofunde ereacala ; impressio patlii sat impressa. 
Testa sub epidermide olivaceo nigrescente alba, margarilacea. Margarita interna alba el iridesens.

lliam umb rentr. (i2 mm; ant. post $98 \mathrm{~mm}$; crassit. 3̈̈ $\mathrm{mm}$.

Coquille ovale allongée, très épaisse et lourde, très renflée en avant et portant deux crêtes diverăentes dont linférieure tlisparaì birntôt, tandis que la postérieure, tout enśattéunant vers l'extrémité poslérieure, limite toutefois nettement le corselet. Surface ornée te plis concentriques irrégulier's el, dans la région dessommets, de plis grossiers chevomnés entremêlés de norlosilés irrégulieres disparaissant dans la région postérieure qui est comprimée et qui devient presque lisse. Le corselet est garni tle forts plis obliques qui rendent le bord dorsal onduleux. Ligament corré, fort, mais peu saillant. Plateau cardinal large et surplombant me cavité prolonte. Charnière tròsépaisse, présentant : dans la valve troite une forte dent cardinale laciniée (située entre une fossette cardinale profonte et l’impression dı musele adducteur antériem également très profonte) el une dent latérale allongée; dans la ralve gauche, deux dents cardinales flantérienre fable situce entre me fosette ardinale profonde et l'impression de ladductenr antérieur également profonde, la postéricure forte, laciniée) et 2 dents latérales allongées, lamelleuses Impression du muscle arducteur postérieur arrontie. superficielle; impression palléale bien accusée. Test hanc nacré sous un épillerme fibreux olivitre foncé. Nacre de l’intérieur blanche, iriılescente.

Tonlin. Bas Luc-Nam, Village de Van-Len (.Y. Blaise).

Celte espèce se distingue de l'U. Leai Gray par son test plus lourl, bien plus renthé dans la région des sommets et par les deux crètes élevées qui partent des crochets. Elle se rapproche également du Guadrula Liedrkei Rolle (Nachrichtsbl. d. d. Malak. (ies. 1901' p. 2.; pl. 3 et pl. 4 fig. a,a) mais celle derniore forme est plus régulièrement ovale, 
ne possède pas les crètes caracléristiques du Blaisei et ne nous paraît être qu’une variété de l'U. nodulosus. W'ood.

Sontila Blasei Daulzenberg el II. Fischer n. sp. (1). $Y, \operatorname{lig} .17,18,19,20$.

Testa orato-subquadrata, solida, tumida, nitida, polita, plicis aliquot inerementi irregularibus seulpta. subinaquilateralis : postice quam antice paullo longior. Lmbones parti, promimuli, contigui antrorsumque leciter incurrati. Margo dorsualis utrinque angustissime hians. Margo temralis fere ree. tus. Cardo debilis: in ralea dextra demtem cardinalem anticum mimutissimum, in valia sinistra dentem quoque cardinalem miuntissimum et dentem lateralem brexem, fere obsoletum marbet.

Color pallide carneolus rersus apicem panhlum pallidior.

Diam. umbono-rentr. $7 \mathrm{1} / 2 \mathrm{~mm}$., antero post. $110 \mathrm{~mm}$., arassit. $5 \mathrm{~mm}$.

Coquille ovale subquadragulaire, peu solide, renllée, luisante, polie, ornée de quelques plis d'accroissement irréguliers; subinéquilatérale; còté postérieur un peu plus grand que l'antérieur. Summets petits, saillants, conligus, légèrement incurvés vers le coté anléricur. Bord dorsal un peu arqué, étroitement baillant de chaque coté des crochets; bord rentral presque horizontal; hord antérieur el bord postéricur faiblement arqués. Charnière très faible (fig. 19 et 20, grossies 6 fois) présentant une dent cardi uale antérieure très faihle sur la valve droile et sur la valte gauche une dent latérale postérieure rourte el fort obsolete.

Coloration d'un ton carnéolé elair, un peu phus clair vers les sommels.

Tonkin. Baie d'Along, Anse du Crine, 2 exemplaires altachés sous les cailloux (M. Blaise).

Nous navons fu identilier cetle espèce arec aucune de 
celles qui figurent dans la monographie du Thesamms: elle se rapproche par sa coloration du Sc. ambigna Desh; mais sa forme est bien plus raccourcie, elle est d'ailleurs bailiante du còté dorsal tandis que le $S$ cambigua est au contraire baillant du eòté ventral.

\section{Libitina reblicata Reeve.}

1S'3. Cymricardir vellicata ReEve. Proc. Zool. Soc., p. 197 (olss.)

18 i.:.

- Reeve, Conch. Roun. pl. 1I, lig. 7.

$1886 . \quad-\quad$ Haxley, Rec. Bir. Sli., p. 370 (obs.).

Tonkin. Inse du Cràne, Baie d'Mong. à marée basse sous des cailloux reposant sur la rase mélangée de gravier et de coquilles brisées. Ces coquilles adhierent aux railloux par un byssus blanchàtre et très pen résistanl ( 11 . Blaise).

\section{Crista gabma Lamarti.}

17:38. Temus pectinata Laxí (ex parte). Syst. Nat., éd. X, p. 689.

1778. - - Boms, Index rer. Mus. Caes. Vind., p. 61 .

1780. - - Boix, Tesl. Mus. Caes. Vindol.. p. T'́

1784. - - Linnaei ete. Comsto(exparte), Conch. Cals. VII, p. 28, p]. 39, fig. 保花 (Iantumi).

1789. Gafrarium Tumidum BoLtex. Mus.lBoltenianmm. 1. 176.

1798. - Angmlalum Bontex,Mus. Boltenianum, p. 177.

1817. Tems pectinata Drums, Deser. Catal. I, p. 198. 
18ı. Cytherea gibbia Lisunck, Anim. s. Vert. $\checkmark, p .877$.

1818. - ramella Lamanck, Inim. s. Vert. I, р. :36.

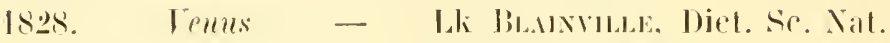
I.VII, I. 此.

I:3so Cytherea gibbia - Sownm, Cienera of Sh.. $\operatorname{lig} . \ddot{3}$.

18:31. - D - Msmones, Enegel. Mélh. II, 1. . ii?.

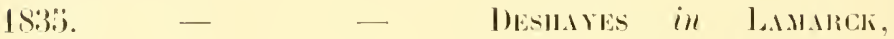
Anim. s. Vert., 2o él. l'l, p. 323 ?3.

1839. Venus (Cytherea) - Axтox, Verzeichniss, p. \&.

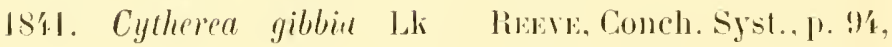
pl. 70 , fig.

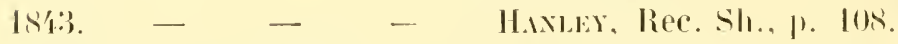
189:. - ranella - Haxter, liec. Sh., p. I0s.

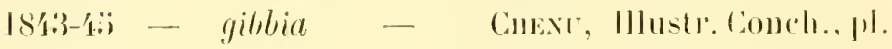
Iㄹ, fig. (i, (6a, (ib), 7, 7a, $7^{1)}$, $8,8^{\prime \prime}, 8^{b}$.

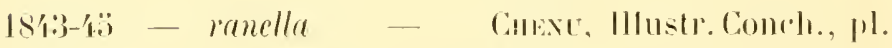
Iㄹ, fig. !), (9a ( $^{\mathrm{b}}$.

18.i3. Ciree gibbia Issmaves, Catal. Conchifera, 131. Mus., p. 90.

Is:3.3. peetinata Mömal (ex parte, non Linne), lat. Voldi II, p. 2i.i.

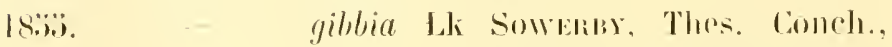

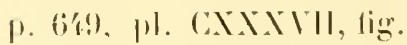
4 i 7 .

15:i7. Cycheren - liöms, liril. linters., p. $9(;$.

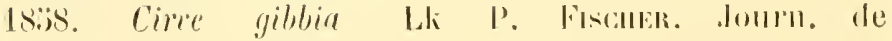
Conch., lill p. 3:3\%. 
18:is. Ciree gibbia

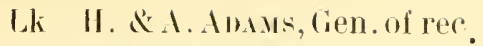
Noll. II, 1. 傮?.

1863. Cycherea (Crista) gibbia Rösen, Malak. 13., p. 17. 186ii3. Circe gibba lik Reves (ex parte), Conch. lcon., pl. I, fig. 29l, , 2le (tantum, exel. fig. 2l:t $=$ C. Menkei Jonas).

1863. Crista gibbia hö̀er, Mon. der Mollus kengatt. Venus 1, 1). 176, pl. XLイll, fig. $2,2^{\mathrm{a}}, 2^{\mathrm{b}}, 2^{\mathrm{e}}$, D. (ranella), 2 .

1869. Cylherea - - Premfer, Mon. Conch. Cab. 2ééd., 1. :il, pl.18, lig. 7-9.

1889. Circe (Cytherea) gibba L. Monuet, Journ. de Conch.,vol.XXXYllp. 16!.

1891. C. (Crista) giblia P. Fiscmer, Catal. IndoCline, loc. cil., p. 2:31 (14.7 du t. à p.).

Tonkin. Ile aux Biches, Bair d'Along (M. Blaise).

II est évident, aussi bien par l'examen des références de Linné que par les observations de llanley (1psa Linu. (Conch.) que Linné a confondu sous le nom de Tenus pectimata la forme à laquelle les auteurs modernes ont con servé ce nom et celle plus gibheuse el phus grossierement sculptée que Lamarck en a séparée sous le nom de Cycherea gibbia (1). Les deux formes ont été lrès bien représentées dans l'Encyelopédie Mlélhodique el nous ne voyons atucun inconvénient à respecter la tradition en conservant le nom de pectimata pour celle fignrée pl. 27/ fig. $1^{\mathrm{a}}, 1^{\mathrm{b}}$ et celui de gibbia pour celle ligurée pl. 27l lig. $4^{\mathrm{a}}$, 价.

(1) Le type du Cytherea ranella Lamarck 1818 que j'ai pu examiner dans la collection Lamarck conservée au Musée de Cipnirrest une forme jeune et blanche du $C$. gibbirl.

Ph. Dautzexheng. 
Merethax MERETIAX Limné.

17is. Tenus Meretrix Laxes, Srst. Nal. éd. X. p. listi.

ITlín.

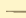

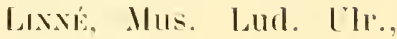
1).

1767

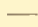

laxié, syst. Nat. él. XII. 1). 1132 .

178.

- L. Boris, Ind. rerum nal. Mus. Cates. Vindols., p. :3l.

178:). lionx, Mus. Cires. Iindoh. 1). (i.j).

178

- ele. Cinsuxitz, Conch. Cab. VI,

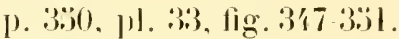

1790.

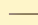
(imelix, sirst. Nal. érl. XIII, 1. 3:2073.

$18(1) 2$ - L. Bosc, Hist. Nil. III, 1. 仵. 1817. Cycherea pouderosa Sculaucuen, Nour. Syst. p. І:̈ (), pl. XV, fig. I.

1818. - impmelica Lamanck, Anim. S. Vert. V. p. :ilil.

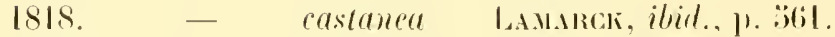

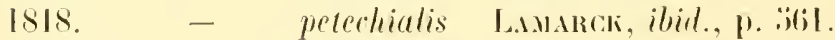

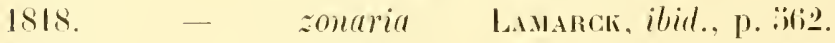

1818. - meretrir l.manck, ibid. p. ïliz.

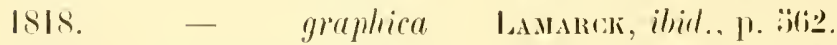

ISIS. - morphima L.susack, ibid., p. ïje.

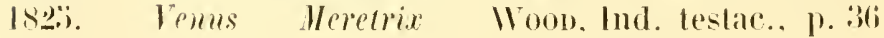
pl. $\overline{7}, \mathrm{fig} .47$.

18:30. Cytherea petechialis lis Sownras, (ienera of sh. II, fig. I, I.

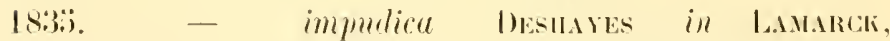
Mnim.s. Verl. Ell. Il p. 2!)!).

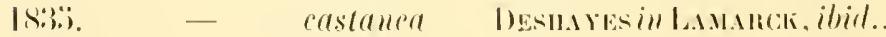
1. $2(1)$ ) 


\begin{tabular}{|c|c|c|c|}
\hline $183 \%$. & Cytherea & zonaria & $\begin{array}{l}\text { DesHaYes in LaMarCK, ibid. } \\
\text { p. 2!!9. }\end{array}$ \\
\hline $18: 3 \%$ & - & petechialis & $\begin{array}{l}\text { DESHA IES in LAMABCK, ibid., } \\
\text { p. D(9). }\end{array}$ \\
\hline $18: 3 \%$ & - & meretrix & $\begin{array}{l}\text { DEsHaYes in LaMARCK, ibid. } \\
\text { 1. :300. }\end{array}$ \\
\hline 18:3:i. & - & graplica & $\begin{array}{l}\text { 1)ESH YES in LaMarack, ibill. } \\
\text { 1. } 3(06) \text {. }\end{array}$ \\
\hline $183 i$ & - & morphina & $\begin{array}{l}\text { DESHAYES in LAMARCK, ibid. } \\
\text { p. } 300 .\end{array}$ \\
\hline
\end{tabular}

1839. Vems (Cytherea) sonaria Axros, Verzeichn., p. 7. 1839. - - morphina Axтox, Verzeichn., p. 7. 189. Cytherea petechialis Lk REEve, Conch. Syst. I, p. 9', 1. LXIX, fig. I; pl. LXX fig. 1.
1811. - meretrix Delessert, Rec.coq..pl.s, lig. $1^{a}, I^{b}, I^{c}$.

1819. - Impudica la Haxues, Ree. biv. Slı., 1. 96i. pl. 11, fig. 10.

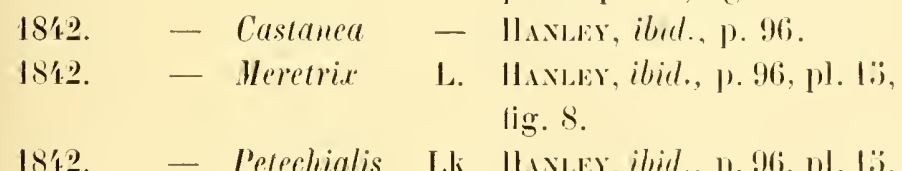
lig. !).

1892. - Graphica - Ilonew, ibil., p. 96, pl. 1:3, lig. 11 .

1842. - Morphina - Haxlst, ibid., p.96.

184. - Zonaria - Ilanew, ibil., p. 96, pl. I:3, fig. I0.

1813-4:̈. - impudica

Is'3-4i.i. - castanea Chext, III. Concli., pl. I, fig, (i, (ja), (ij), (ice.

Cillist, ibil., pl. 2, fig. 1. lat $^{\mathrm{ln}}, \mathrm{I}^{\mathrm{e}}$.

1843-4:- - sonaria Conext, ibid., pl. I, fig. I, $1^{\mathrm{a}}, \mathrm{I}^{\mathrm{b}}, \mathrm{I}^{\mathrm{c}}$. 


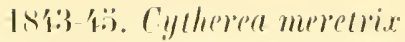

1N13-4:3. - yraphica

18я:3-4:. - morphina

1\$13-4:. - petechialis

18я. Heretrix meretrit Gas, proc. Zool. soe. London, \%. 18:3.

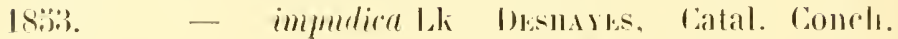
Bir. Mus.. 11.33\%.

18:i3. - Mormhina - DEsHarls, ibirl., p. 37.

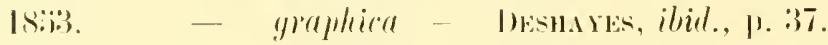

15.i3. - castamea - Destroms, ibid., p. 38.

18:33. - zomaria - DEslares, ibid., p. 38.

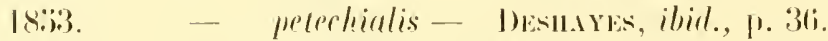

18:33. - impulira Ch. Mörch. Catal. Voldi. II. 1. 28.

18.33. - pomerosa Möncu, Calal. Molali. II. 1. ․ㅛ.

1833. - inseripla Matrex in Mönca, Catal. Yoldi II, I. 29.

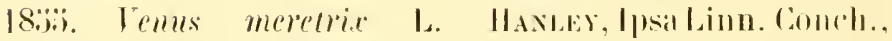
1) 7 (1).

18;i:. Cytherea meretrix L. Sowmas, Thes. Conch. It, p. (il9, pl. CXXIX, dig. 你. 49, : $: 0$.

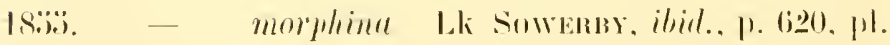
CXXIX, lig. :i), (i).

18:\%i. - smaria - sowsms, ibid. p. (i20, pl. CXXIX, lig. 沙, 茫.

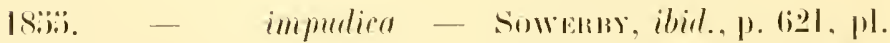
CXXIX, lig. 
18\%.\%. Cycherea petechialis - Sowner, ibid., p. 619, ph.

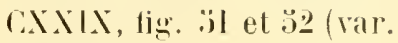
graphica).

18.i7. Tenus meretrix L. Römsn (ex parte), lirit. L'nters., p. 仆.

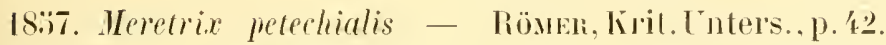
18:58 - impudica L.k II.\& A. Adsus, Genera of rec. Moll. II, p. 1293, pl.107, fig. $6,6,6$

\begin{tabular}{|c|c|c|c|c|}
\hline $8: 38$. & - & castanea & & 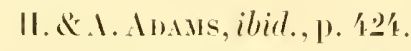 \\
\hline $18: 8$. & - & zonaria & & 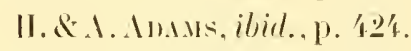 \\
\hline 8:is. & 一 & graphica & & 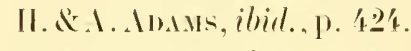 \\
\hline 18.78. & - & morphina & & 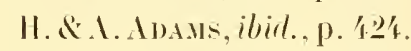 \\
\hline $18: 38$. & -- & petechialis & Lk & 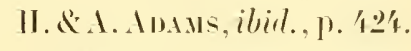 \\
\hline 660. & . $\mathrm{He}$ & rix) zonaria & - & 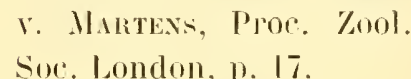 \\
\hline 8600. & & impulica & $1-$ & $\begin{array}{l}\text { ¿. Mantexs, Proc. Zool. } \\
\text { soe. London, D. I7. }\end{array}$ \\
\hline 1861. & Meretrix & imputica & - & DUNKER,Mull.Japon., p.?(i. \\
\hline 1861. & - & meretrix & L. & Röoner, Malak. BL, p. 仿. \\
\hline$|s 6 i|$. & - & petechialis & & Röмек, Malak. ВІ., p. \\
\hline $86 \%$ & Cycherea & graphira & Li & $\begin{array}{l}\text { RiEeve, Coneh. Icon., bl } \\
\text { fig. I. }\end{array}$ \\
\hline 1864. & - & castanea & & RENE, ibid., jl. II, fig. 6. \\
\hline $186 \%$ & - & sonaria & & LiEeve, ibirl. pl. HI. fig. ?. \\
\hline $186 \%$ & - & impudica & & REEVE, ibid. pl. 111 , fig. 10. \\
\hline IS64. & - & morplima & & REEVE, ibid. pl. IV. fig. I?. \\
\hline 1861. & - & petechialis & Lli & Renev, ibiel. pl. II, fig. \\
\hline 1869. & - & meretrix & $\mathrm{L}$. & 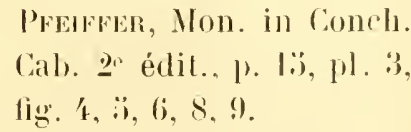 \\
\hline
\end{tabular}

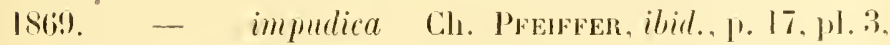
lig. 7.

1869. - petechialis Lk PFEFER, ibid. p. I8 (pl.3, lig. 1?), pl. 4. lig. 1-4. 
1869. Meretrix meretrix

L. Röser, Mon. Molluskeng. Venus, p. 2̄i. pl. VIII, lig. I it $1^{\mathrm{h}}$.

1869. - petechialis Lk Römer, ibirl., 11. 29, [1. IX, fig. I.

185T. Cytherea

1889. Meretrix

18.90

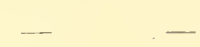

1891.

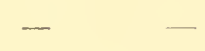

I891. - zonaria

1891. - impudica - P. Fiscuek, ibid., p. 2320 (l 1 s du t. à lo.).

Tonkin. 1) Son, an cote S.-0. de l'entrée du Cua-Cam, sables el vase. Forme lypique el variétés (M. Blajse).

Il ne nous parait pas possible dadmell pe que le Vere. trix petechialis Lamareli (dont $M$. inseripun Nartyn in Mörch est synonyme) soit me espece distincte du $M$. meretrix L. Ce n'en est à notre avis qu'une variété. Cetle opinion est confirméc par les récoltes de M. Blaise qui renferment des spereimens du M. meretrix typinue (coloralion blanche uniforme aree le corselet violacé) ainsi que les varietes castanea li (dim hrun maron miforme), graphica Lk (aver D larges rayons diveregents plus ou moins intermonpus) el entin pechiralis Lk (orne de poncluations disposedes en chevous, surtout dans le voisinage tes sommets). In des caractions qui ont bie invoqués 
pour jusțifier la séparation du $\boldsymbol{M}$. petechialis est la taille beaucoup plus grande que cette forme pourrait atteindre. Or, M. Blaise a rapporté en mème temps que de grands exemplaires de $\boldsymbol{I}$. petechialis des spécimens lout aussi grands de la var. graphica. De plus, certains de ces grands exemplaires présentent dans la région des sommets un mélange des colorations attribuées aux $\%$. petechialis et 11. graphica. La coloration foncée du corselet n'est constante ni chez le $U$. meretrix typique, ni chez le 11 . petechialis.

Meretrix (Pitar) citrixi Lamarek.

1818. Cytherea citrina Lamanck, Anim. s. Vert.
V, p. ̈̈6ї.

1831. - - Lk Deshares, Encyel. méth. II, p. 36 .

1833, - - DESHYES in LaMARCK, Anim. s. Vert., 20 éd., VI, p. 306.

1838. Chione striata Giray, Analyst Ylll, p.306. 1811. Cytherea citrina Decessent, Rec. cof.,pl.8, lig. $8^{\mathrm{a}}, 8^{\mathrm{b}}, \mathrm{s}^{\mathrm{c}}$.

\begin{tabular}{|c|c|c|c|c|}
\hline 181?. & - & - & & $\begin{array}{l}\text { Haxter, Rec. Bir. Sh., } \\
\text { p. } 99 \text {, pl. 13, lig. } 23 .\end{array}$ \\
\hline 1843 184: & . - & - & & $\begin{array}{l}\text { Chexe, Ill. Conch., pl. (i, } \\
\text { fis. } 4, \mathbf{t}^{\mathrm{a}}, \mathbf{4}^{\mathrm{l}} \text {. }\end{array}$ \\
\hline $18: 3$. & Dione & - & Lki & $\begin{array}{l}\text { Deshates, Catal. Br. Mus., } \\
\text { p. T2. }\end{array}$ \\
\hline I8:3:i. & Cytherea & - & & $\begin{array}{l}\text { Sowerby, Thes. Conch., } \\
11 \text { p. } 636 \text {, pl. CXXXIl, fig. } \\
117,118 .\end{array}$ \\
\hline $186: 3$. & Dione & - & Lli & $\begin{array}{l}\text { REEYe, Conch. Icon.,pl. X, } \\
\text { lig.43. }\end{array}$ \\
\hline $186: 3$. & - & striata & & $\begin{array}{l}\text { ReEve, Conch. ICon..pl.X, } \\
\text { fig. 1'́. }\end{array}$ \\
\hline
\end{tabular}


18633.? Dione ustulata lisere,Conch. leon.,pl. XI.

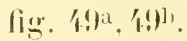

1860. Cytherea citrina l.k Riomer, Mon. Mollusk. (iall. Vellus, J. lon), 1). XXYll, fig. ‥

1881. Callista - deRochemboxe, Documents sur la fante malar. de la Cochinchine u du Cambodge, exlr. Bull. Soc. Iliifomat. Paris, p. Iii.

1891. Meretrix (Pitar) citrina Lk P. Fischer, Calal. IndoChine, loc. rit. 1). 20?3) (1). 1

Tonkin. - Baie d'Along. Anse du Grane sous lus caillomx ì basse mer (M. Blaise).

Vexes (CRrptowimana) sQumosa Limné.

17i.s. Temes squamosa Laxe, syst. Nal. él. X. 1). (iss.

1767. - - Laxe Sysl. Nal. ed. NII. 1). 1113 .

178. - - Linnaei Cinemstz, Conch. Cals. YT,

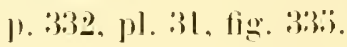

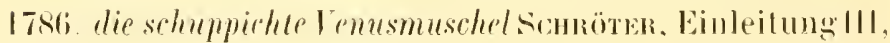
1. 1:3:i.

1790. Lomes squamosa L. Gulem, syst. Mal. id. XIII, 11. 3207.

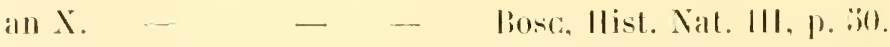

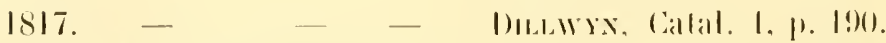
1817. Anomatocardia mgosa somsacmer, Essali noms. sysl., I. 1:3i, fil. 201. lig. 臌, 们。

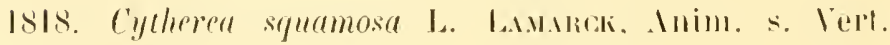
1. 1. 3801 . 
152:- Tenus sfuamosa

$$
\text { Iห⿻上. }
$$

183:. Cytherea -

1839. Temus -
1819. -
1843-184:- Cytherea -

15:3i. Anomalocardia -

18\%3. Cryptogramma -

18\%3.). Temms - I.

18:3.).

$15: 37$.

18:8. Cryphogramma - -

186i;). Tents - -

186ī. Cryptoyramma - -

1869. Temus - -

1869.

18!)1. V.(Cryptogramma)-
Wrood, lud. Iestac., p. 37, 1). 8, fig. 7.

L. Beanville, Dict, Se. Nat. I.VII, p. 27亿.

Deshayes in Lamarck, luim. s. Vert, ㄹe éd. V1, p. $3 \underline{2} 7$.

Ахток, Verzeichn.. p. !.

Haxter, Rer. biy. Sll., p. li:i.).

Chexe, III. Conch., pl. 13. fig. I $2,12^{\mathrm{a}}, \mathrm{I} \mathbf{2}^{\mathrm{b}}$.

Deshases, Cilal. Br. Mus., 1. 117.

Mörch, Catal. Yoldi II, 1. 2.2.

Soweris, Thes. Conch. II. 1. 7l6, pl. CLN1, fig. 83, S' Haxier, lpar linn. Conch., p. 73 .

liimer, lirit.l'nters. p.:̈ir. H. \&. A. Anas, Gen. of reer. Moll. II, p. 但).

hisere, Conch. Icon.. pl. XXI, tig. I(1) I.

Römer, Malak. B1., p. oli.

PFenfer, Mon. Conth. Cab, 20 éd.p. 17s, pl. 17, fig. I2.

L. MonLet, Journ. de Conch. XXXYII, 1) 170.

- P. Fiscilen, Catal. Indo Chine, loc. cit., p. 2033 (p. l'9) (lu t. ¿̀ p.).

Tonkin. lle aux Biches. Baie d'Mlong (M. Blaise). 
TAPES VIRGIXES Linné.

1767. Temes rirginea Laxé, Syst. Nal., éd. Xll, p. 1136.

1sis. - rimularis Lamarck, Anim. sert. Ver, р. $60 \%$.

ISIS. - flammirulala Lasasok, ibid., p. 60:3.

IsIs. - callipyga Lamarck (non Born), ibid., p. (i) 1 .

I8פ8. - rimularis Lk Blanvilee, Dict. Se. Nat. LVII, p. 28I.

189.". - - DESIIAYES ill LanarCK, Inim. sans Vert. 렁. VI, p. $36 \bar{i}$.

183:i. - flammiculata Desmases ibid. p. 36i.

I83:i. - callipyga Desnares (non Borm), ibirl. 1. 3361 .

18:39. - rimularis la Axton, Verzeichn., p. !.

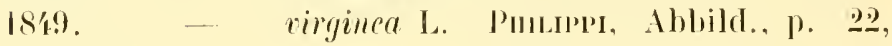
pl. YIII, fig. 2. 3, 3. 亿.

18:i3). Chione rimularis Lk Desmares, Cat. Br. Mus., р. 19!.

Is:3:3. Pullastra rirginea Möвсн. Catal. Yoldi. II, 1. ․․…

18:3i3. ('hione hiamtina var. Deshayes (non lam.), Call. Bir. Mus., 1). I4!!

18:30. Tapes rimularis Lk sowerni, Thes. Conch. II,

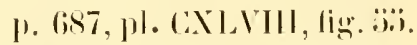

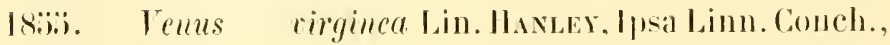
p. 81.

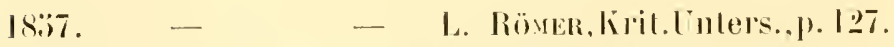
18:37. - flammiculata lek Rï̈mer, ibid.. P. I2s.

Istit. Tapes rimularis Lk lisere, Conch. Icon..pl. I'I, lim. 2 ?.

1slí. - virginea Röser, Malak. B., J. 8:3. 
1869. T'enus virginea Lin. Prenfren, Mon. Conch. Cab.

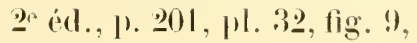
III.

Tonlin, Anse du Crine, Baie d'Along, I exemplaire recueilli à marée basse sous des cailloux reposant sur la vase mélangée de graviers et de coquilles brisées (M. Blaise).

Il est certain que Linné a confondusous le nom de l'enus rirginea l'espece exotique en question et une autre des mers d'Europe. Les deux formes existent, ainsi que nous lapprend llanley dans la collection de Linné. Nais la description et llabitat " in Indiis ") du Systema Vaturae justifient l'identilication de la coquille exotiquedont nous nous occupons, de préférence à celle de l'espèce Européenne à laquelle la plupart des auteurs ont attribué le nom de $T$. rirgineus, mais qui doit porter le nom de $T$. rhomboiles lennant.

Corbicula fluminea Müllel.

177'. Tellina fluminea

1789. Temus - ete.

1886. Corbicula Amamitica
MÜLLR, Historia Yermium, II, p. 206 .

Cuevintz, Conclı. Cab. Vi. p. 3르, p1. 30, fig. 3를, 3로.

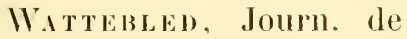
Conch., vol. XXXIY, p. 69, 1). I, fig. 3.

1886. - insularis Pr. L. MonLet (non Prime?), Journ. de Conch. vol. XXXIV, p. 268.

1891. - Ammamitica W. P. Fischer, Catal. IndoChine, loc. cit., p. 236 (1:32 du t. a p.).

1891. - insularis Pr. P. Fiscner (non Prime?), ibid., p. 2038 (1:3̈́ (lu t. à p.). 


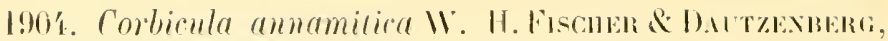
Catal. Indo-lihine in Mission Parie, lor. ril. J. 位 (:ie dul l. ì l\%).

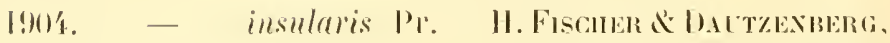
(nom Prime?), ibirl., p. 份 (:ie) dul 1. iो p.).

Ipres m examen allentif des deseriptions el des ligures originaless de Müller el de Chemmilz comparées à une série lrès étendue de Corbieulas de l'Extrême-Orient. nous pensons que le Tellina fluminea Müller et le l'enus fluminea lhemnitz ont été élablis sur des exemplaires jeunes qui. en se développant,auraient produit à l'étal adulte certaines des variélés mentiomées plus loin, sans qu il soil possible de savoir a laquelle de ees variétés le type te Müller doit ère mapporté. Nous ramgeons à la suile de res références typiques le Corbicula Annamitica Walteblet que nous considérons comme une forme jeme de la mème espece, ainsi que les spécimens que nons avons trouvés dans la collection I. Morlet, étiquetés sous le nom Corbicula insularis Prime, mais qui nous paraissent ètre des jemnes fluminea.

Nous nous trouvons donc en prisence diune espere fondée sur des spécimens jemes, dont la forme typique ne peut pas ètre préciséc, et dont les variélés seules sonl bien délinies.

La belle série rapprortie par M. Baise. venanl compléter de nombreux matériaux que nous possédions d'au. l re part, nous a démontré l'existenee de loutes les formes de transition entre plusienrs especes que lon eonsidénit romme distincles el que nous avous réduiles all lang de

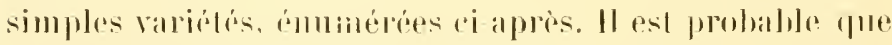

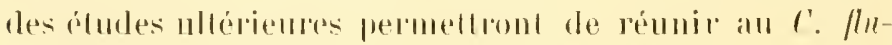
minea beancomp d'aubes formes, el pent edre notamment 
les C. anrea, maeterita, squalida, rariegata, subquadrata, iodina, Cordieriana, lapicida.

Var. tonkiniana, L. Morlet.

1886. Corbicula Tonkiniana L. Mol LET, Journ. de Comeh, vol. XXXIT. p. 26 sis,

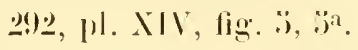

\begin{tabular}{|c|c|c|c|c|}
\hline 1887. & - & - & L. M. & DALTZENBERG \& D'HANON- \\
\hline & & & & $\begin{array}{l}\text { ville, Journ de Conch., } \\
\text { rol. XXXY, 1. 2.29. }\end{array}$ \\
\hline $18 \times 9 \%$ & - & - & & $\begin{array}{l}\text { L. Monlet, Journ. de } \\
\text { Concli., vol. XXXYl, } \\
\text { l7l. }\end{array}$ \\
\hline 1891. & - & - & - & 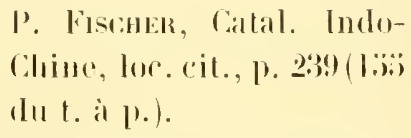 \\
\hline $190 \%$ & - & tonkiniana & - & 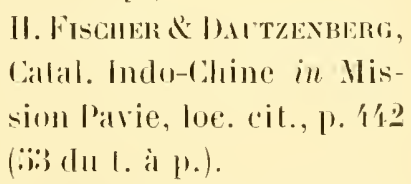 \\
\hline
\end{tabular}

Toukin, Song L.uc-Nam, Tan-Ien (II. Blaise). Spécimens passant à la varieté Bocomrti.

Var. Petiti (Clessin), L. Morlet.

Is86. Corbicula l'étiti Clessin mss L. MarLat, Jomrn. de Concli, vol. XXXIT, p.

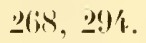

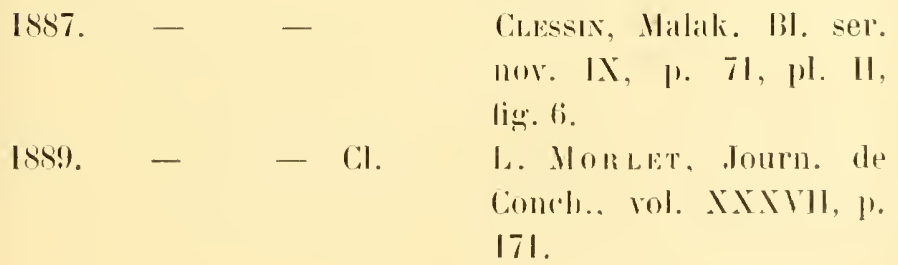


1891. Corbicula Petiti Cl.

1901.
P. Fischer, Catal. Indo(hine. loc. cit., p. 239) (1:3); dı 1. іे p.).

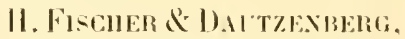
latal. Indo-thine in Mission Pavie, loc. cit., p. 1'旦 (:i.j) du t. à p.).

'Tonkin, Song Lue-Xam, Van-len, un exemplaire (.I. Blaise).

la valiélé Petili est lrès voisine de la variété Moreletiana Prime, mais plus oblique et plus élargie.

\section{Vill. Moreletiana Prime.}

186ī. Corbicula Moreletiona Prine, Ann. Lỵe. X. H. New York YIII. 1. tlit.

1870. Prome, Cital. Corbic. in Amer. Journ. of Conch. $Y$. p. $13 \%$.

187.;. - - \. Morelet, Séries Conch. 11, p. 360 , pl. Xlll, tig. 4.

1876. Cyrena erosa besh. mss Soweks in Reve (non Prime), Conch. Icon., pl. $\mathrm{XI}, \mathrm{fig}$. $4 \mathbf{t}^{\mathrm{i}}, \mathrm{q}^{\mathrm{i}}$.

1579. Corbicula Moreletiana Pr. Clessix, Mon. in Conch. Cab., ㄹéll., p. 1!s.
I889. - - - - L.Monlet,Journ.deCunch. rol. XXX111, p. 171.

18\$:\% - erosa L. МokLet (non Desh. nec l'rime), Journ. de Conch. vol. XXXVl1, p.17\%.

La coquille représentie par Sowerhy in Roeve sous le nom de Cyrena erosa Deshayes mss.. avec ritalion dusyonyme douteux C. triangularis besh., est certainembnt le C. fluminea var. Moreletiana el ne ressemble pas au C. Irian- 
gularis Desh., tel que l'a interprété et figuré Prime. Quant an C. crosa de Prime (186i), il n'a pas de rapport avec l'erosa Desh. mss in Sowerby.

C'est par erreur que Sowerby a indiqué comme référence de Deshayes, a propos de l'erosa: P. Z. S. L. 18:3' p. 3:'́t celte rélérence sapplique en eflet au triangularis, sauf que la page exacte est 3 h.j el non :3.í.

Lid var. Moreletiana n'est mentionnée ici que pour compléter l'étude des dillérentes formes du C. fluminea, car elle ne figure pas dans les récoltes de II. Blaise.

\section{Var. Bocourti A. Norelet.}

186.9. Cyrena(Corbicula) Bocourti Morelet, Journ. deConch. vol. Xlll p. 2208.

1870. Corbicula Bocourti Mor. Prine, Catal. Corbieulidae in Amer. fourn. of Conch. Y. p. 129.

\begin{tabular}{|c|c|c|c|c|}
\hline $187 \%$ & - & - & - & $\begin{array}{l}\text { Monelet, Séries Conch. } \\
\text { IV. p. } 361 \text {, pl. XV1, fig. 2. }\end{array}$ \\
\hline 187 & - & - & - & $\begin{array}{l}\text { Clessix, Conch. Cab. 20 ed. } \\
\text { 1. } 203 .\end{array}$ \\
\hline
\end{tabular}

1883. - gryphaea Hecne, Conch. Huv. Nanling X, pl. Y, fig. 28, 228. 1883. - graris Ilecoe, ibil., pl. IN, hig. 20

1886. - Ciryphaea IIde L. Moncet, d. de Conch. rol. ХХXI, p. 206s.

1887. - Bocourti Mor. Cuessin, Malak. B1., p. 7̈, pl III, fig. 3.

1889. - - - I. Molilet, J. de Conch. rol. XXXY11, p. 170.

1889. - Giracisi IIde L. MolzLet, Ibid. P. 171.

1889. - gryphaea - L. MonLet, ibid., p. 171.

1891. - Rocourti Mor. P'. Fischer, Calal. Indo Chine loc. vit., p. 237 (1;3) lu t. a |l.). 


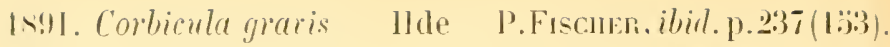
1.9!. - gryphaea - l' Fisamer, ibiel., p. 23\%, (1:3.3).

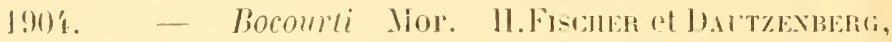
Calal. Indo-Chine in Mission lavie loc. cit. ]. th (:i己 dul. à p.).

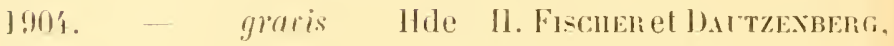
ibirl. ]). 仵 (范).

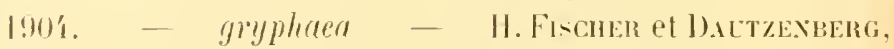

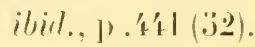

Tonkin. Song Luc-Nam, Van-len. Nombreux spécimens arer passage à la variété tom limiana (М. Blaise).

Jous arons sous les yeux d"autres spécimens de la rar. bocomri qui nous ont élé communiqués jar le lor. Billet 'n Is:T, arec les jororenances suivantes : Song BangCilang a lloxat et près de Can Bang: arroge de Trung-

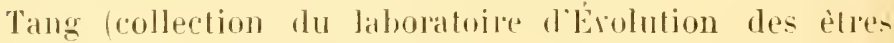
olomises. a la soluonne).

Ial. orientalis lamarek.

IxIs. Cyrena orientalis Lamancis, Inim. $\therefore$. Tert. 1. 1. .3.,.

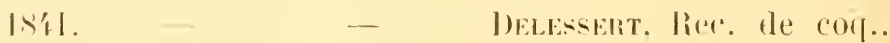
pl. III, tig. S.

18.̈4. Corbicula - Le Desmares, Cátal. Bril. Mus. Conchit., p. 2.27.

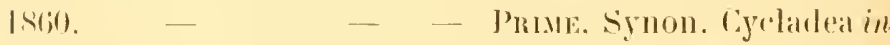
Pror. Arad. x. si. Philad. 1). $2 \div 2$.

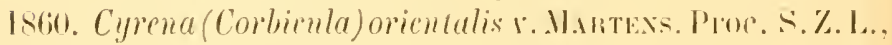
). Ii.

1570. Corbienla orientalis l'muns. Catal. Corbie. in Inter. I. of lionch.. $\mathrm{V}$. 1). 1:3\%. 
1879. Corbicula orientalis Lk Chessix, Mon. in Conch. Cab., 2 éd., p. 130, pl. 느, fig. 1, 2.

1891? - - - P. Fischer. Catal. IndoChine, loc. cit., p. 239 (iijii dul t. à p.).

190'? ? - - - II. Fiscierd DAltzexberg, Catal. Indo-Chine in Mission Parie, loc. cit.,

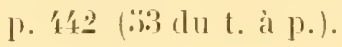

Le Cyrena orientalis a été décit jar Lamarek en ISIS comme ume espece de 17 millimetres de largeur, avec une variété habitant les rivières llu levant, de on millimètres; cette dernière forme a la dent cardinale médiane bifide, caractire qui se retroure tans certains échantillons de l'Intlo-Chine. Delessert figure sous le nom te C. orientalis une coquille bien plus scrande que celle décrite par Lamarek; entin Prime représente. en 1872., sous le mème nom. une coquille du Japon finement strice

Bien qüil subsiste quelque incertitude sur la définition du C. oricmalis, nons pensons que cetle forme doit ètre consilérée comme une simple variété du C. fluminea. Nous ne l’arons pas trourée dans les récoltes de M. Blaise.

Var. indigotina lleude.

I883. Corbicula indigotina Hecoe, Conch. flur. pror. Nanling X. 1l. J. fig. 21, 21.

\begin{tabular}{|c|c|c|c|}
\hline IS89. & - & - & 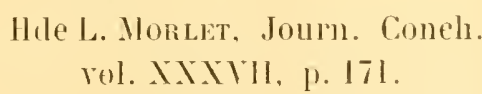 \\
\hline I891. & 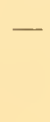 & 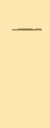 & $\begin{array}{l}\text { - P. Fiscuer, Catal. Indo- } \\
\text { Chine, loc cit., p. } 238 \text { (lït } \\
\text { du t. вे).). }\end{array}$ \\
\hline
\end{tabular}


1901. Corbicula imligotina IIde 1H. Fischer el Dactrexpbais, Catal, Indo Chine, in Mission Pavie, loc. cit., J. 仵

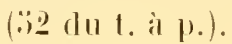

Cette forme est intermédiaire entre les variéles bocomti el Bandoni du C. fluminea, mais elle est plus voisine de la var. Bocontri à cause de ses sillons rapprochés. Elle n'a pas été rencontrée par M. Blaise.

\section{Var. Baudoni L. Morlet.}

1886. Corlicula Bandoni L. Мовьет, Journ. de Conch. rol. XXX11, j. 206, 293, pl. XI, fig. 6, 6".

1887. - L.M. D.nutzexbeng el d'HAMoNVILLE, Journ. de Conch. rol. XXXV, 1. 20.2'.

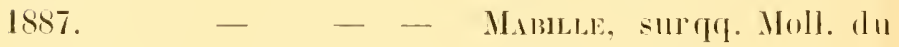
Tonkin. P. [li3).

1891. - - P. Fiscmer, Calal. Imlochine, loce cit., p. 2o:3t; (1:i) du t. a p.).

190'. - - - H. Fischeli el Dattzenbero, Carlal. Indo-Chine in Mission Pavie, loc.cit., p. '倓 (:iz) du t. ì p.).

A cette variété, qui ne figure pas dans les récoltes de $\mathbf{M .}$ blaise, nous pensons qu'il y aurait lieu de rémir comme synonyme la variélé de $C$. graris Heude que cet anteur a représentéeen 1883 dans sa Conch. flur. Prov. Nanking X, 1). IV fig. 20a. Nous possédons d'autre part des spérimens de la collection I. Morlet étiquelés C. insuluris P'rime et (qui nous paraissent appartenir à la var. baudoni du $C$. fluminea. 
Solexoteldixa consomina (1)esh. mss) Reeve.

18:37. Soletellina consobrina Desh. mss. REeve, Conch. Icon., pl. I, fig. 1 .

1891. Solenotellina - - P. Fiscuen, Catal. IndoChine, loc. cit.p. 291 (1:37 (lu t. ̀̀ p.).

Tonkin. Ilaïphong (Il. Blaise).

As.PPIIS DICHOTOMA Anton.

1782. Tellinu anomala Indiae orientulis CHEmxitz, Conch. Cah. 11, p.9\%, pl. 9, fig. 8.3.

1790. Temes deflorata var. g Givelox, Syst. Nat., éd. XIII, 1. 320'1́.

1818. Sanquinoluriarngosa var. b Lamanck, Anim. s. Vert. I, p. B̈l.

183:i. - - Deshayes in Lamarak, Anim.s. Vert, 20 éd. p. I7).

18:38. - dichotoma Axton, Verzeichn., p. 1. 183̈6. Fupse deforate Reve (non. Lin.), Conch. Icon.,pl. I, fig. la (tantum).

18:38. Asaphis Itichotoma Inton Märcn, Journ. de Conch., rol. VIIl, p. 138.

Tonkin. Baie d'Along (It. Blaise).

Mreodesma rilabratey Lamarek.

18I8. Crussatella glabrata L.sмanck, Anim. s. Vert Y, р. 但里.

183:. Mesodesma - Desmares in Lamarck, Inim. s. Vert, 2éd. VI, p. $1: 33$.

18:̈\% - - Lk ReEve, Conch. Icon., pl. III, fig. 20).

Tonkin. Baje d'Along (M. Blaise). 
Tellixi Broguera llanley emend.

184. Tellina Bruguieri Haxley, Proc. \%. S. J., p. 14․․

1847. - - Itaxbex, Mon.in Sow. Thes. Conch., 1. 3리, pl. (i2, lig. 192.

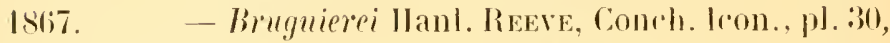
fig. $160 ;$.

1871. - Brugueri Römen, Mon. in Conch. Cab.

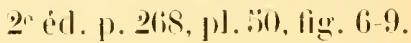

Tonkin. Baie d'Mlong (M. Blaise.)

AMrmbesma hamelosta sowerhy.

18.33. Amphidesma lamellosa Sowner, spec. Conch.. part 2 (inéd.), lig. 9, 19. in ReEve, Conch. Icon., pl. III, fig. 16 .

'Tonkin. Ile aux Biches, baie d'Along (M. Blaise).

Ph. D. et H. F. 


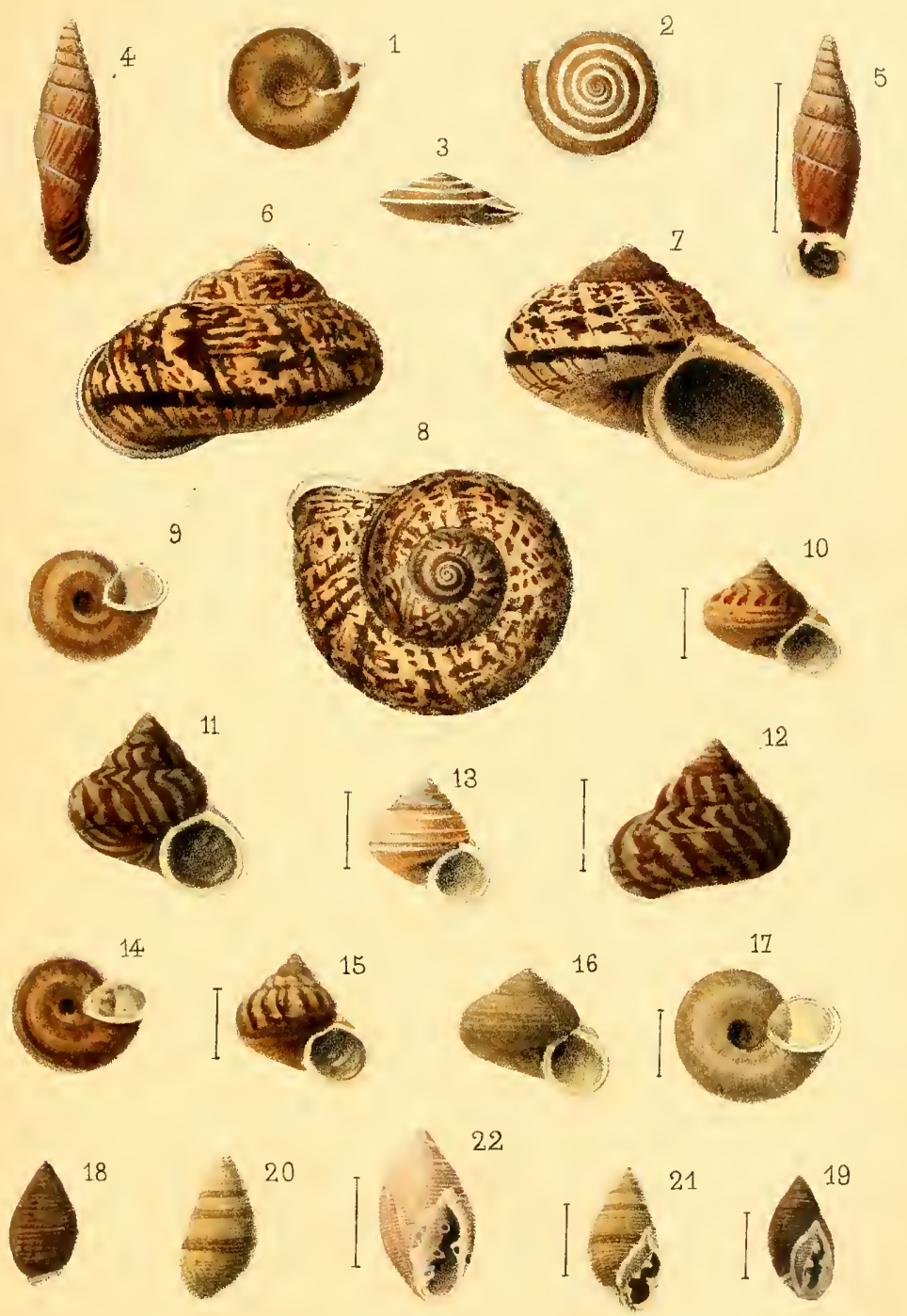

3. Trochomorpha sapeca Heude.

5. Clausilia Ârdouiniana Heude, var minor D \& F 13. Lagochilus scissimargo var. carinata D. \& H.F 8. Cyclophorus unicus Mabille

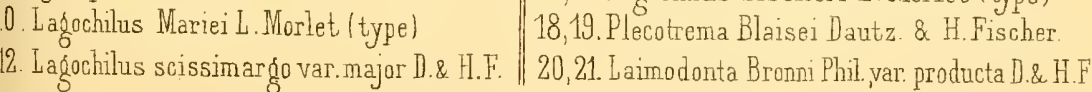
22. Plecotrema siamensis A. Morelet. 

Journal de Conchyliologie. 1905.

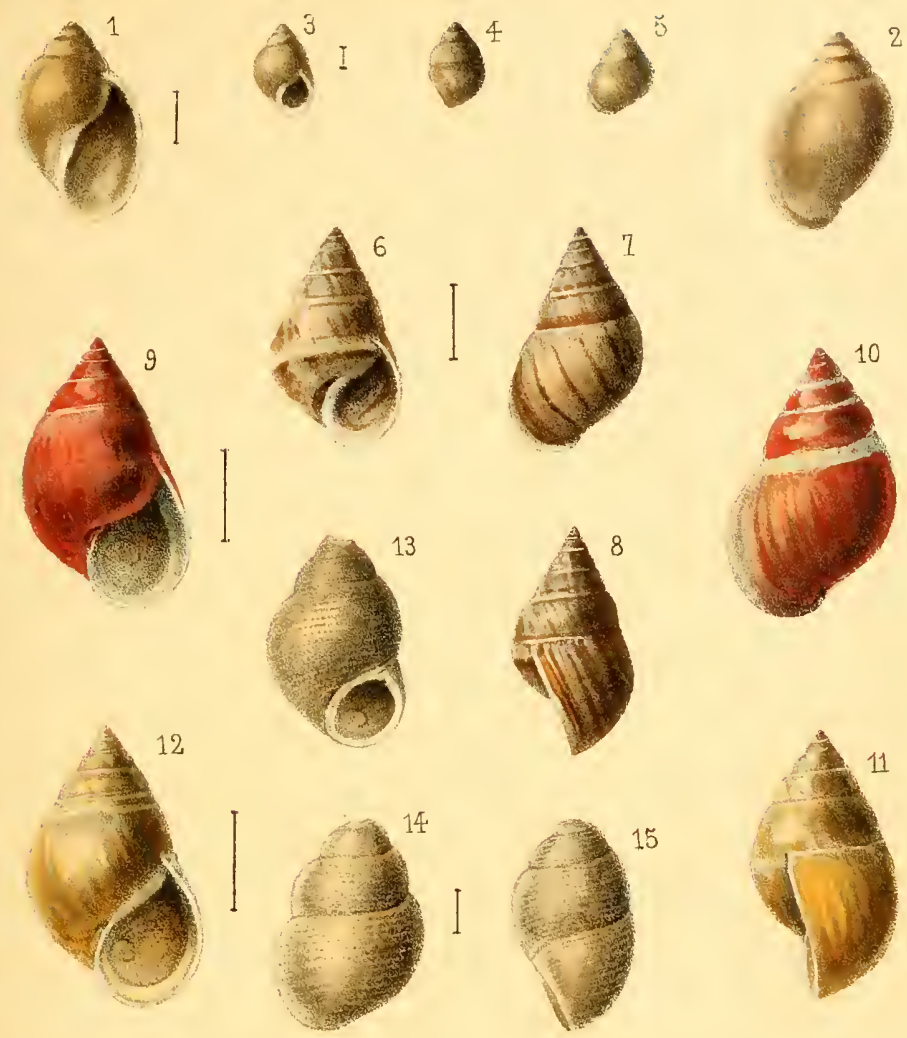

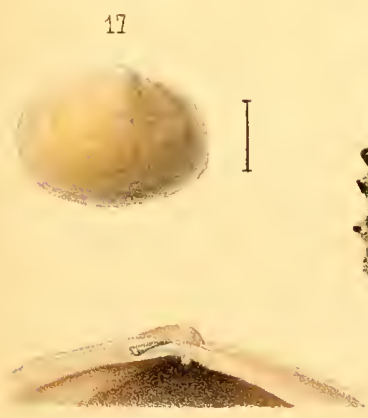

19
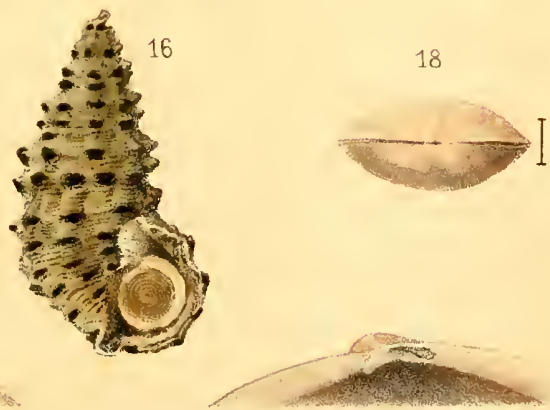

20

G.Reignnier, del. et lith.

Imp. I hafontaine, Pans

1, 2. Limnaa Blaisei Dautzenberd \& H. Fischer. |9,10,11,12. Assiminea brevicula Pfeiffer. 3,4,5. Assimine Francoisi Dautz. \& H. Fischer 13,14,15. Stenothyra monilifera Benson. 6,7,8. Assiminea interrupta Dautz. \& H. Fischer 16. Potamides tuherculatus (Limé) Born. 17,18,19,20. S cintilla Blaisei Dautzenber \& \&. Fischer. 


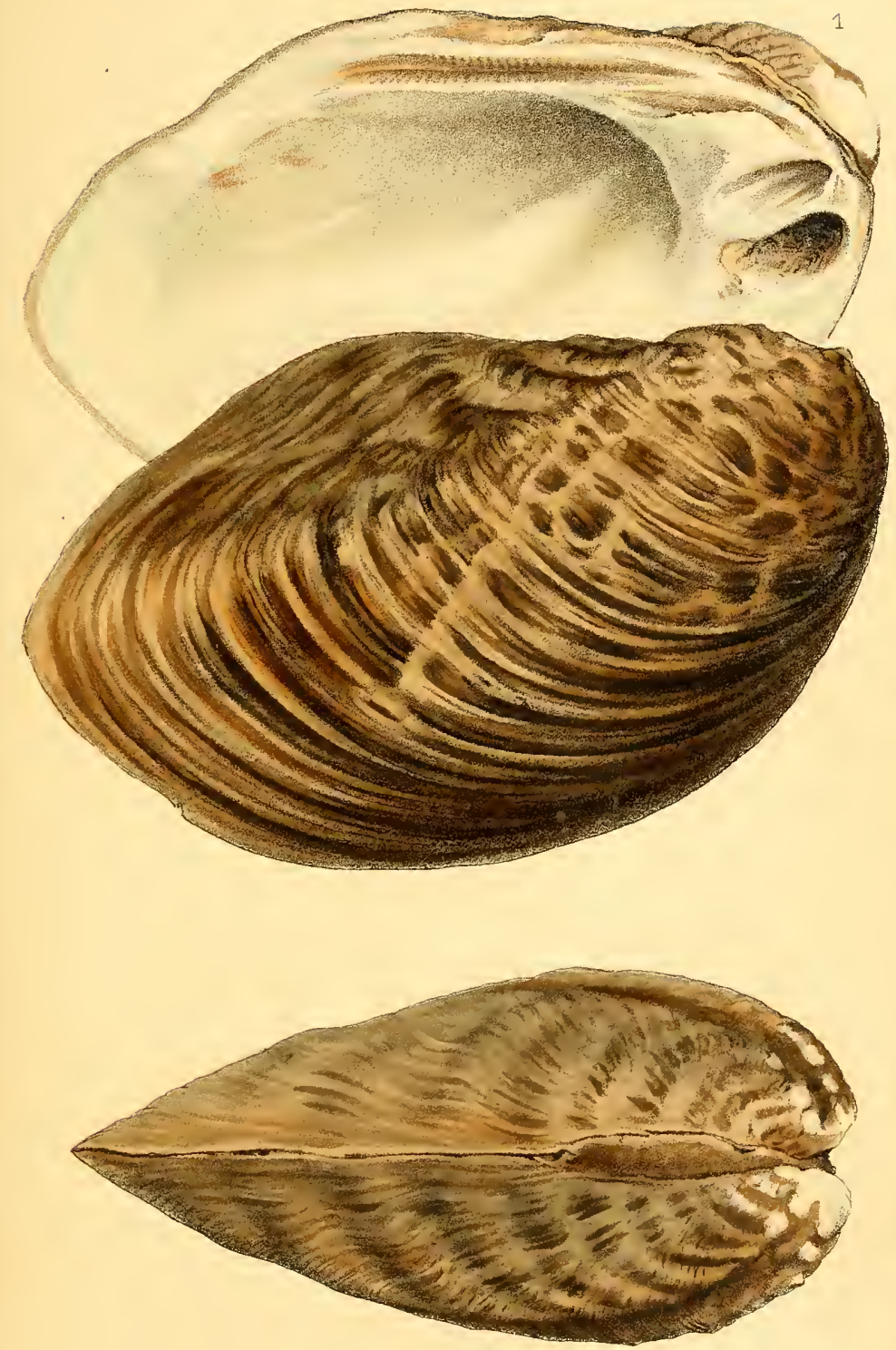

École Professionnelle d'Imprimerie, a Noisy-le-Grand (S.-et-o.) 



$$
\text { . }
$$


Fcole Professionnelle d'Imprimerie à Noisy-le-Grand (S.-et-0.).

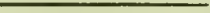




\section{CONTRIBUTION}

A LA

\section{FAUNE MALACOLOGIQUE DE L'INDO-CHINE}

PAR

Ph. DAUTZENBERG et H. FISCHER

(Extrait du Journal de Conchyliologie, Vol. LIV)

\section{PARIS}

Direction et Rédaction:

\section{H. FISGHER}

51, Boulevard Sainl Michel (V") ।
Administration :

F. R. ов RUDEVAL, Éditeur 4, fiue Antoine Dubois ( $\left.\mathrm{II}^{\mathrm{e}}\right)$

1 DOH: 

Extrait du Journal de Conchyliologie. Vol. LIV, 1906, p. 143

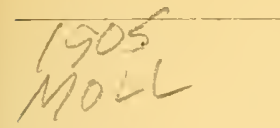

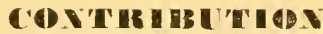

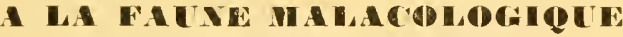

IDE MYNDO-CIIINE

Par Ph. Dautzenberg et H. Fischer

Les matériaux étudiés dans ce travail nous out été obligeamment communiqués par M. Joubin, Professeur de Malacologie au Muséum. Ils ont été recueillis en Annam et au Tonkin par M. Boutan, Directeur de la Mission Scientifique permanente en Indo-Chine.

A première vue ces matériaux" consistant en quelques coquilles mortes recueillies dans les cordons littoraux, semblaient ne présenter qu'un intérêt très relatif ; mais nous avons trouvé dans l'intérieur de certaines de ces coquilles, ramassées sur la plage de Ben-Son, Annam, du sable qui soumis à un triage minutieux nous a fourni toute une série de petites espèces très intéressantes, la plupart nouvelles, et appartenant à des genres qui n'avaient pas encore été signalés dans la région. Il est donc certain que des recherches plus sérieuses aideront à faire connaitre une foule de formes qui ont échappé jusqu'à présent aux observations superficielles.

Aux récoltes de $M$. Boutan se trouvaient jointes quelques espèces terrestres et d'eau douce envoyées du Tonkin par M. Demange, négociant à Hanoï, et par V. Krempf, attaché à la Mission Scientifique en IndoChine. 
Streptaxis (Eustieptaxis) costulatus Mlldff

Var. subcostulata Mlldff

1901. Streptaris(Eustreptaris) costulatus Mlldf, subsp. subcostulatus, MöllendorfF, Diagn. neuer voll $\mathbf{H}$. Frühst. ges. elc. in Nachrichtsbl. d. d. Malak. Ges. p. 6 อั.

1903.

- Mlldff, var. subcostulata Mlldfi, Bavay el DautzenBerg, Deser. coq. nouv. Indo-Chine in J. de Conch. p. 201 , pl. VIII, f. 1, 2, 3.

1904.

- Mlldil subsp. subcostulatus, H. Fischer et Dautzenberg, in Mission Pavie Et. div. III, P. 390 (1 du t. à p.)

Tonkin : Su-Ýut. Rivière noire (M. Demange).

Helicarion Imperator Gould

18599. Vitrina imperator

$\begin{array}{lll}186 \% & - & - \\ 1867 . & -\end{array}$

1868.

18s1. Helicarion - Gld

1885.

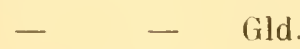

1887. - maleficus
Gould, Proc. Boston Suc. N. H. VI, p. 422.

Goul1, Otia Conch. p. 101. v. Martens, Preuss. Exp. nach Ostasien p. 41. Pfeiffer, Mon.Hel.v.p.14. v. Martens, Mittheilungeu I, p. 73, pl. XIII,f. 1 à 6. Tryon, Man. of Conch. Str. and Syst. I, p. 179, pl. 41, f. 76-7S.

J. Mabille, Moll. Tonk. diagn. p. 2. 
1887. Helicarion maleficus

J. Mabille, Sur quelques Moll. du Tonkin in Bull. Soc. Malac Fr. p. 74.

1904. - (Cryptosoma) imperator Gld., H. Fischer et Dautzenberg, Catal. IndoChine in Mission Pavie Et. div. III, p. $392(3)$.

Tonkiı: Su-Yut, Rivière noire (M. Demange) - BanНаo (M. lírempf).

L'Helicurion maleficus J. Mab., dont nous avons examiné les types récoltés par M. Balansa et conservés au Muséum de Paris, nous paraît identique à l'imperator Gould ; mais la fig. 11 de Mabille est loin d'être satisfaisante : elle présente en effet un tour de spire de plus qui, en réalité, I'existe pas dans les types.

\section{Helix (Eulota) Jourdil L. Morlet}

1906. Helix (Eulota) Jourdyi L. M. Dautzenberg et H. FisCHER, Moll. rec. par M. Mansuy en Indo-Chine, in J. de Conch. LIII, p. 362.

Tonkin : Hanoï (M. Boutan).

Helix (Papuina) Demangei n. $\mathrm{sp}$.

(Pl. V, fig. 1, 2, 3.)

Testa tenuicula anguste rimata. Spira conica, apice obtusiusculo. An/r. 5 rapide crescentes, sutura impressa sejuncti, infra suturam anguste complanati, deinde convexiusculi, striis incrementi tenuilus striisque transversis minutissime decussati. Anfr. ultimus ad extremitatem haud descendens, acute carinatus, basi valde convexus.

A pertura valde transversa et ubi carina desinit, in rostrum producta. Columelli subareuata, obliqua, umbilicum 
fere omnino obtegens. Labrum acutum expansum ac subreflexum. Color albidus: :ona fusca transversa angusta carinam comitatur.

Altit. $14^{\mathrm{mm}}$; diam. max. 19,3̆mm ; diam. $\min .15^{\mathrm{mm}}$. I per. tura $9 \mathrm{~mm}$, alta, $14^{\mathrm{mm}}$ lata.

Coquille assez mince, pourvue d'une perforation ombilicale très étroite. Spire conique à sommet un peu obtus, composée de cinq tours qui croissent rapidement et sont séparés par une suture très accusée. Ces tours présentent immédiatement au dessous de la suture une dépression étroite, aplatie; ils sont ensuite convexes. Leur surface est ornée de stries d'accroissement très fines et de stries décurrentes également fines qui forment un réseau visible seulement à l'aide de la loupe. Deruier tour à base bien convexe, non descendant à l'extrémité, présentant une carène périphériale aiguë.

Ouverture très transverse et présentant un rostre bien accusé à l'endroit oủ aboutit la carène. Columelle subarquée, oblique, dilatée sur la perforation ombilicale qu'elle recouvre presque totalement. Labre dilaté aigu et un peu rétléchi au bord.

Coloration blanchâtre avec une bande périphériale brune étroite, qui accompagne la carène. La moitié de cette bande émerge de la suture des tours précédents.

Tonkin : Su-Yut, Rivière Noire (M. Demange).

Cette espèce diffère de toutes celles qui ont été décrites jusqu'à présent de l'Indo-Chine par la conformation de son ouverture qui ressemble beaucoup à celle des Papuina de la Nouvelle-Guinée et des Iles Salomon. Malheureusement elle n'est représentée que par un exemplaire unique et en mauvais état, recueilli par M. Demange auquel nous dédions cette curieuse forme. 
Clausilia Dokri Bavay et Dautzenberg

1899. Clausilia (Pseudonenia) Dorri Bavay et Dautzenberg, J. de Conch., XLVII p. 283 pl. XII f. 6.

1904. Clausilia Dorri

H. Fischer et DautzenBerg, Catal. Indo-Chine, in Mission Pavie Et. div. III p. $409(20)$.

Tonkin : Su-Yut, Rivière Noire (M. Demange).

\section{Terebra tenera Hinds}

1813. Terebra tenera

181.7

1860. - - Hinds

$188 \%$.
Hinds, Proc.Z.S.L., p. 158. Hinds in Sowerby, Thes. Conch., p. 184, pl. XLV, f. 111 .

Reeve, C. İcon., pl. XXVII. f. 148.

Trion, Man. of Couch. Str. and Syst., VII, p. 33̈. pl. 10, f. 99.

Annam : plage de Ben-Son (M. Boutan).

Cancellaria Bocagei Crosse et Debeaux (emend.)

1S63. Cancellaria bocageana Crosse et Debeaux, J. de Conch., XI, p. 77 ; p. 263 pl. IX f. 3.

1874. - - Cr.et D.Lischie, Japan. Meeres Conch., III, p. 41 pl. 2 , f. $12,13,14$.

1882. - - - Dunker, Ind. Moll. mar. Japonici, p. 103. 
1885. C'ancellaria Thomasiana TuYon, (ex parte, non Cr. et Debeaux), Man. of Conch. Str. and Syst., p. $79, \mathrm{pl} .6$ f. 92 .

Annam: plage de Ben-Son, (II. Boutan).

Nous manquons de matériaux pour contrôler l'opinion de Tryon qui considère comme identiques les $C$. Thomasiana et Bocageana. La première de ces espèces est établie sur les fig. 2כ. 25a du C. Icon. représentant une coquille plus grande que le $C$. Bocagei, avec des côtes plus obliques, et indiquée comme provenant des Indes Occidentales: nous croyons qu'il n'y a pas lieu, pour le moment, de confondre les deux formes.

\section{Olivella Fóktunei A. Adams}

1871. Oliva Fortunei

1871. - pulchra.

1878. - (Olivella) Fortunei A. Ad. Weinkauff, Mon. G. Oliva, in C. Cab., 2 Ed., p. 1534 , pl. 39, f. 20 , 21.

1883. Olivella Fortunei A. Ad. Tryos, Man. of Conch. Str. and Syst., V. p. 69 pl. 16 f. 12-14; (15?)

Annam: plage de ben-Son, nombreux ex. jeunes (M. Boutan).

Cette espèce n'est pas citée dans le Catalogue de P. Fischer. 


\section{Semifusus tuba Gmelin}

1780. Classicum legionis desultoriae Chemnitz, Conch. Cab., IV, p. 171, pl. CXLIII, f. 1333.

1783. die Dragonertrompete

1790. Murex Tuba

1817. - tuba

18⒉ Pyrula -

1825. Buccinum-

1825. Murex -

1832. Pyrula - Gm.

1839. Fusus -

1843. Pyyrula -

1847.

1848. - crassicauda

1852. Melongena tuba

185ว. I'yrula
Schröter, Einleit.I,p. 619. Gmelin, Syst. Nat,, Ed. XIII, p. 3554 .

Dillwyn, Descr. Catal., il, р. 720 .

Lamarck, Anim. s. Vert., VII, p. 139.

Wood, Index testac., p. 108, pl. 23, f. 68.

Woop, Index testac., p. 126, pl. 26, f. 80 (c'est la var. crussicauda).

Deshayes, Encycl. Méth., III, p. 869 (pI. 4의 f. 2).

líener, Icon. coq. viv., p. 51, pl. 26 f. I,1.

Deshayes in LamarcK, Anim. s. Vert., IX, p. ̋07 Reeve, C. Icon., pl. IX, f. 2.2.

Philıpe, Zeitschr. f. Malakoz., p. 28.

Petit de la Saussaye, Nouv. Classif. des Pyrula, in J. de C., III, p. 148.

Eynoux et Souleyet, Voy. Bonite, p. 618, pl. 42, f $4,5,6$ (sub nom. Pyrule trompette). 
1858. Hemifusus tuba

1858. Busycon crassicauda

18599. Fusus (Hemifusus) tuba

1869. Hemifusus

1881. Pyrula

1881. Hemifusus

1889. Semifusus

1891.

H. et A. Abans, Gen. of rec. Moll., I, p. 82, pl. 9, f. 2,2 .

A. Adans, Genera of rec. Moll.. 1, p. 151.

Chenu, Man. de Conch., I, p. 142, f. 61ว.

- Lischke, Jap. Meeres Conch., I, p. 36.

-Gm. Kobelt, C. Cab., 2- Ed., p. 41, pl. 5 , fig 1 ; pl. 7 , fig. 1 (var. crassicauda Phil.).

- Gm. Tryon, Man. of Conch.Str. and Syst., III, p. 112.

- Crosse et Fischer, Note sur la faune conch. mar. de l'Annam, in J. de C., XXXVII, p. 284.

P. Fischer, Catal. IndoChine, in Soc. H. N. Autun p. 139 (วั5).

Annam: plage de Ben-Son (M. Boutan), 1 exemplaire jeune.

\section{Dipsaccus areolates Lamarck}

1758. Buccinum spiratum

1764.

1767.

1780. Nassa oblonga etc.
Linné (ex parte), syst. Nat., Ed. X, p. 739. LINnÉ (ex parte), Mus. Lud. Uir., p. 611.

LINNÉ (ex parte), Syst.Nat. ed. XII, p. 1203.

Chmoxitz, C. Cab. IV, p. $18 \mathrm{pl}$. CXXII. 
1783. Burcinum spiratum var. 2 Schrören,Einleit.I,p. 339.

i789.

1790.

1817.

1821-22. Elnuma tessellata

$\begin{array}{ll}\text { 1822. } & \text { Lanarck, Anim. s. vert, } \\ \text { 1831. } & \text { VII, p. 282. } \\ & \text { Deshayes, Encycl. Méth., } \\ & \text { II, p. 106. }\end{array}$

1833. - - Lk. lírener, lcon. coq. viv., p. $4, \mathrm{pl} .2$, f. 3.3 .

1844.

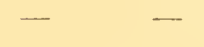

1849.

1850 .

18 รั.

1839.

1839.

1881.

cycl. Méth., p. 263.

GMelin (ex parte), Syst.

Nat., Eil. XIII, p. 3487.

p. 620 .

Swainson, Zool. III., II, pI. 145 .

Deshayes, in Lamarck, An. s. vert, $2 \odot \mathrm{Ed}$. X, p. 235 .

Reeve, C. Icon., pl. I, f. 6.

Adans et Reeve, Samarang Moll, p. 32, pl. VIII, f. 5 .

Küster, Conch.Cab., 2éd.. p. 79, pl. 65 , f. 9 .

Lk. Chenu, Man. de Conch., I, p. 164, f 785 .

Sowerby (non L.), Thes. Conch. III p. 70 pl. CCXV f. 4 .

Tryon, Man. of Conch. Str. and Syst, III, p. 212, pl. 8., f. $476 ; \mu \mathrm{l} .83, \mathrm{f}$ ร20․ 1889. Dipsaccus areolatus - L. MokLet, J. de C., XXXVII, p. 135.

1889.
Crosse et Fischer, Note sur la Faune Conch. mar. 
de l'Annam, in J. de C. XXXVII, p. 285.

1891. Dipsaccus areolatus Lk. P. Fischer, Catal. IndoChine, loc. cit., p. 141 (ร)

Annam: plage de Ben-Son (M. Boutan, 1903), exemplaires roulés.

Il est évident, d'après les descriptions et les références indiquées, que Linné a confondu sous le nom de Buccinum spiratum deux espèces de Dipsaccus. Hanley nous apprend qu'elles existent en effet toutes les deux (et même une troisième D. lutosus) sous ce nom dans sa collection. La description du Mus. Lud. Ulr. semble plutôt viser l'espèce connue actuellement sous le nom d'areolata Lk. puisque la coloration indiquée : albidus maculis ferrugineis triplici serie digestis ne peut s'appliquer qu'à cette espèce.

Toutefois Bruguière avait distingué dès 1789 dans l'Encyclopédie méthodique les deux formes en plaçant dans son $B$. spiratum A la forme à suture canaliculée et dans son $B$. spiratum B celle à suture plane et à taches brunes disposées en 3 séries décurrentes qui est devenue le b. areolatus Lk.

Nous ne croyons pas qu'il y ait lieu de revenir sur l'interprétation des descriptions linnéennes et nous jugeons préférable d'adopter la division, telle qu'elle a été établie d’une manière bien précise par Bruguière et adoptée ensuite par Lamarck et tous les auteurs modernes.

\section{Nassa vittata A. Adams}

183̈1. Nassa (Aciculina) vittata A. Adams, P.Z. S. L.,1) 114. 18533. Nassa vittata A. Ad. Reeve, C. Icon., pl. XXIV f. $160^{\mathrm{a}}, 160^{\mathrm{b}}$. 
1882. Nassa (Aciculina) maculata A. Ad. Tr yon (ex parte), Man. of Conch. Str. and Syst., IV, p. 38, pl. 12, f. 159 (tantum).

Annam : plage de Ben Son (II. Boutan), spécimens brisés.

L'assimilation que Tryon a faite du N. vittata A. Ad. au $N$. maculata du même auteur nous paraît demander confirmation car le $N$. vittata est costulé longitudinalement tandis que le $N$. maculata est lisse ; la coloration est également différente.

\section{Munex Unibentatus Sowerby}

1777. Cochlis volutata, muricata, etc. Martinı, C. Cab., III,

1834. Murex unidentatus

18... - rarispina

1845. - Martinianus

1854 .

1878

1879. - rarispinosus

1880. - - ternispina p. 368, pl. CXIII, f. $103 ̈ 6$. Sowerby, Conch. Illustr. fig, อ๊2.

Sow erby (non Lk.), Conch. Illustr. Catal. p. 1.

Reeve (non L. Pfr. 1840), P. Z. S. L., p. 88.

ReEve (non L. Pfr.), C. Icon., pl. XVIII, f. 72.

Kobelt (non L. Pfr.), C. Cab., ${ }^{\ominus}{ }^{\ominus}$ Ed., p. 59 ; pl. 9 , f. 3 ; pl. 22, f. 7,8 .

Sowerby (non M.rarispina Lk), Thes. Conch., IV, p. 3 , pl. 380 , fig. 2.

Trryon (ex parte, non Reeve), Man. of Conch. Str. and Syst. II, p. 78, pl. 11, fig. 118 (tantum). 
1889. Murex Martinianus Rve

1889

1891
L. Molilet $(n o m$ L. Pfr. $)$, Catal.coq.rec par M.Pavie dans le Cambodge et le Royaumede Siam, in J. C.. XXXVII, p. 137.

Crosse et Fischer (non L. Pfr.), Note sur la Faune conch. mar. de l'Annam, in J. de C., XXXVII, p. 오.

P. Fischer (non L. Pfr), Catal. Indo-Chine, Ioc. cit., p. 146 (62).

Annam: plage de Ben-Son (M. Boulan), 1 exemplaire roulé.

La synonymie de cette espèce est fort difficile à établir. Lamarcki a donné comme référence de son Murex rarispina la fig. 1056 de Martini qui paraît bien représenter l'espèce que nous avons sous les yeux; mais sa description ne concorde pas d'une manière suffisante avec cette figure. Deshayes (Anim. s. Vert. 2e Ed. IX p. ว̆67, note), puis Kiener ont interprété d'une toute autre façon l'espèce de Lamarck en attribuant le nom de rarispina à la coquille nommée plus tard Iurex formosus par Sowerby (pl. I, fig. 6).

Reeve adoptant la manière de voir de Deshayes et de Ǩiener, a donné en 1843 à l'espèce figurée par Martini (fig. 10ă6) le nom de Murex Martiniamus. Malheureusement L. Pfeiffer dans le Register du Conchylien Cabinet avait déjà dénommé en $1840 \mathrm{M}$. Martinianus une tout autre espéce connue sous le nom de pimnatus Wood, de telle sorte que la coquille représentée par Martini fig. 1030 n'a actuellement d'autre nom acceptable que celui de $M$. unidentatus Sowerby indiqué sur l'explication de la planche 
du Conch. Illustr. où se trouve représentée cette espèce (fig. 52).

Sowerby a donc eu tort dans le Catalogue des espèces du Conch. Illustr. publié ultérieurement, de considérer son $M$. unidentatus comme synonyme de 1 . rarispina Lk.

\section{Purpura alveolata Reeve}

1906. P'urpura alveolata Rve. Dautzeneerget H.Fischer, Liste des Moll. rec. par M. Mansuy en Indo-Chine, in J. de Conch. LIII, p. 394.

Annam : plage de Ben-Son, et pointe du Scorpion, sur les ruchers (II. Boutan).

\section{Cuna Carinifera Lamarck}

1906. Cuma carinifera Lk. Dautzeneng et H. Fisciler Liste des Moll. rec. par M. Mansuy en Indo-Chine. in J. de Conch., LIII, p. 394 .

Aunam : plage de Ben-Son (M. Boutan), 1 exemplaire vide.

\section{Sistrum musivum Kiener}

1835. Purpura musiva

\begin{tabular}{|c|c|c|c|c|}
\hline 1844. & - & - & Kien. & $\begin{array}{l}\text { Deshayes in Lamarck, } \\
\text { Anim. s. vert. 2e éd., } \\
\text { p. } 109 .\end{array}$ \\
\hline 1846. & - & - & - & $\begin{array}{l}\text { Reeve, Conch. Icon., pl. } \\
\text { XI, f. } 52 .\end{array}$ \\
\hline 1858. & - & - & - & $\begin{array}{l}\text { Küster, Conch. Cab., 2 } \\
\text { éd., p. } 156, \text { pl. } 25^{\text {f. }} 13 .\end{array}$ \\
\hline
\end{tabular}

Kiener, lcon. coq. viv., p. 38, pl. 9, f. 22, 2 ?.

DeshaYes in LaMarck, p. 109.

ReEve, Conch. Icon., pl. $\mathrm{XI}, \mathrm{I} . \mathrm{J} 2$.

éd., p. 156, pl. $25^{\star}$ f. 13. 
1880. Ricinula Sistrum musiva Kien. Tryon, Man. of Conch. Str. and Syst., II, p. 192, pl. 59, f. 284 .

Annam : pointe du Scorpion, sur les rochers de la côte (M. Boutan).

\section{Bursa natatrix Bolten}

1780. Buccina bufonia compressa Chemni'z (exparte), Conch. Cab., IV, p. 78, pl. CXXVIII, f. 1229, 1230 (tantum).

1790. Murex Giyrimus Gmelin (ex p. non Linné), Syst. Nat.éd. XIII, p. 33̈31.

1798. Triton natator Bolten, Mus. Boltenianum, p. 127 (Chemnitz IV f. 1229, 1230).

183.. Ranella tuberculata

Broderip, Proc. Z. S. L., p. 179.

1835. - - Sowerby jun., Conchol. Illustr., f. 13.

1836. - - Brod. Müller, Synopsis, p. 100. 1842. - - - Kiener, Icon. coq. viv., p. 27 , pl. 12, fig. 2,2.

1843. Ranella tuberculata Brod. Deshayes in LAMarck,

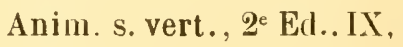
p. 5555 .

1844. - - - - ReEve, C. Icon., pl. VII, f. 36 .

1852. A pollon olivator Meuschen in Mörch, Catal. Yoldi, I, p. 106.

1978. Ranella tuberculata Brod. Kobelt in Küster, C. Cab., $2^{\mathrm{a}}$ Ed., p. 146, pl. 39 , f. 8,9 . 
1881. Rimella tuberculata Brod. Tryon, Man. of Conch. Str. and Syst., III, p. 43, pl. 23, f. 46-47 (tantum).

1889. - - - L. Morler, Catal. coq. rec. par M. Pavie dans le Cambodge et le royaume de Siam, in J. de Conch., XXXVII, p. 138.

1891. - - - $\quad$ P. Fischer, Catal. IndoChine, loc. cit., p. 151 (67).

Annam : plage de Ben-Son, (M. Boutan), 1 ex. fruste.

Nous avons été forcés de reprendre pour cette espèce le nom de natator Bolten, de beaucoup le plus ancien el qui ne prête pas à l'équivoque puisqu'il est basé sur les figures 1229 et $1230 \mathrm{du}$ C. Cab.

Tryon a assimilé à cette espèce le $R$. /uscocostata de Dunker (Novitates pl. XIX f. 1, 2), mais la sculpture nous paraît trop différente pour admettre cette réunion.

\section{Bursa rána Linné}

\section{8 . Мurex rana}

1764.

1767.

1786. Buccina bufonia...

1825. Murex rana Linné
Linné, Syst. Nat., ed. X, p. 748 (excl. var.)

Linné, Mus. Lud. Ulr., p. 629 (excl. var.)

Linné, Syst. Nat., ed.XII, p. 1216 (excl. var.)

Chemnitz(ex. p.), Conch. Cals., IV, p. 106, pl. CXXXIII f. 1270-7I (tantum.)

Wood, Index testac., $p$. 121, pl. 25. f. 21 (excl. syn. plur.) 
1844. Ranella albuvarirosa 1844.

18\%ั. Nurex rana L.
Reeve, Pr. Z. S. L. p. 136. Reeve, C. Icon., pl. I, f. 2. HANLEY,Ipsa Linn.Conch., p. 284 .

1878. Ranella albivaricosa Rve liobelt in Kïüster, C. Cab. 20 éd. p. 133 pl. 38 f. 4, כั, 8,9 .

1881. - - T'RYos, Man. of Conch. Str. and Syst., III, p. 38. pl. 18, f. 5,6 .

Annam : plage de Ben-Son (M. Boutan), 3 ex. morts.

Il nous semble que la reprise, jour cette espèce, du nom linnéen rana s'impose, bien que la description du Systema Naturæ soit fort sommaire. En efiet, la plupart des références s’appliquent à l'espèce qui a été désignée depuis par Reeve sous le nom de Ranella albivaricosa et d'autre part Hanley nous apprend que la collection de Linné renferme bien cette espece et qu'elle y est inscrite sous le nom de Murex rana.

Il est vraj que Linné a considéré la coquille à longues épines connue sous le nom de Bursa spinosa Lk. comme n'étant qu'uue variété de son Murex rana et que cette espèce existe aussi dans sa collection sous le nom de M. rana; mais ces quelques imperfections ne suffisent pas à notre avis pour faire rejeter un nom linnéen qui est meilleur que bien d'autres du mème auteur qui ont été généralement acceptés.

La figure de Wood (Index testac.) sous le nom de Murex rana $\mathrm{L}$. représente bien notre espèce.

\section{Cassis (Semicassis) pila Reeve}

1773. (sans nom)

Martinı, C. Cab, II, p. 10, vign. 18, f. $1,2$. 
1848. Cassis pila

1857. - - Rve
Reeve, C. Icon., pl. IX, fig. 21 .

Küster, C. Cab., 2 Ed., p. 39. pl. ö1, fig. $9,10$.

1885. Cassis( Semicassis) saburon Ad. var. pila Rve. Tr yon, Man. of Conch. Str. and Syst., VII, p. 275, pl. 5, fig. 75 .

1889. Cassis pila Rve

L. Morlet, Catal. coq. rec. par M. Pavie dans le Cambodge et le Roy. de Siam, in J. de C., XXXVli, p. 139.

189J. - - - P. Fischer, Catal. IndoChine, loc. cit., p. 152 (86)

Annam: plage de Ben-Son (11. Boutan), 1 spécimen fruste.

Dolium Lischkeanum Küster

185\%7. Dolium Lischkeanum Küster, Monogr. in C. Cab., 2 Ed., p. 71, pl. 62, f. 1.

1885. - costatum Mke var. fimbriatum Sow. Tryon (ex parte), Man. of Conch. Str. and Syst., p. 264, pl. 3 , fig. 18.

1889. - Lischkeanum Küst. Crosse et Fischer, Note sur la faune Conch. mar. de l'Annam, in J. de C., XXXVII, p. 285.

1891.

- P. Fischer, Catal. IndoChine, loc. cit., p. $152(68)$.

Annam : plage de Ben-Son (M. Boutan). 
Potamides tuberculatus (Linné) Born

1908. Potamides tuberculatus

(Lin.) Born Dautzenberg

et H. Fischer, Liste des

Moll.réc. parle Cap. Blaise au Tonkin, in J. de Conch., LIII, p. 130.

Annam: pointe du Scorpion (M. Boutan, 190马), sur les rochers de la côte.

Trmpanotonus cingulatus Gmelin

1905. Tympanotonus cingulatus Gm. Dautzenberg et H. Fischer, Liste des Moll. réc. par M. Mansuy en Indo-Chine, in J. de C., LIII, p. 410.

Annam: plage de Ben-Son (M. Boutan), 1 ex. fruste.

\section{Planaxis sulcatus Born}

1905. Planaxis sulcatus Born Dautzenberg et H.Frscher Liste des Moll. réc. par le cap. Blaise au Tonkin, in

J. de Conch, LIII, p. 139.

Annam : pointe du Scorpion, sur les rochers de la côte (M. Boutan), $1 \mathrm{ex}$.

\section{Turritella bacillum Kiener}

1845. Turritella bacillum Kíner, Icon. coq. viv., p. 5, pl. 4. fig. 1, 1.

1848. - - Kiener Kirauss, Südafr. Moll., p. 106.

1849. - - - ReEve, C. Icon., pl. II, f. 7 . 
1886. Turritella bacillum Kiener Tryon (ex parte), Man. of Conch. Str. and Syst. VIII, p. 196, pl. 59, fig. 34 (tantum).

\begin{tabular}{|c|c|c|c|c|}
\hline 1891. & - & 一 & - & $\begin{array}{l}\text { (var.)P. Fischer, Cat.Indo- } \\
\text { Chine. loc. cit.,p. } 165(81) \text {. }\end{array}$ \\
\hline 189 & 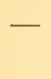 & - & - & $\begin{array}{l}\text { Ковеlt, C. Cab., } 2 \text { Ed., p. } \\
51, \text { pl. } 17, \text { f. } 1 .\end{array}$ \\
\hline
\end{tabular}

Annam: plage de Ben-Son(M. Boutan), 1 ex.

Turritella Reevei nom. nov.

1849. Turritella crocea

Iosi. - bacillum

1897. - sp.
Reeve (non Kiener), C. Icon., pl. VI, 1. 26.

Tryon (ex parte, non Kiener), Man. of Conch. Str. and Syst, VIII, p. 196 , pl. 60 , fig. 42 (tantum). Kовецт,C.Cab., 2 Ed.p.32.

Annam : plage de Ben Son, exemplaires brisés (M. Boutan).

Reeve a assimilé au T. crocea de Kiener (Icon. coq. viv. p. 2/; pl. 11 fig. 2, 2 sub nom. crocata) une forme qui nous paraît en différer absolument par le nombre bien moindre de ses cordons décurrents, par le profil des tours qui est plus plan, et enfin par la coloration.

M. Kobelt s'est aperçu de la discordance des figurations de Kiener et de Reeve. Il accepte toutefois la manière de voir de Tryon qui considère le $T$. crocea Kiener comme une simple variété de coloration du $T$. bacillum Kiener; mais cette opinion nous parait absolument inadmissible. Par contre M. Kobelt représente pl. 8, fig. 3, 4 deux Turritella de Chine qui lui paraisent constituer une espèce voisine des $T$. bacillum et terebra mais pourtant distincte. 
L'une de ces deux figures (f. 3) concorde surlout très bien avec le crocea de Reeve (non liiener) et nous sommes d'avis qu'il y a lieu de lui attribuer un nom spécial. Nous proposons celui de Reevei.

\section{Melania Hamonvillei Brot}

190\%. Melania Ilamomillei Brot Dautzrnese et H. FisCHER, Liste Moll. réc. par le cap. Blaise au Tonkin. in J. de C.. LIII, p. 141.

Tonkin : Su-Yut, Rivière Noire (M. Demange). - Trilé, (M. Krempf). - Van Linh (M. Krempf).

\section{Melania Jullieni Deshayes}

1874. Melania Julieni Desh. (mss.) Brot, Monogr. in C. Cab., 2 Ed., p. 93, pl. 11, fig. 2. 2.

1874. - Jullieni Desh. Drshayes et Jullien, Mém. sur des Moll. nouv. du Cambodge, in Nouv. Arch. du Muséum, p.143, pl. VII. fig. $7,8,9$.

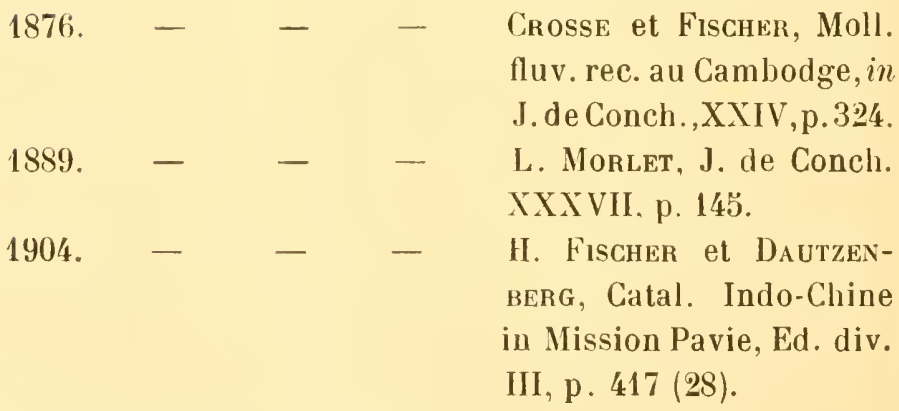

Tonkin : Su-Yut, Rivière Noire (M. Demange). 
Cette espèce. dédiée au $\mathrm{D}^{\mathrm{r}}$ Jullien doit être orthographiée Jullieni et non Julieni comme l'a écrit Brot. Notre exemplaire se rapproche plus du type représenté par Brot, pl. 11 f. 1), que des spécimens figurés par Deshayes et Jullien.

\section{Melania tuberculata Müller}

1906. Melania tuberculata Müll. Dautzenberg et H. Fischer Moll. réc. par M. Mansuy en Indo-Chine, in J. de Conch., LIII, p. 413.

Tonkin : Su-Yut, Rivière Noire (M. Demange).

Melania Krempel n. sp.

(PI. V, fig. 6, 7)

Testa parum solida imperforata elongato-conoidea, spira upice truncata. Anfr. perstantes 5 convexi sutura sat impressa juncti, a plicis incrementi et funiculis transversis obsoletissime granulosim decussati.

A pertura ovuta superne angulata. Columella parum crussa arcuata. I.abrum acutum arcuatum ac sinuosum.

Color corneus flammulis longitudinalibus fuscis, plus miunsve interruptis irregulariter pictus. Columella albescens.

Alt. 26 mill., diam. 11 mill; apert. $11 \mathrm{~mm}$. alta, $8 \mathrm{~mm}$. lata

Coquille peu épaisse imperforée, de forme allongée. spire conoïde tronquée au sommet. Les $ّ$ tours persistants sont convexes, séparés par une suture bien accusée et ornés de plis d'accroissement et de cordons décurrents également faibles qui déterminent un treillis très obsolète et légèrement granuleux qu'on découvre par places à l'aide de la loupe. Ouverture ovale, anguleuse au sommet. Columelle arquée, peu épaisse. Labre aigu, arqué, à profil sinueux. Coloration d'uu jaune corné, orné de flammules 
longitudinales brunes plus ou moins interrompues et irrégulières. Columelle blanchâtre.

Opercule...

Tonkin : Ban-Hao et entre Van-Linh et Trilé (M. Kirempf).

Même espèce que celle rapportée par M. Messager d'un affluent du Song-Ki-Kong. C'est une forme moins allongée que $M$. Schomburgki mais présentant la même coloration.

\section{Solarium Perspectivum Linné}

1758. Trochus perspectivus

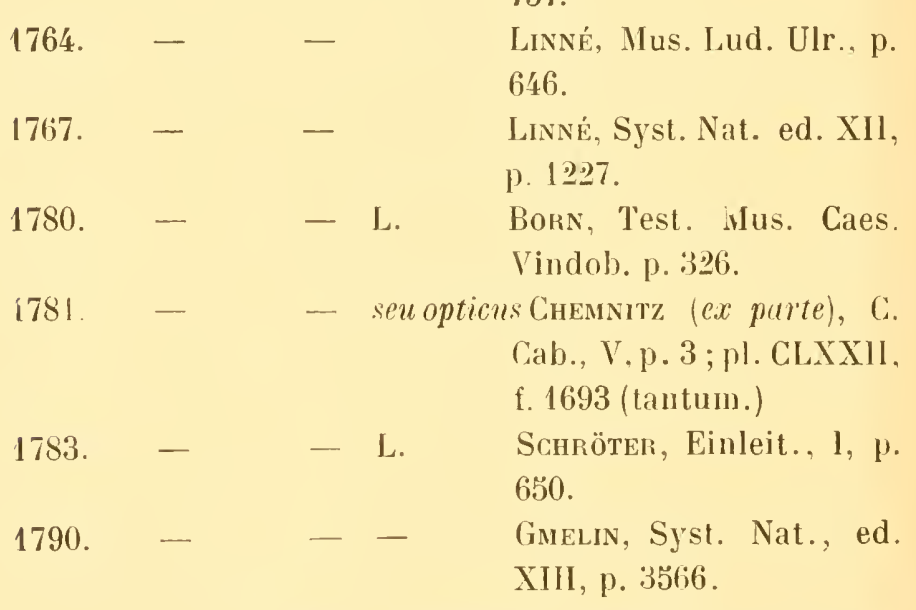

1815. - (Solarium) perspectivus Brookes, Introd. to Conch., p. 123, pl. 7, f. 94 .

1817. - perspectivus Dillwyn, Descr. Cat. II, p. 784 .

1822. Solarium perspectivum L. Lamarck, Anim. s. Vert., VII, p. 3.

182\%. - - - Bow dich, Elem. of Conch., pl. 9, f. 11.

1825. Trochus perspectivus L. Woov, Index testac., p. 137, pl. 29, f. 62. 
1830. Solarium perspectivum Sошквв,, Genera of Shells, I, G. Solarium.

\begin{tabular}{|c|c|c|c|c|}
\hline 1838.? & - & - & L. & $\begin{array}{l}\text { Kiener, Icon. Coq. viv., } \\
\text { p. } 3 \text {, pl. I, fig. } 1,1 .\end{array}$ \\
\hline 1843. & - & - & Lk. & $\begin{array}{l}\text { Deshayes in LamarcK, } \\
\text { Anim. s. Vert., 2e Ed. IX, } \\
\text { p. } 97 .\end{array}$ \\
\hline 1844. & - & formosum & & Hinds, P. Z. S. L., p. 22. \\
\hline 1844. & - & trochleare & & 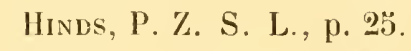 \\
\hline 1848. & - & zonatum & & $\begin{array}{l}\text { Phrlıppı, Zeitschr. f. Ma- } \\
\text { lakoz., p. } 173 .\end{array}$ \\
\hline 1848. & - & austrule & & Philippi, ibid., p. 168. \\
\hline 1848. & - & incisum & & Philıppi, ilid., p. 169. \\
\hline 1852. & - & perspectivum & Lk. & $\begin{array}{l}\text { Eydoux et Souleyet Voy. } \\
\text { Bonite, p. 587, pl. } 37 \text {, fig. } \\
10,11 \text {. }\end{array}$ \\
\hline
\end{tabular}

I85̋3. - trochleare Hinds Philippi, C. Cab., 2e Ed., p. 3, pl. 1, fig. 1 .

1859. - perspectivum L. Chenu, Man. de Conch., I, p. 232, fig. 1352.

1864. - - - - Reeve, C. Icon., pl. II, fig. 11a, $11 \mathrm{~b}$.

1866. - (Architectonica) perspectivum HanLEY in Sowerby, Thes. Conch., III, p. 228 , pl. 253 , fig. 36 , $37,38$.

1878. - perspectivum L. Tryon, Man. of Conch., IX, p. 8, pl. 2, fig. 18-21.
1889.
- Crosse et Fischer, Note sur la faune Conch. mar. de l'Annam, in J.C. XXXVII, p. 287.
1890.
- Crosse et Fischer, Moll. mar. de la Baie d'Along, in J. C. XXXVlI, p. 17. 
1891. Solarium perspectivum L. P. FIScher, Cat. IndoChine, loc. cit, p. 171 (87).

Annam : plage de Ben-Son, (M. Boutan) 2 ex. morts.

Alvania Boutani n. sp.

(PI. VI, fig. 1, grossie 15 fois)

Testa minuta, solidula, nitidula, imperforata, conoideoovata. Spira mediocris, apice obtusissimo. Anfr. $31 / 2-4$ sutura sat impressa juncti, primus levis, ceteri costulis lonyitudinalibus funiculisque transversis 2 regulariter clathrati. In anfr. ultimi basi funiculi 4 accedunt. A pertura subrotundata superne subangulata. Columella arcuata callo tenui adnatoque munita. Labrum extus a funiculis transversis fimbriatum.

Color albus subhyalinus.

Alt. 1,9mm; diam. max. 1,2 $2^{\mathrm{mm}}$. A pertura $0,9 \mathrm{~mm}$ alta, $0,3^{\mathrm{mm}}$ lata.

Coquille de très petite taille, assez solide, luisante, imperforée, de forme ovoïde-conique. Spire médiocrement élevée, très obtuse au sommet, composée de $31 / 2$ à 4 tours séparés par une suture bien marquée. Premier tour (incomplet) lisse, les suivants ornés de costules longitudinales régulièrement et largement espacées et de deux funicules décurrents un peu plus forts que les costules, qui déterminent avec celles-ci un treillis régulier à mailles quadrangulataires légèrement tuberculeuses aux points d'intersection. Sur le dernier tour, quatre autres funicules décurrents règnent entre la périphérie et la base. Les cos. tules longitudinales s'effacent au-dessous de la périphérie.

Ouverture arrondie légèrement anguleuse au sommet. Columelle arquée; bord columellaire pourvu d'une callosité mince, appliquée, mais bien visible. Labre arqué pré- 
sentant, du côté externe, des tubercules correspondant aux extrémités des funicules décurrents.

Coloration blanche uniforme subhyaline.

Annam : plage de Ben-Son (M. Boutan.)

Quoique de fort petite taille, cette coquille paraît bieu adulte. Elle est remarquable par le treillis à mailles la rges dont elle est ornée.

Cingula annamitica $\mathrm{n}$. sp.

(Pl. VI fig. 2, grossie 10̆ fois)

Testa minutissima nitidula imperforata, ovato-conoidea. Spira mediocris, apice obtuso. Anfr. 4 convexi, sutura subcanaliculata juncti, primi 2 leves, ceteri striis incrementi arcuatis parumque conspicuis ornati. Apertura rotundata: peristoma continuum. Columella arcuata, puululum reflexa. Labrum simplex arcuatum.

Color pallide fulvus, zonis saturatioribus 2 parum distinctis, altera in medio anfr. ultimi, altera paullo angustior infra. peripheriam, ornatus. Spira fulva.

Alt. $1,4^{\mathrm{mm}}$; dium. $\max .0,9^{\mathrm{mm}}$. Apert. $0,6^{\mathrm{mm}}$, alta, $0,3^{\mathrm{mm}}$ lita.

Coquille minuscule, luisante imperforée, de forme ovale conoïde. Spire médiocrement élevée, obtuse au sommet, composée de 4 tours convexes séparés par une suture légèrement canaliculée: les deux premiers lisses, les autres ne présentant que des stries d'accroissement faibles arquées. Ouverture arrondie, à péristome continu. Colu. melle arquée, très légèrement réfléchie. Labre simple arqué.

Coloration gris rosé pâle, avec deux bandes décurrentes fauves peu distinctes situées sur le dernier tour, l'une large vers le milieu des tours, l'autre un peu plus étroite 
au-dessous de la périphérie. Les tours supérieurs sont d'un fauve assez foncé.

Annam : plage de Ben-Son (M. Boutan.)

\section{Paluina polyzonata Frauenfeld}

1906. Paludina polyzonata Fr. Dautzenderg et H.Fischer, Moll. réc. par M. Mansuy en Indo-Chine, in J. de Conch., LIII, p. 419.

Tonkin: Ban-Нао (M. Krempf). - Hanoï (M. Boutan), plusieurs spécimens avec passage à la variété Duchieri H. F. (Voyez J. de C., LIII, p. 15̈6).

\section{Paludina ampulliformis Souleyet}

1906. Paludina ampulliformis Soul. Dautzenberg et H. Fischer, Moll. réc., par M. Mansuy en Indo-Chine, in J. de Conch., LIII, p.417.

Tonkin : Hanoï (M. Boutan).

\section{Paludina quadrata Benson}

1906. Paludina quadrata Bens. Dautzenberg el II. Fischer. Moll. rec., par M. Mansuy en Indo-Chine, in J. de Conch., LIII, p. 419.

Tonkin : Hanoï (M. Boutan). Plusieurs spécimens non typiques, mais appartenant aux variétés $\mathbf{m e c v e i} \mathrm{D}$. et H. F. , aruginosa Reeve et hizonalis (MIldff) D. et H.F. 
Ampullaria polita Deshayes

1906. Ampullaria polita Desh. Dautzenberg et H.Fischer, Moll. réc., par M. Mansuy en Indo-Chine, in $J$. de C., LIII, p. 426.

Tonkin : Hanoï (M. Boutan).

\section{Ampullaria gracilis Lea}

1906. Ampullaria gracilis Lea

Dautzenberg et H. Fischer, Moll., réc., par M. Mansuy en Indo-Chine, in J. de C. LIII, p. 426.

Tonkin: Hanoï (M. Boutan).

\section{Cyclophorus Courbet Ancey}

1906. Cyclophorus Courbeti Ancey Dautzenberg et H. FisGHER, Moll. réc. par M. Mansuy en Indo-Chine, in J.de Conch., LIII, p. 431.

Tonkiu : Ban-Hao (M. Krempf). - Su-Yut, Rivière noir $_{\mathrm{i}}(\mathrm{M}$. Krempf), 1 ex. jeune.

Crugibulum (bicathlous) Renovatum Crosse et Fischer

1906. Crucibulum (Bicatillus) renovatum Cr. et F. DautzenBerg et H. Fischer, Moll. réc. par M. Mansuy, in J. de Conch., LIII, p. $445^{\circ}$.

Aunam : Plage de Ben-Sou (M. Boutan). 


\section{NaTICa Lineata Lamarck}

1764. Nerita canrena var. alba lineis undatis ferrugineis Linné, Mus. Lud. Ulr. p. 674 .

1780. Nerita canrena L. var. ? Bonn,Test.Mus.Caes.Vindob., p. 396, pl. 17, f. 1, 2.

1781. Nerita umbilicata laevis etc. Chemnitz, C. Cab., V, p. 2\%4, pl. CLXXXVI, f. 1864, 186 ว.

1784. die liniirte Nerite

1790. Nerita canrena var. \%

1817. - - var. D

1822. Natica lincata

183 ?.

Lk.

1838.

1886.
Schröt er, Einleit. II, p. 335 .

Guelin, Syst. Nat., édit. XIII, p. 3669.

Dillwyn, Descr. Catal., II, ए. 976 .

Lamarci, Anim. s. Vert., VI, $2^{\circ}$ partie, p. 201.

Deshayes, Encycl. Méthod., III, p. 602.

Deshayes in LaMaRcK, Anim. s. Vert., 20 Ed., VIII, p. 640.

184\%. - - - $\quad$ Philippi, C. Cab, 2e Ed., p. 11, pl. I, f. 9, 10.

185i. - - - Reeve, C. Icon., pl. III, f. 24 .

1859. - - - $\quad$ Chesu, Manuel de Conch.. I, p. 213, f. 1164.

Sowerbr, Thes. Conch., V, p. 79, pl. 4.ว̆6, f. 38.

Trion, Man. of Conch. Str. and Syst., VIII, p. 29 , pl. 8, f. 57 . 
1859. Notica lineata Lk.

\section{Natica maroghiensis Gmelin}

1906. Natica marochiensis Gm. Dautzenberg et H. FisCHER, Liste des coq. réc. par M. Mansuy, in J. de C., LIII, p. 44 马̈.

Annam : plage de Ben-Son (M. Boutan), 1 ex. jeune.

Natica (Neverita) didya Bolten

1798. Albula Didyma

Bolten, Mus. Boltenianum, p. 20.

Nous n'avons pas rencontré la forme typique de cette espèce.

\section{var. bieolor Philippi}

1848. Natica bicolor

18ว2. $-\quad-\mathrm{Ph}$.

185ว. - Petiveriana

1886. - licolor Phil.
Philipp1, Zeitschr. f. Malakoz., p. 156.

Philippi, C. Cab. @e Ed., p. $43, \mathrm{pl}, 6, \mathrm{f} .4$.

Recluz mss., in Reeve, C. Icon., pl. V, f. 17a, 17b. Tryon, Man. of Conch. Str. and Syst. VIII, p. 33. 
1886. Natica ampla Phil.

1889

- Petiveriana Recl.
TrYon (ex parte), ibid.. VIII, p. 32, pl. 11, f. 91 (tantum).

Crosse et Fischer, Note sur la faune Conch. mar. de l'Annam, in J. de Conch., XXXVII, p. 288.

1891. P. Fischer, Catal. IndoChine,loc.cit., p.195(111).

Annam : plage de Ben-Sou (M. Boutanj, quelques exemplaires.

La var. bicolor diffère du $N$. didyma typique par sa forme plus déprimée à spire aplatie, son ombilic plus ouvert, sa callosité plus petite, moins saillante, moins largement soudée contre la coquille et enfin par sa spire noirâtre bordée d'une ligne blanche au-dessous de la suture.

Il nous semble que le $N$. papyracea $\mathrm{v}$. d. Busch in Philippi Abbild. 1845̆, p. 45 pl. 2 f. 14 n'est que le jeune âge de la var. bicolor.

\section{Sigaretus neritoldeus Linné}

1758. Helix neritoidea

1764. - -

1767

1790.

1817.
Linné, Syst. Nat. ed. X. p. 775 .

Linné, Mus. Lud. Ulr., p. 678 .

Linné, Syst. Nat., ed. XII, p. 1250.

Gmelin, Syst. Nat. ed. XIII, p. 3663.

Dillwys, Descr. Catal., II, p. 972 . 
1830. Sigaretus halintideus

\begin{tabular}{|c|c|c|c|}
\hline 1850. & - & insculptus & $\begin{array}{l}\text { Adans et Reeve, Voy. } \\
\text { Samarang, p. } 55 \text {, pl. XIII, } \\
\text { f. } 10 \text {. }\end{array}$ \\
\hline 1850. & - & latifasciatus & $\begin{array}{l}\text { Adams et Reeve, ibid.. } \\
\text { p. ว̈5̆, pl. XIII, f. } 12 \\
\text { (non Reeve). }\end{array}$ \\
\hline 1882. & - & neritoideus L. & $\begin{array}{l}\text { Sowerby, Thes. Conch., V, } \\
\text { p. } 40, \text { pl. } 441 \text {, f. } 1 \text { (latifas- } \\
\text { ciatus); pl. } 442 \text {. 1. } 16,17 .\end{array}$ \\
\hline หวดั. & & ritoidea & $\begin{array}{l}\text { H ANLET, Ipsa Linn. Conch., } \\
\text { p. } 390 .\end{array}$ \\
\hline
\end{tabular}

186 4.Sigaretus neritoideus L. Reeve, C. Icon., pl. 1, f. כ้a, ว็b.

$[883$.

$\begin{aligned}-\quad-\quad \text { Weinkauff, C. Cab., 2 éd., } & \text { G. Sigaretus, p. 18, pl. } 3, \\ & \text { f. } 7 \text { à } 11 .\end{aligned}$

1886.

- Tryon, Man. of Conch., VIII, p. 5ั5, pl. 22, f. 35, 38 à 40 (comprenant latifasciatus Ad. et R., Javanicus Gray, insculptus Ad. et R. et Leachii Phil.)

Annam : plage de Ben-Son (M. Boutan), 1 ex

Recluz dans sa monographie des Illustr. Conch. de Chenu a attribué le nom de neritoideus $\mathrm{L}$. à la coquille du Pérou et du Chili nommée cymba par Menke; cette manière de voir pourrait être adoptée car la figure de Gualtiéri pl. 64, fig. 1, bien que très grossière et mauvaise, est d'une taille trop grande pour qu'on puisse la rapporter à une autre espèce. D'autre part les mots testa livida du 
Siystema Naturae et certains termes de la description du Mus. Ludovicae Ulricae: " Testa convexa, altior, mucrone livida, subrotunda, obtuse striata, striis plus quam 40 ; spira depressa terminata puncto calloso. Apertura subrotunda, fauce livida; labium exterius patum interius basi reflexum " conviennent aussi à la grande espèce du Pérou ou du Chili. Mais Hanley nous apprend que la seule coquille de la collection linnéenne à laquelle la description du Systema puisse convenir est celle qui a été représentée par Sowerby, Genera of Shells, Sigaretus, fig. 2, sous le nom de Sigarctus haliotideus et qui est bien l'espèce que la plupart des auteurs ont considérée comme le véritable Helix neritoidea de Linné.

Nous n'hésitons pas à adopter l'opinion de Tryon qui considère les S. latifasciatus Ad. et Rve, insculptus Ad. et Rve, javanicus Gray comme synonymes; mais nous doutons fort qüil en soit de mème du S. Leachi Blainville.

Quant au S. japonicus Lischke, il est certes fort voisin, mais en l'absence de matériaux suffisants, nous ne pouvons savoir s'il s'agit là d'une espèce spéciale ou d'un spécimen peu adulte du S. neritoidens. L'exemplaire envoyé par M. Boutan concorde dans tous les cas avec le S. neritoideus.

Sigaretus (Eunaticina) papilla Gmelin

1781. Papilla seu Ruma felis Chemnitz, Conch. Cab.. V, p. 284, pl. CLXXXIX, f. 1939 .

1790. Nerita papilla

1817. - - Gm.

1832. Natica costulatu
Gmelin, Syst. Nat., ed. XIII. p. $367 \%$.

Dillwyn, Catal.II, p. 985 . Quox et Gaimard, Voy. Astrol., II, p. 235, pl. 66, f. 20,21 . 
1843. Sigaretus papilla

1843-45. - Papillus

1850. - acuminatus

1851. Sigaretus Papillus

1852. Natica papilla

1882. sigaretus - Gm.

1882. - acuminatus

18วั8. Naticina papilla -

Deshayes in Lamarck, An.s.Vert.,2e ed. IX, p. 13. Recluz, Monogr. in Chenu, Illustr. Conch., p. 7. pl. I, f. $1^{\mathrm{a}}, 1^{\mathrm{b}}, 2^{\mathrm{a}}, 2^{\mathrm{b}}$. Adams et Reeve, Voy. Samarang. p. 3̌4,pl.XIII, f.8. Recluz, Catal. des esp.du G. Sigaret, in J. de C., II, p. 181.

Philippi, C. Cab., 2e Ed., Natica, p. 36, pl. 4, f. 18. Sowerby, Thes. Conch., V, p. 43, pl. 142bis, f. 44 . Sowerey, Thes. Conch.. V, p. 44, pl. 142 bis, f. 43. H. et A. Adams, Genera of rec. Moll., I, p. 212, pl. 22, f. 7 .

1859. Sigaretus (Naticina) papillus Gm. Chend, Man. de Conch., I, p. 216, f. 1185 . 1864 - papilla Gm. Reeve, C. Icon., pl. IV, f. $19^{a}, 19^{b}$.

\begin{tabular}{|c|c|c|c|c|}
\hline 1864. & - & \multicolumn{2}{|c|}{ coarctatus } & $\begin{array}{l}\text { ReEve, C. Icon., pl. IV, f. } \\
17^{\mathrm{a}}, 17^{\mathrm{b}} .\end{array}$ \\
\hline 1864. & - & \multicolumn{2}{|l|}{ nitidus } & $\begin{array}{l}\text { ReEve, C. Icon.. pl. IV, f. } \\
20^{\mathrm{a}}, 20^{\mathrm{b}} \text {. }\end{array}$ \\
\hline 1871. & - & papilla & $\mathrm{Gm}$. & $\begin{array}{l}\text { Lischis, Moll. Jap., II, p. } \\
169 .\end{array}$ \\
\hline 1883. & - & - & - & $\begin{array}{l}\text { Wernkauff, C. Cab., } 2 \text { Ed., } \\
\text { G. Sigaretus, p. } 38 \text {; pl. } \\
9 \text {, fig. } 4,6 \text {; pl. } 10 \text {, fig. } 8 .\end{array}$ \\
\hline 1880 & $E u$ & $\operatorname{cin} a)-$ & - & $\begin{array}{l}\text { Tryon, Man. of Conch. } \\
\text { Str. and Syst., VIII, p. כ8; } \\
\text { pl. 2ö, fig. } 78,79,87,88 \text {. }\end{array}$ \\
\hline
\end{tabular}


L. Monlet (non Reeve), Catal. coq. rec. par II.Pa. vie dans le Cambodge et le Roy. de Siam, in J, de C.. p. 156 .

Annam : plage de Ben-Son (M. Boutan).

L. Morlet et P. Fischer ont cité de l'Annam les Eunaticina tumescens Rve et Lamarcliana Recluz qui se distinguent de l'E. papilla par leur forme plus ovale et plus renflée.

Toutefois l'exemplaire de la collection L. Morlet que nous possédons sous le nom de tumescens et qui provient du golfe de Siam n'est pas cette espèce, mais bien le papilla Gmelin. Il y a donc là une erreur de détermination qui doit être rectifiée.

Sigaretus (Eunaticina) perorliquus n. sp.

(pl. V, fig. 4, 3)

Testa sat solida, parum nitens, profunde umbilicata, globoso-depressa. Spira lrevis, ronoidea. An/r. 4 convexiusmli rapille crescentes, sutura impressa juncti. Anfr. primi 2 leves, reteri undique liris spiralibus planis sulcisque interstitialibus inaequalibus transversim sculpti. Anfr. ultimus permagnus basi concexus, circu umbilicum pervinm late excatatus. Apertura valde obliqna et basin versus recedens. Columella obliqua, superne callo crasso, umbilicum semiobtegrnte munita. Callum a sulco transverso lato ac impresso bipartitum. Labrum acutum, arcuatum.

Color lacteus.

Alt. $14 \mathrm{~mm}$; diam. max. $17 \mathrm{~mm}$. Apertura $16^{\mathrm{mm}}$ alta, $12 \mathrm{~mm}$ lata (callo incluso).

Coquille assez solide, peu luisante, profondément ombiliquée, de forme globuleuse déprimée. Spire peu élevée 
conoïde, composée de 4 tours légèrement convexes, croissant très rapidement et séparés par une suture bien accusée. Deux premiers tours lisses, les suivants ornés de stries d'accroissement fines et de cordons décurrents plans séparés par des sillons de largeur fort inégale; la plupart de ceux qui règnent sur la partie supérieure du dernier tour sont plus larges que les cordons, et ceux du reste de la surface beaucoup plus étroits. Le dernier tour,très ample, est convexe à la base et largement excavé autour de la perforation ombilicale qui est profonde. Ouverture très oblique. Columelle oblique, émettant vers le haut une callosité épaisse qui surplombe à moitié l'ombilic et est divisée en son milieu par un sillon transverse assez large et profond. Labre arqué, tranchant.

Coloration d'un blanc laiteux uniforme.

Annam : plage de Ben-Son (M. Boutan).

Cette espèce se distingue de toutes les autres Naticina par sa forme très oblique. Elle est remarquable par sa spire très surbaissée et sa callosité columellaire dlivisée par un sillon médian bien accusé.

Pyramidella (Agatha) Pagei n. $\mathrm{sp}$.

(pl. VI, fig. 3).

Testa minuta imperforata nitidiuscula subhyalina. Anfr. apicales deficientes; normales 4 complanati, rapide crescentes ac sutura valde impressa lateque marginata juncti. Anfr. ultimus magnus, inferne ovatus. A pertura alta, superne angulata. Columella spiraliter torta. Labrum simplex, arcuatum. Color albus.

Altit. (apice excepto) $3,2^{\mathrm{mm}}$; diam. $\max .1,2 \mathrm{~mm}$; apert. $1,3 \mathrm{~mm}$ alta, $0,6^{\mathrm{mm}}$ lata.

Coquille de petite taille, imperforée, un peu luisante, subhyaline. Tours embryonnaires manquant. Tours nor- 
maux au nombre de 4, presque plans, croissant rapidement. séparés par une suture subcanaliculée et largement marginée. Ces tours semblent emboîtés les uns dans les autres. Dernier tour ovalaire. Ouverture haute, anguleuse dans le haut. Columelle fortement tordue en spirale. Labre simple, arqué.

Coloration d'un blanc subhyalin avec une zone plus opa. que, large, bordant la suture.

Annam : plage de Ben-Son (M. Boutan).

Celte espèce que nous dédions à M. S. Pace, ie savant malacologiste anglais, se rapproche, par la forte torsion de sa columelle et la hauteur de son dernier tour, du P. virgo A. Adams, du Japon; mais sa taille est bien plus faible, ses tours sont moins convexes, moins nombreux et moins canaliculés, comme on peut s'en convaincre en comparant notre figure (pl. VI, f. 3) avec celle que MM. Dall et Bartsch viennent de donner de l'espèce d'Adams (Notes on Pyramidellidae, in Proc. Nat. Mus., 1906, p. 335, pl. XVIII, f. 2).

Syrnola Callembrion n. $\mathrm{sp}$.

(pl. VI, fig. 4, grossic 20 fois)

Testa minutissima tenuis nitens pellucida imperforata ovatoelongata. spira elata. Anfr. apicales 2 heterostrophi, normales 5 leves convexiusculi, sutura impressa juncti. Anfr. ultimus basin versus paululum attenuatus. Apertura ovata, superne. angulata, marginibus callo adnato junctis. Columella arcuata, superne in plicam spiralem sat validam desinit. Labrum simplex arcuatum

Color albus hyalinus.

Alt. $2^{\mathrm{mm}}$; diam. max. $0.6^{\mathrm{mm}}$. A pert. $0.6^{\mathrm{mm}}$ alta, $0.4^{\mathrm{mm}}$ lata.

Coquille de très petite taille, luisante, pellucide, imperforée, de forme ovale allongée. Spire élevée. Tours 
embryorinaires hétérostrophes, très saillants. Tours normaux au nombre de כ̆, lisses, très légèrement convexes, séparés par une suture bien accusée. Dernier tour un peu atténué vers la base. Ouverture relativement petite, ovalaire, à bords reliés par une callosité appliquée. Columelle arquée terminée au sommet par un pli spiral assez saillant. Labre simple, arqué.

Coloration d'un blanc hyalin uniforme.

Annam : plage de Ben-Son (M. Boutan), 1 exemplaire.

Cette espèce, remarquable par son embryon très gros, saillant et placé transversalement, ressemble beaucoup, au premier aspect, au Pyramidella (Styloptygma) serotina A. Ads représenté par MM. Dall et Bartsch in Proc. U. S. Nat. Mus. p. 334, pl. XVII, f. 5; mais elle en diffère par son test plus mince, ses tours moins convexes, sa forme plus étroite, son dernier tour plus haut en proportion, par l'absence de bande colorée sur la périphérie du dernier tour, enfin par sa spire non atténuée au sommet comme celle des Styloptygma.

\section{Oscilla Jocosa Melvill}

(Pl. VI fig. 6, 7, grossie 20 fois)

1904. Oscilla jocosa

MeLvill, Journ. of Malac. XI, p. 82, pl. VIII f. 11 .

Annam : Plage de Ben-Son, M. Boutan, 1 exemplaire (pl. VI fig. 6).

Cette espèce se distingue de ses congénères par sa forme conoïde subcarénée à la périphérie. Dans les deux spécimens que nous figurons ici, le pli columellaire est situé un peu plus bas que dans celui représenté par Melvill; mais il est possible que le dessinateur ait relevé un peu plus le sommet, en représeutant le spécimen original. 
Nous avons reçu de $\mathbf{M}$. Preston, sous le nom de 0 . suburbana Preston, une coquille provenant de Ceylan (pl. VI, fig. 7j qui est identique à celle que nous possédons de l'Annam (pl. VI, fig. 6), mais qui ne concorde pas bien avec la figure originale de 0 . suburbana (Journ. of Malac. XIl, p. 6, pl. II, f. 206), qui est plus allongée et non carénée à la périphérie. Il est toutefois possible que les 0 . jocosa et O. suburhana soient l'état jeune et l'état adulte d'une même espèce.

Le genre Oscilla a été établi par A. Adams in P. Z. S. L. 1867, p. 310 .

\section{Odostomia pseudoplicata $\mathrm{n} . \mathrm{sp}$.}

(PI. VI, fig. 5, grossie 10 fois)

Testa solidiuscula, parum nitens, angustissime perforata. Spira conoidea. Anfr. apicales heterostrophi, immersi, normales 6 subplani, sutura impressa juncti, plicis incrementi sub lente conspicuis et striis spiralibus obsoletis ornati : penul. timus ar: antepenultimus prope basin contracti, ultimus basin versus rotundatus. A pertura ovata, superne angulata. Columella as'cuata, superne plicam spirulem validam emittit. Labrum simplex arcuatum, murginibus cullo temi, adnato, supra umbilicum reflexo, junctis.

Color albus.

Alt. $4.6^{\mathrm{mm}}$; diam. max. $1.8^{\mathrm{mm}}$; apertura $1.6^{\mathrm{mm}}$ alta, $1 \mathrm{~mm}$ lata.

Coquille assez solide, peu luisante, très étroitement perforée. Spire conoïde composée d'un embryon hétérostrophe immergé et de 6 tours normaux presque plans, séparés par une suture bien marquée, et ornés de plis d'accroissement bien visibles au microscope. On observe en outre, sous un fort grossissement, des traces de stries décurrentes extrèmement fines. L'avant dernier tour et l'antépénultième sont resserrés à la base. Dernier tour arrondi à 
la base, pourvu d'une perforation umbilicale très étroite. Ouverture ovalaire, anguleuse au sommet. Columelle arquée, terminée au sommet par un pli spiral très saillant et émetlant une lamelle mince qui recouvre eu grande partie la perforation ombilicale. Labre simple, arqué; bord basal arrondi.

Coloration entièrement blanclie.

Annam: plage de Ben.Son (M. Boutan ).

Cette espèce, de taille assez grande pour le senre, est remarquable par la contraction basale des derniers tours, ainsi que par ses plis d'accroissement bien visibles sous le microscope. Elle se rapproche par sa forme générale de l'(). plicata Mtg., des mers d'Europe, mais en diffère par sa taille plus forte ainsi que par sa contraction supra-suturale.

L'O. symrnoloides Melvill (Pr. Mal. S. L. II, 1896, p. 113. pl. VIII, f. 13) ressemble aussi à notre U. subplicutu; mais il possède un tour de moins et ses tours ne sont pas contractés au-dessus de la suture.

Odostoma Boutanı n. sp.

(Pl. VI fig. 8, grossie 10 fois;

Testa tenuis, nitida, pellncida, subrimata. Spira elata turritu. Antr. apicales 2 heterostrophi, normales $\breve{3}$ leves complanati, gradati, sntura valde impressa juncti. Anfr. ultimus ad peripheriam subangulatus. A pertura ovata, superne angulata. Columella arcuata, superne plicam spiralem et onlidam pruebet. Labrum simplex arcuatum.

Color albus hyalimus.

Alt. $3.2 \mathrm{~mm}$; diam. $\max .1 .3 \mathrm{~mm}$. Apertura $1.2 \mathrm{~mm}$ alta, $0.6^{\mathrm{mm}}$ latu.

Coquille mince, luisante, pellucide, ne présentant qu'une faible trace de perforation umbilicale. Spire élevée turri- 
culée, composée de 2 tours embryounaires hétérostrophes et de כ̆ tours normaux iisses, aplatis latéralement, séparés par une suture bien marquée. Dernier tour légèrement auguleux à la périphérie. Ouverture ovalaire, anguleuse au sommet. Columelle arquée, terminée dans le haut par un pli spiral saillant et émettant une lamelle vitreuse, mince, qui recouvre presque entièrement la perforation ombilicale. Labre simple, arqué.

Coloration d'un blanc hyalin uniforme.

Annam : plage de Ben-Son (M. Boutan).

L'Odostomia Boutani est remarquable par l'angle périphérial du dernier tour. Nous ne trouvons à le rapprocher d'aucune des espèces décrites jusqu'à présent.

\section{Odostomia contracta n. sp.}

(Pl. VI fig. 9, grossie 20 fois\}

Testa minuta solidula nitidula imperforata elongataconoidea. Spira mediocriter elata. An/r. apicales 2 heterostrophi, parum prominentes, normales 3 leves, sutura impressa juncti, infra suturam breviter complanati, ultimi in medio paululum coarctati. Anfr. ultimus ad peripheriam obtuse angulatus. A pertura subquadruta. Columella arcuata, superne in plicam spiralem mediocrem desinens. Labrum simplex superne angulatum.

Color albus hyalinus.

Alt. $1,2 \mathrm{~mm}$; diam. $\max .0,6^{\mathrm{mm}}$; apertura $0,,^{\mathrm{mm}}$ ulta, $0,3 \mathrm{~mm}$ lata.

Coquille petite, luisante, imperforée, de forme conoïde allongée. Spire médiocrement élevée, composée de ? tours embryounaires hétérostrophes peu saillants et de 3 tours normaux lisses, séparés par une suture bien marquée, bordés au-dessous de la suture par une rampe étroite et 
contractés en leur milieu. Dernier tour obtusément anguleux à la périphérie. Ouverture subquadrangulaire. Columelle arquée, terminée au sommet par un pli spiral médiocre. Labre simple, anguleux vers le haut.

Coloration d'un blanc hyalin.

Annam : plage de Ben-Son (M. Boutan).

Cette petite espèce se fait remarquer par ses derniers tours contractés au milieu, ainsi que par sa rampe subsuturale plane et étroite.

Odostomia (Pyigulina) pupaeformis Souverbie 1865. Pyramidella pupaeformis Souverbie, Descr. Esp. nouv. Arch.Caléd. in J. de Conch. XIII, p. 152, pl. V, f. 4 .

1867. Odostomia(Parthenia)Kreffti Angas, P.Z.S.L., p. 112, pl. XIII, f. 83.

1875. - pupueformis Souverbie, Descr. Esp. nouv. A rch. Caléd. in J. de Conch. XXIII, p. 291.

1886. - (Pyrgulina)pupeeformis Souv.Tryos, Man. of Conch. Str. and Syst., VIII, p. 362, pl. 79, f. 65,66 .

1887. - mpaeformis Souv. Paetel, Catal.,I, p. 264. Annam : plage de Ben-Son (M. Boutan) 1 exemplaire.

Pyrgulina Meluilli n. sp.

(PI. VI, fig. 10, grossie 20 fois)

Testa minuta nitidiuscula subhyalina, angustissime rimata, subcylindracea. Spira elata. Anfr. apicales... (detriti); normales 5 convexi, sutura impressa juncti, costis longitudina- 
libus olliquis, subureuatis, quam interstitiu angustioribus ac funiculis transrersis mumerosis inter costas tantum conspicuis undique ornata.

Apertura orata, marginibus callo tenui nitido adnatoque juuctis. Columella arcuata. superne in plicam spiralem vix conspicuam desinens. Labrum simplex, arcuatum.

Color albidus.

Alt. $2,3^{\mathrm{mm}} ;$ lat. $0,9^{\mathrm{mm}}$; apert. 0.7mm alta, 0, $\mathrm{5}^{\mathrm{mm}}$ lata.

Coquille de très petite taille un peu luisante, subhỹaline, pourvue d'une perforation étroite, allongée. Spire èlevée composée d'un sommet ...(usé) et de ŏ tours nornaux convexes. séparés par une suture bien accusée. surface ornée partout de côtes longitudinales obliques, légèrement arquées, plus étroites que leurs intervalles, et de cordons décurrents nombreux, visibles seulement dans les espaces intercostaux. Sur le dernier tour les còtes longitudinales se prolongent jusquà la base où elles s’atténuent toutefois un peu.

Ouverture ovalaire. à bords réunis par une callosité mince appliquée et luisante. Columelle arquée, terminée au sommet en un pli spiral à peine visible. Labre simple, arqué.

Coloration blanche uniforme.

Annam : plage de Belı-Son (.M. Boutan), 1 exemplaire.

Cette jolie espèce se rapproche beaucoup par sa sculpture, la faiblesse de son pli columellaire ainsi que par sa forme, de l'Odostomiu (Trabecula) tantilla A. Adams, du Japou, représenté par .III. Dall et Bartsch (Proc. L.S. Nat. Mus. XXX, p. 348, pl. XXIl. f. 3); mais elle en diffère par ses cordons decurrents bien plus nombreux puisqu'on en compte une dizaine au lieu de š sur l’avant-dernier tour. 
PyRguliNa SyKesi II. sp.

(PI. VI, fig. 11, grossie 20 fois)

Testa minuta nitidula subhyatina imperforata, oblongoovata. Spira elata. Antr. apicales heterostrophi, immersi, normales 5 gradati subplani, sutura subcanaliculata juncti, costis longitudinalibus tere rectis, quam interstitia angustioribus, ac funiculis transversis sat validis parum numerosis, inter costas tantum conspicuis ornati.

Apertura ovata, marginibus callo tenui adnatoque junctis. Columella arcuta. Labrum urcuatum acutum. Plica columellaris inconspicua.

Color altus .

Alt. 2,3̈m ; diam. max. $0,8 \mathrm{~mm}$; apert. $0,8 \mathrm{~mm}$ altu, $0.4^{\mathrm{mm}}$ lata.

Coquille de petite taille, un peu luisante, subhyaline, imperforée, de forme ovale très allongẻe. Spire élevée composée d'un embryon hẻtérostrophe immergé et de כ̆ tours normaux emboîtés, presque plans, séparés par une suture subcanaliculée. Toute la surface est ornée de côtes longitudinales à peu près verticales, plus étroites que leurs intervalles et de funicules décurrents peu nombreux (10 sur l'avant-dernier tour) qui ne se voient que dans les espaces intercostaux.

Ouverture ovalaire à bords reliés par une callosité mince appliquée. Columelle arquée ne montrant pas de pli spiral. Labre simple arqué.

Coloration blanche uniforme.

Annam : plage de Ben-Son(M. Boutan), 1 ex et fragments.

Cette espèce est remarquable par la hauteur de ses tours qui sont presque emboîtés et séparés par une suture profonde, légèrement canaliculée. Nous ne pouvons la comparer à aucune de celles qui ont été figurées jusqu'à présent. 


\section{Pyrgulina Standeni n. sp.}

(Pl. VI fig. 12, grossie 15 fois)

Testa solidula, imperforata, parum nitens, mupaeformis. Spira sat elata, apice obtuso. Anfr. apicales heterostrophi, immersi, normales 4 convexi, sutura impressa juncti, longitudinaliter costati. Costae validae, regulares, interstitia subaequantes. Striae transversae approximatae, inter costas tantum conspiciuntur, sed in medio unfractum deficiunt.

A pertura subrotunda. Columella arcuata superne plicam spiralem validam emittit. Labrum simplex arcuatum, intus vero liris transversis ornatum.

Color albus.

Alt. $2,7 \mathrm{~mm}$. ; diam. $\max .1,2 \mathrm{~mm}$.; apertura $1 \mathrm{~mm}$. alta, $0,7 \mathrm{~mm}$. lata.

Coquille assez solide, imperforée, peu luisante. pupiforme. Spire assez élevée, obtuse au sommet. Tours embryonnaires héterostrophes immergés ; tours normaux au nombre de 4, convexes, séparés par une suture bien marquée et ornés de côtes longitudinales fortes, régulièrement espacées et séparées par des intervalles qui sont sensibiement de même largeur que les côtes elles-mêmes. Dans ces intervalles, on observe des stries décurrentes régnant au sommet et à la base des tour's mais qui manquent dans leur région médiane. Ouverture arrondie. Columelle arquée, terminée au sommet par un pli spiral fort et saillant, émettant une lamelle mince qui recouvre en partie la perforation ombilicale. Labre simple arqué, orné sur sa face interne de costulestransversales qui se prolongent dans le fond de l'ouverture.

Coloratiou d'un blanc uniforme.

Annam : plage de Ben-Son (M. Boutan), 1 ex.

Ce Pyrgulina se fait remarquer par sa sculpture longitu. 
dinale bien développée et sa forme pupoïde. Nous ne trouvons rien d'analogue parmi les espèces déjà décrites.

\section{Prrgulina Claudoni n. $\mathrm{sp}$.}

(PI. VI, fig. 14, grossie 20 fois)

Testa nitidula solidu imperforata conoideo-ovata. Spira mediocris. Anfr. apicales heterostrophi; normales 4 parum convexi, sutura impressa juncti, costis longitudinalibus validis, obliquis, interstitia fere aequantibus instructi, inter costus transversim tenuiter sed distincte striati. Funiculus transversus in 2 anfr. ultimis e sutura emergit et alter iu anfr. ultimo paullo intra peripheriam accedit. Anfr. ultimi basis concentrice tenuiter striata.

A pertura subquadrata. Columella subarcuata, superne in dentem spiralem validum desinens. Labrum simpler.

Color albus.

Alt. $2 \mathrm{~mm}$; diam. $\max .1 \mathrm{~mm}$.; apertura $0,7 \mathrm{~mm}$. alta, $0,6 \mathrm{~mm}$. lata.

Coquille assez luisante, solide, imperforée, de forme conoïde ovale. Spire médiocrement élevée, composée d'un embryon hétérostrophe lisse et de 4 tours normaux peu convexes, séparés par une suture bien accusée. Surface ornée de costules longitudinales obliques, fortes, égalant à peu près les intervalles qui les séparent et de stries. décurrentes nombreuses, fines, mais bien visibles dans les espaces intercostaux. Un funicule décurrent émerge de la suture des deux derniers tour; sur le dernier tour, un autre funicule décurrent règne un peu au-dessous de la périphérie et est séparé du premier par un sillon assez accusé.La base du dernier tour est striée concentriquement et on y distingue aussi quelques plis d'accroissement rayonnants.

Ouverture subquadrangulaire. Columelle légèrement 
arquée et terminée dans le haut par un pli spiral bien développé. Labre simple.

Coloration blanche uniforme.

Annam : plage de Ben-Son (M. Boutan).

Cette espèce est caractérisée par ses côtes obliques, les stries de ses espaces intercostaux et son sillon périphérique bordé de deux funicules décurrents. Nous prions notıe confrère M. Claudon, auteur d'un excellent catalogue des Mollusques de Saint-Raphaël, d'en accepter la dédicace.

\section{Prbgulana Lamyi 11. sp.}

(PI. VI fig. 10., grossie 20 fois)

Testa mimuta solidula imperforata ovato-conoidea. Spira mediocriter clata. Anfr. apicales heterostrophi immersi parum prominentes: normales 4 planulati, inferne breviter rontracti, costis longitudinalibus rectis ac funiculo transverso prope basin ornati. In anfr. nltimi basi funiculi 2 accedunt. Funiculus transversus insupra in anfractum parte supera. inter costas conspicitur.

A pertura subquadrata. Cohmella subarcuata, superne in plicam spiralem deisinit. Labrum simplex.

Color albus.

Alt. $1.7 \mathrm{~mm}$. :diam. $\max .0,8 \mathrm{~mm}$.; apertura $0,7 \mathrm{~mm}$. alta, $0,5 \mathrm{~mm}$. lata.

Coquille de petite taille, assez solide, imperforée de forme ovalaire conoïde. Spire médiocrement élevée, composée d'un embryon hétérostrophe immergé et de 4 tours normaux presque plans, étroitement contractés à la base et ornés de côtes longitudinales droites ainsi que d'un funicule qui règne immédiatement au-dessus de la contracture, oủ il détermine une série d'alvéoles suprasuturales. Sur le dernier tour on observe encore 2 cordons décurrents au-dessous de celui qui entoure la périphérie. Eıfin 
vers le sommet des tours règne un cordon décurrent très faible, visible seulement dans les espaces intercostaux.

Ouverture subquadrangulaire. Columelle légèrement arquée, terminée au sommet parun pli spiral assez développé. Labre simple.

Coloration blanche uniforme.

Annam : plage de Ben-Son (M. Boutan), 1 ex.

Par sa taille, sa forme et sa sculpture, cette espèce que nous dédions à notre confrère M. Ed. Lamy se rapproche beaucoup du Pyrgulina turbonilloides Brusina, de la Méditerranée; mais un examen attentif ne permet pas de la lui assimiler: l'ornementation est en effet beaucoup plus nette et plus saillante chez le $P$. Lamyi que chez son congénère eurọéen.

Pyrgulina Bartschi n. sp.

(PI. VII fig. 1, grossie 20 fois)

Testa minuta nitida subhyalina imperforata ronoideo-elonguta. Spira elata. Anfr. apicales 2 heterostrophi, normales 5 complanati, sutura ralde impressa sejuncti, /uniculo trans. verso suprasuturali et costis longitudinulibus bituberculatis, quam interstitia multo angustioribus, sculpti. In anfr. ultimi lorsi funiculi a transversi accedunt.

A pertura subquadrata. Columella snbrecta, superne in plicam spiralem validam desinens. Labrum acutum polygonatum.

Coior albus.

Alt. 2,2 mm; dirm. max. I mm.; apertura $0,6 \mathrm{~mm}$. alta. $0,5 \mathrm{~mm}$. lata.

Coquille de petite taille, luisante, subhyaline, imperforée, conoïde allongée. Spire élevée, composée d'un embryon hétérostrophe et de 5 toursnormaux a platis et même légèr ement excavés au milieu, séparés par une suture très 
profonde. Surface ornée d'un cordon suprasutural et de costules longitudinales beaucoup plus étroites que leurs intervalles, renflées à leur extrémité supérieure ainsi que vers le milieu des tours, déterminant ainsi une double rangée décurrente de tubercules. Sur les tours supérieurs, ces costules se prolongent au-delà du cordon suprasutural. tandis que sur les derniers elles s'arrêtent contre ce cordon qui est tout à fait lisse, très saillant et continu. Trois autres cordons décurrents ornent la base du dernier tour: les deux inférieurs sont plus faibles.

Ouverture subquadrangulaire. Columelle presque rectiligne terminée au sommet par un pli spiral bien développé. Labre tranchant, rendu polygonal par les extrémités des cordons décurrents.

Coloration blanche uniforme.

\section{Aunam : plage de Ben-Son (M. Boutan).}

Cette espèce ne peut être comparée à aucune de celles qui ont été figurées jusqu'à présent. Nous la dédions à M. Paul Bartsch qui vieut de publieravec M. Dall une excellente étude sur les Pyramidellidés.

Pyrgulina Prestoni n. $\mathrm{sp}$.

(PI. VI, fig. 13, grossie 20 fois).

Testa minutissima nitidula imperforata, conoideo-ovata. Spira mediocris. Anfr. anicales heterostrophi, immersi, normales 3 sulphni. supra suturam impressam breviter contracti, costis longitudinalibus rectis, interstitia aequantibus, ac funiculo basali transverso ornati ; funiculus alter paullo infra situs anfr. ultimum exornat; denique sub lente valido funiculi aliquot transversi obsoleti inter costas detectuntur.

A pertura subquadrata marginibus callo adnato junctis. Columella paululum arcuata superne in plicam spiralem debilissimam desinit. Labrum simplex arruatum. 
Color allus.

Alı. $1,3 \mathrm{~mm}$.; diam. max. 0,7 mm. ; apertma 0,6 $\mathrm{mm}$. alta, $0,4 \mathrm{~mm}$. Inta.

Coquille minuscule, assez luisante, imperforée, conoïde ovalaire. Spire médiocrement élevée, composée d'un embryon hétérostrophe immergé et de 3 tours normaux presque plans, mais brièvement et brusquement contrac tés au-dessus de la sıture qui est bien accusée. Surface ornée de côtes longitudinales droites, égales en largeur à leurs intervalles, et d'un funicule décurrent qui borde la contraction basale des tours. Sur le dernier tour, un $2^{e}$ funicule décurrent règne un peu au-dessous du premier. Eufin à l'aide d'un fort grossissement, on décourre, entre les côtes longitudinales, des traces de plusieurs cordons décurrents extrèmement faibles.

Ouverture subquadrangulaire, à bords reliés par une callosité appliquée. Columelle légèrement arquée, terminée au sommet par un pli spiral très faible. Labre simple, arqué.

Coloration blanche uniforme.

Annam : plage de Ben-Son (M. Boutan).

Cette petite espèce appartient au même groupe que les P. Imrbonilloides Brus. et Penchynati B. D. D. des mers d'Europe; mais elle se distingue du premier, par l'absence de cordons saillants sur la base du dernier tour, et du second par ses tours moins convexes et ses côtes longitudinales plus droites.

Pyrgulina Germaini n. sp.

(PI. VII, fig. 3, 4, grossies 20 fois).

Testa minuta, solidula, parum nitens, imperforata, conoiteo ovata. Spira mediocris. Anfr. apicales heterostrophi; normales 3 gradati, sutura impressa ac marginata juncti, costis 
longitudinalibus crassis, superne tuberculosis, ornati. In anfr. ultimo, in medio paululum contracto costre paullo infra peripheriam cranescunt. Apertura ovata. Columella arcuata. superne in plicam spiralem desinens.

Color allous.

Alt. $1,5 \mathrm{~mm}$. : diam. max. $0,8 \mathrm{~mm}$.; apertura $0,7 \mathrm{~mm}$. alta, $0,5 \mathrm{~mm}$. lata.

Coquille de petite taille, assez solide, peu luisante, imperforée, de forme conoïde-ovale. Spire médiocre composée de 2 tours embryonnaires hétérostrophes et de 3 tours normaux étagés, séparés par une suture bien accusée et marginée par un bourrelet saillant. Ces tours sont ornés de fortes còtes longitudinales plus larges que leurs intervalles et se terminant chacune dans le haut par un gros tubercule. Le dernier tour est légèrement contracté au milieu et les côtes longitudinales s'effacent sur sa base un peu au dessous de la périphérie. Ouverture ovalaire, légèrement anguleuse au sommet. Columelle arquée, se terminant dans le haut par un pli spiral bien développé. Labre simple, faiblement anguleux dans le haut.

Coloration d'un blanc uniforme.

Anuam : plage de Ben-Son (M. Boutan), 2 ex.

Cette espèce, que nous dédions à M. Germain, bien connu par ses travaux sur les mollusques terrestres et tluviatiles. est nettement distincte de toutes les formes décrites jus. qu'à présent.

Pyrgulina gemmifera n. $\mathrm{sp}$.

(PI. VI, fig̣. 2, grossie 20 fois).

Testa minutissima, nitidula, imper/orata, conoideo-ovata. Spira mediocris: anfr. apicales heterostrophi; normales 3 sutura valde impressa juncti, superne costis longiturlinalibus 
brevibus et funiculis transversis .3 sculpti. Costæ, ubi funiculos superiores 2 transgrediuntur, tuberculatæ; funiculus vero inferus continuus, haud tuberculatus. In anfr. nltimi basi, funiculi transversi 3 accedunt.

A pertura ocata, maryinibus callo tenui adnatoqne junctis. Columella arcuatula. plicam spiralem immersam ostendit. Labrum a funiculis polygonutum.

Color altus.

Alt. $1,3 \mathrm{~mm}$. ; diam. max. $0,6 \mathrm{~mm}$. ; apertura $0,5 \mathrm{~mm}$. alta. $0,3 \mathrm{~mm}$. lata.

Coquille minuscule, assez luisante, imperforée, de forme conoïde-ovale. Spire médiocrement élevée, composée d'un sommet hétérostrophe et de 3 tours normaux séparés par une suture bien accusée. Surface ornée de 3 cordons décurrents dont l'inférieur très saillant est lisse, tandis que les deux supérieurs sont garnis de gros tubercules arrondis, produits par la rencontre de côtes longitudinales espacées. Au-dessous du cordon médian, les côtes longitudinales se divisent en costules plus nombreuses et faibles qui sont bien apparentes dans les intervalles des cordons décurrents. La base du dernier tour est ornée de 3 cordons décurrents supplémentaires non tuberculeux.

Ouverture ovalaire, à bords reliés par une callosité mince et appliquée. Columelle arquée et terminée au sommet par un pli spiral suffisamment immergé pour qu'il soit difficile de l'apercevoir lorsque la coquille est placée de face, mais qui devient bien apparent lorsqu'on l'incline vers la gauche. Labre reudu polygonal par les extrémités des cordons décurrents.

Coloration blanche uniforme.

Annam : plage de Ben-Son (M. Boutan), 1 ex.

Espèce voisine de notre Parthenina Bartschi décrit plus laaut, mais différent par sa taille plus faible, sa sculpture plus saillaute, et ses deux rangées de tubercules plus rap- 
prochées. Il est possible que l'exemplaire unique recueilli par M. Boutan ne soit pas complètement adulte.

\section{Pyrgulina eximia n. sp. \\ (Pl. YII, fig. 5, grossie 20 fois.)}

Testa minutissima, sat solida, nitidula. imperforata, bieviter conoideo-ovata. Spira parum elata. Anfr. apicales 2 heterostrophi, normales 3 convexi, sutura impressa juncti, antepenultimus transversim bicarinatus, penultimus superne costis longitudinalibus crassis, quam interstitia latiorilnus, in.. ferne vero /uniculo transverso valido ornatus. In basi anfr. ultimi, funiculi 3 insupra accedunt.

A pertura subquadratu. Columella parum arcuata, superne in plicam spiralem debilem desinens. Labrum simplex arcuatum.

Color albidus, subhyulimus.

Alt. $1.2 \mathrm{~mm}$. ; diam. max. $0,6 \mathrm{~mm}$. ; apertura $0.5 \mathrm{~mm}$. alta, $0,3 \mathrm{~mm}$. lata.

Coquille minuscule, assez solide, un peu luisante, imperforée, de forme conoïde-ovale raccourcie. Spire peu élevée, composée de 2 tours embryonnaires hétérostrophes et de 3 tours normaux convexes séparés par une suture bien marquée. Premier tour normal pourvu de 2 carènes décurrentes, l'avant-dernicr présentant sous la suture, des côtes longitudinales épaisses, plus larges que leurs intervalles, et au-dessous, un cordon décurrent large et saillant. Sur le dernier tour, les côtes longitudinales qui ornent la région supérieure, sont rétrécies au milieu et renflées aux deux bouts : au-dessous de ces côtes, règnent 4 cordons décurrents très gros et saillants.

Ouverture subquadrangulaire, subanguleuse au som. met et à la base de la columelle. Columelle faiblement arquée et terminée au sommet par un pli spiral médiocrement développé. Labre simple légèrement arqué.

Coloration blanche subhyaline. 
Annam : plage de Ben-Son (M. Boutan).

Cette espèce se rapproche de l'Odostomia (Chrysallida) dux Dall et Bartsch (Proc. U. S. Nat. Mus. XXX, 1906, p. 330 , pl. XVII, f. 4), du Japon ; mais elle en diffère par les cótes longitudinales du sommet des derniers tours continues et non partagées par le milieu, ainsi que par ses cordons décurrents plus saillants.

\section{Pyrgulina pretiosa n. sp.}

(P). VIl, fig. 6, grossie 20 fois).

Testa minutissima, nitidule uc subhyalina, subcylindrica pupoidea imperforata. Spira sat elata. Anfr. apicales heterostrophi, normales 4 sutura valde impressa sejuncti: primus intra unicarinatus; ceteri costis longitudinalibus valde obliquis, simuosis, superne inflatis et infra funiculo transverso prominente ornati. Inter costas striæ transversæ conspiciuntur. In basi unfr. ultimi, funiculi 4 insupra accedunt.

Apertura parva, subquadrata. Columella rectiuscula, superne in plicam spiralem mediocrem desinens. Labrum arcuatum et in medio sinuosum.

Color albidus.

Alt. 1,5 mm.; diam. max. $0,6 \mathrm{~mm}$. ; apertura $0,6 \mathrm{~mm}$ alta, $0,3 \mathrm{~mm}$. lata.

Coquille minuscule, assez luisante, subhyaline, imperforée, de forme subcylindrique pupoïde. Spire assez élevée, composée d'un sommet liétérostrophe et de 4 tours normaux, dont le premier est orné d'une carène subbasale saillante, tandis que les suivants présentent sur leur région supérieure de fortes côtes longitudinales-obliques, sinueuses et renflées au sommet. Dans les intervalles des côtes on observe quelques stries décurrentes. Au-dessous des côtes longitudinales, règne un cordon décurrent fort et saillant ; sur la base du dernier tour, on remarque en outre 4 autres cordons décurrents. 
Ouverture petite, subquadrangulaire. Columelle presque rectiligne, terminée au sommet par un pli spiral peu développé. Labre simple, sinueux au milieu.

Coloratiou blanche uniforme.

Anuam : plage de Ben-Son (M. Boutan), 1 ex.

Cette espèce, remarquable par l'élégance de sa sculpture, se rapproche de l'Odostomia (Miralda) gemma A. Adams, du Japon, figuré récemment par MM. Dall et Bartsch (Proc. U. S. Nat. Mus. XXX, 1906, p. 35้6, pl. XXII, f. 1); mais elle s'en distingue par sa taille plus faible (haut. $1^{\mathrm{mm}}, \breve{5} \mathrm{au}$ lieu de $\left.3^{\mathrm{mm}}, 2\right)$, par ses côtes longitudinales obliques sinueuses et continues, tandis que chez l'espèce d'Adams elles sont verticales et découpées en 2 séries de tubercules quadrangulaires.

\section{Turbonilla sp.}

Annam : plage de Ben-Son (M. Boutan).

Nous avons rencontré dans les récoltes de M. Boutan, une demi-douzaine de formes différentes de Turbonilla, que leur jeune âge ne nous permet pas de décrire ou d'identifier à des espèces connues.

L'une d'entre elles, caractérisée par ses tours convexes, ornés de côtes longitudinales fortes, légèrement arquées et s'arrêtant à la phériphérie du dernier tour, nous semble voisine du T. abseida Dali et Bartsch (P. U. S. Nat. Mus. XXX, 1906, p. 337, pl. XXI, f. 4).

Eulinella progoides n. $\mathrm{s} p$.

(PI. VII, fig. 7, grossie 15 fois)

Testa minula, angustissime rimata, elongato-urrita. Anfi. npicales heterostrophi, normales 4 leves subplani, prope basin valde coarctati, sutura conspicua juncti. A pertura subqua- 
drata, marginibus callo adnato crassiusculo junctis. Columella parum arcuata, subplicata. Labrum arcuatum in medio subangulatum,

Color albus .

Alt. $2 \mathrm{~mm}$; diam. $\max .0,7^{\mathrm{mm}}$; apertura $0,6^{\mathrm{mm}}$ alta, $0,4^{\mathrm{mm}}$. lata.

Coquille de petite taille, pourvue d'une fissure ombilicale à peine visible. Spire allongée, turriculée, composée d'un embryon hétérostrophe et de 4 tours normaux séparés par une suture bien marquée. Ces tours sont plans à partir de la suture jusque près de leur base où ils sont anguleux et brusquement contractés. Ouverture subquadrangulaire, à bords reliés par une callosité appliquée assez épaisse. Collumelle presque verticale, pourvue d'un pli à peine visible. Labre simple, légèrement anguleux au milieu.

Coloration entièrement blanche.

Annam : plage de Ben-Son (II. Boutan) $2 \mathrm{ex}$.

Cette espèce est fort remarquable par la contraction brusque qui règne à la base de ses tours et qui lui donne un aspect scalaroïde. Elle peut être comparée sous ce rapport à l'Eulimella eugonia Bush et à la variété teres Bush, de cette même espèce, des côtes orientales d'Amérique, dont elle diffère toutefois par sa forme plus cylindrique et son sommet plus obtus (Trans. Conn. Acad. VI, pl. 45, fig. 9).

On peut aussi la rapprocher de l'Eulimella venusta Melvill (Proc. Mal. Soc. L. VI, 1904, p. ǒ6, pl. V, fig. 15), mais à taille égale, l'espèce de Melvill aurait 7 tours aulieu de 5 .

Il est probable que le plus grand des deux exemplaires de notre espèce, représenté pl. VII, fig. 7, n'est pas encore adulte et qu'on trouvera ultérieurement des spécimens plus grands et possédant un nombre plus élevé de tours de spire. 


\section{Nerita albicilla Linné}

1758. Nerita Albicilla

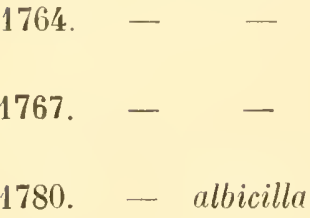

Linné, Syst. Nat. Ed. X, p. 778 .

Linné, Mus. Lud. Ulr. p. 679 .

Linné, Syst. Nat. Ed. XII, p. 1234.

Bors, Test. Mus. Caes. Vindob. p. 406,

1781. Palatum bovis Albicilla Linnaei Chemnitz, C. Cab. V, p. 313, Pl. CLXXXXIII, fig. 2000a à 2000h. (numéroté par erreur 200a. à $200 \mathrm{~h}$.

1784. Nerita albicilla

L. Sснвӧтеr,Einleit.II.p. 296.

1790. - Albicilla

- Gmelin, Syst. Nat. ed. XII, p. 3681 .

1817. - albicilla Dillwyn, Descr. Catal. II, p. 998.

\begin{tabular}{|c|c|c|}
\hline 1822. & - & $\begin{array}{l}\text { Lanarck, Anim. s. vert. } \\
\text { VI, 2e p.,p. 192. }\end{array}$ \\
\hline 1832. & - & $\begin{array}{l}\text { Deshayes, Encycl. Mé- } \\
\text { thod., III, p. } 621 \text {. }\end{array}$ \\
\hline 1834. & - & $\begin{array}{l}\text { Quoy et Gaimard, Voy. } \\
\text { Astrolabe } 111, \text { p. } 182 \text {, pl. } \\
65 \text {, fig. } 17,18 .\end{array}$ \\
\hline 1838. & - & $\begin{array}{l}\text { Deshayes in Lamakci, } \\
\text { Anim. s. Vert. 2e Ed. } \\
\text { VII, p. } 605 .\end{array}$ \\
\hline 1840. & - & $\begin{array}{l}\text { Dufo, Moll. Séchelles et } \\
\text { Amirantes in Ann. des } \\
\text { Sc. Nat., XIV, p. } 194 \text {. }\end{array}$ \\
\hline 1844. & - venusta & $\begin{array}{l}\text { Dunier in Phillpl, } \\
\text { Abbild., p. } 6, \mathrm{pl} \text {. I, f. } \mathbf{1 1} .\end{array}$ \\
\hline
\end{tabular}


1848. Nerita albicilla

1850.

1 รวั2.

185ว5. - - -

185ว5. - - -

18 อัร.

1 Sอัง.

1859.

1866.

1869

1871

1874 .

1883.

1883.

ustulata

1889. - albicilla
Krauss, Südafr. Moll., p. 89.

Recluz, Catal. des Nérites in J. de C., I, p. 286.

Gould, Univ. St. Expl. Exp. Moll., p. 167, pl. 11, f. 194, 194a.

L. Reeve, C. Icon., Pl. XV, f. 64 a à 64 d.

- Hanley, Ipsa Linn. Conch., p. 405. Gray, Obs. on the G. Nerita and its operculum in P. Z. S. L., p. 94, f. 6 (opercule).

Gray, Ann. and Mag. N. H. 3d Ser., II, p. 66 . f. 6 (operc.).

L. Chenu, Man de Conch., 1 , p. 334, f. 2423 à 2425 .

Mabille et le Mesle, Obs. sur la faune malac. de la Cochinchine et du Cambodge in J. de C., XIV, p. 119.

Lischise, Japan. Meeresconch., I. p. 85 (excl. var.). Lischí, ibid., II, p. 81. Lischise, ibid., III, p. 60. Sowerby, Thes. Conch., V, p. 112,; pl. 464, fig. 26 , 27, 28; pl. 468, fig. 113. Sowerby, Thes. Conch., pl. 468, fig. 122 (juv.)

L. L. Monlet, Catal. coq. 
1889. Nerita albicilla

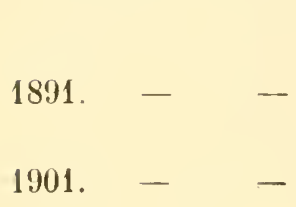

rec. par M. Pavie dans le Cambodge et le Roy. de Siam in J. de C., XXXVII, p. 157.

L. von Martens, C. Cab., 2 Ed., p. 25, pl. 8, fig. 1, 2; p. 110 , pl. 3,fig. 1-4;6-9. - P. Fischer, Catal. IndoChine, loc.cit, p. 199 (115).

- H. Fischer, Liste coq. rec. par M. de Gennes à Djibouti in J. de C., XLIX, p. 121 .

Annam : plage de Ben-Son, (M. Boutan), 1 ex. vivant.

Nerita lineata (Chemnitz) Gmelin

1905. Nerita lineata (Ch.) Gm. Dautzenberg et H. Fischer, Liste des Moll. rec. par I. le Cap. Blaise en Indo. Chine, in J. de Conch., LIII, p. 179.

Toukin : Hanoï (M. Boutan).

Nerita chamaeleon Linné

1905. Nerita chamaeleon L. Dautzenberg et H. Fischer, Liste des Moll. rec. par M. Blaise au Tonkin in J. de C., LIIl, p. 177.

Annam : plage de Ben-Son (M. Boutan), $1 \mathrm{ex}$. 
Turbo coronatus Gmelin

Var. granulata Gmelin

1900̈. Turbo coronatus Gm. var. granulata Gm. DautzenBErG et H. FISChER, Liste des Moll. rec. par M. le Cap. Blaise au Tonkin, in J. de Conch., LIII, p. 186.

Aunam : Pointe du Scorpion (M. Boutan).

Monodonta labio Linné

1905. Monodonta labio L. Dautzenberg et H. Fischer, Liste des Moll. rec. par M. Blaise au Tonkin in J. de C., LIII, p. 187.

Annam : plage de Ben-Son (M. Boutan), 1 ex.

Umbonium vestiarium Linné

1758. Trochus vestiarius

1767.

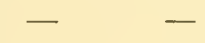

1790.

1822. Rotella lineolata

1822. - rosea

1827. - lineolata

1838
Linné (ex parte), Syst. Nat. X, p. 758.

Linné, Syst. Nat. édition XII, p. 1230.

Gurelin, Syst. Nat. edit. XIII, p. 3578.

Lamarck, Anim. s. Vert. VII, p. 7.

Lamarck, Anim. s. Vert. VII, p. 8.

Crouch, Introd. to Conch. pl. 16, f. 14, 14a.

Kiener, Iconogr. coq. viv. G. Roulette, p. 13, pl. I. 
1838. Rotella elegans

18:30. Trochus vestiarius $\mathrm{L}$.

1853. Globulus -

1853. - elegans Beck

1853. - australis

1853. - parvulus Ant.

1853. Umbonium depressum

1859. Rotella lineolata Lk.

1878. Rotella vestiaria $\mathrm{L}$.

1878. - depressa

1878. - elegans

188I. Umbonium - Beck

1883. Rotella vestiaria L.

1889. Umbonium vestiarium $\mathrm{I}$.
Beck in Kiener, ibid. p. 4. pl. III, fig. 6, 6, 6a. Hanley, I psa Linn. Coneh. p. 320 .

Philrppi, Conch. Cab. 2o Ed. p. 45, pl. 7, fig. 1-10. Philippi, ibid.p. 46, pl. 7, fig. 16-20.

Philippl, ibid. p. 48, pl. 7 , fig. 21-23.

Philippl, ibill. p. 48, pl. 7, fig. 11-12.

A. Adans, P. Z. S. L. p. 188.

Chenu, Man. de Conch. I, p. 353, fig. 2592-2603, (comprenant la var. elegans, fig. 2592).

Sowerby in Reeve, C. lcon. pl. III, f. $12 \mathrm{a}$ à $12 \mathrm{t}$. Sowerby in Reeve, C. Icon. pl. I, fig. 2.

Sowerby in Reeve, C. Icon. pl. II, fig. 6a, 6b, $6 \mathrm{c}, 6 \mathrm{~d}$.

de Ruchebiune, Doc. sur la faune Malac. Cochiuchine et Cambodge in Bull. Soc. Philom. p. 19 du tiré à part.

Sowerby, Thes. Conch. V, p. 13əั, pl. 4i2, fig. 1-ว. Pilsbry in Tryon, Man. of Conch. Str. and Syst. XI, p. 450, pl. 58, f. 1-8. 
1889. Umbonium elegans

1891.
L. Morlet, Cat. eoq. rec. par M. Pavie dans le Cambodge et le Roy. du Siam in J.de C. XXXVII, p. 160.

P. Fischer, Catal. IndoChine,loc. cit.p. 205(121).

Annam. Plage de Ben-Son (II. Boutan), très abondant.

Linné a confondu, sous le nom de Trochus vestiurius, la coquille à laquelle on attribue généralement ce nom aujourd'hui et celle distinguée depuis par Sowerby sous le nom de monilifera, qui est costulée transversalement et ornée de tubercules sous la suture. La première référence, celle de Petiver, pl. XI, fig. 6, représente une coquille lisse, tandis que les figurations de Gualtieri, pl. 63̆, fig. E, E représentent des coquilles sillonnées et ornées de tubercules sous la suture. Les fig. G, G de Gualtieri représentent aussi des formes lisses. Enfin, nous supposons, d'après la forme de la callosité que la fig. F du même auteur, représente probablement l'Umbonium costatum.

D'autre part Hanley nous apprend que les deux espèces lineolata Lk et monilifera Sow. sont réunies dans la collection de Linné, sous le non de Trochus vestiarius.

Gmelin, au lieu d'élucider la question, l'a encore compliquée en ajoutant diverses références dont la première, Lister, pl. 649, fig. 44 et 47 , représente l' $U$. costatum tandis que les fig. 45, 46, 48, 49 et 30 représentent l'U. vestiarum.

Lamarck a substitué au nom de vestiaria celui de lineolata sans aucune utilité, puisqu'il cite comme synonyme la référence de Linné. 
Cyclostrema Goneti n. sp.

(PI. VII, fig. 8, 9, 10, grossies 10 fois)

Testa nitidula, crassissima, sat late ac pervir umbilicata, turbinata, depressa. Spira parum elata. Anfr. I gradati, sutura conspicua juncti: primi 2 leves ac conceri, ceteri superne complanati, funiculo transverso ac costis longitudi. nalibus validis interstitia subæquantibus et ubi funiculum transgrediuntur tuberculatis, ornati. In anfr. ultimo funi culi 3: unus peripherialis, alter basin cingens, infimus umbilicum rircumdans, accedunt. Anfe. ultimus ad extremitatem valde descendens.

Apertura circularis, peristoma crassissimum et continuит.

Color allous.

Alt. 1,4 mm., diam. max. 2,1 mm.; apertuea $0,9 \mathrm{~mm}$. alta, $1 \mathrm{~mm}$. lata.

Coquille luisante très épaisse, assez largement et profondément ombiliquée, de forme transversalement turbinée. Spire peu élevée, composée de tours étagés: les deux premiers lisses et convexes, les autres présentant au-dessous de la suture une rampe aplatie, bordée d'un cordon décurrent et des côtes longitudinales fortes, égalant à peu près leurs intervalles; ces côtes déterminent sur le cordon décurrent une série de gros tubercules; sur le dernier tour, les còtes longitudinales se prolongent jusque dans l'ombilic. Trois autres cordons décurrents viennent s'ajouter au premier; ils sont situés : l’un à la périphérie, un autre un peu plus bas, et le troisième en bordure de la cavité ombilicale. Dernier tour très descendant à son extrémité.

Ouverture circulaire, à péristome très épais et continu.

Coloration d'un blanc uniforme.

Annam : plage de Ben-Son (M. Boutan). 
Cette jolie espèce, que nous sommes heureux de dédier au savant professeur Paul Godet, Directeur du Musée d'Histoire Naturelle de Neuchatel, est remarquable par son ornementation très saillante. Les tubercules que forment les côtes longitudinales en passant sur le cordon périphérique, donnent au pourtour de la coquille, vu d'en haut ou d'en bas, un aspect régulièrement festonné.

C'est du Cyclostrema micans A. Adams, (Pr. Z. S. L. 1850 , p. 44; Thes. Conch., pl. CCLV, fig. 7, 8), qu'elle se rapproche le plus par l'épaisseur du test et la forme générale; mais sa taille est plus faible, ses côtes longitu dinales sont beaucoup plus fortes, plus espacées et plus saillantes, ses cordons décurrents sont moins nombreux, le cordon inférieur est plus rapproché de la cavité ombilicale, etc.

\section{Cyclostrema Bushi n. $\mathrm{sp}$.}

(PI. VII, fig. 11, 12, 13, grossies 10 fois)

Testa nitidula, crassa, late ac pervie umbilicatu, turbinata, depressa. Spira vix prominula. Anfr. 4 sutura impressa juncti ac prope suturam breviter excavati; primi leves, convexi, ceteri funiculo transverso et costis longitudinalibus, quam interstitia multo angustioribus sculpti. Anfr. ultimus ad extremitatem lreviter descendens, in peripheriam carinam valde prominentem ostendit; in basi funiculi insupra 3 cre.. nulati accedunt: infimus umbilicum cingit. A pertura circularis, valde obliqua, a peristomate incrassato coarctata.

Color albus.

Alt. $1,1 \mathrm{~mm}$; diam. max. $2 \mathrm{~mm}$; apert. $0,9 \mathrm{~mm}$. alta, $0,9 \mathrm{~mm}$. lata.

Coquille assez luisante, épaisse, largement et profondément ombiliquée, de forme turbinée aplatie. Spire à peine saillante, composée de quatre tours séparés par une suture bien accusée, accompagnée d'un sillon profond 
bordé par un cordon décurrent assez saillant; un deuxième cordon encore plus saillant entoure la périphérie; on observe sur la base du dernier tour. deux cordons bien saillants dont l'inférieur borde l'ombilic et il en existe encore deux ou trois autres, plus faibles. sur la paroi même de la cavité ombilicale. Tous ces cordons sont traversés par des côtes longitudinales plus faibles qui rendent les points d'intersection tuberculeux, et qui se prolongent jusqu'au fond de l'ombilic. Ouverture circulaire, très oblique par rapport à l'axe de la coquille et rétrécie par l'épaississement du péristome.

Coloration blanche uniforme.

Cette espèce, que nous dédions à Mlle $\mathrm{K}$. Bush, de Washington, se rapproche, au premier aspect, du C. alveolatum Jousseaume (Revue et Mag. de Zoologie 1872, p. 392, pl. XIX, fig. 4), mais elle diffère par les détails de la sculpture, notamment par la disposition des carènes et des cordons décurrents.

Teinostoma multisulcatum $\mathrm{n}$. $\mathrm{sp}$.

(PI. VII, tig. 14, 15, 16, grossies 15 fois)

Testa minuta, nitilula, sat solila, imperforata, depressoturbinata. Spira vix prominula. Anfr. 3 1/2 rapide crescentes, sutura sat impressa juncti, undique regulariter sulcati. Anfr. ultimus hand descendens nec uscendens, basi late excavatus; umbilicum a callo omnino clausum. A perturn circularis obliqua, peristomate continuo.

Color albus.

Alt. $0,7 \mathrm{~mm}$; diam. max. $1,5 \mathrm{~mm}$.: apertura $0,7 \mathrm{~mm}$. alta, $0, \overline{\mathrm{mm}}$. lata.

Coquille de petite taille, assez luisante el solide, imperforée, de forme turbinée déprimée. Spire très surbaissée, composée de $31 / 2$ tours croissant rapidement et séparés 
par une suture assez marquée. Toute la surface est régulièrement sillonnée. Le dernier tour ne descend pas et ne remonte pas non plus à son extrémité; sa base est largement excavée et présente une région ombilicale recouverte d'une callosité peu étendue. Ouverture circulaire, oblique par rapport à l'axe de la coquille, à péristome continu, peu épais, si ce n'est du côté columellaire.

Coloration blanche uniforme.

C'ette espèce ressemble, par sa sculpture, au $C$. concentricum A. Adams (Ann. and Mag. N. H. 3 Ser. XI (1863), p. 267, mais en diffère par son dernier tour non ascendant à son extrémité. Adams dit dans sa description, que chez le concentricum, le dernier tour remonte de manière à envelopper les autres presque jusqu'au sommet de la spire.

\section{Dentaliun sp.}

Nous avons trouvé dans le sable qui s'échappait des coquilles recueillies sur la plage de Ben-Son, Annam, par M. Boutan, deux espèces de Dentalium, qui ne sont pas suffisamment représentées pour nous permettre des déterminations certaines. Toutefois, comme aucun Dentalium n’a encore été cité jusqu'à présent de l'Indo-Chine, nous croyons intéressant de les signaler.

L'une de ces deux espèces est représentée par un exemplaire unique de $20 \mathrm{~mm}$ de longueur, assez arqué, sans trace de fissure, orné de 9 côtes médiocrement saillantes et séparées par des intervalles à peu près plans qui ne préseutent que des stries d'accroissement sans trace de costules ni de stries longitudinales. MM. Pilsbry et Sharpe ont coustaté que chez le $D$. octangulatum Donovan, le nombre des côtes qui est normalement de 8, est parfois aussi de 9 et nous nous serions décidés à identifier 
l'exemplaire de M. Boutan à cet octangulatum si les côtes n'étaient sensiblement moins saillantes.

L'autre espèce de $14^{\mathrm{mm}}$ de long, peu arquée, est pourvue d'une courte fissure du côté convexe, ornée de 25 côtes subégales à l'extrémité antérieure et seulement d'environ 12 à l'extrémité postérieure. Nous croyons qu'il s'agit là d'un exemplaire jeune, peut-être du D. Vernedi Hanley.

ANomia Lischici nov. sp.

(PI. V, fig. 8, 9, 10, 11)

Cette espèce qui a été bien caraclérisée, mais non dénommée par Lischke (Japanische Meeres-Conchylien, I, p. 180), se distingue de tous les autres Anomia par la sculpture de sa valve gauche (supérieure): elle consiste en costules fines, très nombreuses qui ont une direction générale rayonnante, mais qui, au lieu d'être parallèles et continues, s'entrecroisent très irrégulièrement et s“interrompent, tantôt se divisaut, tantôt confluant entre elles.

M. Hirase nous a envoyé cette espèce de Kumihama Tango sous le nom de $A$. elyros Gray; mais le vérilable elyros (P. Z. S. L. 1849, p. 118, pl. 4, fig. 1) est fort différent.

D'autre part, l'abbé Culliéret a recueilli en 1890, à Yokohama, des spécimens de la même espèce et nous en avons reçu de nombreuses valves d'Oho-Shima (R. P. Ferrié).

Nous représentons ici (fig. 8, 9), un des spécimens japonais d'Yokohama, ainsi que les deux valves recueillies par M. Boutan (fig. 10, 11).

Les seuls Anomia qui aient été cites de l'Indo-Chine, sont l'A. sol Reeve (Crosse et Fischer, J. de Conch. XXXVII, p. 290), et I'A. wnigmatica Chemnitz.

Annam : plage de Ben-Son (M. Boutan), 2 valves supérieures. 


\section{MYtilus viridis Linné}

1798. Mytilus viridis

1764

1767. - -

1788. - smaragdinus

1790.

1819.

1819. - opalus

1825. - smaragdinus

183I. - opalus

1836

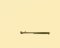

1836 .

- smaragdinus

1843.

- viridis

1855.
Linné, Syst. Nat. Ed. X, p. 706 .

Linné, Mus. Lud. Ulr., p. วั42.

Linné, Syst. Nat. Ed. XII, p. 1158.

Chemnitz, Conch. Cab. VIII, p. 12马, 166, pI. 83, fig. 745 ('Tranquebar!), pl. 84 fig. 7146 (Guinée). Schröter in Martini et Chemnitz, Namen Register, p. 69.

Guelin, Syst. Nat. Ed. XIII, p. 3359.

Gm. Lamarcis, Anim. s. Vert. VI, Irø p., p. 12飞. LAMARCK, ibid. Wood, Ind. Testac., p. 58, pl. 12, fig. 26.

Deshayes, Encycl. Mèthod. II, p. כ̋61.

Lk. Desilayes in Lamarck, Anim. s. Vert. $2^{e}$ éd., VII, p. 43 (= smaragdinus in notis).

Deshayes in Lamarcis, ibid.

Hanley, Rec. biv. Sh., p. 247.

H ANLEY, I psa Linn . Conch., p. 14 . 
1857. Mytilus smaragdinus Ch. Reeve, C. Icon., pl. VII, fig. 28 (Bornéo).

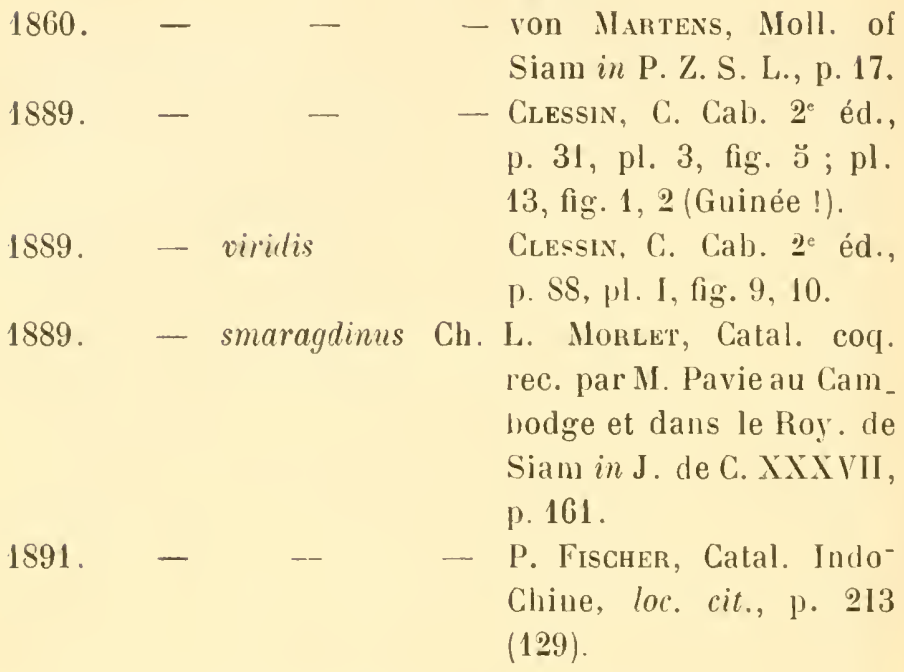

Annam : plage de Ben-Son (M. Boutan).

D’après les renseignements fournis par Hanley sur la collection de Linué, il est certain que le Mytilus viridis de Linné et le $\boldsymbol{M}$. smaragdinus de Chemnitz sont la même espèce. Quant au M. opalıs Lk., il est établi sur des spécimens vieux et décortiqués de la même également.

Linné n’a d'abord connu que des spécimens très jeunes puisqu'il le dit être plus petit qu'un ongle. (Mus. Lud. Ulr., p. 542).

\section{MYrILUs HiRsutus Lamarck}

1819. Mytilus hirsutus

1836.
Lamarck, Anim. s. Vert. $\mathrm{VI}(\mathrm{I})$, p. 120.

Lk. Desilayes in Lamarck, Anim. s. Vert. ํo éd. VII, p. 38 . 
1857. Mytilus hirsutus

1882.

1889.

1890.
Lk. Reeve, Conch. Icon., pl. III, fig. 8.

- Dunker, Index Moll. Maris Japon., p. 222.

- Clessin, Conch. Cab. $2^{\circ}$ éd., p. 40, pl. 7, fig. 6 .

- Paeter, Catal. der Conchy. lien-Sammlung IlI, p. 192.

Annam : pointe du Scorpion (M. Boutan).

Cette espèce bien caractérisée n'est pas signalée dans le catalogue de P. Fischer.

\section{Anudonta Jourdyi L. Morlet}

1906. Anodonta Jourdyi L. M. DAutzenberg et H. Fischer, Liste des Moll. réc. par M. Mansuy en IndoChine, in J. de Conch. LIII, p. 451 .

Tonkin : Hanoï (M. Boutan).

\section{Dipsas plicatus Leach}

1906. Dipsas plicatus Leach Dautzenberg et H. FisCHER, Liste des Moll. réc. par M. Mansuy en IndoChine, in J. de Conch. LIII, p. 4 3ั3.

Tonkin : Hanoï (M. Boutan).

\section{Unio Jourdyi L. Morlet}

1906. Unio Jourdyi

L. M. Dautzenberg et H. FisCHer, Liste des Moll. réc. par M. Mansuy en Indo- 
Chine, in J. de Conch. LIII, p. 452 .

Tonkin : Hanoï (M. Boutan).

\section{Cardium sinense Sowerby}

1840. Cardium Sinense

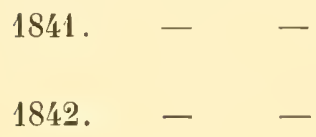

1844. - sinense Sow.

1869. - Sinense

1889. - sinense

1891 .
Sowerby, Pr. Z. S. L. p. 105.

Sowerby, Conchol. Illust. fig. 35 .

Hanley, Rec. Biv. Sh.

p. 135, pl. 17, fig. 2马.

Reeve, C. Icon. pl. I, fig. 3.

Römer, C. Cab. 2e éd., p. 73, pl. 12, fig. 11, 12. Crosse et Fischer, Note sur la faune conch. mar. de l'Annam, in J. de C. XXXVII, p. 293.

P. Fischer, Cat. IndoChine, p. 14 ว̆.

Annam : Plage de Ben-Son (M. Boutan), une valve.

\section{Cardium coronatum (Splenger) Schröter}

1782. Cardium costatum Indix orientalis var. Chemnitz, C. Cab. VI, p. 161.

1786. - coronatum (Spengler) Schröter, Einleit. Ill, p. 53, pl. 7, fig. 13$13^{\mathrm{b} .}$

1790. - Lima var. $\beta$. Guelix, Syst. Nat. éd. XIlI, p. 3253 .

1815. - fimbriatum Wood, General Conch. pl. 56 , figg. 4, 5. 


\begin{tabular}{|c|c|c|c|}
\hline 39. & Cardic & riat & $\begin{array}{l}\text { Lamakick, Anim. s. Vert. } \\
\text { VI, 2e part. p. כ̆. }\end{array}$ \\
\hline 1825. & - & - & $\begin{array}{l}\text { Wood, Ind. Test. p. } 29 \text {, } \\
\text { pl. } 5 \text {, fig. } 39 .\end{array}$ \\
\hline 1839. & - & - & $\begin{array}{l}\text { Gray, in Beechey's Voyage } \\
\text { p. Iว̆2, pl. XLII, fig. } 1 \text {. }\end{array}$ \\
\hline 1842. & - & $\mathrm{Lk}$ & $\begin{array}{l}\text { Hanley, Rec. biv. Sh., } \\
\text { p. } 130 .\end{array}$ \\
\hline 1845. & -- & Lk & $\begin{array}{l}\text { Reeve, C. Icon. pl. XVIII, } \\
\text { fig. } 91 \text {. }\end{array}$ \\
\hline 1869. & - & coronatum Sp. & $\begin{array}{l}\text { Römer, C. Cab. } 2^{3} \text { éd., } \\
\text { p. } 68 \text {, pl. } 12 \text {, fig. } 3,4,5 .\end{array}$ \\
\hline 1889. & - & fimbriatum W. & $\begin{array}{l}\text { L. Morlet, Catal. coq. } \\
\text { rec. par M. Pavie dans le } \\
\text { Cambodge et le Roy. de } \\
\text { Siam, in J. de C. XXXVII. } \\
\text { p. } 168 .\end{array}$ \\
\hline 1889. & - & - & $\begin{array}{l}\text { Crosse et Fischer, Note } \\
\text { sur la faune conch. mar. } \\
\text { de l'Anuam in J. de C. } \\
\text { XXXVII, p. } 293 .\end{array}$ \\
\hline 1891. & - & - & $\begin{array}{l}\text { P. Fischer, Catal. Indo- } \\
\text { Chine, loc. cit. p. } 229 \\
\text { (145). }\end{array}$ \\
\hline
\end{tabular}

Annam : plage de Ben-Son (M. Boutan), 4 valves.

Crista divaricata Chemnitz

1906. Crista divaricata Ch. Dautzenberg et H. FisCHer, Liste des coq. réc. par M. Mansuy en IndoChine, in J. de C. LIII, p. 458.

Annam: plage de Ben-Son (M. Boutan), 1 ex, 


\section{Dosınia Grönnerı Philippi}

1847. Cytherea (Artemis) Grïneri Philıp', Abbild. p.

$$
\text { pl. VIIl, fig. 2. }
$$

1848. Artemis Grüneri Ph. PııLıpp, Zeitschr. f. Malak., p. 132.

1850. - - Reeve, C. Icon. pl. V, fig. 31.

1853. Dosinia - Deshayes, Cat. Br. Mus., p. 27.

1855. Artemis - SowkrBy, Thes. Conch. II, p. 666 , p. CXLH, fig. 47 .

1862. Dosini - Röмer, Monogr. Dosinia, P. 61 .

Annam : plage de Ben-Son (M. Boutan), 1 ex.

Il esı probable que c'est cette espèce qui a été indiquée par L. Morlet sous le nom de D. contusa.

\section{Cyclina chinensis Chemnitz}

1906. Cyclina chinensis

Ch. Dautzenberg et H. Fischer, Liste des Moll. réc., par M. Mansuy en IndoChine in J. de C. LIII, p. 460 .

Annam : Plage de Ben-Son (II. Boutan) 1 valve.

\section{Meletrix meretrix Liuné}

1906. Meretrix meretrix

L.Dautzenberg et H.Fischer, Liste des Moll. réc. par M. Mansuy en Indo-Chine in J. de C. LIII, p. 461.

Annam: Plage de Ben-Son, nombr. valves et $1 \mathrm{ex}$. pointe du Scorpion (M. Boutan). 
Venus (Cryptogramma) impressa Anton

1906. Venus (Cryptogramma) impressa Ant. Dautzenberg et H. Fischer, Liste des Moll. réc. par M. Mansuy en Indo-Chine, in J. de C. LIII, p. 462.

Tonkin : Hanoï (M. Boutan).

\section{Venus (Anaitis) roseotingta Baird}

1873. Venus roseo-tincta

Baird in Brenchley, Jottings during the Cruize of H. M. S. Curaçao among the South Sea Islands, p. 452, pl. 42, fig. 1, 2, 3.

Annam : Plage de Ben-Son (M. Boutan), 1 ex. et 1 valve.

Cette espèce n'avait été signalée jusqu’à présent que de la Nouvelle-Calédonie. Les monographies n'en font pas mention.

\section{Venus (Chione) scabra Hanley}

1844. Venus scabra.

1853. Chione -

1855. Venus -

1856. - - -

1863.
Hanley, P. Z. S. L., p. 161. Desha yes, Catal. Venerida? in the British Museum, p. 130.

Hanl. Sowerby, Thes. Conch. II, p. 718, pl. CLVII f. 101, 102.

Hanley, Rec. biv. Sh. Append., p. 361, pl. 16. f. 24 .

Hanl. Reeve, C. Icon, pl. XXI, f. $97^{\mathrm{a}}, 97^{\mathrm{b}}$. 
Annam: Plage de Ben-Son (M. Boutan), nombreuses valves.

Gomphina semicancellata (Koch) Philippi

1843! Venus semicancellata

185วั! - donacina

1837.

-

Kосн in Philippi, Abbild. I, p. 40(2), pl. I f. 2-3. Soweri (non Gmelin), Thes. Conch. II, p. 739, pl. CLIX, f. 16马̈-166-167 (excl. syn. veneriformis Lamarek.)

Ch. Röner (non Gmelin), Kririt. Unters., p. 12 (note) et p. 134.

1858? Meretrix (Gomphina) donacina H. et A. Adaus (non Gmelin), Gen. of rec. Moll., II, p. 424.

1865. Gomphina donacina Clı. Römer (non Gmeliu), Malak. BI., p. 142.

1866? Venus donacifornis Mabille et Le Mesle, 0 bs. sur la faune malac. de la Cochinchine et du Cambodge in J. de Conch., XIV, p. 118.

1891? Gomphina donacina Ch. P. Fiscuer (non Gmelin), Indo-Chine loc. cit., p. $234(150)$.

Annam: Plage de Ben Son(M. Boutan), I valve jeune.

Pfeiffer dit que le nom de Venus donacina attribué par Chemnitz à cette espèce peut être conservé parce que le Venus donacina Gmelin (plus ancien) s'applique à un Sunetta: mais cette manière de voir ne peut être admise car les règles adoptées pour la nomenclature n'admettent 
aucun double emploi, même lorsque le nom le plus ancien se trouve transposé dans un autre genre. Il faut donc reprendre pour l'espèce en question le nom de G. semicancellata (Koch) Phil.

\section{Cokbicula fluminea Müller}

Var. Bocourti A. Norelet

1906. Corbicula fluminea M. var. Bocourti A. M. Dautzenberg et H. Fischer, Liste des Moll. réc. par M. Man. suy en Indo-Chine, in J. de Couch. LIII, p. 466.

Tonkin : Hanoï (M. Boutan).

Donax incarnatus Chemnitz

1906. Donax incarnatus

Ch. Dautzenberg et H. Fischer, Liste des Moll. réc. par M. Mansuy en Indo-Chine, in J. de C. LIII, p. 468.

Annam : plage de Ben-Son (M. Boutan).

Le D. saigonensis $\mathrm{Cr}$. et $\mathrm{F}$. est voisin de cette espèce mais il est plus allongé transversalement.

\section{Donax semigranosus Dunker}

1871. Donax Dysoni

1877. - semigranosus

1882
Lischке(non Desh.)Japan. Meeres conch. Il, p. 112; III, p. 91.

DUNKer, Malakoz. Bl. XXIV, p. 68.

Dunker, Ind. MolI. Maris Japonici, p. 193. 
Annam : plage de Ben-Son (M. Boutan).

Cette espèce,commune au Japon, qui avait été confondue avec le $D$. Dysoni par Lischke, en a été séparée avec raison par Dunker : elle est plus allongée, moins trigone et les plis transversaux de sa région postérieure sont moins développés.

\section{Solen Lamarckil Chenu}

1843-45̆ Solen Lamarckii

1874.

1888.
Chenu, Illustr. Conch. pl.I, f. 2, 2a, $2^{\mathrm{b}}$. 2c $, 3,4$.

ReEvk, C. Icon. pl. $\mathrm{IV}$, fig. 16 (Ceylan).

Chemnitz Clessin, Mon. in C. Cab. 2 Ed. p. 23 pl. 8, f. 3.

Annam : plage de Ben-Son (M. Boutan).

\section{Siliqua radiata Linné}

1758. Solen radiatus

$\begin{array}{llll}\text { 1764. } & - & - & \\ 1767 . & - & \ldots & \\ \text { 1780. } & - & - & \\ \text { 1782. } & - & - & \text { etc. } \\ \text { 1784. } & - & - & \text { Linn } \\ \text { 1790. } & - & - & - \\ 1810 . & - & - & -\end{array}$

Linné, Syst. Nat. éd. X, p. 673.

Linné, Mus. Lud. Ulr., p. 474 .

Linné, Syst. Nat. éd. XII, p. 1114.

Bons, Test. Mus. Caes. Vindob., p. 26.

Chemnitz, C. Cab. I'I, р. 39, 54; р. วั, 1. 38, 39. Schröter, Einleit., II, p. 628 .

Gmelın, Syst. Nat.éd. XIII, p. 3220.

Wood, General Conclı., p. $128, \mathrm{pl}$. XXXI, f. 1. 2. 


\begin{tabular}{|c|c|c|c|c|}
\hline 1817. & Solen rae & udiatus $\mathrm{Li}$ & inné & $\begin{array}{l}\text { Dilliwyn, Descr. Catal. I, } \\
\text { p. } 63 .\end{array}$ \\
\hline $181 §$. & - & - & - & $\begin{array}{l}\text { Lamaвск, Anim. s. Vert., } \\
\text { p. } 4335 .\end{array}$ \\
\hline 182.ั. & - & - & - & $\begin{array}{l}\text { Wood, IndexTestac.,p. } 15 \text {, } \\
\text { pl. 3, f. } 14 .\end{array}$ \\
\hline 1832. & - & - & -- & $\begin{array}{l}\text { Desha yes, Encycl. Méth., } \\
\text { III, p. } 962 \text { (pl. } 220 \text {, f. } 2 \text { ) }\end{array}$ \\
\hline 1833. & - & - & - & $\begin{array}{l}\text { Deshayes in LaMarck, An. } \\
\text { s. vert., } 2{ }^{\ominus} \text { él., VI. p. } 60 .\end{array}$ \\
\hline 1842. & - & - & - & Hanley, Rec.Biv.Sh.,p.14. \\
\hline $185 ั 50$. & - & - & - & $\begin{array}{l}\text { HANLEY, I psa Linn. Conch., } \\
\text { p. } 30 .\end{array}$ \\
\hline 1862. & Machaera & aradiata & - & $\begin{array}{l}\text { Chenu, Man. de Conch., } \\
\text { 11, p. 23, fig. 102. }\end{array}$ \\
\hline 1874. & Cultellus & radiatu: & & Reeve, C.Icon., pl.IV, f.13 \\
\hline 1888. & Machaera & a radiata & $\ell \mathrm{L}$. & $\begin{array}{l}\text { Clessin, Mon. Solenacea } \\
\text { in C. Cab. 2e éd., p. oั9, } \\
\text { pl. 5, f. 9-10. }\end{array}$ \\
\hline 1889. & Siliqua & - & - & $\begin{array}{l}\text { L. Morlet, Cat. Coq. rec. } \\
\text { par M. Pavie dans le } \\
\text { Cambodge et le Roy. de } \\
\text { Siam, in J. C., XXXVII, } \\
\text { p. 172. }\end{array}$ \\
\hline 1891. & - & - & - & $\begin{array}{l}\text { P. Fischen, Catal. Indo-Chine } \\
\text { loc. cit.. p. } 212 \text { (138). }\end{array}$ \\
\hline
\end{tabular}

Solenocurtus exaratus Philippi

1874. Solenocurtus exaratus Philip pi in Reeve, C. Icon.. pl. I, f. 1.

1888. - - - Ph. Clessin, C. Cab., 2 éd., p. 96, pl. 24, f. 5 . 
Annam : plage de Ben-Son (M. Boutan), 1 ex.

Cette espèce, non citée dans le catalogue de P. Fischer, est une intéressante addition à la faune malacologique de l'Indo-Chine.

\section{Mactra dissimilis Deshayes}

1854. Mactra dissimilis

1854.

1853. Trigonella -

1867.

1884. Mactra
Deshayes, P. Z. S. L. p. 63. ReEve, C. Icon. pl. XIII, f. 59 .

Desh. H. et A. Adams, Geu. of rec. Moll. il, p. 376.

Conirad, Catal. fam. Mac. tride in Amer. Journ. of Couch. III, p. 36.

- Weinkauff, C. Cab. 2e Ed. p. 113 .

Annam : Plage de Ben-Son (M. Boutan), nombreuses valves.

Cette espèce, non citée dans le Catalogue de P. Fischer, a peut être déjà été indiquée de la régiou, par M. de Rochebrune, sous le nom de lurida Phil., à moins que cet auteur n'ait eu en vue que le $M$. aphrodina Desh.

\section{Magtra aphrodina Deshayes}

1854. Mactra Aphrodina 1854

1858. Trigonella -

1867.
Deshayes, P. Z. S. L. p. 62. Desh. Reeve, C. Icon., pl. XIX, f. 103 .

H. el A. Adams, Gell. of rec. Moll. II, p. 375. Conrad, Cat. Fam. Mactrida in Amer. Journ. of Conch. III, p. 33 . 
1884. Mactra aphrodina

- Weinkauff, C. Cab. $2^{e}$ Ed.

p. 65, pl. 23, f. 2,2 .

Annam : Plage de Ben-Son (M. Boutan), valves.

Cette espèce n'a pas été citée par P. Fischer dans son Catalogue de l'Indo-Chine.

\section{Mactra violacea Chemnitz}

1782. Mactra violacea

Chemnitz, C. Cab. VI, p. 208, 220; pl. 22, f. 213, 214.

1786. der Violetblaue Backtrog Sснвöтев, Einleit. in die Conch. III, P. 82.

1788. Mactra violacea

Schröter in Martini et Chemitz, Namen Register C. Cab. p. 60.

1790. - - GMelin, Syst. Nat.Ed.X!II,

1798. - - Chtz. Bolten,Mus. Boltenianum, p. 170.

1817. - - - - Dillivyn, Descr. Catal. I. p. 135 (excl. var.)

1818. - - Gm. Lamarck, Anim. s. Vert, V. p. 475.

1825. -- - $\quad-\quad$ Wood, Index. test. p. 29, pl. 6, f. 9.

183\%̆. - - Chtz. Deshayes in Lamarck, An. s. Vert. ${ }^{2}$ Ed. VI, p. 101. 1842. - - Gm. Hanley, Rec. biv. Sh. p. 30 .

1834. - - Chtz. Reeve, C. Icon, pI. XII, f. 57 .

1858. Trigonella - $\quad-\quad$ H. et A. Adams, Genera of rec. Moll. II, p. 376. 
1867.

1870. Mactra

1884.
Conrad, Cat. Fam. Mactri. da, in Amer. Journ. of Conch. III, p. 40.

- Mörcı, Malakoz, Blätter, XVII, p. 12?.

Weinisauff, C. Cab. 2e Ed. p. $12, \mathrm{pl} .4$, f. 1, 2 .

Annam: Plage de Ben-Son (M. Boutan).

Cette espèce ne figure pas dans le catalogue de P. Fischer.

Tellina lamyi nov. s. p.

1782. Tellina angulata Linuzi

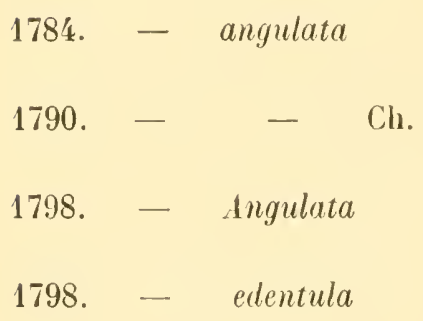

1815. - angulata

1817. Scrobicularia inflata

1818? Lutraria tellinoides

1825. Tellina angulata

1847. - $-\mathrm{Ch}$
Chementz (non Linné), C. Cab. VI, p. 89, pl. 9, f. 74, 75 .

Schröter, (non L.), Einleit. II, p. 63, pl. T, f. 8 . Gmelis (non L.) Syst. Nat. ed. XIII, p. 3229.

Bolten (non L.), Museum Boltenianum, p. 197.

Spengler, Nat. Selsk. Skr. IV. p. 96 (non Brod. et Sow.

Wood (non L.), Gen. Conch. p. 146, pl. 43, f. 1. Schumacher (non L.) Essai nouv. Syst. p. 128. Lamarck, An. s. Vert. V, p. 470.

Wood (non L.), Index Testac. p. 17, pl. 3, f. 3 Hanley in Sowerby (non 
L. )Thes.I, p. 324, pl.LXV, 1. 250 .

1867. Tellina angulata

1871
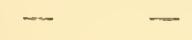

1878 Metis
Reeve (non L.), C. Icon., pl. $26,1,141$.

Ch. Römer (noll. L.), C. Cab. 2 ed., p. 209, pl 40, 1.4, 5,6 .

Bertin (non.L.), Revis.des Tellinidés du Mus. in Nouv. Arch. du Mus. p. 330 (excl, réf. de Limné).

Annam : Plage de Ben-Son (M. Boutan), I valve.

Comme l’a fort bien démontré Hanley (Ipsa Linnaei Conch. p. 33), le Tellina angulata de Linné a été mal conmpris par Chemnitz. En effet les tirmes de la description originale : " testa subovuta striis transversis recurvatis aff. nis $T$. virgatx... etc. "n ne conviennent pas du tout à la coquille figurée par Chemnitz, mais sembleraient s'appliquer au 'T. plicata dont il existe un exemplaire dans la collection linnénue. Malheureusement Linné ajoute " dentibus lateralibus nullis ") ce qui n'est pas le cas chez le T. plicata qui est pourvu de dents latérales, mais si faibles, qu'elles ont pu échapper à l'observation de Linué. Qnoi qu'il en soit, le T. angulata de linné est une espèce ornée de lamelles concentriques et non une espéce à surface lisse pourvue seulement de lignes d'accroissement comme le $T$. angulata de Chemnitz. Il n'est done pas possible d'adopter le $110 m$ de Chemnitz puisqu'il a été employé précédemment par Linné pour une espéce diflérente. Le nom de $T$. inflatu Schumacher, basé sur les figures 7í el 75 de Chemnitz, ne peut ètre employé nou plus à cause de l'existence d'un Tellina inflata Chemnitz C. Cab. VI, p 7J, 9I, pl. 9, f. 76 qui est tout différeut. Quant au Lutraria tellinoides Lk qui est considćré comme synonyme 
par Römer, su description insuffisante et l'absence de références ne permettent guère de le reconnaître. Dans ces circonstances nous nous voyons forcés d'altribuer à l'espéce représentée par Chemuitz pl. 9, f. 74, 75 un nom nouveau et nous proposons relui de Telliua L.am!yi.

\section{Strigllla splendiud Anton}

1839. Tellina splendida 1846.

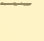

15\%. - -

1878. Strigilla -
Anton, V'erzeichn. p. 5 .

Hanley in Sowerby, Thes. Conch. I, p. 259, pl LVI, f. 39 .

Röмен, C. Cab. 2е Ed. p. 192, pl. 37, t. 13, 16. Bentin, Revis. 'Tellinidés du Muséum in Nouv. Arch. Mus. p. 312 .

Annam : Plage de Ben-Son (M. Boutan).

\section{Strigillina lactea Dunker}

1861. Strigillina lactea

1865. Syndosmya strigilloides
DunKkr, Mal. Bl. p. 43.

VaILlant, J. de C. XIII, p. 125, pl. VI, f. 1.

1866. Strigillina lactea Dkr. Semper, J. de C. XIV, p. 166.

1890. - - - PAETEL, Catal. III, p. 53.
PH. D. et H. F. 
Journal de Conchylioloǵie. 1906.

P1.V
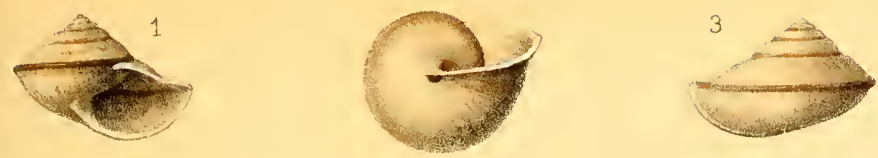

4

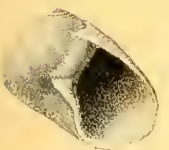

8

5
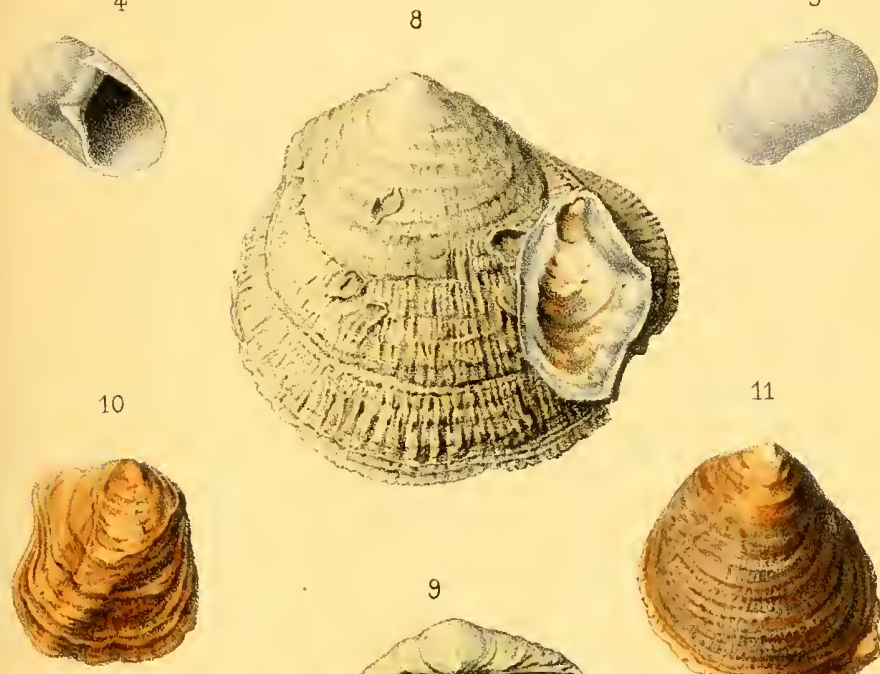

6
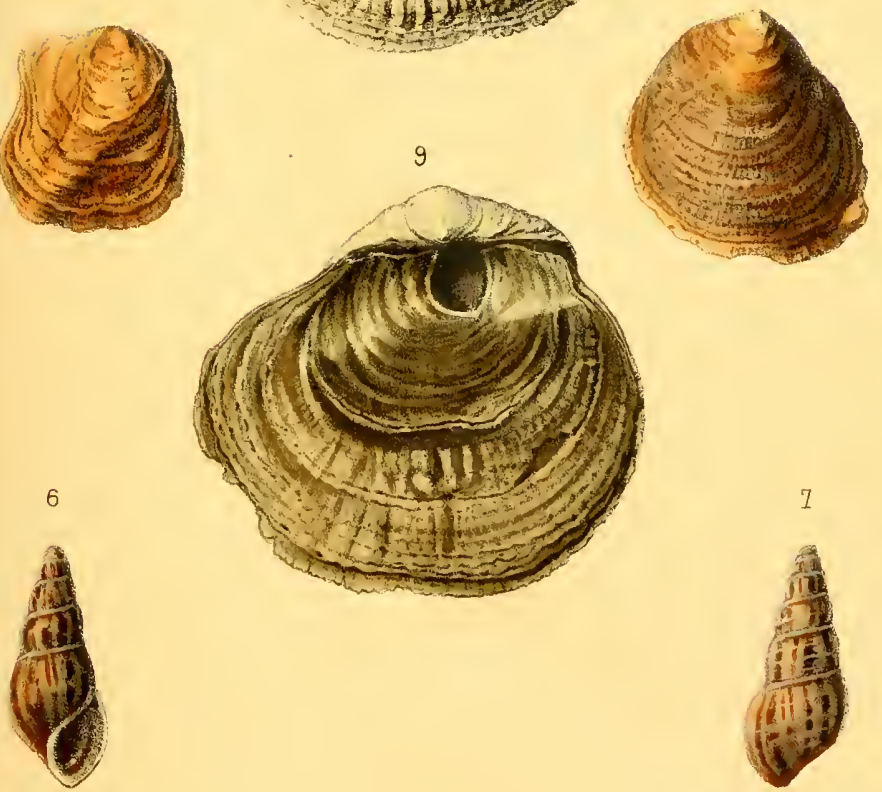

C Reignter, del. \& lith

Imp. L. Lafontane, Parıs

\section{2. 3. Helix Demangei D \& H.F.}

4. 5. Sigaretus (Naticina) perobliquus D.\& H.F.

6. 7. Melania Krempfi D. \& H.F.

8.9. Anomia Lischkei D.\& H. . Japon.

10.11. Annam. 

Journal de Conchyliologie. 1906.

P1. VI
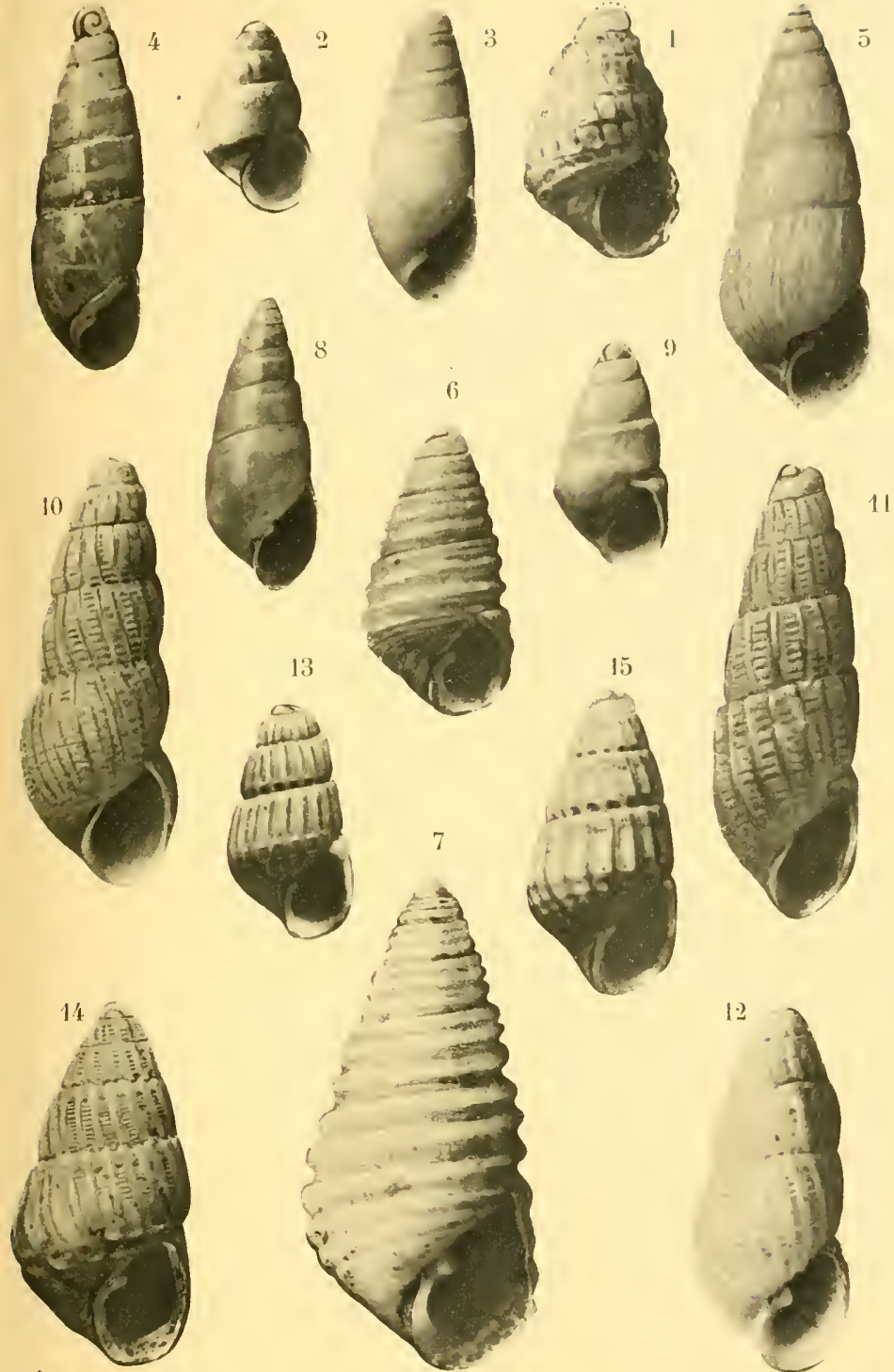

11. Fischer, phot.
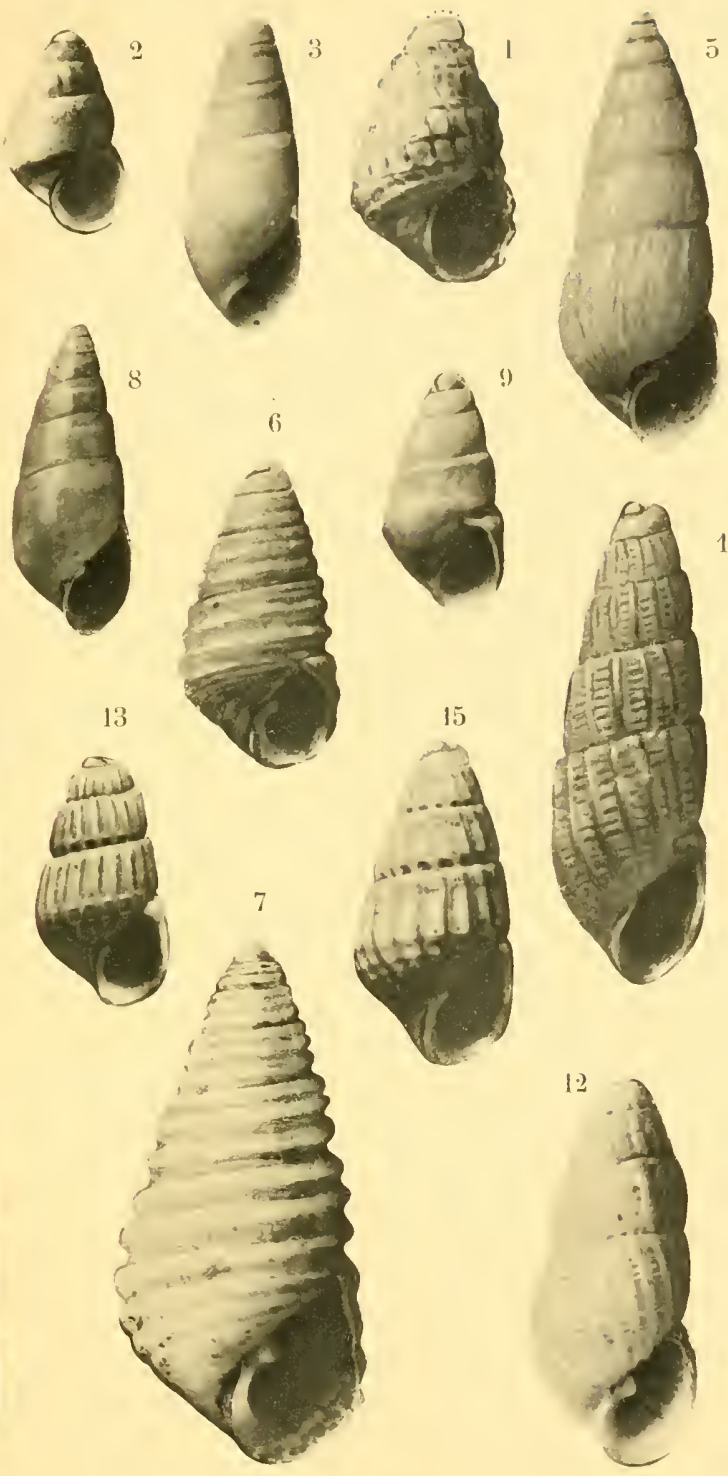

I'hototypie G. Pissary
1. Alvania Boutani D. et H. F. $メ$ I 5 2. Cingula annamitica do $\times 15$ 3. Pyram. (Agatha) Pacei do $\times 10$ 4. Syrnola callembryon d $\quad \times 20$ 5. Odostomia pseudoplicata ${ }^{\circ} \times$ ro $(6,7$. Oscilla jocosa Melvill 8. Odostomia Boutani D. et H.F. $\times$ io
9. Odostomia contracta D. et H.F. $\times 20$ 10. Pyrgulina Melvilli d' $\times 20$ 11. Pyrgulina Sykesi d $\times 20$ 12. Pyrgulina Standeni d" $X$ IS 13. Pyrgulina Prestoni d. $\times 20$ 11. Pyrgulina Claudoni d $\times 20$ 15. Pyrgulina Lamyi do $\times$ io 



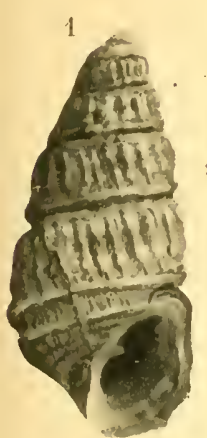

S
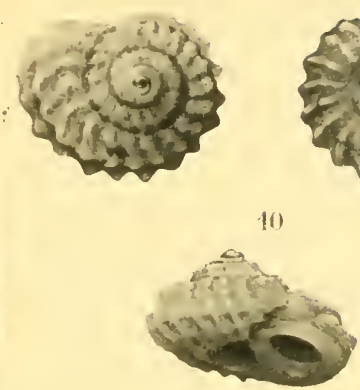

1

$$
3
$$
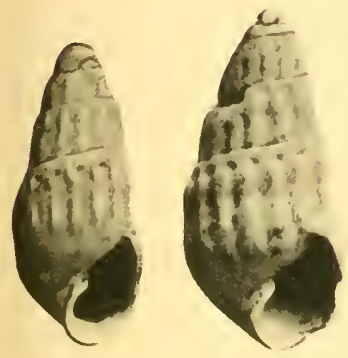

11

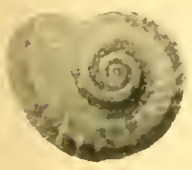

14

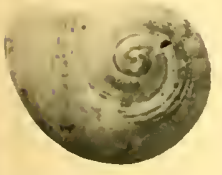

II. Fiselher, phot.

5

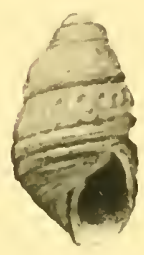

9

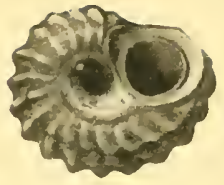

6

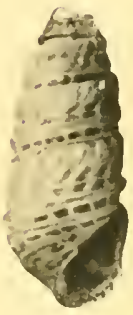

2

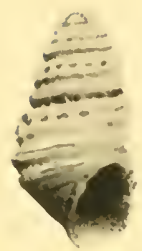

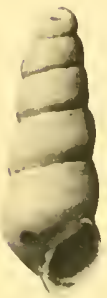

13

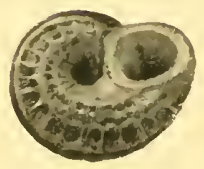

16

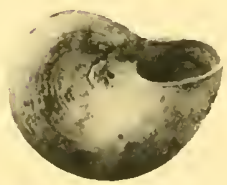

Hototypite fi, lissatre

1. Pyrgulina Bartschi D. et H. F. $\times 20$.

2. Pyrgulina gemmifera D. et H. F. $\times 20$.

3,4 . Pyrgulina Germaini D. et H. F. $\times 20$.

5. I'Jrgulina eximia D. et H. F. $\times 20$.

(i. J'yrgulina pretiosa D. ct H. F. $\times 20$.

7. Eulimella pyrgoides D. et H. F. $\times 15$.

\$, 9, 10. Cyclostrema Godeti D. et H. F. X ro.

11, 12, 13. Cyclostrema Bushi D. et H. F. Х

$14,15,16$. Teinostoma multisulcatum D. et H. F. $\times 15$. 





\section{I S T E \\ DES \\ MOLLUSQUES}

Récoltés par M. H. MANSUY en Indo-Ghine et au Yunnan

E'I'

DESCRIPTION D'ESPËCES NOUTELLES

PAR

P II. DA I T Z EX B ERG ET H. FISC II ER

(Extrait du Journal de Conchylinlogis, Vol. Iall)

s

$$
\text { PA.RIS }
$$

1906 



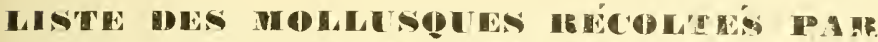

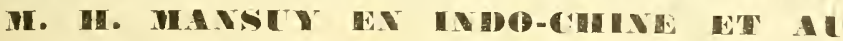

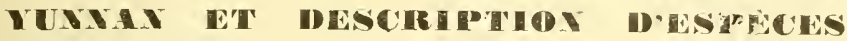 TOUTELLES.}

Par Ph. Dautzenberg et H. Fischer

M. H. Mansuy, géologue attaclıé au Service géologıque, à la Direction des T'ravaux Publics de l'Indo-Chine et qui a fail partie, à ce titre, de la Mission des Etudes géologiques et minières ayant pour but le choix du tracé du chemin de fer de Lao Kay à Yunnan-Sen, a bien voulu nous communiquer à son retour, de nombreux exemplaires des co. quilles qu'il a recueillies, soil pendant ce voyage, soit pendant ses séjours dans diflérentes localités de l'IndoChine; ses recherches malacologiques ont été faites principalement à Saïgon, Pnom-Roang, Hong-Chon, Chaudoc, Hatien en Cochinchine; dans l'ìle de Poulo-Condore ; à Pnom Penh, Somron-Seng, Kampot et dans l'Archipel des Pirates au Cambodge ; à Phan-Ran en Annam ; à Hanoï, Ha-Giang et dans la Baie d’Along au Tonkin ; à Mong-Tsé, Yunnan-Sen et dans la vallée du Sin-Chiem au Yunnan. Parmi ses récoltes figurent des coquilles provenant des gisements préhistoriques de Somron-Seng (Cambodge) et de Mong.'Tsé (Yunnan), enfin quelques espèces intéressantes et des indications concernant certaines stations malacologiques à visiter ont été fournies à M. Mansuy par 
M. Lantenois, Ingénieur en chef au corps des nines, chef du Service gréologique à la Direction des Travaux Publics de I'Indo-Chine, par M. Monod, chef adjoint du Service géologique el par II. Jacquet, Directeur de l'Agriculture a fonkin.

L’importance des matériaux réunis par M. Mansuy, montre avec quel zèle el quelle largeur d'esprit ce distingué naturaliste, absorbé cependant par le service géologique dont il était chargé, a entrepris celle longue exploration qu'il a su rendre si fructueuse; nous lui sommes très reconnaissants de nous avoir confié l'étude de ses récoltes où figurent beaucoup de nouveautés et qui nous ont permis de mieux apprécier nombre d'espéces de ces régions dont la faune malacologique est encore si incomplètement connue.

Nous adressons aussi tous nos remerciments à .I. Douvillé, Professeur de Paléontologie à l'Ecole Nalionale des Mines, qui a mis à notre disposition les collections de cel élablissement, où les types décrits ci-après sont déposés.

Exvea (Elma) Messagerı Bavay et Dalutzenberg

1903. Ennea (Elma) Messageri Bavay et Dautzenberg, Journ. de Conch., vol. LI, p. 20̈̈, pl. VIll, fig. 3-4.

Cette espèce diffère de l'E. sinensis Müllendorfí par son sinus beaucoup plus profond et par ses tours moius convexes.

Tonkin : Ha-Riang (M. Mansuy).

Ensea (Elala) tonliniana bavay et Dantzenberg

1903. Emea (Elma) Ionliniana Bavay et Dautzenbling, Jouru, de Conch., vol. I.I, p. 204, pl. VIII, lig. 1-2 
Cette espèce diflère de l'E. Swinhoei H. Adams, par sa spire moins haute, son sommet moins obtus, son sinus moins profond.

Tonkin : Ha Giang (M. Mansuy).

Ennea (Elma) Maxsuy Dautzenberg et H. Fischer 11, sp. (PI. VIIl, fig. 8-9)

Testa tenuis, subhyalina, nitida, late rimata, valde elongata, subcylindrica, ad apicem obtusa, basin versus vix dilatata. Anfr. S convexiusculi, sutura impressa sejuncti, lineis incrementi flexuosis ornati; ultimus prope aperturam valde ascendens. Apertura edentuli, subquadiata. Columella rectiuscula, crassa; labrum flexuosum incrassatum breviterque reflexum, ad insertionem in sinulum profundum desinens.

Coquille mince, subhyaline, luisante, pourvue d'une perforation ombilicale située à l'exirémité d'une fente allongée. Spire allongée, turriculée, subcylindrique, composée de 8 tours un peu convexes, séparés par une suture assez profonde et ornés de lignes d'accroissement flexueuses. Dernier tour très ascendant à son extrémité. Ouverture non dentée, subquadrangulaire. Columelle presque perpendiculaire, épaisse. Labre flexueux, épais et réfléchi, terminé au sommet par un sinus profond et haut.

Hauteur $10^{\mathrm{mm}}$, largeur $3^{\mathrm{mm}}$. Ouverture : hauteur $3 \mathrm{~mm} 1 / 2$, largeur $2 \mathrm{~mm} 1 / 2$.

Cette espèce appartient au mème groupe que les $E$. tonliniana et Messageri. C'est de ce dernier qu'elle se rapproche le plus ; mais elle est bien plus allongée, plus cylindrique, bien plus largement ombiliquée; son dernier tour est très ascendaut à l'extrémité, tandis que celui de l'E. Messageri l'est à peine, le sinus du labre est beaucoup plus grand, etc.

Tonkin : Ha-Giang (M. Mansuy). . 


\section{Nanixa (Rhysota) distincta Pfeilfer}

180ั0. Helix distincta

$1852 .-$
$18: 33 .-$
$1853 .-$

18ว̈3̆. Vanina --

1839. Helix

1860. Nanina -

1863. Heli.r -

1866.

1867. Tamina (lihyssota) distincta von Mantexs, Preuss. Exp. nach Ostasien II, p. 69 , pl. 6, fig. 8. (arec l'animal).

1868. Helir distincta

1870. Xesta -

1876. Helix -
Premfer, Zeitschrift für Malakoz. 1.69.

Pfr. Reeve Cohch. Icon., pl. LXXXYl, fig. 46. .

Pfeiferi, Mon. Hel. III, p. 81 .

Pfeifeer, Conch. Cab. ze éd. p. 346, pl. 134. fig. 1, ?.

Gray, Catal. Pulm. Brit. Mus. p. 118.

Pfeiffer. Mon. Hel. IV. p. 69.

von Martens, Moll. of Siam in P. Z. S. L. P. 7. Pfr. Crosse et P. Fischeli, Journ. Conch. XI, p. 348.

- J. Mabille et Le Mesle, Journ. Conch. XII, p. I2S. p. 133.

Senper, Reisen im Archi . pel der Philippinen, I. p. 62.

Pfeiffer, Mon. Hel. viv. VII, p. 133̈.

1886. Nanina (Rhysota) distincta Pfr. Trios, Man. of Conch. II, p. 30, pl. 8, 1. 26. 
1889. Hemiplecta distincta

L. Morlet, Catal. Cambodge el Siam (Pavie) in Journ. de Conch.. vol. XXXVil. p. 124.

1859. Nunina (Helix) distinctu PAeteL. Catal. II, p. 24. 1894. Ariophanta (Hemiplecta) distincta Pfr. L. Mondet, Contr. Faune mal. IndoChine in J. de Conch. XXXix, p. 231.

IS91. - _ - distincta Pir. P. Fischer. Catal. Indo-Chine in Soc. Hist. Nat. Autun, p. 107 (p. $23 d u$ tiré à part).

1896. Tanina distincte Smuth, Landsh. from Vanbu in Ann. and IIag. N. H. $6^{\text {th }}$ Ser. XVII, p. 128.

1903. Hemiplecte - B La NFord, Land and Freshw. Moll. from Siam in Proc. Mal. Soc. Lon. don, p. 277

1904. Nanina (Rhysota) distincta Pfr. H. Fischer et DautZFnberg, Catal. Indo-Chine in . Mission Pavie, loc. cit. p. 393 (p. 4 du tiré à part).

La forme typique de cette espèce n’est pas représentée dans les récoltes de $\mathbf{M}$. Mansuy.

\section{Var. Neptunus Pieiffer}

1861. Heii.r Teptumus

1861. -
Pfeiffer, Proc. Zool. Soc. p. 190.

Pfeifrer. Novit. Conch., p. 176, pl. XLVIII, fig. $1,2$. 
1868. Helix Neptunus

Pfeiffer, Mon. Hel, I, p. 160.

1876. - - P PfeIfFer, Mon. Hel. VII, p. 172.

1886. Nanina (Rhysota) Teptumus Pfr. TrYox, Man. of Conch. II, p. 34, pl. 8, fig. 27.

1891. Ariophanta (Hemiplecta) Teptunns Pfr. P. Fischer, Catal. Indo-Chine, in Soc. Hist. Nat. Autun. p. 106 (22 du tiré à part).

1903. Hemiplecta distincta Pfr. var. Neptums Blanford, Land and Freshwater Moll. from Siam in P. Z. S. L. p. 277.

190'. Nanina (Rhysota) Neptumus Pfr. H. Fiscuer el Dautzenbrag, Cat. Indo Chine in Mission Pavie, loc. cit. p. 393 (p. 4 du tiré à part).

Cochinchine: Hatien (M. Mansuy).

II résulte de l'étude que nous avons faite, non seulement sur les exemplaires de M. Mansuy, mais encore sur une nombreuse série d'autres spécimens de diverses provenances, que les Helix Teptumus et I'lnto de Pfeiffer ne sont que des variétés de l'Helix distincta.

La variété Neptumus est plus grande, plus large, a la spire plus obtuse et sa coloration est plus uniforme.

La variété Pluto est d'une coloration plus foncée avec les bandes plus distinctes et a une carène périphérique bien visible.

L'II. pernobilis Férussac. = Limax lampas Martyn parait être un II. distincta var. Neptunus de coloration brun marron uniforme.

L'Il, pernobilis Pfr nous parait bien voisin de l'Il. 
Pluto dont il ne nous semble différer que par l'absence de carène.

Le Nanina funerea Smith et sa variété pallitior sont très déprimés, ont l'ombilic sensiblement plus étroit que chez toutes les variétés de $N$. listincta. leur carène est aussi bien accusée. Il s'agit peut-ètre là d'une espèce spéciale ; mais nous ne pourons l'atîirmer, car la variété Pluto semble la relier à la variété Neptunus et de lì au $N$. distinctu typique.

Hemiplecta Weinkauriana Crosse el Fischer

1863. Helix Weinlianfiana

Crosse el Fischer, Faune mal. Cochinchine in Jourı. Conch., Vol. XI, I. 330 .

IS6't. - -

Crosse el Fischer, Jouın. de Conch., Vol.XII, p.36, j.l. Xll, fig. 7 .

1866.

- Cr.elf. Mabille el Le Mesle, Journ.de Conch., Vol. XIV, p. 127 .

1868. --

- - Pfeiffer, Mon. Hel., V, p. S8.

1876. - - - $\quad$ - Pfinfra, Mon. Hel. VII, p. 100.

1889. Hemiplecta - - L. Morter, Catal. Cambodge et Siam (Pavie) in Journ. Conch., p. 12't.

1889. Nanina (Testa) - - PAetel, Catal. II p. 50.

1891. A. (Hemiplecta) -. - P. Fischer, Catal. IndoChine, in Soc. Hist. nat. Autun, p. 103 (p. 2 ) du tirage à parl). 
1904. Hemiplecta Weinlianffiana Cr.el F.II. Fiscuen el D.eetzenbelig, Catal.Indo-Chine in Mission Pavie, p. 393 (p. 4 du tiré à part).

Cochinchine : Saïgon (II Mansuy). - Cambodge: PnomPenh (M. Mansuy).

Xestina tenera Möllendorlf

190I. Lestina tenera

Möllendorfa, Nachrichıts Malt d. d. Mal. Gies., p. 4ti. 1904. - - Möll. H. Fischer, Cátal. IndoChine in Mission Pavie, p. 394 (כ̆ du liré à part).

Var. elata Dautzenberg et H. Fisclier, n. var. (PI. VIII, fig. 1, 2, 3.)

Le type de notre variété nouvelle clata, que nous représentons ici, a les dimensions suivantes : diam. maj. $27 \mathrm{~mm}$ diam. min. $25 \mathrm{~mm}$, alt. $20 \mathrm{~mm}$. Le type de l'espèce, qui n’a pas été figuré, est plus déprimé puisque l'auleur donue les dimensions suivantes : diam. $27 \mathrm{~mm} 7$, alt. $15 \mathrm{~mm} 6$.

Annam : Plian-Ran (M. Mausuy).

Nanina (Macrochlamis) Despecta J. Mabille

1887. Nanina despecta

1887.

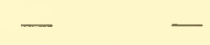

1891. Ariophanta-
J. MabHlde, Mo!l, tonkinorum diagı. p. 2.

J. Mabille, Sur quelques Mollusques du Tonkin, p. 79, pl. I, fig. 13, 14.

Mab. P. lischer, Catal. IndoChine in Soc. H. N. Autuu, p. 107 (p. 23 du t. à p.). 
1900. Macrochlamys Alluandi

1904.

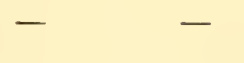

Bavay el Dautzenbelig, Descr. coq. nouv. IndoChine in Journ. Conch., Vol.XLVIII, p. 444, pl. XI, tig. 22. 23, 24.

H. Fischei et Dautzenberg, Catal. Indo-Chiue in Mission Pavie, loc. cit., p. 394 (p. 5̋ du t. à p.)

Tonkin : Ha-Giang (M. Mansuy.)

Macrochlamys Douville Dautzenberget II. Fischel n. sp. (Pl. VIII, fig. 4, 5, 6.)

Testa tennissima et fragilis, anguste umbilicata, spira depresse conoidea. Anfr. 5 convexiusculi, rapide crescentes, plicis incrementi ureuatis infra suturam magis conspicuis ornati. Anfi. ultimus vatde inflatns, haud descendens. Basis convexa, circa umbilicum excavata. Apertura ampla, marginibus callo tenuissimo adnatoque junctis. Columella temuis, arcuatu, haud incrassata, inferne brevissime reflexa, umbilicum ex parte obtegit. Labrum tenue, arcuatum, acutum.

Altit. 16, diam. maj. 27, min. 23 mill. A pertura $12 \mathrm{~mm}$ alta, $15 \mathrm{~mm}$ lata.

Coquille très mince et fragile, pourvue d'un ombilic étroit et profond. Spire conoïle, peu élevée, composée de 5 tours un peu convexes, croissant rapidement et séparés par une suture bien visible. Surface ornée de plis d'accroissement arqués, plus développés au-dessous de la suture. Sur la partie supérieure de la seconde moitié dlu dernier tour on apercoit quelques stries décurrentes obsolètes. Dernier tour très renflé, ne descendant pas à l'extrémité, à base convexe, excavée autour de l'ombilic. Ouverture grande; bords reliés par une callosité très mince, 
appliquée. Colunelle mince, arquée, un peu réfléchie dans le haut, au dessus de la perforation ombilicale. Labre arqué, mince et tranchant.

Tonkin: Ha-Giang (M. Mansuy).

Cette espèce, que nous dédions à M. Dourillé, professeur de Paléontologie à l'Ecole Nationale des llines, ressemble un peu à un Helicurion par son test mince et son dernier tour très renflé. C'est du Macrnchlamys derlivis qu'elle se rapproche le plus, mais elle en diffère par sa taille plus forte, son dernier tour plus renllé, son ouverture plus ample.

\section{Trochomomina Paviei L. Morlet}

18s4. Helir P'ariei

L. Monı:т, Journ. Conch. XXXII. 1. 386, 川. XI, lig. I, Ia.

1887. Trochomorpha Tonkinormm Mabiles. Moll. tonk. diagn. p. 7 .

1587.

Ilabille. sur quelques Moll. du Toukin, in Bull. soc. Mal. Fr. 17, p. 97. pl. III. lig. 1-3.

1589. I'lectotropis Pariei

L. Monlet, Calal. Cambodge et siam (Parie). in Journ. Conch. XXXYH. р. 1 อัว.

1591. Trochomorphe Tontinorum Mab. P. Fischen Catal. Indo-Chine, in Soc. Ilist. nat., Autun. p. 108 (p. 2'i dı 1. à p.).

1891. H. (Plectotropis) P'utiei L. M. P. Fiscusis, Catal. Indo-Chine, in Soc. II N. Autun, p. 110 (p. 26 du 1. (1).). 
1891. Trochomorpha Tonkinorum Mab. L. Monlet, Journ. Conch. XXXIX, p. 294. 1904. - Paviei L.M. H. Fischer el DaltzexiBERG, in Mission Pavie, loc.cit.p. 398(9 du t.à p.).

Cochinchine : Hong.Chon (Mansuy).

\section{Candexa cicatricosa Müller}

177'́ Helix cicatricosa

1786. - senegalensis etc.

1786. - cicatricosa etc.

$17 \Sigma 6$ Müll. Kämerer Calal. Rudols.

1783. - cicatricosa sinistrorsa Chemsitz, Conch. Cab.

1790. - cicatricosa Müll. Gments, Syst. Nat. édit.

1790. - romu venatorium rar. Gasels (non Chemnitz)

1793̆. - cicatricosa Müll. Chemsitz, Conch. Cab. XI.

1817.

- Dillwry, Descr. Catal. II,

1820-5ั1.-

1822. - senegalensis p. 173, pl. Xl. fig. 6 . Register, p. 44. XIII, p. 3614. Syst. Nat.eu..XIII, p.3614.

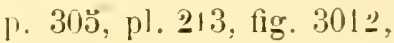
3013 . p. 888 .

MÜLlek, Hist. Vermium II. p. 42 .

Chemsitz, Conch. Cab.IX p. 83̈. pl. 109, fig. 917 . 918.

p. 23,90 , pl. 109, fig. 2923 ; rign. 19 Jit. A (p. 1). Férl'ssac, Hist. Yat. Moll. I, p. 168, pl. 78, fig. 1. 2. Ch. LAMARCk, dnim. sans vert. II, 2 e parlie, p. 74. 
18ㄹ․ Helircicatricosa Müll. Woon, Indextestac. p.1.ั4. p]. 32, fig. כ̈.

1820̈. - senegalensis Ch. Férussac, Tabl.Syst.,p.43.

1831. - - - Deshares, Encyel. Néthod. II, p. 끙.

1834. - Chimensis - Vorgt in Cuvin Das Thierreich 111, I. 61 .

1837. Ariophanta cicatricosa Müll. Beck, Index, p. З.

183s. Helix cicutrosa

1838. - senegalensis

1843̈. - cicatricosa

1846. - - -

1948. - -
1842-58 -

Mïll. DESHAYES in LAMA BCK Anim. s. vert., ¿e éd. V'III, p. 89 .

Ch. DESHAYEs in LaMARCK Anim. s. vert. Q édit. VIII, p. 4:.

- Chesu, lllustr. Conch., pl. S, fig. ö, כّa.

Müll. Catlow el Reeve, Conch. Nomelicl, p. 12 I.

- Jaetfaer, Monogr, in Couch. Cab., „2 édit., p.93. pl. 12, fig. 1, 2, 7 à 9 .

- Preiffer Mon. Hel. vir. 1., p. 330 .

1850. Camana cicatricosa Müll. Albers, Di e Heliceen, p. 8 .

1852. Helix

- - Reeve, Conch. Icon. pl. LXXII, fig. 37I.

183̈3. - - - Pfeiffer, Mon, Hel. viv. III, P. 223.

1858. - (Camæna $)$ - H. et A. Andms, Genera of rec. Moll. II, p. 189.

1859. Camrena

- Preiffer, Mon. Hel. vir. IV, p. 261 .

1861. Camiena édit. p. 165. 
1868. Helix cicatricosa Müll. Pfeiffer, Mon. Hel, viv. V, p. 338 .

1869. Camina - - Frauenfeld, Verh. zool. bot. Ges. Wien, XIX, p. 873 .

1876. Helix - - Pfeiffer, Mon. Hel. viv. YH, p. 393.

1878. - (C'amana) - - Peeifere el Clessix, Nomencl. Hel. viv., p. 188.

1886. - - - L. MorLet, Journ, de Conch. XXXIT, p. 258.

1890. - (Camæna) - - P'ilsbri, Man. of Conch., Helicida IV, p. 198, pl. 2) , fig. 4 3̈. 46,47 .

1891. - - - - P. Fischer, Catal. IndoChine, Joc. cit., p. 112 ( 28 du 1. à p.).

1904. (Camæra) - - H. Fischer el Dautzenberg Catal. Indo-Chine, in Mis. sion Pavie, loc.cit., p. 399 ( $10 \mathrm{du} \mathrm{t.} \mathrm{à} \mathrm{p.).}$

La forme typique de cette espèce n’a pas été recueillie par II. Mansuy.

\section{Var. inflata Möllendorff}

188:. Helix cicatricosa Müller, var. inflata MölcendonfF Jahrb. d. Malak. Ges., p. 393, pl. Xı, fig. 20.

1\$90. - (Camena) cicatricosa Müll., var. inflata Mlldf. H.-A. Pilsbry, Man. of Conch. Str. and Syst. Vl, p. 198. 
1891. Melix (Hadra) subgibbera L. Monlet (non Mlldfi), Journ. de Conch. XXXIX, p. 244.

1898. - (Camena) cicutricosa Müll. var. inflata Mldıf. H. Fischer, Liste des Moll. rec. par le $\mathrm{D}^{\mathrm{r}} \mathrm{A}$. Billet in Bull. Sc. France et Belgique XXVIII, p. 3l4 et var. obtecta, p. 315, pl. XVII, fig. $3-6$.

1904. - - - - var. inflatu MIdif. H. Fischer et Dautzenberg, Catal. Indo-Chine in Mission Pavie, loc. cil., p. 399 (II) du t. à p.).

Tonkin: Ha-Giang (M. Mansuy).

Ainsi que l'un de nous le prévoyait en 1898 , nous pouvons établir, grâce aux spécimens recueillis par M. Mansuy, que la variété obtecta à ombilic complètement fermé par une callosité dilatée, se relie intimement à la var. $i$. flata, à ombilic encore un peu apparent.

Nous avons constaté, d'après l'examen de la collection L. Morlet, que la citation de l'Helix (Hadra) subyibbera mentionnée par cet auteur en 1891, est erronée et que le spécimen ainsi étiquetẻ se rapporte au Camrena cicutricosu var. inflata Mldff.

Var. connectens Dautz. et H. Fischer n. var.

Cette variété nouvelle (diam. maj. $50 \mathrm{~mm}$, minor $43^{\mathrm{mm}}$, alt. $34^{\mathrm{mm}}$ ) a la même forme que le C. cicatricosu typique : spire obtuse en forme de dome, base dı dernier tour renflé autour d'un ombilic assez étroit. Par contre, elle se rapproche du $C$. Hahni Mabille (sur quelques Mollusques 
du Tonkin, in Bull. Soc. Mal. Fr. 1887, p. 82, pl. 4, fig. 9, 10) par sa sculpture composée de granulatious fines el serrées.

Chez la variété inflatu Möllendorff du C. cicatricosa, on rencontre parfois de place en place des granulatious; mais elles sont toujours plus espacées et plus allongées que celles de notre var. comectens.

Ha-Giang (M. Mansuy).

Nous possédons un autre spécimen de cetle variété ré. colté au Tonkin, par l'ablué Vathelet (Collect. Dautzenberg).

Camaxa Mansuy Dautzenberg el H. Fischer n. $\mathrm{sp}$. (PI. IX, fig. 1, 2, 3)

Testu solida, globosa, turbinata angusteque rimatu, parum nitens. Spiru conexa, olsusa. Anfr. 5 : primi subplani, regulariter et sat rupide crescentes; penultimus concexior, ultimus haud descemlens utrinque valde convexus et medio obtuse sed tamen conspicue angulatus. Sutura anfir. superiorum superficialis, in ultimo vero magis impressa. Testa areuatim striata et undique oblique et transverse irregnlariter malleatorugosa. A pertura obliqua semilnnaris, peristoma marginibus remotis callo adnuto junctis. Columella obliqua umbilicum fere omnino obtegit. Labum expansum paululumque reflexum.

Color fulvus linea peripheriali castaneu carinam connitante et lineis pluribus plus mimusve obscuris in basi anfr. ultimi ornatus. P'eristomu et aperturæ faux ex albido carulescentes et nitentes.

Diam.maj. $50^{\mathrm{mm}}$; minor $41^{\mathrm{mm}}$; alt. $35^{\mathrm{mm}}$; upertura (cum peristomate) $27 \mathrm{~mm}$ alta, $34 \mathrm{~mm}$ luta.

Coquille solide subglobuleuse, peu luisante, pourvue d'une fente ombilicale étroite. Spire convere en forme de dòme, obluse au sommet, composée de 5 tours croissant régulièrement et assez rapidement, les premiers presque 
plaus, l'avant-dernier plus convexe, le dernier non descendant, bien convexe au-dessus ainsi qu'au-dessous de la périphérie qui est accompagnée d'une carène obtuse mais bien sensible au toucher. La suture des tours supérieurs est superficielle et celle du dernier tour plus accusée. Surface ornée de nombreuses stries d'accroissement arquées et de malléations obliques irrégulières, qui donnent au test un aspect rugueux. Ouverture oblipue, semilunaire. Péristome à bords éeartés, reliés par une callosité luisante, appliquée. Columelle oblique, peu épaisse mais remplissant presque complètement la cavité ombilicale. Labre dilaté et étroitement réfléchi au bord.

Coloration d'un brun fauve, ornée à la périphérie d'une ligne brun-marron qui accompagne la carène el, sur la base du dernier tour, de quelques linéoles concentriques de même couleur, plus ou moins marquées. Péristome et fond de l'ouverture luisants, d'un blanc bleuâtre.

Tonlin : Ila-Giang (M. Mansuy).

Cette espece se rapproche du C. illustris Pfr., mais en diffère par sa forme plus globuleuse, son péristome moins épais, la base du dernier lour un peu concave et ne présentant dans le voisinage de la région ombilicale aucune trace du renflement si caractéristique chez le $C$. illustris typique aussi bien que chez sa variété vanbuensis Smith. Enfin nous n'avons observé chez aucuu des spécimens du C. illustris que nous avons examinés, les linéoles concen. triques qui règnent sur la base de notre nouvelle espèce.

Var. depressa Dautzenberg et H. Fischer n. var. (PI. VIII, fig. 7)

Moins globuleuse, plus déprimée que le type et avec la carène périphériale un peu plus accusée.

Tonkin : Vi Loai (M. Dorr) ; An-Cham à Vi-Loai (M. Dorr). 
Helix (Chlonitis) Norodomana L. Morlet

1883. Helix Norodomiana L.MonLenJourn.de Conch. vol. XXXI, p. 106 pl. 4, fig. $3,3 \mathrm{a}, 3 \mathrm{~b}$.

\begin{tabular}{|c|c|c|c|}
\hline 1889. & Fruticicola & - & $\begin{array}{l}\text { L.Moncet Journ.de Conch. } \\
\text { vol. XXXVII, p. } 123 .\end{array}$ \\
\hline 1891. & H. (Fruticicola) & - & $\begin{array}{l}\text { L..MorLET Journ. de Conch. } \\
\text { vol. XXXIX, p. } 232 \text {. }\end{array}$ \\
\hline 1891. & - & - L. M. & $\begin{array}{l}\text { P. Fischer, Catal. Indo } \\
\text { Chine loc. cit. p. } 111 \\
\text { (27 du t. à p.) }\end{array}$ \\
\hline 1904. & Chloritis & - & $\begin{array}{l}\text { H. Fischer et Dautzenberg } \\
\text { Catal. Iudo-Chine in Mis- } \\
\text { sion Pavie loc. cit. p. } 401 \\
\text { (I2 du t. à p.) }\end{array}$ \\
\hline
\end{tabular}

Cochinchine : Houg-Chon (M. Mansuy).

Celte espèce est bien voisine de l'H. Tanquereyi Crosse et Fischer (Journ. de Conch. 1863, pl. XIV, fig. 2) qui n'en diffère que par sa taille plus faible, sa forme plus aplatie, so: ombilic plus ouvert. L'H. condoriana Crosse et Fischer (Journ, de Conch. 1863, pl. XIV, fig. 1) est une forme plus grande, plus globuleuse, à carène très atténuée autour de l'ombilic. L'H. Fouresi L. Morlet (Journ. de Conch. 1889, pl. VI, fig. 3), est une forme biconvexe et portant au-dessus de la carène une dépression très accusée.

La sculpture microscopique est identiquement la mème dans ces quatre formes qui présentent d'étroites affinités. Il nous semble probable que des études ultérieures permettront de les réunir en une seule et même espèce à laquelle reviendra le nom le plus ancien, $H$. condoriana, les trois autres noms tombant dès lors au rang de simples variétés. 


\section{Plectopylis Fischeri Gude}

1901. Plectop!lis Fischeri

1904.

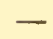

Gude, Sur une collection de Plectopylis du Tonkin in Journ. de Conch., rol. XLIX, p. 204, pl. rl, fig. 4a, 4b. 4c.

H. Fischer et Dautzenberg Catal. Indo-Chine in Mission Pavie loc. cit.. p. 401 (12 du t. à p.).

Tonkin : Ha-Giang (M. Mansuy).

Chalepotaxis infantilis Gredler

1905. Chalepotaxis infantilis Gredler Dattzenberg et H.

Fisciler, Journ. de Conch., vol. LIII p. 94.

Tonkin : Ha-Giang (M. Mansuy).

Satsuma Lantenoisi Dautzenberg et H. Fischer n. sp. (Pl. IX fig. 10, 11)

Testu trochiformis solidiuscula. Spira conica elatu, apice obtusulo. Anfr. 9 1/2 plani, sutura anguste marginata juncti et striis incrementi valde obliquis sculpti. Anfr. ultimus paululum descendens, acutissime carinatus, basi convexus sed infra carinam impressus striisque concentricis numerosis undulatisque ornatus. Umbilicus angustus et fere omnino obtectus. A pertura valde obliqua, subqualrata, marginibus expansis vix reflexis, calloque adnato, temuissimo junctis. Columella arcuata superne cxpansa umbilicumque obtegens. Labrum, ubi carina desinit, angulatum.

Color pallide fulous, apicem versus paullo suturatior; linea 
angusta fusca suturam carinamque anfr. ultimi comitatur. Peristoma albidum.

Altit. $18^{\mathrm{nm}}$ : diam. maj. $14^{\mathrm{mm}} ; \min .13^{\mathrm{mm}} \cdot$ Apert. $5^{\mathrm{mm}}$ alta, $9 \mathrm{~mm}$ lata.

Coquille trochiforme médiocrement solide, à spire conique élevée, un peu obtuse au sommet, composée de 9 tours 1/2 plans séparés par une suture étroitement marginée et ornés de stries d'accroissement très obliques, irrégulières. Dernier tour descendant lẻgèrement à son extrémité, pourvu à la périphérie d’une carène aiguë et saillante. Base convexe mais présentant immédiatement au-dessous de la carène une dépression sur laquelle on distingue quelques stries concentriques. Ombilic très étroit presque entièrement recouvert par l'expansion du bord columellaire.

Ouverture très oblique, subquadrangulaire; bords dilatés, à peine réfléchis et reliés par une callosité très mince, appliquée. Columelle arquée, dilatée au sommet en une lame qui recouvre l'ombilic. Labre dilaté, anguleux à l'endroit où aboutit la carène périphériale.

Coloration d'un fauve clair un peu plus foncé au sommet; suture accompagnée d'un filet brun clair très étroit qui règne également sur la carène du dernier tour qu'il déborde un peu de chaque côté. Péristome blanchâtre.

Tonkin : Ha-Giang (M. Mansuy), exemplaire figuré pl. IX fig. 10. - Siam : (M. Frühstorfer), exemplaire figuré pl. IX fig. 1t.

Cette espèce est très voisine du $S$. peraliensis Crosse, de Pérak. Elle en diffère par sa forme plus étroite, sa spire plus élevée et sa coloration un peu plus foncée. Le $S$. perakiensis, dont nous avons examiné le type et plusieurs exemplaires bien frais présente sur la base du dernier tour des poils courts, caducs, mais dont la trace se distingue toujours, tandis que chez le S. Lantenoisi nous 
n'en voyons aucun vestige; les stries concentriques de la base sont en outre bien moins visibles chez le $S$. perakensis.

Nous avons reçu de M. Frülıstorfer, sous le nom de Satsuma filosa Möllendorff, un exemplaire que nous figurons p]. IX fig. I1, provenant du Siam et qui appartient bien à l'espèce que nous venons de décrire; il est toutefois moins élevé que le spécimen de Ha-Giang. Le nom de Satsuma filosa est sans doute resté manuscrit, car nous n’avons trouvé aucune publication où Möllendorff ait décrit cette forme; il existe d'ailleurs un Helix filosa Deshayes qui a la priorité. Nous sommes donc obligés de donner un nom nouveauà cette espèce, que nous dédions à M. Lantenois, Ingénieur en chef au corps des Mines, chef du Service géologique à la Direction des travaux publics de l'Indo-Chine.

\section{Helix (Eulota) Jourdy L. Morlet}

1905. Helix (Eulota) Jourdyi Dautzennerg et H. Fischen Journ. de Conch. vol. LIIl p. 98 .

La forme typique n'a pas été rencontrée par M. Mansuy.

Var. minor Lautzenberg et H. Fischer n. var.

Tonkin. Hanoï (M. Mansuy).

Amphidronus polymorphus Tapparone Canefri

1891. Amphidromus perversus

P. Fischer (non Lin.), Catalog. Indo-Chine, loc. cit. p. 116 (32 du 1. à p.). 
1894. Cochlostyla polymorpha Tapparone Canefri, Malac. viag. della Freg. Magenta in Mem. Accad. Rle delle Sc. di Torino p. 186, pl.II, fig. 4a, 4b.

1896. Amphidromus polymorphus Tapp. C.Fult on, Ann. and Mag. N. 11. 2d Ser. XVIl, p. 71 .

1898. - perversus C. F. Ancey, (non Lin.).
Bull. Mus. Marseille,
p. 133.

1904. - polymorphus Tapp. C. H. Fischer et DautzenBerg Catal. Indo-Chine, in Mission Pavie loc. cit., p. 406 ( 17 du t. à p.).

1863. Bulimus perversus

Crosse et Fischer, inon Lin.) Jour. de Conch. Xl, p. 359 .

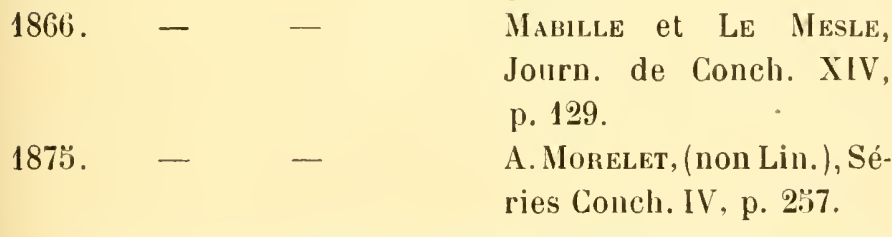

Cochinchine : Saïgon, plusieur's exemplaires dextres et senestres (M. Mansuy).

Cambodge: Gisement préhistorique de Somron-Seıg, 1 exemplaire très allongé, haut. $55 \mathrm{~mm}$, diam. $27 \mathrm{~mm}$ ( M. Mansuy).

\section{Amphidromus Semitessellatus L. Morlet}

1884. Bulimus (Amphidromus) semitessellatus L. Mor LET, J. de Conch., vol. XXXII, p. 387, pl. XI, fig. 2, 2a. 
1889. A mphidromus semitessellatus L. Mortet, Journ. de Conch., vol. XXXVll, p. 128.

1891

- L.M. P. Fiscuen, Catal. IndoChine, loc. cit., p. 117 (33 du t. à p.).

1896.

- - Fulton, Ann. and Mag. of Nat. Hist. 2d. Ser. XVll, p. 87 .

1904.

H. Fiscula et Diutzenberg, Catal. Indo Chine in Mission Pavie, loc. cit. p. 407 (18 du t. ̀̀ p.).

Cambodye : liampot, chaine de l'Eléphant, à $200 \mathrm{~m}$ d'altitude (M. Mansuy).

\section{A.MPIIDRonus RHoDostrlus Möllendorfi}

1901. Amphidromus thodustylus NöLLexdorfF. Nachrichtshl. der deutsch. Mal. Ges., p. 47.

1904. - - Möll. H. Fischen et DautzexBeis, Calal. Indo-Chine in Mission Pavie, loc. cit. p. 407 ( 18 du t. à p.).

Cambodge : Kampot, chaîne de l'Eléphant, à :00m d'altitu de (M. Mansuy).

Anuam : Plıan-Ran, un exemplaire plus verdàtre que le type (M. Mansuy).

Ampitidromus xiengensis L. Morlet

1891. Amphidromus Viengensis L. Monuer, Journ. de Conch. Vol. XXXIX, p. 27, 23른. 20, pl. $Y$, fig. 4, ́ril. 
1891. Amphidromus liengensis L. M. P. Fıscher, Catal. Indo-Chine, loc.cit.p. 117 (33 du t. à p.).

1896. - porcellumus Mousson, var. xiengensis L. M. Fulton, Anu. and Mag. N. H. 6th Ser. XVII, p. 79 .

1898. - Tiengensis L. M. C. F. Ancer, Bull. Mus. Marseille, p. 134.

1903. - xiengensis - Blanford, Land \& Freshw. Moll. from Siam, in Proc. Mal. S. L. p. 279 et var. Tryoni,ibid.

1904. - porcellanus Mousson, var. xiengensis L. M. H. Fischer el Dautzenberg, Catal. Indo Chine in Mission Pavie, loc. cit. p. 406 (17 du t. à p.).

Anuam : Phan-Ran (M. Mansuy).

Anphidronus Bülow Frühstorfer

(PI. VIII fig. 10, 11, 12)

1903̈. Amphidromus (Goniodromus) Bïlowi FrüHSTORFER Nachrichtsblatt der deutschen Malakoz. Ges. p. 83, pl. I, fig. 2, 2.

Annam : Lang-Biang. Exemplaires recueillis par M. Jacquet, Directeur de l'Agriculture au Tonkin.

Les spécimens qui ont servi à la description de cette espèce ont été récoltés par M. Frühstorfer, dans l'ouest de Sumatra. Cet auteur dit qu'il a également rencontré à Lang-Biang, dans le sud de l'Annam, une espèce trèss voisine de son A. Büloui, mais seulement à l'état d'exemplaires morts. 
11 est bien évident que les individus frais trouvés par M. Jacquet, dans la même localité de l'Annam, appartiennent à la même espèce que les spécimens morts signalés par M. Frühstorfer. D'autre part, nous ne croyons pas que cette forme de l'Annam puisse être séparée de celle de Sumatra.

Bulminus Rnombostomus Pfeiffer

1861. Buliminus rhombostomus Pfeifrer, Proc. Zool.Soc. p. 194.

1868. - - - Pfeiffer, Mon. Hel. viv. VI, p. 33.

1877

Pfeiffer, Mon. Hel. viv. VII, p. 47.

1889. Amphidromns

- Pfr. L. Mohlet, Journ. de Conch. vol. XXXVIl, p. 127.

1890.

- - L. Morlet, Journ. de Conch. vol. XXXVili, p. 122, pl. IlI, fig. 6, 6a, 6b. 1891.

1904.

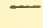
(32 du t. à p.) - - P. Fischer, Catal. Indo- Chine, loc.cit. p. 116 H.Fischer et DAUTzenBerG, Catal. Indo-Chine in Mis. sion Pavie, loc.-cit.p. 407 (18 du t. à p.)

Cochinchine: Hong-Chon (M. Mansuy).

La forme représentée par L. Morlet est bien conforme, comme dimensions, à celle indiquée par Pfeifter. Cette espèce est presque aussi variable que le $B$. siumensis : le type tel que l'a figuré L. Morlet est plus élevé et de forme trochoïde avec des tours convexes; il existe une variété bien plus élevée à laquelle nous donnous le nom de: 
Var. pupoistea Dautzenberg et H. Fischer, n. var.

Mais les récoltes de M. Mansuy présentent des intermédiaires. La variété pupoidea se distingue toujours des formes courtes du $B$. siamensis par sa columelle plus oblique.

Buliminus pharangensis Frühstorier mss.

(Pl. IX, fig. 8, 9).

Testa sinistrorsa, anguste umbilicatu, parum solida, pupoidea, sat elongata, Anfr. \& convexiusculi, sutura distincta juncti; primi 4 leve's, ceteri rudissime ac valde irregulariter malleuti; ultimus carina peripheriali obsoleta munitus. A per. tura subqualratu, obliqua, marginibus callo tenuissimo parum. que conspicuo junctis. Columella recta superne expansa umbilicumque obtegens. Labrum arcuatulum, cum margine busali expansiusculo angulum obtusum efformans.

Color undique albus.

Altit. 92, diam. maj. 10 millim. A pertura $71 / 2$ millim. alta, 7 mill. lata.

Coquille senestre, étroitement ombiliquée, peu épaisse, de forme pupoïde assez allongée. Spire conoïde, composée de 8 tours légèrement convexes séparés par une suture bien visible mais peu enfoncée. 4 premiers tours lisses, les autres très grossièrement et irrégulièrement malléolés. Dernier tour pourvu à la périphérie d'une carène mousse qui s’accuse un peu sur la dernière moitié de ce tour. Ouverture subquadrangulaire, oblique. Bord columellaire perpendiculaire, élargi vers le haut où il masque presque entièrement la cavité ombilicale. Labre un peu arqué, plus dilaté vers le bas et formant un angle obtus à son point de rencontre avec le bord basal qui est également un peu dilaté et évasé.

Coloration entièrement blanche.

Cochinchine : Hong-Chon (M. Mansuy). 
Cette espéce se rapproche du $B$. siamensis Redf., par sa taille et sa forme, mais elle s'en éloigne par sa surface malléolée et sa coloration blanche. Elle a été récoltée à Pha-Rang (Annam) par M. Frühstorfer qui nous en a envoyé un exemplaire sous le nom que nous lui conser. vons ici, bien qu'il n’ait pas été publié.

\section{Clausilia vanbuensis Bavay et Dautzenberg}

(PI. IX fig. 12, 13).

1899. Clansilia vanbuensis

Bayay et Dautzenberg, Journ, de Conch. vol. XI.VII, p. 38 pl. II fig. 1 , $1 \mathrm{a}, 1 \mathrm{~b}$.

Tonkin : Ha-Giang (II. Mansuy).

Le type du Clausilia venbuensis nous paraissant un peu anormal, nous faisons représenter un spécimen de la même espèce, rapporté par M. Mansuy, chez lequel le pli columellaire est plus lamelleux el plus oblique, ce qui nous semble être l'état habituel.

Cette espèce est voisine du Cl. l'aviei dont elle a la taille el la coloration; elle en diffère par sa spire plıs atténuée, plus cylindrique et plus eftilée au sommet, ainsi que par la présence d'un pli subcolumellaire bien visible lorsqu'on observe l'ouverture par le bas.

Clausilia Lantenoisi Dautzenberg et H. Fischer n. sp. (PI. IX fig. 6, 7).

Testu sinistrorsu, solida, imperforata, fusiformis, sat obesu, parum nitens. Spira integra, apicem versus attenuatt. Anfr. 10 converi, sutura impressa temuiterque erenulata juncti; primi 2 leves, sequences striis longitudinalibus obliquis, in ultimis tribus rero evanescentibus ornati. A pertura ovata: jeristoma continum, brevissime solutum, expunsum ac subduplicutum, lamellu parietalis mediorris, tere marginalis. La- 
mella columellaris debilis, valde immersa. I'lica palatalis principalis longa, oparitate conspirna. Plica palatales serundarix inconspicux.

Color fulvus, apicem versus albescens. Peristoma albidum : apertura intus fulca.

Longit. 25 mill., latid. 7 mill., apertwa 6 millim. alta, $51 / 2$ millim. lata.

Coquille seuestre, solide, imperforée, fusiforme, assez obèse, peu luisante. Spire entière, atténuée vers le sommet qui est légèrement papilleux, composée de 10 tours convexes séparés par une suture bien accusée et finement crénelée. Deux premiers tours lisses, les suivants ornés de costules longitudinales obliques qui s'effacent graduellement sur les trois derniers tours. Ouverture ovalaire; péristome épais, continu, faiblement détaché, élalé el dédoublé en arrière. Lamelle pariétale-médiocre, presque marginale; lamelle columellaire faible, profondément immergée. Pli palatal long, visible par transparence; plis palataux secondaires indistincts.

Coloration d'un brun rougeâtre passant au blanc vers le sommet de laspire, péristome blanchàtre, fond de l'ouverture brun.

Chine: province de Yunnan, vallée du Sin-Chiem (M. Mansuy).

Cetle espèce ressemble un peu au $\mathrm{Cl}$. Elisabethae Möllend., mais a le périslome dédoublé, le pli columellaire moins saillaut, la suture crénelée, etc. Nous n’avons rien trouvé de semblable dans les espèces décrites de Chine par le R.P. Heurle.

Clausilia Lavillea Dantzenberg et H. Fischer n. sp. (PI. IX fig. 5, 6.)

Testa sinistror'sa solidula, imperforatu, fusiformis, parum nitens. Spira integra, apicem rersus attennata. An/r. 10 
convexi, sutura impressa tenuiterque crenulata juncti: primi leves, sequentes striis longitudinalibus in anfr. ultimis vero cva. nescentibus ornati. Apertura ovata; peristoma continum brevissime solutum, expansum. Lamella parietalis marginalis, sat valida. Lamella columellaris debilis, immersa; lamella subcolumellaris marginalis brevis spiraliterque ascendens. Plica palatalis principalis horizontalis, sat longa; Innella falriformis; plicae palatul's secundariae nullae.

Color pallide fulous apicem versus allescens. P'eristoma allidum.

Longit. 22 mill., latid. i $1 / 2$ mill., apertura ij $1 / 2$ mill. alta, $41 / 2$ mill. lata.

Coquille senestre assez solide, imperforée, fusiforme, peu luisante. Spire entière, atténuée vers le sommet, composée de 10 tours convexes séparés par une suture bien accusée et très finement crénelée; les premiers lisses, les suivants ornés de costules longitudinales obliques qui s'effacent sur les derniers tours. Ouverture ovale, un peu oblique. Péristome continu faiblement détaché, élalé. Lamelle pariétale marginale assez forte. Lamelle columellaire faible, immergée; lamelle subcolumellaire bien visible, marginale, courte, s'élevant en spirale. Pli palatal principal horizontal, assez long; lunelle falciforme, bien visible par transparence. Pas de plis palataux secondaires.

Coloration d'un fauve clair passantau blanc vers le sommet. Péristome blanchâtre.

Tonkin: Ha-Giang (M. Mạnsuy).

Cette espèce que nous dédions à M. Laville, préparateur à l'Ecole Nationale des Mines de Paris, ressemble au $\mathrm{Cl}$. Lantenoisi D. et H. F. par sa sculpture, mais elle en dilfère par sa forme moins obèse, son pli subcolumellaire, sa lunelle, son péristome non doublé, etc. Elle diffère du Cl. gısota Möll. par sa spire plus atténuée au sommet, ses der- 
niers tours plus renflés, son pli columellaire plus saillant, ses plis longitudinaux plus saillants; elle s'éloigne encore plus des $\mathrm{Cl}$. montana Möll. et $\mathrm{Cl}$. platyloma Möll. Le $\mathrm{Cl}$. Billeti $\mathrm{H}$. Fisclıer est une espèce bieu différente, beaucoup plus petite.

Siphonaria atra Quoy el Gaimard

1833. Siphonaria atra

Quor el Gamalid, Voy. Astrolabe II, p. 337, pl. 25, fig. 41, 4ㄴ, (Siphonaire de Vanikoro).
1856 .
-- Q. et G. Rever, Conch. Icon. pl. III, fig. 14a, 14b.
1881. - - - - Rochebrune, Docum. faune malac. Cochin- chine et Cambodge, extr. du Bull. Soc. Philom. Paris, p. 31 .
1891. - - - P. Fischer, Catal. Indo- Chine, loc. cit. p. 122. (38 dı t. à p.)

Cochinchine: Hong-Chon (M. Mansuy).

\section{Auricula auris Midez Linné}

1758. Bulla A uris-Mida

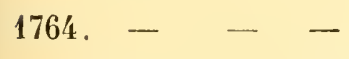

1767. Voluta- -

1773. Cochlis volutata, etc.
Linné, Syst. Nat. Ed. X, p. 728 .

Linné, Mus. Lud. Ulr. p. วั89.

Linné, Syst. Nat. Ed. XII, p. 1186.

Martini, Conch. Cab. II, 1. 119. pl. XLIII, fig. 436438. 
1774. Helix anris Midx

1780. Voluta Auris -

1783. - anris -

1789. Bulimus- -

1790. Volutu Amis -

1798. Ellobium Midde

1898. - Cerumense

1798. - tumidum

1801. Auricula mide

1814. - Midie

1816. - auris Midx

1817. Voluta - -

1821. A uricula Midie L.

1822. - -

182\%. Voluta Auris Midie

1830. Auricula Midie

1832.
Müller, Hist. Verm. II. p. 110.

Born, Test Mus. Cies. l'indob. p. 211.

Schröter, Einleit. in die Conchylienk. 1, p. 196.

Bruguiera, Encycl. Méth.

I, p. 3'2, pl. 460, fig. 6 a et $6 \mathrm{~b}$.

Gmelin, Syst. Nat. Eil. XIII, p. $343 \%$.

Bolten, Mus. Boltenianum p. 105 .

Bolten, ibid. p. 105.

Boltes, ibid. 105.

Lamarcli, Syst. d. anim. p. 92.

LEaCH, Zool. Miscellan. I, p. 74, pl. 32 .

Brainville, Dict. Sc. nat. IIl, suppl. p. 132.

DuL,Wyn, Deser. Catal. I, p. 499 .

Férussac, Tabl. Syst. p. 106.

Lamarck, Anim.s. Vert., VI, 2e p., p. 137.

Wood, Index testac. p. 89, pl. 19, f. 1 .

Lesson, Voy. Coquille p. 337, pl.9, fig. 1, 1a, 1b, 1c Quor et Gammad, Voy. Astrolabe II, p. 156, pl. 1/, p. 1 à 14. 
1838. Auricula Midie

1838. - -
1839. -
1840. Geovula -

1842. Auricula -

1844. - -

1847. Auricule de Midas

1850. Anricula Midx

1852. - auris Midx

1852. - Milix

18วั4. Ellobium anris Midle

1856. Auricula - -

1853. Ellobium - - L.

1876. Auriculus - - - Pfelffer, Mon. Pneum.,

1887. Auricula - -

1889.
Deshayes in Lamarck, Anim.s. Vert, $2 \mathrm{e}$ Ed. VIII, p. 323.

L. Potiez el Michaud, Gal. de Douai, 1, p. 204.

Lk. Anron, Verzeichu. p. 48. Swainson, Treatise on Malac. p. 34 4.

Lk. Reeve, Conch. Syst. II, pl. CLXXXVII, fig. 10.

- Köüster Mon. in Conch. Cab. 2e Ed. p. 12, pl. A, fig. I, pl. 2, fig. 1, 2, 3. Chenu, Leçons élém. p. 243, fig. 907 .

M. E. Gray, fig. Moll. Anim. pl. 306, lig. 12.

Mörcil, Catal. Yoldi, I, p. 37.

Eydoux et Souleyet, Voy. Bonite, p. 515.

H. et A. Adans, Proc. Zool. Soc. p. 7.

Pfeiffer, Mon. Auricul. p. 125.

H. et A. Adams, Gen. of rec. Moll. II, p. 237, pl. 82, fig. $\mathbf{1}^{\mathrm{a}}$. $3^{\circ}$ Suppl., p. 337 .

J. Mabille, sur quelques Moll. du Tonkin, in Bull Soc. Mal. Fr. p. 159. Petel, Catal. II, p. 385. 
1889. Auricula auris Midæ 1891.

1904.
L. Morlet, Journ. de Conch. p. 129.

L. P. Fischer, Catal. IndoChine, loc. cit. p. 123 (39 du t. à p.)

II. Fischer el Dautzen. BERG, Catal. Indo-Chine, in Mission Pavie, loc. cit. p. 413 (24 du t. à p.)

Cochinchine : Hong-Chon, Golfe de Siam(M. Mansuy).
1758. Bulla Auris Judie

176'. - - -

1767. Voluta - -

1773. Auris Midæ trillentata

1774. Helix auris Judx

1779. Voluta auris Midre

1783. - - Judw

1789. Bulimus - _ -

1790. Voluta I uris Judx
Linvé, Syst. Nat. Ed. X p. 728 .

LinNé, Mus. Lud. Uli. p. $\ddot{3} 90$.

Linné, Syst. Nat. Ed. XII p. 1187.

Martini, Conch. Cab. II, p. 119 et 128, pl. XLIV fig. 449, 450, 451 .

Muller, Hist. verm. II, p. 109.

Schroeter, (non L.) Flussconch. p. 314, pl. IX.f. J0 (var minor.)

SCHROETER, Einleit. in die Conchylienk. I, 1 . 197, pl. I, fig. 9.

Bruguière, Eucycl. Nétl. I. p. 344 .

Ginelin, Syst. Nat. Ed. XIII, p. 3437. 
1798. Ellobium Labrosum

1798. - Subtile

1813. Voluta Auris Mide

1816. Auricula auris Judie

1817. Voluta auris Judx

1817. Auricula reticulata

1821. - Judx L.
1821. - Simii
1822. - Judæ

1820ั. Toluta Auris Judx

1830. Auriculu Judx

1838

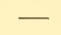

1838.

1939. - reticulata

1840. Geovula .......

1842. Auricula Judx
Bolten, Mus. Boltenianum, p. 105ั.

Bolten, Mus. Boltenianum, p. 105.

Burrow, (non L.) Elem. of Conch. p. 133, pl. XV , fig. 1.

Blainville, Dict. des Sc. nat. III, suppl. p. 132 ; pl. 55, fig. $1,1^{\text {b }}$, sub nom. Auricule de Juda. Dillwyn, Descr. Catal. I p. 500 .

Schumacher, Essai nouv. syst. p. 229.

Férussac, Tabl. Syst. p. 106.

Férussac, ibid. (teste Beck).

Lamarcis, Anim. s. Vert, VI, 20 p. p. 137.

Woon, Index testac. p. 89. pl. 19, fig. 2.

Lesson, Voy. Coquille, p. 339 .

Lk. Deshayes in Lanarck Anim. s. Vert. 2me Ed., VIII, p. 324.

L. Potiez el Michaud, Gal. de Douai, 1, p. 204. Anton, Verz. p. 48.

Sirainson, Malac. p. 344, fig. 105 .

lk. Reeve, Conch. Syst. II. pl. CLXXXVII, fig. 4. 
1844. Auricula Juelie

Lk Küster, Mon. in Conch. Cilb. 2e Ed. p. 15, pl. 3, fig. 1, 1, 2.

1844. - - var. reticulata Küster ibid. p. 68, pl. :3, fig. 1, 2.

1844. Voluta Auris Midie

Burkow (non L.) Elem. of. Conch. new ed.p. 143, pl. XV, fig. 1.

1847. Luricule de Judas Chenu, Leçons élem. p. 243, fig. 908.

1850. - - -

1852. Auricula Judee

185\%. - -

1852. Auriculu auris Judæ
Mrs E. GhaY, Moll. An. pl. 304, fig. 4, $4^{\mathrm{a}}$.

Sowenbr, Conch. Man. 4 th. Ed. p. 79, fig. 297.

Eydoux et Souleyet, Voy. Bonite p. 5l5, pl. 23, fig. 19, 20.

Möнсн, Catal. Yoldi I, p. 37 .

1852. - tridentata Martini Mörch, Catal. Yoldi I, p. 37 .

1854. Ellobium auris Judx H. et .A. AdaMs, Proc. Z. S. L. p. 7.

1856. Auricula - - L. PFEIFFer, Mon. Auricul. p. 130.

1858. Ellobium - - - H. el A. Adams, Gen. of rec. Moll. II, p. 237.

1866. Auricula Jude

- W. T. Blanford, Contrib. to. Indian Malac. VIII, p. 13.

1870. - - WoodWard, Man. de Malac. trad. fr. p. 315, pl. 12, fig. 3 .̈.

1875. - auris Judæ Monelet, Séries Conch. IV, p. 270. 


\begin{tabular}{|c|c|c|c|c|}
\hline 36. & - & - & & $\begin{array}{l}\text { Tapparone Canefri, Mal. } \\
\text { Viaggio Magenta in Mem. } \\
\text { Accad. Rle delle Sc. di } \\
\text { Torino XXVIII, p. } 208 \text {. }\end{array}$ \\
\hline 1876. & - & - & L. & $\begin{array}{l}\text { Pfeiffer, Moll. Pneum. } \\
\text { viv. } 3^{\text {e }} \text { suppl. p. } 357 .\end{array}$ \\
\hline 1889 & - & dactylus & & $\begin{array}{l}\text { L. Morlet (non Pfr. !) J. } \\
\text { de Conch. vol. XXXVII } \\
\text { p. } 129 .\end{array}$ \\
\hline 889. & - & auris Judæ & $\mathrm{L}$. & PAetel, Catal. II, p. 383. \\
\hline 391 & - & & & $\begin{array}{l}\text { P. Fischer, Catal. Indo- } \\
\text { Chine loc. cit. p. } 123 \text { (39 } \\
\text { du t. à p.) }\end{array}$ \\
\hline 1891. & - & dactylus & & $\begin{array}{l}\text { P. Fischer, (non Pfr !) } \\
\text { ibid. }\end{array}$ \\
\hline 1904. & - & auris Julde & & $\begin{array}{l}\text { H. Fischer et Dautzen- } \\
\text { Berg, Catal. Indo-Chine } \\
\text { in Mission Pavie, loc. cit. } \\
\text { p. } 412 \text { (23 du t. à p.) }\end{array}$ \\
\hline
\end{tabular}

Cochinchine (MI. Mansuy).

Il est possible que l'Auriculä dactylus Pfeiffer, de Bornéo, ne soit qu'une variété de l'A. auris Judx. Quant aux spécimens de l'Iudo-Chine rapportés par L. Morlet à l'A. dactylus, ils appartiennent certainement, ainsi que nousavons pu le vérifier, à l'A. auris Judx.

Cassidula auris-Fells Bruguière

1903. Cassidula auris-felis Br. Dautzenberg et H. FisCHER, Journ. de Conch. Vol. LIII, p. 108.

Cambodge. Rivière de Kampot (M. Mansuy). 


\section{Scarabus trigorus Troschel}

183s. Scarabus trigonus

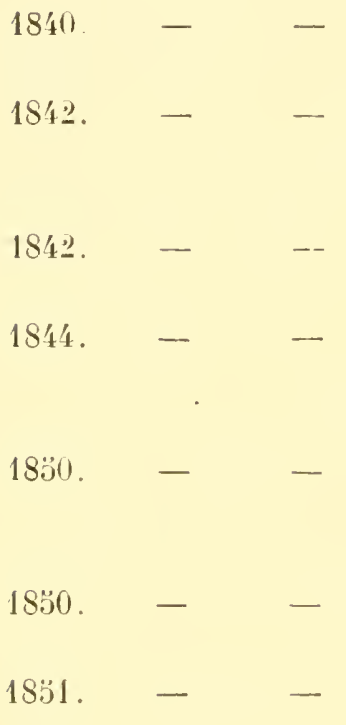

1832. Pythia trigona

1836 .

18.38.

1859. Scarabus trigonus

1860.

1874.
Troschel, Archiv für Naturg. 1, p. 207, pl. IV, fig. 3.

Troschel, Archir für $\mathrm{Na}$ turg. p. I24.

Tr. Pieeve, Ann. and Mag. of Nat. Hist. Ist Ser. IX, p. 2I9, pl. IV, fig. 2.

- Reere, Conch. Syst. II, pl. CLXXXVIII, fig. 2.

- Küster, Monogr. in Conclı. Cab., 2édit.. p. 10, pl. I, fig. 6, $6^{*}$.

- Adans et Reeve, loy. Samarang, p. 5́6, pl. XIV, fig. I 2 .

- A. Adans Proc.Zool. Soc. of London, p. 148.

- A. Adams, Ann. and Mag. of Nat. Hist., 2d Ser. VIll, p. $6 \overline{\%}$.

- Môrch, Catal. Yoldi. p.37

- Pfeiffer, Monogr. Auricul. viv., p. T⿱宀.

- 1I. el A. Aonus, Genera of rec. Moll. Il, p. 240.

- Curno, Manuel de Conch. I, p. 476, fig. 33it.

- Reeve, Conch. lcon. pl. III, fig. 22.

- Isser, Molluschi Borneensi, [). 61 . 
1876. I'ythia trigona

\begin{tabular}{|c|c|c|c|}
\hline 1881. & - & - & $\begin{array}{l}\text { de Rochebrune, Docum. } \\
\text { sur la faune malac. de la } \\
\text { Cochinchine et du Cam- } \\
\text { bodge, p. } 33 \text {. }\end{array}$ \\
\hline 1889. & Scarabus trigonns & $\rightarrow$ & $\begin{array}{l}\text { L. Monlet, Journ. de } \\
\text { Conch. XXXVII, p. } 129 .\end{array}$ \\
\hline 1889 & Pythia trigona & - & Patel, Catal. Il, p. 383. \\
\hline 189 I & Scarabus trigonus & - & $\begin{array}{l}\text { P. Fiscier, Catal. Indo- } \\
\text { Chine loc. cit. p. } 123 \text { ( } 41 \\
\text { du t. à p.) }\end{array}$ \\
\hline
\end{tabular}

Cochinchine : Hong-Chon (littoral du Golfe de Siam) (MI. Mansuy).

\section{Linnaea discreta J. Mabille}

4887. Limnaea discreta

1887.

1889.

- unica

1891. - discreta

1904.
J. MaBiLle, Moll. Tonk. diagn. p. 11.

J. Mabille, sur quelques Moll. du Tonkin in Bull. Soc. Malac. Fr. p. 133, pl. III, fig. 6.

J. Mabille, Moll. Tonk. diagn. p. 13.

J. M. P. Fischer, Catal. IndoChine loc. cit. p. 126 (42 dı t. à p.)

H. Fischer el Dautzenberg, Catal. Indo Chine in Mission Pavie loc. cit. p. 414 (25 du t. à p.)

Tonkin : Hanoï (M. Mansuy). 
Le Limnaea unica Mabille dont nous avons vu le type au Muséum de Paris, ne nous paraît être que le jeune âge du L. discreta.

\section{LinnaA spadicea A. Morelet}

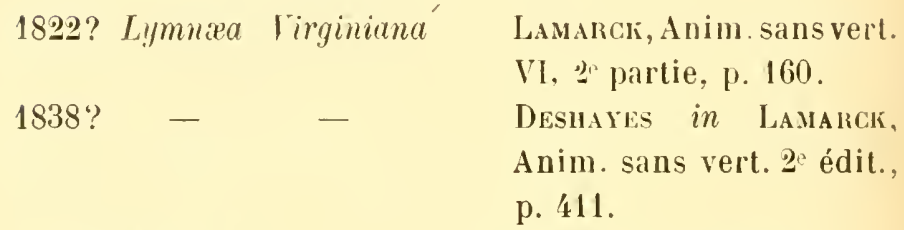

1841? Lymncea - Lk. Delessert, Recueil de Coq., pl. 30, fig. 4.

1862. Limniea spadicea

A. Morelet, Revue el Mag. de Zool., p. 478.

1863. - - A.-M. Crosse el Fischer, Journ. de Conch. XI, p. 362.

1866. - Crosseana Mabille et Le MesLli, Journ. de Conch. XIY, p. 130, pl. VII, fig. ö.

1875. - spadicea A. Moreret, Séries Conch. II, p. 279, pl. XIll, fig. 10.

1886. Limnaus spadiceus A.-M. Chessin, Monogr. in Conch. Cab), 2. édit., p. 399.

1886. - Crosseana M. et Le M. Chessix, ibid. p. 376, pl. ว̈0, fig. 8 .

1891. Limnee - - P. Fiscuer, Catal. IndoChine, loc. cit. p. 12 (4) du t. à p.).

1891. - spadicea A.-M. P. Fischer, Catal. IndoChine, loc, cit. p. 125 (4) du t. à p.).

1891. - - - L. MonLit, Journ. de Conch. XXXIV, p. 233. 
1904. Limnea spadicea

A. M. H. Fischer el DautzenBERg, Catal . Indo Chine in Mission Pavie, p. 414 (2气)

1904. - Crosseana M. et Le M. H. Fischer et Dautzenberg, ibid.

Cochinchine: Saigon (M. Mansuy).

Le $L$. Crosseana ne nous paraît être qu'une légère variété du spadicea, ayant le dernier tour moins renflé vers le haut et ne présentant pas de trace de la carène obsolète qui caractérise les exemplaires très adultes.

Planorbis exustus Deshayes

1773. Planorbis purpura juniore e littore Coromandelico 0. MüLlen, Hist. Verm. p. $15 \%$

1786. Helix cornea Limnei var. Chemnitz, Conch. Cab. IX, p. 96, pl. 127, fig. 1116, 1117.

1834. Planorbis exustus

Desuayes in Belanger, Voy.del'Inde,p.417, pl.1, fig. 11, 12, 13.

\begin{tabular}{|c|c|c|c|}
\hline 1836. & - & - & $\begin{array}{l}\text { Desh. Müller, Synops. test. } \\
\text { p. } 34 \text {. }\end{array}$ \\
\hline 1836. & - & Indicus & $\begin{array}{l}\text { Benson, Journ. Asiat. Soc. } \\
\text { of Bengal } \mathrm{V}, \mathrm{p} .743 \text {. }\end{array}$ \\
\hline 1838. & - & erustus & $\begin{array}{l}\text { Deshayes in Lamarck, } \\
\text { Anim.s. Vert. VIII, p. } 392 .\end{array}$ \\
\hline 1840. & - & cornews & $\begin{array}{l}\text { Pfeiffer (ex. p. non L. } \\
\text { Regist. zu Mart. \& Chemn. } \\
\text { p. } 87 .\end{array}$ \\
\hline
\end{tabular}

1860. - coromandelicus Beck V. Martens (non 0. Fabr.) Proc. Z. S. L., p. 12. 
1862. Planorbis rircumspissus

1863.

1867. - Indirus

1870.

- exustus

Deslr.

Conch. Indica, p. 18, pl. XXXIX, fig. 10.

1871. - rircumspissus J. Mabille, Rev. el Mag. de \%ool. XXIII, p. 30 .

1875. - exustus Desh. A. Morelet, Séries Conch. IV, p. 274 .

1876.
1877. C. Icon. pl. IV, fig. 34 .

1889. - exmstus Desh. L. Morler, J. de Conch. XXXYH, p. 130.

\begin{tabular}{|c|c|c|c|c|}
\hline 1891. & $\ldots$ & - & - & $\begin{array}{l}\text { L. Monlet, J. de Conch. } \\
\text { XXXIX, p. } 233 \text {. }\end{array}$ \\
\hline 1891. & - & - & - & $\begin{array}{l}\text { P. Fischer, Calal. Indo- } \\
\text { Chine loc. cil. P. } 126 \text { (42 } \\
\text { du t. à p.) }\end{array}$ \\
\hline 1903. & - & .- & - & $\begin{array}{l}\text { BLANForn, Land and } \\
\text { Freshw. Moll. from. Siam } \\
\text { in Proc. Nal. Soc. London } \\
\text { p. } 280 \text {. }\end{array}$ \\
\hline 1904. & - & - & - & $\begin{array}{l}\text { H. Fischer el Dautzen- } \\
\text { Berg, Catal. Indo-Chine in } \\
\text { Mission Pavie, loc. cit. p. } \\
414 \text { ( } 23 \text { du t. à } 1 \text {.) }\end{array}$ \\
\hline
\end{tabular}

Cochinchine: Hong-Chon (M. Mansuy). 


\section{Conus textile Linne}

1757. Stombus Le Loman

1758. Comus textile

1767. - Tertile

1780. - textile

1790. - - -

179ะ. - -

1811. - gloria maris

1811. - rete anrenm

1815. - Textile

1813 - textile $\mathrm{L}$.

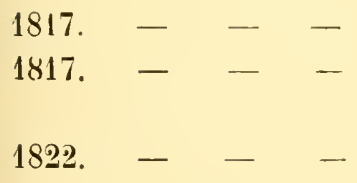

1825. - Textile -

1825. - textile -

1834. - - -
Adanson Voy, au Sénégal, p. 96, pl. 6 , fig. 7 .

Linné Syst. Nat., Ed. X, p. 717 .

Linné, Syst. Nat, Ed. XII, p. 1171 .

Bonn Test. Mus. Cas. Vindob. p. 163.

Gmelix Syst. Nat, Edit. XIII, p. 3393.

Hwass in Bruguière Encycl. Méth. p. 730 (pl. 344, fig. 5 ).

Perry (non Hwass) (Conchology pl. 25, fig. 1 .

Perry, Conchology, pl. 25, fig. 5 .

Burrow, Elem. of. Conch., pI. XIIl, fig. 3.

Rolssy, Hist. Nat. V, p. 408.

Dillwyn, Catal. I, p. 424. Schumacher, Nouv. Syst. p. 20 วั.

Lamarck, Anim s. Vert, VII, p. 5ั23.

Woov, Ind., Testac., p. 77, pl. 16. fig. 136.

Blainvilee, Man. de MaI. p. 415, pl. 26. fig. 4. Quor et Gaimard, Voy. Astrolabe III, p. 100, pl. 53, fig. 1 . à 17. 
1843. Conus textile L.

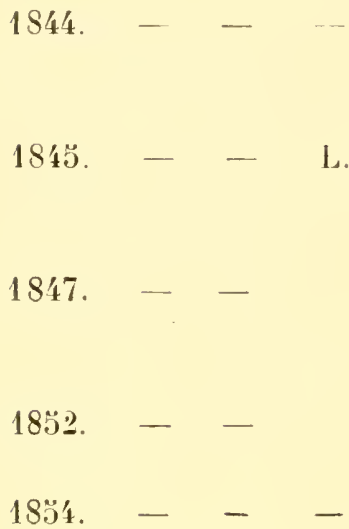

18วั5ั. - - -

1858. - - -

1859.

1864

$187 \%$

1884.

1889. Cylindrus textile L.
Reeve, Conch. lcon. pl. XXXVll, fig. 209.

Burrow, Elem. of Conch. new Ed., p. 138. pl. XIII, fig. 3.

Deshayes in Lamarck, Anim. s. Vert., 2e Ed., p. 123.

hiener, Icon. Coq. vir. p. 328 , pl. $90 ;$ pl. 102 , fig. 4 (var).

Sowerbr, Conch. Man. $4^{\text {th }}$, Ed., pl. 2I, fig. 46 I.

líüster (ex. p.), Mon. in Conclı, Cab. 2e éd., p. ö!, pl. 7, fig, $10 ;$ pl. 8, fig. 4, כ̈, 6 (excl. pl. 4, fig. 9). Haxley, I psa Linn. Conch. p. 176.

Sowerbi, Thes. Conch. [11, p. 41, pl. 23, fig. 567. Chexu, Man, de Conch., I, p. 247. fig. 1497.

Chosse el Fischen, Jouru. de Conch.. XIl, p. 335.

Weinkaufr, Mon, in Concls. Cah., 2e éd., p. $15 \%$.

Tryon, (ex. p. excl, var. pluribus) Man. of Conch. Str. and Syst. VI, p. 89, pl. 29, fig. 92, 93, 94, $98,99$.

L. Morleit, J. de Conch., XXXVII, p. 133. 
1891. Conus tertile I.
P. Fischer, Cat. IndoChine, loc. cit., p. 135 , (马̊l du t. à p.)

Mitra (Turricula) Gruneri Reeve

1844. Mitra Gruneri

1867. Turricula modesta

1874. Hitra Gruneri Reeve

1874 - lrvicostata

1882 - (Turricula) Cruneri Tryos, Man. of Conch. IY, p. 168, pl. 49, fig. 416 , $418,419$.

Poulo-Condore (M. Mansuy).

Celte espèce qui est commune en Nouvelle-Calédonie n'avait pas encore été mentionnée jusqu'à présent dans la faune de l'Indo-Chine. Le Mitra laevirostata Sowerby est établi sur un spécimen jeune de cette espèce et le Turricula modesta Pease en est une variété à côtes longitudina. les un peu plus nombreuses.

\section{Mitra (Turricula) Sanguisuga Linné}

17อั8. Toluta Sanguisuga

Livné, Syst. Nat. éd. X p. 732 .

1767.

LINNÉ, Syst. Nat. édit. XII p. 1192 ?.

1780. - sanguisuga L. Borx, Test. Mus. Cæs. rindob., p. 226. 
1780. Turricula granulnta

1788. Tolutagranosa etc. Chemnitz, Conch. Cab. X,

1788. Tolutagranosa etc. Chemnirz, Conch. Cab. X,

Chemsitz, Conch. Cab. IV, p. 215, pl. CXIVIII, fig. 1367, 1368, 1374. p. 173, pl. 151, fig. 1419. 1443.

1790. - Samguisuga L. Guelin. Syst. Nat. éd. XIII, p. 34300 .

1811. Mitra sanguisuga

- Lamarck, Annales du Muséum XVII, p. 208.

1817. Tolnta -
1822. Mitra -
1822. - stigmutari
1823. Voluta sanguisugu

1838. Mitra

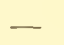

1838. - stigmataria

1841. - sanguisuga

- Dillwyn, Deser. Cat. I p. 346.

- Lamarcí, Anim, s. Vert. VII, P. 310.

Lamarci, Anim. s. Vert. VII I). 31 I.

Woov, Ind. test. p. 97, pl. 20, fig. 105̆.

Lk. KIENER, Icon. Coq. viv. p. 80, pl. 24, fig, 75, 7 5. liener, ibid. p. 79, pl. 24, fig. 74, 74 .

lï̈ster, Mon. C. Cab. p. 58 , pl. 10, fig. 10, 11 .

1841. - stigmataria Lk. Küsten, Mon. C. Cab. p. 48, pl. 8, fig. 17.18; pl. 9, fig. $\check{2}, 6 ; \mathrm{pl} .10$, fig. $3, \ddot{3}$.

1844. - sangnisuga Lk. Reeve. C. Icon. pl. XIV, fig. 99.

1844. - stigmataria Lk. Reeve,C.Icon.pI.III,fig.13̈.

1864. - - - - Crosse el Fischer, Journ. de Conch. XII, p. 336.

1874. - sanguisuga

L. Sowerbr, Thes. Conch. IV , p, 29, pl. 38ั9, fig. 9l*, 92*, 93*, 94*. 
1874. Mitra stigmaturia

1882. - sanguisug"

1891. - stigmataria
Lk. Sowelzв, ibid. p. \&9, pl. 3ว̆5, fig. ' $\mathbf{7}, 48,49$.

L. Thyon, Man. of Conch. IV, p. 165, pl. 48, fig. 393 , 397.

Lk. P. Fiscuer, Catal. IndoChine, loc. cit. p. 138 (כ̈4 du t. à p.)

Nous n'avons pas trouvé la forme typique dans les ré. coltes de M. Mansuy.

Subsp. condoriana Dautzenberg et H. Fischer, n. subsp.

Jolie forme qui semble localisée à Poulo-Condore d'où nous avions déjà reçu un spécimen récolté par le Directeur du Pénitencier. Elle se distingue non seulement par sa coloration gris-bleuâtre, avec une zòne blanchâtre peu distincte, mais aussi par sa sculpture longitudinale consistant seulement en sillons étroits qui coupent les cordons décurrents, tandis que chez les formes déjà décrites du $\boldsymbol{M}$. sanguisuga il existe de véritables côtes longitudinales séparées par des espaces aussi larges qu'elles mêmes.

Poulo Condore (M. Mansuy).

P. Fischer a cité le $\boldsymbol{\mu}$. stigmalaria Lk de cette mêue provenance (Poulo-Condore), mais sans dire de quelle variété il s'agissait.

Mitia (Turricula) vulpecula Linné

1758. Voluta vulpecula

1767.

1780
Lısné, Syst. Nat. édit. X, p. 732 .

Linné, Syst. Nat. éd. XII, p. 1193.

L. Born, Test. Mus. Caes. Vindols., p. 227. 
1780. Turricula Íulpecula - Chesnitz, Conch. Cab. IV, p. 213, pl. CXLVIII, fig. 1366 .

1790. Voluta vulpecula

- Guelin, Syst. Nat. éd. XIII, p. $343 ̈ 1$.

1811. Mitra

- Lamarci, Annales du Muséum X VII, p. 207.

1817

- Dillwyn, Deser. Cat. I. p. 547.

1822. - -

- Lamarck, Anim. s. Vert. VII. p. 308.

1825. Voluta -

Woon, Ind. testac. p. 97, pl. 20, fig. 109.

1838. Mitra -

L. Kiener, Icon. Coq. viv. p. 76, pl. 2I, fig. 64, 64 .

1841

- Küster, Mon. C. Cab. 2e éd. p. 58, pl. 10, fig. 12, 13 ; pl. 1 I , fig. 1, 2, 3.

1844. - - - - Rieve, C. Icon. pl. VIII,

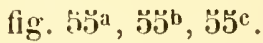

1864. -

- Crosse et Fischer, Journ. de Conch. XII, p. 337.

1874. - - -

- Sowerby (ex parte), Thes. Conch. IV, p. 30, pl. 353, fig. 11 (tantum).

1882. Mitra(Turricula) vulpecula L. Tryon, Man. of Conch. IV, p. 167, pl. 49, fig. 410-413.

1891. - - - - P. Fischer, Catal. IndoChine, loc. cit. p. 138 (马゙́t du t. à p.).

Poulo-Condore (M. Mansuy). 
Canthanus rumosus (Sol.) Dillwyn

Var. rubiginosa Reeve

1790. Buccinum strigosum

1817. - fimosum

1833. - - undosum

1834.

1846. - rubiginosum

1846. - Proteus
Gublin (ex p.). Syst. Nat. éd. XIII, p. 3494.

Solander mss. in Dillwy, Descr. Catal. II, p. 629, élabli sur les fig. 1145 et 1146 de Martini. Conch. Cab.

Quoy (non L.), Voy. Astrolabe II, p. 411, pl. 30, fig. 1, 2, 3, 4 .

Kiener (non L.) lcon. Coq viv. pl. 12, fig. $41^{\mathrm{a}}, 4 !^{\mathrm{a}}$, (juv.)

Reeve, C. Icon. pl. VII, fig. 47.

ReEve, C. Icon. pl. VII,

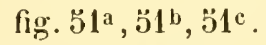

1881. Cantharus fumosus Dillw. Tryon, Man. of Conch. III, p. 15วอ, pl. 73, fig. 247-25ว.

Poulo-Condore (M. Mansuy).

P. Fischer, dans son Catalogue de l'Indo Chine, a cité le C. undosus, mais non le $C$. fumosus.

Nous n'avons pas cru devoir reprendre pour cette espèce l'ancien nom: strigosus Gmelin, parce qu'il a été foudé sur des figurations médiocres de Bonanni et sur la vignette 39 (p. 49) du Conchylien Cabinet, qui représente une autre espèce. Au contraire, les figures 1145 et $11 \frac{1}{4}$ du Conch. Cab., sur lesquelles Solander a établi son B. fumosum sont très satisfaisantes. Tryon indique comme synonymes les 
Buccinum biliratum et nigricostatum de Reeve et le Pisunia Desmoulinsi de Montrouzier; mais ces assimilations sont bien douteuses.

\section{Nassa corosata Bruguiere}

1783. Buccinum mutabile
1789. - coronatum Bruguière, Encycl. Mé- thod., p. $27 \overline{7}$.
1817. - _ Brug. Dillwys, Descr. Catal. Il, p. 603.
1829. - - - LamarCK, Anim. sans Vert. VII, p. $2 \overline{7} 6$.
182ว. - - - Wood, Index testac., p. 106, pl. 22, fig. 41 .
1833. - - - Quor et Gainard (ex p.). Voy. Astrolabe II, p. 440. pl. 32 , fig. 8, 9, 10 (excl. var. fig. 11, 12).
183อ̆. - - - líiexer, Icon. Coq. viv., p. $9 \overline{7}$, pl. 28, fig. $112,1[2$.
184年 - - - Deshates in Lamarck, Anim. sans Vert. 2e édit. $\mathrm{X}, \mathrm{p}, 180$.
1848. - Bronnii Philıpr, Zeitschr. f. Mala- koz., p. $13 \overline{7}$.
1849. - $\quad$ - $\quad$ Philippi, Abbildungen, $p$. $49(9), \mathrm{pl}$. I, fig. $1 \overline{7}$.

18533. Tassa coronata Brug. Reeve, Conch. Icon., pl. III, fig. $20^{\mathrm{a}}, 20^{\mathrm{b}}, 20^{\mathrm{c}}$.

18333. - Bronni Phil. Reeve, Conch. Icon., pl., XXII, fig. 149. 
185̆8. Buccinumcoronatum Brug. Küster, Monogr in Conch. Calı., 2 édit., p. 36, pl. 8. fig. 4, כै.

1864. Nassa coronata - Crosse et Fischer, Journ. de Conch. XII, p. 332.

1851. - Bromnii Pliil. de Rochebruxe, Suppl. aux Docum. sur la Faune malac. de la Cochinchine et du Cambodge, p. 18.

1882. - coronata Brug. Triox, Man. of Conch. IV. p. 23, pl. 7, fig. 7. 8 .

1891. - - $\quad$ - P. Fischer, Catal. IndoChine, loc. cit. p. 141 (ว̆7 du t. à p.).

1891 - Bronni Phil. P. Fischer. Catal. IndoChine, loc. cit., p. 141 (วั̆ du t. à p.).

Poulo Condore (M. Mansuy).

D'après Philippi (Abbildungen), le N. Bronnii différerait du $Y$. coronata par sa coloration, ses tubercules plus faibles, les stries de I’intérieur du labre plus nombreuses, le grand développement du bord columellaire ; mais aucun de ces caractères ne présente la moindre constance.

\section{Nassa seclusa P. Fischer}

18ว3. Nassa marginulatu

1Sรั3. - margaritifera

18วั3. - Isabellei
REEVE (non Lk.) C. Icon. pl. VII, fig. 43.

Reere (non Dkr.) C. Icon.. pl. IX, fig. כั9.

Reeve (non d'orb.) C. Icon., pl. VII, fig. 47 (= var. de $\mathrm{Y}$. marginulatu teste Reeve.) 
1863. Nassa Kieneri

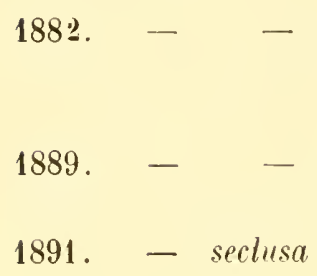

Deshayes (noll Anton). Moll. Réunion, p. 129. Desh. Tryon (non Anton). Man. of Conch. IV, p. 53, pl. 16, fig. 301-303.

- L. Montex, Journal de Conch. XXXVIl, p. 13ว̆. P. Fischer, Catal. IndoChine, loc. cil., p. 143 (59 du t. à p.).

Poulo-Condore (M. Mansuy).

\section{Canidia cambodgensis Reeve}

1861. Melania cambodjensis

Reeve, C. Icon, pl. LIS, fig. 468 (juv.).

1866. Hemisinuscambodjensis Rve. Mabille et Le Mesce, Journ. de Conch. XIV, p. 132, pl. VII, fig. 2.

1874. Canidia

- Brot, Monogr. Melanide in Conch.Cab. 2éd., p.467.

1870. Hemisimus - - A.Morelet, Séries Conch. IV, p 321 .

1889. Semisinus Cambodgensis - L. Montet, Journ. de Conch. XXXVII, p. 146.

1891. Canidia Cambojiensis - P. Fischer, Catal. IndoChine, loc. cit., p. 143 (61 du t. à p.).

19j4. - cambojiensis - H. Fischer el Dautzexberg, Catal. Indo-Chine in Mission Pavie, loc. cit., p. 415 ( 26 du t. à p.).

Cambodge: Gisement préhistorique de Somron Seng (M. Mansuy). 


\section{Murex capucinus Lamarck}

1793. Murex Monuchus Capucinns Cuemnitz, Conch. Cab. Xl, p. 123, pl. 192, fig. 1849, 1850 .

1817. - ramosus var. C. DiLwwyn, Descr. Cat. II, p. 687 .

1822. - capucimus Ch. Lamakck, Anim. s. Vert. VII, p. 164

\begin{tabular}{|c|c|c|c|c|}
\hline 1832. & - & - & & $\begin{array}{l}\text { Deshayes, Encycl. Méth. } \\
\text { III, p. } 900 \text {. }\end{array}$ \\
\hline 1842. & - & -- & & $\begin{array}{l}\text { Kiener, Icon Coq. viv., } \\
\text { p. } 42, \text { pl. } 4 \text {, fig. } 2,2 \text {. }\end{array}$ \\
\hline $18 i 3$. & - & $\cdot-$ & & $\begin{array}{l}\text { Deshayes in Lamarck, } \\
\text { Anim. s. Vert., } 20 \text { éd. } 1 X \text {, } \\
\text { p. 5̆ } 7 j .\end{array}$ \\
\hline 1844. & - & - & - & $\begin{array}{l}\text { Küster, C. Cab., ¿e édit., } \\
\text { p. } 32, \mathrm{pl} .13, \text { fig. } 6,7 .\end{array}$ \\
\hline 1843 . & - & - & - & $\begin{array}{l}\text { Reeve, C. Icon., pl. II, } \\
\text { fig. } 10 .\end{array}$ \\
\hline 1866. & - & - & Lk. & $\begin{array}{l}\text { MaBille et LE MESLE, } \\
\text { Journ. de Conch. XIV, } \\
\text { p. } 120 \text {. }\end{array}$ \\
\hline
\end{tabular}

1879. - - Ch. Sowerвy, Thes. Conch. IV, p. 18, pl. 383, fig. 40 .

1880. Murex (Chiroreus) capucimus Lk. TrYos, Man. of Conch. Il, p. 9', pl. 19, fig. 174.

1889. Murex capncinus Ch. I. Monlet, Journ. de Conch., XXXVII, p. 137. 1891. - - Lk. P. Fischer, Catal. IndoChine, loc. cit., p. 146 (62 du t. à p.).

Cochinchine: Hong-Chon, golfe de Siam (M. Mansuy). 


\section{Punpura alveolata Reeve}

1905. Purpura alveolata Rve Dautzenberg et H. FisCHER, Journ. de Conch.' vol. LHI, p. 121.

Cochinchine : Hong-Chon, golfe de Siam (M. Mansuy).

\section{Cuma Carinifera Lk}

1822. Purpura carinifera

1829.

carinata

\begin{tabular}{|c|c|c|c|}
\hline 832. & - & Lk. & $\begin{array}{l}\text { Deshayes, Eucycl. Méth. } \\
\text { III, p. \$41. }\end{array}$ \\
\hline 36. & - & - & $\begin{array}{l}\text { línener, Icon. Coq. viv., } \\
\text { p. } 62 \text {, pl. 14, fig. } 38,38 \text {, } \\
38^{\mathrm{a}} \text {. }\end{array}$ \\
\hline 44. & - & & $\begin{array}{l}\text { Des на YEs, in LamarGK, } \\
\text { Anim. s. Vert., 2e édit., } \\
\text { X, p. } 73 .\end{array}$ \\
\hline - & - & Lk. & $\begin{array}{l}\text { REEve, C. Icon., pl. Vl, } \\
\text { fig. } 26 .\end{array}$ \\
\hline- & - & - & $\begin{array}{l}\text { Kï̈ster, Mon. Conch., } \\
\text { Cab. } 2 \text { Ed., p. } 97 \text {, pl. } 17 \text {, } \\
\text { fig. } 3,6,7 ; 1 \text { l. 18, fig. } \\
3 \text { (var). }\end{array}$ \\
\hline
\end{tabular}

1880. Cuma

1889.
Lamarck, Anim. s. Vert. VII, p. 241 .

Schubert el Wagner, Conch. Cab. Xll, p. ^41,pl. 232. fig. 4078, et pl. 233, fig. 4091, 4092. III, p. 84 '́

líiener, Icon. Coq. viv. p. 62, pl. 14, fig. 38, 38,

Anim. s. Vert., 2e édit., fig. 26 .

Küster, Mon. Conch., Cab. 2e Ed., p. 97, pl. 17, Tryon (ex-parte), Man. of Conch., Str. and Syst. II, p. $200, \mathrm{pl}$. 62, fig. 319 (tantum).

L. Morlet, Journ. de Conch., XXXVII, p. 138. 
1889. Purpura carinifera Lk. Crosse et Fischkik, Journ. de Conch.,XXXVH, p. 286.

1890. - - - - C Crosse el Fischer, Journ. de Conch., XXXVIII, p. 1 .ั.

1894 .

P. Fischer, Catal. Indo-
Chine, loc. cit., p. 147
(63 du t. à p.)

Cochinchine: Hong-Chon, golfe de Siam (H. Mansuy).

Tryon a réuni au C. carinifera les $C$. imperialis Blainv., Purpura Ilelena Quoy, $P$. diadema Rve et $P$. thiarella Lk. La forme récoltée par M. Mansuy n'a pas les tubercules de la carène supérieure des tours très développés.

\section{Sistium undatum Chemnitz}

1900̈. Sistrum undatum Ch. Dautzenberg et H. FisCHER, Journ. de Conch., LIII, p. 122.

Nous n'avons pas, dans les récoltes de M. Mansuy, la forme typique de cette espèce.

Var. Kieneri Dautzenberg et H. Fischer, n. var.

1833. Purpura fiscella Lk. var. Kiener, Icon. Coq. viv., p. 30, pl. 6 , fig. $12^{\text {b }}$.

Poulo-Condore (II. Mansuy).

L'exemplaire rapporté par M. Mansuy correspond à la fig. $12^{\mathrm{b}}$ de Kiener, citée plus haut. Il se rapproche par la disposition de ses tubercules du $S$. concatenatum, mais sa forme est bien celle du $S$. undatum. Ne trouvant aucun nom qui lui convienne, nous nous décidons à lui attribuer celui de var Kieneri.

Cette variété diffère du Sistrum undatum typique par ses côtes longitudinales plus accusées, découpant les cordons 
décurrents principaux en rangées de tubercules; les intervalles des cordons principaux sont parfois blancs, c'est le cas dans l'exemplaire de M. Mansuy.

SIstrum bicinuloides Quoy et Gaimard

1833. Murex ricinuloides

1844. - -
1845. - decussatus

1850. - Ricinuloides (1.

1880. Sistrum fiscellum Ch.
Quoy et Galmard, Voy. Astrolabe II, p. 53i, pl. $36, \mathrm{fig} .13,14,15,16$.

Q. Küster, Mon. in Conch.,

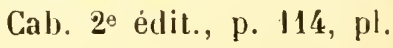
35, fig. 2.

Reeve C. Icon, pl. XXXI. fig. 153.

JAY, Cat., p. 333.

Tryov (ex.p. non Reeve), Man. of . Conch. II, p. 188, pl. ว̋8, fig. : ร5้ (tantum).

Cochinchine : Hong-Chon, golfe de Siam (M. Mansuy).
1758. Cypraea arabica

1767.

1836 .

1845.

1816.

1860 .
Linné, Syst. Nat. éd. X, p. 718 .

Linné, Syst. Nat. éd. XII, p. 1173.

Sowerby, Conch. Illust.

p. I, fig. 85, 85 .

Reeve, Conch. Icon., pl. I, fig. 2.

Kiener, Icon. Coq. viv., p. 105, pl. 17, fig. 1, 1.

V. Martens, Proc. Zool.

Soc. of London, p. 17. 


\begin{tabular}{|c|c|c|c|c|}
\hline 1866. & $\ldots$ & - & - & $\begin{array}{l}\text { Mabille et le Mesqe, } \\
\text { Jour. de Conch. XIV, p. } \\
120 \text {. }\end{array}$ \\
\hline 1869. & - & -- & - & $\begin{array}{l}\text { Crosse, Catal. in J. de } \\
\text { Conch. XVII, p. } 39 .\end{array}$ \\
\hline 1870. & - & - & - & $\begin{array}{l}\text { Sowerby (ex. p.), Thes. } \\
\text { Conch. IV, p. } 15 \text {, pl. X, } \\
\text { fig. } 60,61 \text { (tantum). }\end{array}$ \\
\hline 1881. & - & - & - & $\begin{array}{l}\text { Whinkuff, Mon. in } \\
\text { Conch. Cab. 2e éd., p. 51, } \\
\text { pl. } 16, \text { fig. } 3,4,5,6 .\end{array}$ \\
\hline 1882. & Aricia & & - & $\begin{array}{l}\text { Rossiter, Catal. Cypr. of } \\
\text { N. Cal. in Proc. Linn. Soc. } \\
\text { N. S. W., p. } 828 \text {. }\end{array}$ \\
\hline 1885. & Cypraea & - & - & $\begin{array}{l}\text { Roberts in Tryon (ex. p.) } \\
\text { Man. of Conch. Vli, p. } \\
\text { 174, pl. 8, fig. 18, 19, } \\
\text { (tantum), excl. fig. 23, 24, } \\
\text { (= eglantina et nigra). }\end{array}$ \\
\hline 1889. & - & - & - & $\begin{array}{l}\text { L. Monlet, Journ. de } \\
\text { Conch. XXXVII, p. } 139 .\end{array}$ \\
\hline 1891. & - & - & - & $\begin{array}{l}\text { P. Fischer, Catal, Indo- } \\
\text { Chine, loc. cit. p. } 154(70 \\
\text { du t. à p.) }\end{array}$ \\
\hline 1902. & C. (Aricia) & - & - & $\begin{array}{l}\text { Da Utzenberg, Revis. } \\
\text { Cypr. N. Cal. in Journ. } \\
\text { Conch. Xlix. p. } 325 .\end{array}$ \\
\hline
\end{tabular}

\section{Cypraea caurica Linné}

1758. Cypraea canrica

1767.
Linné, Syst. Nat. éd. X. p. 723 .

LINNÉ, Syst. Nat. éd. XII, p. 1179. 
1837. Cypraea caurica L.

\begin{tabular}{|c|c|c|c|c|}
\hline $845 \%$ & - & - & - & $\begin{array}{l}\text { ReEve, Conch. Icon., pl. } \\
\mathrm{Xl}, \text { fig. } 46 .\end{array}$ \\
\hline 1846. & - & - & - & $\begin{array}{l}\text { Kiener, Icon. Coq. viv. } \\
\text { p. } 54, \text { pl. 10, fig. } 22,23 \text {. }\end{array}$ \\
\hline 1869. & - & - & - & $\begin{array}{l}\text { Crosse, Catal. in J. Conch. } \\
\text { XVII, p. } 45 .\end{array}$ \\
\hline 1870. & - & - & - & $\begin{array}{l}\text { Sowerby, Thes. Conch. } \\
\text { IV, p. 8, pl. XXIII, fig. } \\
\text { 188-193 (tantum). }\end{array}$ \\
\hline 1881. & - & - & - & $\begin{array}{l}\text { Weinkauf, Mon. in } \\
\text { Conch. Cab. } 2^{\mathrm{e}} \text { éd. p. } 12, \\
\text { pl. } 3 \text {, fig. } 4,5, \text { p. } 34 \text { pl. } 10 \\
\text { fig. } 2,3 .\end{array}$ \\
\hline 1881. & Aricia & - & - & $\begin{array}{l}\text { DE Rochebrune, Suppl. aux } \\
\text { docum. sur la faune malac. } \\
\text { de la Cochinchine et du } \\
\text { Cambodge, p. } 13 \text {. }\end{array}$ \\
\hline 1882. & Luponia & - & - & $\begin{array}{l}\text { Rossiter, Catal. Cypræida } \\
\text { of N. Cal. in Proc. Linn. } \\
\text { Soc. N. S. W. p. } 8 \text { } 1 \text {. }\end{array}$ \\
\hline 1885. & Cypraea & - & - & $\begin{array}{l}\text { Roberts, in Tryon, Man. } \\
\text { of Conch. VII, p. 171. pl. } \\
5 \text {, fig. } 88,89,90 \text {. }\end{array}$ \\
\hline 1889. & - & - & - & $\begin{array}{l}\text { L. Morlet, Journ. de } \\
\text { Conch. XXXVII, p. } 140 \text {. }\end{array}$ \\
\hline 1891. & 一 & - & - & $\begin{array}{l}\text { P. Fischer, Catal. Indo- } \\
\text { Chiue, loc. cit. p. } 10 ّ 4,(70 \\
\text { du t. à p.) }\end{array}$ \\
\hline
\end{tabular}

Poulo-Condore (M. Mansuy), 1 exemplaire de forme allongée, correspondant à la fig. 90 de Tryon.

Sowerby, (ex. p.), Conch. Illustr., p. 8, fig. 158, 158, 160, 160, excl. fig. Iŏ9. Reeve, Conch. Icon., pl. $1,19.46$. Crosse, Catal. in J. Conch. IV, p. 8, pl. XXIII, fig. 188-133 (tantum) pl. 3, fig. 4, ร̆, p. 34 pl. 10 , fig. 2, 3 . docum. sur la faune malac. de la Cochinchine et du Cambodge, p. 13.

Rossiren, Catal. Cypre Soc. N. S. W. p. 8ㄴ.1.

Roberts, in Tryon, Man. of Conch. VII, p. 171, pl 5, fig. $88,89,90$.

Conch. XXXVII, p. 140.

- Pischer, Catal. MndoChime, Joc. cit. p. 15h, (70 du t. à p.) 


\section{Cypraea (Luponia) erosa Limné}

1758. Cypraea erosa 1767.

1837.

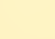

1845 .

1846.

1866

1869

1870

1881

1882.

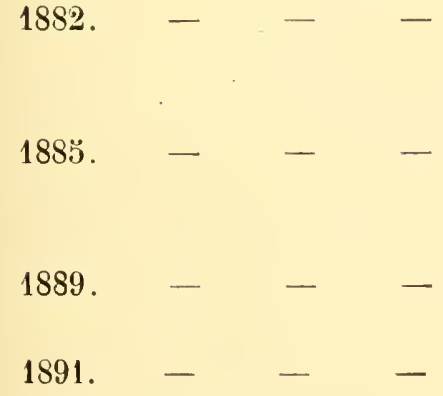

Linné, Syst. Nat. éd. X. p. 723 .

Linné, Syst. Nat. éd. XII p. 1179.

Sowerby, Conch. III. p. 6, fig. $119,119$.

L. Reeve, C. Icon. pl. XI fig. 43.

Kiener, Icon. Coq. viv. p. 23, pl. IX, fig. $2,2,3,3$. Ma bille et Le Mesle, Jour'. de Conch. XIV, p. 120.

- Crosse, Catal. in J. Conch. XVII, p. 4 5̆.

Sowerbi, Thes. Conch . IV, p. 37: pl. XVIll, fig. 110 à 110 .

Weinkauff, Mon. in Conch. Cab. 2 éd. p. 107, pl. 33, fig. 1, 2, 3, 4.

Rossiter, Catal. Cypr. of. $\mathrm{N}$. Cal, in Pr. Linn. Soc. N. S. W. p. 822

RoBerts in Tryon Man. of Conch. VII, p. 192, pl. 18, fig. 90, 100, 1.

L. Morlet, Journ, de Conch. XXXVII, p. 140. P. Fischer, Catal. IudoChine loc. cit. p. 137 (73 du t. à p.) 
1902. C. (Luponia) erosa L.

Dautzenвeиg, Revis. Cypr. N. Cal. in J. Conch. XLIX, p. 366.

Poulo-Condore (M. Mansy.)

$$
\text { Cypraea (Luponia) vitelots Linné }
$$

1758. Cypraea vitellus

1767.

Vitellus

1836. - vitellus L.

$\begin{array}{cccc}1843 . & - & - & \\ 1846 . & - & - & - \\ 1866 . & - & - & -\end{array}$

1869.

1870.

1881.

I882. Luponia

1885. Cypraea
Linné, syst. Nat. éd. X, p. 721 .

Linvé, syst. Nat. éd. XII, p. 1176.

Sowerby, Conch. Illustr. p. 2, fig. 66, 66 .

Reeve. C. Icon. pl. V, fig. 14.

líever, Icon. Coq. viv. p. 12, pl. 19, fig. 1, I.

Mabille et he Mesce, Journ de Conch. XIV, $\mathrm{p}$. 120.

Crosse, Catal. in Journ. de Conch. XVII, p. 41. Sowerby, Thes. Conch. IV, p. 13, pl. VI, fig. 31 , 32,33 .

Weinkauff, Mon, in Conch. Cab. ${ }^{\circ}$ éd. p. 38 , pl. 11, fig. 6, 7, 10, 11 .

Rossiter, Catal. Cypraeidie of N. Cal. in Proc. Linn. Soc. N. S. WV. p. 826.

Roberts in 'Tryon, Man. of Conch. VII, p. 182, pl. 13, fig. 72,73 . 
1889. Cypraea vitellus L.

1891.

1902. C. (Luponia $)--$

Poulo-Condore (M. Mansuy).
L. MonLte Journ. de Conch. XXXVII, p. 141. P. Fischer, Catal. Indo Chine loc. cit. p. $154 \quad(70$ du I. à p.)

Dautzenheri;, Rev. Cypr. N. Cal, in Jour. de Conch. XIIX, p. 343.

\section{Cypraea (Luposia) lynx Linné}

1758. Cypraea L.ynx

1767.

\begin{tabular}{|c|c|c|c|c|}
\hline 1836. & - & - & I. & $\begin{array}{l}\text { Sowenвy, Conch. III. p. 2, } \\
\text { fig. } 107,107 .\end{array}$ \\
\hline $184 \breve{.}$ & - & - & - & $\begin{array}{l}\text { ReEve, C. Icon, pl. IX, } \\
\text { fig. } 33 \text {. }\end{array}$ \\
\hline 1846. & - & - & -- & $\begin{array}{l}\text { Kínen, Icon. Coq. viv. } \\
\text { p. 10, pl. 25, fig. } 2,2 \text {; } \\
\text { pl. } 38 \text {, fig. 2, 2. }\end{array}$ \\
\hline 1866. & - & - & - & $\begin{array}{l}\text { Mabille et le Mesle, J. } \\
\text { de Conch. XIV, p. } 120 .\end{array}$ \\
\hline 1869. & - & - & - & $\begin{array}{l}\text { Crosse, Catal. in Journ. } \\
\text { de Conch. XVII, p. } 41 \text {. }\end{array}$ \\
\hline 1870 & - & - & - & $\begin{array}{l}\text { Sowerby, Thes. Conch. IV } \\
\text { p. } 21 \text {, pl. XV, fig. } 85^{*} 86^{*} \\
87^{*} 88^{*}\end{array}$ \\
\hline 1881. & - & - & - & $\begin{array}{l}\text { W еinka Uf , Mon. in } \\
\text { Conch. Cab. 2e éd. p. } 79 \text {, } \\
\text { pl. 23, fig. 6, 7, 10, } 11 \text {. }\end{array}$ \\
\hline
\end{tabular}

Linné. Syst. Nat. éd. $\mathrm{X}$, p. 721 .

LINNÉ, Syst. Nat. éd. XII, p. 1176.

Sowerby, Conch. IH. p. 2,

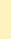


1882. Luponia lynx L.

1883. Cypraea - -

1902. C. (Luponia) - -
Rossiren, Cypr. of N. CaI. in Proc. Linn. Soc. N. S. W. p. $8: 4$.

Roberts in Tryon, Man. of Conch. VII, p. 183, pl. 14, fig. 86,87 (et fig. 98 var. caledonica).

\begin{tabular}{|c|c|c|c|}
\hline 1889. & - & - & $\begin{array}{l}\text { L. Morlet, J. de Conch. } \\
\text { XXXViI, p. } 141 \text {. }\end{array}$ \\
\hline 1891. & - & - & $\begin{array}{l}\text { P. Fischer, Catal. Indo- } \\
\text { Chine loc. cit. p. } 153 \text { ( } 71 \\
\text { du tà p.) }\end{array}$ \\
\hline 02 & C. (Luponia) & ) - - & $\begin{array}{l}\text { Dautzenberg, Rev. Cypr. } \\
\text { N. Cal. in Jour. de Conch. } \\
\text { Xl.IX, p. } 344 \text {. }\end{array}$ \\
\hline
\end{tabular}

Poulo-Condore (M. Mansuy).
1832. Cypraea Walkeri

1838.

$\begin{array}{llll}\text { 1838. } & - & - & \\ \text { 1845. } & - & - & \text { Gray } \\ \text { 1845. } & - & - & -\end{array}$

1845

1868. - Bregeriana

1869. - Walkeri Gray

1869. - Bregeriana
Gray in Sowerby, Conch. III. p. 7, fig. 22*.

Gray, Desc. Catal. Cypraeadæ, p. 11.

Reeve, C. Icon. pl. XlI, fig. $50^{\mathrm{a}}, 50^{\mathrm{b}}$.

Í́mener, Icon. Coq. viv. p. 33 , pl. 14, fig. 3.

Crosse, Journ. de Conch . XV1, p. 277.

Crosse, Catal. in J. de Conch. XVII, p. 43.

Crosse, ibid. p. 46, pl. 1, fig. 2 . 
1870. Cypraca Walkeri Gray Sowenвs, Thes. Conch. IV, p. 2:5, pl. XVIII, fig. 123 à 125; pl. XXXVllı, fig. : "336.

1882

- Welnkauff, Monogr. in Conch. Cab. 2éd. p. 78. pl. 23, fig. 1, 4 .

1882. - Biegeriana Cr. IVernliauff, ibid.p.77, pl. 23, fig. 2, 3 .

1885. - Walleri Gray Roberts in Tryos, Man. of Conch. VII, p. 18\%, pl. 15, fig. 10 ; pl. 17, fig. 68 ; pl. 14, fig. 1, 2.

1902. С. (Lиролia $)$ - - Dautzenberg, Rev. Cypr. N. Cal. in J. de Conch. p. 331 .

Poulo-Condore (M. Mansuy).

\section{Ojula lactea Lamarck}

1810. Ovula lactea

1822

1830. Ovulum lactenm

1832. Ovula lactea

1844. - -

1845

1855. Ovulum lacteum
Lamarck, Ann. du Muséum XVI, p. 111.

Lamarck, Anim. s. Vert. VII, p. 368 .

Lk. Sowkкву, Śpecies Conchyl. p. 30 , fig. 13, 14.

- Deshayes, Encycl. Méth. 111, p. 685 .

DeshaYes in LAMARCK, Anim. s. Vert. 2 éd. X, p. 469 .

Lk. Kiener, lcon. Coq. viv. p. 8, pl. 6, fig. 1, 1 .

Sowerby, Thes. Conch. II, p. 468, pl. CI, fig. 67-69. 
183̈8. Amphiperas lactea

1839. Ooula

1865. Ovulum lacteum

1881. Ovula lactea

1885. - -

H. el A. Adams, Gell. of rec. Moll. I, p. 270.

- Lli. Chenu, Man. de Conclı. i, p. $2 i 2$, fig. 1773 .

Reeve, C. Icon. pl. I, fig. 1 .

Weinkauff, Mon. in Conch. Cab. 2 éd. p. 170, ol. 44, fig. 1, 3 .

- Tryon (ex p.), Man. of Conch. Str. and Syst. VII, p. 247, pl. 2, fig. 23, 24 (excl. var.).

Poulo-Condore (M. Mansuy).

Celte espèce n'est pas signalée dans le Catalogue de P. Fischer.

Stronbus succincrus Linné

1767. Strombus succinctus

1780. - accinctus

1790. - succinctus

1817. - accinctus

1822. - sucrinctus

1825.

1832.
LINvé, Syst. Nat., éd. XII, p. 1212 .

Bonx, Mus. Cas. Vind. p. 283, pl. 10, fig. 14, 15. Gmelin, Syst. Nat., éd. XIII, p. 33̈l8.

L. Dillwye, Descr. Cat. il, p. 672 .

Lamarci, Anim. s. Vert. p. 208.

Wood, Ind. Test. p. 118, pl. 25, fig. 30 .

Deshayes, Encycl. Méth. III, p. 992. 
1840. Strombus septimus

\begin{tabular}{|c|c|c|c|c|}
\hline 1843. & - & succinceus & L. & Kiener, Icon. Coq. viv. \\
\hline 1813. & - & 一 & - & $\begin{array}{l}\text { Deshayes in Lamarck, } \\
\text { Anim. s. Vert, } 2 \text { édit. IX, } \\
\text { p. } 703 \text {. }\end{array}$ \\
\hline
\end{tabular}

1845

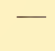

- Küster, Mon. Concli., Cab. p. 39, pl. 7, fig. 1.
1847. - - - - Sowerby, Thes. Conch. I, p. 28, pl. VI, fig. 20, 21.
1851. -- - - Reeve, Conch. Icon. pl. XVII, fig. 43.
1839. - - - Chenu, Man. de Conch. I, p. 2ö6, fig. 1589, 1592.
1874. - robustus Sowerir, Proc. Z. S. L, p. 599, pl. LXXII, fig. 3, 3 ( (var.)

1883. - sucrinctus - Triox, Man. of Conch. VII, p. $116, \mathrm{pl} .6, \mathrm{fig} .56,57$.

1889. - septimus Duclos Crosse et Fischer, Journ. de Conch. XXXVII, p. 287. 1891. - - - P. Fischer, Catal. IndoChine, loe cit., p. 158 (74 du t. à $\mathrm{p})$.

Poulo-Condore (M. Mansuy).

Strombus unceus Linné

1758. Strombus urcens

Laxné, Syst. Nat., édit. X, p. $74 \%$.

1767. - Urceus

Linné, Syst. Nat. édit. XII, p. 1212. 
1780. Strombus urceus

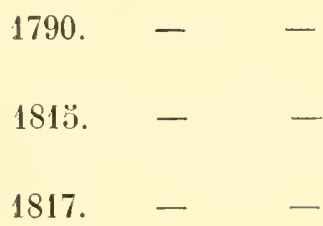

1817. Canarium ustulatum

1822. Strombus urcens

1825.
1832.
1810.

1843.

1843.

1844. - Urceus

1847

1845. - urceus
L. Born, Mus. Cres. Vindob. p. 283 .

- Guelin, Syst. Nat., éd. XIII, p. 3518 .

- Burrow, Elem. of Conch. p. 162 , pl. 17, fig. 3 .

- Dilwys, Descr. Catal. I, p. 673.

Schumacher, Essai Nouv. Système, p. 219.

Lin. Lamarck, Anim. s. Vert, VIl, p. 210.

- Wood, Ind. test. p. 118, pl. 25 , fig. 32.

- Deshayes, Encycl. Métl. III, p. 993.

- Duclos in Chenu, lllustr. Conchyl., p. 4, pl.5, fig. 8 à 15 ; pl. 24, fig. $\ddot{j}, 6$.

- líiener, Icon. Coq. viv. p. 60, pl. 30, fig. 2, 2, 3 ; pl. 15, fig. 2.

- Deshares in Lamarck, Anim. s. Vert. 2eéd. p. 705.

- Burrow, Elem. of Conch. new édit. p. 1500, pl. 17, fig. 3.

- Küster, Mon. in Conch. Cab. 2éd. p. 51, pl. 9, fig. 4, bै, 6, 7 ; pI. 14, fig. 9 (juv.)

- Sowerby, Thes. Conch. I, p. 30, pl. VII, fig. 3', 35, $36,37,41,42$. 
1850. Strombus urceus

18909.

1883. S. (Canarium) urceus
L. Reeve, Conch . lcon, pl. XI. fig. $24^{\mathrm{a}}, 2 \mathbf{4}^{\mathrm{b}}, 2 \mathbf{4}^{\mathrm{c}}$.

- Cunnu, Man. de Conch. I, p. פอ๊ 7, fig. 1606.

L. Trixon, Man. of Conch. VII, p. 118, pl. 6, fig. (i⿱⺈-67.

Cochinchine : Hong Chon (M. Mansuy).

Cette espèce n’a pas été signalée dans le Catalogue de P. Fischer.

Ceritthium purpurascens Sowerby
Sowerby, Thes. Conch. II, p. 872, pl. CLXXXIII, fig. $182-186$,

\begin{tabular}{|c|c|c|c|}
\hline 1859. & - & nitidum & $\begin{array}{l}\text { Sowerby, (non Hombron } \\
\text { et J., nec Hanley, nec } \\
\text { Zelieli) Thes. Conch. II, p } \\
\text { 872, pl. CLXXXII, fig. } \\
\text { 180, } 181 \text {. }\end{array}$ \\
\hline 1855 & - & splendens & $\begin{array}{l}\text { Sowerbi, Thes. Conch. } \\
\text { II, p. } 872 \text {, pI. CLXXXIII, } \\
\text { fig. } 191 \text { (var.) }\end{array}$ \\
\hline 1865. & - & purpurascens Sow. & $\begin{array}{l}\text { ReEve, Conch. ICon. pl. } \\
\text { VI, fig. } 36 .\end{array}$ \\
\hline 1860 . & - & nitidum Sow. & $\begin{array}{l}\text { Reeve, (non Hombron et } \\
\text { Jacq. nec Hanley, nec } \\
\text { Zekeli) Conch. Icon. pl } \\
\text { V, fig. } 28 .\end{array}$ \\
\hline 1880 & - & gentile & $\begin{array}{l}\text { BaYle, Journ. de Conch. } \\
\text { XXYIII, p. 248. }\end{array}$ \\
\hline
\end{tabular}


1887. Cerithium Trailli var. splendens Sow. Tryon, Man. of Conch. Str. and Syst. IX, p. 13əั, pl. 25, fig. 5ั1, 53.

Poulo-Condore ( M. Mansuy).

P. Fischer n’a pas signalé cette espèce dans son Catalogue de l'Indo-Chine.

Nous ne pouvons accepter l'opinion de Tryon qui considère cette forme comme n'étant qu'une variété du C. Trailli Sow. Le C. Trailli possède en effet une sculpture bien plus fine et son labre n'est pas polygonécomme celui du $C$. purpurascens. Le C. splendens Sow. est une variété plus grande et plus allongée que le $C$. purpurascens typique.

Cerithium concisum Hombron et Jacquinot

1905. Cerithium concisum H. et J. Dautzenberg et H. Fischer Journ. de Conch. LIII, p. 124.

Poulo-Condore (M. Mansuy).

\section{Cerithium (Vertagus) Koch Philippi}

1848. Cerithium Kochi

1849. - -

185ว. - Kochii

1866. Vertagus -

1869. Cerithium Kochi

1875. C.(Vertagus)-
Philıppi, Zeitschr. f. Malakoz. p. 21.

Philippi, Abbildungen, pl, 1, fig. 3.

Sowerby, Thes. Conch. Il, p. 853, pl. CLXXVI, fig. 13, 15 .

Reeve, Conch. Icon., pl. V fig. $26^{\text {a }}, 26^{\text {b }}$.

Lischke, Japan. Meeresconch. I, p. 72.

Smith, Ann. and Mag. N.

H. $4^{\text {th }}$ Ser. XVI, p. 105 . 
1878. C. (Vertagus) Kochi Ph. Suith, Proc. Z. S. L. p. 817, pl. L., fig. 18 (juv.) 1880. Cerithium - - v. Martens, Moll. del Maskarenen u. Seychellen, p. 10\}.

1882. Vertagus Kochii - Dunker, Ind. Moll. Jap. p. 108 .

1886. C.(Vertagus) Kochi Ph. Watson, Challenger Gastrop. p. ๖37.

1887. - - Kochii Trox, Man. of Conch. p. 147, pl. 28, fig. 48, 49. 1898. Cerithium Kochi Phil. Kobezt, Mon. in Conch. Cab. $2^{\circ}$ éd. p. 105, pl. 21, fig. 3,4 (radula).

Poulo-Condore (M. Mansuy).

Cette espèce ne figure pas dans le Catalogue de P. Fischer.

Potamides (Terebralia) sulcatus Born

1903. Potamiles (Terebralia) sulcatus B. Dautzenberg et II. Fischer, Journ. de Conch. vol. LIll, p. 128.

Annam : Tourane (M. Monod).

Potamides (Terebralia) tuberculatus (Linné) Born 1905. Potamides (Terebralia) tuberculitus(L.) Born DautzenBERG et H. Fischer, Journ. de Conch. vol. LIII, p. 130.

Poulo-Condore (M. Mansuy). 
Trmpanotonls cingleates Gmelin

190วั. Tympanotomus cingulatus Gm. Daltzenberg et H. FisCHER, Journ. de Conch. vol. LIII, p. 132.

Cochinchine: Hatien (.I. Mansuy).

\section{Var. microptera Kiener}

190\%. Tympanotomus cingulatus var microptera DAUTzeNBerg et H. Fischer, Journ.de Conch. vol.LIII. p. $13 \dot{4}$.

Cochinchine: Hatien (M. Mansuy).

\section{Cerithidea Qcadrata Sowerby}

1s66. Cerithidea quadrata

Sowerbi in Reeve, Conch. Icon. pl. I, fig. 5 .

15S1. - - Sow. DE Rochebruse, Docum. sur la faune malac. de la Cochinchine et du Cambodge. p. 23.

18si. - obtusa Lkvar. quadruta Sow. Tryos,Man. of Conch. Str. and Syst. IX, p. 161, pl. 33, fig. 63 .

1859. Cerithidea quadrata Sow. L. Morlet, Journ. de Conch. XXXVII, p. 144. 1891. Potamides (Cerithidea) obtusa var. quadrata Sow. P. FisCHer, Catal. Indo-Chine loc. cit. p. 163 (79 du t.à p.) 1s9s. Cerithium (Cerithidea) Kieneri var. Dohrni Kobelt. Mon. in Conch. Cab., p. 49: pl. 10, fig. 4, כ. 
1904. Potamides (Cerithidea) obtusa Lam. H. Fischer et Dactze.sberg (ex parte). Catal. Indo-Chine in Mission Pavie loc. cit. p. 416 (2ৃ du t. à p.)

Cochinchine : Saigon (II. Mansuy); Kangiou (Collect. Dautzenberg, ex D. Dupur).

II. Kobelt a mal compris le C. quadrata, puisqu'il repré. sente sous ce nom une coquille fort différente de celle figurée dans le Conchologia Iconica. D'autre part. il a décrit le véritable $C$. quadrata sous le nom de $C$. Kieneri var. Dohrni.

Nous ne partageons par l'avis de Tryon qui considérait le $C$. qualrata comme une simple variété du $C$. obtusa Wood.

\section{Melaxia Hegelim Philippi}

1S43. ISelania Hügelii PhilıPpi Abbild. p. $61(9)$ pl. II, fig. S.

1SวัS. Hemisinus Hugelii Phil. H. et A. Aduys, Genera of гес. Moll. I. p. 303.

1860. Melania siphonatu ReEve, Conch. Icon. pl. IX, fig. 143 .

1870. Melunia Hugeli Phil. Hasley et Throbald. Conch. Indica. p. 31.pl. L.XXI. fig. כ้. 6 .

157'. - - Brot. Conch. Cab. 2= èd. p. 17. pl. I. fig. 6. $6^{\mathrm{a}}$.

Tontin : Ha-Giang (.I. Mansuy), exemplaires jeunes.

Nous possédons également de jeunes spécimens de la me̊me espèce. récoltés en 1903. par. II. Blaise. capitaine de frẻgate. dans la régrion de Ifai-Iu. Haut Song Bo (Tonkin). 
Cette espèce, non signalée dans le catalogue de P. Fischer, est une acquisition nouvelle pour la faune IndoChinoise.

\section{Melania scabra Müller}

\section{Var. spinulosa Lamarck}

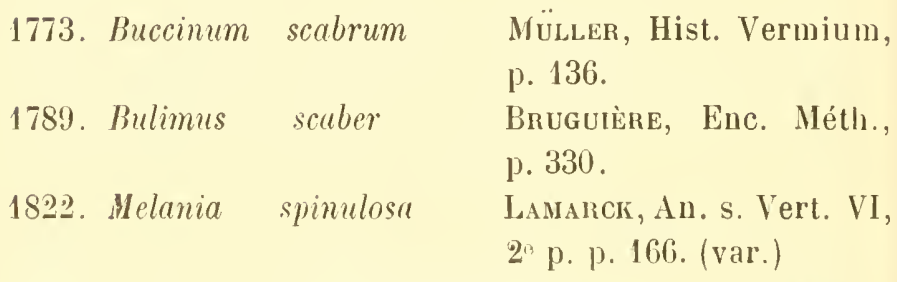

1838. - scabra Fér. Deshayes in Lamarcik, Anim. s. Vert. $2^{\ominus}$ éd. VIII, p. 443 .

1841. - spinulosa Lk. Delessert, Rec. Coq. pl. $30, \quad$ fig. $15^{\mathrm{a}}, 15^{\mathrm{b}}, \quad 15^{\mathrm{c}}$ (var.).

1849. - - - $\quad$ - Philippi, Abbild. p. 6, pl. I, fig. 20 (var.)

1849. - - - Mousson, Moll. Java, p. 76 , pl. XI, fig. 11, 12 (var.)

1858. I'lotia - $\quad$ H. et A. Adaus, Genera of rec. Moll. I, p. 295 (var.)

18วั8. - tenuis Lea. H. et A. ADAms, ibid. (var.)

1859. M. (Plotia) spinulosa Lk. Chenu, Man. de Conch. p. 288, fig. 1943 (var.).

1868. Melamia - - Broт, Mat. II, p. 38 (var.). 1870. - scabra Hanley et Theobald, Conch. Ind. p. 31, pl. LXXIII, fig. 1, 4. 
1872. Melania spinulosa

Brot, Mat. III, p. 19, pl.

I, fig. 15 (var.).

1874. - scabra Müll. Brot. Mon. Conch. Cab. p. 266, pl. 27, fig. 14, $14^{\mathrm{a}}, 14^{\mathrm{b}}, 14^{\mathrm{c}}, 15,15^{\mathrm{a}}$.

1875. - spinulosa Lk. A. Monelet, Séries Conch. IV, p. 319 (var.).

1891. - - - L. Morlet, Journ. de Conch. XXXIX, p. 234 (var).

1891. - - - P. Fischer, Catal. IndoChine, loc. cit, p. $168(84$ du t. à p.).

1897. - scabra var. a spimulosa Lk. Max Weber, Die Süss u. Brackw. Moll. d. Ind. Archip. p. 62.

1904. - spinulosa Lk. H.Fischer et Dautzenberg, Catal. Indo-Chine in Mission Pavie loc. cit. p. 418 (29 du t. à p.).

Tonkin : Ha-Giang (M. Mansuy).

\section{Melania tuberculata Müller}

1905. Melania tuberculata Müll. Dautzenberg et H. Fischer Journ. de Conch, vol. LIII, p. 141.

Tonkin : Hanoi, Grand Lac ; Ha-Giang (M. Mansuy).

Melania Jacqueti Dautzenberg et H. Fischer n. sp.

$$
\text { (PI. X, fig. 16) }
$$

Testa orato-conoidea, solidula, imperforata. Spira conica sat elata, apice acuminato. Anfr. 7 planiusculi sutura impressa angusteque canaliculata juncti, funiculis obsoletis 
remotisque, in anfr. ultimo circiter 5, ornati. I pertura ovalis supernebrevitergeniculata, inferne paululum effusa. Columella callosa, arcuata ; labrum arcuatum et acutum.

Color sub cpidermide fusco albidus. Columella albida. Apertura intus fuscescens. Alt. 27 mill. ; latit. 13 mill.; apertura 19 mill. alta, 7 mill. lata.

Coquille ovale-conoïde assez solide, imperforée. Spire conique assez élevée, acuminée au sommet, composée de 7 tours presque plans séparés par une suture bien accusée et étroitement canaliculée. Surface ornée de cordons obsolètes. écartés, ne se distinguant que sur les deux derniers tours, ils sont au nombre de 5 environ sur le dernier.

Ouverlure ovale, légèrement géuiculée au sommet, un peu évasée à la base. Columelle calleuse. Labre arqué, simple, tranchant.

Coloration blanchâtre sous un épiderme brun très adhérent. Collumelle blanchâtre ; fond de l'ouverture teinté de brun.

Nous dédions cette intéressante espèce à M. Jacquet, Directeur de l'Agriculture au Tonkin, qui a fait parvenir à M. Mansuy diverses formes étudiées dans ce mémoire. Tonkin : Ha-Giang (M. Mansuy).

Littorina (Melaraphe) carinifera Menke

1830. Phasianella carinifera

1831. Littorina perdir

1847. - sarinifera

$183 \% 7$.

18:38. L. (Neluraphe) -
Menke, Synopsis, 2e éd. p. $51,141$.

King, Zool. Journal, V, p. 345 (teste Reeve).

Philippi, Abbild. p. 4.3, pl. V, fig. 22-23(tantum).

Reeve, Collch. Icon. pl. VI, fig. 29a $, 29^{\mathrm{b}}, 29^{\mathrm{c}}$.

H. et A. Adams, Genera of rec. Moll. I, p. 314. 
1882? Litorina carinifera Mke Weinkauff,Mon.in Conch. Cab. 2 ed. p. 48. pl. 6, fig. 2, 3.

1887. L. (Melaraphe) scabra L. var. filosa Tryon, (ex parte non Sow.) Man. of Conch. IX, p. 244, pl. 42, fig. 48 (tantum).

1889. Littorina carinifera Mke L. MonLet, Journ. de Conch. XXXVII, p. 146. 1891. P. Fiscierr, Catal. IndoChine, loc. cit. p. 170 ( 86 du t. à $p)$.

Cochinchine: Hatien (M. Mansuy).

Cette forme que Tryon rattache au $L$. filos $a$ Sow. et dont il ne fait qu'une variété du L. scabra, nous paraìt suffisamment caractérisée par sa forme trochoüde, son dernier tour court et ses carènes décurrentes bien développées pour mériter d'être maintenue au rang d'espèce spéciale.

Littorina (Melaraphe) intermedia Philippi

190\%. Littorina (Melaraphe) intermedia Phil. Dautzenbera et H. Fischer, Journ. de Conch. LIII, p. 246.

Cochinchine: Hatien (M. Mansuy).

Trctarius nodulosus Gmelin

190כ. Tectarius nodulosus Gm. Dautzenberg et H. Fischer Journ. de Conch. LIII. p. 149.

Cochinchine : Hatien (M. Mansuy). 
Bithinia Dautzenbergi Wattebled. (emend).

1905. Bithinia Dautsenbergi Watt.(em.) Dautzenberg et H. Fischer Journ. de Conch. LIII, p. 152.

Tonkin. Hanoï, Grand Lac (M. Mansuy).

\section{Bithinia Chaperi L. Norlet}

1886. Bithinia Chaperi

1891.

1904
L. MorLet, Journ. de Couch. XXXIV, p. 263, 2S6, pl. XIV, fig. 3, $3^{\text {a }}$, $3^{\text {b. }}$

P. Fischer, Catal. IndoChine, loc. cit. p. 172, (88 du t.à p.)

H. Fischer et DautzenBerg, Catal. Indo-Chine, in Mission Pavie, loc. cit. p. 419 (30 du t. à p.)

Tonkin : Hanoï, (irand Lac (M. Mansuy).

Il nous semble difficile de séparer cette espèce de celles de Chine auxquelles Heude a attribué les noms de $: B$. chinensis Heude, B. spiralis Heude, B. scalaris Heude et $B$. striatula Bensun (Mémoire Empire Chinois, p. 171, pl. XLII, fig. $8,9,10,11$.) Ce ne sont probablement là que des variations d'une même espèce.

\section{Bithinia tonisiniana L. Morlet}

1886. Stenothyra Tonkiniana

I. Morlet, Journ. Conch. XXXIV p. 263, 287, pl. XIV, fig. $4,4^{\mathrm{a}}, 4^{\mathrm{b}}$.

1887. - - L. M. J. Mabille, Sur quelques Moll. du Tonkin in Bull. Soc. Mal. Fr. p. 157. 
1891. Stenothyra Tonkiniana L. M. P. Fischer, Catal. IndoChine, loc. cit. p. 173 (89 du t. à p.)

1904. - tonkiniana - H. Fischer el DautzenBERg, Catal. Indo-Chine in Mission Pavie loc. cit. p. 420 (31 du t.à p.)

Tonkin: Hanoï, Grand Lac (M. Mansuy),

Cette espèce, décrite par L. Morlet comme appartenant au genre Stenothyra, ne présente pas la forte contraction de l'ouverture caractéristique des Stenothyra. La valeur de ce genre nous paraît d'ailleurs peu importante car plusieurs Bithinia de l'lnde, telles que B. orcula Benson, etc., ont une légère tendance à cette contraction.

\section{Paludina ampuldiformis Souleyet}

1905. Paludina ampulliformis Soul. Dautzenberg et H. Fischen, Journ. de Conch. LIII. p. 154.

Tonkin: Grand Lac d'Hanoï (M. Mansuy).

\section{Paludina lecytholdes Benson}

1842. Paludina lecythoides

1846.

1852.

1862.
Benson, Ann. and Mag. N. H. $1^{\text {st }}$ Ser. IX p. 488.

Phililpi Abbild.II. p. 133.

pl. II, fig. 1.

Bens. Küster Mon. in Conch. Cab. 2éd. p. 23, pl. 5, fig. 1, ?.

- Reeve, Conch. Icon., pl. IV, fig. 21 . 
1863. P. (Vivipara)lecythoides Bens. Frauenfeld, Verz.Paludina in Verh. K. K. Zool. Bot. Ges., p. 620 (60 du t. à p.)

1865. ? Paludina - - v. Martens, Ueber Ostasiat. u. Neuholländische Paludinen in Malakoz. Bl., p. 146.

1866. Paludina - - Mabille et Le Mesle, Jour de Conch., p. 134.

\begin{tabular}{|c|c|c|c|c|}
\hline 1890 . & - & - & - & $\begin{array}{l}\text { Heude, Mém. H. N. Emp. } \\
\text { Chinois. Moll. de la Vallée } \\
\text { du Fleuve Bleu, p. } 174 \text {, } \\
\text { pl. XXXIX, fig. 6. }\end{array}$ \\
\hline 1891. & - & - & - & $\begin{array}{l}\text { P. Fischer, Catal. Indo- } \\
\text { Chine, loc. cit., p. } 177 \text { (93 } \\
\text { du t. à p.) }\end{array}$ \\
\hline 1904. & - & - & - & $\begin{array}{l}\text { H. Fischer et Dautzen- } \\
\text { BErg, Catal. Indo-Chine } \\
\text { in Mission Pavie, loc. cit., } \\
\text { p. } 423 \text { ( } 34 \text { du t. à p.) }\end{array}$ \\
\hline
\end{tabular}

Chine: Mong-Tsé (Yunnan), entre la ville et la concession européenne (M. Mansuy).

Var. Iatissima Daulzenberg et H. Fischer, n. var. (PI. X, fig. 17)

L'exemplaire de cette variété que nous avons fait figurer, est remarquable par le renflement excessif de la partie supérieure des tours, qui détermine un aplatissement subsutural très marqué. Nous avous observé tous les intermédiaires entre cette variété et le $P$. lecythoides typique. Mème localité. 
Paludina polyzonata Frauenfeld

1903. Palurlina polyzonata Frauenf. Dautzenberg et H. Fischer, Journ. de Conch. LIII, p. 15ั.

Tonkin: Hanoï, Grand Lac (M. Mansuy). Forme typique.

Paludina Quadrata lienson

1905. I'aludina quadrata Benson Dautzenberg et H. FisGHer, Journ. de Conch. Vol. LIII, p. 157.

La forme typique n'est pas représentée chez les spécimens recueillis par M. Mansuy.

Var. bizonalis (Möllendorff) Dautzenberg et H. Fischer 1905. Paludina quadrata var. bizonalis Möll. mss. DautzenBERg et H.Fischer, loc. cit. p. 160.

Tonkin : Hanoï, grand lac (M. Mansuy).

Var. Reevei Dautzenberg et H. Fischer 1905. Paludina quadrata var. Reevei Dautzenberg et H. FisCHer, loc. cit. p. 160.

Tonkin: Hanoï, Grand Lac (M. Mansuy). Cambodge : gisement préhistorique de Somron-Seng (M. Mansuy).

\section{Var, neruminosa Reeve}

190马. Paludina quadrata var. æruginosa Rve. Dautzenberg et H. Fischer, loc. cit. p. 160 .

'Tonkin : Hanoï, Grand Lac. (M. Mansuy). 


\section{Paludina Ratrei Crosse et Fischer}

187'. Paludina Frauenfeldi

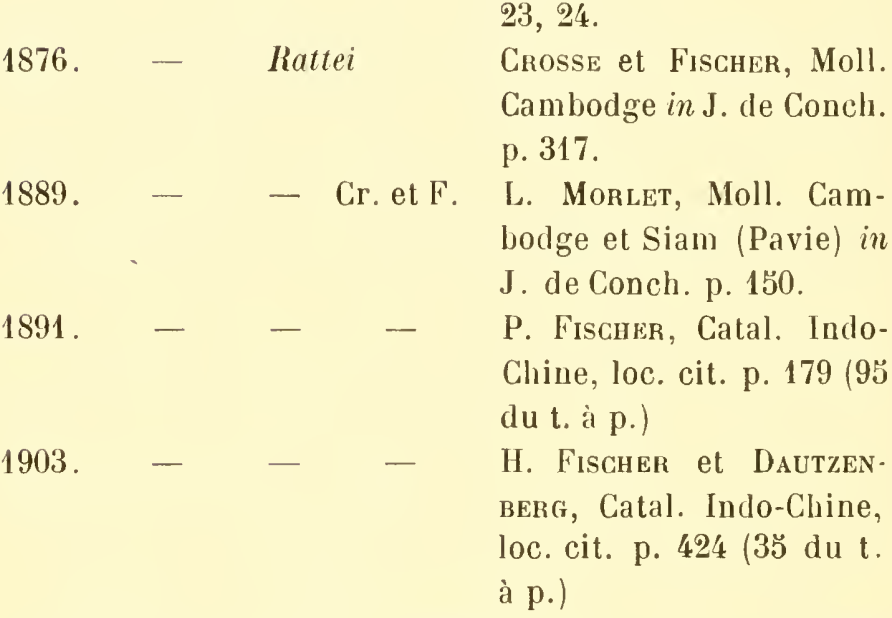

Deshayes in Deshayes et Juldien (non A. Morelet 1869). Moll. nouv. du Cambodge in. Nouv. Arch, du Mus. p. 134, pl. 7, fig. 23, 24.

Moll. J.
Cambodge : Gisement prélıistorique de Somron-Seng (M. Mansuy).

Var. elomgata Dautzenberg et H. Fischer, n. var.

(PI. X, fig. 18)

Forme de grande taille, plus allongéeque le type. Dimensions du plus grand spécimen : hauteur $45^{\mathrm{mm}}$ diamètre $32 \mathrm{~mm}$.

Cambodge : Gisement préhistorique de Somron-Seng (M. Mansuy).

\section{Margarya melanioides Nevill}

Les nombreux Margarya vivants et fossiles rapportés par M. Mansuy nous ont permis de constater que le M. me- 
lanioides est extrêmement polymorphe sous le rapport de la forme aussi bien que de la sculpture et que les diverses figurations de Nevill, Neumayr, Mabille et Heude que nous avons citées dans la synonymie, doivent être rapporlées à une seule espèce. M. Mansuy a récolté aussi quelques formes qui s'écartent de celles représentées jusqu’à présent. La bibliographie de cette espèce peut être groupée comme suit :

\section{Forme typique:}

1877. Margarya melanioides

1881. Margaria melanioides

1879.

$$
\text { - }
$$

Nevill, Journ. Asiat. Soc. Beng. XLVI, p. 30.

Nev. Nevill in Anderson, Exp. West Yunnan, p. 891, pl. LXXX, fig. ๖̊ (sub. nom. Paludina Margariana).

Nevild, New or little known, Moll. of the IndoMalayan Fauna in J. As. Soc. of Beng. L, part II, p. 155, pl. 5, fig. 1 (avec les var. tuberculata, carinata, rotundata.

1883. Vivipara Margeriana Nev. Neum A Y R, Über einige Süssw. Conch. aus China in Neues Jahrb. für Mineralogie 1883, II, p. 24, 25.

1884. Margarya melanoides G. Nevill, Hand List Moll. Ind. Mus., p. 33.

1885. Paludina (Margarya) melanoides Nev. P. Fischer, Man. de Conch., p. 753.

1890. Margarya melanioides Nev. Heude, Mem. Emp. Chinois, p. 178, pl. XXXIX, fig. 1 (tantum). 
1898. Vivipara (Tulotoma) Margariana Nev. var. tuberculata Neumayr, Ergeb.der Reise desGrafen Béla Széchenyi in 0stasien, 1877-1880. II, Süssw. Moll., p. 649. pl. III, fig. 2a, 2'

Le type décrit par Nevill a la spire médiocrement élevée, le dernier tour très large et des carènes décurrentes bien saillantes, coupées par des plis longitudiuaux qui déterminent des lamelles variqueuses et des tubercules. La coquille représentée par Heude, pl. XXXIX, fig. 1, concorde avec le type. La variété tuberculata de Neumayr ne peut être distinguée du type, son auteurl'ayant créée pour la forme typique elle-mếme.

Var, Delavayi J. Mabille

1886. Vivipara Delavayi

J.Mabille, Descr. de Vivipares nouvelles du Lac Ta-Li in Bull. Soc. Mal. Fr., p. 66, pl. II, fig. $1^{\mathrm{a}}$, $1^{\mathrm{a}}, 1^{\mathrm{b}}$.

Cette forme est intermédiaire entre le type et la variété carinata Neumayr.

\section{Var. conrinara Neumayr}

1883. Vivipara Maryeriana Nev. Neumayr, loc. supra cit., p. 25, fig. de gauche.

1890. Margarya melanioides Nev. Heude, loc. supra cit., pl. XXXIX, fig. 2 (tantum).

1898. Vivipara (Tulotoma) Margariana Nev. var. carinatu Neumayr, loco supra cit. pl. III, fig. $3^{\mathrm{a}}, 3^{\mathrm{b}}$. 


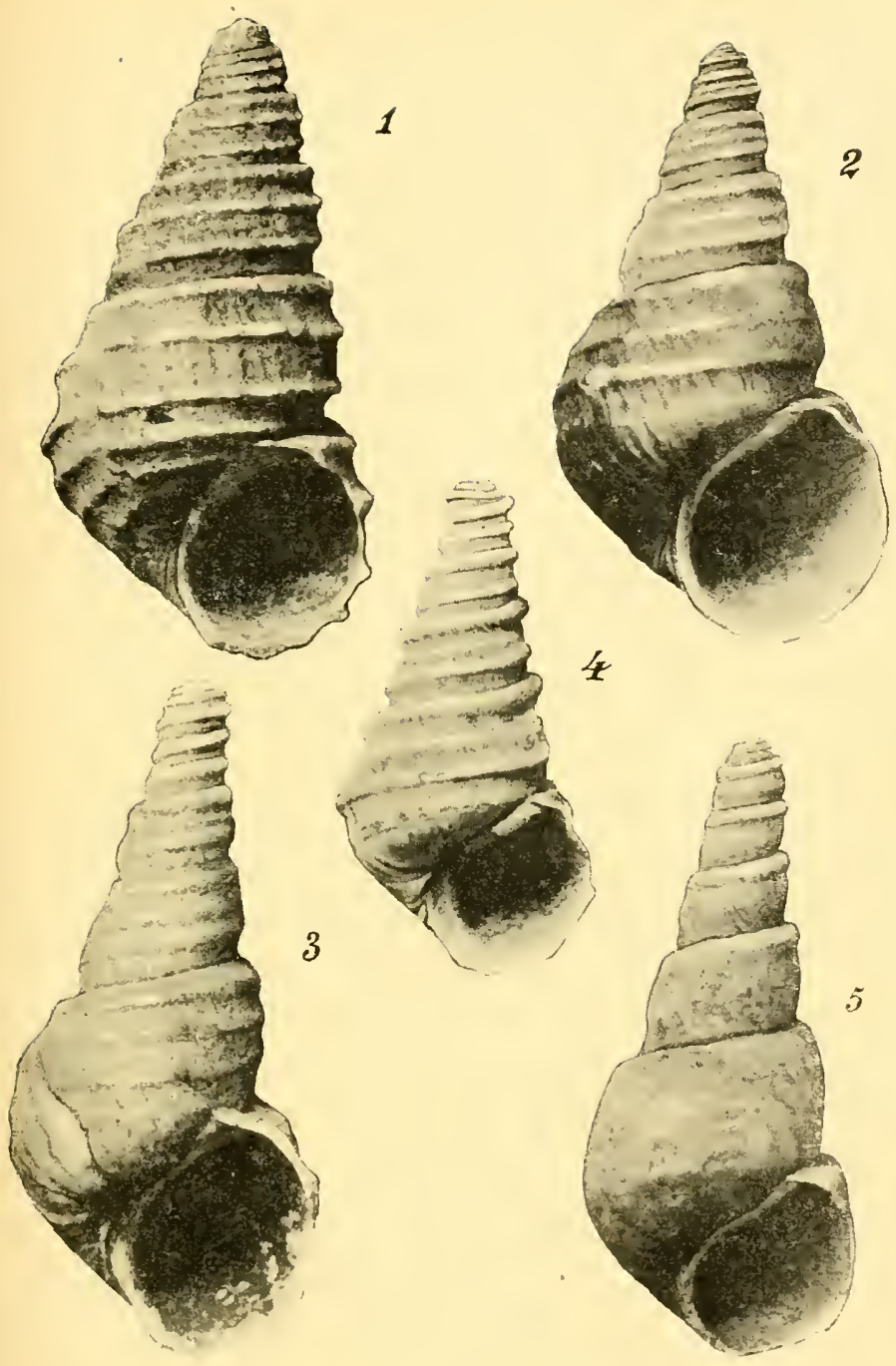

Fig. 1-3. - Margarya melanioides et variétés. 
Se distingue du type par la disparition des plis longitudinaux.

Var. Nonodi Dautzenberg et H. Fischer, n. var. (fig. 1)

Cette variété se distingue de toutes les autres par sa spire conique, son dernier tour non renflé, ses carèues décurrentes bien développées, au nombre de trois au lieu de deux sur chaque tour, et de six sur le dernier tour, y compris celles de la base qui sont bien développées; les carènes sont garnies de tubercules assez réguliers sur les tours supérieurs.

Nous dédions celte forme à M. Monod, Chef adjoint du Service Géologique, qui a fourni à M. Mansuy des renseignements utiles sur certaines stations de Margarya.

Var. Mansugi Dautzenberg et H. Fischer n. var. (fig. $2,3,4$ )

Cette variété est remarquable par sa spire très étroite et très allongée, son dernier tour médiocrement renflé, ses carènes décurrentes très saillantes et dépourvues de tubercules, ainsi que l'absence de plis longitudinaux. Par sa sculpture elle se rapproche de la variété carinata, mais sa forme est bien plus haute en proportion et presque scalaroïde.

Var. olssoleta Dautzeuberg et H. Fischer, n. var. (lig. 5)

De même forme très allongée que la var. Mansuyi, mais à carènes décurrentes presque entièrement effacées.

\section{Var. Francheti Mabille}

1883. Vivipara Margeriana Nev. Neumayr, loc. supra cit. p. 26, figure de droite. 
1886. Vivipara livancheti

J. Mabille, loc. supra cit.

p. 68, pl. II, fig. $2,2$.

1898. - ('Tulotoma) Margariana Nev., var. rotundata

Neumarr, loc. supra cit.

pl. III, fig. $4^{\mathrm{a}}, 4^{\mathrm{b}}$.

Celte variété ne possède qu'une seule carène décurrente située à la partie supérieure des tours. La var. rotunduta Neumayr est strictement syuonyme de cette var. Francheti.

\section{Var. tropidophora Mabille}

1886. Tivipara tropidophora

Mabille, loc. supra cit.

p. 70, pl. II, fig. 3,3 .

Décrite comme espèce spéciale par Mabille, cette variété est caractérisée par l'oblitération complète des carènes décurrents.

La forme typique du Margarya melanioides et les variétés Delavayi et Monodi sont représentées dans les récoltes de M. Mansuy par des exemplaires provenant du gisement quaternaire du déversoir du lac de Yunnan-Sen, à Koui-An.

La var. Mansuyi est plus abondante que les autres formes : M. Mansuy en a récolté des spécimens actuels (fig. 2) dans les lacs de la Chaussée, à Mong-Tsé; il l'a trouvée également dans les gisements quaternaires de Tong-Hai (fig. 3), jusqu'à 50 mètres d'altitude au-dessus du niveau du lac actuel, et dans le quaternaire de Mong-Tsé, près la ville (fig. 4).

La var. obsoleta n'a été recueillie que dans les gisements quaternaires des lacs de la Chaussée, plaine de Mong-Tsé (fig. כ̈) et de Tong-Hai.

Les var. Francheti Mab. et tropidophora Mab. n'ont pas été rapportées par M. Mansuy. 
Ampullaria gracilis Lea

190ว. Ampullaria gracilis Lea Dautzenberg el H. FıSCHEl, Journ. de Conch. LIII, p. 161.

Tonkin : Hanoï, Grand Lac (M. Mansuy).

Cette espèce est fort variable sous le rapport de la coloration : chez certains exemplaires, on voit de nombreuses bandes brunes très nettes, tandis que chez d'autres, ces bandes sont complètement effacées; mais on en aperçoit presque toujours des traces sur la paroi interne du dernier tour.

Ampullaria polita Deshayes

1905. Ampullaria polita Desh. Dautzenberg et H. FischEi, Journ. de Conch., LIII, p. $16 \mathrm{I}$.

Cochinchine : Mékong à Chaudoc (M. Mansuy).

Cambodge : Kampot (M. Mansuy).

Tonkin : Hanoï, Grand Lac (M. Mansuy).

Var. major Dautzenberg et H. Fischer n. var.

De grande taille, atteignant $90 \mathrm{~mm}$ de haut et $72 \mathrm{~mm}$ de diametre, un peu plus globuleuse que le type et avec la fente ombilicale plus accusée.

Cambodge: Gisement préhistorique de Somron-Seng (M. Mansuy).

\section{Ampullaria sumatrensis Philippi}

1851. Ampullaria sumatrensis Phusppl, Mon. in Conch. Cab., 2 édit., p. 59, pl. 19, fig. 1, 2, a.

1856. - ampullacea Reeve (non Linné) C. Icon., pl. X, fig. 48. 
Cambodge: Gisement préhistorique de Somron-Seng (M. Mansuy).

Cette espèce, signalée pour la première fois en IndoChine, est très voisine de l'A. turbinis, mais sa spire est plus saillante, et son dernier tour est plus globuleux. Le véritable $A$. ampullacea de Linnè, devenu A. Linnaei Philippi (pl. 20, fig. 6) est une espèce à spire très élevée, acuminée, très voisine et peut-être même synonyme de l'A celebensis Quoy et Gaimard.

\section{Ampullaria turbinis Lea}

185̋6. Ampullaria turbinis

1860. - celebensis

1864 .

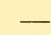

turbinis

$187 \ddot{3}$.

$18 i 6$.

1889.

1891.

1904.
LeA, Proc. Acad. N. Sc. Philad, vol. VIII, p. 110. v. MaR'Tens (non Quoy el G.), Proc. Zool. Soc., p. 12 (teste A. Morelet).

LeA, Obs. G. Unio, XI, p. 70, PI. XXII, fig. 2.

- Lea A. Morelet, Séries Conch. IV, p. 288 .

Crosse et Fischer, Journ. de Conch., XXIV, p. 313

L. Monlet, J. de Conch., p. 151.

P. Fischer, Catal. IndoChine, loc. cit., p. 180 (96 du t. à p.).

1891. - celehensis P. Fischer (non Quoy et G. Catal. Indo-Chine, loc. cit.,p. 182 (98 du t. à p.). H. Fischer et DautzenBerg, Catal. Indo Chine in Mission Pavie, loc. cil., p. $\$ 6$. 
Cambodge: Gisement préhistorique de Somron-Seng (M. Mansuy).

Var. erythroehila Dautzenberg et H. Fischer n. var.

Nous proposons ce nom pour une forme qui diffère du type par la coloration rouge du péristome.

Cochinchine : Mékong à Chaudoc (M. Mansuy).

Cralotus (Siphonociclotus) tener Menke

185̋6. Pterocyclos tener Meske, Malak. BI. III, p. 69 .

\begin{tabular}{|c|c|c|c|}
\hline $854-60$. & - & & $\begin{array}{l}\text { Pfeiffer, Novitates I, } \\
\text { p. } 90 \text {, pl. XXV, fig. } 13-15 \\
\text { (mala, juv.). }\end{array}$ \\
\hline 1858. & 一 & - & $\begin{array}{l}\text { - Preiffer, Mon. Pneum., } \\
\text { 1erSuppl., p. } 32 .\end{array}$ \\
\hline 1860. & Rhiostoma & & $\begin{array}{l}\text { - Benson, Ann. \& Mag. N. } \\
\text { H., } 3^{\text {d }} \text { ser., V, p. } 97 .\end{array}$ \\
\hline 1863. & Pterocyclos & & $\begin{array}{l}\text { - Reeve, Conch. Icon., pl. } \\
\quad \text { r, fig. } 28 .\end{array}$ \\
\hline $186 弓$. & Rhiostoma tene & rum & $\begin{array}{l}\text { - Pfeiffer, Mon. Pneum., } \\
\text { 2e Suppl., p. } 40 .\end{array}$ \\
\hline 1876. & - & - & $\begin{array}{l}\text { - Preiffer, Mon. Pneum., } \\
\text { 3० Suppl., p. } 46 .\end{array}$ \\
\hline 1891. & - & & $\begin{array}{l}\text { - P. Fischer, Catal. Iıdo- } \\
\text { Chine, loc. cit., p. } 185 \\
\text { (101 du t. à p.). }\end{array}$ \\
\hline 1897. & Opisthoporus $t$ & ner & $\begin{array}{l}\text { - Kobelt \& MöllendorfF, } \\
\text { Nachrichtsbl., p. } 119 .\end{array}$ \\
\hline $1898 ?$ & Opisthoporus & - & $\begin{array}{l}\text { - v. Möllendorf, Die Bin- } \\
\text { nenmoll. Annams in } \\
\text { Nachrichtsbl. d. d. Mal. } \\
\text { Ges., p. } 83 \text {. }\end{array}$ \\
\hline
\end{tabular}


1900. Cyclotus (Siphonocyclotus) tener v. MöLlendorfa, Zur Binnenmoll. Fauna Annams III, in Malak. BI., p. 133.

1901. Cyclotus (Siphonocyclotus) tenఃr v. MöllendorfF, Zur Binuenmoll. Fauna Annams, IV, in Malak. Bl., p. 50 .

1904. Rhiostoma tener Mke H. Fischer et DautzenBERg, Catal. Indo-Chine in Mission Pavie, loc. cit., p. 427 (38 du t. à p.).

Cochinchine : Hong-Chon (M. Mansuy).

Rhiostoma Monleti Dautzenberg et H. Fischer n. sp.

(Pl. X. fig. 1, 2, 3. 4)

1891. 'Pterocyclus planorbulus

L. МORLET (non Lk), Jour. de Conch., p. 247.

1891. ? Pterocychus - L.-M. H. Fischer et Dautzenberg (ex parte, non Lk). Catal. Indo-Chine in Mission Pavie loc. cit., p. 430 (41 du t. à p.)

Testa solida, discoidea, latissime umbilicata. Spira depressa complanata, apice vix prominulo. Anfr. 5 convexi sat celeriter accrescentes, sutura profunda et canaliculata juncti; ultimus prope suturam subplanatus, deinde convexus et antice rapide descendens ac breviter solutus. Superficies striis incrementi valde approximatis satque irregularibus sculpta. Pars supera anfr. ultimi insupra obscure malleata. A pertura obli. qua circularis. Peristoma incrassatum, breviter expansum, superne in tubulum latum et recurvatum productum.

Operculum calcareum, crassum, cyathiforme, extus concen- 
trice acute multispiratum, intus levis profundeque excavatum.

Color sub epidermide flavido allidus, superne fulvoflammulatus et linea peripheriali nigricunte circumdatus. P'eristoma et tubuhum albescentia.

Diam. maj. 30, min. 24, alt. 15; diam. apert. $111 / 2 \mathrm{~mm}$. (fig. 1, 2). Diam. maj. 36, min. 27, alt. 19 ; diam. apert. $14 \mathrm{~mm}$ (fig. 3, 4).

Coquille solide discoïde aplatie, très largement ombiliquée. Spire déprimée, plane, à sommet à peine saillant. Tours convexes, au nombre de $\ddot{3}$, croissant assez rapidement et séparés par ume suture profonde et canaliculée. Dernier tour aplati au-dessous de la suture, ensuite convexe, descendant rapidement et légèrement détaché ì son extrémité. Surface ornée de nombreuses stries d'accroissement assez irrégulières. La partie supérjeure du dernier tour présente en outre des malléations peu accusées. Ouverture oblique, circulaire. Péristome épaissi, étroitement dilaté et terminé au sommet par un tube large, recourbé, dont les bords sont rapprochés à la base et s'écartent vers le haut.

Opercule calcaire épais, cyathiforme : face externe multispirée et portant une carène lamelleuse ; face interne cupuliforme et lisse.

Coloration blanchàtre ornée, au-dessus, de flammules brunes très irrégulières plus ou moins disposées en zigzags et d'une bande périphériale étroite et d'un brun noirâtre. Base du dernier tour blanchâtre, dépourvue de taches. Un épiderme jaunâtre recouvre toute la coquille ainsi que la face externe de l'opercule. Péristome et tube blancs.

Laos: Luang-Prabang (Massie) — Tonkin : Ha-Giang (M. Mansuy).

- Grande forme se distinguant du Rh. Housci Haines par son dernier tour moins détaché, son péristome ordinairement plus évasé, son tube plus court et constam- 
ment ouvert en avant. Elle a été confondue par diversauteurs notamment par L. Morlet avec le Cyclophorus planorbulus Lamarck, de Poulo-Condore, mais elle s'en distingue par sa taille plus faible et ses tours croissant plus rapidement; en outre, chez le C.planorbulıs, le sommet de l'ouverture neforme pas de tube et ne présente qu'une légère expansion triangulaire.

Nous figurons, pl. X, fig. 1, 2, un des spécimens de la collection L. Morlet, provenant du Laos, étiquetés $C$. planorbulus. Les figures 3 el 4 représentent un individu récolté à Ha-Giang, par M. Mansuy, et appartenant à cette même espèce à laquelle nous donnons le nom de Rhiostoma Morleti pour la distinguer du véritable $C$. planorbulus Lamarck.

Le groupe des Rhiostoma de l'Indo-Chine a été bien mal précisé par les auteurs. Haines nous parait avoir confondu sous le nom de Housei, deux formes distinctes; Pfeiffer a décrit sous le nom de $R$ h. Hainesi une forme à dernier tour très détaché qui ne nous paraît différer en aucune façon de la figure 14 de Haines représentant le Rh. Housei. Il a de plus créé un $R h$. simplicilabre qui ne nous paraît pas autre chose qu'un jeune Rh. Hainesi à péristome moins épaissi ; enfin le I't. Marioni Ancey nous paraît identique au Ilainesi Pfr.

\section{Cyclophorus Courbetr Ancey}

1888. Cyclophorus Courbeti

Ancey, Mollusques du Haut-Tonkin, in Le Natuliste, p. $15 \mathrm{du} \mathrm{t}$. à $\mathrm{p}$.

1891 - Anc. P. Fischer, Catal. IndoChine, loc. cit., p. 188 (104 du t. à p.)

1904.

- Anc. H. Fischer et Dautzenberg, Catal. Indo Chine in Mission Pavie, p. 39. 
Cette espèce a été si bien décrite par M. Ancey, qu'on la reconnaît aisément, malgré la médiocrité de la figure de texte, qui ne montre ni le dessin ni le système de coloration signalés par l'auteur.

Très voisin du C. l'earsoni Benson, tel qu'il est représenté par Pfeilfer (Conch. Cab. 2e édit., p. 380, pl. 49, fig. 36, 36, 36) et par Hanley et Theobald (Conch. Indica p. 1, pl. I, fig. 6; pl. XLVlII, fig. 5), le C. Courbeti s'en distingue par sa spire plus élevée, ses tours plus étagés, son dernier tour moins aplati et plus caréné à la périphérie, sa surface plus lisse et plus luisante. Sa coloration présente aussi quelques différences: tandis que le Peursoni est orné, immédiatement au-dessous de la périphérie, d'un filet noir étroit et que la base du dernier tour est jaune clair uniforme ou traversé seulement par quelques linéoles décurrentes, chez le Courbeti, la bande subpériphérique est très large et accompagnée sur la base du dernier tour de plusieurs autres bandes larges et bien appa. rentes.

Ha-Giang (M, Mansuy).

Var. Ieucostoma Dautzenberg et H. Fischer, n. var.

Chez cette variété, le péristome est blanc, alors qu’il est d'un rouge vif chez le Courbeti typique. Nous possédons toute une série d'exemplaires recueillis dans le HautTonkin par le Colonel Messager, montrant que la teinte du péristome varie du rouge vif au blanc, en passant par l'orangé et un jaune plus ou moins clair. Parmi les spécimens rapportés par M. Mansuy, il s'en trouve également qui ont le péristome jaunâtre.

Ha-Giang (M. Mansuy). 
Crclophorus dodrans J. Mabille

1903. Cyclophorus dodrans J.Mab. Dautzenberg et H.Fischer, Journ, de Conch. LIII, p. 166.

Tonkin: Ha-Giang (M. Mansuy).

\section{Crclorhorus Jourdyi L. Morlet}

1905. Cyclophorus Jourdyi L. M. Dautzenberg et H. Fischer Journ. de Conch. LIII, p. 168.

Annam (M. Mansuy).

\section{Cyclophorus speciosus Philippi}

1847. Cyclophorus speciosus

Philippr mss. in Pfeiffer, Zeitschr. für Malakoz. p. 107.

1847. - $\quad-\quad$ Philippi, Zeitschr.für Ma lakoz. p. 123.

1850. Cyclostoma speciosum Ph. Pfeiffer, Monogr. in Couch. Cab. $2^{\text {o }}$ édit., p. 170, pl. 25, fig. 1 à 3 .

1852. Cyclophorus speciosus - Pfeiffer, Monogr. Pneumonop. viv., p. 56 .

1857. - Theobaldianus Benson, Ann. and Mag. of Nat. Hist. $2^{4}$ ser. XIX, p. 206.

1838. - speciosus Ph. Pfeiffer, Monogr. Pneum. suppl. I, p. 41.

1861. - - - Reeve, Conchol. Iconica, pl. I, fig. 4.

1863. - $\quad-\quad$ - Pfeiffer, Monogr.Pneum. suppl. II, p. 61 . 
1870. Cyclophorus speciosus

Ph. Hanley et Theobald, Collchol. Indica, p. 42, pl. CIV, fig. 4.

1870. - Theobaldianus Bens. Hanley et Theobald Conch. Indica, p. 1 pl. I, fig. 2 et var. p. $37, \mathrm{pl}$. CXLIV, fig. 2 .

1876. - speciosus Ph. Pfeiffer Monogr. Pneum. suppl, III, p. 404.

1889. - edulis J. MabiLle, Moll. Tonk. diagn. p. 14.

1891. - speciosus Ph. P. Fischer, Catal. IndoChine, loc. cit., p. 186 (p. 102 du t. à p.)

1903. - - - - Blanford, Land \& Frehsw. Moll. from Siam, in Proc. Mal. Soc. of Lond., p. 280.

1904. - - $\quad$ - $\quad-$ H. Fischer et DautzenBerg, Catal. Indo-Chine in Mission Pavie, loc. cit., p. 427 ( $40 \mathrm{du} \mathrm{t}$. à p.).

A notre avis, le $C$. Theobaldianus Benson est strictement synonyme du $C$. speciosus Philippi : la sculpture est identique chez les deux, composée de stries décurrentes nombreuses, fines et ondulées qui s'atténuent sur la base du dernier tour.

Le C. edulis Mabille, ne nous paraîtêtre qu'unevariétẻ du speciosus à sculpture plus faible, à péristome un peu inoins dilaté et à carène periphériale un peu plus accusée. Les nombreux exemplaires que nous avons sous les yeux, comprenant les spécimens typiques de l'edulis qui nous ont été obligeamment communiqués par le Muséum, démontrent qu'il ne s'agit là que de variations trop peu importantes pour justifier une séparation spécifique.

Ha-Giang (M. Mansuy). 


\section{Cyclophorus Klobukowskil}

1884. Cyclophorus Klobukowskii L. Mornet, Journ. de Conch. vol. XXXII. p. 39I, pl. XII, fig. 1.

1889. - $\quad$ L. Morlet, Journ. de
Conch. vol. XXXVII, p.
1891. - 153.

Cambodge : Pnom Roang (M. Mansuy).

\section{Myostoma breve Martyn}

1789. Lituus brevis

1828. Turbo Petiverianus

1828. Cyclostoma Petiverianum

1842.

1845. Lituus brevis
Marty, Fig. of non descr. shells, pl. 28 G.

Wood, Ind. test. Suppl. p. 18 , pl. 6 fig. 2 a.

Gray, in IVood, Suppl. p. 36 .

Reeve, Concl.. Syst. II, pl. 18', fig. $1 \%$.

Martin, Ed. Chenu p. 2I, pl. 8, fig. 2, 2 .

1846. Cycl. (Lituus) breve Mart. Pfeiffer, in Conch. Cab. 2éd.p. 166, pl. 24, fig. 1, 2. 1847. Myxostoma Petiverianum Gray Troschel, Zeitschr. für Malak., p. 44.

1847. - breve Mart. Pfeifler, Zeitschr. f. Malak., p. 111. 
1847. Cyclostoma Petiverianum Sowerbi, Thes. Conch. p. $1 \mid 6$, pl. XXV, fig. 100 , 101 .

1830. - - Petit, Catal. des Cyclostomes in J. de Conch. I, p. 43.

1851. I'terocyclos brevis Martyn Preiffer, Zeitschr. f. Malak., p.9.

18ว̌2. Pt. (Lituus) - - Preiffer, Mon. Pneum. p. 42 .

185ัŏ. Cyclophorus (Lituus) brevis Mart. Benson, Ann. and Mag. of Nat. Hist., 2d Ser. $\mathrm{XV}$, p. 16.

1858. - brecis Mart. PFemfer, Mon. Pneum. Ier suppl. p. 40.

1862. Cyclostoma lychnus Moreler, Rev. et Mag. de Zool. p. 478 .

1863. Pterocyclos brevis Crosse et Fischer, Journ. de Conch. XI, p. 364.

1863 Reeve, Conch. Icon. pl. I fig. 4a, 4b.

186io. Cyclophorus Pfeilfer, Mon. Pneum. 2 suppl. p. 60.

1866. Pterocyclos - Mart. Mabille et le Mesle, J. de Conch. XIV, p. 131.

1869. Myxostoma breve Frauenfeld, Verh. Zool. Bot. Ges. Wien XIX, p. 878.

1876. Cyclophorus irevis

Pfeiffer, Mon. Pneum. 3e suppl. p. 99.

1901. Myxostoma breve Mart. P. Fischer, Catal. IndoChine loc. cit. p. ISS, (104 du t. à p.)

190'. - - - H.Fischer et Dautzesiberg, Catal. Indo-Chine in Mis- 
sion Pavie loc. cit. p. 429 (40 du L. à p.)

Archipel des Pirates, Golfe de Siam (M. Mansuy). Forme un peu plus petite que le type: diam. maj. 26, min. 21, alt. $15^{\mathrm{mm}}$.

Leptopona vitreum Lesson (non Sow. Thes.)

Var, nitida Sowerby

1830. Cyclostoma vitrea Lesson, Voy. Coquille, p. 346, pl. 13, fig. 6. 1832. - luted Quor et Gamard, Voy. Astrolabe I1, p. 180, pl. 12, fig. 11.12 (non Lesson), 13, 14 var.

1838 vitrea Less. Deshayes in Lamarck, Anim. s. Vert., 2 éd., VIII, p. 367.

1842. - nitidum Sow. Reeve, Conch. Syst., pl. 183, fig. 2 (mala). 1843. - - Sowerar, Proc.Zool.Soc., p. 60 .

1846. - - Sow. Pfeiferer, Conch. Cab., 2. édit,, p. 96, pl. I6, fig. 10.

1846. - vitreum Pfelfrer, Conch, Cab., 2e édit., p. 158, pl. 28, fig. 16-18 (ex Quoy).

1847. - mitidum Sowerbi, Thes. Conch. I, p. 133, pl. 29, fig. 225227.

1847. Leptopoma vitrea Less. Preiffer, Zeitschr.f. Mal., p. 108.

1847. - nitidum Sow. Preirfen, Zeitschr. f. Mal., p. 108. 
1849. Cyclostoma vitreum Less. Mousson, Java Moll., p. 57 , pl. 6, fig. 4, 4, 4 (et var. fragilis Sow. mss.).

1852. Leptopoma - $\quad$ - PfelfFer, Mon. Pneum., p. 101 .

18วั2. Cyclophorus vitreus MörсH, Catal Yoldi, p. 42.

1858. Dermatocera vitrea Less. PFeiffer, Mon. Pneum., 1er Suppl., p. 78.

1858. - - - Addus, Genera of rec. Mol. II, p. 282, pl. LXXXV, fig. $7,7^{\mathrm{a}}, 7^{\mathrm{b}}, 7^{\mathrm{c}}$.

1862. Leptopoma vitreum Less. Reeve, Conch. Icon., pl. III, fig. 15a $15^{\mathrm{b}}$ et var. lutea, pl. VI, fig. 32 .

1863. Dermatocera vitrea - Wallace, Proc. Zool. Soc., p. 414.

1865. - - - Pfeifrer, Mon. Pneum., 2. Suppl.,p. 8ว̆.

1867. Leptopoma vitreum I. Martens (ex p.)Preuss. Exp. II, p. 66, 143, pl. 4, fig. $2^{a}, 2^{b}, 2^{c}$ (tantum).

1868. Dermatocera vitrea - Cox, Mon. Austr. landsh., p. 98. pl. 16, fig. 2, 2a, 3 .

1872. Leptopoma firagile Sow. Мӧвсн, J. de Conch., p. 317.

1876. - vitreum Less. Pfeiffer, Mon. Pneum., 3 Suppl., p. 127.

1881. - - D DE RocherRune, Doc. Faune Mal. Cochinchine et Cambodge, p. 29.

1889. - - Less. P.тец, Catal., p. 458 et var. luteum et nitidum.

1889. - - - L. Mlorier. J. de Conch., XXXVII, p. 152. 
1891. Leptopoma vitreum Less. P. Fischer, Catal. IndoChine, loc. cit., p. 190 (106 du t. à p.).

1904. - - - H. Fischer el Dautzerberg, Catal. Indo Chine, in Mission Pavie, loc. cit., p. 430 (4.1 du t à p.).

Cochinchine: Hong-Chon (M. Mansuy).

\section{Lagochilus Cros̀sei L. Morlet}

1886. Lagocheilus Crossei

1886 .

1887.

$-$

- L. II. 1891. Lagochilus - - P. Fischer, Catal. Indo-

L. Morlet, Diagn. Moll. Tonkin,p. 3.

L. Morlet, Journ. Conch, p. 260. 277, pl. XIII, fig. 2, $2^{\mathrm{a}}, 2^{\mathrm{b}}$.

J. Mabille, sur quelques Mollusques du Tonkin in Bull. Soc. Mal. Fr., p. 149. Chine, ioc. cit., p. 191 (107 du t. à p.).

1904. - - - H. Fischer et DautzexBerg, Catal. Indo-Chine in Mission Pavie, loc. cit., p. 431 (42 du t. à p.).

Tonkin : Ha-Giang (M. Mansuy).

\section{Lagochilus scissimargo Benson}

et var. major D, et H. F.

1903̈. Lagocheilus scissimurgo Bens. et var. mujor. DALTzeNBerg et H. Fischel, Journ. de Conch. LIII, p. 169.

Tonkin : Ha-Giang (M. Mansuy). 
Pupixa Douvillei Dautzeuberg et H. Fischer n. s. p. (Pl. $\mathrm{X}$ fig. 10, 11, 12)

Testa ouato-conoidea, ventrosa, tenuis, nitilissima, subpelln. cida. Spira brevis, conoidea, apice obtusiusculo. Anfr. 5 converiusculi, striis obliquis parum conspicuis ornati ac sutura simplici sejuncti. Anfr. ultimus vix desccudens. Apertma circularis, bicanaliculata. Canalis superus a lamina parietali valida formatus; canulis inferus angustus, sat elongatus. Columella callosa ; labrum superne pauluhm emarginatus.

Color albido hyalinus, peristoma album opacum.

Longit. 8 mill., latit. 5 mill., apertura 3 mill. alta, 3 mill. lata.

Coquille ovale-conoïde, mince, très luisante, subpel. Iucide. Spire courte, conoïde, obtuse au sommet, composée de š tours légèrement convexes, ornés de stries obliques peu apparentes et séparés par une suture simple. Dernier tour à peine descendant à son extrémité. Ouverture circulaire, bicanaliculée ; canal supérieur limité par une lamelle pariétale forte. Canal inférieur très étroit, allongé et limité par deux bourrelets calleux. Columelle calleuse. Labre peu épais, uu peu sinueux au-dessous du canal supérieur.

Tonkin : Ha-Giang (M. Mansuy).

Nous prions M. Douvillé, professeur à l'Ecole Nationale des Mines, d'accepter la dédicace de cette nouvelle espèce dont nous ne connaissons qu'un seul exemplaire.

\section{Pupina Verneaui Dautzenberg et H. Fischer n. sp.}

(Pl. X, fig. 13, 14, 15)

Testa ovato conoidea, nitida, solidinscula, sulprellucida. Spira conoilen, apice obtusiuscula. Anfi. 5 parum convexi, striis obliquis fere inconspicuis ornati suturaqne simplici juncti. Anfr. ultimus valde descendens; apertura circularis 
bicanaliculata, basi antice provecta; canalis superus angustus arcuatus, inferus angustissimus, sat elongutus ac callo circumscriptus. Columella areuata callosa. Labrum arcuatum, inferne callosum siperneque sinulum efformans.

Color pallide fulous, peristoma allum.

Longit. 7 mill., latit. 4 mill., apertura $21 / 2$ mill., alta, $21 / 2$ mill. lata.

Coquille ovale-conoïde assez solide, luisante, subpellucide. Spire conoïde, obtuse au sommet, composée de 5 tour's peu convexes, ornés de stries obliques à peine visibles à l'aide de la loupe, séparés par une suture simple, superficielle. Dernier tour Jien descendant à son extrémité. Ouverture circulaire, projetée en avant à la base, bicanaliculée, canal supérieur étroit, arqué, se prolongeant un peu en arrière du plan de l'ouverture ; canal inférieur extrèmement étroit, arqué et entouré d'une callosité; columelle arquée, calleuse. Labre arrondi, calleux à la base et sinueux au sommet.

Coloration d'un fauve très pâle, péristome blanc.

Toukin : Ha-Giang (M. Mansuy),

Cette espèce, que nous dédions à M. Verneau, assistant au Muséum de Paris, diffère de l'E. Donvillei par sa forme plus allongée, moins ventrue, par son dernier tour bien descendant àl'extrémité, par le canal supérieur de l'ouverture plus étroil, par le canal inférieur également beaucoup plus étroit, enfin par sa taille plus faible et sa coloration jaunâtre.

Hrbocrstis gravida Benson

190כ. Hybocystis gravida Bens. Dautzenberg et H. Fisснек, Jourı. de Conch. LIII, p. 171.

Tonkin: Ha-Giang (M. Mansuy). 


\section{Diplommatina Balansai L. Morlel}

1886. Diplomatina (Palaina) Balansai L. Morlet, Diagn. Moll. Tonkin. p. 5.

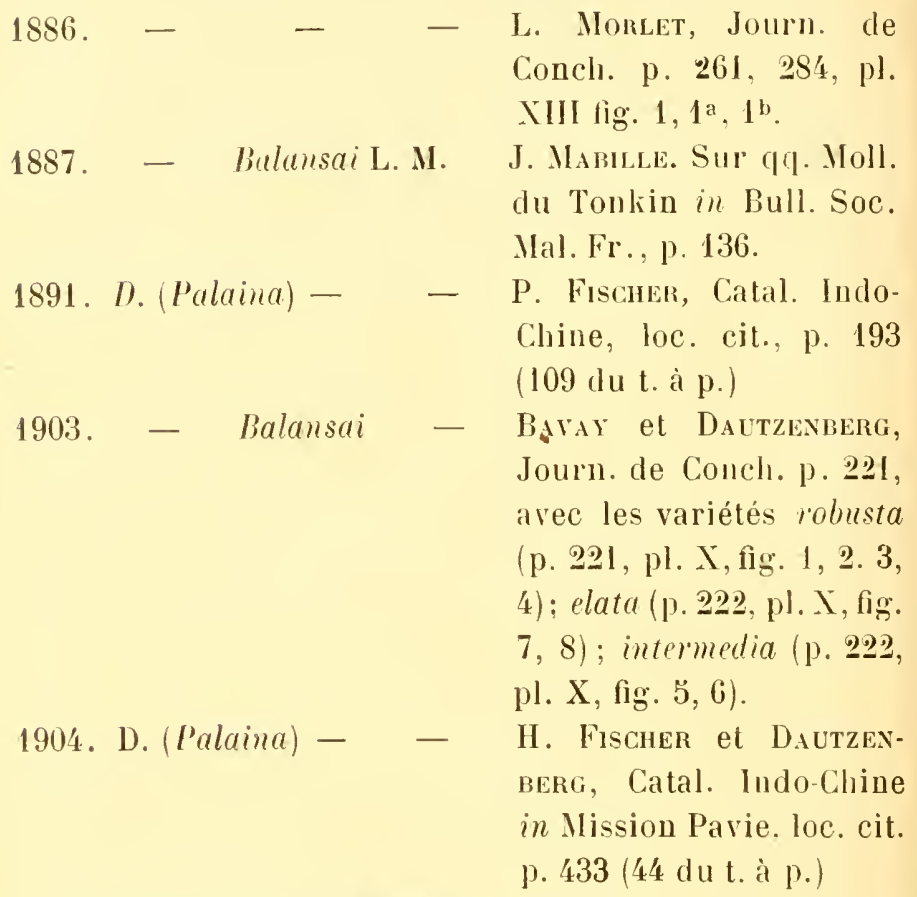

Tonkin: Ha-Giang (M. Mansuy). La forme recueillie par M. Mansuy appartient à la var. olbust a Bavay et Dautzenberg.

Diplomatixa Lavillei Dautzenbers et H. Fischer n. sp. (Pl. X, fig. 8, 9)

Testa parva dextrorsa, pupoidea, sat tumida, temieula subhyalina, rimata et anyuste perforata. Anfr. 7 valde convexi medioque angulati; anfr. penultimus quam ultimus tumidior; ultimus prope aperturam ascendens. Anfr. primi? 
leves, ceteri lamellis longitudinalibus temuissimis sat elevatis sigmoideisque orunti. Lamellarum interstitia transversim minutissime confertimque strinta. A pertura circularis, peristoma paululum incrassatum, haul continuum, marginibus callo debili adnatoque junctis. Columella armata, haud dentuta; labrum areuatum, extus subduplicatum.

Color albidus versus upicem fulvescens.

1 lt. $43 / 10$ mill., latit. $31 / 2$ mill., apertura $11 / 2$ mill., alta, $1 / / 2$ mill. lata.

Coquille de petite taille, mince, subhyaline, de forme pupoide assez renllée, pourvue d'une fente ombilicale large, terminée par une perforation étroite. Spire composée de 7 tours hien convexes, étagés, anguleux au milieu. Avant-dernier tour plus renflé que le dernier; dernier tour ascendaut à sou extrémité. Deux premiers tours lisses, les suivants ormés de lamelles longitudinales très délicates et sigmoïdes. Intervalles des lamelles sculptés de stries décurrentes fines et nombreuses. Ouverture circulaire. Péristome un peu épaissi, non continu, à bords reliés par une callosité faible. Columelle arquée non dentée. Labre arrondi, bordé extérieurement d'une lamelle plussaillante que les autres et qui le fait paraître dédoublé.

Coloration blanchàtre, sommet fauve.

Cambodge: Pnom Roang, daus une caverme (M. Mansuy).

Ce Diplommatina que nous dédions à M. Laville, prèparateur à l'Ecole Nationale des Mines à Paris, est remarquable par sa taille relativement grande, ses tours supérieurs anguleux et ornés de lamelles longitudinales délicates et sigmoïdes. 
Opisthostona ronkinunum Dautzenberg et H. Fischer n. $\mathrm{sp}$.

(PI. X, fig. $0,6,7$ )

Testa tenuis, imperforata. Spira pyramidalis. Anfr.6 convexi, sutura impressa sejuncti : primi 2 leves, ceteri lamellis filiformibus undulatis, numerosis, quam interstitia tamen multo angustioribus ornati. Anfr. ultimi dimidia pars ultima retorta, denique pauhulum ascendit. Apertura circularis; peristoma simplex continum.

Altit. 4 1/2 mill., diam. 3 mill. Apertura /1/2 mill. alta, $11 / 2$ mill. lata.

Coquille mince, imperforée. Spire pyramidale-conoïde composée de 6 tours courexes, séparés par une suture bien accusée. Deux premiers tours lisses. les autres ornès de lamelles filiformes ondulées, preu saillantes, nombreuses, séparées par des intervalles beaucoup plus larges qu'elles-mêmes, sauf à l'extrémité du dernier tour où elles se rapprochent au point d'être presque contiguës. A partir de la moitié environ de son développement, le dernier tour se replie brusquement en arrière. Il résulte de cette déviation que la coquille adulte semble être senestre, lorsqu'on la regarde du còté de l'ouverture, alors, qu'en réalité, l'enroulement des tours est dextre. Le dernier tour remonte un peu à son extrémité, de telle sorte que le haut de l'ouverture est situé à peu près à la moitié de la hauteur de l'avant-dernier tour. Ouverture circulaire; péristome simple, continu.

Les spécimens rapportés par M. Mansuy étant morts et défraîchis, nous ne pouvons donner aucun renseignement surr la coloration de cette espèce.

Tonkin (M. Mansuy).

La découverte de cette nouvelle forme est d'autant plus intéressante que la présence du genre Opisthostoma n'avait pas encore été signalée au Toukin. 
Crucibulum (Bicatillus) Renovatum Crosse et Fischer

1825. Calyptraea extinctorium Blanville (non Lamarcli) Man. de Mal. p. $506, \mathrm{pl}$. 48, fig. 8 .

1859. Crucibulum - Reeve (non Lamarck), Conch. Icon, pl. V, fig. $14^{\mathrm{a}}, 14^{\mathrm{b}}$.

1886. Cr. (Bicatillus) - Tryos (non Lamarck), Man. of Conch. VIII, p. 119 pl. 33, fig. 46-öl.

1887. Crucibulum - Sowerby (non Lamarck), Thes. Conch. V, p. 61, pl. 449, fig. 5ว3̆-60.

1889. - - - L Morlet (non Lamarck), Journ. de Conch. XXXV1I, p. $15 \%$.

1389. - - renocatum Crosse et Fisciler, Journ. de Conch. XXXYil, p. 288. 1891. - - Cr.et F. P. Fischer, Catal. IndoChine, loc. cit. p. 193, (109 du t. à p.)

Cochinchine: Hong-Chon (II. Mansuy).

Cambodge: Gisement préhistorique de Somron-Seng (M. Mansuy).

Natica Marochiensis Gmelin

1781. Neritæ maroccanæ

1790. Nerita marochiensis

1817. Natica maroccana

1822. - marochiensis
Chemnitz, C. Cab. V.p. 270, pl. 188, fig. 190弓-1910.

Guelin, Syst. Nat. Ed. XIII, p. 3673.

Dillwyn, Catal. I1, p. 983.

Lamarck, Anim. s. Vert. VI, 2 p. p. 203. 
1822. Natica unifusciata

1832. - marochiensis

1838. - unifasciata

1841. -- - -

18ว2. - maroccana Ch. Philippi, Mon.Conch. Cab.

18วั5. - marochiensis Gm.

1838. - - Ch.
pl. 32, fig. 13, 13․ Qอ éd. p. 78, pl. 12, fig. 1-5. Anim. s. Vert. 2 éd., VIII, p. 642.

Deshayes in Lamarck, Anim.s. Vert. 2 éd., VIII, p. 640.

Delessert, Recueil de Coq.

Reeve, Conch. Icon. pl. X!II, fig. 弓̈2.

1886. - - - - $\quad-$ Tryon, Man. of Conch.VIII p. 22, pl. 3 fig. $74-96, \mathrm{pl} .7$, fig. $36, \mathrm{pl}$. 8 fig. 49 .

Poulo-Condore (M. Mansuy).

Cette espèce n'a pas été signalée par P. Fischer dans son Catalogue de l'Indo-Chine.

Pyramidella (Otopleura) auris cati Ch.

1795. Voluta Auris Cati

Chennitz, Conch. Cab. Xl, p. 20, pl. 177, fig. 1711, 1712.

1817. - auris-cati

1822. Pyramidella plicata

1825. Voluta spiralis

1829. Pyramidella plicata Lk. Schubent\&Wagner, Conch. 
Cab. XII, p. 153, pl. 234, fig. $4100^{\mathrm{a}}, 4100^{\mathrm{b}}$.

1832. Pyramidella plicata Lk. Deshayes, Encycl. Méth. III, p. 863 .

1835 .
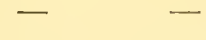

kíener, icon. Coq. viv. p. $\breve{3}, \mathrm{pl} .1$, fig. 4,4 .

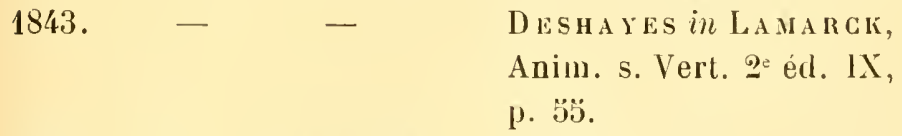

1855. - auris-cati Sowerby, Thes. Conch. II, p. 8I2, pl. CLXXII, fig. 1, 2.

\begin{tabular}{|c|c|c|c|c|}
\hline $1863 ّ$. & 一 & - & & $\begin{array}{l}\text { ReEve, C. Icon. pI. III, } \\
\text { fig. } 21 .\end{array}$ \\
\hline 1881. & 一 & 一 & Ch. & $\begin{array}{l}\text { De Rocherrune, Docum. } \\
\text { sur la faune malac. de } \\
\text { la Cochinchine et du } \\
\text { Cambodge, p. } 24 \text {. }\end{array}$ \\
\hline 1886. & P. (Otoplewra) & - & -- & $\begin{array}{l}\text { Tryon, Man. of Conch. } \\
\text { VIII, p. } 305, \text { pl. } 73 \text {, fig. } \\
93 .\end{array}$ \\
\hline 1891. & 一 & - & - & $\begin{array}{l}\text { P. Fischer, CataI. Indo- } \\
\text { Chine, loc. cit. p. } 197 \text { (113 } \\
\text { du t. à p.) }\end{array}$ \\
\hline
\end{tabular}

Poulo-Condore (M. Mansuy).

Neritina (Dostia) violacea Gmelin

1903. Neritina (Iostia) violacea Gm. Dautzenberg et H. Fiscuer, Journ. de Conch. LIII, p. 182.

Cambodge: Rivière de Kampot. Grande forme typique, à péristome sanguin sale. 


\section{Var. melanostoma Troschel}

1837. Neritina melanostoma Troschel, Archiv für Naturgeschichte, p. 179.

Cochinchine: Le Mékong, à Chaudoc (M. Mausuy). Exemplaires bien caractérisés par leur péristome noir.

\section{Patella eucosmia Pilsbry}

183̈́. Putella variegata

\begin{tabular}{|c|c|c|c|}
\hline 1870. & - & Rve & $\begin{array}{l}\text { P. Fischer, Journ. Conch. } \\
\text { p. } 167 .\end{array}$ \\
\hline 1871. & Helcioniscus & variegatus & $\begin{array}{l}\text { DALL, Amer. Journ. of } \\
\text { Conch. VI, p. } 277 \text {, pl. } 16 \text {, } \\
\text { fig. } 27 \text { (radula). }\end{array}$ \\
\hline 1891. & - & encosmia & $\begin{array}{l}\text { Pilsiry, Mall. of Conch. } \\
\text { XIII, p. 148, pl. 7I, fig. } \\
6 \mathrm{I}, 62,63,64 .\end{array}$ \\
\hline
\end{tabular}

Cochinchine: Hong-Chon, golfe de Siam (M. Mansuy).

\section{Patella chitonoides Reeve}

183̈4. Patella chitonoides

ReEve, Conchol. Iconica, pl. XX1, fig. $52^{\mathrm{a}}, 52^{\mathrm{b}}$.

1863.

Rve Deshayes, Moll. Réunion, p. 43.

1891. - pica (Rve) Pilsbry (ex parte) Manual of Conch. Str. and Syst. XIII, p. 97, pl. 26, fig. $28,29$.

Cochinchine: Hong-Chon (M. Mansuy). 
M. Pilșbry assimile le $P$. chitonoides Reeve au $I$. pica du même auteur; mais l'absence de matériaux suffisants ne nous permet pas de vérifier cette identification et les figures des deux formes dans le "Conchologia Iconica» nous paraissent bien différentes.

\section{Ostrea crenulifera Sowerby}

1871. Ostrea cremulifera

1881. Lopha

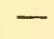

1891. Ostrea
Sowerbi, C. Icon. pl. XXVil, fig. $67^{\mathrm{a}}, 67^{\mathrm{b}}$.

Sow. De Rochebrune, Documents sur la faune malac. de la Cochinchine et du Cambodge, 1.4.

- P. Fischer, Catal. IndoChine, loc. cit, p. 207 123 du t. à p.)

Cochinchine: Hong-Clıon (M. Mansuy).

\section{Ostrea cucullata Borm}

1780. Ostrea cucullata

1785. Cornu copiae Ostrea

178ว. Ustrea Forskalii

1790 - Cornu copix

1790. - Forshalii

1797. - purpurea

1797. - Cornucopia

1817. - cucullate
Born, Mus. Caes. Vindob. p. 114, pl. 6, fig. 11, 12.

Chemitz, C. Cab. VIII, p. 41, pl. 74, fig. 679 .

Cheminz, C. Cab. VIII, p. 30 , pl. 72, fig. $671^{\mathrm{a}}, 671^{\mathrm{b}}$, $671^{\mathrm{c}}$.

Guetin, Syst. Nat., éd. XIII, p. 3336.

Ganeln, ibid.

Humphrex, Mus. Calonn. p. 83.

Humphrey, ibid.

Dillwye, Descr. Cat. I, p. 277. 
1817. Ostrea Forshalii

1819. - cucullata Born

1819. - cornucopix

1831. - cucullata

1831. - cornucopize

1836. - cucullata Born.

1836. - cornucopix

1842-58. - Cornucopia

1856. - Cucullata Born.
Dilliwys, ibid.

Lamarck, Anim. s. Vert. VI, p. 210.

LaMarci, ibid.

Deshayes, Encycl. Méthod. II, p. 296.

Deshayes, ibid.

Deshayes in Lamarck, Anim. s. Vert. 2e éd. VII, p. 230 .

Deshayes, ibid.

Chesu; Ill. Conch. pl. 3, fig. 3.

Hancey, Rec. biv. sh. p. 306.

1862. Alectryoniucucullata Born. Cuenu, Man. de Conch. II, p. 197, fig. 1000, 1003.

1871. Ostrea - Sowerby in Reeve Conch. lcon. pl. XVI, fig. $34^{\mathrm{a}}$, $34^{\mathrm{b}}, 34^{\mathrm{c}}$.

1891. - Forstali Ch. P. Fischer, Catal. IndoChine loc. cit. p. 207 (123 du t. à p.)

Cochinchine: Hong-Chou (M. Mansuy).

\section{Septifer bilocularis Linné}

1758. Mytilus bilocularis Linné, Syst. Nat., éd. X, p. $70 \%$.

1764. - - Linné, Mus. Lud. Ulr., p. 540 (excl. var.).

1767. - - - LinNé, Syst. Nat., éd. XII, p. 11336.

1785. - Nicobaricus, etc.Chennitz, Conch. Cab. VIII, p. 15马̆, pl. 82, fig. $737^{\mathrm{a}}, 737^{\mathrm{b}}$. 
1786. Mytilus bilocularis L. Schroeter, Einleit. in die Conchylienk. III, p. 431.

1790. - - Gueur, Syst. Nat., ed.

1819. - - L. Lamakck, Anim. s. Vert. II, p. 121 .

1836. - - Deshayes in Lamarck, Anim.s. Vert, 2e éd. V1I, p. 39 .

1839. Tichogonia - Anton, Verz., p. 17.

18... - - Küster, Mon. in Conch. Cab., 2 éd., p. 10, pl. 2, fig. $11-17$ (excl. var. pl. 3, fig. 6,7$)$.

18308. Mytilus Nicobaricus Ch. Reeve, Conch. Icon., pl. IX, fig. 42.

1881. Septifer bilocularis L. DE Rochebrune, Doc. sur la faune malac. de la Cochinchine et du Cambodge in Bull. Soc. Philom. p. 6.

1891. - - - P. Fischer, Catal. IndoChine, loc. cit., p. 214 (130 du t. à p.).

Tonkin: Baie d'Along (M. Mansuy), un exemplaire jeune.

\section{Anodonta Jourdy L. Morlet}

190弓. Anodonta Jourdyi L. M. Dautzenberg et H. FisCHER, J. de Conch., vol. LIII, p. $19 \%$.

Tonkin : Hanoï, Grand Lac (M. Mansuy). 


\section{Anodonta lugida Heude}

1905. Anodonta lucida Heude Dautzenserg et H. Fischer, J. de Conch., vol. LIII, p. 196.

Tonkin : Hanoï, Grand Lac (M. Mansuy).

\section{Pseudodon Moreleti Crosse et Fischer}

1874. Monocondylaea tumida Deshayes (non A. Morelet) Nouv. Arch. Mus., p. 117; pl. 5, fig. 1, 2, 3 .

1876. Pseudodon Moreleti Chosse el Fischer, J. de Conch. XXV, p. 330.

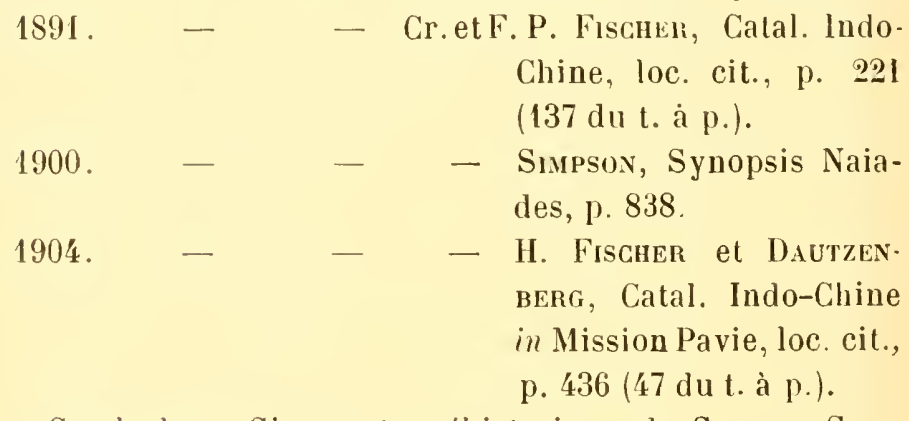

Cambodge: Gisement préhistorique de Somron-Seng (M. Mansuy).

Le $P s$. Moreleti est remarquable par sa grande taille, sa forme transversale et l'épaisseur de ses valves. Il se rapproche du Ps. Vondembuschianus Lea (= Zollingeri Mouss. = crispatus Mouss.) de Java, mais est moins ovale, plus trapézoïde, plus épais.

Nous possédons également du Cambodge des spécimens étiquetés par le $\mathrm{C}^{\mathrm{t}}$ Morlet sous le nom de P's. Moreleti mais qui nous paraissent identiques à la forme de Ps. Vondem. buschianus figurée par Clessin dans le Conch. Cab., pl. 98, fig. 3. Cette forme diffère du type de Lea par son diamètre 
antéro-postérieur plus grand par rapport au diamètre umbono-ventral.

\section{Dipsas plicatus Leach}

190כ. Dipsas plicatus Leach Diutzenberg et H. Fischer Journ. de Conch, vol. LIII, p. 196.

Cambodge: Somron-Seng et gisement préhistorique de la même localité (M. Mansuy).

\section{Unio Jourdy L. Morlel}

1905. Unio Jourdyi L. M. Dautzenberg et H. Fischer Journ. de Conch., vol. LIII, p. 20 '.

La forme typicque de celte espèce n'est pas représentée dans les récoltes de M. Mansuy.

Var. ponderosin Dautzenberg et H. Fischer n. var.

Cette variété se distingue de l'U. Jourdyi typique par son test beaucoup plus épais, sa région antérieurs plus haute et bien plus renflée, ses crochets plus proéminents.

Tonkin : Hanoï, Grand Lac (M. Mansuy).

\section{Unio (Nobularia) Dautzeniergi L. Morlet}

1889. Unio Dautzenbergi

L. Morlet, Journ. de Conch. vol. XXXVII, p. 190, pl. VIII, fig. כั.

1891

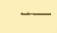

1900. Nodularia -
. P. Fisciner, Catal. IndoChine, loc. cit. p. $224(140$ du t. à p.).

- Simpson, Synopsis Naiades p. 819 . 
1904. Unio - - H.Fischer el Dautzenberg Catal. Indo-Chine, in Mission Parie, loc. cit. p. 437 ( 48 du t. à p.)

Cambodge : gisement préhistorique de Somron-Seng (II. Mansuy),

Nous possédons de la rivière de Sutrang (qu’on doit écrire Patrang suivant M. Pavie) un exemplaire de la collection L. Morlet qui est bien l'U. Dautzenbergi mais qui est étiqueté $U$. msticus Lam.

\section{Uxio (Nodularia) scorinatus Lea}

1S3̈6. Unio scobinata

183̈\%. - scobinatus
1858. $-\quad-$
1860. $-\quad-$
1864. - mandarinus
$1863 . \quad-\quad$ pellis-lacerti
$1866 . \quad-\quad$ venustus

LEA. Proc. Acad. N. Sc. Philad. VIII, p. 93.

LEA, Obs. G. Unio V1, p. 19. pl. 26, fig. 3.

LEA, Journ. Acad. N. Sc. Philad. III, p. 299, p]. 66, tig. 13 .

v. Martens, Proc. Zool. Soc. Lond. p. 15.

Morelet, Jour, de Conch. XIl, p. 159 (teste ipso: Séries Conch.).

MoRelet, Journ. de Conch. XIII, p. 2.2.

Morelet (non Lea) J. de Conch. XIT, p. 63 (rar. teste ipso: Séries Conch.).

1S68. - scobinatus Lea Sowerbi, Conch. Icon. pl. LxIII, fig. 313 .

1868. - pellis-lacerti
Soweris, Conch. Icon. pl. LXXV1, fig. 457 (= scobinutus L.ea) 
1870. Margaron (Unio) scobinatus LEA, Synopsis [.. 32.

1870. Unio oblitus

LEA, Synopsis p. 6\%, note (nom. subst. à $U$. venustus Morelet).

1875. - scobinatus Lea Morelet, Séries Conch.IV, p. 3ว̈4 pl. XVII, fig. 2, 6.

1875. - pellis-lacerti Morelet, ibil. p. 3:\%, pl. XVII, fig.

1876. - scobinatus Lea Crosse et Fischer, Journ. de Conch. XXIV, p. 326 . 1876. - pellis-lacerti Chosse et Fischer, Journ. de Conch. XXIV, p. 327.

1889. - - - Morel. L. Morter, Journal de Conch. XXXVII, p. 164.

1889. - scobinatus Lea L. Morlet, Journ. de Conch. XXXVII, p. 164.

1890. - (Iridea) - - Petel, Catál. III, p. 167. 1890. - mandarinus Mor. P.ETEL, Catal. III, p, 168. 1890. - (Iridea) pellis-lacerti M. Petel, Catal. III, p. 162.

1890. - venuslus Mor. Petel, Catal. III, p. 171. 1891. - scobinatus Lea P. Fischer, Cátal. IndoChine loc. cit. p. 22:) (141 du t. à p.)

1891. - pellis-lacerti Mor. P. Fischer, ibidem.

1960. Nodularia scobinala Lea Simpsos, Synopsis of the Naiades, p. $81 \%$.

1904. Unio scobinalus Lea

H. Fischer EL DAUTZENBERG Catal. Indo-Chine in Mis. sion Pavie loc. cit. p. 4.39 (วั0 du t. à p.).

1904. - pellis-lacerti Morelet H. Fischer et Dactzenberg, ibid.

Cambodge : Somron-Seng et gisernent préhistorique de Somron-Seng (M. Mansuy). 
Unio delphinopterus Dautzenberg et H. Fischer nom. subst. 184. Unio delphims

E. Ch. L. Grüner (non Spengler) Arch.f. Naturg. I, p. 276, pl. LX, fig. 1, $1 \mathrm{a}, 1 \mathrm{~b}, 1 \mathrm{c}$.

\begin{tabular}{|c|c|c|c|}
\hline 1841. & - & - & $\begin{array}{l}\text { Gr. Delessert, Rec. de Coq. } \\
\text { pl. XIX, fig. } 3,3 \mathrm{a}, 3 \mathrm{~b} \text {. }\end{array}$ \\
\hline 1842. & - & - & 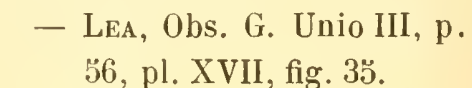 \\
\hline 1843. & - & 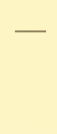 & $\begin{array}{l}\text { - Lea, Trans. Am. Phil. } \\
\text { Soc. VIII, p. } 218, \mathrm{pl} . \\
\text { XVII, fig. } 35 .\end{array}$ \\
\hline
\end{tabular}

1852. - Delphinus - Küster, Conch. Cab. 20 Ed., p. 18, pl. 2, fig. 2-4.

1852. Margaron (Unio) delphinus Gr. LEA, Synopsis p. 19.

185\%6. Unio delphinus

Gr. Hanley, Rec. Bir. Sh. p. 381, pl. XX, fig. 44.

1857. Unio (Metaptera) delphinus Gr. H. \& A. Adams, Gen. of rec. Moll. Il, p. 498.

\begin{tabular}{|c|c|c|c|c|}
\hline 859 . & - & - & - & $\begin{array}{l}\text { Chenu, Man. de Conch. p. } \\
\text { 143, fig. } 707 .\end{array}$ \\
\hline 1864. & - & megapteru. & & $\begin{array}{l}\text { Morelet (non Metaptera } \\
\text { megaptera Rafinesque nec }\end{array}$ \\
\hline$:$ & & & & $\begin{array}{l}\text { Unio megaptera Chenu } \\
\text { 1845) J. de Conch. XII, p. } \\
159 \text { (juv., teste ipso: Séries } \\
\text { Conch.) }\end{array}$ \\
\hline 1864. & - & 一 & Mor. & $\begin{array}{l}\text { Crosse et Fischer J. Conch. } \\
\text { XII. p. } 325 .\end{array}$ \\
\hline 1866. & 一 & deiphinus & Gr. & $\begin{array}{l}\text { Mabille et Le Mesle, Journ } \\
\text { de Conch. XIV, p. } 121 .\end{array}$ \\
\hline 1866. & - & 一 & - & $\begin{array}{l}\text { Sowerby, Conch. lcon. pl. } \\
\text { XLIII, fig. } 238 .\end{array}$ \\
\hline 870. & - & - & - & LeA, Synopsis, p. 28. \\
\hline
\end{tabular}




\begin{abstract}
1875. Unio delphinus Gr. Morelet, Séries Conch. IV, p. 345.

1889. Metaptera - - L. MorLet, Journ. de Conch. XXXVII, p. 163. 1890. Unio $\quad-\quad-$ Pdetel, Catal. III, p. 150. 1890. - megapterus Mor. Pareu, Catal. III, p. 158. 1891. - delphinus Gr. P. Fischen, Catal. IndoChine, loc. cit. p. 222 (138 du t. à p.)

1900. Myriopsis bialatus Simpson (non Unio bialatus Deshayes 1839) Synopsis Naiades, p. 579 .
\end{abstract}

1904. Unio delphinus Gr. H. Fischer et DautzenBerg, Catal. Indo-Chine in Mission Pavie loc. cit. p. 437 (48 du t. à p.)

Cambodge : Gisement préhistorique de Somron-Seng (M. Mansuy).

M. Simpson a remplacé le nom bien connu d'Unio delphinus à cause de l'existence d'un $U$. delphinus de Spengler différent de celui de Grüner et plus ancien. Il n'a pu reprendre le nom megapterus Morelet, à cause de l'existence d'un Metıptera megaptera Rafinesque cité par Chenu sous le'nom d'Unio megaptera. Dans ces circonstances, il a proposé le nouveau nom de bialatus.

- Malheureusement le nom de Unio bialatus ayant déjà été employé dès 1839 par Deshayes, pour un Dipsas, nous croyons préférable de lui substituer le nom de delphinopterus, car nous ne pensons pas que le genre Iyjriopsis s'isole assez des autres groupes d'Unionidés pour qu'il soit possible d'y admettre des noms spécifiques déjà employés dans ces groupes. 


\section{Chama fibula Reeve}

1846. Chama fibula

1889. - -
ReEve, Conchol. Iconica pl. V, fig. 27.

Rve Clessin, Monogr. in Conch. Cab. 2e Ed. p. 50, pl. 20, fig. 4 (mala).

Cochinchine : Hong-Chon (M. Mansuy).

\section{Crista divaricata Chemnitz}

1778. Venus incrustata

1787. Chamæformis Persicites
Born (non Linné), Index rer. Mus. Cæs. Vindob., p. 60 .

1780. - - Bors (non Linné), Test. Mus. Cæs. Vindob., p. 73.

1782. - divaricata etc. Chemnitz, Conch. Cab. VI, p. 317, pl. 30, fig. 316 .

Meuschen, Mus. Gevers. p. 450 .

1790. Venus divaricata

Ch. Gmelin, Syst. Nat., édit. XIII, p. 3277.

An $\mathrm{X}$

1815. Paphia

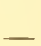

1817. Venus

1818. Cythera

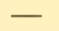

1818. - testudinalis

1825. Venus divaricata

1828.
- Bosc, Hist. Nat., p. วั2.

- B R 0 o Kes, Introd, to Conch., p. 66, pl. 2 , fig. 24.

- Dillwyn, Descr. Catal. I, p. 200.

- Lamarck, Anim. s. Vert. V, p. 578.

LAMARCK, ibid.

Wood, Ind. testac. p. 39, pl. 8, fig. 94 .

Gm. Blainville, Dict. des Sc. Nat., LVII, p. 272. 
1835. Cytherea divaricata

DESHAYEs in LAMARCK, Anim. s. Vert. 2e édit. VI, p. 324 .

1835. - testudinalis DeshaYes, ibid. p. 320ั.

1839. Venus (Cytherea) dixaricata Ch. Anron, Verzeichn. p. 8.

1842. Cytherea divaricata Ch. Hanler, Rec. biv. Sh., p. 108.

1842-58. - - - Chenu, Illustr. Conch., pl. 13, fig. 1, $1^{\mathrm{a}}, 1^{\mathrm{b}}, 2$, $2^{\mathrm{a}}, 3,3^{\mathrm{a}}$.

1842-58. - testudinalis Lk. Chenu, Illustr. Conch., pl. 13, fig. 4, 4a, 4b.

1853. Venus divaricata

Deshayes, Cat. Brit. Mus. p. 88.

1853. Circe Mörch, Catal. Yoldi II, p. 25.

185̌5. ! Circe æquivoca Sowerby (non Ch.), Thes. Concin II, p. 65̆0, pl. CXXXVII, fig. 12-15.

1857. Venus divaricata RöMER, Krit. Unters. p. 66 .

1858. Circe - $\quad$ Ch. H. \& A. Adams, Gen. of rec. Moll. II, p. 429.

1863. Cytherea (Crista) divaricata Ch. Römer, Malak. Bl. X, p. 20.

1863. Circe divaricata Ch. ReEve, Conch. Iconica, pl. VI, fig. $23^{\mathrm{a}}$ à $23^{\mathrm{d}}$.

1869. Cytherea (Crista) divaricata Römer, Monogr. der Molluskengattung Venus I, p. 181, pl. XLIX, fig. $1^{\text {a }}$ à $1^{\mathrm{d}}$, pl. LI, fig. 5 .

1869. - divaricata Ch. PFelpFer, Mon. Conch. Cab., $2 \circ$ édit., p. 44, pl. 16, fig. 8. 
1881. Circe divaricata

1889.

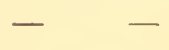

Ch. De Rochebrune, Docum. sur la faune malac. de la Cochinchine et du Cambodge, p. 14 .

- L. Morlet, Journ. de Conch. XXXVII, p. 169.

1891. Circe (Crista) divaricata Ch. P. Fischen, Catal. IndoChine, loc. cit., p. 231 (147 du t. à p.).

Poulo-Condore (M. Mansuy).

Dosinia Fibula Reeve var.

1862. Dosinia fibula Reeve var. Röner, Monogr. der Molluskeng. Dosinia, p. 45, pl. XII, fig. 2, 2a, $2^{\mathrm{b}}$.

Poulo-Condore (M. Mansuy).

Le type de cette espèce, non encore signalée en IndoChine, a été décrit par Reeve comme provenant de la Sénégambie, tandis que la variété indiquée par Römer l'a été, d'après des spécimens du Musée de Copenhague étiquetés comme provenant de Tranquebar. Cette variété ne se distingue du type que par ses cordons concentriques pluslamelleux aux extrémités latérales et par l'angle postérieur plus visible. Nous avons reçu la même forme des mers de Chine.

\section{Cyclina Chinensis Chemnitz}

1788. Venus chinensis

1790. - sinensis

1817. - Chinensis
Chemnitz, Conch. Cab. X, p. 356, pl. 171, fig. 1663 . Gmelin, Syst. Nat. éd. XIII, p. 3285.

Dillwyn, Descr. Catal. I, p. 192. 
1818. Cyprina tenui-stria

1825. Venus Sinensis

1833. - chinensis Ch.

183ว̈. Cyprina tenui-stria

1842. Venus Sinensis

1843-כั0. Cyclina Chinensis

185̆0. Artemis

1833. Cyclina

1855. Artemis

1866. Cyclina chinensis

1886. - Chinensis

1891

\section{Meretrix meretrix Linné}

1905. Meretrix meretrix L. 116. fig. 20-22. p. 30 . p. 118. du t. à p.)
Lamarck, Anim. s. Vert, $V$, p. วૅอัS (วั68).

Wood, Index testac. p. 38, pl. 8, fig. 76.

Desilayes in Lamarck Anim. s. Vert, $2^{\ominus}$ éd. VI, p. 291 (note).

DESHAYES in LAMARGK, ibid. p. 291.

Ch. Hanlex, Rec. Biv. Sh., p.

Deshayes, Traité Elém. 2e p., p. 626, pl. 14 bis,

Reeve, C. Icon, pl. I, fig. 6. Deshayes, Catal. Br. Mus.,

Ch. Sowerby, Thes. Conch. p. 661, pl. CXLI, fig. 23, 24.

- Mabille el le Mesle, Journ. de Conch. XIV,

- Dautzenberg et D'HamonVILLE, Journ. de Conch. XXXV, p. 224.

- P. Fischer, CataI. IndoChine, loc. cit. p. 332 (148

Tonkin : Baie d'Along, golfe du Tonkin (M. Mansuy).

Dautzenberg et H. FisCHнR, Journ. de Conch., vol. LIII, p. 216. 
Toulin: Baie d'Along (M. Mansuy). La forme typique et les variétés castanea Lamarck, graphica Lamarck, zonaria Lamarck.

\section{Meretrix lyrata Sowerby}

1853. Cytherea lyrata

Sowerby, Thes. Conch., p. $621, \mathrm{pl}$. CXXIX, fig. 61 (fig. médiocre).

1853. Meretrix - Sow. Deshayes, Catal. Br. Mus., p. 40 .

1864. Cytherea - Reeve, Conch. Icon., pl. IV, fig. 13 (fig. bonne).

1864. Cytherea (Meretrix) lyrata Sow . Crosse et Fischen, Journ. de Conch. XII, p. 324.

1869. Meretrix lyrata Sow.

Römer, Mon. der Molluskeng. Venus, p. 36. pl. $\mathrm{XI}$, fig. $3,3^{\mathrm{a}}, 3^{\mathrm{b}}$

1899. - - L. Morlet, Journ. de
1891. Conch. XXXVII, p. 168.
P. Fischer, Catal. Indo-
Chine, loc. cit., p. 233
(149 du t. à p.).

Tonkin: Baie d'Along (M. Mansuy).

\section{Venus (Cryptogramma) impressa Anton}

1837. Venus impressa

1839.

1853. Cryptogramma -
Anton, Wiegman's Arch.

f. Naturg. III, vol. I, p. 284 .

Anton, Verzeichn. der Conch., p. 9.

Mörce, Catal. Yoldi II, p. 22. 
18333. Anomalocardia Sow.

185ว. Venus flexuosa

1856. impressa

Deshayes, Catal. Brit. Mus., p. 118.

Sowerby (non L.) Thes. Conch., p. 716, pl. CLVI, fig. 85,86 .

\begin{tabular}{|c|c|c|c|}
\hline 18536. & - & impressa & $\begin{array}{l}\text { Hanley, Biv. Shells, Ap- } \\
\text { pend., p. } 362 \text {, pl. } 16 \text {, fig. } \\
27 \text {. }\end{array}$ \\
\hline 1857. & - & - & $\begin{array}{l}\text { Römer, Krit. Unters., p. } 34 \\
\text { (Obs.). }\end{array}$ \\
\hline $185 \%$. & Cryptogramma & - & $\begin{array}{l}\text { H. \& A. AdaMs, Gen. of } \\
\text { rec. Moll. II, p. } 420 \text {. }\end{array}$ \\
\hline $\begin{array}{l}1867 . \\
1869 .\end{array}$ & Venus impressa & $\begin{array}{r}- \text { Ant. } \\
\text { Ant. }\end{array}$ & $\begin{array}{l}\text { Römer, Malak. Bl. p. } 27 . \\
\text { Pfeiffer, Conch. Cab. } \\
\text { Veneracea, p. } 200 \text {, pl. } 32 \text {, } \\
\text { fig. } 7,8 \text {. }\end{array}$ \\
\hline
\end{tabular}

Poulo-Condore (M. Mansuy).

Cette espèce n'avait pas été signalée jusqu’à présent sur les côtes de l'Indo-Chine.

\section{Venus (Chyptograma) squamosa Linné}

1903. Venus (Cryptogramma) squamosa L. Dautzenberg et H. Fischer, Journ. de Conch., vol. LIII, p. 222.

Poulo Condore (M. Mansuy).

\section{Tapes striatus (Chemnitz) Gmelin}

1782. Venus literata, etc.

1782. Venus striata, etc.

1786. die gestreifte Venus
Chemnitz, Conch. Cib. VI, p. 33̆7, pl. 34, fig. 364 .

Снемnitz, ibid. p. 358, pl. 34 , fig. $365,366$.

Schröter, Einleitung, III, p. 164. 
1789. Venus striata Chemnitzii Karsten, Museum Leskeanum, p. 16 .

1790. - -
1790. - japonica
anX. - striata
an X. - japonica
$1817 . \quad-$ striata
$1817 . \quad-$ japonica
$1818 . \quad-$ eleyantina
$1818 . \quad-$ vermiculosa
$1825 . \quad-$ striata
18 25.

1828. - aurisiaca

1841. - elegantina

1842. - striata

1842. - japonica

1842. - Vermiculosa lk. Hanley, ibid. p. 125.

1849. - striata

1850. - Labuana

1853. Chione striata

Ch. Gulizin, Syst. Nat. éd. XIII p. 3279 .

Gmelis, ibid.

Bosc, Hist. Nat. III, p. כ็วั. Bosc, ibid.

Ch. Dillwyn, Catal. I, p. 183. Dillwyn, Catal. I, p. 182. Lamarck, Anim.s. Vert., V, p. 606.

LaMARCK, ibid. p. 604.

Wood, Ind. test. p. 36, pl. 7, fig. 36 .

Woov, ibid. p. 36, pl. 7 fig. รัวั.

GraY in Wood, Ind. test. Suppl. p. 5, pl. 2, fig. 12.

Delessert, Rec. Coq. pl. 11, fig. 3.

Ch. Hanlex, Rec. Biv. Sh.p. 127.

HANLEY, ibid. p. 127, pl. 13, fig. 46 .

Gm. Philippi, Abbild. III, p. 29

pl. VIII, fig. 5, 丂 6.

Adans \& Reeve, Voy. Samarang, p. 79, pl. XXI, fig. 16.

Ch. Deshayes, Catal. Br. Mus., p. 14.5 .

18333. - elegantina Lk. Deshases, ibid., p. 148.

1853 ? - vermiculosa Deshayes, ibid., p. 1309. 
1853. - Philippii

1853. - Japonica

1853. - regularis

1853. Venus striata

185ว. - Labuana

1856. Tapes caledonica

1857. Venus striata

1864. Tapes

1864. - Philippii

1864. - striata

1869. Venus

1872. Tapes
Deshayes, ibid., p. 146 (= striata s. s.t. Römer). Deshayes, ibid., p. 146. Deshayes, P. Z. S. L., p. 7 .

Ch. Sowerby, Thes. Conch. II, p. 735, pl. CLII, fig. 207, 209, 211.

Sowerby, Thes. Conch., p. 735, pl. CLXII, fig. 214. Bernardi, Journ. Conch. V. p. 82, pl. 3, fig. 7 .

Ch. Römer, Kritische Untersuch., p. 74 (excl, syn. tristis Lamarck).

- Römer, Malak. Bl. XI, p. 86 .

Reeve, C. Icon., pl. VIII, fig. $36^{\mathrm{a}}, 36^{\mathrm{b}}$.

- Reeve, C. Icon., pl. IX, fig. $45^{\mathrm{a}}, 45^{\mathrm{b}}$.

- Pfeiffer, Mon. in Conch. Cab., 2॰ éd., p. 127, pl. כ̌, fig. 12, 14 et var. fig. 13. - Römer, Monogr. der Molluskeng. Venus II, p. 101, pl. XXXV, fig. 1, $1^{\mathrm{a}}$ à $1^{\mathrm{k}}$.

Poulo-Condore (M. Mansuy).

Cette espèce ne figure pas dans le Catalogue de P. Fischer. Nous la possédons également de Tourane (Annam). 
Corbicula fluminea Müller

1903. Corbicula fluminea Müller Dautzenberg et H. FisCHER, Journ. de Conch. vol. LIII, p. 220.

Cochinchine : Saigon (M. Mansuy). Un spécimen complet et quelques valves isolées correspondant assez bien à la forme figurée par Chemnitz en 1782, et que nous considérons comme jeune.

Var. tonkiniana L. Morlet

1903̈. Corbicula fluminea Müller var. tonkiniana L. M. Dautzenberg et H. FisCHer, Journ. de Conch. vol. LIII, p. 227.

Tonkin : Hanoï, Grand Lac (M. Mansuy).

Var. Petiti (Clessin) L. Morlet, Moreletiana Prime Bocourti A. Morelet

1905. Corbicula fluminea Müller var. Petiti (Cl.) L. M., Moreletiana Pr., Bocourti A. II. Dautzenberg et H. FisCHER, Journ. de Conch. vol. LIII, p. 227 .

Cambodge: Gisement préhistorique de Somron-Seng. Exemplaires complets et valves des variétés Moreletiana et Petiri qui se relient intimement l'une à l'autre; une valve de la variété Bocourti.

Corbicula Souverbieana Wattebled (emend.)

1886. Corbicula Souverbicana

WATTEBLED, Jour n . Conch. XXXIV, p. 70, pl. V, fig. 4, 4, 4. 
1891. Corbicula Souverbiana IV. P. Fischer, Catal. IndoChine, loc. cit. p. 239 (15ว du t. à p.)

1904. - Souverbieana W. H.Fischer et Dautzenberg, Catal. Indo-Chine, in Mission Pavie, loc. cit. p. 442 (53 du t. à p.)

Cochinchine: Saigon (M. Mansuy).

Cette espèce nous paraît bien caractérisée par sa forme de Vénéridé. C'est une coquille plutôt mince et chez laquelle les sillons s'effacent le long des bords.

\section{Donax BICOLOR Gmelin}

1790. Donax bicolor

$\begin{array}{llll}1818 & - & - & \\ 1835 . & - & - & \\ 1842 . & - & - & \text { Lk. } \\ 1854 . & - & - & - \\ 1866 . & - & - & - \\ 1869 . & - & - & \mathrm{Gm} .\end{array}$

1881. Latona - Lk.
GMelin, Syst. Nat. Ed. XIII, p. 3265 (ref. Gualtieri pl. 88 , fig. s.). Lamarck, Anim. s. Vert. V, p. 548 .

Deshayfs in Lamarck, An. s. Vert. 2e éd. VI, p. 243. Hanley, Rec. Biv. Sh. p. 81 (an D. bicolor Gm. ?) Reeve, C. Icon.pl. V, fig. $28^{\mathrm{a}}, 28^{\mathrm{b}}$.

Sowerby, Thes. Conch. III, p. 311, pl. 283 (Donax IV), fig. 102, 103.)

Römer, Mon. Conch. Cab. 2 e Ed, p. 23, pl. 16, fig. $12-16$.

de Rochebrune, Suppl. atix docum. sur la faune malac. de la Cochinchine et du Cambodge, p. 8 . 
1891. Donax bicolor Lk.

P. Fischer, Catal. IndoChine, loc. cit. p. 241 (157 du t. à p.)

- Cochinchine : Hong-Chon (M. Mansuy).

Nous adoptons la manière de voir de Roemer qui attribue à Gmelin la paternité de cette espèce, car il ne nous paraît pas douteux que la figuration de Gualtieri (pl. 88 fig. S.) citée comme référence par Gmelin, soit la même espèce que le $D$. bicolor de Lamarck.

\section{Donax incarnatus Chemnitz}

1782. Donax incarnata etc. Chemnitz, Conch. Cab. VI, p. 265, pl. 26, fig. 2อั9. 1786. Die röthliche oder fleisch farbige Dreyeclimuschel ScnröTER Einleit. III, p. 102.

1790. Donax incarnata Ch.
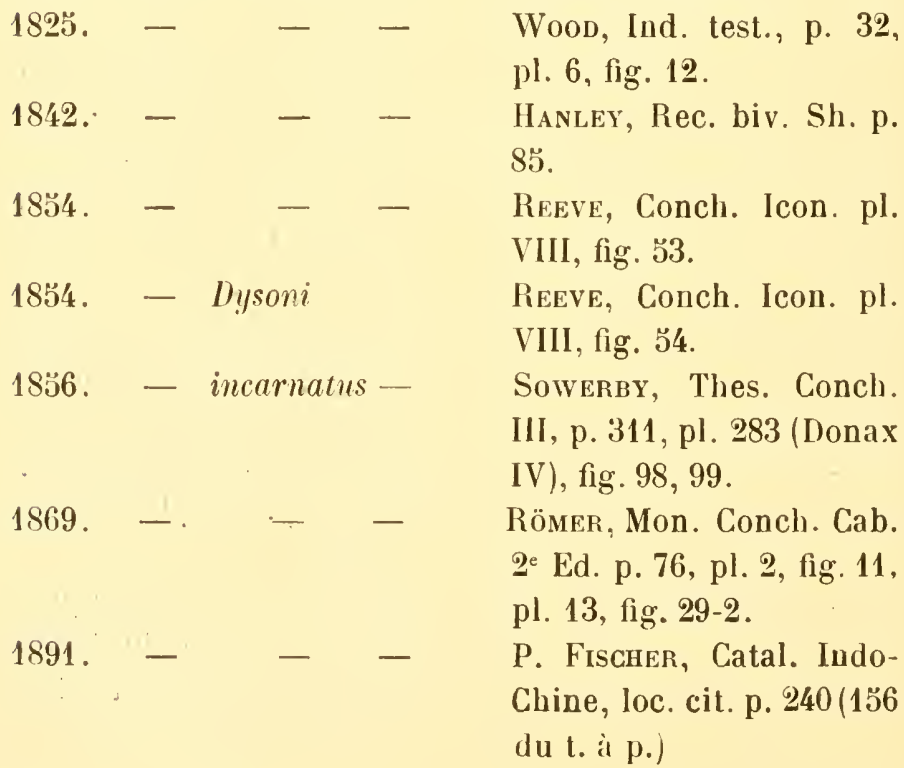

Guelin, Syst. Nat. Ed. XIII, p. 3265 .

Wood, Ind. test., p. 32, pl. 6, fig. 12.

Hanley, Rec. biv. Sh. p. 85.

Reeve, Conch. Icon. pl. VIII, fig. 53.

ReEve, Conch. Icon. pl. VIII, fig. 5̌4.

Sowerby, Thes. Conch. III, p. 311, pl. 283 (Donax IV), fig. 98, 99 .

Römer, Mon. Conch. Cab. $2^{\text {e }}$ Ed. p. 76, pl. 2, fig. 11, pl. 13, fig. 29-2.

P. Fischer, Catal. Indodu t. à p.) 
1891. Donax Dysoni Desh. P. Fischer, ibid. p, 240 (10ั6 du t. à p.)

Cochinchine: Hong-Chon (M. Mansuy).

II n'y a d'autres différences entre le $D$. incarnatus et le D. Dysoni que la coloration. Ce dernier est gris à l'extérieur et violet foncé à l'intérieur, tandis que le $D$. incarnatus est rosé, avec l'intérieur rosé vif et des rayous violets.

\section{Donax faba Chemnitz}

1782. Donax faba etc.

1786. die Bohne

1790. Donax Faba

$$
\begin{aligned}
& \text { 1817. - faba } \\
& \text { 182ว. - Faba }
\end{aligned}
$$

1842. - - Ch.

1866. - - -

1866. - faba

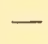

1869.

1887
Chemnitz, Conch. Cab. VI, p. 270, pl. 26, fig. 266, 267.

Schröter, Einleit. III, p. 102.

Gmelin, Syst. Nat. éd. XIII, p. 3264.

Dillwyn, Catal. I, p. 1 รัวั. Wood, lind. test. p. 32, pl. 6, fig. 16.

Hanley, Rec. biv. Sh. p. 80.

Sowerby, Thes. Conch. III, p. 312, pl. 283, Donax IV, fig. 108, 109.

Mabille et Le Mesle, Journ. de Conch. XIV, p. 118.

Römer, Mon. Conch. Cab. 2 Ed. p. 88, pl. 2, fig. 12 à 17.

DAUTZENBERg et D'HAMONviLLE, Journ. de Conch. XXXV, p. 224. 
1889. Donax faba $\mathrm{Ch}$.

1891 .

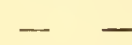

1818. Donax radians

1819. - -

1835. - - -

1854. - -

1866. - -

1891. - faba var. radians
L. Morlet, Journ. de Conch. XXXVII, p. 171.

P. Fischer, CataI. IndoChine loc. cit. p. 241 (157 du t. à p.)

Cochinchine: Hong-Chon (M. Mansuy).

\section{Variété radians Lamarck}

LAMARCK, Anim.s. Vert. V, p. 547 .

\begin{tabular}{|c|c|c|c|c|}
\hline 819. & - & - & Lk. & $\begin{array}{l}\text { Blainville, Dict. les Sc. } \\
\text { Nat. XIII, p. } 421 .\end{array}$ \\
\hline 183วั. & - & - & & $\begin{array}{l}\text { Deshayes } i n \text { Lamarck, An. } \\
\text { s. Vert. 2e Ed. VI, p. } 241 .\end{array}$ \\
\hline 1854 & - & - & Lk. & $\begin{array}{l}\text { Reeve, Conch. Icon. pl. V, } \\
\text { fig. } 26^{\mathrm{a}}, 26^{\mathrm{b}}, 26^{\mathrm{c}} \text {. }\end{array}$ \\
\hline 1866. & - & - & & $\begin{array}{l}\text { Sowerby, Thes. Conch. } \\
\text { III, p. } 312 \text {, pI. } 283 \text { (IV) fig. }\end{array}$ \\
\hline 1891. & - & $b a \mathrm{v}$ & lians & $\begin{array}{l}91,92,93,94,95 . \\
\text { P. Fischer, Catal. Indo- } \\
\text { Chine loc. cit. p. } 157 \text {. }\end{array}$ \\
\hline
\end{tabular}

Cochinchine : Hong-Chon (M. Mansuy).

1 exemplaire d'une forme plus transverse que le $D$. radians de Lamarck. Nous pensons que ce dernier nom peut-être conservé pour désigner la variété plus haute et moins large: on rencontre tous les intermédiaires possibles entre la variété et la forme typique.

\section{? Martesia ob'tecta Sowerby}

1849. Pholas obtecta

18:1. Martesia -
Sowerby, Thes. Conch. II, p. 496 , pl. CVIII, fig. 80, 81 . Sow. Gray, Ann. \& Mag. N. H. @id ser. VIII, p. $38 \dot{4}$. 
183̈8. Martesia obtecta Soll. H. et A. Abans, lien. of. rec. Moll. II, p. 331.

18:8. - - - P. Fiscaler, Journ. de Conch. III, p. 320.

186. - - - Trion, Monog, of the Order Pholadacea, p. 90.

1872. Pholas - Sowrerse, Conch. Icon. pl. IX, fig. 3̈a, 3öb.

1893. Martesia - Clessin, Conch. Cab. $2{ }^{\circ}$ éd. p. 43. pl. 11, fig. 9, 10.

Poulo-Condore (M. Mansuy).

C'est, avec quelque hésitation que nous rapportons à cette espèce, non encore signalée en Indo-Chine, 4 valves en médiocre état, recueillies à Poulo-Condore par M. Maısuy .

Ph. D. et H. F. 


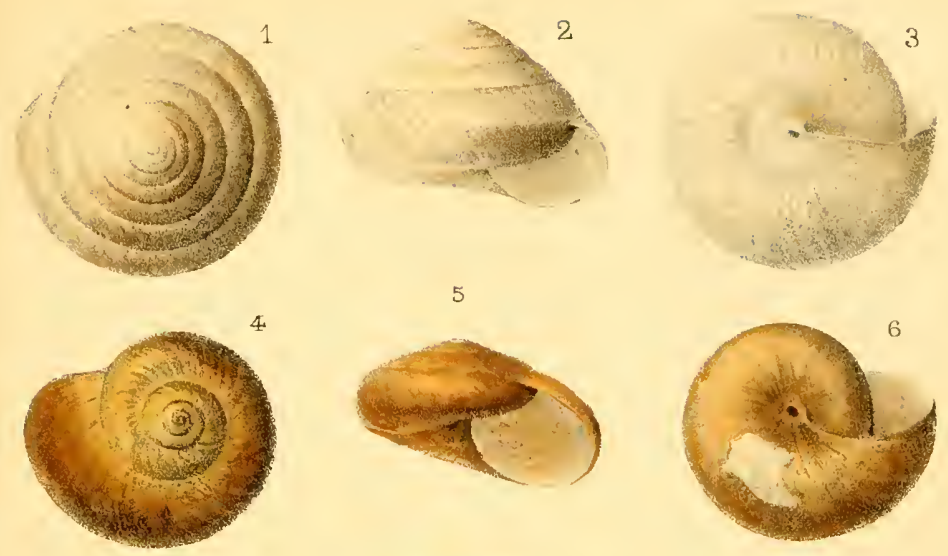

5
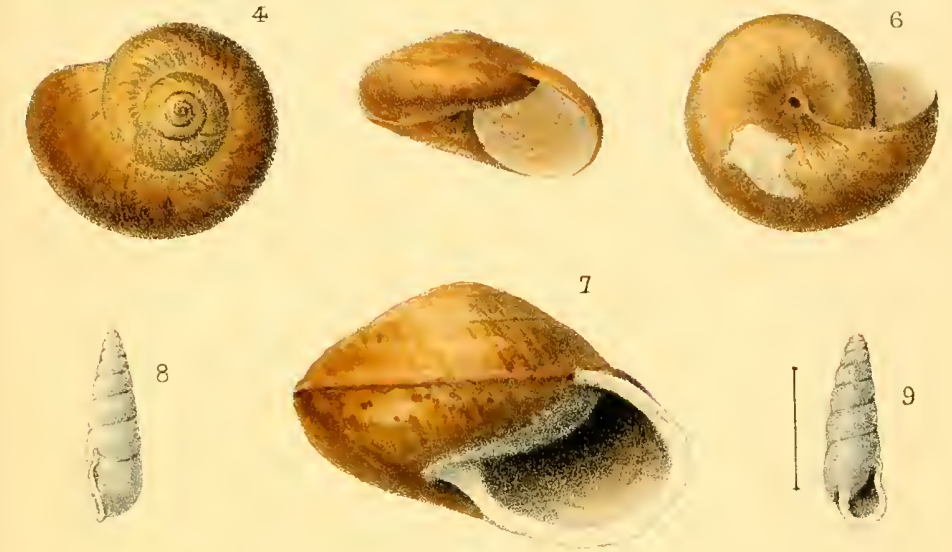

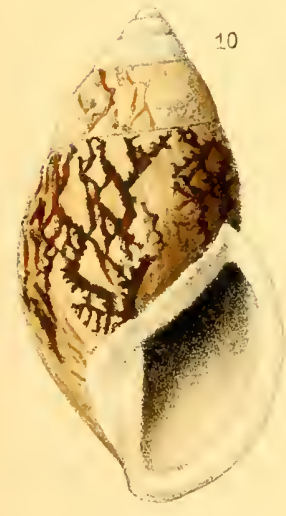

C Reignier, del \& lith

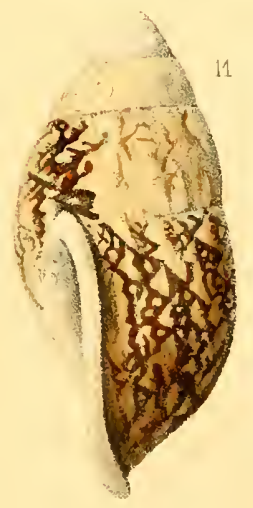

Imp. L. Latontaine, Paris

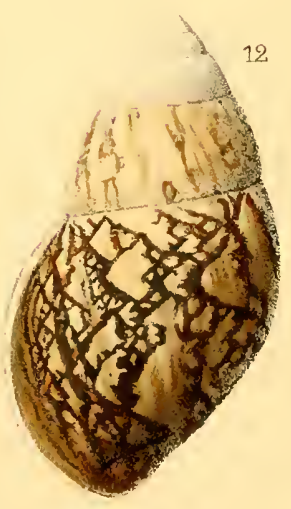

\section{1,2,3. Xestina tenera Möllendorff \\ 4,5.6. Macrochlamys Douvillei Dautz. \& H.F. \\ 1. Camana Mansuyi D. et H. F. var. depressa D. et H.F. \\ 8,9. Ennea (Elna) Mansuyi Dautz. et H. Fischer. \\ 10,11,12. Amphidromus Bulowi Fruhstorfer.}





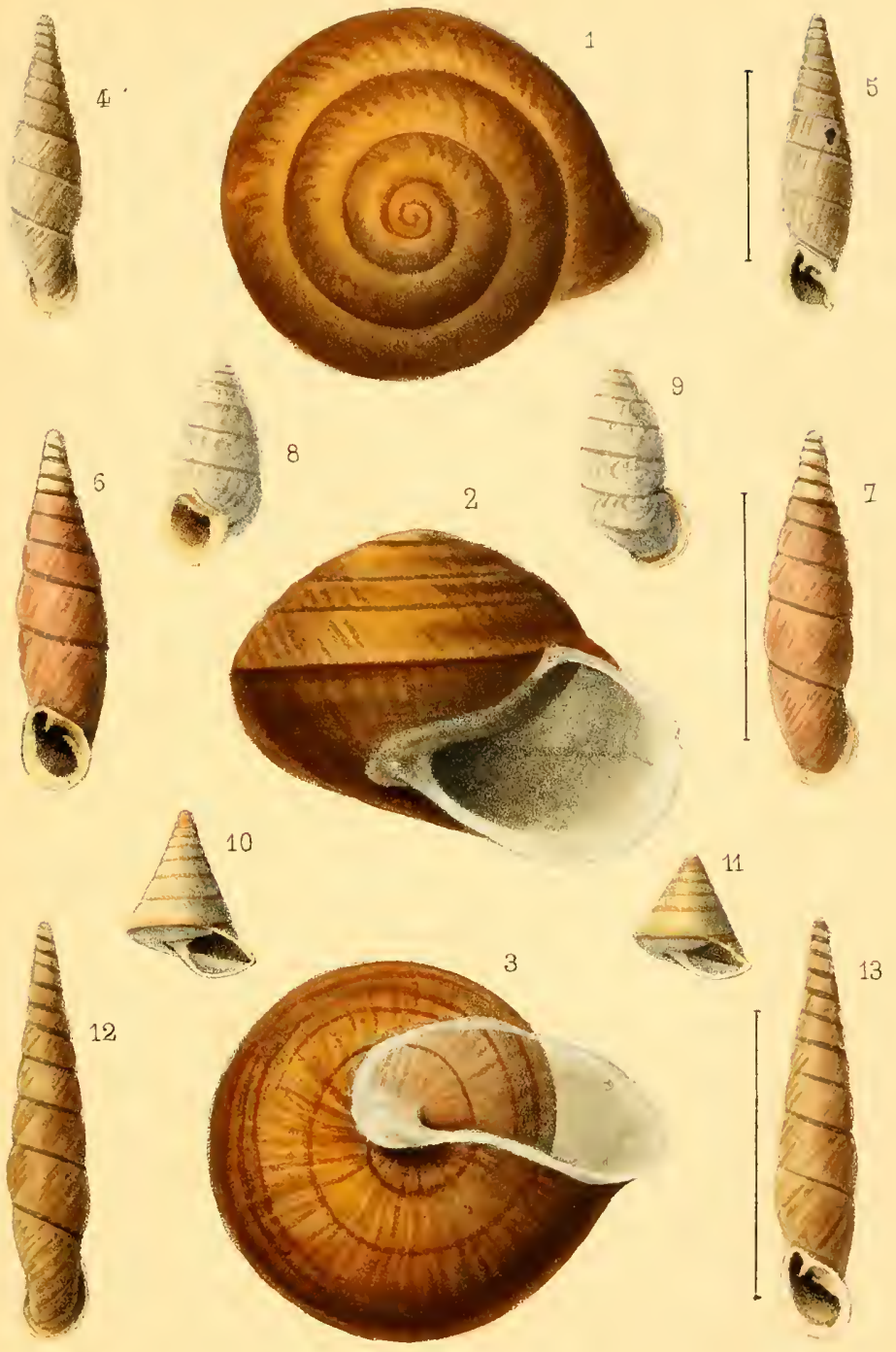

\section{1, 2, 3. Camæna Mansuyi Dautzenberg et H. Fischer}

4, 5. Clausilia Lavillei Dautzenberó et H. Fischer

6, 7. Clausilia Lantenoisi Dautz et H. Fischer.

8,9 Buliminus pharandensis (Fruhstorfer) Dautz. \& H. Fischer.

10,11 Satsuma Lantenoisi Dautz. \&. H. Fischer.

12,13 Clausilia vanbuensıs Bavay \& Dautz 

Journal de Conchyliologie. 1905
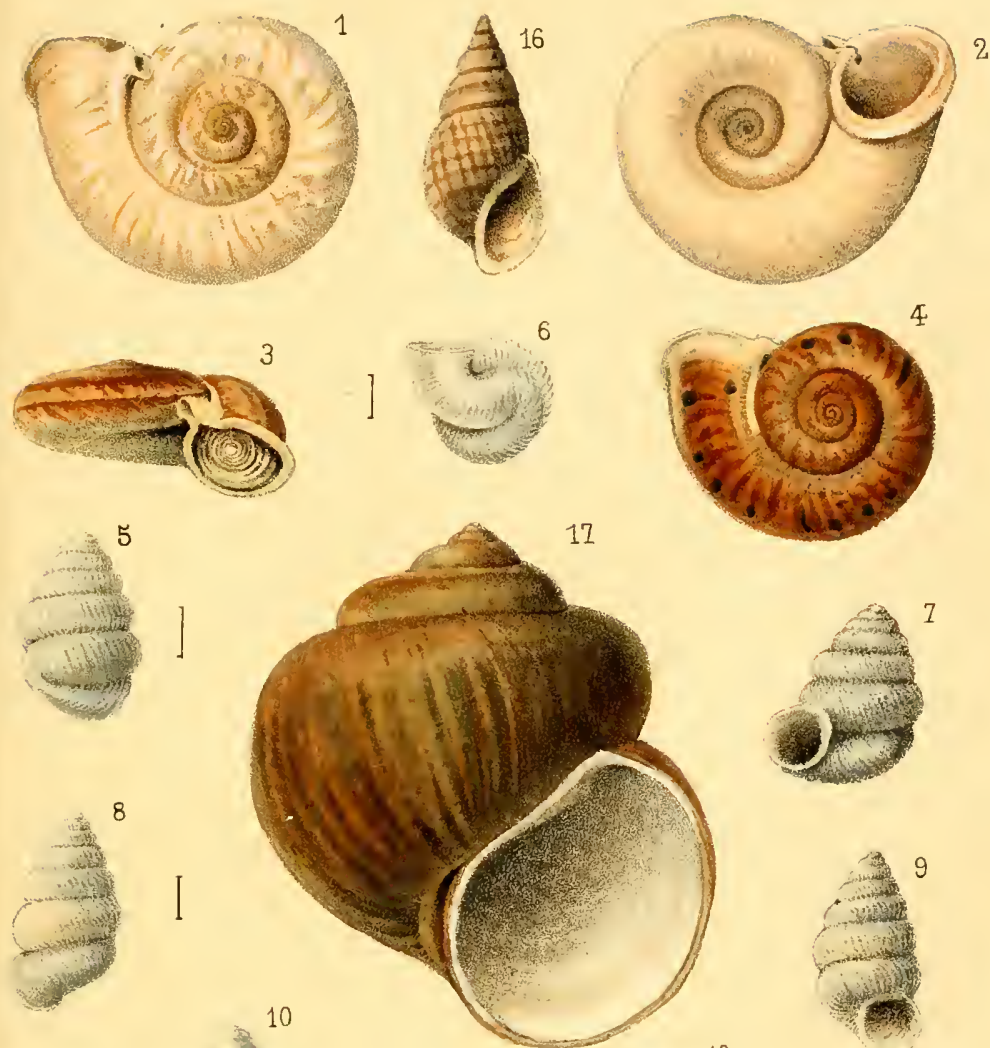

13
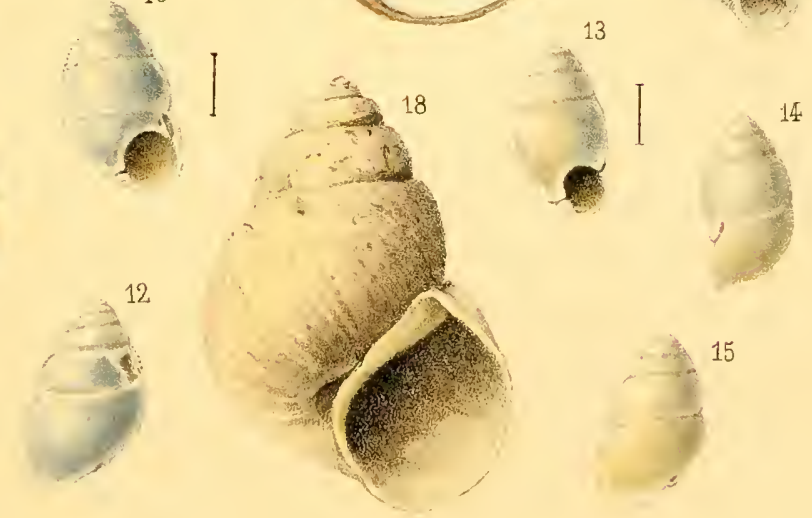

G. Reigner, del et lith.

Iup. I. Lafortaine, Parts

2,3,4. Rhrostoma Morleti Dautzenberó \& H. Fischer |13,14,15. Pupina Verneaui Dautzenberé \& H. Fischer. 5, 6, 7. Opisthostoma tonkinianum Dautz. \& H.F. 8,9. Diplommatina Lavillei Dautz.\& H.F. 10,11,12. Pupina Douvillei Dautzenbero \& H Fischer. 16. Melania Jacqueti Dautzenber \& \&. Fischer. 17. Paludina lecythoides Benson var: latissima D.\&H.F 18. Paludina Rattei Cr. \& F. var. elongata D.\& H.F. 





\section{LISTE DES MOLLUSOUES}

Récoltés par II. Ylansuy en Indo-Chine

ET DESCRIPTION D'ESPĖCE NOUVELLES

II

PAR

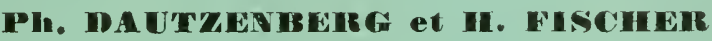

(Extrait du Journal de Conchyliologie, Vol. LVI)

\section{PARIS}

Direction et Rédaction:

H. FISGHER

$.5 \%$, Boulevard Saint-Michel (
Administration:

F. R. D\& RUDEVAL, Éditeur 4, Kue Antoine Dubois $\left(\mathrm{VI}^{\mathrm{C}}\right)$

\section{8}





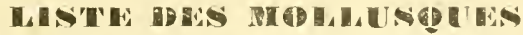

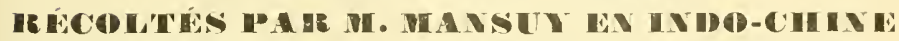

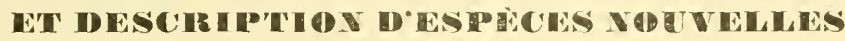

Par Ph. Dautzenberg et H. Fischer

Nous avons déjà publié dans ce Recueil (1) les découvertes malacologiques que M. Mansuy avait faites au cour s de son premier voyage en Indo Chine. Nous sommes heureux de pouvoir étudier également aujourd'hui les matériaux que ce zélé naturaliste a réunis peudant son deuxième séjour dans la même contrée. Cette seconde récolte, au moins aussi importante que la première, renferme également de nombreuses formes nouvelles, dont les types, déposés dans les collections de l'Ecole des Mines, nous ont été obligeamment communiqués par M. le Profr Douvillé.

Presque toutes les espèces dont il est question dans ce mémoire ont été capturées par M. Mansuy, en 1906 et 1907, dans le Haut-Tonkin oriental et notamment dans la région de Cao Bang: vers l'Est, à Trung Khan-Phu, Ban-Cra, Ha-

(1) Journ. de Conchyliologie, LIII, 1905, p. 341-471, pl. VHI, IX, X. 
Lang et Quang-Huyen, qu'il ne faut pas confondre avec Quang-Yen, ville des environs de Haiphong; vers l'Ouest à Mo Xat et vers le Sud à Nam-Nhang et à Long-Phoi près de That-Khé ainsi que dans le massif du Bac-Son, au NordOuest de Lang-Son. La plupart des espèces aquatiques ont été pêchées dans le Song-Bang-Giang, à Cao-Bang. Enfin, quelques-unes, provenant du Royaume de Luang-Prabang, n'ont pas été recueillies par M. Mansuy, mais lui ont été données par M. Monod.

Les Plectopylis rapportés par M. Mansuy ont été étudiés par notre savant confrère de Londres, M. G. K. Gude, dans ce Journal: vol. LV, 1907, p. 345337 , pl. VII.

Streptaxis (Eustreptaxis) costulatus Möllendorff

\section{Var. majö Bavay et Dautzenberg}

1903. Streptaxis (Eustreptaxis) costulatus Mōllend. var. major Bavay et Dautz., Dautzenberg et H. Fischer, Liste coq. rec. par Blaise, in Journ. de Conch., LIIl, p. 86.

Loc. : Trung-Khan-Phu (M. Mansuy 1906).

Streptaxis (Odontartemon) Mabillei Bavay et Dautzenberg 1903. Streptaxis (Odontartemon) Mabillei Bavay et Dautzenberg, Descr. coq. nouv. Indo-Chine, in Journ. Conch., LI, p. 203, pl. VII, fig. 13,14, 15 .

Loc. : Trung-Khan-Phu (M. Mansuy, 1906).

Ennea (Microstrophia) plagiostoma von Möllendorff (PI. IV, fig. 1, 2,3)

1901. Ennea (Microstrophia) plagiostoma MöLlendonfF. Nachrichtsbl. d. deutschen Malak. Ges., p. 110.

Loc. : Ha Lang (M. Mansuy).

Cette espèce n’ayant pas été représentée par Möllendorff, nous la figurons ici. 
Ennea (Elma) tonkiniana Bavay et Dautzenberg 1906. Ennea (Elma) tonkiniana Bav. et Dautz., Dautzenberg et $\mathrm{H}$. Fiscier, Liste Moll. rec. par Mansuy, in Journ. de Conch., LJV, p. 344 .

Loc. : Quang-Huyen (M. Mansuy).

\section{Helicarion imperator Gould}

1906. Heticarion imperator Gould, Dautzengerg et II. Fischer, Contrib. Faune Malac. Indo-Chine, in Journ. de Conch., LIV, p. 146

Loc. : Trung-Khan-Phu; Quang Huyen (M. Mansuy, 1906); Massif du Bac-Son (M. Mansuy, 1907).

\section{Nanina (Rhysota) distincta Pfeiffer}

\section{Var. Neptumus Pfeiffer}

1906. Nanina (Rhysota) distincta Pfr., var. Neptunus PIr., DautzenBERg et H. Fischer, Liste Moll. rec. par Mansuy, in Journ. de Conch., LIII, p.347.

Loc. : Royaume de Luang Prabang (M. Monod).

\section{Macrochlamys Despecta J. Mabille}

1906. Nanina (Macrochlamys) despecta J. Mab., Dautzenberg et H. Fischer, Liste Coq. rec. par Mansuy, in Journ. de Conch., LIII, p. 350.

Loc.: Quang-Huyen; Massif du Bac-Son (M. Mansuy).

\section{Camaena Billeti H. Fischer}

(Pl. IV, fig. 5).

1898. Helix (Camaena) Billeti H. Fischer, Notes faune Haut-Tonkin, III, Liste des Moll. rec. par Ie $\mathrm{D}^{\mathrm{r}} \mathrm{A}$. Billet, in Bull. scient. France et Belgique, XXVIII, p. 316, pl. XVII, fig. 7-11.

1904. Camaena - H. F., H. Fischer et Dautzenberg, Catal. Indo. Chine Mission Pavie, p. 399. 
Loc.: Quang-Huyen (M. Mansuy).

La taille est assez variable chez cette espèce : parmi les spécimens rapportés par M. Mansuy, nous en trouvons qui ont, les uns $32 \mathrm{~mm}$. diamètre et d'autres 2כ mm. seulement.

Le $C$. Billeti n'ayant été représenté jusqu'à présent qu'en noir, nous en donnons ici une figure coloriée.

\section{Camaena cicatricosa Müller}

Var. inflata von Möllendorff

1906. Camaena cicalricosa Mūll, var. inflata v. Möll., Dautzenbekg et H. Fischer, Liste Moll. rec. par Mansuy, in Journ. de Conch., LIII, p. 3ว̆.

Loc.: Quang Huyen (M. Mansuy).

Chez certains spécimens rapportés par M. Mansuy, on observe sur une partie du test les granulations qui caractérisent notre var. connectens Dautz. et H. Fisch. (Journal de Conch., LIII, p. 33̈6).

Camaena Gabriellae Dautzenberg et d'Hamonville.

Var. platytaenia von Möllendorff (mss.).

(PI. IV, fig. 4).

Loc.: Quang.Huyen (M. Mansuy, 1906).

Nous avons représenté celte variété, caractérisée par une large zône périphériale brune, d'après un exemplaire récolté par M. Frühstorfer et déterminé par von Möllendorff ; mais nous croyons qu'elle n'a jamais été publiée.

Camaena Yorvonga Bavay et Dautzenberg.

1900. Helix (Camaena) Vorvonga Bavay et Dautzenberg, Diagn. coq. nouv. Indo-Chine, in Journ. de Conch, XLVIII, p. 109. 
1900. $\rightarrow-$

1904. Camaena
- Bavay et Dautzenberg, Descript. coq. nouv. Indo-Chine, in Journ. de Conch., XLVIII, p. 436, pl. X, fig. 1, 2, 3 .

- B. et D., I1. Fisciner et Dautzenberg, Catal. Indo-Chine, Mission Pavie, p. 399.

Loc.: Quang-Huyen (M. Mansuy).

Nous remarquons que chez cette espèce, la bande brune qui accompagne la carène est plus ou moins apparente et parfois obsolète.

\section{Kaliella ordinaria Ancey}

1903. Kaliella ordinaria

Ancey, in Bavay et Dautzenbehg, Coq. nouv. Indo-Chine, in Journ. de Conch., LI, p. 210, pl. VIII, fig. 18, 19.

Loc.: Trung-Khan-Phu (M. Mansuy).

Kaliella tongkingensis von Möllendorff.

1901. Kaliella tongkingensis von Móchendorff, Diagn. neuer, von H. Frühstorfer in Tongking Gesamm. Landschoecken, in Nachrichtsbl. d. d. Malak. Ges., p. 70.

1903. - - - v. Móll., Bavay et Dautzenberg, Coq. nouv. IndoChine, in Journ. de Conch., LI, p. 209, pl. VIII, fig. 14, 15, 16, 17.

Loc.: Trung-Khan-Phu (M. Mansuy).

Trochomorpha Paviei L. Morlet.

190'. Trochomorpha Paviei L. M., H. Fischer et Dautzenberg, Liste Indo-Chine, in Mission Pavie, p. 398.

1906. - - - Dautzenberg el H. Fischer, Liste Moll. rec. par Mansuy, in Journ. de Conch., LIV, p. 3.3.

Loc.: Quang-Huyen; Trung-Khan-Phu (M. Mansuy) 
Neocepolis Cherrieri Bavay (1).

(PI. IV, fig. 6-12).

1899. Helix (Obba) langsonensis Bavay et Dautzenberg, Journ. de Conch., XLVII, p. 29, pl. I, fig. 1, $1^{\circ}$.

1904. Camaena (Neocepolis) - B. et D., H. Fischer et Dautzenberg, Catal. Indo-Chine, Mission Pavie, p. 400.

Var. . edentula nov, var.

forme typique, mais dépourvue de dent à la base de la columelle. (PI. IV, fig. 6).

Var. $\beta$. scrobiculata nov. var.

pourvue d'une scrobiculation transversale en arrière du labre. Cette scrobiculation correspond à un pli saillant dans l'intérieur de l'ouverture (PI. IV. fig. 7).

\section{Var. \% depressa nov. var.}

forme déprimée: haut. $19 \mathrm{~mm}$., diam. $28 \mathrm{~mm}$. (Pl. IV, fig. 8).

Var. $\delta$, depressa-serobiculata nov, var.

semblable à la précédente par sa forme surbaissée; mais pourvue d'une scrobiculation comme la var. $\beta$. (Pl. IV, fig. 9,10$)$.

Var. s. careinata nov. var.

Cette variété se distingue de toutes les autres formes du $N$. Cherrieri par son dernier tour nettement caréné à la

(1) L'Helıx longsonensis L. Morlet, indiqué par son auteur comme provenant de Long-Son (Tonkin), est une tout autre espèce. Comme il est probable que c'est par erreur que le commandant L. Morlet a écrit Long-Son au lieu de Lang.Son, je propose, afin d'éviter toute confusion, de remplacer pour notre espèce le nom de Langsonensis par celui de Cherrieri.

A. Bavay. 
périphérie, par son ombilic plus ouvert et par sa sculpture qui consiste sur toute la surface, au-dessus de la carène, en costules très irrégulières, dont quelques-unes sont très saillantes. Sur la base du deruier tour, les costules sont beaucoup plus faibles, (Pl. IV, fig. 11, 12).

Loc.: Quang-Huyen (M. Mansuy).

\section{Chloritis diestalmena nov. sp.}

(PI. IV, fig. 13, 14, 15, 16).

Testa tenuis, depressa, snbdiscoideu sat late ac pervie umbilicata. Spira planala. An/r. $41 / 2$ convexinsculi, rapide accrescentes, sutura impressa juncti striis incrementi farum conspicuis sculpti ac, sub lente, insupra, minutissime puncticulati. Anfr. ultimus ad extremitatem vix descendens, ad peripherium. obtusissime angul:atus, basi convexus. Apertura obliqua, transverse ovata, ampla, marginibus callo adnato junctis. Margo columellaris obliqua et margo basalis subarcuata, paululum incrassata atque breviter reflexa. Labrum expansum et acutum.

Color pallide corneus; zonula intense fulva basin anfr. superiorum occupat et in anfr. ultimo peripheriam superat. Altit. $9 \mathrm{~mm}$; diam. maj. 21, $\min .16 \mathrm{~mm}$. Apertura $81 / 2 \mathrm{~mm}$. alta, $111 / 2 \mathrm{~mm}$. lata.

Coquille mince, déprimée, subdiscoïde, assez largement et profondément ombiliquée. Spire plane, composé de 4 1/2 tours légèrement convexes, croissant rapidement et séparés par une suture bien accusée. La surface est ornée de stries d'accroissement fines et, lorsqu'on l'examine sous la loupe, on remarque qu'elle est parsemée de très petites ponctuations, disposées en quinconces et qui semblent être des cicatrices de poils. Dernier tour descendant à peine à son extrémité, très obtusément auguleux à la périphérie, bien convexe à la base. Ouverture oblique, trausversalement uvale, ample. Burds du péristome reliés 
par une callosité mince, appliquée et nettement limitée. Le bord collumellaire oblique, et le bord basal légèrement aiqué, sont étroitement épaissis et réfléchis, tandis que le labre est dilaté, mais tranchant.

Coloration d'une teinte cornée très claire, avec une zone brune régnant sur la base des premiers tours et se prolongeant sur le dernier, immédiatement au-dessus de la périphérie.

Ciette espèce ressemble un peu à l'Helix rhinocerotica Heude (Mém. Empire chinois, pl. 38, fig. 14), de Tay-Ninh, Cochinchine; mais elle est bien plus aplatie et a l'ouverture bien plus dilatée.

Loc.: Massif du Bac Son, Tonkin (M. Mansuy, 1907).

\section{Chloritis Durandi Bavay et Dautzenberg}

1900. Helix (Chloritis) Durandi Bayay el Dautzenbeng, Diagn. Coq. nouv. Indo-Chine, in Journ. de Conch., XLVIII, p. 111.

1900. - - - Bavay el Dautzenbehg, Descr. Coq. nouv. Indo-Chine, in Journ. de Conch. XLVIII, p. 441, pl. XI, fig. 1, 2, 3.

Loc : Ha-Lang (M. Mansuy).

Chloritis Lambineti Bavay et Dautzenberg

1899. Helix (Chlorilis) Lambineli Bavay el Dautzenberó, Descr. Coq. nouv. Indo-Chine, in Journ. de Conch., XLVII, p. 28, pl. I, fig. $3,3^{\mathrm{a}}, 3^{\mathrm{b}}$.

1904. Chloritis - H. Fischer el Dautzenberg, Catal. IndoChine, Mission Pavie, p. 400.

Loc. Ban-Cra ; Quang-Huyen (M. Mansuy, 1906).

\section{Chloritis maka J. Mabille}

1887. Helix miara 1887
J. Mabille, Moll. Tonk. Diagn., p. 6. J. Mabille, Sur quelques Moll. du Tonkin, in Bull. Soc. Malac. France, p. 8 o, pl. I, fig. $6,7$. 
Tonkin (collection André Bonnet).

Les exemplaires qui nous ont été communiqués par M. Bonnet sans indication de localité précise, ont la microsculpture granuleuse un peu moins accusée que la forme typique de l'Ile des Merveilles, notamment sur la base du dernier tour.

Le Chloritis insuiaris Möllendorff (Nachrichtsbl., 1901, p. 113), non Crosse et Fischer, qui provient également de l'Ile des Merveilles, nous paraît, d'après sa description, devoir tomber en synonymie de miara J. Mabille.

\section{Plectopylis Bayayi Gude}

1901. Plectopylis Bavayi Gude, Sur une Collect. de Plectopylis du Tonkin. in Journ. de Conch., XLIX, p. 200,201 , fig. 2a 2 b, 2c, $2^{d}, 2^{e} ;$ pl. VI, fig. $2 \mathrm{a}, 2^{b}, 2^{c}$.

Loc. : Quang-Huyen (M. Mansuy), un seul exemplaire.

\section{Plectopylis Glandi H. Fischer}

1907. Plectopylis Giardi H.F., Gude, Obs. on a number of Plectopylis collect. by M. Mansuy, in Journ. de Conch., LV, p. 346, 347, fig. 1a, 1h.

Loc. Quang Huyen (M. Mansuy).

\section{Plectopylis infralevis Gude}

1907. Plectopylis infralevis Guoe, Obs. on a number of Plectopylis collect. hy M. Mansuy, in Journ. de

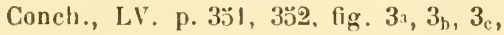
3d, 3 ; ; pl. VIl, fig. $4,5,6$.

Loc. : Quang-Huyen (M. Mansuy) 


\section{Plectopylis Mansuyl Gude}

1907. Plectopylis Mansuyi Gude, Obs. on a number of Plectopylis collect. by $M$. Mansuy, in Journ, de Conch., LV, p. 348,6 g. $2^{\mathrm{a}}, 2^{\mathrm{b}}, 2^{\mathrm{c}}, 2^{\mathrm{d}}, 2^{\mathrm{e}}$; pl. VII, fig. 1, 2, 3 .

Loc. : Ha-Lang (M. Mansuy).

\section{Plectopylis sonor Gude}

1907. Plectopylis soror

Gude, Obs. on a number of Plectopylis collect. by M. Mansuy, in Iourn. de Conch.. LV, p. 3ว̌s, 3ว̈6, fig. $5^{a}, 5^{b}, 5^{c}$, $5^{d}, 5^{e}$; pl. VII, fig. 10, 11, 12.

Loc. : Quang-Huyen (M. Mansuy).

\section{Plectopylis suprafilaris Gude}

1907. Plectopylis suprafilaris GUDE, Obs, on a number of Plectopylis collect. by M. Mansuy, in Journ. de Conch., LV, p. 3วั3, 3ว̆4, fig. $4^{\mathrm{a}}, 4^{\mathrm{b}}, 4^{\mathrm{c}}$, $4^{\mathrm{d}}, 4^{\mathrm{e}} ;$ pl. VII, fig. $7,8,9$.

Loc. : Quang-Huyen (M. Mansuy).

\section{Chalepotaxis infantilis Gredler}

1906. Chalepotaxis infantilis Gredl., Dautzenberg et H. Fischer, Liste Moll. rec. par Mansuy, in Journ. de Conch., LIV, p. 360.

Loc. : Ha-Lang ; Quang-Huyen (M. Mansuy 1906).

\section{Ganesella Saurivonga Bavay el Dautzenterg}

1900. Helix (Gunesella) Saurivonga Bavay et Dautzenberg, Diagn. Coq. nouv. Indo-Chine, in Journ. de Conch., XLVIII, p. 112.
1900. - - - - Bavayel Dautzenberg, Descr. coq. nouv. Indo-Chine, in Journ. de Cinch., XLVIII, p. 4 4 i2, pl. IX, fig. 10, 11, 12.
1904. Ganesella
- B. et D., H. Fischer et Diutzenberg, Catal. Indo-Chine, Mission Pavie, p. 403.


Var. lepressa nov. var.

Plus surbaissée que le type : haut. $9 \mathrm{~mm}$., diam. maj. $16 \mathrm{~mm}$., min. $14 \mathrm{~mm}$.

Loc. : Ha-Lang (M. Mansuy)

Satsuma fulvescens nov. sp.

(Pl. V, fig. 7, 8, 9)

Testa tenuis, nitidiuscula, subtranslucida, trochiformis, anguste ac pervie umbilicata. Spira conica apice obtusulo. Anfr. 6 subplani, sutura lineari juncti. Anfr. primi leves, nitentes, ceteri striis incrementi obliquis et valde irregularibus sculpti. Anfr. ultimus acute carinatus, ad extremitatem sat abrupte descendens, basi convexus. Apertura perobliqua, ovata, marginibus conniventibus. Columella obliqua, superne dilatata et umbilicum ex parte obtegens. Labrum breviter expansum ac in medio subangulatum.

Color pallide fulvus.

Altit. 12 diam. $11 \mathrm{~mm}$.; apertura $4 \mathrm{~mm}$. alta; $71 / 2 \mathrm{~mm}$. lata.

Coquille mince, peu luisante, subtranslucide, trochiforme, étroitement et profondément ombiliquée. Spire conique, un peu obtuse au sommet, composée de 6 tours presque plans (très légèrement convexes), séparés par une suture linéaire. Premiers tours lisses, les autres ornés de stries d'accroissement obliques, très irrégulières. Dernier tour caréné à la périphérie, descendant assez brus. quement à son extrémité, convexe à la base. Ouverture très oblique, à bords rapprochés mais non reliés par une callosité. Columelle oblique, dilatée vera le haut où elle masque en partie l'ombilic. Labre étroitement étalé, non réfléchi, subanguleux à l'endroit où aboutit la carène périphériale.

Coloration d'un fauve clair uniforme. 
Loc. : Nam-Nhang (M. Mansuy).

Cette espèce diffère du Satsuma acris Benson = perakensis Crosse et de ses variétés par son dernier tour plus descendant à l'extrémité, par sou bord collumellaire très dilaté postérieurement, de sorte que les bords de l'ouverture sont beaucoup plus rapprochès. De plus, sa coloration est uniforme, sans aucune trace de baude brune sur la carène.

\section{Satsuma leptoponopsis nov. $\mathrm{sp}$.}

(Pl. IV, fig. 17, 18, 19)

Testa tenuis, subtranslucida, turbinata, sat anguste sed pervie umbilicata. Spira conoidea, apice obtusulo. Anfr. 5 1/2 convexiusculi, sutura parum impressa juncti; primi leves, ceteri striis incrementi obliquis irregulariter et subolsolete sculpti. Anfr. ultimus ad extremitatem descendens, ad peripheriam olutuse subangulatus, basi convexus. Apertura peroliligua, transverse ovata, marginibus connicentibus ac callo tenui adnutoque junctis. Peristoma undique expansum, paullo incrassatum sed vix reflexum. Murgo columellaris superne dilatatus, umbilicum fere onnino obtegit.

Color pallide corneus, zonula fusca in peripheria anfr. ultimi ornatus. Peristoma allnum.

Altit. 14, diam. $15 \mathrm{~mm}$. ; apertura $9 \mathrm{~mm}$. alta, $10 \mathrm{~mm}$. lata.

Coquille mince, subtranslucide, turbinée, assez étroitement, mais profondément ombiliquée. Spire conoïde, obtuse au sommet, composée de 5 1/2 tours un peu couvexes, séparés par une suture peu profonde: premiers tours lisses, les autres ornés de stries d'accroissement obliques, irrégulières et subobsolètes. Dernier tour descendant à son extrémité, trẻs obtusément subanguleux à la périphérie, convexe à la base. Ouverture très oblique, transversalement ovale, à bords rapprochés et reliés par 
une callosité mince, appliquée. Péristome dilaté, un peu épaissi, mais à peine réfléchi. Bord columellaire dilaté vers le haut, masquant presque entièrement l'ombilic.

Coloration cornée très claire avec une zone brune sur la périphérie du dernier tour. Péristome blanc.

Loc. : Lung-Phoi, près That-líhé (M. Mansuy, 1906).

Cette espèce est plus grande que le $S$. Vatheleti, moins carénée à la périphérie et moins élevée. Son ombilic est plus large et son labre plus dilaté. Elle a un peu l'aspect d'un Leptopoma.

\section{Satsuma acris Benson}

1906. Satsuma Lantenoisi

Dautzenberg el H Fischer, Liste Moll. rec. par Mansuy, in Journ. de Conch., LIII, p. 360, pl. IX, fig. 10, 11.

Loc. : Ha-Lang (M. Mansuy).

M. le colonel Beddome nous a informés qu'il possède de l'Inde et aussi de Bornéo, des spécimens de $S$. acris Benson (Annals and Magazine of Nat. Hist., $3^{\mathrm{d}}$ Ser. III, 1859, p. 387 et Conchologia Indica, pl. LIV, fig. 6) et que notre S. Lantenoisi est identique à cette forme. En comparant la figure du Conchologia Indica à nos spécimens, nous avons reconnu le bien fondé de son observation.

Il résulte, d'autre part, de l'examen des nouveaux matériaux rapportés par $M$. Nansuy, que le principal caractère qui nous avait décidés à séparer le $S$. Lantenoisi du S. perakensis Crosse, n'existe pas en réalité, puisque nous avons observé sur la base du dernier tour de plusieurs spécimens des cicatrices de poils.

Enfin, étant convaincus aujourd'hui de l'identité spécifique des $S$. acris (= Lantenoisi) et peraliensis, il y a lieu de rejeter en synonymie ce dernier nom, qui est le plus récent, ou plutôt de le conserver pour désiguer une variété un peu plus petite et un peu moins élevée que l'acris typique. 


\section{Satsuma producta nov. sp.}

(PI. V, fig. 1, 2. 3, 4, 5, 6)

Testa tenuis, nitidiuscula, subtranslucida, elongato-trochiformis, angustessimeque perforata. Spira elata, conica, apice obtusulo. Anfr. 9 subplani, sutura lineari juncti. Anfr. primi leves, ceteri striis incrementi obliquis irregularibusque sculpti. Anfr. ultimus hand descendens, ad peripheriam carinatus, basi convexus. Apertura perobliqua, subquadrata, marginibus callo tenuissimo adnatoque junctis. Columella rectiuscula, subtorta, superne paululum dilatata. Labrum expansiusculum, medio subangulatum.

Color pallide fulvus.

Altit. 17, diam. $9 \mathrm{~mm}$. Apertura $5 \mathrm{~mm}$. alta, $5 \mathrm{~mm}$. lata.

Coquille mince, à peine luisante, subtranslucide, trochiforme allongée, très étroitement perforée. Spire élevée, conique, à sommet un peu obtus, composé de 9 tours presque plans, séparés par une suture linéaire. Premiers tours lisses, les autres ornés de stries d'accroissement obliques, irrégulières. Dernier tour ne descendant pas à son extrémité, caréné à la périphérie, convexe à la base.

Ouverture très oblique, subquadrangulaire, bords du péristome reliés par une callosite mince, appliquée et luisante. Columelle presque verticale, un peu tordue, légèrement dilatée vers le haut. Labre étroitement étalé, subanguleux à l'endroit où aboutit la carène périphériale.

Coloration d'un fauve clair uniforme.

Loc : Nam-Nhang (M. Mansuy, 1906).

Le S. producta diffère du S. straminea von Möllendorff (Nachrichtsbl., 1901, p. 73), par sa forme plus étroite, ses tours plus plans, son dernier tour nettement caréné à la périphérie, enfin, par son ombilic plus étroit. Le S. straminea n'ayant pas été figuré, nous eu avons repré- 
senté, pour comparaison, pl. V, fig. 10, 11, 12, un exemplaire recueilli à Than-Mö̈ par M. Frühstorfer et déterminé par von Möllendorff.

\section{Eulota Jourdyi L. Morlet}

\section{Var. montis•ola von Möllendorff}

1901. Eulola (Eulotella) Jourdyi L. M. subsp. monticola v. Môt.ENDORFF, Nachrichtsbl.d.deulschen Malak. Ges., p. 74.

1904. - Jourdyi subsp. monticola v. Mōll, H. Fischer et Dautzen. BERG, Liste Moll. Indo-Chlne in Mission Pavie, p. 403.

Loc. : Ha-Lang (M. Mansuy).

Cette forme diffère du Jourdyi typique par sa taille plus faible, son ombilic moins ouvert, son labre plus épais et sa coloration fauve uniforme, mais elle ne mérite pas, selon nous, d'être considérée comme une sous-espèce: c'est tout au plus une variété. L'exemplaire que nous avons sous les yeux a $10 \mathrm{~mm}$. de haut et $14 \mathrm{~mm}$. de diamètre.

\section{Plectotropis Bonvieri H. Fischer}

I898. Helix (Plectotropis ?) Bonnieri H. Fiscuer, Moll. du Haut-Tonkin, in Bull. Sc. France et Belgique, XXVIII, p. 319 , pl. XVII, fig. 12 à 16.

1900. - (Eulota) Vignali Bavay el Dautzenberg. Descr. coq. nour. Indo-Chine, in Journ. de Conch., XLVIII, p. 439, pl. 1X, fig. 4, 5, 6.

1904. Eulota (Plectotropis) Bonnieri H. Fischer el Dautzenberg,

Catal. Moll. Indo-Chine, in Mission Pavie, p. 403.

Loc. : Ha-Lang ; Quang-Huyen ; Trung-Khan-Phu, (M. Mansuy).

Nous avons été amenés par la comparaison d'un grand nombre d'exemplaires, à réunir l' $H$. Vignali à l'H. Bonnieri. Le type de l'H. Vignali était dépourvu de squamules 
épidermiques; mais nous avons constaté que ces squamules s'enlèvent très facilement et qu'elles ne sont visibles que chez les spécimens frais et qui n'ont pas subi de frottement.

Le type du $P$. Bonnieri a $81 / 2 \mathrm{~mm}$. de hauteur et $12 \mathrm{~mm}$. de diam. maximum. La taille des spécimens récoltés par M. Mansuy varie de 5 à $11 \mathrm{~mm}$. de haut et de 7 à $15 \mathrm{~mm}$. de diamètre. La spire est plus ou moins élevée ou aplatie et l'ombilic est plus ou moins grand.

\section{Euhadra Fauveli Bavay et Dautzenberg}

1900. Helix (Euhadra) Fauveli Bavay et Dautzenberg, Diagn. coq. nouv. Indo.Chine, in Journ. de Conch., XLVIII, p. 110.
1900. - - - - Bavay el Dautzenberg, Descr. coq. nouv. Indo-Chine, in Journ. de Conch., XLVIII, p. 437, pl. IX, fig. 16, 17, 18.
1904. Eulota (Euhadra) - B. et D., 11. Fiscuer et Dautzenberg, Catal. Moll. Indo-Chine, Mission Pavie, p. 404 .

Loc.: Massif du Luc-Khu (M. Mansuy, 1906).

\section{Möllendorffia Messagerı Bavay et Dautzenberg}

1899. Helix (Möllendorffia) Messageri Bavay el Dattzenberg, Descr. coq. nouv. Indo-Chine, in Journ. de Conch., XLVII, p. 33, pl. I, fig. $5,5^{a}, 5^{b}$.

1904. Helicodonta - - B. et D., H. Fischer et Dautzenberg, Catal. Indo-Chine, in Mission Pavie, p. 40 \%.

Loc.: Nam-Nhang (M. Mansuy).

\section{Helix (?) Onestera J. Mabille.}

1887. Helix onestera 1887 .

1904. - - Mab.
J. Mabille, Moll. Tonk. Diagn., p. 3. J. MablLle, Surquelques Moll. du Tonkin, in Bull. Soc. Malac. France, IV, p. 89, pl. II, fig. 4, 5.

H. Fischer et Dactzenberg, Catal. IndoChine, Mission Pavie, p. 404. 
Loc.: Nui-Con-Voi, près de Haiphong (montagne de l'Éléphant) (M. Mansuy).

\section{Bulimines (?) SIamensis Redfield}

18วั3. Hulimus siamensis

Redfield, Deser. new sp. of Helicidae, in Ann. Lyc. Nat. Hist. of New-York, VI, p. 15.

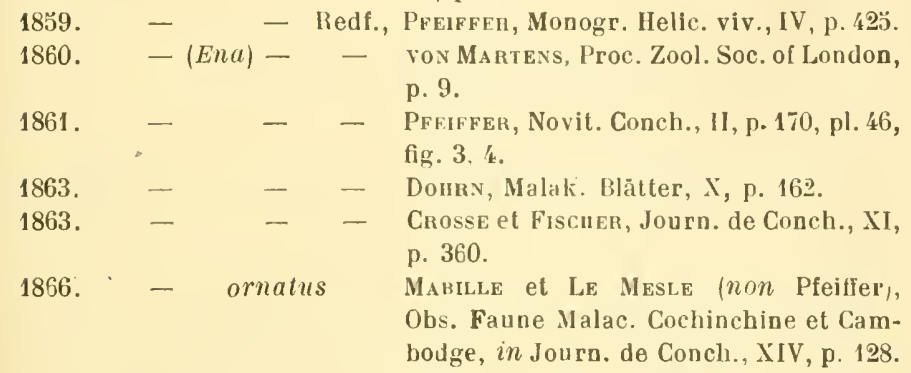

1867. Buliminus (Napaeus) siamensis Redf., von Martens, Preuss. Exp. nacl Ostasien, II, p. 81, pl. 19, fig. 7.

1868. Bulimus

1875. -

1859. Petraeus

1891. Buliminus

1899. - (Napaeus)

1902. Satsuma

1902. Ganesella p. 65.

- - A. Morelet, Séries Conch., IV, p. 266.

- - L. Morlet, Catal. coq. rec. par Pavie, in Journ. de Conch., XXXVII, p. 128.

- - P. Fischer, Catal. Indo.Chine, p. 34.

- - Kobelt, Monogr. in Conch. Cab., 2॰ édit., p. 559, pl. 83, fig. 1-5.

- Kobelt, Monogr. in Conch. Cab., $2^{\text {e }}$ édit., Register, p. 1048.

- Pilsbry, in Tryon, Manual of Conch. Struct. a. Syst., Index Bulimulidæ, p. XCIV.

1904. Buliminus siamensis Redf., H. Fischer et Dautzenberg, Catal. Indo-Chine, Mission Pavie, p. 407.

Loc.: Royaume de Luang-Prabang (M. Monod).

Cette espèce n'est représentée dans la collection de M. Mansuy que par un exemplaire unique, exceptionnellement grand et allongé : hauteur 31 diam. maj. 
9 mm., qui correspond à la var. 1 de Dohrn (Malak. Bl. X, p. 163.).

La position de ce Mollusque ne paraît pas encore bien définitivement fixée: M. Kobelt dans la table de la Monogr. des Buliminidæ du Conchylien Cabinet l'a classée parmi les Satsuma tandis que M. Pilsbry la place dans le genre Ganesella.

\section{Buliminus tenuistriatus nov. $\mathrm{sp}$.}

(PI. $\mathrm{V}$, fig. 13, 14)

Testa anguste rimata, parum solida, ovato-elongata. Spira conoidea apice obtusiusculo. An/r. 7 1/2 sat convexi, sutura impressa juncti, striis incrementi obliquis, irregularibus striisque trunsversis, sub lente valido tantum conspicuis ornati. Apertura obliqua, subquadrato-ovata ; peristoma paululum expansum, marginibus connicentibus, callo tenuissimo adnatoque junctis. Margo columellaris superne dilatata rimam umbilicalem obtegens. Labrum expansum sed haud reflexum, basin versus subeffusum.

Color sordide fulrus.

Altit. 13, diam. maj. $5 \mathrm{~mm}$; apertura $51 / 2 \mathrm{~mm}$. alta, $4 \mathrm{~mm}$. lata.

Coquille pourvue d'une fente ombilicale étroite, peu solide, ovale-allongée. Spire conoïde, obtuse au sommet, composée de 7 1/2 tours assez convexes, séparés par une suture bien accusée et ornés de stries d'accroissement obliques, irrégulières et de stries décurrentes extrême. ment fines, visibles seulement à l'aide d'une forte loupe. Ouverture oblique, ovale-subquadrangulaire. Péristome légèrement dilaté, à bords rapprochés et reliés par une callosité très mince, appliquée. Bord columellaire dilaté au sommet et masquant la fente ombilicale. Labre dilaté mais non réfléchi, un peu versant à la base.

Coloration d'un fauve sale uniforme. 
Loc.: Quang-Huyen (M. Mansuy).

Cette espèce diffère du B. Messageri B. et D., par sa sculpture composée de stries d'accroissement et de stries décurrentes très fines, tandis que la surface du Messageri est malléolée.

\section{Clausilia Auregani Bavay et Dautzenberg.}

1899. Clausilia Fargesiana Bavay et Dautzenberg (non Heude), Descr. coq. nouv. Indo-Chine, in Journ. de Conch., XLVIII, p. 45̆, pl. II, fig. 6, 6a .

1904. - $\quad$ - $\quad$ I. Fisghfr el Dautzenberg (non Heude), Catal. Indo-Chine, Mission Pavle, p. 409. Bavar el Dautzenbeng, Descr. coq. nouv. Indo-Chine, $3^{\circ}$ suite, in Journ. de Conch., LI, p. 213, pl. IX, fig. 9, 10, 11.

Loc.: Massif du Bac Son, Tonkin (M. Mansuy)

\section{Clausilia backanensis Bavay et Dautzenberg}

1899. Clausilia backanensis Bayay et Dautzenberg, Deser. coq. nouv. Indo.Chine, in Journ. de Conch., XLVII, p. 28I, pl. XII, fig. 5.

1904. - - B. el D., H. Fischer el Dautzenbeng, Catal. Indo Chine, Mission Pavie, p. 408.

Loc.: Quang-Huyen (M. Mansuy).

Clausilia Gereti Bavay et Dautzenberg.

1900. Clausilia Gereti

Bavay et Dautzenberg, Diagn. coq. nouv. Indo-Chine, in Journ. de Conch., XLVIII, p. 115, 447, pl. X, fig. 8 .

1904. - - B. et D., H. Fischer el Dautzenbert, Catal. IndoChine, in Mission Pavie, p. 409.

Loc. : Quang-Huyen (M. Mansuy).

Clausilia Giardi H. Fischer.

18y8. Clausilia Giardi

H. Fischer, Notos sur la Faune du Haut Tonkin, in Bull. Scient. France et Belgique, XXVIII, p. 14, pl. XVIII, fig. 22-27. 
1899. Clausilia Giardi II. F., Bavay et Dautzenberg, Descr. coq. nouv. Indo.Chine, in Journ. de Conch., XlVII, p. ö0, pl. III, fig. 2, 2a.

Loc. : Quang-Huyen; Trung-Khan-Phu (M. Mansuy).

Clausilia Grangerı Bavay et Dautzenberg.

1899. Clausilia Grangeri Bavay et Dautzenberg, Descr. coq. nouv. Indo-Chine, in Journ. de Conch., XLVII, p. 46 , pl. III, fig. $8,8^{\mathrm{a}}, 8^{\mathrm{b}}$.

1904. - - B. et D., H. Fischer et Dautzenberg, Catal. Indo. Chine, in Nission Pavie, p. 409.

Loc. : Ha-Lang (M. Mansuy).

Clausilia Mansuyi nov. sp.

(Pl. VI, fig. 1, 2, 3)

Testa sinistrorsa, solida, parum nitida, imperforata, fusiformis, superne ac inferne attenuata. Spira decollata. Anfr. superstites j 1/2 convexiusculi, sutura parum impressa juncti, oblique tenerrime confertissimeque striati. A pertura suberti. calis, piriformis. I'eristoma duplicatum : anticum quam posticum multo validius, continuum, incrassatum, expansum, paululum reflexum breviterque solutum; posticum circiter 1 millim. ab antico recedens. Lamella supera mediocris, marginalis et introrsum attenuata; lamella infera marginem haud attingens ac spiraliter ascendens. P'lica collumellaris prolunde immersa, plicae palatales opacitate conspicuae: principalis elongata a sutura recedit, plica secundaria brevis, unica, lunellam falciformem superat.

Color pallide /ulvus, peristoma albidum.

Altit. 25, diam. S millim.; apertura 7 1/2 millim. alta, 6 millim. lata.

Coquille senestre, solide, peu luisante, imperforée, fusiforme, atténuée aux deux extrémités. Spire tronquée. Tours subsistants au nombre de $301 / 2$, assez convexes, sé- 
parés par une suture bien marquée et ornés de stries obliques très fines et serrées. Ourerture subverticale, subpiriforme. Péristome double: l’antérieur, beaucoup plus épais que le postérieur, est continu et un peu détaché dans le haut; le postérieur, situé à peu près à 1 millimètre en arrière de l'antérieur, en est séparé par une rainure bien accusée. Lamelle supérieure médiocre, marginale, s'atténuant dans le fond de l'ouverture; lamelle inférieure submarginale, montant en spirale. Pli columellaire situé très profondément. Plis palataux visibles par transparence: le supérieur allongé et très éloigné de la suture. Un pli secondaire unique surmonte une lunelle falciforme.

Coloration d'un fauve clair, péristome blanchâtre.

Loc.: Quang-Huyen (M. Mansuy).

Le $C l$. Mansuyi diffère du pseudauregani par sa forme plus courte, moins atténuée dans le haut, son dernier tour plus rétréci, son pli subcolumellaire profondément enfoncé, son péristome détaché, sa coloration plus claire et ses plis palataux au nombre de trois seulement ( $\mathrm{y}$ compris la lunelle), au lieu de quatre.

\section{Clausilia orientalis J. Mabille}

(Pl. VI, fig. 4, 5े, 6)

1887. Nenia orientalis

1887

1904. - - J. Mab.,
J. Mabille, Moll. Tonk. Diagn., p. 17. J. Mabille, Sur quelques Moll. du Tonkin, in Bull. Soc. Malac. de France, p. 108.

H. Fischer et Dautzenberg, Catal. IndoChine, in Mission Pavie, p. 410.

Loc. : Massif du Bac Son, Tonkin (M. Mansuy).

Nous représentons ici cette espèce, qui n’avait pas encore été figurée, d’après un spécimen recueilli à Than Moi par II. Dujardin-Beaumetz. 
Clausilia pseudauregani nov. sp.

(PI. VI, fig. 7, 8, 9).

Testa sinistrorsa, solida, parum nitida, imperforata, fusiformis. Spira decollata, superne attenuata, medio ventrosula. Anfr. superstites 7 convexiusculi, sutura parum impressa juncti, oblique confertim tenuistriati. Anfr. ultimus attenuatus. A pertura subverticalis, oblique subpiriformis. Peristoma duplicatum: anticum quam posticum multo validius, continuum, incrassatum, expansum et vix refiexum; externum ab antico rima minime profunda sejunctum. Lamella supera mediocris, marginalis et introrsum attenuata ; lamella infera submarginalis spiraliterque ascendens; lamella columellaris sat valida ac marginalis. Plicae palatales opacitate extus conspicuae: principalis debilis, profunde immersa, secunda. riae duo breves, obliquae, lunellam brevem et falciformem superant.

Color castaneo-fuscus. Peristoma album.

Altit. 27, diam. $81 / 2 \mathrm{~mm}$. Apertura $8 \mathrm{~mm}$. alta, $6 \mathrm{~mm}$. lata.

Coquille senestre, solide, peu luisante, imperforée, allongée fusiforme. Spire tronquée, atténuée ver's le haut, ventrue au milieu. Sept tours persistants, légèrement convexes, séparés par une suture peu accusée. Surface ornée de stries d'accroissement obliques, fines et serrées. Dernier tour rétréci. Ouverture subverticale, obliquement subpiriforme. Péristome double: l'antérieur continu, étalé, à peine réfléchi, est beaucoup plus épais que le postérieur dont il n’est séparé que par une rainure étroite, peu profonde. Lamelle supérieure médiocre, marginale, atténuée dans le fond de l'ouverture. Lamelle inférieure submarginale, forte, montant en spirale. Pli columellaire marginal et assez fort. Plis palataux visibles par transparence : le principal est très étroit, allongé, les deux 
secondaires sont courts, obliques et surmontent une lunelle falciforme courte.

Coloration d'un brun marron foncé. Péristome blanc. Loc. : Quan-Huyen (M. Mansuy).

Cette espèce diffère du Cl. Auregani Bavay et Dautzen. berg (Journal de Conch. 1903, LI, p. 213, PJ. IX, fig. 9, 10, 11), par sa taille plus faible, son péristome moins épaissi, par la présence d'un pli columellaire marginal. Les plis palalaux sont semblables. Les deux péristomes sont un peu moins écartés.

\section{Clausilia thatkeana Bavay et Dautzenberg}

1899. Clausilia thatkeana

Bavay el Dautzenberg, Descr. coq. nouv. Indo-Chine, in Journ. de Conch., XLVII, p. 285, pl. XII, fig. 7.

190\%. - - B. el D., H. Fischer el Dautzenberig, Catal, IndoChine, in Mission Pavie, p. 410.

Loc. : Quan-Huyen (M. Mansuy).

\section{Prosopeas Lavillei nov. sp.}

(Pl. V, fig. 10๊, 16.)

Testu parum solida, nitidula, imperforata, elongatoturvita. Spirce elate, apice obtusiusculo. An/r. 11 subplani sutura impressa irregulariterque laceratu juncti : primi 2 lævigati, ceteri plicis longitudinalibus arcuutis et jurta suturam validioribus ornati. Anfr. nltimus basin versus attenuatus. Apertura sat elonguta, superne angulatu, marginibus callo tenui adnatoque junctis. Columella recta, ad basin attenuata; labrum simplex et acutum.

Color, sub epidermide sordide hutescente, allidus.

Altit. 32 1/2. diam. 8 millim.; apertura $9 \mathrm{~mm}$. alta, 5) mm. lata.

Coquille peu épaisse, légèrement luisante, imperforée, de forme allongée-turriculée. Spire élevée un peu obtuse 
au sommet, composée de 11 tours presque plans, séparés par ıne suture irrégulièrement crénelée : les deux premiers lisses; les autres ornés de plislongitudinaux arqués, plus accusés sous la suture. Dernier tour at ténué à la base. Ouverture assez allongée, anguleuse au sommet; bords reliés par une callosité mince, appliquée. Columelle verticale, atténuée à la base ; labre simple, iranchant.

Coloration blanchâtre sous un épiderme d'un jaune sale.

Luc. : Nam-Nhang (M. Mansuy).

Plus grande et moins allongée que le P. Fagoti Mabille, cette espèce que nous dédions à $\mathbf{M}$. Laville, préparateur à l'École des Nines, est aussi plus fortemeut sculptée, comme nous avons pu nous en rendre compte en la comparant au type du $P$. Fagoti, conservé au Muséum d'Histoire Nalurelle et que M. le Professeur Joubin a bien voulu nous communiquer.

\section{Pseudopeas Douvillei nov. sp.}

(Pl. V, fig. 17, 18;

Testa tennicula, haud nitens, imperforata, elongato-turrita. Spira elongata, apice mugno et obtuso. Anfr. 7 convexi, sutura valde impressa juncti : primi 2 spiraliter striati; ceteri plicis arcuatis, irregularibus densissimisque longitudinaliter ornati. Anfr. ultimus basin versus attenuatus. Apertura anguste elongata: columella recta, arl basin attenuata; labrum simplex et acutum.

Color undique sordide hutescens.

Altit. 12, diam. 31/2 mm. ; apertura $4 \mathrm{~mm}$. alta, $9 \mathrm{~mm}$. lata.

Coquille mince, non luisante. imperforée, de forme allongée turriculée. Spireélevée, à sommetrenflé et obtus, composée de 7 tours couvexes, séparés par une suture très accusée. Deux premiers tours (embryonnaires), ornés 
de stries décurrentes nombreuses; les autres, au contraire, de plis longitudinaux arqués, irréguliers et très serrés. Dernier tour atténué vers la base. Ouverture étroite, allongée ; columelle verticale, atténuée à la base. Labre simple, tranchant.

Coloration d'un gris jaunâtre uniforme.

Loc. : Massif du Luc-Khu (M. Mánsuy).

Le genre Pseudopeas n’a été signalé, jusqu'à présent, qu'ell Afrique et dans l'Australie centrale. If diffère de Prosopeas par ses tours embryonnaires striés spiralement. Chez l'espèce que nous décrivons ici, ce caractère est très net. Nous prions M. Douvillé, le savant Professeur de l'École des Mines, d'eu accepter la dédicace.

\section{Glessula Paviei L. Morlet}

1892. Glessula Paviei

L. Morlet, Descr. esp. nouv. IndoChine, in Journ. de Conch., XL, p. 321, pl. VII, fig. $4,4^{\mathrm{a}}, 4^{\mathrm{b}}$.

1904. - - L. Morl., H. Fischer el Dautzenberg, Catal. IndoChine, in Mission Davie, p. 411.

Loc. : Quang-Huyen (M. Mansuy).

TorTaxis papillosa nov. sp.

(PI. V. fig. 19, 20)

Testa solidula, nitens, imperforata, elongato turrita. Spira elatu apice valde tumido ac obtuso. Anfr. 12 concexi, sutura impressa juncti: mimi leves, ceteri plicis longitudinalibus obliquis virque conspicuis ornati. A pertura obliqua, ovuto-oblonga : columella arcuata, ad basin verticaliter truncato-plicata ; labrum simplex, arenatum.

Color undique virescente cornens.

Altit. $361 / 2$, diam. $\& 1 / 2 \mathrm{~mm}$. apertura $s \mathrm{~mm}$. alta, $5 \mathrm{~mm}$. lata. 
Coquille assez solide, luisante, imperforée, allongéeturriculée. Spire élevée, à sommet très renflé et obtus, composée de 12 tours convexes, séparés par une suture bien accusée : les premiers lisses, les autres ornés de plis longitudiuaux obliques très superficiels, à peine visibles à l'œil nu Ouverture oblique, ovale allongée ; columelle arquée, tordue et tronquée verticalement à la base; labre arqué, simple, tranchant.

Coloration d'uu jaune verdàtre sale, uniforme.

Loc. : Nam-Nhang (M. Mansuy).

Cette espèce est remarquable par ses tours embryonnaires très renflés, formant au sommet de la coquille une sorte de papille obtuse.

\section{Tortaxis permira Ancey}

1903. Spiraxis permira

Ancey, in Bavay et Dautzenberg, Descr. coq. nouv. Indo-Chine, in Journ. do Conch., LI, p. 219, pl. IX, fig. 17-20.

1906. Torlaxis permirus Ancey, Pilsbry, Man. of Conch. Struct. and Syst., XVIII, p. 10, pl. 2, fig. 15, 16, 17.

Loc. : Quang-Huyen; Nam Nhang (M. Mansuy).

\section{Var. multiplicata Ancey}

1903. Spiraxis permira var. multiplicala ANCEY in Bavay et DAUTzenBERg, Descr, coq. nouv. Indo-Chine, in Journ. de Conch., LI, P. 220 .

1906. Tortaxis permirus var. multiplicalus Ancey, PILsBry, Man. of Conch. Struct. and Syst., XVIII, p. 10, pl. 2, fig. 23.

Loc. : Quang-Huyen (M. Mansuy).

\section{Tontaxls Pfelfferi Menke}

18วั6. Spiraxis Pfeifferi

Menke, Malakoz. Blătter, III, p. 68 (Tourane).

1859. - - Menke, PfeifFr R, Monogr. Helic. viventium, IV, p. วั73. 

1860. - - - Pfeiffer, Novit. Conch., I, p. 103, pl. XXIX, fig. 7, 8 .
1900. Prosopeas - - v. Móllfndorf, Nachrichtsbl. der deutschen Malakoz. Ges., p. 134.
1904. Spiraxis - - H. Fischer et Dautzenberg, Catal. Indo- Chine, in Mission Pavie, p. 411.
1906. Tortaxis - - Pilsbry, Manual of Conch. Struct. and Syst., XVIII, p. 12, pl. 4, fig. 21, 22.

Loc. : Nam-Nhang; Massif du Luc-Khu ; Quang-Huyen (M. Mansuy).

\section{LimnaEa anNamitica Wattebled}

1886. Limnæa annamitica IVattebled, Descr. Moll. Annam rec. par Dorr, in Journ. de Conch., XXXIV, p. 57 , pl. IV, fig. 4 .

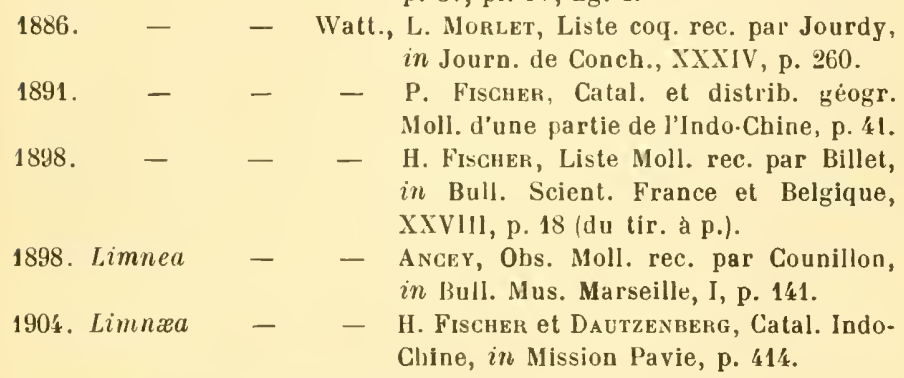

Loc. : Quang-Huyen (M. Mansuy).

\section{Limnaea spadicea A. Morelet}

1906. Limnza spadicea A. Mor., Dautzenberg et H. Fischer, Liste coq. rec. par Mansuy, in Journ. de Conch., LIII, p. 380.

Loc. : Royaume de Luang-Prabang (M. Monod).

\section{Canidia Helena Meder}

1817. Melania (Melanopsis) Helena Mener in PhiLIPPI, Abbildungen, p. $20(170)$, pl. IV, fig. 4 (Java).

1849. Melanopsis

- Meder, Moussox, Moll. de Java, p. 6', pl. X, tig. 2. 
1860

1860. Hemisinus

1868. Canidia

1875. Hemisimus

1886. Canidia Baudoniana
- - von Martens, On the Moll. of Siam, in Proc. Zool. Soc, of London, p. 14.

- - Reeve, Conch. Icon., pl. VI, fig. 24a $24^{\mathrm{b}}$

- - Ввот, Catal. Syst. fam. Mélaniens, p. 33.

- - Monelet, Séries Conch., IV, p. 320. Mabille et Le Mesie, in Journ. de Coneh., X1Y, p. 133, pl. VIII, fig. 1.

1891. - Helena Meder, L. Morlet, Contrib. faune malac. Indo. Chine, in Journ. de Coneh., Xxxix, р. 233 .

1891. - - - P. Fischer, Calal. el distrib. géogr. Moll. d'une partie de l'Indo-Chine, p. 60.

1904. - - - - H. Fischer el Dautzenberg, Catal. IndoChine, in Mission Pavie, p. 415.

Loc. : Royaume de Luang-Prabang (M. Monod).

\section{Melania Aubryana Heude}

1890. Melania Aubryana

Heude, Mém. Emp. Chinois, 4* cahier, p. 166, pl. XLI, fig. 27 (non fig. 28, 28).

1898. - - Heude, II. Fischer. Note sur la Faune du Haut Tonkin, in Bull. Seient. France et Belgique, p. 18, pl. XVIIr, fig. 42 à 44.

1904. - - - H. Fischer et Dautzenberg, Catal. IndoChine, in Mission Pavie, III, Hist. Nat. Indo-Chine Orient, , p. 417.

1006. - Hugeli Dautzenberg et H. Fischer ( $n o n$ Philippi), Liste Moll, rec. par Mansuy, in Journ. de Conch., LIII, p. 411.

Var. obliterata nov. var.

Chez cette forme, la sculpture décurrente est beaucoup plus atténuée que chez le type, les cordons étant même parfois presque entièrement effacés sur le haut du dernier tour. C'est par erreur que nous avons assimilé au M. Hugeli des spécimens jeunes récoltés par M. Mansuy à Ha-Giang et par M. Blaise dans la région de Mai-Xu, Haut-Song-Bo (Tonkin), ils appartiennent tous, en réalité, à la présente variété du $M$. Aubryana. Quant au véritable 
11. Hugeli Philippi, c'est une forme plus lisse, très finement striée, sauf sur la base du dernier tour, qui porte quelques sillons bien marqués. Sa surface est plus luisante, son test est plus mince, sa coloration est d'un vert noirâtre et l'intérieur de son ouverture est d'un brun foncé.

Loc. : Song Bang-Giang à Cao-Bang, Mansuy ; Nam-Luat (M. Mansuy).

\section{Melania proteus Bavay (nom. mut.)}

1906. Melania Jacqueti

Dautzenberg et H. Fischer (non M. Jac. quetiana Heude), Liste Moll. rec. par Mansuy, in Journ. de Conclı., LIII, p. 413, pl. X, fig. 16.

Loc. : Ban-Cra (M. Mansuy).

M. Bavay ayant remarqué qu'il existait déjà un Melunia Jacquetiana Heude : Moll. Empire Chinois, p. 163, pl. XLI, fig. $7,8,8^{\text {a }}, 9$ et pl. XLIII, fig. 5 , propose de substituer au nom que nous avions employé, celui de $\boldsymbol{M}$. proteus.

\section{Melania Schomburgii (Hanley) Reeve}

1839. Melania Schomburgki Hanley in Reeve, Conch. Icon., pl. XIV, fìg. 93 .

$\begin{array}{llll}1866 . & - & - & \text { Hanl., Mabile et Le Mesce, Obs. sur Ia faune } \\ \text { malac. de la Cochinchine et du Cam- } \\ \text { 1874. }\end{array}$

Loc. : Royaume de Luang-Prabang (M. Monod). 


\section{Melania tuberculata Müller}

1906. Melania tuberculata Mäll, Diutzenrerg el H. Fischer, Contrib. Faune malac. Indo-Chine, in Journ. de Conch., LIV, p. 165.

Loc. : Quang-Huyen, Mansuy; Royaume de Luang-Prabang (M. Monod).

Les spécimens recueillis par M. Mansuy à Quang-Huyen, sont remarquablement grands: haut. 36 , diam.121/2 mm.

\section{Cremnoconchus Messageri Baray et Dautzenberg}

1900. Cremnoconchus Messageri Bavay et Dautzenberg, Diagn. et Descr. coq. nouv. Indo-Chine, in Journ. de Conch., XLVIII, p. 116 ; p. 449, pl. X, fig. 10 .

Loc. : Ban-Cra (M. Mansuy).

\section{Paludina ampulliformis Souleyet}

1906. Paludina ampulliformis Soul., Dautzenberg et H. Fischer, Contrib. faune malac. Indo-Chine, in Journ. de Conch., LIV, p. 170.

Loc. : Ha-Lang (M. Mansuy).

Nous sommes amenés à croire aujourd'hui, en présence de nombreux spécimens, que la forme rapportée du Yunnan par M. Mansuy et que nous avions rattachée au lecythoides Philippi, n'est qu'une variété de l'ampulliformis. Notre $P$. lecythoides var. latissima deviendrait donc $P$. ampulliformis var. latissima.

\section{Paludina lithophaga Heude}

1889. Paludina lilhophaga Heude, Diagn. Moll. nov. in Sinis collect., in Journ. de Conch., XXXVII, p. 49.

1890. - - - Heune, Moll. Empire Chinois, $4^{e}$ cahier, p. 177, pl. XL, fig. 13, 13a. 
Loc. : Ha-Lang (M. Mansuy).

Il existe une variété à bandes transversales brunes et on observe aussi parfois, sur certains exemplaires, des traces de carène.

\section{Paludina polyzonata Frauenfeld}

\section{subsp. Duchient H. Fischer}

190万. Paludina polyzonata Fr. var. Duchieri H. F. Dautzenberg et H. Fischeli, Liste Moll. rec. par Blaise, in Journ. de Conch., LIII, p. 156.

\section{Var. obsoleta nov. var.}

(PI. VI, fig. 16)

Loc. : Cao-Bang (M. Mansuy).

Les spécimens rapportés decette localité par M. Mansuy, ont les cordons décurrents très faibles, parfois même obsolètes et remplacés, dans ce cas, par des lignes brunes. Nous avons représenté pour comparaison, pl. VI, fig. 17, un spécimen de la subsp. Duchieri de forme typique; mais à péristome rouge, tandis que le type a l'ouverture blanchâtre. Nous désignons cette variété sous le nom de var.

erythrostoma, nov. var.

\section{Paludina Tiranti L. Morlet}

1884. Paludina Tiranti

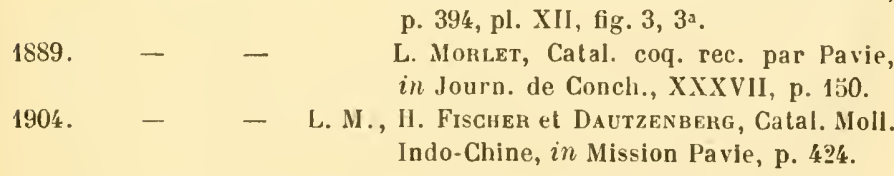

Loc. : Royaume de Luang-Prabang (M. Monod, 
A upullaria polita Deshayes

1906. Ampullaria polita Desh., Dautzenberg et II. Fischer, Liste Moll. rec. par Mansuy, in Journ. de Conch., LIII, p. 426.

Loc. : Royaume de Luang-Prabang (M. Monod).

Opisthoporus Beddomei nov. $\mathrm{sp}$.

(Pl. VII, fig. 1, 2, 3, 4)

Testa solida latissime ac pervie umbilicata. Spira depressa complanata, apice prominulo. Anfr. 4 convexi, sat celeriter accrescentes, sutura mofunda juncti; ultimus ad extremitatem solutus, descendens et tubulo, circiter $2 \mathrm{~mm}$. post aperturam, munitus. Anfr. primi leves, ceteri plicis incrementi lamellosis, confertissimisque ornati. Apertura circuluris. Peristoma duplicatum : internum continum; externum breviter expansum atque inflexum, superne in aliculam productum.

Color, sub epidermide fuscescente ac setoso sordide griseus et flammulis luscis depictus. Peristoma internum albidum.

Altit. $\$ 1 / 2$, diam. maj. 16, $\mathrm{min} .12 \mathrm{~mm}$. ; apertura $7 \mathrm{~mm}$. alta, $7 \mathrm{~mm}$. lata.

Coquille Iargement et profondément ombiliquée. Spire déprimée, subplane, à sommet légèrement saillant, composé de 4 tours convexes, croissant assez rapidement et séparés par une suture très profonde. Dernier tour détaché et descendant à l'extrémité, pourvu, à 2 mm. environ, en arrière du péristome, d'un tube de $2 \mathrm{~mm}$. de longueur. Premiers tours lisses, les suivants ornés de plis d'accroissement lámelleux, très serrés. Ouverture circulaire. Péristome interne continu; l'externe, un peu dilaté, est infléchi du côté basal et le long du labre; il est terminé, dans le haut, par une très légère expansion aliforme.

Coloration d'un gris sale, orné de flammules brunes sous un épiderme brunâtre et soyeux. Péristome interne blanchâtre. 
Loc: Quang-Huyen (M. Mansuy).

Nous prions notre savant confrère de Londres, M. le colonel Beddome, qui s'est spécialement adonné à l'étude des operculés terrestres, d'accepter la dédicace de cette espèce.

Opisthoporus lubricus nov. sp.

(PI. VII, fig. 5, 6, 7, 8)

Testa solida, nitida, depresse turbinata, late ac pervie umbilicata. Spira parum elata. Anfr. 5 convexi, sat celeriter accrescentes suturaque profunda juncti. Anfr. ultimus ad ertremitatem paululum descendens, sed haud solutum et tubulo brcvi, rirciter $2 \mathrm{~mm}$. post aperturam munitus. Anfr. primi leves, ceteri striis incrementi tenuissimis obsolete ornati. A pertura circularis ; peristoma duplicatum : internum continuum, externum breviter expansum atque inflcxum; superne truncatum.

Color pallide fulvus, flammulis fuscescentibus, fulguratis, pictus; anfr. ultimus in peripheria zonula fusca angusta et interrupta cinctus. Operculum normale.

Altit. 9, diam. maj. 16, min. $12 \mathrm{~mm}$.; apertura $7 \mathrm{~mm}$. alta, $7 \mathrm{~mm}$. lata.

Coquille solide, Iuisante, comme vernissée, de forme déprimée, turbinée, largement et profondément ombiliquée. Spire peu élevée, composée de 5 tours convexes, séparés par une suture profonde. Dernier tour descendant légèrement à son extrémité mais non détaché et pourvu, à $2 \mathrm{~mm}$. environ, en arrière du bord de l'ouverture, d'une tubulure courte et étroite. Premiers tours lisses, les suivants ornés de stries d'accroissement fines et très peu apparentes. Ouverture circulaire. Péristome double: l'intérieur continu, l'extérieur étroitement dilaté et infléchi, tronqué au sommet où il se termine par une très légère expansion. 
Coloration d'un fauve clair, orné de flammules disposées en zigzags. A la périphérie du dernier tour, règne une zone brune, étroite et interrompue. Opercule normal.

Loc. : Quang-Huyen (M. Mansuy).

Cette espèce diffère de l'( $)$. Beddomei par sa forme un peu plus haute, moins aplatie, son dernier tour non détaché à l'extrémité, son ouverture plus ample, son épiderme non fibreux, sa surface lisse et très luisante, ses flammules plus délicates et plus nombreuses.

Mrxostoma paradoxum nov. sp.

(PI. VII, fig. 9. 10, 11 et opercule, fig. 12, 13)

Testa solida, parum nitens, latissime ac pervie umbilicata. Spira depressa, complunata, apice vix prominulo. Anfr. 5 convexi, sat celeriter accrescentes, sutura impressa juncti; ultimus ad extremitutem paululum descendens et dimidio tnbulo, postice recurco, juxta aperturam munitus. Anfr. primi leves, ceteri plicis capilluribus arcualis, in basi anfr. ultimi attenuatis, ornati. A pertura circularis; peristoma subduplicatum, superne incisum ac sinum rotundatum, extus in tubulum productum, efformans. Operculum tenue, corneum, extus planatum, lamellam helicoidalem primo deciduam, deinde late expansum radiatimque striatam emittit ; pagina interna paululum concaca, in medio pustulam praebet.

Color sordide albidus, flammulis fuscis /nlguratis profuse depictus, in peripheria zonula albida angusta puncticulatim interrupta, zonulam alteram saturate fuscam superat. Peristoma album.

Altit. 13, diam. maj. 30, min. 24 mm.; upertura $11 \mathrm{~mm}$. alta $11 \mathrm{~mm}$. lata.

Coquille solide, peu luisante, très largement et profondément ombiliquée. Spire déprimée, aplatie, à sommet à peine saillant, composée de 5̆ tours convexes, croissant assez rapidement et séparés par une suture profonde. 
Dernier tour descendant un peu à l'extrémité et pourvu, contre le péristome, d'une demi-tubulure large et recourbée en arrière. Premiers tours lisses, les suivants ornés de plis capillaires arqués qui s'atténuent sur la base du dernier tour. Ouverture circulaire, péristome double du còté du labre el dans le haut, chez les exemplaires bien adultes; bords du péristome disjoints au sommet, oủ ils forment un sinus arrondi d'où part la demi-tubulure externe.

Opercule corné, mince, aplati, présentant au centre une petite dépression circulaire et orné d’une lamelle lébicoïde qui ne subsiste que le long du bord où elle s'étale largement dans l'intérieur de l'ouverture. Celte lamelle est ornée de stries rayonnantes très visibles. La face interne de l'opercule présente, au centre, un petit mamelon arrondi correspondant à la dépression circulaire de l'intérieur.

Coloration d'un blanc sale profusément couvert de flammules brunes rayonnantes et de linéoles en zigzags de même couleur. A la périphérie, règne une zône blanche, étroite, ponctuée de brun et immédiatement au-dessous, on observe une large bande brun foncé. La base du dernier tour est ornée de linéoles brunes en zigzags, très serrées. Péristome blanc.

Loc. Quang-Huyen (M. Mansuy).

Cette espèce ressemble au premier aspect au Rhiostoma Morleti Dautz. et H. Fischer (Journ. de Conch., 1905\%, p. 429, pl. X, fig. 1 à 4); mais sa spire est plus plane et son dernier tour descend moins à l'extrémité. Son ouverture est partiellement entourée d'un péristome double : l’intérieur sinfléchit vers le sommet en formant un sinus arrondi, tandis que l'extérieur enveloppe l'autre et vient toucher lavant dernier tour sans, toutefois, se souder au bord columellaire. La tubulure est produite, soit par une prolongation du bourrelet du labre, soit par la partie dlu test 
qui se ${ }^{*}$ rouve immédiatement en arrière. La conformation de l'opercule fixe la place de ce mollusque parmi les Myxostoma.

\section{Cyclophorus Mansuyi nov. sp.}

(PI. VIII, fig. 1, 2, 3, 4)

Testa solida, late ac pervie umbilicata, turbinato-depressa. Spira parum elata, apice obtusulo. Anfr. 5 convexi, rapide crescentes, sutura impressa juncti, striis incrementi irregularibus et striis transversis tenuissimis, sub lente valido tantum conspicuis ornati. Anfi. ultimus paululum dilatatus, haul descendens et ad peripheriam vix subangulatus. Apertura obliqua, cirularis. Peristoma continuum, callosum, expansum atque reflexum.

Color castaneus, albo pro/use irregulariter variegatus; ad peripheriam zonula albidu exilissima vittam latissimam nigrocastaneam superante cinctus. Regio collumellaris alba. Peristoma albidum

Altit. 31, diam. maj. $48, \min .36 \mathrm{~mm}$.; apertura $22 \mathrm{~mm}$. alta, $25 \mathrm{rm}$. Lata.

Coquille solide, largement et profondément ombiliquée, de forme turbiuée, assez déprimée. Spire peu élevée, à sommet légèrement obtus, composée de 5 tours convexes, croissant rapidement, sẻparés par une suture bien accusée et ornés de stries d'accroissement irrégulières aiusi que de stries décurrentes extrêmement fines et visibles seulement avec l'aide d'une forte loupe. Dernier tour un peu dilaté, non descendant et à peine subanguleux à la périphérie. Ouverture oblique, circulaire; péristome continu, calleux, étalé et réfléchi.

Coloration d'un brun foncé parsemé, au-dessus de la périphérie, de flammules blanches irrégulières. A la péri phérie règne une zône blanche étroite qui surmonte une 
bande très large, d'un brun noirâtre. Région columellaire blanche. Péristome blanchâtre.

Loc. : Quang-Huyen (M. Mansuy).

Cette espèce est relativement très aplatie. Elle a beaucoup d'analogie avec le $C$. Theodori Aneey; mais son test est plus épais, son dernier tour est moins haut, en proportion, se dilate moins vers l'ouverture et est encore plus faiblement carénée à la périphérie; son onbilic est plus largement ouvert. Enfin, on distingue sur la surface du C. Mansuyj, à l'aide de la loupe, des stries décurrentes très fines dont nous n'avons pu découvrir la moindre trace sur l'exemplaire type du C. Theodori qui fait actuellement partie de notre collection.

De même que chez ses congénères, la taille du C. Mansuyi est fort variable. Le plus pelit specimen de la récolte de M. Mansuy n’a effet, que $20 \mathrm{~mm}$. de laauteur, $36 \mathrm{~mm}$. de diam. max. et de 27 de diam. miu. La coloration varie aussi beaucoup; mais le sommet de la spire est toujours noirâtre et la région ombilicale blanche.

\section{Cyclophorus Courbeti Ancey}

1906. Cyclophorus Courbeli Anc., Dautzenberg et H. Fischer, Liste Moll. rec. par Nansuy, in Journ. de Conch., LIII, p. 431.

Loc. : Quang-Huyen (M. Mansuy); formal minor : CaoBang (Mansuy).

\section{Cyclophorus dodrans J. Mabille}

1906. Cyclophorus dodrans J. Mab., Dautzenberg et H. Fischer, Liste Moll. rec. par Mansuy, in Journ. de Conch., p. 433.

Loc. : Quang-Huyen (M. Mansuy). 


\section{Cyclophorus speciosus Philippi}

1906. Cyclophorus speciosus Phil., Dautzenberg et H. Fischer, Liste Moll. rec. par Mansuy, in Journ. de Conch , LIII, p. 433.

Loc. : Quang-Huyen (M. Mansuy).

\section{Pseudoponiatias fulvus von Möllendorif}

(Pl. VI, fig. 10-11)

1901. Pseudopomatias fulvus von Môllendorff, Diagn. neuer von Frühstorfer ges. Landschn., in Na. chrichtsbl. d. Malak. Ges., p. 81.

1904. - - v. Möll, H. Fischer et Dautzeniberg, Catal. Moll. Indo-Chine, in Mission Pavie, p. 429.

Loc. : Deux-Ponts (M. Mansuy).

Nous arons représenté ici cette espèce qui n'avait pas encore étẻ figurée.

\section{Platyrhaphe Leucacme von Möllendorff}

1901. Platyrhaphe leucacme v. MöllendorfF, Diagn. in Tonkin ges. Landschn., in Nachrichtsbl. d. Malak. Ges., p. 80 .

1903. - - v. Mōll., Bavay et Dautzenberg, Descr. coq. nouv. Indo-Chine, in Journ. de Conch., LI, p. 233, pl. XI, fig. 15, 16.

1904. - - - - H. Fischir el Dactzen Beng, Catal. Moll. Indo-Chine, in Mission Pavle, p. 429.

Loc. : Quang-Huyen (M. Mansuy).

\section{Platrihaphe sordida Pfeiffer}

18วัD̆. Cyclostoma (Cyclotus) sordidum PretrFen, Proc. Zool. Soc. of London, p. 103.

1863. Cyclolus sordidus Pfr., Reeve, Conch. lcon., pl. IX, fig. S.2.

1903. Platyrhaphe sordida Baval et Dautzenberg, Deser. coq. nouv. Indo-Chine, in fourn. de Conch., LI, p. 232, pl. X1, fig. 13, 14.

Loc. : Quang-Huyen (M. Mansuy). 


\section{Lagochilus scissimargo Benson}

1906. Lagochilus scissimargo Bens., Dautzenberg el H. Fischer, Liste Moll. rec. par Mansuy, in Journ. de Conch., LIII, p. 439.

1906. Japonia (Lagochilus) - - KoBelt, Monogr. Cyclophoridx, in Syst. Conch. Cab., $2^{2}$ édit., p. ö0̋̈, pl. 63 , tig. 12, 13, 14, 23.

Loc. : Quang-Huyen (M. Mansuy).

\section{Eupupina Angeyl Bavay et Dautzenberg}

1899. P'upina Anceyi

Bavay et Diutzenberg, Deser. coq. nouv. Indo-Chine, in Journ. de Conch.,

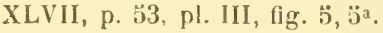

190\%. - - B. et D., H. Fischer et Dautzenberg, Catal. Moll. Indo. Chine, in Mission Pavie, p. 431.

Loc. : Mo-X̃at (M. Mansuy),

Eupupina Mansuri nov, sp.

(Pl. VI, fig. 12, 13, 14, 15)

Testa subpellucida, tenuis, nitidissima, ocato-conoidea et sat ventrosa. Spira mediocris, conoidea, apice subacuto. Anfr. 6 convexiusculi, sutura conspicun juncti, striis incrementi lenuissimis etiam sub lente vix conspicuis ornati. Anfr. ulimus versus aperturam descendens. Apertura circularis. Peristoma value callosum, inferne pauluhum provectum, superne emarginatum et sinulum ascendentem efformans. Ad columellae basin simulus alter rotundalus, brevis culloque circumilatus conspicitur. Margo columellaris arcuata. Labrum arcuatum, expansum et reflexum.

Color pullide fulrus, ad extremitatem an/r. ultimi rufescens. Peristoma albidum. Operculum tenue, corneum.

1ltit. 10, diam. maj. $5 \mathrm{~mm}$, apertura $4 \mathrm{~mm}$. alua, \& $\mathrm{mm}$. lata. 
Coquille assez mince, subpellucide, très luisante, de forme ovale-conoïde. assez ventrue. Spire conoïde, médio crement élevée, subacuminée au sommet, composée de 6 tours un peu convexes séparés par une suture bien visible et ornés de stries d'accroissement qu'on aperçoit à peine, même sous la loupe. Dernier tour descendant vers l'ouverture. Ouverture circulaire. Péristome très calleux, un peu projeté en avant à la base et présentant, dans le haut, un sinus ascendant, bordé extérieurement et limité, en avant, par une forte lamelle pariétale trigone. La base de la columelle est échancrée par un sinus arrondi, peu profond, circonscrit par une callosité. Bord columellaire régulièrement arqué. Labre arqué, largement étalé et réfléchi.

Coloration d'un fauve pâle, passant au bruu rougeàtre à l'extrémité du dernier tuur. Péristome blanchâtre. Opercule mince, corné.

Loc.: Deux-Ponts; Quang-Huyeu (M. Mansuy).

$\mathrm{Au}$ premier aspect, cette espèce ressemble assez au tonkiniana Bavay et Dautzenberg (Journ. de Conch.1899, p. 54) mais la conformation de son ouverture est fort différente : tandis que chezle tonkiniana, la lamelle pariétale est très faible, elle est, au contraire, tres forte chez le Mansuyi et elle forme avec le haut du labre un long sinus. Le sinus de la base est aussi plus grand chez le Mansuyi. Au contraire, chez ce dernier, l'ouverture est plus petite et limitée dans le haut par une callosité saillante et nettement limitée, tandis que chez le tonkiniana, les bords ne sont reliés que par une callosité mince et appliquée.

Eupupina $V$ erneaui Dautz, et H. Fisch. 
Loc.: Mo-Xat; Quang-Huyen (M. Mansuy).

La récolte de $M$. Mansuy renferme une forme de grande taille : haut. 9 diam. $\breve{~} \mathrm{~mm}$., qui peut être dèsignée comme var. major.

\section{Hybocystis gravida Benson}

1906. Hybocystis gravida Bens,, Dautzenber; et H. Fischer, Liste Moll. rec. par Mansuy, in Journ. de Conch., LIII, p. 441.

Loc.: Quang-Huyen (M. Mansuy).

Alycaeus (Chakax) Fraterculus Bavay et Dautzenberg 1900. Alycaeus (Chatax) fraterculus Bavay et Dautzexblerg, Diagn. et descr. coq. nouv. Indo-Chine, in Journ. de Conch., XLVIII, p. 120, 450 pl. XI, fig. 11, 12,13, 14 .

1904. - - - B. et D., H. Fischer et Dautzenberg, Catal. Moll. Indo Chine, in Mission Pavie, p. 432.

Loc.: Quang-Huyen (M. Mansuy).

\section{Alygaeus (Diorix) pilula Gould}

1900. Alycaeus pilula Gld., Baray et Dautzenberg, Descr. coq. nouv. Indo-Chine, in Journ. de Conch., XLVIII, p. 4 כ̆6.

190\%. - - - H. Fischer et Dautzenberg, Catal. Moll. Indo-Chine, in Mission Pavie, p. 432 .

Loc.: Quang-Huyen (M. Mansuy).

Diplommatina Balansai L. Morlet

Var. Musuta Bavay et Dautzenberg

1903. Diplommatina Balansai L. M. var. robusla Bavay et DavtzenBerg, Descr. coq. nouv. Indo-Chine, in Journ. de Conch., LI, p. 221, pl. X, fig. $1,2,3,4$. 
1906.

- - - - Dattzexberg et H. Fischer, Liste Moll. rec. par Mansuy, in Journ. de Conch., LIII, p. 4 \&2.

Loc.: Quang-Huyen (M. Mansuy).

\section{Diplomintina Lemyrei Bavay et Dautzenberg}

1903. Diplommatina Lemyrei Baray et Dattzenberg, Descr. coq. nouv. Indo.Chine, in Joura. de Conrb., LI, p. 227, pl. XI, fig. วั, 6 .

Loc.: Quang-Huyen ; massif du Luc-Khu (M. Mansuy).

Helicisa (Aphaxoconia) Huxgerfordiaxa von Möllendorff 1882. Helicina Hungerfordiana vox Möllexdorff, Jahrb. d. deutschen Malakoz. Ges., p. 182, 33̆'t.

1903. Aphanoconia - v.Möll., A.-J. Wagxer, Helicinenstudien, in Denk. Ak. Wien, LXXVII, p. 389, pl. IN, fig. $10^{\mathrm{a}}, 10^{\mathrm{b}}, 10^{\mathrm{c}}$.

Loc.: Quang-Huyen (M. Mansuy).

Cette espèce a été décrite d'après des spécimens provenant de Hong-Kong; mais elle a été également recueillie dans l'île liebao par. II. Frühstorfer.

\section{Pseudotrochatella Gredltaiana von Möllendorff}

188'. Helicina (Troihatella) Mouholi Pfeiffer var. Martensi Greder (non Jssel), Jahrb. d. Malakoz. Ges., XI, p. 141.

1895. - Gredleriana vox Mölendonff, Jahrb. d. Malakoz. Ges., XII, p. 3iı.

1૪86. - (Trochatella) - v. Möll., L. MonLet, Liste coq. rec. au Tonkin par Juurdy, in Journ. de Conch., XXXIN, p. 261.

1831. - Geotrochatella) - - P. Fischer, Catal. et distr. géogr. Mull. dune partie de I'InjoChine, p. 113.

1s93̈. Festrochatella - - Daltzesberg, Revis, rsp. g. Gintrochatella, in Journ. de Conca., XLIII, p. 22. 
1898. Pseudotrochatella

- - H. Fischer, Notes Faune $\mathbf{H}^{\mathbf{2}}$ Tonkin, in Bull. Scient. France el Belgique, p. 24 .

190\%. - - - H. Fischer el Dictzexberg, Catal. íoll. Indo.Chine, in Mission Pavie, p. $\$ 33$.

1903̆. - Martensi Gredl., A. J. WAgrer (non Issel), Denk. Ak. Wien, LXXVIl, p. 367, pl. I, fig. 23a, $23^{\text {b }}, 23$.

1908. Geolrochatella - - Koвelt (non Inel), Monogr. in Conch. Cab., 2' édit., p. 13, pl. 2, fig. 1, 2.

Loc.: Trung-Kihan-Phu ; Quang-Huyen (M. Mansuy).

Psecdotrochatella Nogieri Dautzenberg et d'Hamonville (PI. III, fig. 14, 15ั, 16, 17, 18)

1887. Trochatella Nogieri Dattzexberg et d’Hamorimlle, Descr. de 2 esp. nouv. du Tonkin, in Journ. de Conch, XXXV, p. 301.

1892. Geolrochatella - D. et d'H., L. Mortet, Descr. esp. nour. Indo Chine, in Journ. de Conch, XL, p. 32S, pl V'III, fig. $1,1^{\mathrm{a}}, 1^{\mathrm{b}}$.

1895. - - - - Dactzexbarg, Revis. esp. G. Geolrochatella, in Journ. de Conch., XLIII, p. 23.

1904. Pseudotrochatella - - H. Fischer et Dattzriberg. Catal. Noll. Indo-Cluine, in Mission Pavie, p. $\mathbf{4 3 3}$

490כ. - - - A.-J. Wagxer, Denk. Ak. Wien, LXXYI, p. 368 .

1908. Geotrochatella - - Kobelt, Monogr. in Conih. Cah., 2e edit., p. 14, pl. I, 0.2. 16, 17 .

Loc.: Massif du Bac-Son. au X.-O. de Lang-Son (.M. Mansuy).

Le Ps. Mogieri a été décrit d'après un spécimen unique recueilli mort à Than-ıloï et qui était décoloré.

Nous en faisons figurer aujourdhui deux individus récoltés vivants par M. Mansuy. l’un est d'une teinte jaune citron, l'autre rosé. Ils présentent tous deux des taches blanches opaques trés irrégulières, de mème que les autres Pseudotrochatella. 


\section{Anodonta Jourdyi L. Morlet}

1903. Anodonla Jourdyi L. M., Dautzenberg et H. Fiscier, Liste Moll. rec. par Blaise, in Journ. de Conch., LIII, p. 19 .

Loc.: Song Bang-Giang, à Caro-Bang (M. Mansuy).

\section{Pseunodon eluipticus Cunrad}

186ä. Pseudodon ellipticum Conran, Descr. of a new sp. of Pseudodon, in Amer. Journ. of Conch., I, p. 35้2, pl. 25, fig. 1.

1900. - - - - S Simpson, Synopsis of the Naiades, p. 836. 1904. - elliplicus H. Fischeis et Da utzenberg, Catal. IndoChine, in Mission Pavie, p. 436.

Loc.: Royaume de Luang-Prabang (M. Monod).

Nos spécimens sont plus grands que le type: diam. antéro-post. 80, diam. umbono ventral 48, épaisseur $23 \mathrm{~mm}$; ils sont aussi d'une coloration plus foncée.

\section{Pseudodon exilis A. Morelet}

1866. Microcondylus exilis A. Monelet, Descr. esp. Indo-Chine, in Journ. de Conch., XIV, p. 63.
1875. Pseudodon -
A. Monelet, Séries Conch., IV, p. 3i0, pl. XVII, fig. 1.
1886. - - A.M., L. Mortet, Liste coq. rec. par Jourdy, in Journ. de Conch., XXXIV, p. 266.
1900. - _ _ - Simpson, Synopsis of the Naiades, p. 839. Chine, in Mission Pavie, p. 436.
1904. - - - - H. Fiscier el Dautzen berg, Catal. Indo-

Loc.: Song Bang-Giang, à Cao-Bang (M. Mansuy).

\section{Unio Frühstorferi Dautzenberg}

1900. Unio Frïhstorferi

1901
Dautzenberg, Diagn. nouv. esp. Unio Indo-Chine, in Journ. de Conch., XLVIII, p. 429.

Dautzenberg, Deser. 2 Unio, in Journ de Conch., XLIX, p. כ̈, pl. I, tig. 1, 2. 
1903

Dautzenberg, Liste Moll. rec. pár Blalse, in Journ. de Conch., LIII, p. 204 (obs.).

Loc.: Song Bang-Giang, à Cao-Bang (M. Mansuy).

\section{Unio (Quadrula) Leai Gray}

1905. Unio (Quadrula) Leai Gray, Dautzenberg et H. Fischer, Liste Moll. rec. par Blaise, in Journ. de Conch., LIII, p. 206.

Loc.: Song Bang-Giang, à Cao-Bang (M. Mansuy).

Unio Messageri Bavay et Daulzenberg

1901. Unio Messageri Bayay el Dautzenbeng, Descr. 2 Unio, in Journ. de Conch., XLIX, p. 7, pl. I, fig. 3,4 .

1904. - - B. ei D., H. Fischer et Dautzenberg, Catal. IndoChine, in Mission Pavie, p. 439.

Loc.: Song Bang Giang, à Cao-Bang (M. Mansuy).

\section{Unio (Nodularia) Micheloti L. Morlet}

1886. Unio Micheloti

1886

1900. Nodularia - L. M., 1904. Unio
L. Morlet, Diagn. Moll. nov. Tonk., in Journ. de Conch., XXXiv, p. 77.

L. Morlet, Liste coq. rec. par Jourdy, in Journ. de Conch., Xxxiv, p. 267.

Simpson, Synopsis of the Naiades, p. 814. H. Fischeli el Dautzenberg, Catal. Indo. Chine, in Mission Pavle, p. 439.

Loc.: Song Bang-Giang, à Cao-Bang (M. Mansuy).

\section{Unio (Quadrula) nodulosus Wood}

181อั. Mya nodulosa

1817. - - IVood,

1825. - -

183. Margarila

1838. - 2 - $\quad$ - $\quad$ - LeA, Synopsis, p. 15.

1842. Unio nodulosus IV. Hanley, Test. Moll., p. 180.

1843. - - - Hanley, Recent Biv. Sh., p. 180. 
1845. - nodulosa - Catlow and Rever, Conch. Nom., p. 61. 1852. Margaron (Prisodon) nodulosus W. LEA, Synopsis, p. 22.

185̋6. Mya nodulosa Woov, Index testac. Rev., p. 16, pI. II, fig. 29.

1857. Unio nodulosus Wood H. et A. Adsus, Genera of rec. MolI., II, p. 496.

1862. - grandidens Lfa, Proc. Acad. N. Sc. Philad., p. IG8.

1862. - - LFA, Journ dead. N. Sc. Philad., V,

p. $20 \%$, pl. XXX, fig. 274.

1863. - - Lea, Obs. G. Unio, IX, p. 27, pl. 30, fig. 274.

1868. - nodulosus Wood Sowerby, in Reeve, Conch. Icon., p]. LXXXIII, fig. 439.

1870. - (Prisodon) - - Lea, Synopsis, p. 27.

1870. Margaron (Unio) grandideus LEA, Synopsis, p. 34.

1890. Unio nodulosus Wood PaEtel, Catal. Conch.Samml., III, p. 161. 1900. Quadrula nodulosi - Simpson, Synops. of the Naiades, p. 798.

Loc.: Song Bang-Giang, à Cao-Bang (M. Mausuy).

\section{Unio (Quadrula) Mansuri nov. sp.}

(Pl. vIlI, fig. ว.)

Testa crassissima 't ponderosa, oblongo-trapezoidalis, an. tice rotundatu, postice declivis et angulata, striis incrementi irregularibus plicisque aliquot ab umbonibus radiantibus, in medio subrectis postice vero multo validioribus ac dicaricantibus ornata. Umbones contigui, valde erosi. Pagina interna margaritacea, parum nitida, sub lente minutissime granulosa. Cardo perincrassatus, in valva dextra dentes cardinales duo laciniatos, antiro quam postico multo validiore, dentemque lateralem posticum unicum crassumque; in valva sinistra dentes cardinales duo laciniatos, postico quam antico validiore, dentesque laterales duo a sulco profundo sejunctos praebet. In ntraque valva dentes laterales a margine recedunt. Impressiones musculares conspicuae, rotundatae; impressio pallii integra, antrorsum ascendit et a margine recedit. Hargo integer et acutus.

Color, sub epidermide /usco, albus; margarita alba, vix iridescens. 
Diam. umbono-ventralis 61, antico-posterior 100. crassit. $37 \mathrm{~mm}$.

Coquille trís épaisse et lourde, d'une forme trapezoïde allongée, arrondie en avant, anguleuse en arrière, ornée de stries d'accroissement irrégulières et, sur la région médiane, de quelques plis rayonnants subverticaux plus ou moins confluents. Sur la région postérieure, on observe des plis beaucoup plus gros, un pen nodulenx et divergents. La région antérieure est dépourvue de plis rayonnants. Sommets contigus, érodés, situés un peu en avant du tiers du diamètre antéro-posterieur de la coquille.

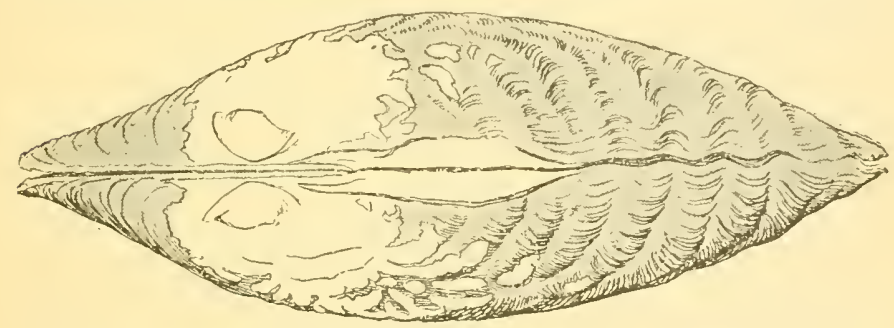

Unio (Quadrula) Mansuyi Dautzenberg et H. Fischer.

Intérieur des valves nacré, peu luisant, couverı de granulations microscopiques. Charnière très forte, présentaut, dans ia valve droite, deux dents cardinales laciniées, dont l'antérieure est la plus forte et la plus saillante et une dent latérale unique, forte, saillante, anguleuse au sommet et séparée du bord dorsal de la coquille par un espace assez large, profondément creusé; dans la valve gauche, deux dents cardinales laciniées dont la postérieure est la plus forte et deux dents latérales lamelleuses séparées l'une de l'autre par un sillon profond et, du bord de la coquille, par un espace assez large, profoudément creusé. Impressions des muscles adducteurs médiocres, arrondies, bien marquées. Impression paléale entière, ascendante du 
côté anlérieur où elle s'écarte graduellement du bord de la coquille. Bords des valves simples, tranchants.

Coloration blanche sous un épiderme d'un brun fauve foncé, légèrement fibreux vers les bords. Nacre blanche peu brillante et à peine irisée.

Loc. : Song Bang-Giang à Cao-Bang (M. Mansuy).

Celte espèce diffère de l'U. Leai par sa région postérieure tronquée obliquement et acuminée à l'extrémité inférieure; son ornementation est aussi différente ; elle consiste en plis subverticaux sur la région médiane et nettement divariqués sur la région postérieure; on n'observe pas de nodosités isolées comme celles qui ornent l'U. Leai.

\section{Unio (Nodularia) sagitTarius Lea}

1856. Inio sagillarius

1857. - - -

1857. - -

1870. Margaron (Unio)

1892. Unio Dugasti

1893. - -
Lea, Proc. Acad. N. Sc. Philad., VIII, p. 93.

LeA, Journ. Acad. N. Sc. Philad., III, p. 298, pl. 26, fig. 12.

LEA, Obs. G. Unio, VI, p. 18, pl. 26, fig. 12. LEA, Synopsis, p. 38.

L. Moklet, Diagn. Moll. nov. in IndoChina collect., in Journ. de Conch., XL, p. 86.

L. Morlet, Descr. esp. nouv. Indo.Chine in Journ. de Conch., XLI, p. 15\%6, pl. VI, fig 4 .

1900. Nodularia sagiltaria Lea, Simpson, Synopsis of the Naiades, p. 811 .

1904. Unio Dugasti L. M., H. Fischer et Dautzenserg, Catal. IndoChine, in Mission Pavie, p. 438.

Loc. : Royaume de Luang-Prabang (M. Monod).

\section{Unio (Nodularia) scobinatus Lea}

1906. Unio (Nodularia) scobinalus Lea, Dautzenbera et H. Fischer, Liste Moll. rec. par Mansuy, in Journ. de Conch., LIII, p. 454.

Loc. : Royaume de Luang-Prabang (M. Monod). 


\section{Corbicula Lamarchiana T. Prime}

1864. Corbicula Lamarckiana T. Phime, Ann. Lyc. Nat. Hist. of N. York, VIII, p. 69, fig. 16.

1869. - - T. Prime, Catal. Corbic., n ${ }^{\circ} 50$.

1879. - - T. P., Clessin, Die fam. der Cycladeen, in Syst. Conch. Cab., 2' édit., p. 170, pl. 30 , fig. 9 .

1891. - $\quad$ - L. Morcet, Contrib. Faune Malac. IndoChine, in Journ. de Conch., XXXIX, p. 239.

1904. - - - - H. Fischer et Dautzengerg, Catal. IndoChine, in Mission P'avie, p. 441.

Loc. : Ha-Lang (M. Mansuy).

Ph. D. et H. F. 



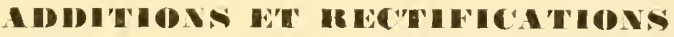 à la liste des Mollusques régoltés par M. Mansus ent Indo-chine
}

\author{
Par Ph. Dautzenbebg et H. Fischer
}

Ennea (Microstrophia) plagiostoma v. Möllendorff., Journal de Conchyliologie, vol. LVI, p. 170. Le type figuré a été recueilli à Bah-Mun (Tonkin), par M. Frühstorfer.

Camaena Gabriellae Dautz. et d'Hamonville, var'. platytaenia v. Möllendorff, ibid, p. 172. C'est par erreur que nous avons dit que l'exemplaire figuré provenait d'un envoi de M. Frühstorfer : cet exemplaire a élé récolté à Quang-Huyen par M. Mansuy; il est d'ailleurs tout à fait conforme à ceux de M. Frühstorfer.

Neocepolis Cherrieri Bavay, var. edentula, scrobiculata, lepressa, nov. var., ibid., p 174. Ces trois variétés proviennent de Quang-Huyen (M. Mansuy); la var. depressoscrobiculata nov. var. provient du Haut-Tonkin (C' Messager).

Paludina polyzonata Frauenfeld subsp. Duchieri H. Fis cher, var. erythrostoma nov. var., ibid., p. 199. Le spécimen figuré provient de Bac-Kan (Cl Messager). 

Journal de Conchyliologge 1908.
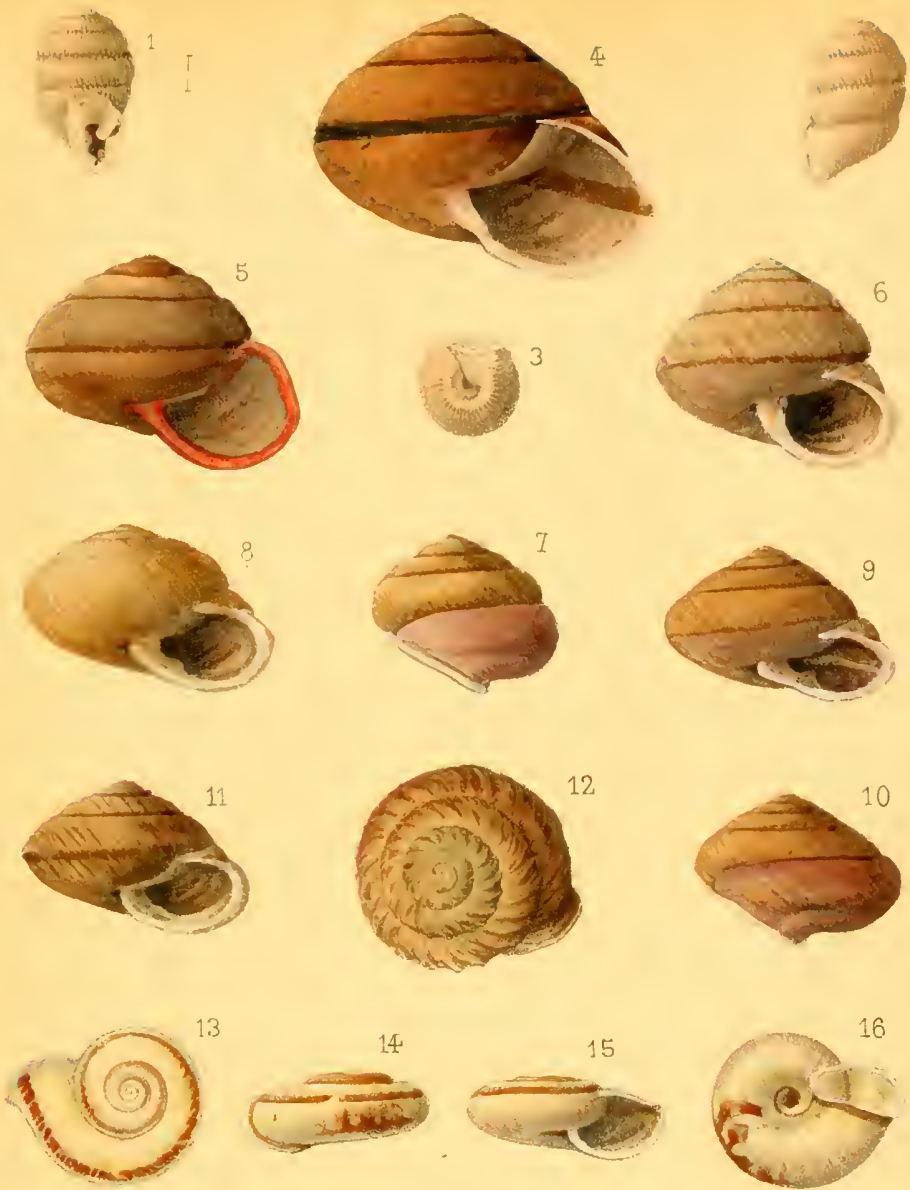

18
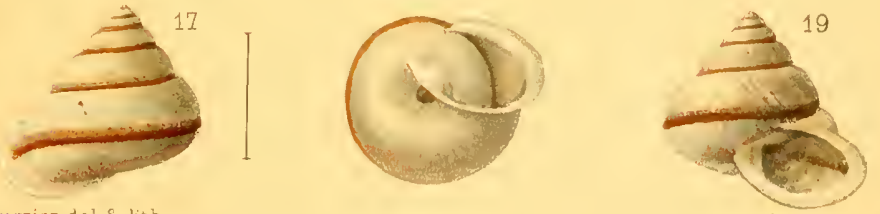

(10) Keignier del. \&: lith

1, 2, 3. Ennea plagiostoma von Mïllendorff

4. Camaera Gabriellae Dautz et d'Hanı. var platytaema von Möllendorff.

5. .... BilletiH. Fischer.

6. Neocepolis Cherrier Bavay var. edentula Dautz.et H. Fischer

7. ... " " var. scrobiculata Dautz et H. Flscher

9,10 ......... var. depressa Dautza et H. Fischer:

11,12 "... " var. depressa scrobiculata. Dautz. et, H. Fischer $13,14,15,16 . \quad$ " var. carinata Dautz el H. Fischer.

17, 18,19. Satsuma leptopomopsis Dautz.et H. Hischer. 

Journal de Conchylioloǵic. 1908.

PI.V.
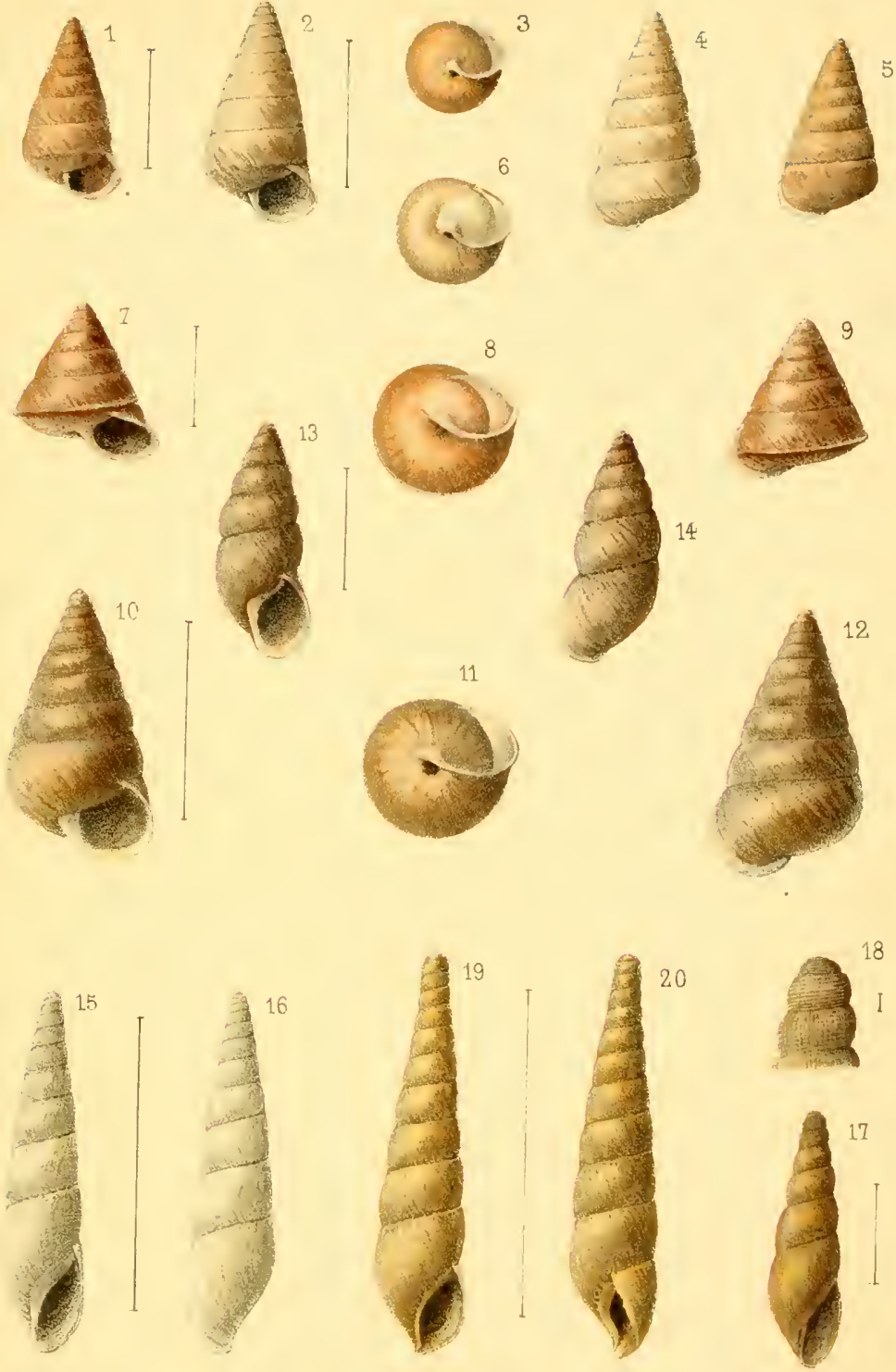

G Reignier del \&. lith.

$1,2,3,4,5,6$. Satsuma producta Dautz. et H Fisch. 7. 8, 9. . fulvescens Dautz. \& H.Fisch $10,11,12$. ..... straminea von Mollendorff 13, 14. Bulminus temustriatus Dautz. \& H. Fisch. 15, 16. Prosopeas Lavillei Dautz \& H. Fisch

17. Pseudopeas Douvillei Dautz. \& H. Fisch. 18. .... "... sommet grossi 6 fois 19, 20. Tortaxis papillosa Dautz. \& H. Fisch

Inp. L Lafontaine, Parts 

Tourtal de Jononyioloǵte 1908
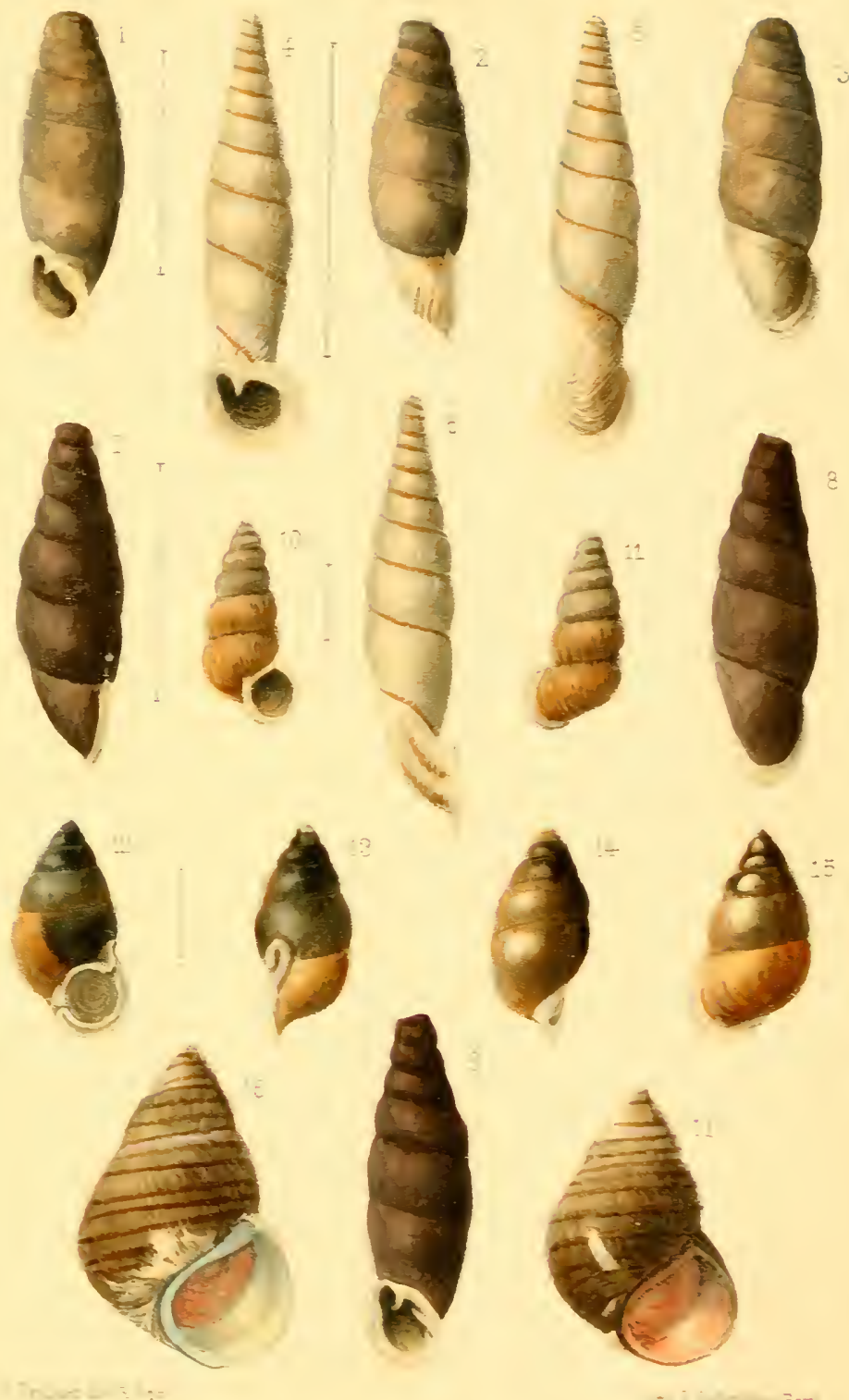

Journal de Conchylioloǵıe 1908.
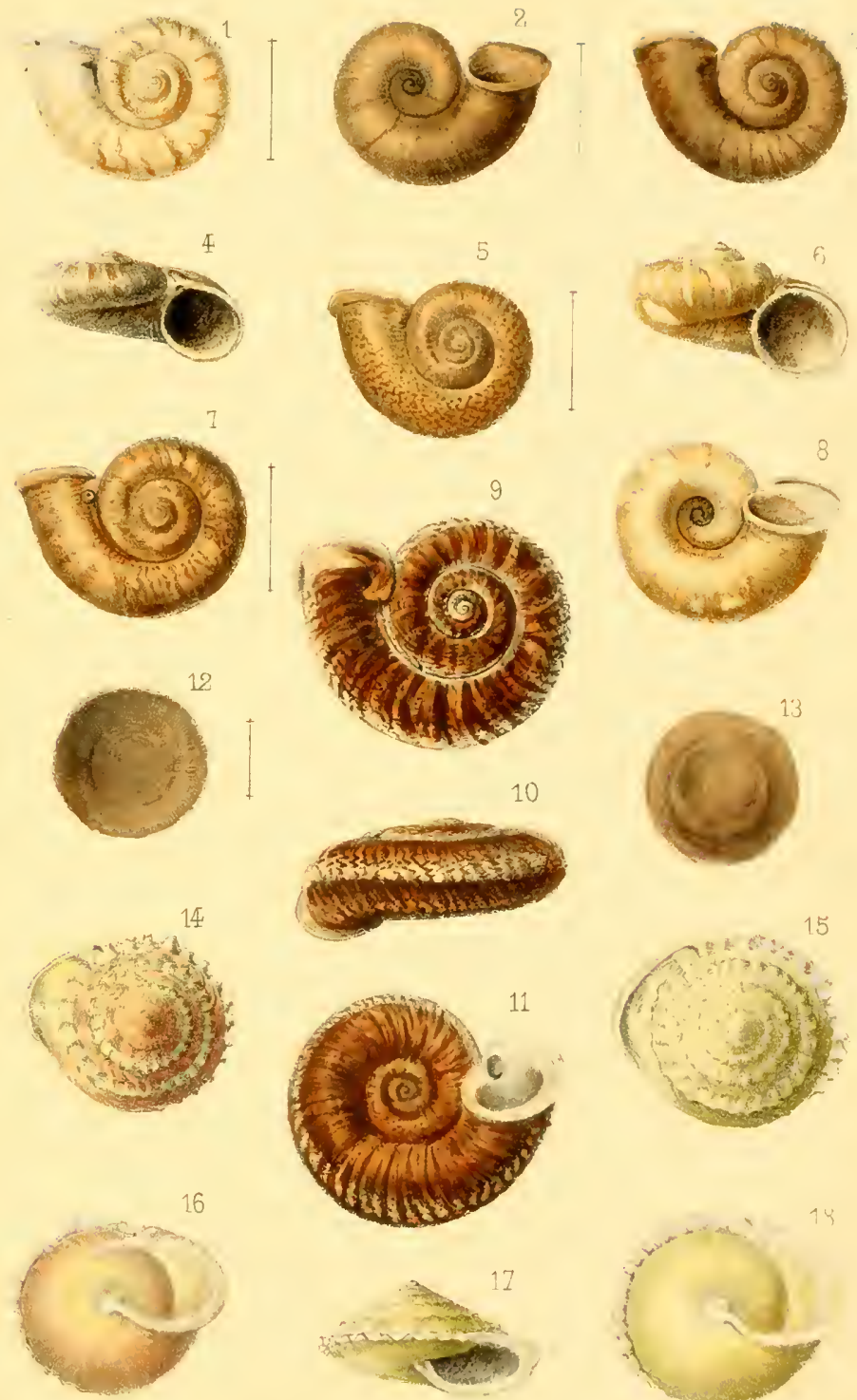

¿ Beigner del. \& lith
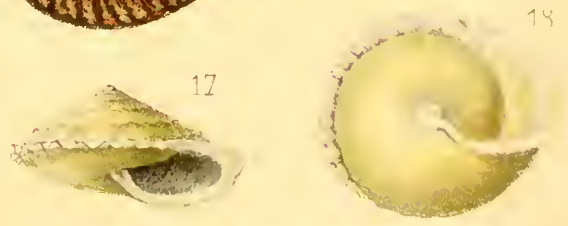

Imp L Lafentame í âr!

1. 2, 3, 4 Opisthoporus Beddomei Dautz. et H. Fisch

$5,6,7,8$ lubricus Dautz et H. Fisch

$9,10,11$. Nyxostoma paradoxa Dautz. et H. Fisch.

12. " " opercule, face externe, grossi 1 tois $1 / 2$

$14,15,16,17,18$. Pseudotrochatella "No óieri Dautz et d'Hamonville. 

Journal de Conchyliologit 1908
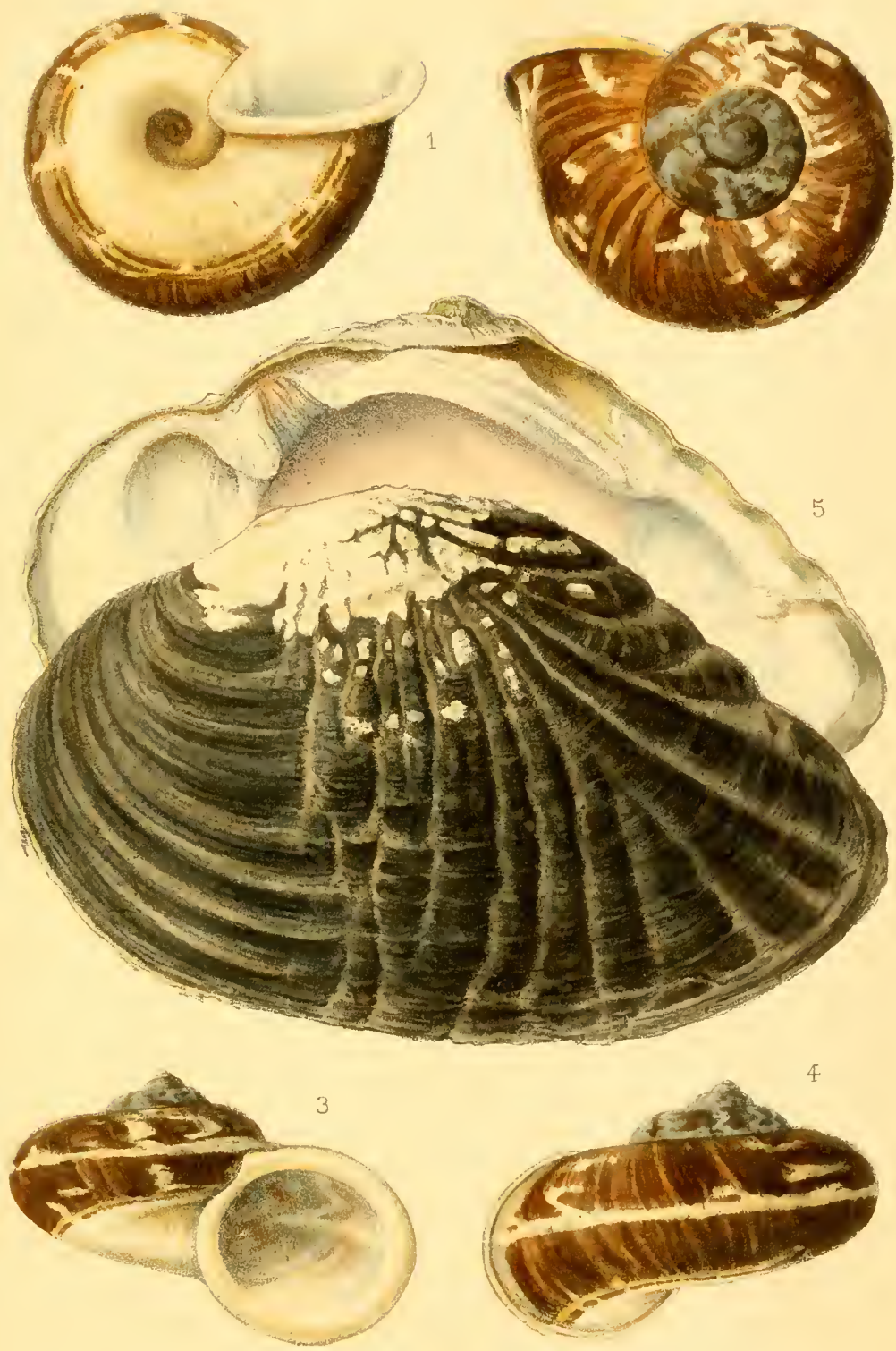

G. Reignier alel. \& itth

$\operatorname{Imp} . \mathcal{L}$ L a fontarne, $\vec{P}$ aris

1,2,3,4. Cyclophorus Mansuyi Dautz. et H. Fisch

5. Unio (Quadrula) Mansuyi Dautz. et H. Fisch. 






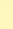

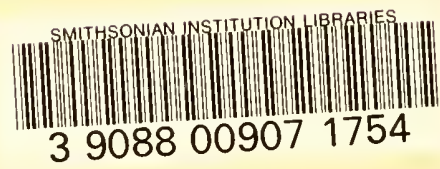





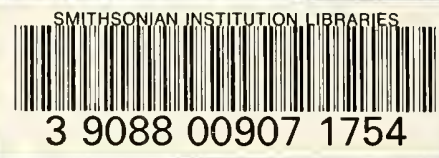

